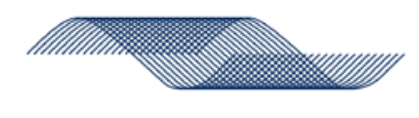

COLUMBIA POWER

\title{
SeaRay Experiment
}

\section{A Scaled Prototype Wave Energy Converter \\ Deployment in the Puget Sound}

January 20, 2013

Rev 2.11 


\section{Table of Contents}

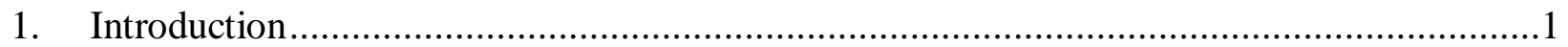

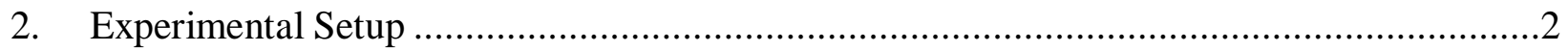

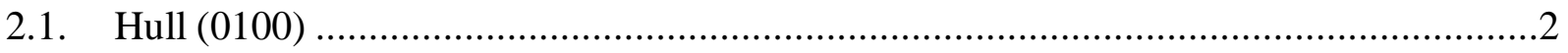

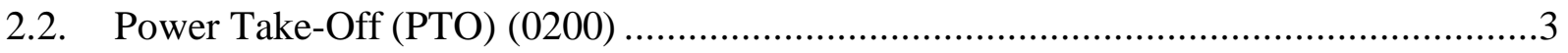

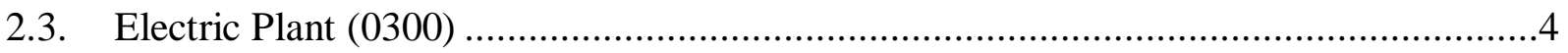

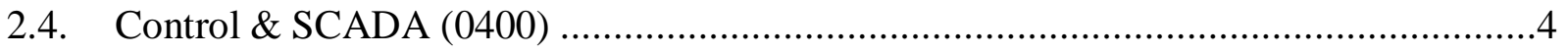

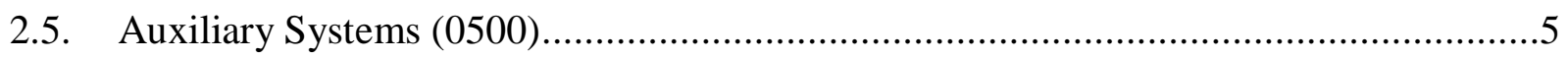

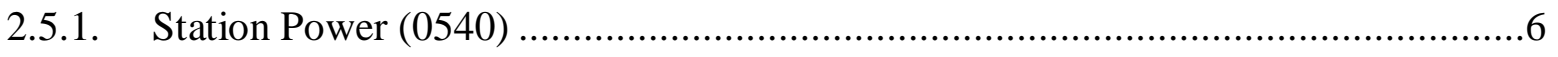

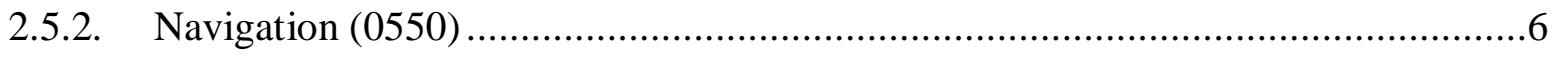

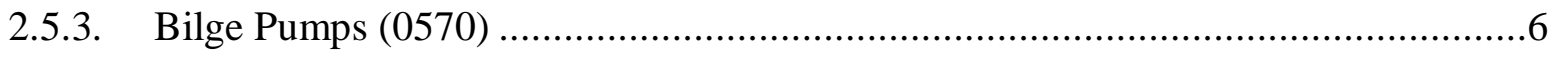

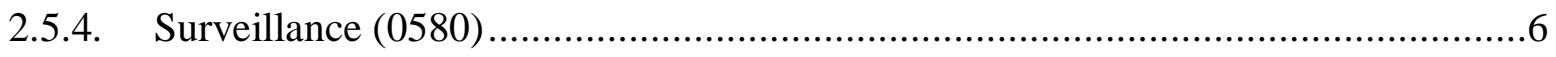

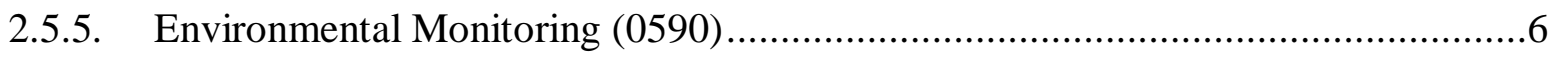

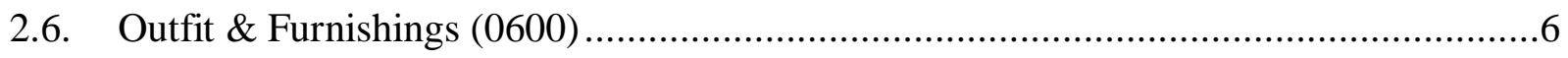

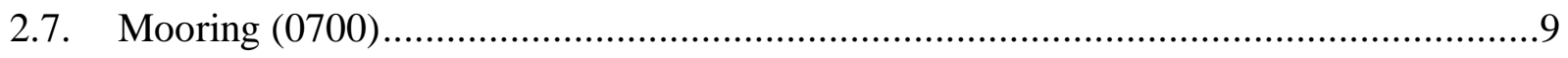

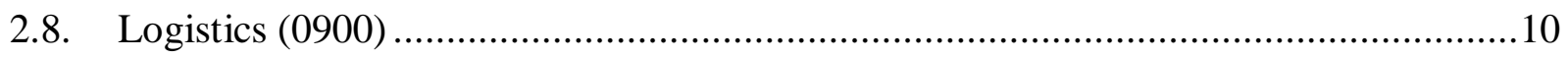

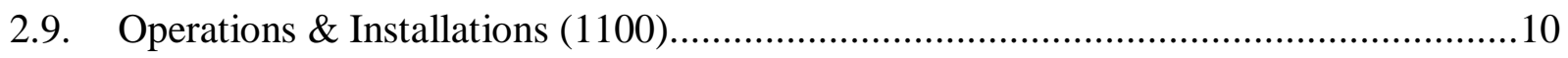

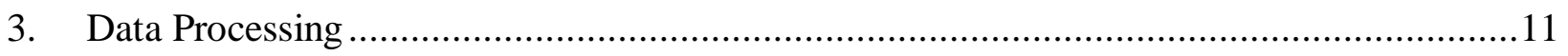

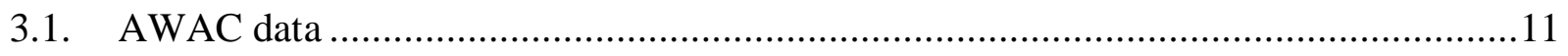

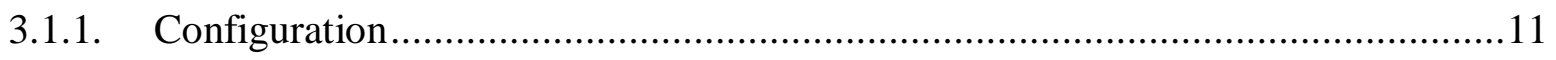

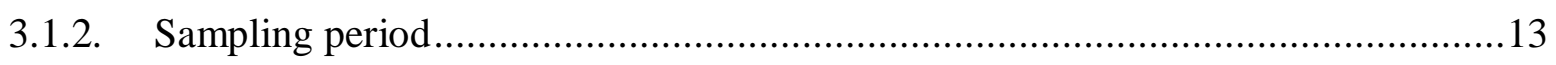

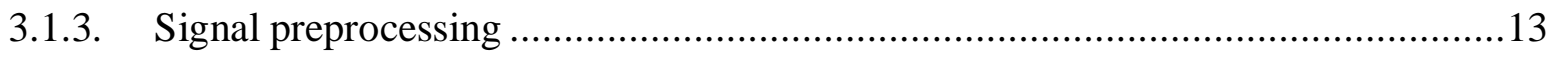

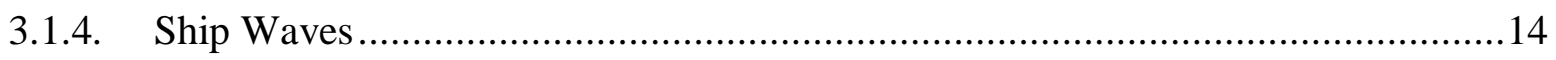

3.1.5. Observed and intrinsic spectra frequency spectra .............................................14

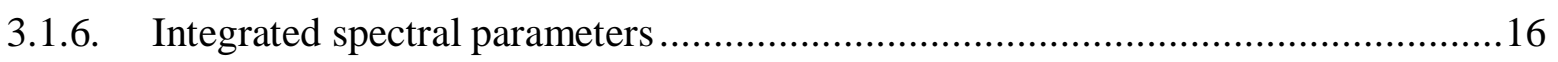

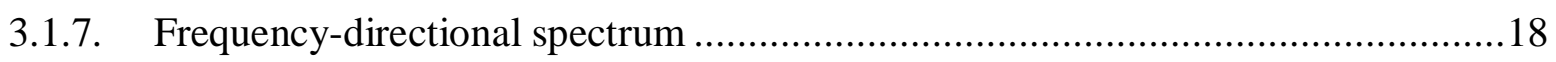

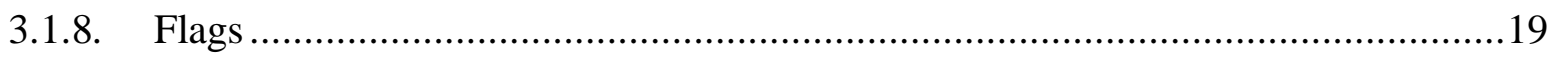

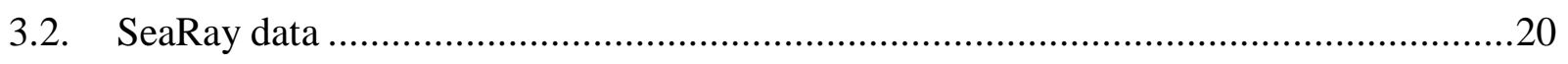

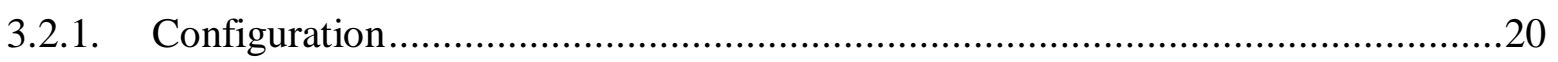

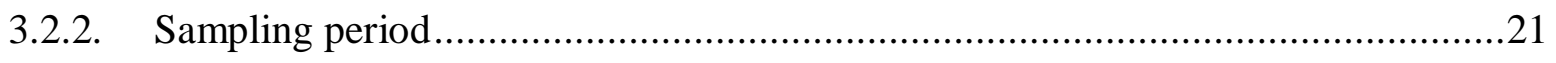

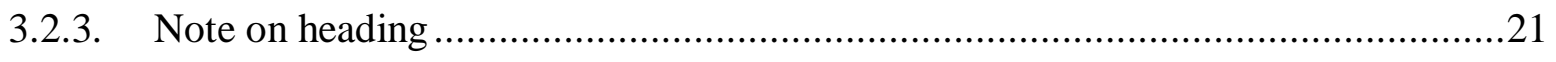

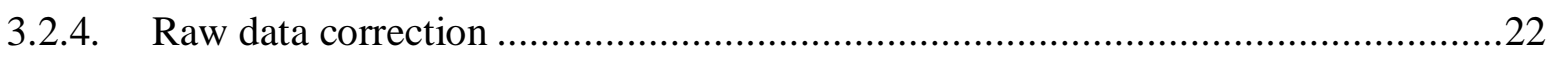

Columbia Power Technologies, LLC $•$ 4920A SW $3^{\text {rd }}$ St, Corvallis, Oregon 97333 Phone: (541) 368-5033 • Fax (541) 230-1498 • www.columbiapowertechnologies.com 


\section{- \\ COLUMBIA POWER}

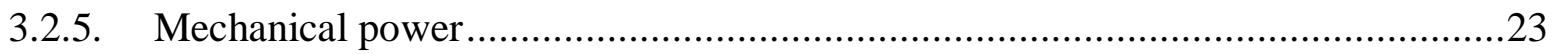

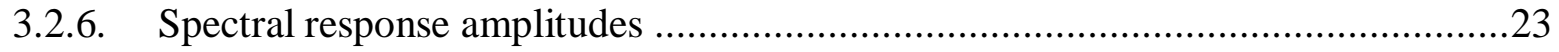

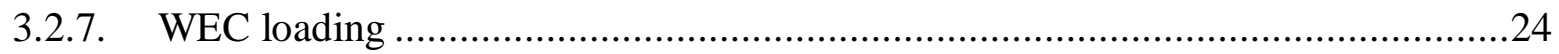

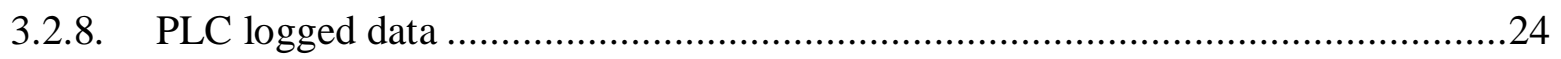

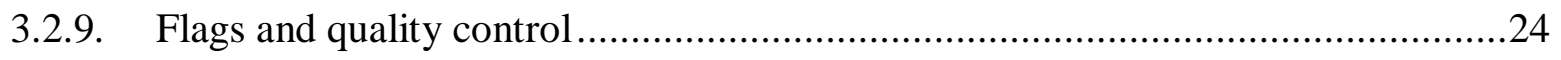

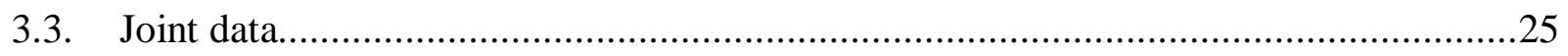

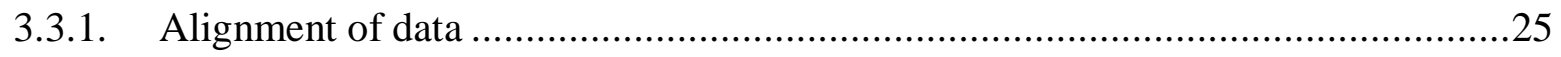

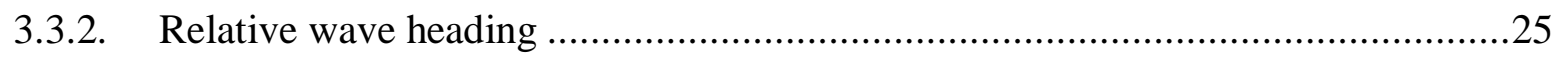

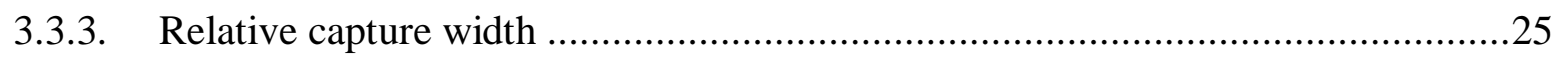

3.3.4. Spectral Response Amplitude Operator (RAO) …………...................................25

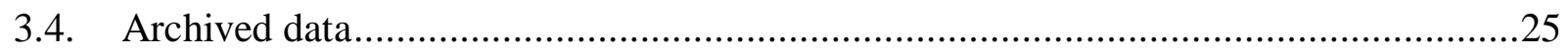

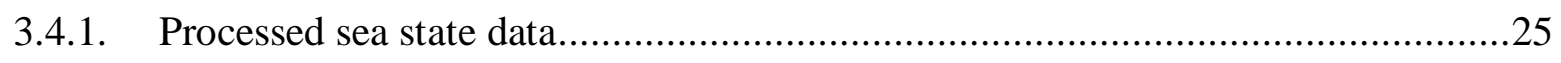

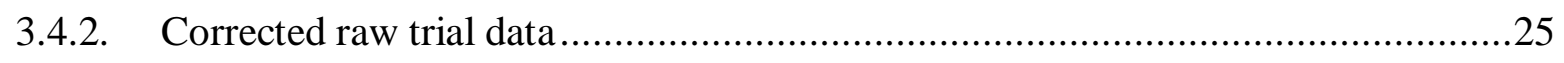

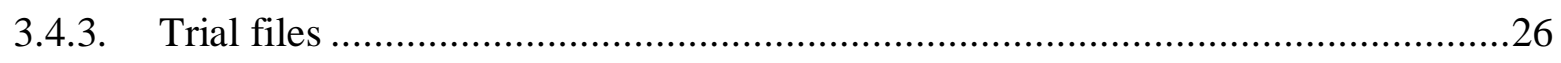

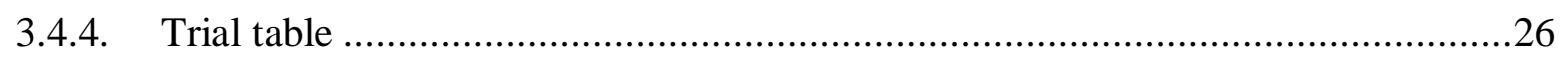

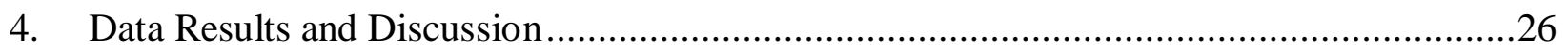

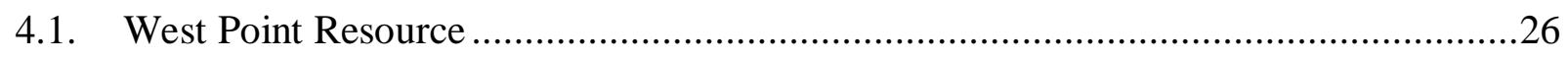

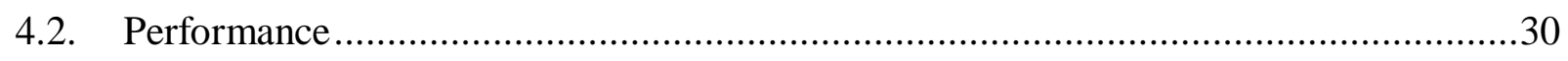

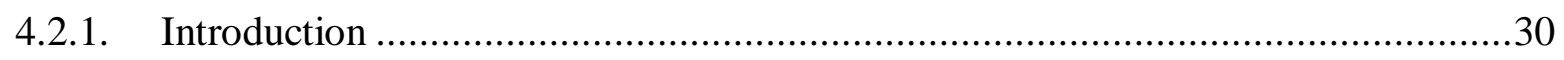

4.2.2. Sensitivity to significant wave height and energy period .....................................31

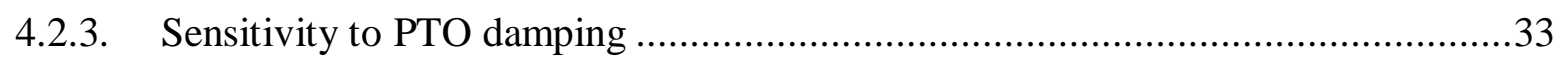

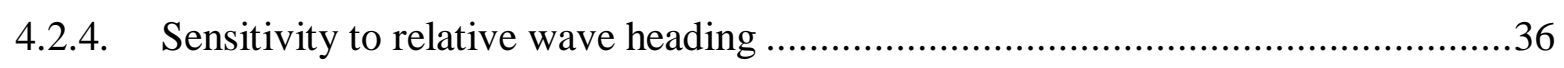

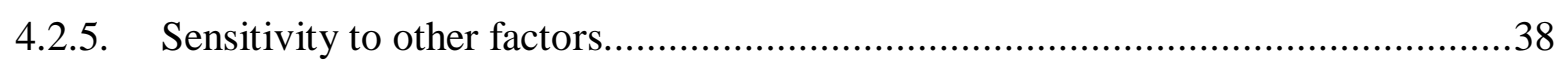

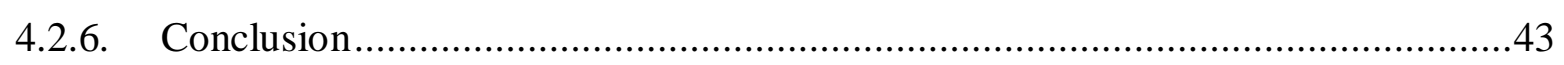

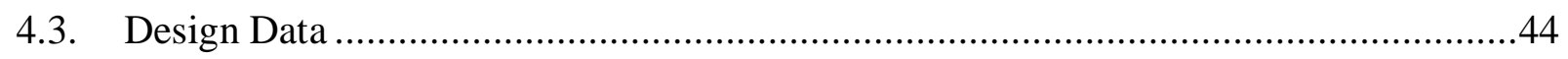

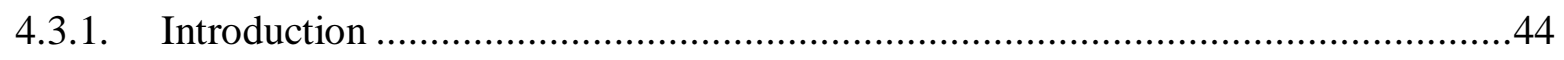

4.3.2. Mooring loads..........................................................................................

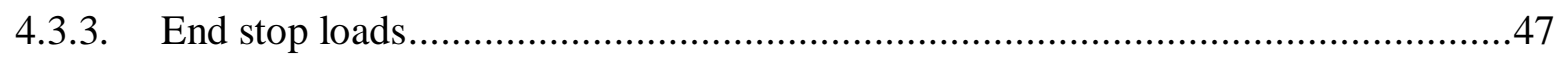

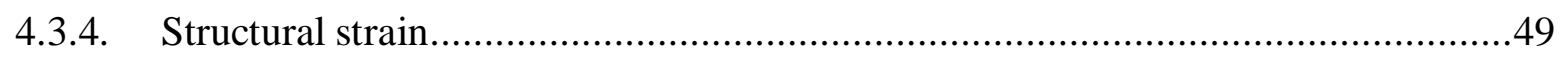

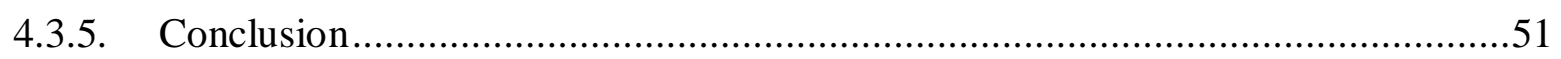

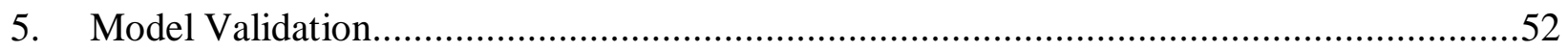

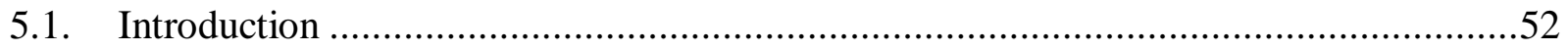

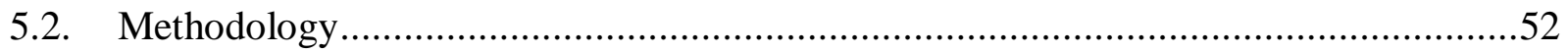




\section{- \\ COLUMBIA POWER}

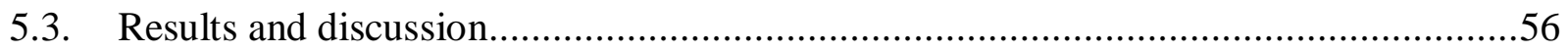

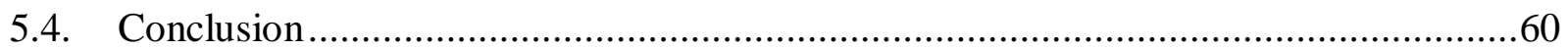

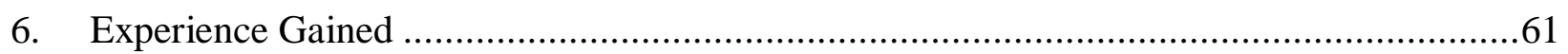

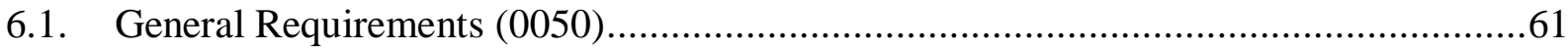

6.1.1. Assembly, Operations, \& Maintenance (0054) .....................................................61

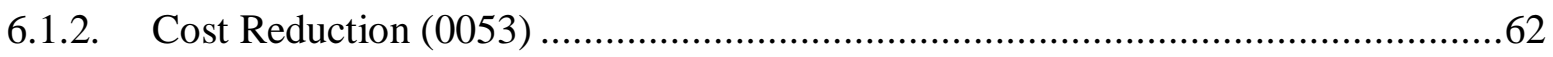

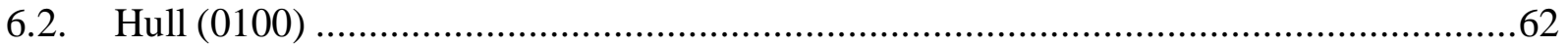

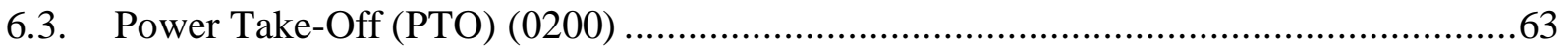

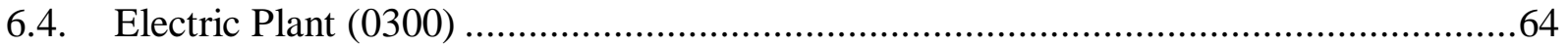

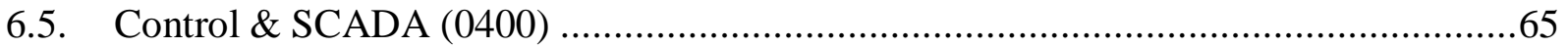

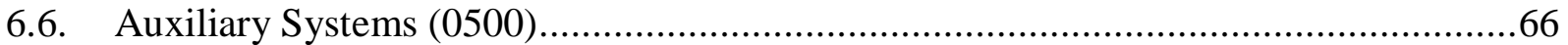

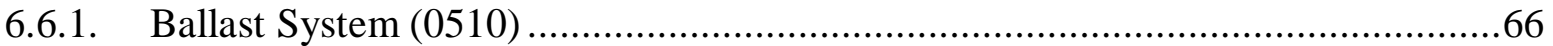

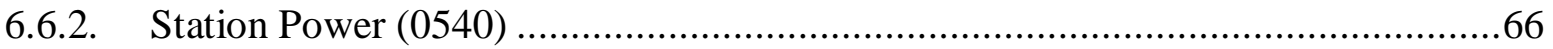

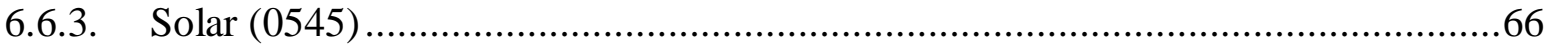

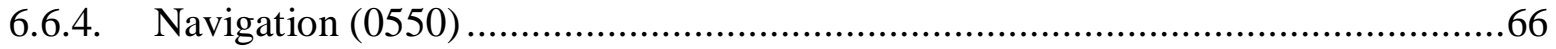

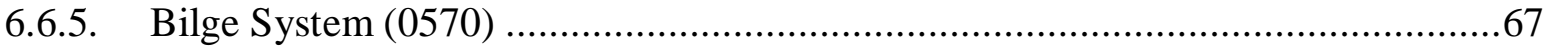

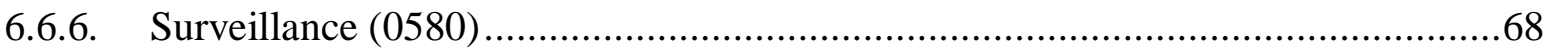

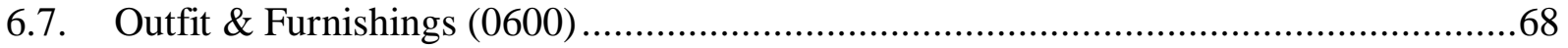

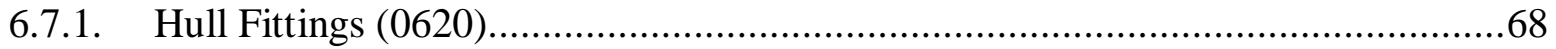

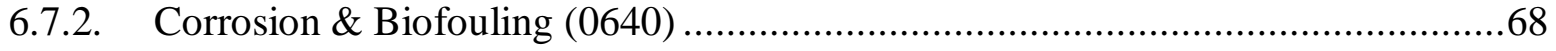

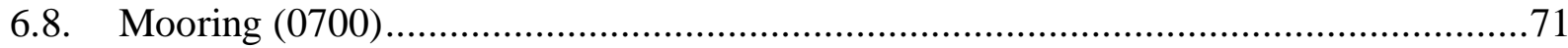

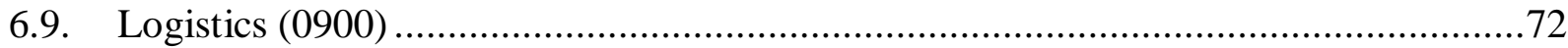

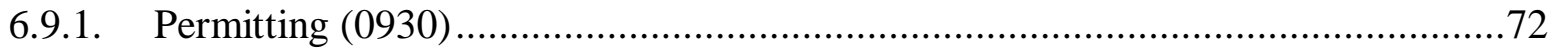

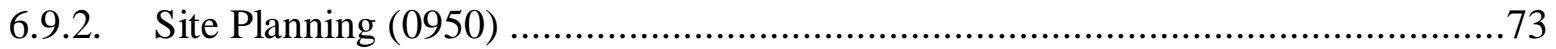

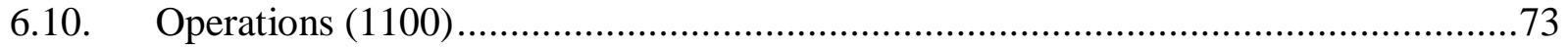

6.10.1. WEC and Mooring Installation (1110) ..........................................................73

6.10.2. Emergency Operations (1130) …………........................................................73

6.10.3. Maintenance, Repair \& Overhaul (MRO) (1140) ..............................................74

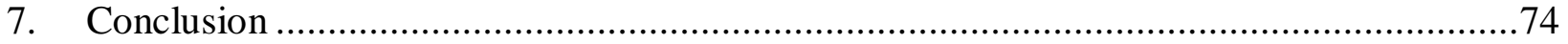

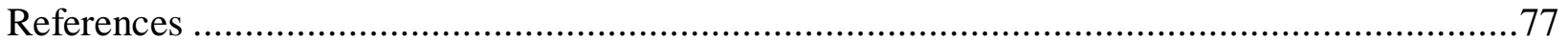

Appendix A. Prototype WEC dimensions .........................................................................

Appendix B. Prototype data collection equipment and sensors ………..................................79

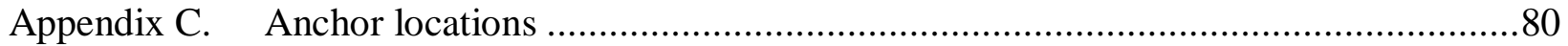




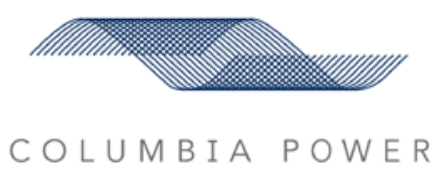

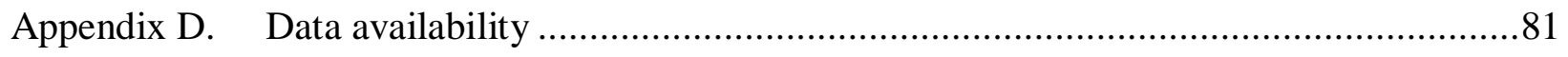

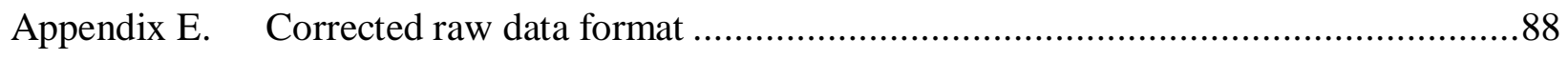

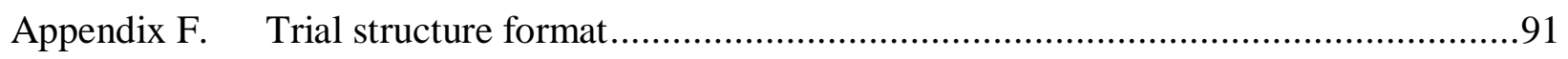

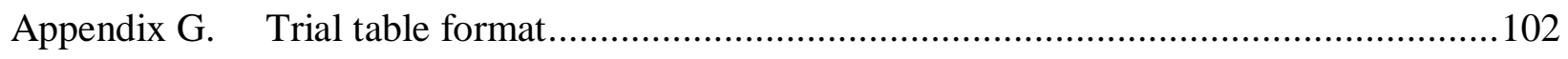

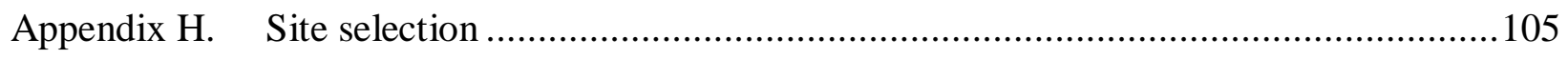

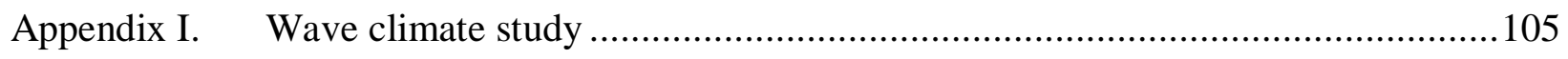

Appendix J. Wave climate study and scale selection .........................................................105

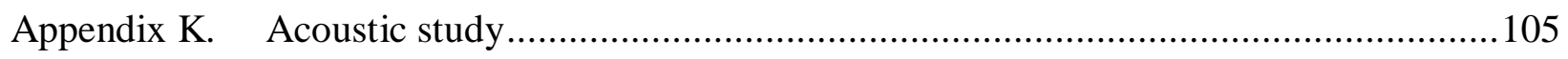

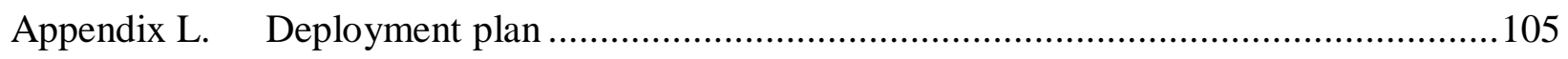

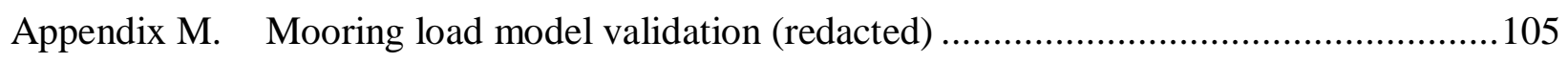

Appendix N. Site bathymetry survey ................................................................................. 105 


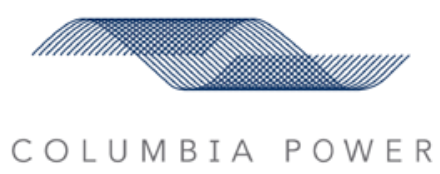

\section{List of Figures}

Figure 1. SeaRay deployed at West Point, Seattle (left) and as assembly drawing (right)............1

Figure 2 - Fully assembled SeaRay WEC on transport cradle at Ershigs................................2

Figure 3 - Dual PTOs installed in the SeaRay beneath the main access hatch...........................3

Figure 4 - SeaRay Electric Plant enclosure showing top layer only.....................................4

Figure 5 - SeaRay's SCADA and control hardware in a watertight enclosure. ..........................5

Figure 6 - SeaRay WEC after deployment in Puget Sound, WA. .......................................

Figure 7 - Main structural frame rail installation into nacelle.........................................8

Figure 8 - Watertight aluminum hatch manufactured by Freeman Marine, Gold Beach, OR. .......8

Figure 9 - Float arms connected to PTO shaft with zinc anodes and depth gauge on nacelle. .......9

Figure 10 - Isometric view of SeaRay mooring system. ...................................................10

Figure 11. AWAC as installed on taught-moored subsurface buoy. .....................................12

Figure 12. West Point wave resource characterization over WEC deployment period (88.9\%

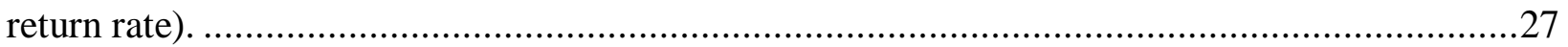

Figure 13. Wave rose for Puget Sound, indicating proportional occurrence for directional bins of

$22.5^{\circ}$ width. Direction is w.r.t. true north....................................................................28

Figure 14. Current rose for Puget Sound, indicating proportional occurrence for directional bins of $22.5^{\circ}$ width. Direction is w.r.t. true north. .....................................................................28

Figure 15. SeaRay test site, note significant north/south fetch. ........................................28

Figure 16. West Point bathymetry (in units of feet), with deployed location of SeaRay indicated.

Figure 17. Histograms for wave and current parameters during West Point trials....................29

Figure 18. Primary performance matrix... Number of trials..............................................32

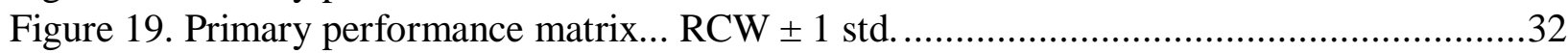

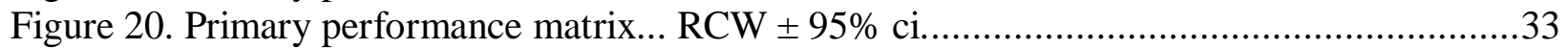

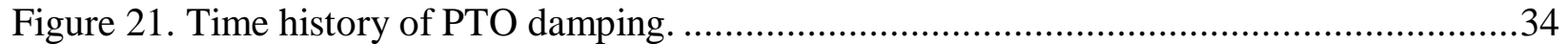

Figure 22. Sensitivity of WEC performance to PTO damping..........................................35

Figure 23. Sensitivity of WEC performance to wave heading. ...........................................37

Figure 24. Sensitivity of RCW to UI, for head waves and operationalLowAft damping.............39

Figure 25. Sensitivity of RCW to fitQ, for head waves and operationalLowAft damping...........40

Figure 26. Performance matrix: head on, low spreading, operationalHighAft damping.............41

Figure 27. Performance matrix: head on, high spreading, operationalHighAft damping............42

Figure 28. Performance matrix: head on, low spreading, operationalLowAft damping..............42

Figure 29. Mean mooring loads versus current speed................................................46

Figure 30. RMS mooring loads versus significant wave height. .......................................47

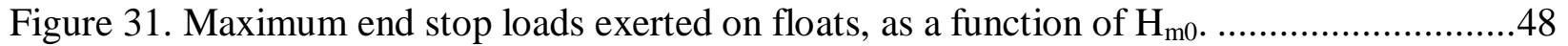

Figure 32. Sealing and waterproofing of structural strain gauges. .........................................49

Figure 33. Drift in mean strain over time, for the fore spar gauge. .......................................50

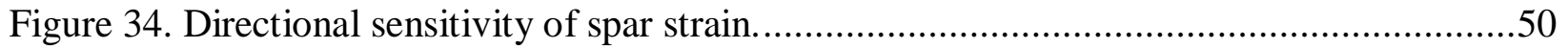

Figure 35. Mean RMS fore spar strain response matrix, head seas.....................................51

Figure 36. Surface mesh for AQWA model, with a total of 10,500 diffracting panels. .............53

Figure 37. Example mean observed /AQWA spectra pair. ...............................................55

Figure 29. Percent error of total RCW, for simulation w.r.t. experimental. ..............................57

Figure 30. WEC power versus wave power, comparison of experiment and simulation. ............58

Columbia Power Technologies, LLC • 4920A SW $3^{\text {rd }}$ St, Corvallis, Oregon 97333

Phone: (541) 368-5033 • Fax (541) 230-1498 • www.columbiapowertechnologies.com 


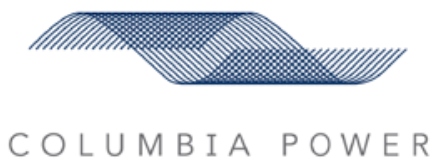

Figure 31. RCW versus Te, comparison of experiment and simulation. .....................................59

Figure 38 - Collision damage to the aft float FRP structure.....................................................63

Figure 39 - Power Electronic components inside the waterproof Fix Box. ................................65

Figure 40 - Upgraded navigational aids to increase visibility against a grey Seattle sky.............67

Figure 41 - Webcam image from lighthouse 500 yards away....................................................68

Figure 42 - Degraded zinc anode after deployment.................................................................69

Figure 43 - Corrosion caused failure of mooring load cell amplifier enclosure............................70

Figure 44 - Typical biofouling on acoustic modem and mooring hardware. …….......................70

Figure 45 - SeaRay during recovery, not a sea monster.....................................................

Figure 46 - SeaRay damper tank showing the yaw control system and yaw drive motor.............72 


\section{Introduction}

Columbia Power Technologies deployed a scaled prototype wave energy converter (WEC) in the Puget Sound in February 2011. Other than a brief period (10 days) in which the WEC was removed for repair, it was in the water from Feb. 15, 2011 until Mar. 21, 2012. The SeaRay, as this WEC is known, consists of three rigid bodies which are constrained to move in a total of eight degrees of freedom (DOF). The freeboard portions of the three bodies are seen in the left hand side of Figure 1, as deployed in the Puget Sound. A detailed assembly drawing is shown in the right hand side of Figure 1. Any position can be described as a surge/sway/heave/pitch/roll/yaw of the central body (consisting of nacelle, spar and damper tank) and a pitching of both the forward and the aft floats with respect to (w.r.t.) the nacelle. Each of these relative pitching motions actuates a permanent magnet generator, converting the mechanical energy of the sea into electrical energy.
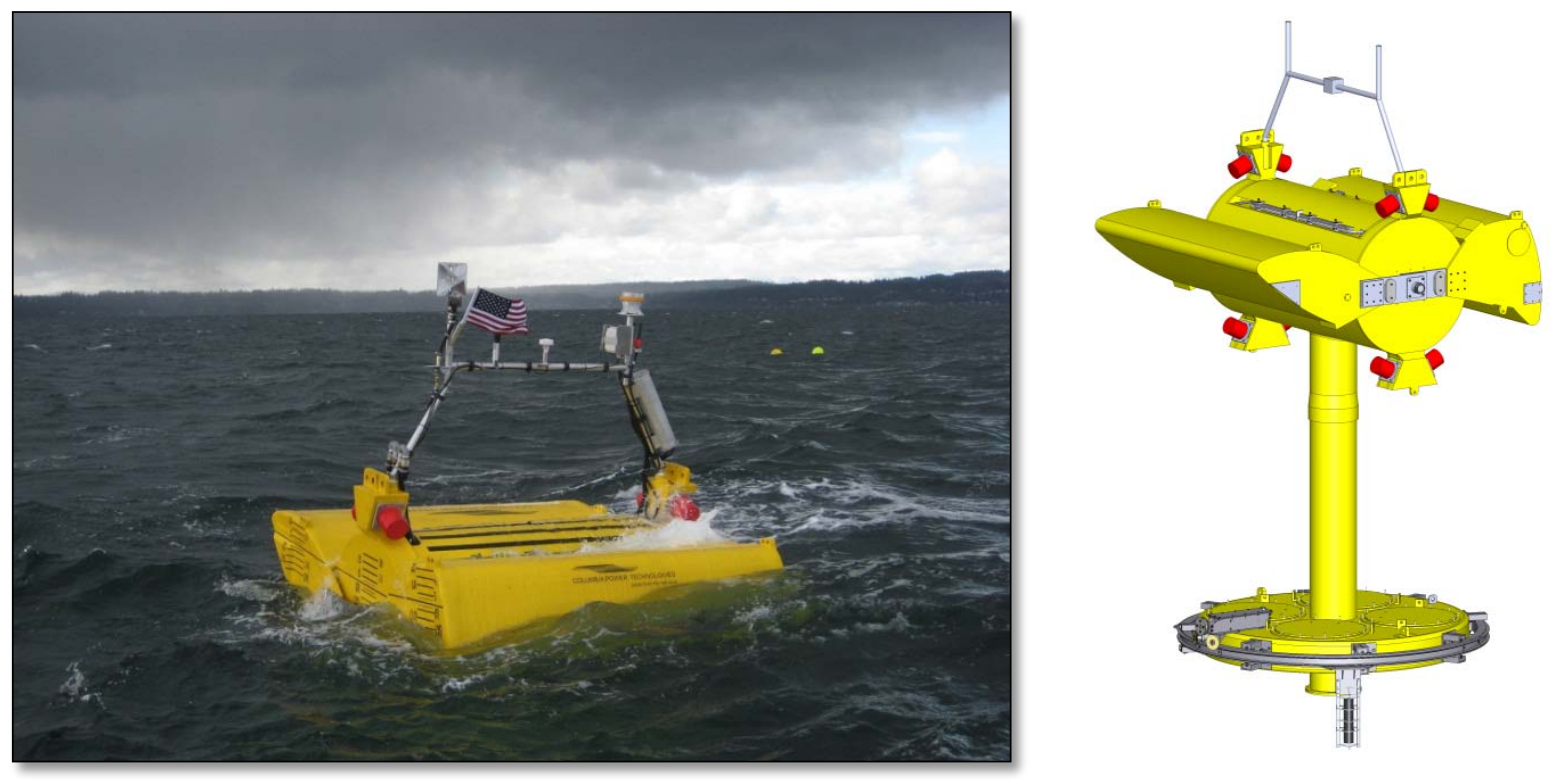

Figure 1. SeaRay deployed at West Point, Seattle (left) and as assembly drawing (right).

The SeaRay is kept on station with a spread, three-point mooring system. This prototype WEC is heavily instrumented, including but not limited to torque transducers and encoders reporting generator torque applied to and relative pitch of the floats, an inertial measurement unit (IMU) reporting translational acceleration and rotational position of the spar/nacelle, a GPS sensor reporting position, load cells reporting mooring loads at the WEC connection points and a number of strain gauges embedded in the fiberglass reinforced plastic (FRP) hull. Additionally, wave and current data are collected using an Acoustic Wave And Current Profiler (AWAC), allowing performance and design data to be correlated to environmental input conditions. This data - quality controlled, processed and analyzed - is used to characterize the metocean conditions (i.e. sea states). The WEC response will be correlated to the metocean conditions. These results will primarily be used to validate numerical models. The validated numerical models will be used optimize the commercial scale WEC and inform the design process. This document details the SeaRay experiment, including the quality control, processing and 
subsequent analysis of the data. Furthermore, the methodology and the results of numerical model validation will be described.

\section{Experimental Setup}

The SeaRay was developed as a 1:7 ${ }^{\text {th }}$ scale prototype of the Generation 3.1 Ray series WEC. The SeaRay design used the same organizational structure for the full-scale process, which proved highly valuable and demonstrated a successful design process. Each subsystem is known by a representative ' 100 s' series identifier (i.e. 0100, 0200, 0300, etc.) and is broken down into finer resolution as necessary.

\subsection{Hull (0100)}

The WEC hull design was a collaboration between Columbia Power and Ershigs. Columbia Power engineers designed the external shape based on optimization work and provided Ershigs with static and dynamic loading data. Ershigs then performed load analysis to design the structural requirements for each of the three bodies. Five main components comprise the entire WEC: Nacelle, spar, damper, forward float, and aft float. The nacelle houses the PTO's and the all of the electronics. The spar connects the nacelle to the damper tank which houses onboard batteries. The damper tank provides the mooring connection points and ballast components. It is filled with seawater, foam, and steel to achieve the desired waterline, center of gravity, and inertia. The forward and aft floats move relative to the nacelle to generate electricity. Each float was ballasted independently of the nacelle with a combination of freshwater and foam.

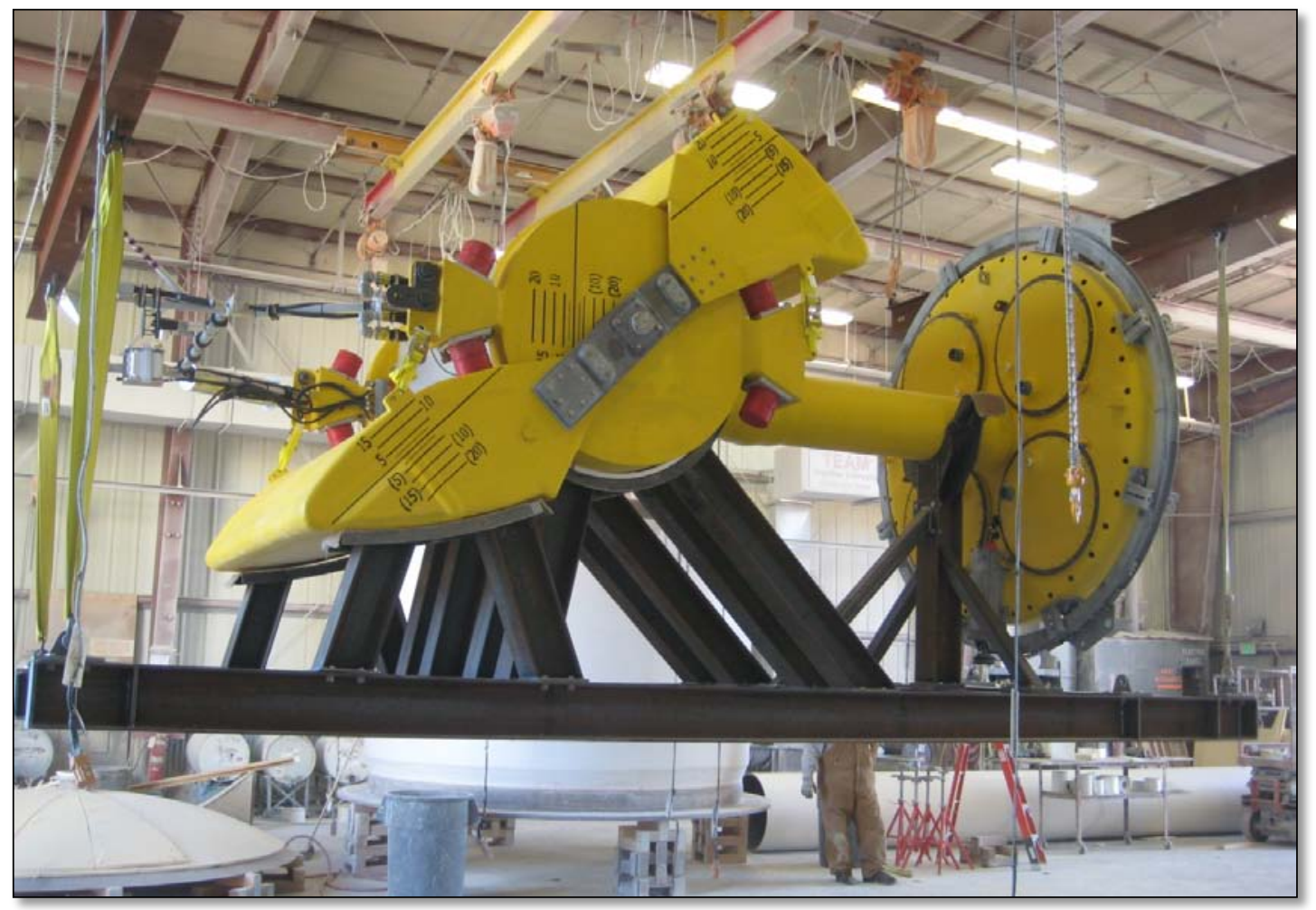

Figure 2 - Fully assembled SeaRay WEC on transport cradle at Ershigs.

Columbia Power Technologies, LLC • 4920A SW $3^{\text {rd }}$ St, Corvallis, Oregon 97333 Phone: (541) 368-5033 • Fax (541) 230-1498 • www.columbiapowertechnologies.com 


\subsection{Power Take-Off (PTO) (0200)}

The Power Take-Off (PTO) systems onboard the SeaRay were designed to provide damping up to the saturation limit of the torque transducer. In the event that the electronic torque control limit did not function properly, a slip clutch was mounted between the primary drive shaft and the torque sensor to protect the system from over torque. The PTO systems where designed as mirrored systems, one starboard driven by the fore float and one port driven by the aft float. The gearbox, which was installed between the torque transducer and the $250 \mathrm{rpm}$ permanent magnet generator (PMG), increased the low relative speed of the floats motion and allowed the PMGs to function more efficiently. The 3-phase PMG electrical outputs were connected to the 0300 Electric Plant. To handle the operational loads and achieve alignment, a back to back bearing housing was designed and mounted to the main shaft flange which mounted to the inside of the nacelle wall. To maintain the watertight integrity of the nacelle, dual shaft seals where installed between the solid stainless steel main shaft and the flange housing. The flange was packed with marine grade silicon and the main shaft protruded outside of the nacelle wall.

Over all the PTO design was a huge success. The durable and reliable design was capable of providing a wide range of damping values with peak torque capabilities beyond that required for the scaled model validation.

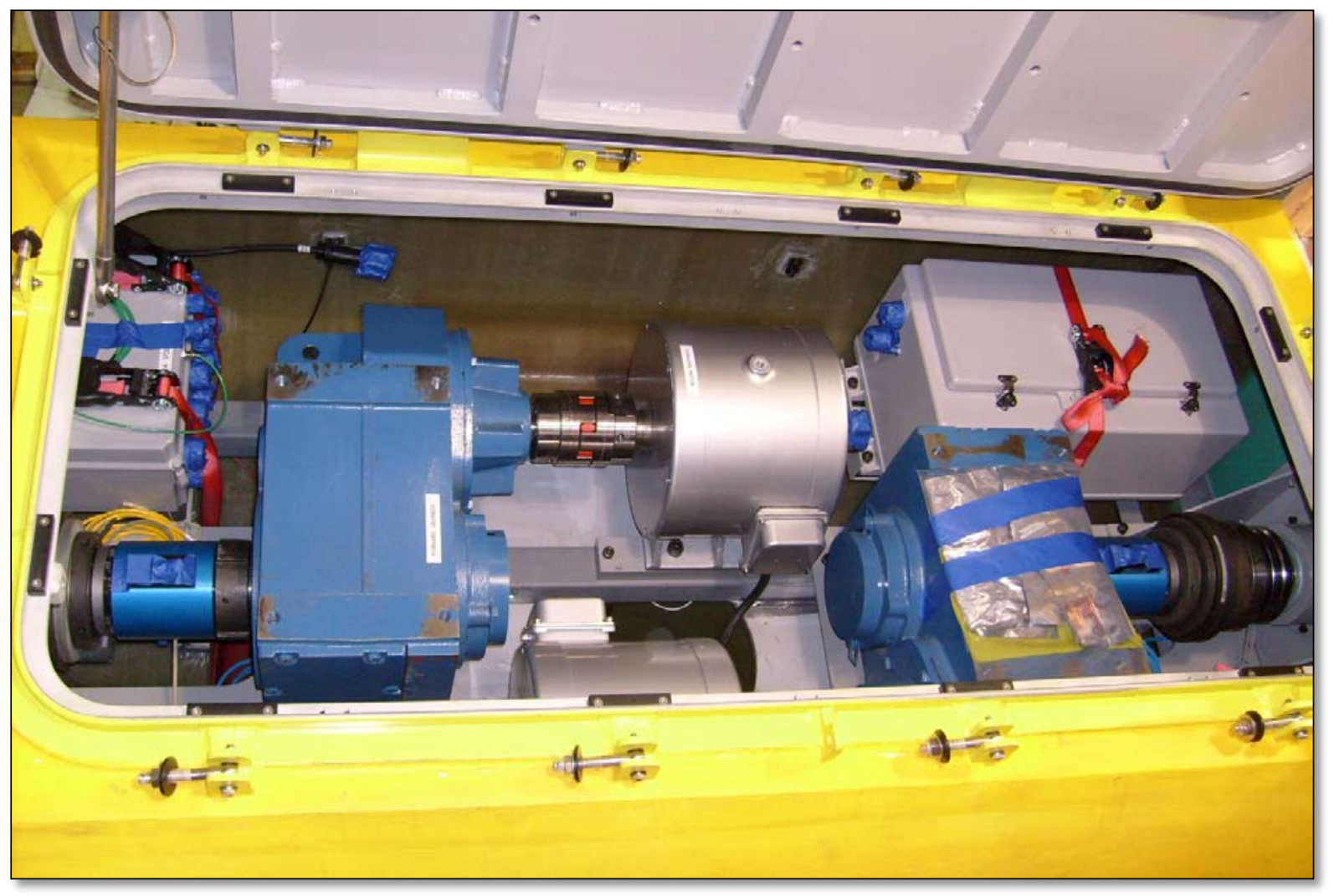

Figure 3 - Dual PTOs installed in the SeaRay beneath the main access hatch. 


\subsection{Electric Plant (0300)}

The Electric Plant serves several critical functions for the WEC systems. Primarily it controls the PTO damping allowing the WEC to optimize energy capture for any given wave condition. It also collects power from the PTOs and smoothes it into usable electrical power which is then delivered to all the WEC systems. In the case of SeaRay the primary load was the onboard battery bank used to power the Station Power system. At times the Electric Plant worked so well that the onboard battery bank was filled and additional power needed to be burned off using the onboard load resistor.

The Electric Plant is composed of numerous power electronic and control components. Initially the variable frequency, variable amplitude (stochastic AC) current from each of the generators is sent to a rectifier transforming it into pulsed DC power. An inline DC to DC converter bucks or boosts the generator voltage to effectively throttle the current and control the load. The DC/DC converter is controlled in real-time by the onboard control system to achieve exactly the specified PTO damping. The power is then merged from the two PTOs and is sent to a single large onboard bank of capacitors which stores and filters the incoming power. The battery charger can then draw continuous power from the relatively stable capacitor bank bus and charge the batteries.

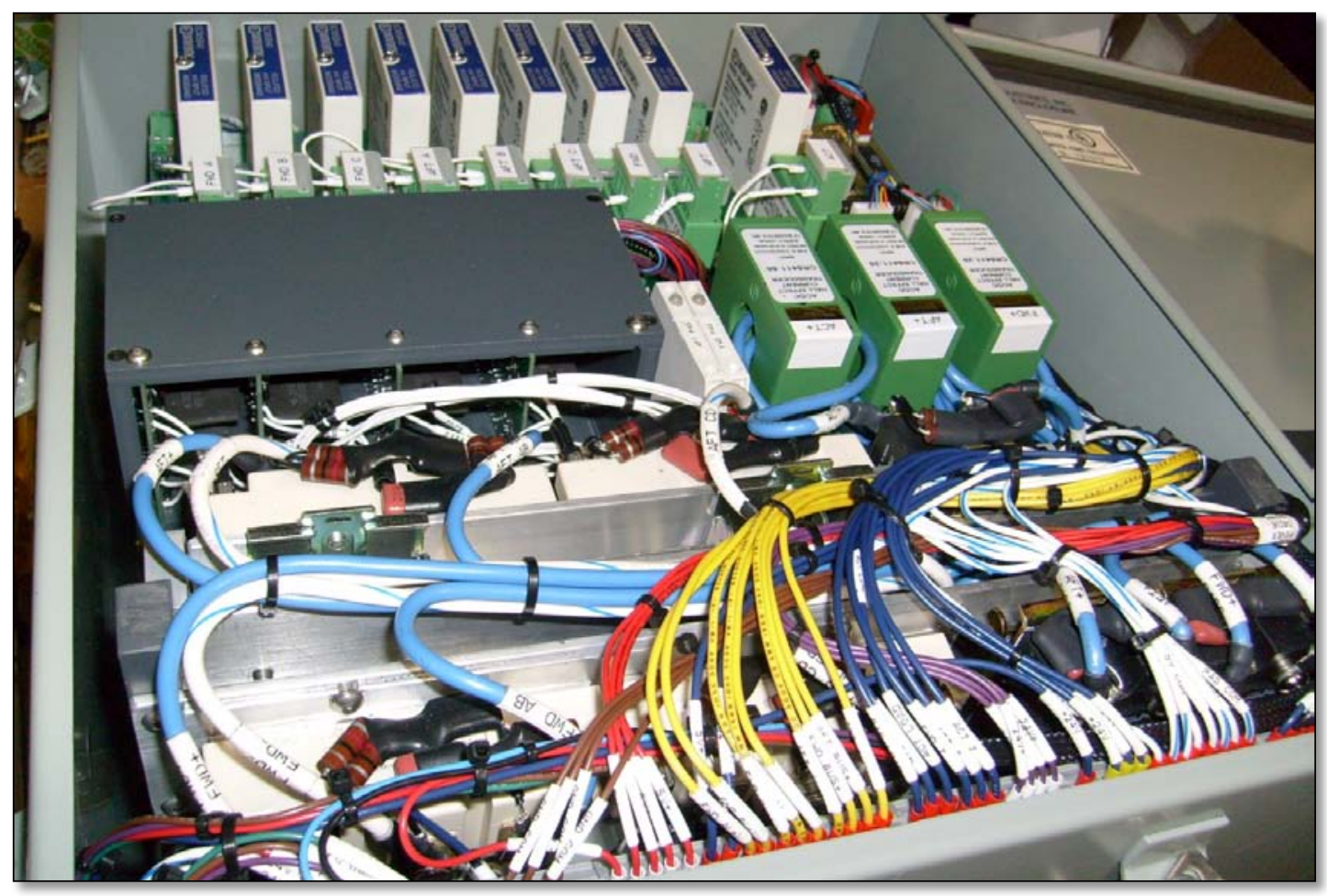

Figure 4 - SeaRay Electric Plant enclosure showing top layer only.

\subsection{Control \& SCADA (0400)}

The SeaRay WEC is a dedicated research platform instrumented with a suite of onboard sensors to collect operational, performance, and load data during the deployment. The highly instrumented nature of the SeaRay made it an extremely valuable asset for understanding WEC

Columbia Power Technologies, LLC • 4920A SW $3^{\text {rd }}$ St, Corvallis, Oregon 97333

Phone: (541) 368-5033 • Fax (541) 230-1498 • www.columbiapowertechnologies.com 
performance and determining relationships between different parametric influences. Data from each sensor is collected by onboard data collection equipment and then duplicated to onshore servers via a wireless data link.

Each sensor was calibrated and then validated through a documented process conducted by Columbia Power to test sensor accuracy over its entire range of operation. The sensor network is organized into the major WEC subsystems. A complete sensor list is shown in the appendix section. In total there are 98 unique data series collected by these sensors and onboard data variables. Each sensor or sensor group is fused independently to prevent a single sensor failure from affecting others.

Data collection is accomplished with dSPACE's compact rapid prototyping hardware known as the MicroAutoBox. The hardware takes inputs from all field sensors, the software makes control decisions, and provides synchronized data collection. The MicroAutoBox is programmed with a special real-time interface through Matlab Simulink to accomplish all of the WEC control operations and generator control algorithms. The combination of dSPACE ControlDesk and Simulink allows control and processing codes to be developed and tested offline and quickly programmed into hardware for use in the SeaRay WEC. An onboard embedded PC is loaded with Matlab, Simulink, and ControlDesk software which gives access, diagnostics, and code development capabilities while deployed. ControlDesk has a convenient HMI to visualize and store data collected by the MicroAutoBox. Together the embedded PC, the MicroAutoBox, and various signal conditioning modules make up the SCADA and Control systems. The system is secured in an IP 67 watertight enclosure to ensure safe operation during the SeaRay deployment.

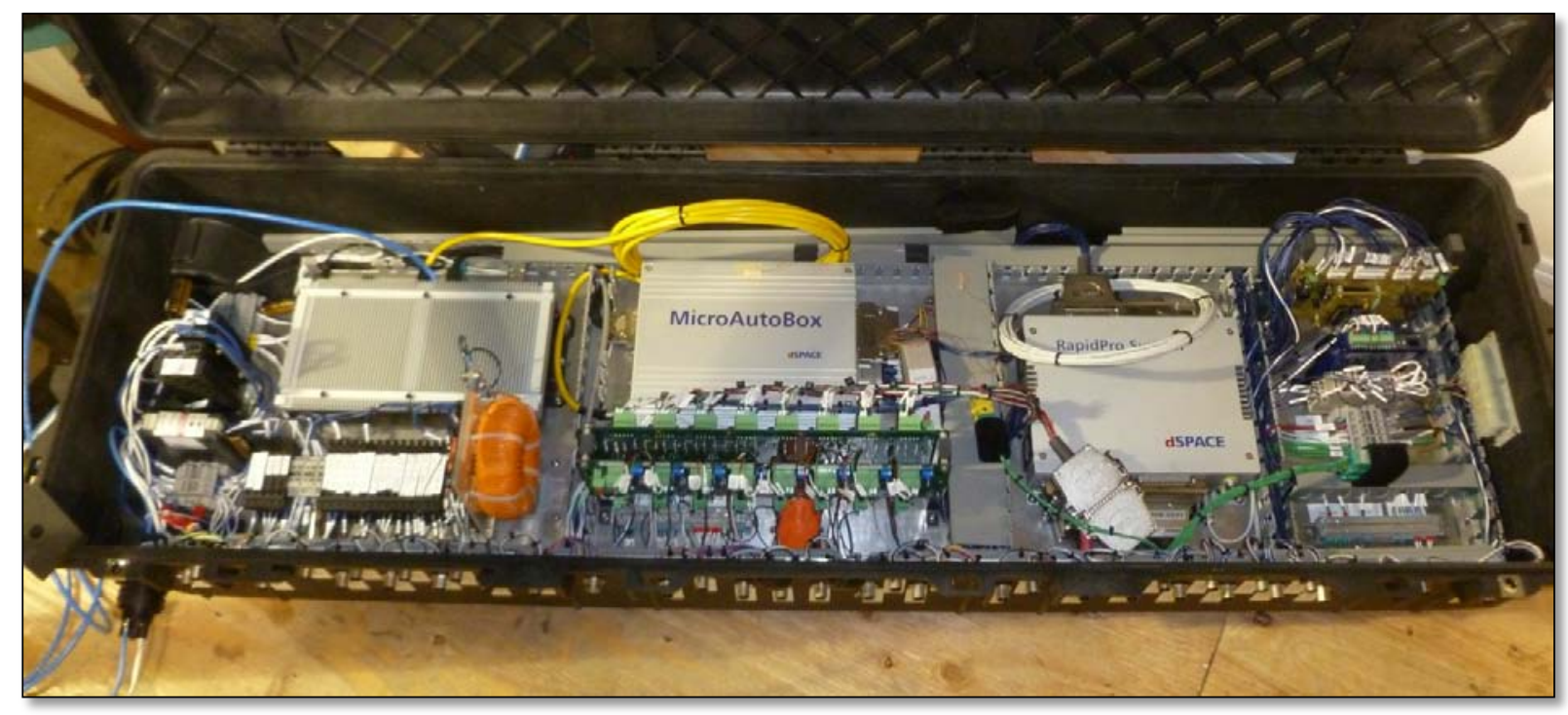

Figure 5 - SeaRay’s SCADA and control hardware in a watertight enclosure.

\subsection{Auxiliary Systems (0500)}

The Auxiliary Systems comprise all of the buoy systems that are required for a successful WEC deployment but are not part of the critical energy harvesting path. This is a diverse set of systems that each played a key role in ensuring proper operation.

Columbia Power Technologies, LLC • 4920A SW $3^{\text {rd }}$ St, Corvallis, Oregon 97333

Phone: (541) 368-5033 • Fax (541) 230-1498 • www.columbiapowertechnologies.com 


\subsubsection{Station Power (0540)}

The Station Power system receives energy from the WEC's main PTO bus and stores it in the onboard battery bank which is used to power all of the WEC systems at required voltage and current levels. The onboard battery bank consisting of 16 marine lead-acid batteries and can store enough energy to power the WEC for a full week from battery operation alone. A battery charger, several power supplies, distribution wiring, and over current fuses compose the rest of the Station Power system.

\subsubsection{Navigation (0550)}

A solar powered amber navigational beacon blinking every 4 seconds and a set of radar reflectors provide navigational aids to mariners. Additional details can be found in the Experience Gained section.

\subsubsection{Bilge Pumps (0570)}

Two 24VDC marine bilge pumps were installed to remove water from the sump of the nacelle in the event of a leak. Each pump had its own power line, float switch, discharge tube, and check valve providing maximum redundancy. The bilge system provided added confidence and security during the entire deployment.

\subsubsection{Surveillance (0580)}

Shortly after deployment, a web based telescopic surveillance camera was installed 500 yards on-shore from the WEC at the West Point Lighthouse. The web-cam allowed Columbia Power personnel to assess the general condition of the WEC at any time from any location worldwide. This remote live monitoring proved to be an essential system and saved expense in costly inspection visits.

\subsubsection{Environmental Monitoring (0590)}

An acoustic wave and current (AWAC) measurement device was deployed concurrently with the WEC. The AWAC provided the primary resource assessment data during the deployment. The AWAC was equipped with an underwater acoustic data link to the SeaRay providing up to date wave condition information to operators which aided in control decisions. The AWAC required periodic battery changes and data downloads to span the 13 month deployment.

\subsection{Outfit \& Furnishings (0600)}

The Outfit \& Furnishings on SeaRay include hull fittings, hull compartmenting, and corrosion \& biofouling prevention. This included external attachment points, a main hatch, waterproof enclosures, internal structural mounts, and zinc anodes. Attachment points on each side of the main bodies were used during deployment and recovery for crane and line tenders to attach. These attachment points were also used to tie off vessels when work was being performed on the deployed WEC. Every system on the SeaRay prototype was designed as a marine application with proper waterproofing and corrosion protection.

Internally a steel frame rail weldment acted as a mounting structure for the PTOs and all of the internal electronic enclosures. The weldment also served as a critical alignment tool between Columbia Power and Ershigs for construction of the FRP nacelle. 
The main nacelle hatch was designed to allow access inside the WEC during maintenance and repair operations. The aluminum hatch was large enough to allow installation of major system components through the opening. The hatch uses dog bolts and nuts to clamp the lid tight to a neoprene edge seal ensuring an IP 67 waterproof rating. The hatch was well made and served its purpose well.

All of the internal electronics were in waterproof IP 66 and IP67 enclosures to protect critical systems in the event of hull leakage or splashing events when the hatch was open. All enclosures used outside of the nacelle were rated IP 67 for prolonged submergence. A minimum amount of steel components were used in the design to reduce susceptibility to corrosion. All steel exposed to sea water was hot dipped galvanized to reduce corrosion. Additionally, sacrificial zinc anodes were placed on the steel float arms to help mitigate corrosion on these electrically connected steel components. A surface area calculation was used to determine the minimum size of the zinc anodes in order to last the project duration.

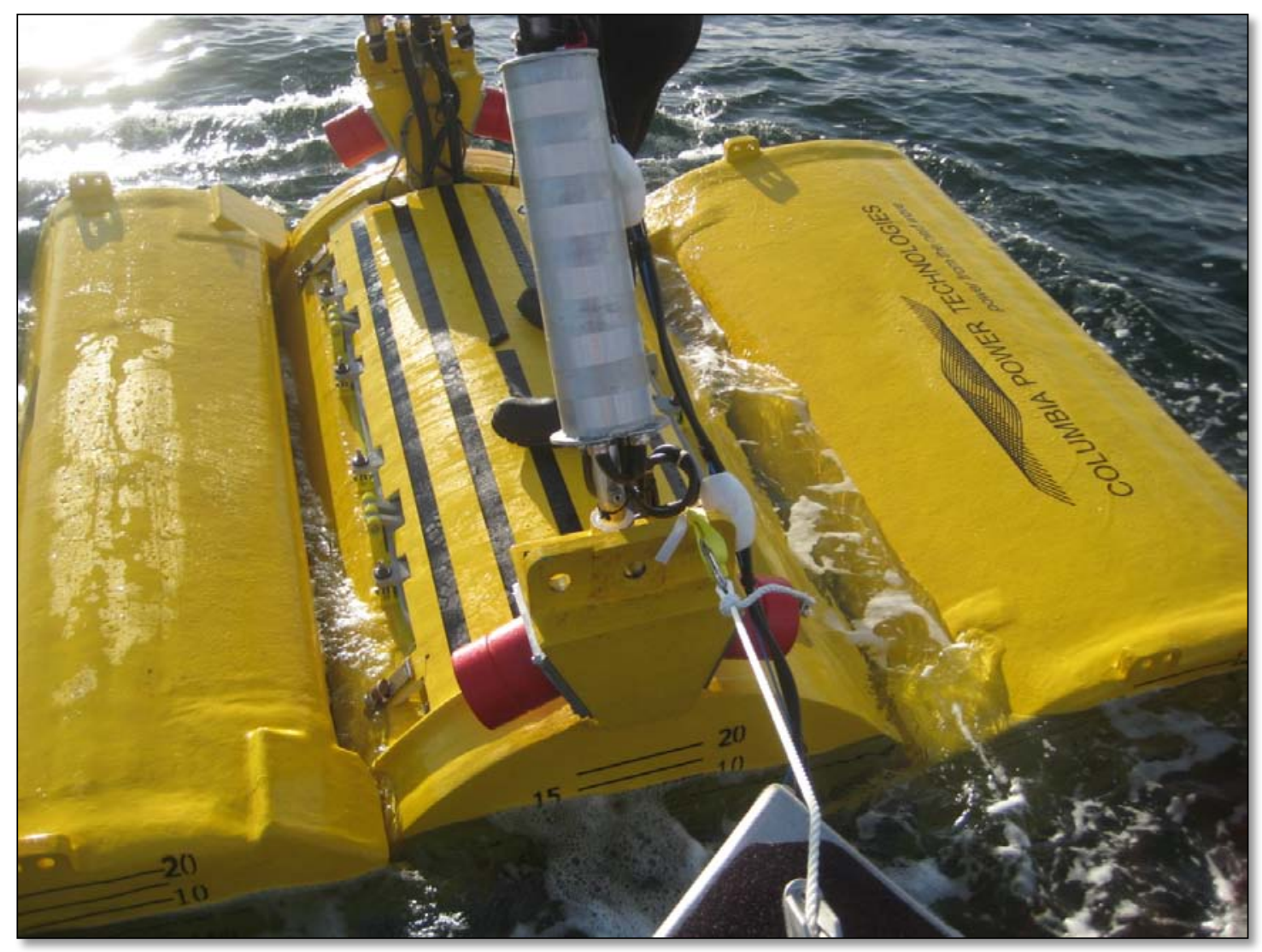

Figure 6 - SeaRay WEC after deployment in Puget Sound, WA. 


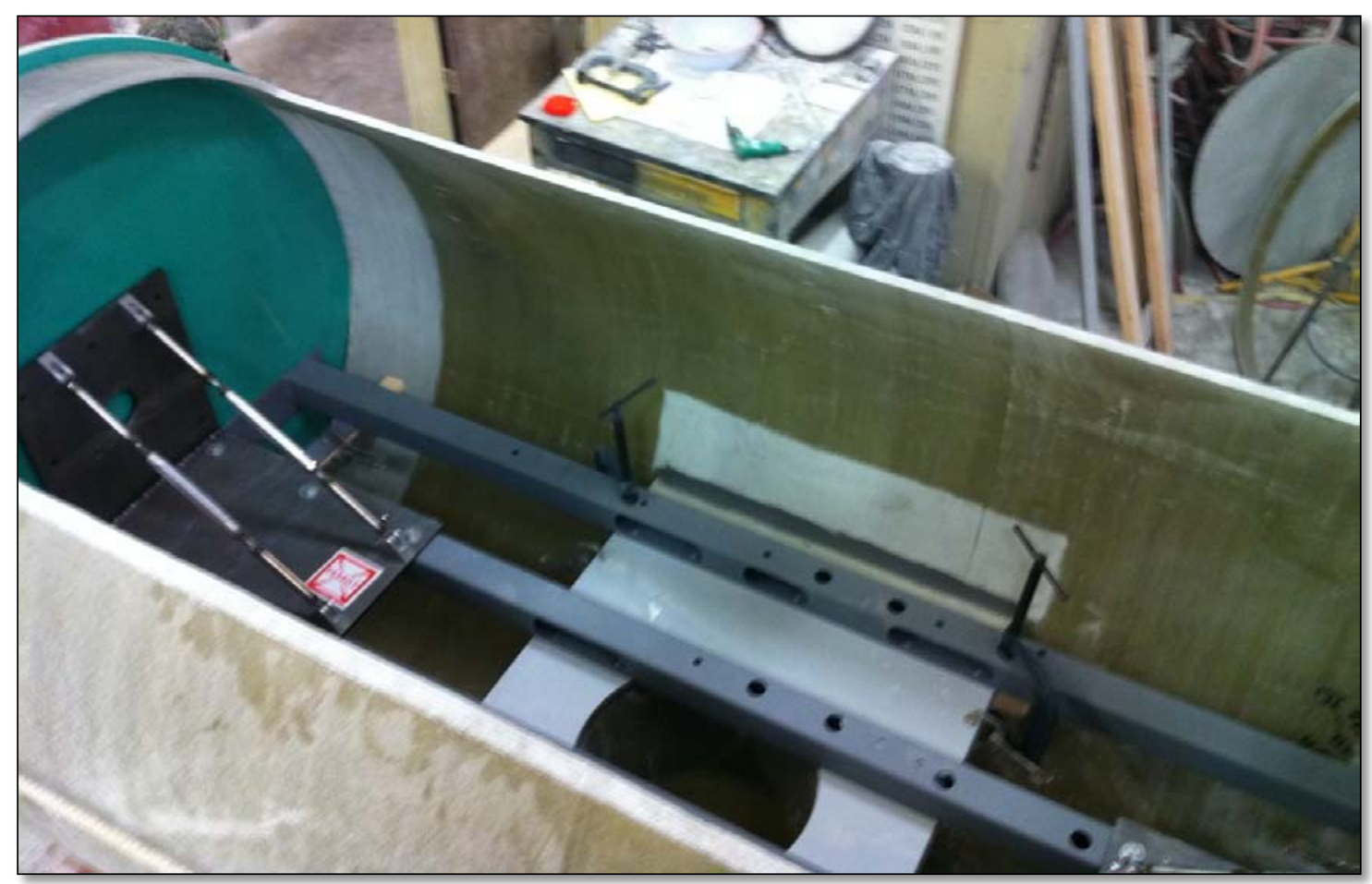

Figure 7 - Main structural frame rail installation into nacelle.

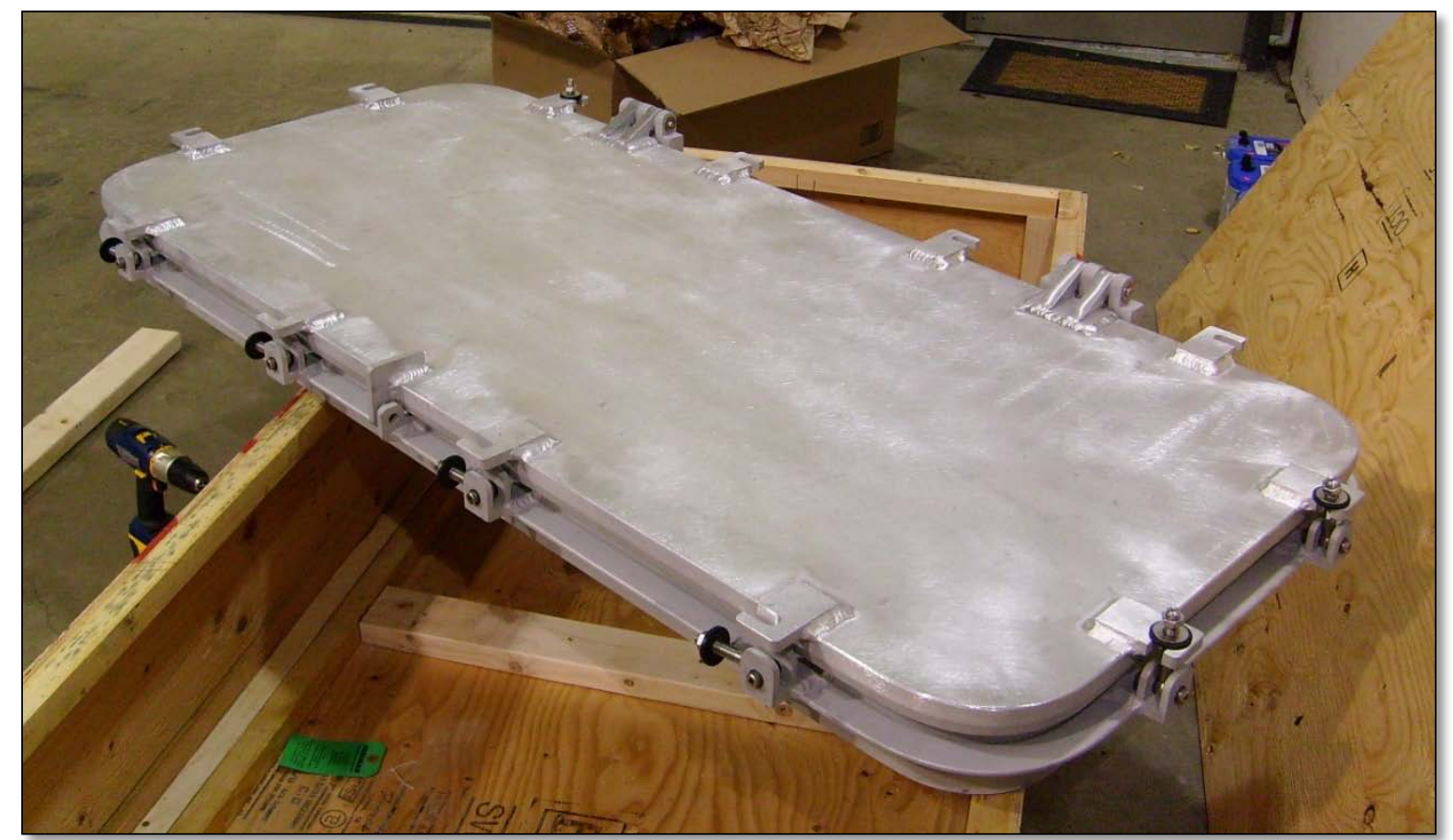

Figure 8 - Watertight aluminum hatch manufactured by Freeman Marine, Gold Beach, OR.

Columbia Power Technologies, LLC • 4920A SW $3^{\text {rd }}$ St, Corvallis, Oregon 97333 Phone: (541) 368-5033 • Fax (541) 230-1498 • www.columbiapowertechnologies.com 


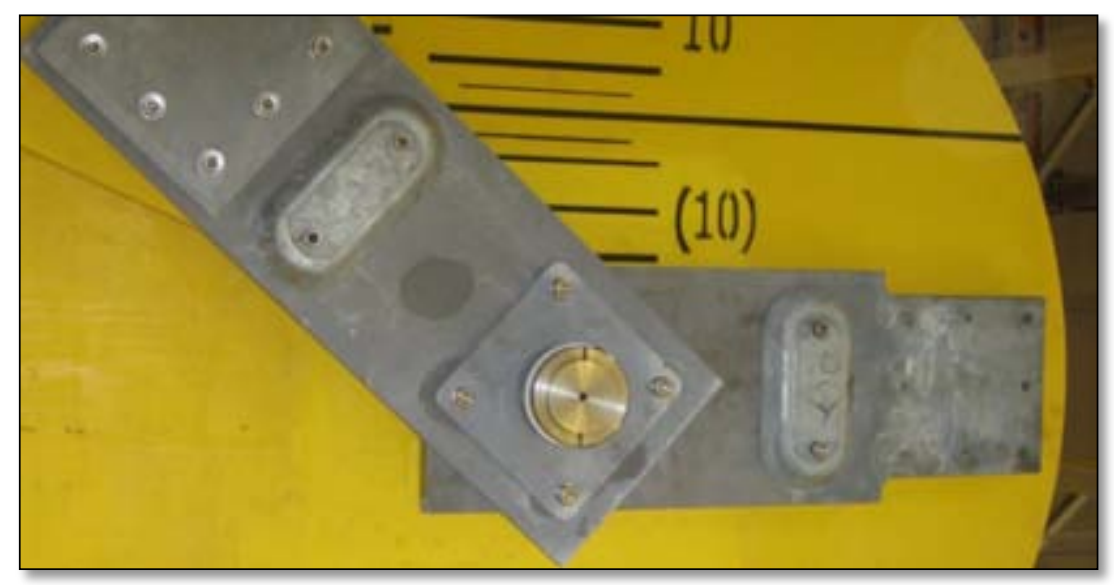

Figure 9 - Float arms connected to PTO shaft with zinc anodes and depth gauge on nacelle.

\subsection{Mooring (0700)}

The SeaRay mooring system was developed in cooperation with Sound \& Sea Technologies, Glosten and Associates, Sound Ocean Systems, and Columbia Power. Initially this team began by investigating mooring solutions for the full scale design. Modeling parameters including wave conditions and WEC descriptions were provided by Columbia Power. Glosten took these inputs and ran numerical models in OrcaFlex for a variety of wave conditions. The mooring design went through many design iterations in an effort to find a mooring that could provide low loads at low cost and with a relatively small footprint. During these design iterations Sound and Sea investigated various mooring components which were fed back into the design. Once the fullscale design converged on a practical solution, the SeaRay mooring design kicked off.

Some variance from full-scale was experienced in the $1: 7^{\text {th }}$ scale mooring; the SeaRay mooring was in deeper "scaled" water depths, currents were larger than full scale, and wave energy in the Puget Sound could come from nearly any direction. To maximize experimental value from the wave conditions, a yaw control system was used to control WEC heading into the waves. Columbia Power contracted Sound Ocean Systems to design and build an active yaw control system. This large steel structure acted as a turret allowing engineers to yaw the WEC into the desired direction. The yaw control system attached to the damper tank of the WEC and the mooring attached to the movable outer ring of the yaw control system.

In the end, a three point mooring design using mid-column floats was established and hardware components were specified. Three mooring load cells were used to collect mooring load data for analysis and model validation. Whitehill Manufacturing provided all of the synthetic line at no cost as a way of testing new lines currently under development. At the end of the deployment these lines were returned to Whitehill for inspection and analysis.

Three 6,000 lb Pearl Harbor type lead anchors were used as the seabed connection for the three mooring points. These anchors and all of the mooring equipment were installed using the Seahorse crane barge at the same time that SeaRay was installed. The mooring system worked extremely well at keeping the WEC on station and limiting mooring loads. 


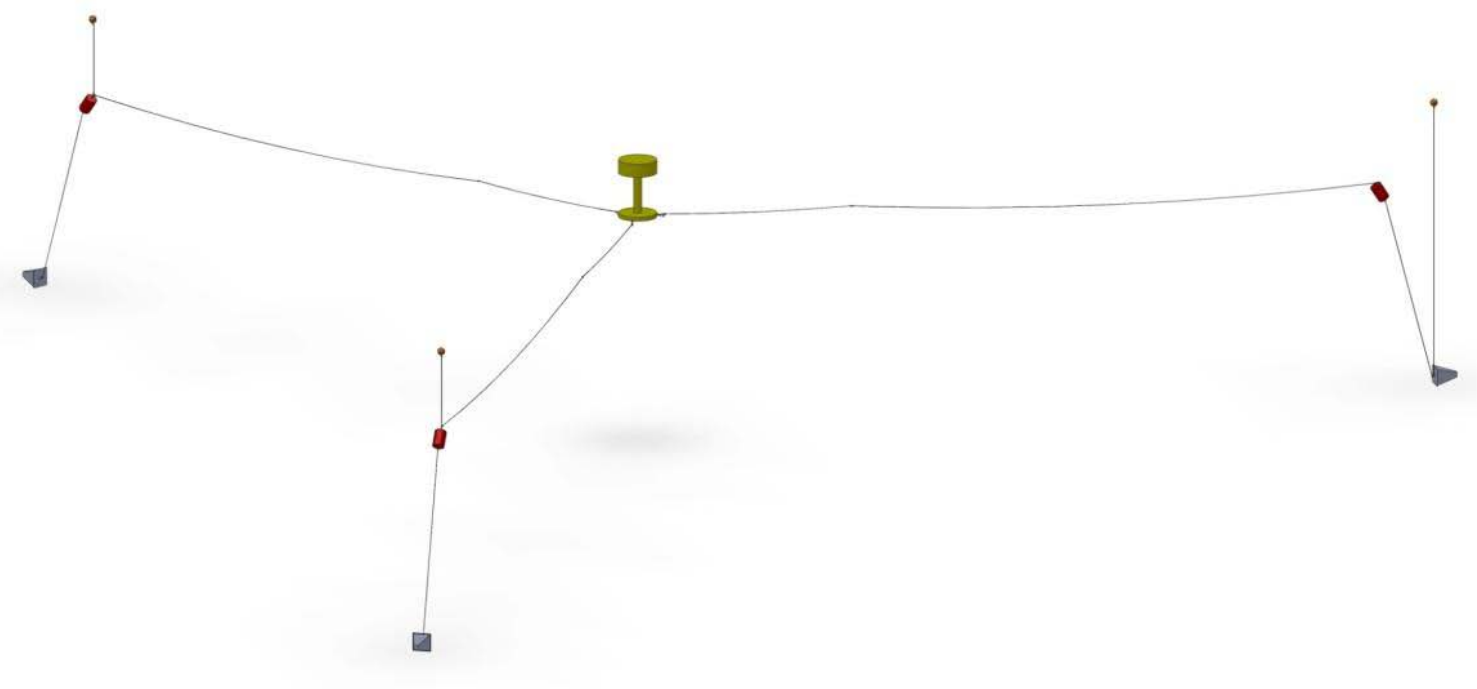

Figure 10 - Isometric view of SeaRay mooring system.

\subsection{Logistics (0900)}

The logistics of permitting, site surveys, collection of met-ocean data, transportation, manufacturing/assembly site planning, hiring of labor, acoustic monitoring of WEC to meet permit requirements, extended deployment permits, travel and lodging for remote engineering and oversight were all notable activities even for this relatively small project. Adding to the cost and magnitude of these logistical efforts was the extension of the deployment period from four months to thirteen months. Cost estimates and labor forecasts to organize and plan these activities should be included in future projects with contingencies considered for project extension.

\subsection{Operations \& Installations (1100)}

The WEC and mooring installation and recovery was outsourced to Sound and Sea Technologies (SST) and is covered in detail in the "1143 Deployment Plan SBG 1-24-2010.pdf". This plan assured a detailed description of deployment and recovery procedures and minimized the potential for unexpected events.

Extended deployment throughout the summer of 2011 established a need for additional maintenance support and as much as bi-weekly battery charge requirements when the wave conditions were insufficient to charge batteries. Operations and battery charging was performed by All Star Fishing Charters of Everett, Washington.

Scuba diver services were hired to perform a mooring inspection six months into deployment, to clean excess bio-fouling on WEC, to repair the yaw control system and post deployment anchor recovery. These services were hired out to local Seattle dive services. 
Regular and frequent observations, log taking and record keeping of WEC status is an essential aspect of system operations. These processes were evaluated and revised several times throughout the deployment period and included continuous monitoring at times of critical evolutions or intense weather, hourly monitoring when frequent updates were needed and no-less than every eight hours during less intensive periods or over-night. Revisiting this deployment experience from a monitoring perspective, it is essential to have frequent (hourly) observations of system status; if less frequent monitoring by personnel is desired, then automated warning/notification systems should be included as part of the instrumentation package in order to notify on-call personnel of abnormal conditions.

\section{Data Processing}

\subsection{AWAC data}

\subsubsection{Configuration}

A 2MHz AWAC (http://www.nortek-as.com/en/products/wave-systems/awac), manufactured by Nortek, was mounted on a taught-moored subsurface buoy approximately $50 \mathrm{~m}$ southeast of the WEC. As most of the waves arrive from the north or the south, shadowing of the wave energy by the WEC is assumed to be negligible. Also, the small separation distance along with the relative depth of the site (20 m MLLW, 3 s average wave period) allows the assumption that the seas incident upon the WEC are statistically equivalent to those measured by the AWAC. Note that although the AWAC and WEC clocks were synchronized before deployment, there was no common trigger and thus AWAC and WEC signals cannot be correlated in the time domain, but rather through statistical characterizations (e.g. mean wave power and mean mechanical power).

The AWAC is outfitted with one upward looking transducer for Acoustic Surface Tracking (AST), three off angle transducers for measuring particle velocity, and a pressure sensor. Additionally, a compass and a tilt sensor allow for transformation of measurements to an earth referenced axes system while the AWAC is mounted on a moving platform. Thus particle velocities measured along the three slanted beams are internally processed to yield three earthreferenced orthogonal velocity components at the mean water level. Note that for logistical reasons the AWAC was not fitted with its batteries and frame during calibration, and as such any distortion of the magnetic field by the batteries is unaccounted for and may affect the accuracy of the AWAC's compass. This seems to be standard practice. 


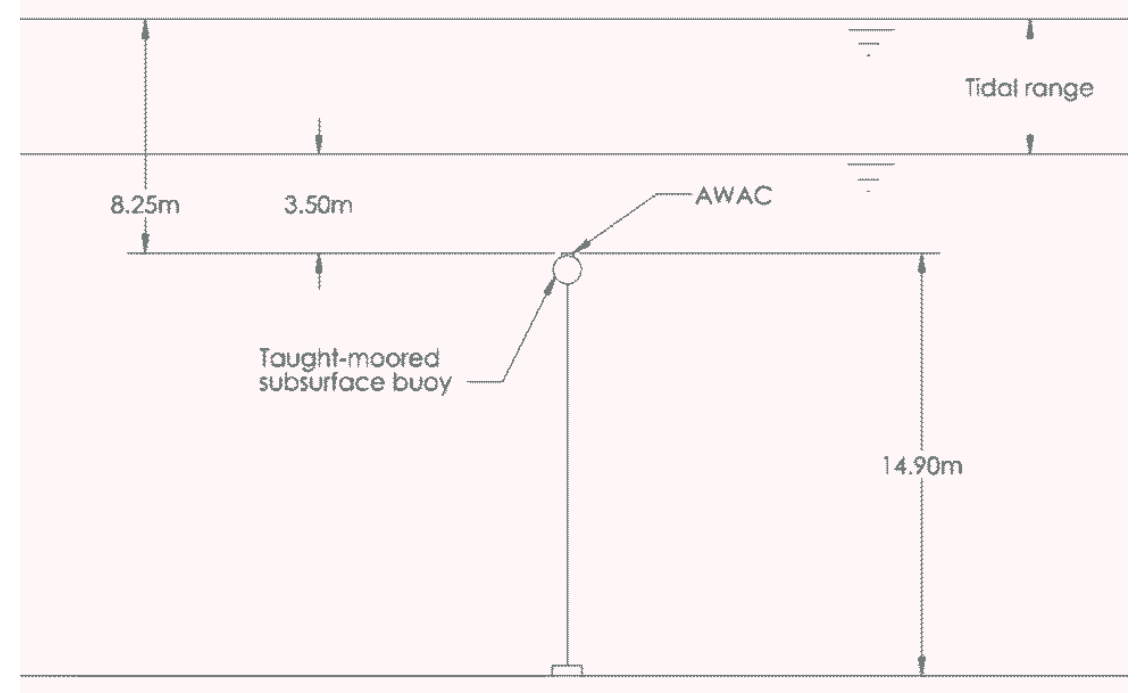

Figure 11. AWAC as installed on taught-moored subsurface buoy.

The AWAC was deployed such that the sensor heads (transducers) were $14.9 \mathrm{~m}$ from the sea floor, and depending on the tidal variation their depth below the mean sea surface varied between 3.5 and $8.25 \mathrm{~m}$ (see Figure 11). Note that the AWAC reports direction with a magnetic reference, and that a $+16.8^{\circ}$ declination is applied in the post processing (http://www.ndbc.noaa.gov/geomagmodels/struts/calcDeclination).

The AWAC was configured to sample current speed and direction for $60 \mathrm{~s}$, every $20 \mathrm{~min}$, on the hour. Immediately thereafter the AWAC samples for waves for $1024 \mathrm{~s}$. Thus wave sampling begins at time XX:01:00, XX:21:00 and XX:41:00 (HH:MM:SS). Wave elevation (AST) is sampled at $4 \mathrm{~Hz}$ while the off angle beams and pressure transducer are sampled at $2 \mathrm{~Hz}$.

Table 1. Froude scaling

\begin{tabular}{lc}
\hline Physical Parameter & Multiplication Factor \\
\hline Scale factor, $\lambda$, equal to model scale (e.g. 7 for the SeaRay) \\
\hline Length & $\lambda$ \\
Mass & $\lambda^{3}$ \\
Force & $\lambda^{3}$ \\
Moment & $\lambda^{4}$ \\
Acceleration & 1 \\
Time & $\sqrt{\lambda}$ \\
Pressure & $\lambda$ \\
Linear velocity & $\sqrt{\lambda}$ \\
Rotational velocity & $1 / \sqrt{\lambda}$ \\
Rotational inertia & $\lambda^{5}$ \\
Power & $\lambda^{3.5}$ \\
Damping & $\lambda^{4.5}$ \\
Torque & $\lambda^{4}$ \\
Wave power & $\lambda^{2.5}$ \\
\hline
\end{tabular}

Columbia Power Technologies, LLC $\bullet 4920$ A SW $3^{\text {rd }}$ St, Corvallis, Oregon 97333 Phone: (541) 368-5033 • Fax (541) 230-1498 • www.columbiapowertechnologies.com 


\subsubsection{Sampling period}

After observing a number of wave elevation time histories it became clear that significant trending of wave height often occurred during a single $1024 \mathrm{~s}$ trial. To increase the statistical stationarity of the characterized sea states, as well as to increase the number of trials in the experiment, each $1024 \mathrm{~s}$ record is split into two separate $512 \mathrm{~s}$ trials in the post processing. At full scale, seas are typically sampled for 20 to $30 \mathrm{~min}$ [1], which is roughly 150 to 200 waves for a representative mean zero-crossing period of $8 \mathrm{~s}$. Preliminary observation of the mean wave period at the Puget Sound test site shows it to typically fall between 2.5 and $3.5 \mathrm{~s}$, which for a 512 s sampling period is roughly 150 to 200 waves. One can also observe that 512 s, when Froude scaled up (see Table 1 ), is equivalent to $\sqrt{7} * 512 \mathrm{~s} \approx 23 \mathrm{~min}$ at full scale. Thus $512 \mathrm{~s}$ is deemed an appropriate sample length. The effect of this trial length on the uncertainty associated with the spectral estimate will discussed in 3.1.5. After splitting the $1024 \mathrm{~s}$ records in half there are six 512 s records per hour, with start times of $X X: 01: 00, X X: 09: 32, X X: 21: 00, X X: 29: 32$, XX:41:00 and XX:49:32.

\subsubsection{Signal preprocessing}

As previously noted the sensor heads of the AWAC are located $14.9 \mathrm{~m}$ above the sea floor. The mean pressure signal over a $512 \mathrm{~s}$ sampling period is used to determine the position of the mean sea surface position with respect to the AWAC for each trial, and thus the mean water depth.

There are three time series signals that are the primary data used for estimating waves and currents: surface elevation (AST) and two orthogonal particle velocity components in the horizontal plane (U, V). The two particle velocity components are the result of internal processing by the AWAC which transforms velocity measurements in beam coordinates to an earth-referenced coordinate system. The mean values of the two velocity signals, over one $512 \mathrm{~s}$ trial, are used to determine the speed and direction of the current at a near surface elevation. The direction is then corrected for magnetic declination.

Prior to spectral analysis, these three signals (AST, U, and V) are demeaned, detrended, low-pass filtered and despiked. For the AST signal, which is sampled at $4 \mathrm{~Hz}$, the signal is cleaned up as follows. After demeaning, detrending and filtering (zero-phase low-pass Butterworth filter with a pass frequency of $1.8 \mathrm{~Hz}$ ), the despiking is achieved by identifying and setting to zero any values in the time series which fall outside of the range of \pm five times the standard deviation of the signal. To reduce the effect of outliers in calculating this threshold, an estimation of the sample standard deviation, $\operatorname{std}_{\mathrm{iqr}}$, based on the interquartile range (IQR) will be used

$$
\operatorname{std}_{\mathrm{iqr}}=0.7413 \mathrm{IQR}
$$

Additionally, any surface elevation data point associated with acceleration of magnitude greater than gravitational acceleration is considered erroneous and set to zero. Finally, all indices which were set to zero are replaced using linear interpolation between the nearest non-erroneous neighbors. A sensitivity study was performed using randomly selected AWAC records out of a pool in which no spikes were detected. Relative errors in significant wave height and energy 
period were calculated after simulating the effects of multiple spikes (by selecting points and resetting the values using linear interpolation between neighbors). As a result of this sensitivity study a threshold for number of corrected samples was established; any 512 s trial with greater than 125 corrected samples or a single contiguous block of corrected samples in excess of 40 samples will be flagged (see 3.1.8). A record of which indices were replaced with interpolated values is retained with the data.

A similar methodology is followed with the velocity signals. The velocity signals are sampled at $2 \mathrm{~Hz}$ (Nyquist frequency of $1 \mathrm{~Hz}$ ), however wave energy with a wavelength of less than twice the beam separation distance (near the sea surface) cannot be resolved directionally [2]. With a beam separation of $25^{\circ}$ and a maximum distance from sensor head to sea surface (at High High Water) of $8.25 \mathrm{~m}$ the threshold wavelength is equal to $2 \cdot 8.25 \mathrm{~m} \cdot \tan \left(25^{\circ}\right)=7.69 \mathrm{~m}$. With a deep water assumption, this equates to a frequency of $0.45 \mathrm{~Hz}$, or $0.17 \mathrm{~Hz}$ full scale equivalent. The particle velocity signals are low-pass filtered with a pass frequency of $0.8 \mathrm{~Hz}$. The vertical beam used to measure surface elevation has a width of $1.7^{\circ}$.

Following the above analysis, wave energy with a frequency greater than $1.8 \mathrm{~Hz}$ cannot be properly resolved for the elevation variance density spectrum. A more limiting cutoff of $1.5 \mathrm{~Hz}$ was selected to avoid aliasing.

\subsubsection{Ship Waves}

For statistical characterization of sea states, it is essential that they display stationarity in time (i.e. they are characterized by a consistent random process throughout the analysis window). An otherwise stationary sea state punctuated by a relatively large amplitude wave train from a passing ship's wake can no longer be considered stationary - and should not be included in the results of this experiment.

Suspected ship wave incidents are detected within a given trial period by comparing the variance of the surface elevation signal inside and outside of a moving window. The window has a width of $64 \mathrm{~s}$ and is moved in intervals of $16 \mathrm{~s}$, and as the window is moved through the data a series of variance ratios is generated. The maximum value thus generated (maximum variance ratio) is recorded and will be used to flag records suspected of containing substantial ship waves.

\subsubsection{Observed and intrinsic spectra frequency spectra}

Having demeaned, detrended, low-pass filtered and despiked the surface elevation signal, it can now be analyzed in the frequency domain to yield the surface elevation variance density spectrum, referred to here as the frequency spectrum. To reduce spectral leakage, a one-eight cosine taper is applied to the $512 \mathrm{~s}$ AST time series before performing the Fast Fourier Transform. The resulting raw spectrum is scaled up (returning the energy lost by the tapering) and is smoothed by averaging the variance density of a number of adjacent spectral estimates in non-overlapping windows. To achieve a balance between frequency resolution and uncertainty in the smoothed frequency spectrum, groups of 13 adjacent spectral estimates are averaged together. This results in a frequency resolution of $0.025 \mathrm{~Hz}$ (equivalent to $0.096 \mathrm{~Hz}$ at full scale). Raw spectral estimates are statistical measures with a large uncertainty associated with them. In 
fact, the proportional standard deviation (pstd) of a raw estimate is equal to 1. By averaging together $\mathrm{N}$ raw estimates the pstd is reduced by a factor of $1 / \sqrt{\mathrm{N}}$, and so with 13 spectral estimates averaged together the pstd of the resulting smoothed estimates is reduced to 0.28 . See Tucker and Pitt [3] for details on spectral analysis. Note that this analysis yields the un-truncated, observed spectrum.

The presence of a significant current complicates the spectral analysis. Although ocean waves are essentially unaffected by a steady state current, the water in which the waves are propagating is itself being transported by the current. An observer travelling with the current can utilize standard linear wave theory to calculate the typical wave parameters (e.g. wave length, wave power, etc.). However, the Doppler shift effect of the current must be accounted for if an observer is stationary with respect to earth. The AWAC and the WEC are both such stationary observers. Although a stationary observer experiences the wave crests (for example) at the observed frequencies, $\omega$, the wavelengths and group velocities are related to the intrinsic frequencies, $\sigma$.

The observed and intrinsic frequencies are related by the following equation

$$
\omega=\sigma+k U_{n}
$$

where $k$ is the wave number and $U_{n}$ is the component of the current velocity in the direction of wave propagation. Note that the wave number must be calculated using the intrinsic frequency, and thus an iterative algorithm is needed to solve for the intrinsic frequency. In a frame of reference that travels with the current, the wave energy will be observed to propagate at the group velocity associated with the intrinsic frequency. But in a fixed frame of reference, the wave energy is seen to propagate at the effective group velocity

$$
c_{g, e f f}=c_{g, i n t}+U_{n}
$$

where $c_{g, \text { int }}$ is the group velocity calculated from the intrinsic frequency [4].

Both the observed and intrinsic spectra are utilized in the analysis. First the observed spectrum is calculated, followed by the directional spectral analysis (see 3.1.7). The directional estimates produced are not tied to particular frequencies so much as to the estimate's index. Upon Doppler shifting the directional estimates apply to the same indices; only the associated frequencies are changed. Once the directional analysis is completed, the Doppler shifting (which yields the intrinsic frequencies) is performed. For the Doppler shifting, it is assumed that the mean direction associated with the peak of the variance density spectrum is valid for all wave energy.

The next step is the determination of a high frequency cutoff. The high frequency spectrum has two limitations. First, the Nyquist frequency is $2 \mathrm{~Hz}$ for the wave elevation spectrum (and it is 1 $\mathrm{Hz}$ for the directional spectrum, which is calculated using data sampled at $2 \mathrm{~Hz}$ ). This limit applies to the observed spectrum. Second, a cutoff frequency of $1.5 \mathrm{~Hz}$ has been selected due to the spatial Nyquist frequency $(0.45 \mathrm{~Hz}$ for the directional spectrum, see 3.1.3). This limit applies to the intrinsic spectrum. The upper cutoff index is set to the more limiting of these two. To give

Columbia Power Technologies, LLC • 4920A SW $3^{\text {rd }}$ St, Corvallis, Oregon 97333

Phone: (541) 368-5033 • Fax (541) 230-1498 • www.columbiapowertechnologies.com 
an example, a $1 \mathrm{~Hz}$ wave with a strong favorable current has an observed frequency of $2.1 \mathrm{~Hz}$; although this estimate has a long enough wave length to be resolved (for wave elevation), it is observed at a frequency which is not resolvable, and as such this estimate is above the cutoff. For the directional spectrum, this high frequency cutoff is just that. However for the variance density spectrum, a high frequency tail is fit to the data above the 'cutoff', and integration for the determination of characteristic wave parameters is carried out up to $2 \mathrm{~Hz}$.

Next, the observed variance density spectrum must be modified to properly represent the intrinsic spectrum. The total energy is the same for the observed and intrinsic spectra, but the frequency spacing in general is different. At each frequency index, the intrinsic variance density estimate is multiplied by the ratio of frequency widths such that the energy is the same. After this, a theoretical high frequency tail is fitted to the intrinsic and observed spectra, from the high frequency 'cutoff' up to $2 \mathrm{~Hz}$. The fitted tail decays with increasing frequency as $f^{-4.5}$.

Finally, to avoid the inclusion of low frequency noise in the integrated spectral estimates, an appropriate low frequency cutoff is determined. The lowest frequency spectral estimate is disregarded outright. Thus the second spectral index is considered the lowest frequency estimate that may be valid. If the variance density of this estimate is less than $10 \%$ of the peak value, this estimate is taken to be the low frequency cutoff. If it exceeds the $10 \%$ threshold, then the lowest variance density at a frequency below the peak frequency is taken as the low frequency cutoff. Integration is carried out from the low frequency cutoff up to $2 \mathrm{~Hz}$ for the non-directional integrated spectral parameters.

\subsubsection{Integrated spectral parameters}

Having obtained the frequency spectrum, a number of parameters are calculated which will serve to characterize the sea state. Omnidirectional wave power, $J$ or simply wave power, is calculated from the intrinsic spectrum as

$$
J=\rho g \sum_{i} c_{g, e f f, i} S_{i} \Delta f
$$

where $\rho$ is the density of sea water, $g$ is the acceleration of gravity, $c_{g, e f f, i}$ is the effective group velocity of the $i^{\text {th }}$ spectral estimate, $S_{i} \equiv S\left(f_{i}\right)$ is the variance of the $i^{\text {th }}$ spectral estimate and $\Delta f$ is the frequency resolution of the smoothed spectrum. The group velocity is calculated from the intrinsic spectrum as

$$
c_{g, i}=\frac{\pi f_{i}}{k_{i}}\left(1+\frac{2 k_{i} h}{\sinh 2 k_{i} h}\right)
$$

where $k_{i}$ is the wave number associated with the $i^{\text {th }}$ spectral estimate at the mean water depth, $h$. The wave number associated with a given frequency and water depth is implicitly defined through the dispersion relation

$$
\left(2 \pi f_{i}\right)^{2}=g k_{i} \tanh k_{i} h
$$


As mentioned previously, the wave number is correlated with the intrinsic frequency. Significant wave height, $H_{m 0}$, is calculated as

$$
H_{m 0}=4 \sqrt{m_{0}}
$$

where $m_{0}$ is the zeroth moment of the frequency spectrum, and any $n^{\text {th }}$ order moment is calculated as

$$
m_{n}=\sum_{i} f_{i}^{n} S_{i} \Delta f
$$

where $f_{i}$ is the frequency of the $i^{\text {th }}$ spectral estimate and. The significant wave height is the same regardless of whether intrinsic or observed spectrum is used. The mean frequency content of the sea state is characterized using the energy period, $T_{e}$, which is calculated as

$$
T_{e}=m_{-1} / m_{0}
$$

The energy period is calculated separately using both the observed and the intrinsic spectra. As the WEC is influenced by the timing and the geometry of the waves, it is unclear at this stage which parameter is better suited to predicting WEC response. It is desirable to characterize the shape of the spectrum in some manner, as the response of the WEC may in some way be sensitive to the spectral shape. The spectral width [5], $\epsilon_{0}$, will be calculated as

$$
\epsilon_{0}=\sqrt{\frac{m_{0} m_{-2}}{m_{-1}^{2}}-1}
$$

A spectral width is calculated separately using both spectral representations. A characteristic measure of the steepness can be found via the significant steepness, $S_{s Z}$, which is calculated as

$$
S_{s Z}=\frac{2 \pi}{g} \frac{H_{m 0}}{T_{Z}^{2}}
$$

where $T_{z}$ is the mean zero-crossing wave period and is estimated from the moments of the frequency spectrum as

$$
T_{z}=\sqrt{m_{0} / m_{2}}
$$

A similar steepness parameter will be calculated using the energy period $\left(S_{s e}\right)$. The significant steepness parameters are calculated using the intrinsic spectrum, as these parameters are related to wave geometry.

As explained in 4.2.1, sensitivity studies will be performed to determine the suitability of any characteristic parameter (beyond $H_{m 0}$ and $T_{e}$ ) for estimating WEC response. 


\subsubsection{Frequency-directional spectrum}

The frequency-directional spectrum is defined such that the variance at a given frequency is distributed by a directional spreading function, $D(f, \theta)$, such that

$$
S(f, \theta)=S(f) D(f, \theta)
$$

As the directional spreading function is periodic in $\theta$ with a period of $2 \pi$, it can be described by a Fourier series.

$$
D(f, \theta)=\frac{1}{\pi}\left[\frac{1}{2}+\sum_{n}\left\{a_{n} \cos n \theta+b_{n} \sin n \theta\right\}\right]
$$

Triplet methods (such as the commonly used PUV method) are often used to estimate the coefficients of the first two harmonics of this Fourier series. With the taught moored subsurface AWAC the appropriate input signals are the surface elevation (S) and horizontal, orthogonal particle velocity signals $(\mathrm{U}, \mathrm{V})$, and the method is termed SUV [2] and the relevant Fourier coefficients are calculated as

and

$$
A_{1}(f)=\frac{C_{S U}}{\sqrt{C_{S S}\left(C_{U U}+C_{V V}\right)}}
$$

$$
B_{1}(f)=\frac{C_{S V}}{\sqrt{C_{S S}\left(C_{U U}+C_{V V}\right)}}
$$

where $C_{* *}$ is the indicated cospectra, and is understood to be frequency dependent. Groups of 13 adjacent spectral estimates are averaged together to achieve a relatively smooth spectrum.

The wave energy at a given frequency is characterized with a mean direction, $\theta_{1}(f)$, and a directional spreading, $\sigma_{1}(f)$, as

and

$$
\theta_{1}(f)=\operatorname{atan} 2\left(B_{1}(f), A_{1}(f)\right)
$$

where

$$
\sigma_{1}(f)=\sqrt{2\left(1-M_{1}(f)\right)}
$$

$$
M_{1}(f)=\sqrt{A_{1}^{2}(f)+B_{1}^{2}(f)}
$$

The directional spreading parameter can also be represented as a non-dimensional spreading index, $s(f)$, as

$$
s(f)=\frac{M_{1}}{1-M_{1}}
$$

These frequency dependent quantities are calculated over the entirety of the un-truncated spectra. However, the high and low cutoffs discussed above are utilized when calculating the integrated 
parameters below. Wave power weighted directionality parameters are calculated to characterize the sea state as a whole. Although the estimated spectra are truncated above $0.45 \mathrm{~Hz}$ (ignoring the directional characteristics of a potentially significant portion of the spectrum), averaged parameters are likely to be more stable than parameters associated only with peak. The direction of the wave power vector sum of the components of the directional spectrum is used to characterize the predominant directionality of a given sea state, and this parameter is designated $\theta_{J}$.

As it was not clear at this stage which will best predict WEC response, the directional spreading of wave energy for the sea state as a whole is characterized by three separate parameters. Unidirectivity Index, $U I$, is the ratio of the magnitude of the wave power vector sum of the components of the directional spectrum to the omnidirectional wave power (integrated over the directional spectrum's frequency limits). Additionally, a wave power weighted mean directional spreading is calculated for each sea state, as well as a wave power weighted mean spreading index. These are designated $\sigma_{J}$ and $s_{J}$ respectively. Directional spreading and the spreading index are two alternate parameters used to describe the same thing. They are both determined by the same factor (see above), but are not linearly related. UI accounts for spreading of energy frequency bins, as well as variation of mean angles between frequency bins. As such UI is more descriptive of the total variability of direction observed in a sea state.

\subsubsection{Flags}

A number of flags may be generated for any given record to alert the data handlers to potentially erroneous data. These flags do not necessitate rejection of the record, but manual inspection is warranted. Upon manual inspection the flags may be cleared. A cleared flag is given a value of 2 and an inspected trial whose flag is found to be warranted is given a value of 3 . This is to differentiate inspected trials from those that have not been inspected. All flags marked with an asterisk $\left(^{*}\right)$ must have a value of 0 or 2 for the trial to be included in the final data analysis. Flags and their respective criteria are outline below.

Unusual depth * - Mean pressure indicates that the AWAC is at a depth of less than $2.5 \mathrm{~m}$ or greater than $8.5 \mathrm{~m}$

Wave crests clipped * - Wave elevation (absolute value) in excess of $19.5 \mathrm{~m}$ (AST has a range of $\pm 20 \mathrm{~m}$, and the signal is clipped beyond that)

High AST data loss * - More than 125 samples (6.1\% of record) replaced during despiking process, or the replacement of a contiguous block of more than 40 samples (10 s)

Excessive tilt * - Tilt in excess of $10^{\circ}$ (excessive tilt results in poor return of the AST beam)

White noise $*-\frac{\max \left(S_{i}\right)}{\operatorname{mean}\left(S_{i}\right)}<1.2$

Low frequency noise $*-S\left(f_{2}\right)>0.1 \cdot \max (S(f))$ 
Unusual wave conditions $*-H_{m 0}>1.2 \mathrm{~m}, T_{e}<1.1 \mathrm{~s}, T_{e}>5.3 \mathrm{~s}$

Ship waves * - Maximum variance ratio (see 3.1.4) in excess of a threshold value of 4 . Investigation has indicated that ship waves do not necessarily lie outside of the frequency range of local wind waves, thus a spectral approach to detection is not indicated.

Low high cutoff * - Energy period lies above the high frequency 'cutoff' (i.e. within the fitted high frequency tail)

\subsection{SeaRay data}

\subsubsection{Configuration}

All attempts were made to design the SeaRay such that it was a 1:7 Froude scaled model of the utility scale Ray series WEC Generation 3.1 (see Appendix A for a simple dimensioned drawing). However, there are certain aspects in which the SeaRay differs from the target. The asbuilt mass distribution is given in Table 2, along with the relative error of as-built with respect to target. Note in particular that the vertical location of the center of gravity of the spar is not nearly as low as targeted, a factor which modeling has clearly identified as reducing performance. Furthermore, there are significant compromises in the as-built moment of inertia.

Table 2. SeaRay mass distribution: as-built and relative error.

\begin{tabular}{|c|c|c|c|c|c|c|}
\hline & \multicolumn{2}{|c|}{ Mass } & \multicolumn{2}{|c|}{ Center of gravity } & \multicolumn{2}{|c|}{$\begin{array}{l}\text { Moment of inertia wrt } \\
\text { PTO axis of rotation }\end{array}$} \\
\hline & $\begin{array}{c}\text { As-built } \\
\text { [kg] }\end{array}$ & $\begin{array}{l}\text { Error wrt } \\
\text { target [\%] }\end{array}$ & $\begin{array}{c}\text { As-built } \\
\text { [m] }\end{array}$ & $\begin{array}{l}\text { Error wrt } \\
\text { target [\%] }\end{array}$ & $\begin{array}{c}\text { As-built } \\
{\left[\mathrm{kg} \mathrm{m}^{2}\right]}\end{array}$ & $\begin{array}{l}\text { Error wrt } \\
\text { target [\%] }\end{array}$ \\
\hline Total system & 6530 & 8 & \multicolumn{2}{|c|}{$\mathbf{x}, \mathbf{y}, \mathbf{z}$} & \multicolumn{2}{|c|}{$\mathbf{I}_{\mathrm{xx}}, \mathbf{I}_{\mathrm{yy}}, \mathbf{I}_{\mathrm{zz}}$} \\
\hline Spar & & & & & & \\
\hline Fore float & & & & & & \\
\hline Aft float & & & & & & \\
\hline
\end{tabular}

As described in the Experiment Setup section the SeaRay prototype is a heavily instrumented research platform. A suite of sensors provides historical data from the deployment period. Originally the signals were sampled at $100 \mathrm{~Hz}$. However the sampling rate was briefly reduced to $50 \mathrm{~Hz}$, and finally settled at $25 \mathrm{~Hz}$. Additionally, the number of signals sampled was decreased 
during the experiment. Decisions to downsample and drop data signals were driven by processor overload and dSPACE data capture errors.

\subsubsection{Sampling period}

Sampling begins every 20 minutes on the hour, and signals are recorded for 19.5 minutes with a 30 s quiescent period between trials.

\subsubsection{Note on heading}

To determine the orientation of the mooring system itself, a vessel was positioned over each anchor using a fish finder, and a GPS unit was used to determine the location of each anchor. See Appendix $C$ for documentation of mooring orientation and anchor locations for each of the two deployments (Feb 15, 2011 and March 11, 2011).

While the SeaRay is kept on station with a spread three-point mooring system, an integral Yaw Control System (YCS) allows the SeaRay to change its yaw heading with respect to the mooring system. This allows the SeaRay to be turned into the waves regardless of where they are coming from. This capability also adds a degree of complexity to the post-processing. To quantify the relative heading of the waves one must quantify the WEC heading as well as that of the wave system. The YCS encoder failed on June $29^{\text {th }}$ and was not replaced or repaired. There were also a few periods in which the yaw control signal is known to be in error (e.g. when the drive chain was broken). In these instances the signal will be corrected using manual heading measurements.

The GPS sensor originally installed on the mast of the SeaRay did not yield a reliable signal. Two sensors of the same make and model were tested with the same results: an erratic and unreliable signal. A dual GPS heading sensor was later installed on September 7, 2011. This instrument yields a reliable heading signal. Another, previously installed, sensor was investigated with hope that it could provide the instantaneous heading signal up to that time. The inertial motion unit (IMU) installed within the nacelle is designed to report pitch, roll and yaw position via a combination of measurements made with the accelerometers, rate gyroscopes, and magnetic sensor. The yaw position is stabilized via the magnetometer signal, which was not calibrated after installation in the SeaRay. Efforts to derive a correction function for this heading signal have yielded some success, but the results are deemed unsatisfactory.

Examination of the instantaneous WEC heading signal from the dual GPS sensor indicates that under the influence of waves and currents the WEC heading oscillates about the mean position on the order of $\pm 5^{\circ}$. This level of WEC directional variability seems fairly insignificant, when considering the uncertainty associated with determination of the wave heading and the significant directional spreading generally observed in the wave systems at the deployment site. Thus the two types of WEC headings (i.e. the orientation of the YCS, and the measured WEC heading) will be used interchangeably in determining the mean orientation of the WEC during a given trial. The YCS orientation will be used up until the time at which the dual GPS sensor was installed.

Columbia Power Technologies, LLC • 4920A SW $3^{\text {rd }}$ St, Corvallis, Oregon 97333 Phone: (541) 368-5033 • Fax (541) 230-1498 • www.columbiapowertechnologies.com 


\subsubsection{Raw data correction}

The raw SeaRay data is preprocessed to a 'corrected raw data' state, and archived, before proceeding with the primary data processing. Note that the raw data is unaffected by the creation of the 'corrected raw data', as new data files are created and both sets are archived.

Firstly, any records that have no time stamp, have an erroneous time stamp, are incomplete (i.e. file size $<10 \mathrm{kB}$ ), or were recorded during known downtime are ignored. Next, all records sampled at 50 or $100 \mathrm{~Hz}$ are downsampled to the common $25 \mathrm{~Hz}$.

As noted in 3.2.3 there are instances of erroneous or missing yaw encoder data. Erroneous data exists during times that the YCS was not yet online or after encoder failure on June $29^{\text {th }}$. Missing data is manually replaced, where possible, with records kept thrice daily in a hand-written paper log from the live data stream. Yaw data from the period before the yaw control was online is generated using known installation configuration and GPS measurements of the positions of the three anchors. Instantaneous WEC heading data from before the dual GPS sensor was installed will not be used.

The anemometer installed on the mast of the SeaRay was originally referenced to the GPS heading signal, which is now known to be erroneous. It was then referenced to the yaw position encoder, which has since failed. The wind direction reported in the corrected raw data archive is referenced to the WEC. The wind direction reported in the $512 \mathrm{~s}$ trial file archive is referenced to true north, by accounting for the mean WEC heading.

The generator encoder speed signal has a number of erroneous spikes. Despiking is achieved by identifying and replacing with linear interpolation any values in the time series which fall outside of the range of \pm eight times the standard deviation of the signal, and have a reported speed (absolute value) in excess of $1.5 \mathrm{rad} / \mathrm{s}$. The absolute value threshold was added after observing the inappropriate 'despiking' of high speed events in relatively calm seas. A record of the raw (uncorrected) speed signal is retained.

To achieve the required resolution and accuracy of generator torque measurements under typical conditions, a torque transducer whose saturation limit $\quad \mathrm{Nm}$ ) is less than the maximum experienced torque was selected. There are also slip clutches installed on either shaft between the float and the generator that is set to slip at torque limits of $\quad \mathrm{Nm}$ for the fore and aft floats respectively. Once the slip clutch disengages the torque rapidly drops (the system is freewheeling) and is once again accurately measureable. Thus generator torque between the transducer saturation and slip clutch threshold must be estimated. As the generator torque was controlled such that it was linearly related to generator speed, a linear damping coefficient was calculated and used to estimate torque. From Dec. 22, 2011 on, an actively controlled torque limit was imposed via a PLC. The actively controlled torque limits were $\quad$ Nm for the fore and aft generators, respectively.

Only those data points for which the absolute value of the reported torque was less than Nm were used to estimate the damping coefficient. A least squares linear fit algorithm is applied (MATLAB function regress) to the speed/torque data for each generator separately, yielding a fore and aft linear damping coefficient. These parameters are then used to estimate torque (i.e.

Columbia Power Technologies, LLC • 4920A SW $3^{\text {rd }}$ St, Corvallis, Oregon 97333

Phone: (541) 368-5033 • Fax (541) 230-1498 • www.columbiapowertechnologies.com 
torque is equal to damping times speed) for all data points in which the raw torque measurement is greater than Nm. These estimates are saturated at torque limits detailed in the preceding paragraph. A record of the raw (uncorrected) torque signal is retained. Damping estimation and torque signal correction is done for each 19.5 minute record.

At this stage in the data processing the raw data has been 'corrected' and is saved in a new folder as 'corrected raw data'. See Appendix E for a listing and brief explanation of all data stored in the corrected raw data archive.

At this point two $512 \mathrm{~s}$ records are extracted from the 'corrected raw data'. The $512 \mathrm{~s}$ windows are co-temporal with the $512 \mathrm{~s}$ AWAC records. Due to data drop outs, not all SeaRay records are the full 19.5 minutes. If less than $410 \mathrm{~s}$ exists within the desired $512 \mathrm{~s}$ window, that record is ignored. If at least $410 \mathrm{~s}$ are available, but not the entire $512 \mathrm{~s}$, then the incomplete data series is processed, but will not be utilized in the spectral analysis. These nominally $512 \mathrm{~s}$ time series records, along with the characteristic parameters that will be calculated from them, will be archived in trial files described in detail in 3.4. The associated sea state data will also archived alongside the WEC data in each trial file.

\subsubsection{Mechanical power}

Instantaneous mechanical power is calculated for the fore and aft generators as the product of the torque and speed signals. The total instantaneous mechanical power is simply the sum of the fore and aft instantaneous powers. The mean mechanical power (fore, aft and total) for each $512 \mathrm{~s}$ trial period is calculated as well.

\subsubsection{Spectral response amplitudes}

As was mentioned previously, the SeaRay is a system comprised of three rigid bodies constrained to move in eight DOF. The IMU installed in the nacelle is measuring acceleration in the three translational DOFs and position in the three rotational DOFs. The final two DOFs, rotation of the floats with respect to the nacelle, are measured by encoders. Double integration of the acceleration signals yields position. As absolute position is not needed for calculation of response amplitude, no initial conditions are required.

For each valid motion signal, response amplitudes as a function of frequency can be estimated using spectral analysis. While the two relative pitch motions are the most important (these motions are directly responsible for power generation), spar pitch and heave are also integrally related to WEC performance. The motions in the other degrees of freedom may yield some insight into the system and may also be used to validate numerical models. It should be noted that some of the motions may be very small compared to noise and as such may not yield useful information.

Each signal will be treated quite similarly to the surface elevation signal. They will be demeaned, detrended, band-pass filtered and subjected to a 1/8 cosine taper before spectral analysis. Rather than estimating the variance density, however, it will be the amplitude spectrum that is estimated. Additionally, the raw spectra will be averaged over 13 adjacent estimates. To insure 
that the estimates are at the same frequencies as the wave amplitude spectrum the record lengths should be identical. Therefore, if motion record shorter than $512 \mathrm{~s}$ it will not be utilized in the spectral analysis. The variable pass band is set for each trial such that energy outside of the range of observed wave excitation is filtered out. Lower and upper thresholds are established where the wave elevation variance density is $2 \%$ of the peak value. These lower and upper thresholds are then modified by a factor of 0.9 and 1.05 , respectively.

\subsubsection{WEC loading}

A number of strains and loads were recorded during the SeaRay deployment. These include mooring line tension at the three connection points, compression force on each of the eight end stops, and structural strain in a total of 8 DOFs from strain gauges embedded at five locations in the hull structure (see 4.3). This data will all be quality controlled and archived in the trial files.

\subsection{8. $\quad$ PLC logged data}

On the $15^{\text {th }}$ of January, 2012, dSPACE crashed and never recovered. At this point, the PLC that was controlling the PTO generators was also used to log what data it could. The only relevant data that the PLC was aware of was voltage and current for both generators, and time. Whereas dSPACE was sampling at $25 \mathrm{~Hz}$, the PLC was only capable of sampling on the order of $6 \mathrm{~Hz}$. Additionally the sampling rate was variable and channels were not synchronized.

The voltage constant, $\mathrm{K}_{\mathrm{e}}$, and torque constant, $\mathrm{K}_{\mathrm{v}}$, of each generator was estimated using dSPACE logged data. This allowed for the voltage and current data to be converted to torque and speed. As stated previously, the signals were not synchronized. As the relationship was controlled to be linear (i.e. torque is equal to a constant times speed), the maximum power will be realized when these signals are properly aligned. To estimate power, the speed signal was shifted in time until maximum power was realized. Furthermore, the current signal saturated low at a positive value which meant that low torque values (below that saturation value) were exaggerated. To remedy this, damping was estimated from the torque (current) data and used to estimate the low torque data from the low speed (voltage) signal.

\subsection{9. $\quad$ Flags and quality control}

A number of flags may be generated for any given record to alert the data handler to potentially erroneous data.

fYawPositionSource - 'ycs' for yaw encoder signal (including corrected signal), 'gps' for dual GPS heading sensor

dampingFitR2 - Coefficient of determination (e.g. $\mathrm{R}^{2}$ ) from linear regression on torque and speed. Low values typically come when PTO nonlinearities are significant, such is in very small seas where friction dominates the PTO. Records with R2 values less than 0.92 will not be included in the performance results. For PLC logged data the threshold was 0.7 and only the fore PTO was considered. The lower threshold is justified because of the low, variable and asynchronous sampling rate of the PLC logged data. 


\subsection{Joint data}

\subsubsection{Alignment of data}

Based on the earliest time stamp for a given 512s record (AWAC or SeaRay), a nominal start time will be assigned. The nominal start times will all fall on the 10 minute mark (e.g. XX:00:00, XX:10:00, XX:20:00, etc.). All times are UTC. AWAC records are associated with SeaRay records via the nominal start time.

\subsubsection{Relative wave heading}

The relative wave direction is calculated as the characteristic wave direction minus the yaw position of the SeaRay. The relative current direction is calculated as the direction of the near surface currents minus the yaw position of the SeaRay.

\subsubsection{Relative capture width}

The relative capture width (RCW) is calculated as the ratio of the mean mechanical power to the omnidirectional wave energy incident upon an imaginary vertical plane that is the width of the SeaRay and extending from the sea floor to the surface. This width is the width of the forward float as seen head on and is $2.57 \mathrm{~m}$. For each valid $512 \mathrm{~s}$ trial, an RCW is calculated for the forward float, the aft float and the total system (fore plus aft).

\subsubsection{Spectral Response Amplitude Operator (RAO)}

For each trial a frequency dependent response amplitude operator (RAO) can be calculated, for each measured degree of freedom. This is simply an element-wise ratio of the response amplitude spectrum to the wave amplitude spectrum.

\subsection{Archived data}

\subsubsection{Processed sea state data}

The raw and processed $512 \mathrm{~s}$ AWAC data is archived in an AWAC data folder. The AWAC data covers periods of time in which the WEC was not fully operational and/or not collecting data. The AWAC data which coincides with SeaRay trials will be merged into the appropriate trial files.

\subsubsection{Corrected raw trial data}

Once the raw data is 'corrected', as outlined in 3.2.4, it is archived in a 'CorrectedRawData' folder. These records are all nominally 19.5 minutes. Records that have no time stamps, were recorded during known down time, or are less than $10 \mathrm{kB}$ (a complete record is approximately $5 \mathrm{MB}$ ) are not archived. Signals that were never used (e.g. S093_Control_Mode) or are irrelevant at this stage (such as signals telemetered from AWAC, e.g. S084_AWAC_Hs) will not be archived. The archived signals, along with a brief description and units, are listed in Appendix E. 


\subsubsection{Trial files}

All relevant signals and derived parameters discussed in 3.1 to 3.3 will be stored in a MATLAB data file. There will be one .mat file per $512 \mathrm{~s}$ trial and it will include all measured and derived data (including flags) regarding an individual trial. With standardized trial file format, if a new parameter is considered at some future time (e.g. an alternate spectral width parameter) it will be easy to loop through each file, calculate the new parameter, and save the new parameter to the file. The format of the data files, along with brief descriptions and units used is described in Appendix F.

\subsubsection{Trial table}

File names, characteristic parameters and flags for all 512 s trials will also be stored in a MATLAB dataset array. This will allow for easy querying of data (e.g. finding all file names of trials with $H_{m 0}$ within a specified range and no flags). A dataset array allows for matrix style organization of data, with a horizontal row representing a trial and each named column representing a parameter. Units are also declared and stored in the dataset array. Two trial tables are archived - one includes only dSPACE logged trials and the other includes PLC logged trials as well. The second covers a greater time frame, but includes fewer parameters as the PLC did not log e.g. WEC motion. The format of the trial tables, along with brief descriptions and units use, are described in Appendix G.

\section{Data Results and Discussion}

\subsection{West Point Resource}

The AWAC was installed and operating for 400 days (the length of the WEC deployment). Accounting for data loss due to quality control and down time between battery swaps, a return rate of $88.9 \%$ is realized. While quality controlled WEC data is not available for correlation with all of the AWAC samples, it is still quite useful to understand the entirety of the resource measured by the AWAC as the WEC was exposed to and survived this environment. An occurrence table over the period of WEC deployment is presented in Figure 12. The occurrence is given as a number of $512 \mathrm{~s}$ samples, each of which accounts for 10 minutes of time. In total, more than 51,000 quality controlled samples are represented in the occurrence table. A quality controlled maximum significant wave height of $1.55 \mathrm{~m}$ was observed.

Wave and current roses are depicted in Figures 13 and 14. These figures indicate the proportional occurrence of waves (or currents) arriving from directional bins of $22.5^{\circ}$ width. Waves tended to arrive from the north and south, as was expected based on available fetch (see Figure 15). Furthermore, nearly all of the larger seas $\left(H_{m 0}>0.3 \mathrm{~m}\right)$ arrived from the north or south. Recall that the YCS allowed for the SeaRay to reorient to the incident wave system. The current primarily arrived from a bearing of approximately $135^{\circ}$. The bathymetry at the West Point test site (see Figure 16) likely had a significant influence on the local currents, such that flood tides (from the north) were shadowed and ebb tides (from the south) flowed around the point.

Columbia Power Technologies, LLC • 4920A SW $3^{\text {rd }}$ St, Corvallis, Oregon 97333

Phone: (541) 368-5033 • Fax (541) 230-1498 • www.columbiapowertechnologies.com 


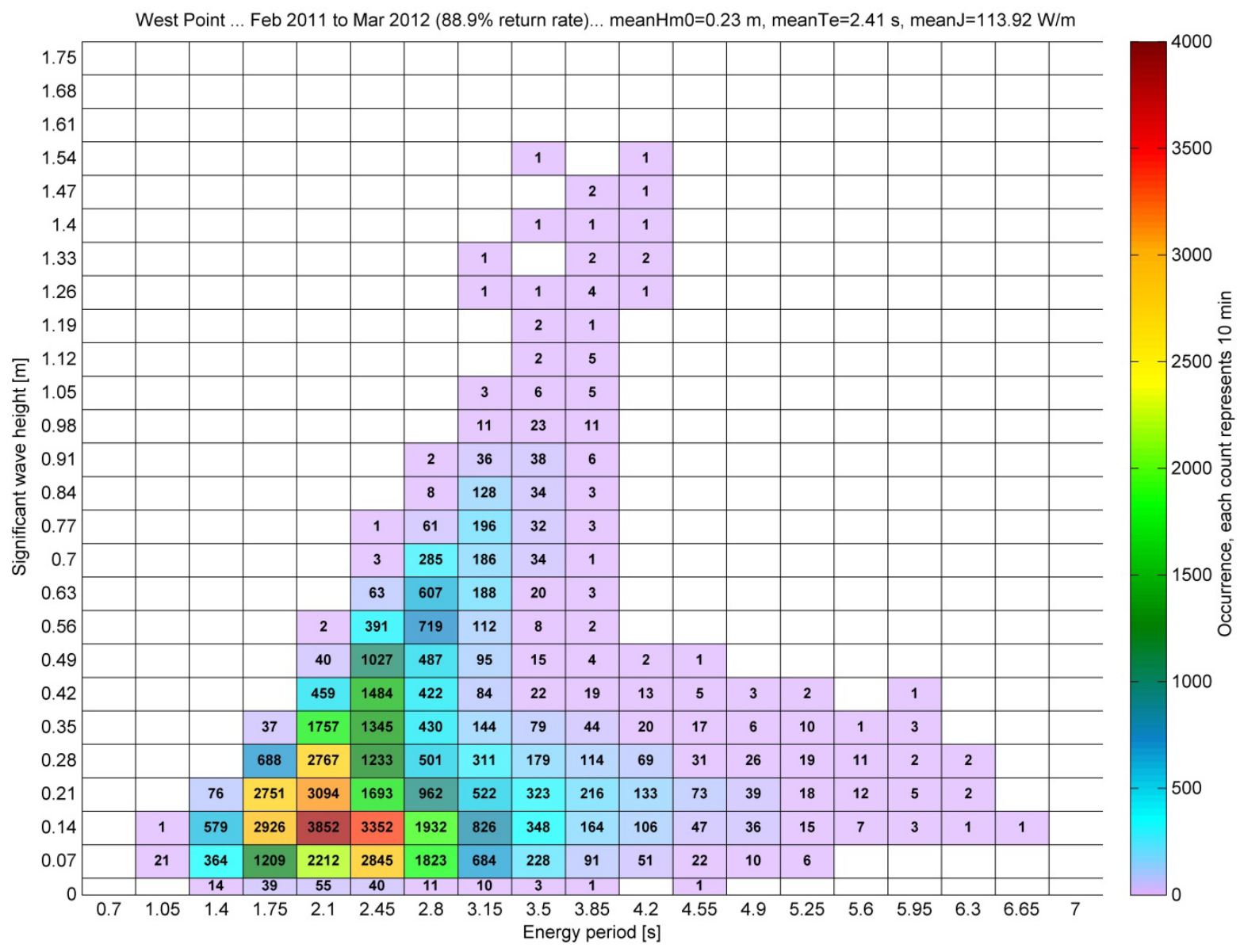

Figure 12. West Point wave resource characterization over WEC deployment period (88.9\% return rate).

Columbia Power Technologies, LLC • 4920A SW $3^{\text {rd }}$ St, Corvallis, Oregon 97333 Phone: (541) 368-5033 • Fax (541) 230-1498 • www.columbiapowertechnologies.com 


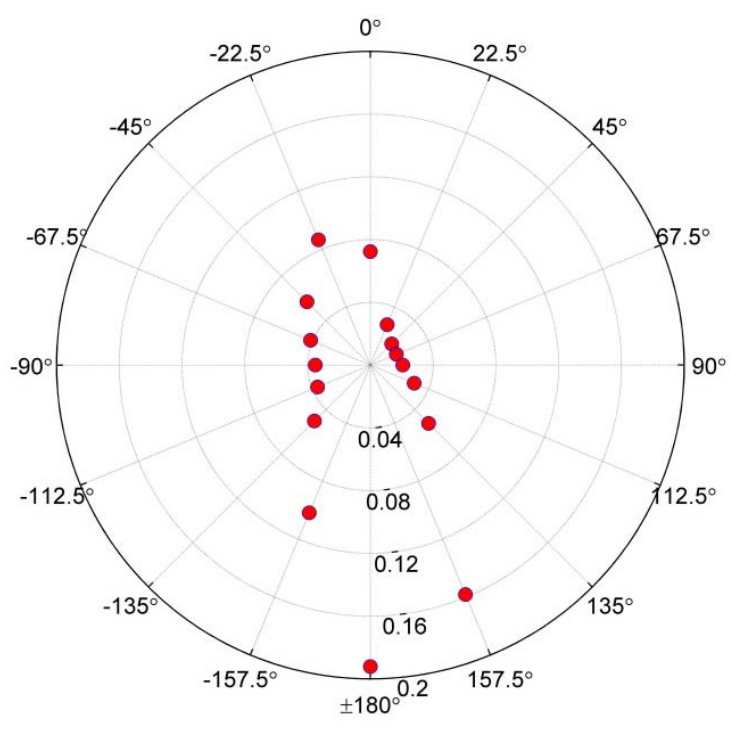

Figure 13. Wave rose for Puget Sound, indicating proportional occurrence for directional bins of $22.5^{\circ}$ width. Direction is w.r.t. true north.

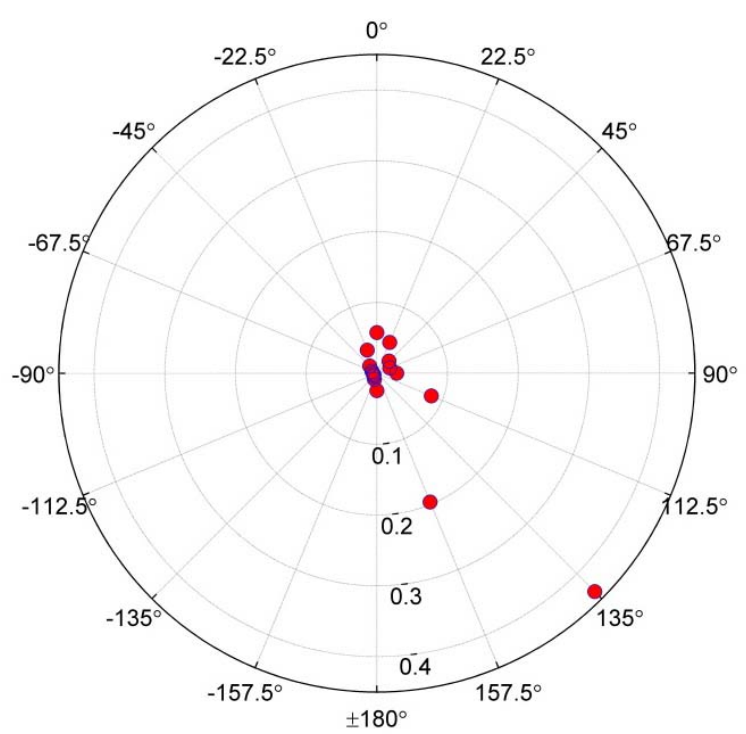

Figure 14. Current rose for Puget Sound, indicating proportional occurrence for directional bins of $22.5^{\circ}$ width. Direction is w.r.t. true north.

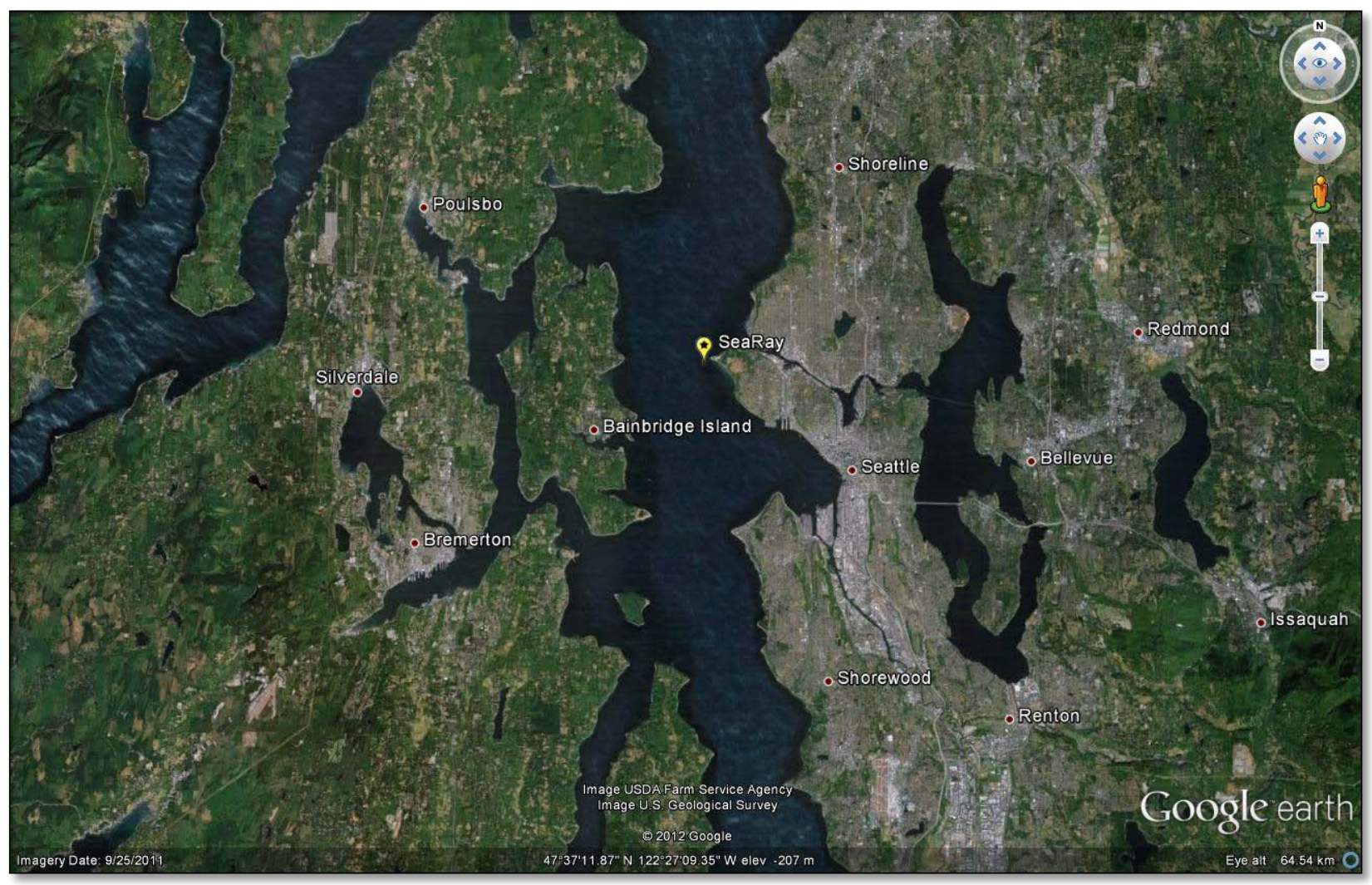

Figure 15. SeaRay test site, note significant north/south fetch.

Columbia Power Technologies, LLC • 4920A SW $3^{\text {rd }}$ St, Corvallis, Oregon 97333 Phone: (541) 368-5033 • Fax (541) 230-1498 • www.columbiapowertechnologies.com 


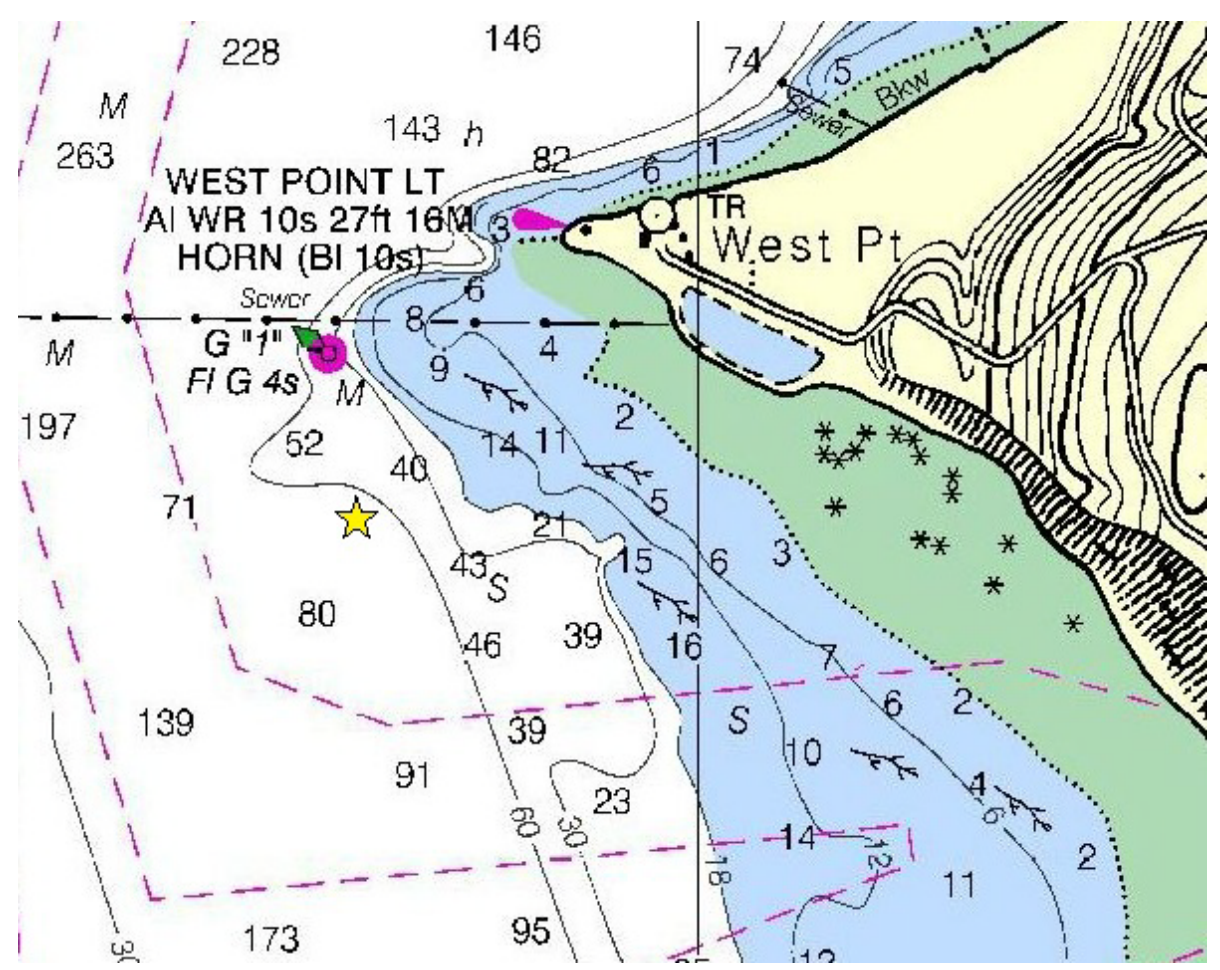

Figure 16. West Point bathymetry (in units of feet), with deployed location of SeaRay indicated.
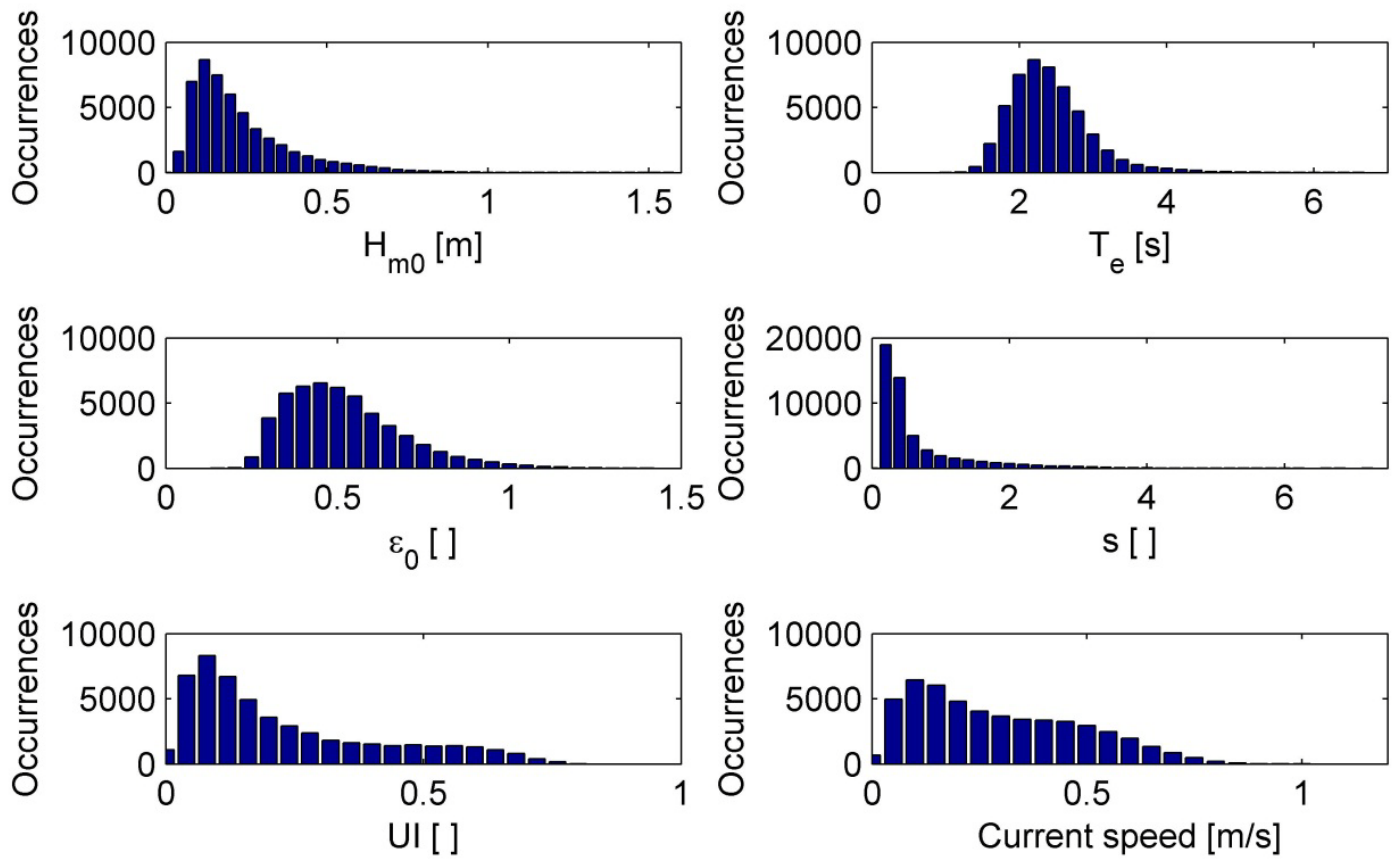

Figure 17. Histograms for wave and current parameters during West Point trials. 
Histograms depicting the distribution of several relevant parameters describing the waves and currents can be found in Figure 17. Taking the scale factor as 7, typical full scale equivalent (FSE) $H_{m 0}$ values range from roughly 0.5 to $3.5 \mathrm{~m}$, covering the range of operational seas expected in open oceans. The maximum observed value of $1.55 \mathrm{~m}$ scales to $10.9 \mathrm{~m}$, which is on the order of design wave height expected in open oceans. FSE $T_{e}$ is typically between 5 and $9 \mathrm{~s}$, which is marginally higher frequency than what would be considered representative of expected open ocean conditions. Thus the waves on the whole tend to be steeper than expected open ocean conditions. UI and s do not scale, and they are both biased more towards lower numbers than would be typical of open ocean conditions, indicating very "confused" and short-crested directional distributions. Although it is not depicted here, analysis shows that increasing wave height correlates with increasing UI and s values. It may be that reflections off the ubiquitous shorelines results in a redistribution of wave energy across directions. The observed spectral width values typically range between 0.3 and $0.8 . \epsilon_{0}$ does not scale, and these values are fairly typical of open ocean environments. Finally, FSE current speeds range from 0.1 to $2.0 \mathrm{~m} / \mathrm{s}$. These values are in excess of what would be considered typical in open ocean conditions.

\subsection{Performance}

\subsubsection{Introduction}

An n-dimensional space will be delineated into a number of cells, each dimension being a parameter to which power performance is significantly sensitive. $H_{m 0}$ and $T_{e}$ are known to have a strong influence on power performance and will be the primary dimensions. Use of these two independent dimensions alone will constitute the primary performance matrix. Cell size for $H_{m 0}$ and $T_{e}$ respectively are $0.07 \mathrm{~m}$ and $0.35 \mathrm{~s}$ (which corresponds approximately to $0.5 \mathrm{~m}$ and $1.0 \mathrm{~s}$ FSE). Sensitivity studies will be performed to determine other relevant dimensions.

Power performance will be understood primarily through the RCW. Although electrical power was generated on the SeaRay, the power electronics do not scale properly and so the WEC power will be understood through the mechanical power. Mechanical power may vary substantially within a cell (e.g. with the square of $H_{m 0}$ ), but by normalizing the mechanical power by the incident wave power to establish the RCW, there should be significantly less variability within a given cell and low variability will increase confidence in the results.

A measure of performance (i.e. RCW) will be presented for each sufficiently populated cell. A minimum of five trials in a given cell is desired for statistical relevance; however, if fewer trials are available for a given cell the information may still be presented along with an indication that fewer than five trials were available.

The central tendency indicated for a given cell is the mean value of either the RCW or mean mechanical power for each of the trials within the bounds of the cell. A measure of dispersion of performance within the cell is also calculated. The metric used is the standard deviation (std) of each performance metric for all trials within the bounds of the cell. Additionally, the uncertainty associated with the estimated central tendency will be calculated. This uncertainty is represented by the $95 \%$ confidence interval (ci) which is calculated as

$$
\mathrm{ci}=t^{*} \frac{\text { std }}{\sqrt{n}}
$$

Columbia Power Technologies, LLC $\bullet$ 4920A SW $3^{\text {rd }}$ St, Corvallis, Oregon 97333

Phone: (541) 368-5033 • Fax (541) 230-1498 • www.columbiapowertechnologies.com 
where $t^{*}$ is taken from a Student's t-distribution table with a two-sided confidence level of 95\% and $n$ - 1 degrees of freedom, and $n$ is the number of trials in the matrix cell. To be clear, the std quantifies the variability of the results within the matrix cell and the ci quantifies the uncertainty associated with the estimation of the central tendency.

Note that, due to practical limitations associated with scale and the data requirements, the mass distribution of the SeaRay differs substantially from the commercial scale design (see Table 2). Extensive numerical modeling indicates that these changes in system mass and mass distribution have a significant effect on WEC response. As such, the observed performance is indicative, but does not accurately describe the response of the commercial scale device.

\subsubsection{Sensitivity to significant wave height and energy period}

The results of trials which pass quality control are placed in a 'primary' RCW matrix with only two independent dimensions (i.e. 2-D matrix). As stated previously, these two primary dimensions are significant wave height and energy period. Performance was found to correlate with the observed energy period to a significantly greater degree than the intrinsic energy period. As such unless otherwise noted all references to $T_{e}$ will be understood to be the observed energy period. The total number of trials for each cell of the primary matrix is given in Figure 18. Note that the majority of the trials are contained in a relatively small area within $H_{m 0}-T_{e}$ space. Primary performance matrices depicting total RCW \pm one std and $\pm 95 \%$ ci are given in Figures 19 and 20, respectively. Note that the standard deviation is quite large for many cells, often on the order of $1 / 3$ or more of the mean value, indicating that there is considerable dispersion amongst the individual trial results. On the other hand the 95\% confidence intervals are relatively small, indicating that the central tendency of the performance response for this data set has been quantified with little uncertainty. Although the variation within each cell is considerable the mean response follows a clear pattern, with the RCW decreasing with increasing $T_{e}$ and to a lesser extent with increasing $H_{m 0}$. From previous experiments as well as numerical modeling, this pattern of dependence upon $H_{m 0}$ and $T_{e}$ was expected. Sensitivity of performance to parameters other than $H_{m 0}$ and $T_{e}$ will be discussed in the following sections. 

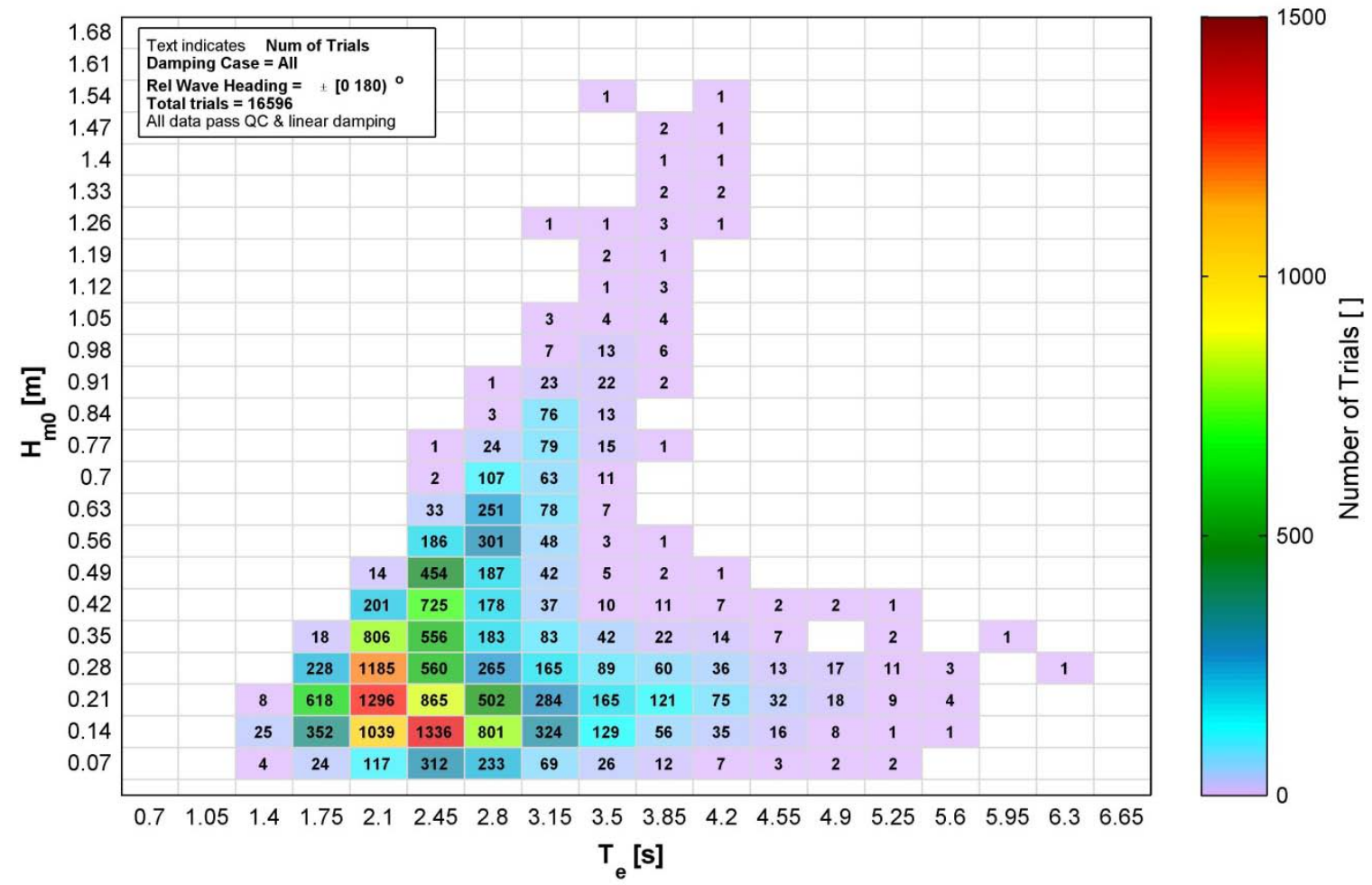

Figure 18. Primary performance matrix... Number of trials.

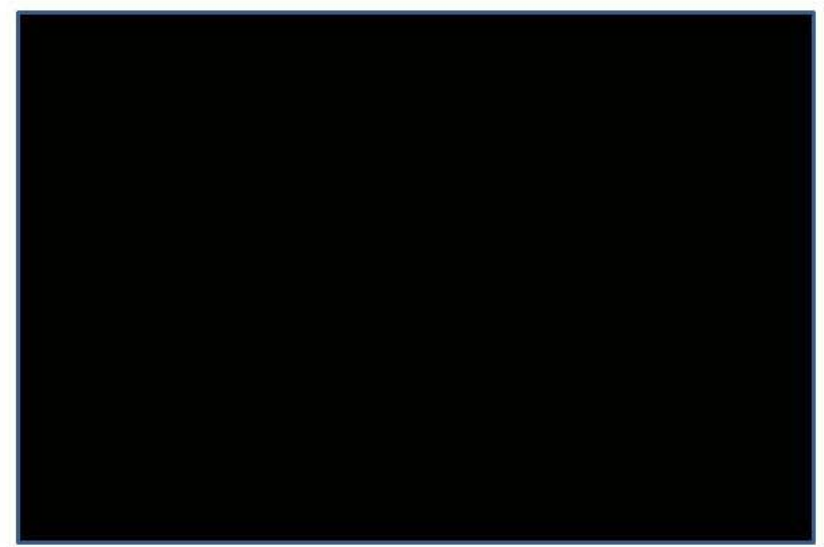

Figure 19. Primary performance matrix... RCW \pm 1 std.

Columbia Power Technologies, LLC • 4920A SW $3^{\text {rd }}$ St, Corvallis, Oregon 97333 Phone: (541) 368-5033 • Fax (541) 230-1498 • www.columbiapowertechnologies.com 


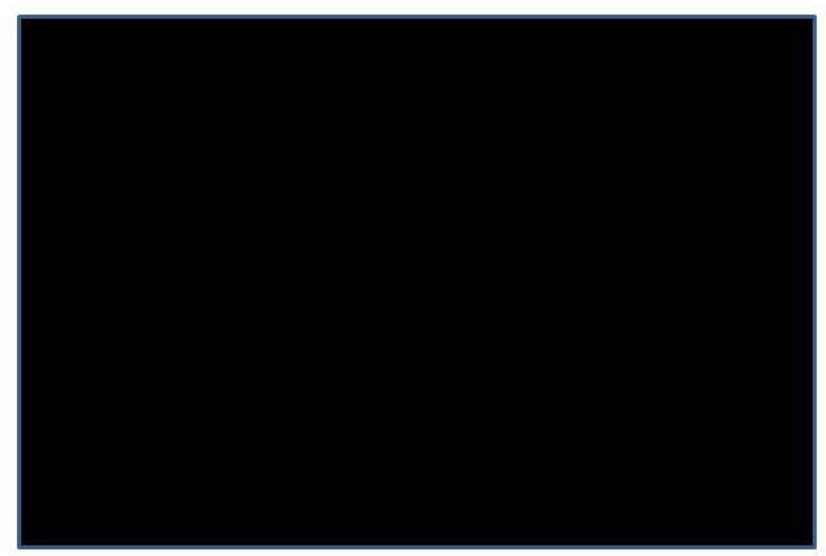

Figure 20. Primary performance matrix... RCW $\pm 95 \%$ ci.

\subsubsection{Sensitivity to PTO damping}

The PTO damping estimated via least squares linear fit of measured torque and speed data is depicted in Figure 21. Overdamping (large damping designed as a backup and for extreme seas) was applied for a few days at the beginning of the test, and again in May. An operational damping level was applied for the remaining trials. Various control strategies were implemented with differing power electronic control hardware during the 13 month long test. This is the reason that different regions of damping (or damping cases) are observed over time. Four broad regions were identified, and are listed in Table 3 . The bounds on damping regions are somewhat arbitrary, and serve to group trials with similar damping while excluding trials whose observed damping deviated significantly from the majority. Only those trials for which the observed damping was sufficiently linear (see 3.2.9) are included in the performance analysis. As is apparent in the Figure 21, there is very little data for the two overdamped cases. Also, operationalLowAft was primarily applied in the winter months, while operationalHighAft was primarily applied in the relatively calm summer months. 


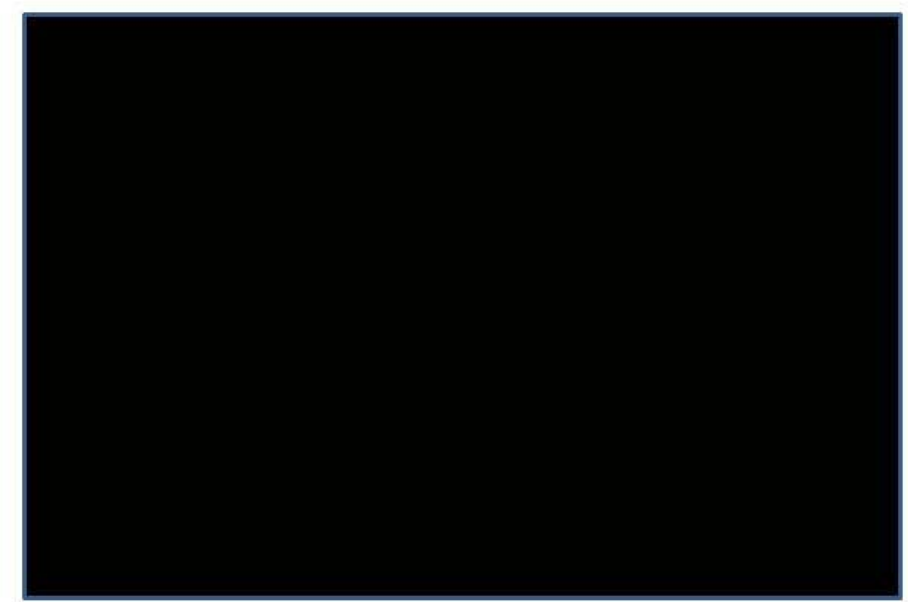

Figure 21. Time history of PTO damping.

Table 3. Damping cases.

\begin{tabular}{ll|l}
\hline & Fore [kN m s] & \multicolumn{1}{c}{ Aft[kN m s] } \\
\hline $\begin{array}{l}\text { operationalLowAft } \\
\text { operationalHighAft } \\
\text { overdampedLow } \\
\text { overdampedHigh }\end{array}$ & & \\
\hline
\end{tabular}

Figure 22 depicts WEC performance in head on seas as a function of observed energy period for each of four damping cases. The mean RCW for a given $T_{e} / H_{m 0}$ bin, along with error bars indicating the $95 \% \mathrm{ci}$, is used to show the trends. The three plots depict WEC performance as the total RCW, the fore generator RCW and the aft generator RCW. Data points for which there are fewer than 5 trials are indicated, as there may be insufficient data to draw strong conclusions. A single, well-populated, $H_{m 0}$ value (over one bin width) is selected as representative. Head on seas are here defined as a relative wave heading of less than $\pm 22.5^{\circ}$. Thus, each point on the plot (mean and confidence interval) is calculated from quality controlled data from a single bin of a performance matrix with four independent dimensions: $H_{m 0}, T_{e}$, relative wave heading and damping case. 

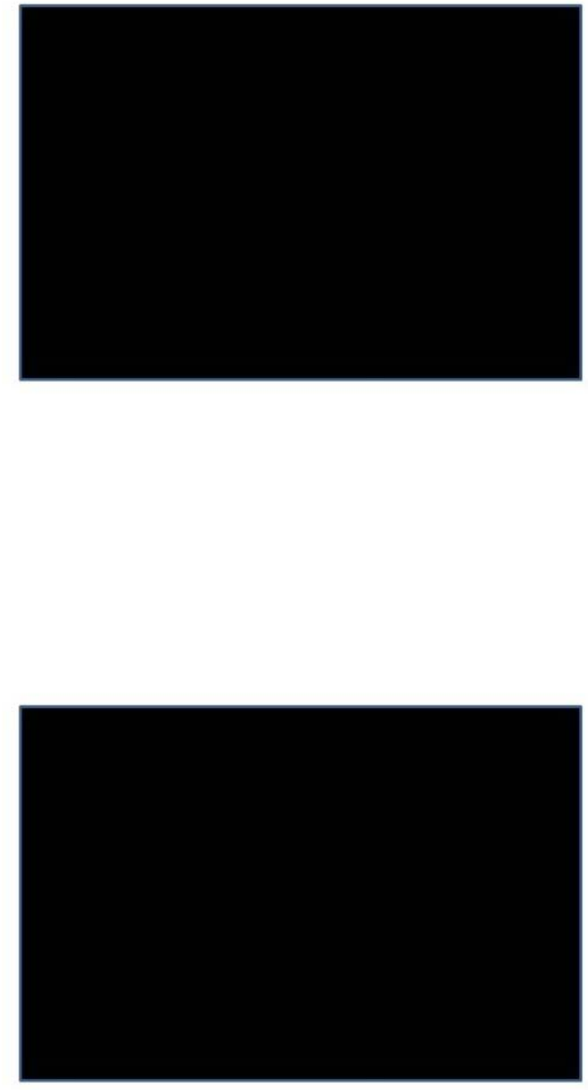

Figure 22. Sensitivity of WEC performance to PTO damping.

Columbia Power Technologies, LLC • 4920A SW $3^{\text {rd }}$ St, Corvallis, Oregon 97333 Phone: (541) 368-5033 • Fax (541) 230-1498 • www.columbiapowertechnologies.com 
Looking first at the total RCW, the expected WEC performance for the overdampedHigh case shows the greatest deviation from the rest of the damping cases. The differences in expected performance between the other three cases are generally small, and may be considered insignificant on their own, at least over the range of energy periods for which there is sufficient data for all four damping cases. However, looking at the individual performance of the fore and aft generators, the sensitivity to damping case becomes more apparent. There is not sufficient data over a great enough damping range to draw much in the way of general conclusions about WEC performance and generator damping. That being said, it is clear that the most heavily damped case (overdampedHigh) resulted in relatively poor performance. Furthermore, the observed sensitivity of performance to the four identified damping cases justifies segregation of data along this dimension. This plot also further illustrates the sensitivity of performance on energy period.

\subsubsection{Sensitivity to relative wave heading}

Having accepted $H_{m 0}$, Te, and PTO damping as performance indicators, the next dimension investigated was relative wave heading. Damping case operationalHighAft has the most data points, at a variety of wave headings, and is thus most useful in understanding the sensitivity to wave heading. To retain sufficient data points in each bin and smooth out variation, a wave heading bin size of $45^{\circ}$ is selected (i.e. nominal value $\pm 22.5^{\circ}$ ). Furthermore, the symmetry of the WEC allows us to consider only the absolute value of relative wave heading. In other words, we assume that the WEC responds to waves from a $+60^{\circ}$ heading identically to waves from a $-60^{\circ}$ heading.

Figure 23 depicts WEC performance as a function of wave heading for a variety of observed energy periods. The mean RCW for a given $T_{e} / H_{m 0}$ bin, along with error bars indicating the 95\% ci, is used to show the trends. The three plots depict WEC performance as the total RCW, the fore generator RCW and the aft generator RCW. Data points for which there are fewer than 5 trials are indicated, as there may be insufficient data to draw strong conclusions. Thus, each point on the plot (mean and confidence interval) is calculated from the quality controlled data contained in a single bin of a matrix with four independent dimensions: $H_{m 0}$, Te, relative wave heading and damping case. To illustrate how the sensitivity to direction is influenced by the frequency content of the sea state, data for a number of energy periods are overlaid. 

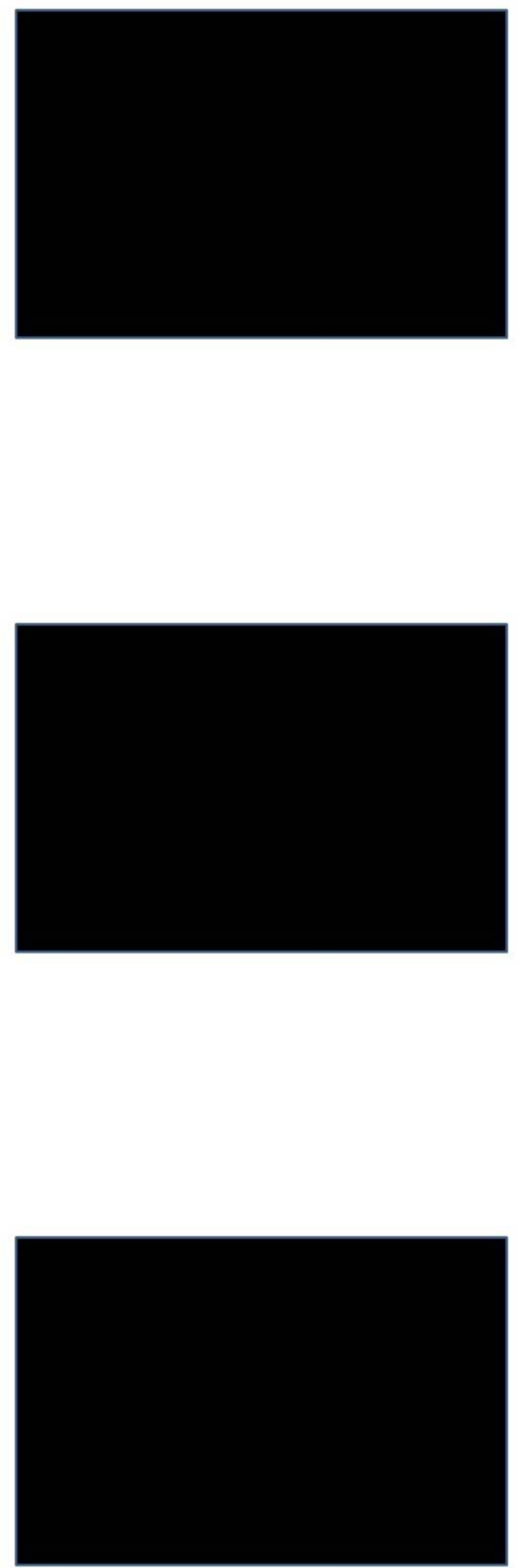

Figure 23. Sensitivity of WEC performance to wave heading.

Columbia Power Technologies, LLC • 4920A SW $3^{\text {rd }}$ St, Corvallis, Oregon 97333 Phone: (541) 368-5033 • Fax (541) 230-1498 • www.columbiapowertechnologies.com 
Focusing first on the total RCW, we see that for shorter period waves there is a marked reduction in RCW as the wave's transition from head, to beam, to following. This performance trend lessens as the energy period increases, and the wave lengths become longer; eventually the trend flattens out, with similar RCW for head and following waves and somewhat of a reduction in RCW for beam waves. The performance of the aft float increases with following waves, for all but the shortest period sea states. Over a range of energy periods this increased aft performance with following wave's balances out the decrease in fore performance, resulting in a relatively flat total performance. A notable exception is the shorter periods $\left(T_{e} \lesssim 2.45 \mathrm{~s}\right.$ ), where the drastic reduction in fore RCW is accompanied by an aft RCW reduction or perhaps slight improvement. It is clear from Figure 23 that there is in general performance sensitivity to relative wave heading, and it is assumed that a bin width of $45^{\circ}$ strikes an appropriate balance between resolution and the available number of data points.

Note that the waves observed in the Puget Sound were exceptionally short-crested when compared to what is expected in the open ocean (see ), and as such the sensitivity of performance to relative wave heading observed in the SeaRay experiment may be less than would be expected for an open ocean deployment.

\subsubsection{Sensitivity to other factors}

After verifying that $H_{m 0}, T_{e}$, PTO damping and relative wave direction are parameters to which performance is significantly sensitive, other parameters were investigated. A total of 12 bins in a four dimensional matrix were selected. The four dimensions are described above, and the selection was as follows:

- $H_{m 0} / T_{e}$ : a 3 by 4 block of neighboring bins, with 18 to $229 \mathrm{QC}$ trials in each

- relative wave heading: head on waves $\left(0 \pm 22.5^{\circ}\right)$

- PTO damping: operational high aft damping (run for the majority of the experiment)

For each bin, a selected parameter was plotted versus total RCW. A linear regression was performed (using MATLAB regress function), and the p-value and correlation coefficient $\left(\mathrm{R}^{2}\right)$ was calculated. A significance level of 0.05 was assumed for the p-value, meaning that a p-value less than 0.05 indicated that the null hypothesis could be rejected (i.e. apparent correlation was not a result of pure chance). The $\mathrm{R}^{2}$ value indicates the proportion of variability accounted for by the independent variable. An $\mathrm{R}^{2}$ value greater than 0.10 was taken to indicate that the parameter in question accounted for a significant amount of the variability remaining after binning by $H_{m 0}$, $T_{e}$, relative wave heading and PTO damping. Table 4 indicates, for each parameter investigated, how many of the 12 data sets (defined by a four dimensional bin) passed the p-value threshold and how many passed the $\mathrm{p}$-value and $\mathrm{R}^{2}$ threshold. Based on an interpretation of these results only two of these parameters were seen as significant: UI and spectral fit quality.

Table 4. Significance of various parameters on performance.

\begin{tabular}{lllr}
\hline Parameter & Name in trial table & $\begin{array}{l}\text { \# passing p- } \\
\text { value }\end{array}$ & $\begin{array}{l}\text { \# passing p- } \\
\text { value } \text { and R }^{2}\end{array}$ \\
\hline Relative current speed & relCurrSpeed_Jweight & 4 & 1 \\
Unidirectivity index & UI & 8 & 5 \\
\hline
\end{tabular}

Columbia Power Technologies, LLC $\bullet$ 4920A SW $3^{\text {rd }}$ St, Corvallis, Oregon 97333

Phone: (541) 368-5033 • Fax (541) 230-1498 • www.columbiapowertechnologies.com 


\begin{tabular}{llll}
\hline Spreading angle & spreadAngle_Jweight & 8 & 5 \\
Spreading index & spreadIndex_Jweight & 6 & 5 \\
Gamma of fitted JONSWAP spectra & gamma_fit & 3 & 0 \\
Spectral fit quality & fitQ & 9 & 6 \\
Spectral width & eps0_obs & 4 & 1 \\
Significant steepness & Ss & 4 & 2 \\
Water depth & waterDepth & 1 & 0 \\
\hline
\end{tabular}

Any of three parameters describing directionality could be seen as significant, but UI accounts more completely for the directional variation observed in a sea state (see 3.1.7), and as such is preferentially selected. As expected, RCW is positively correlated with UI; as more of the wave energy is incident from head on, the WEC captures a greater share of the power.

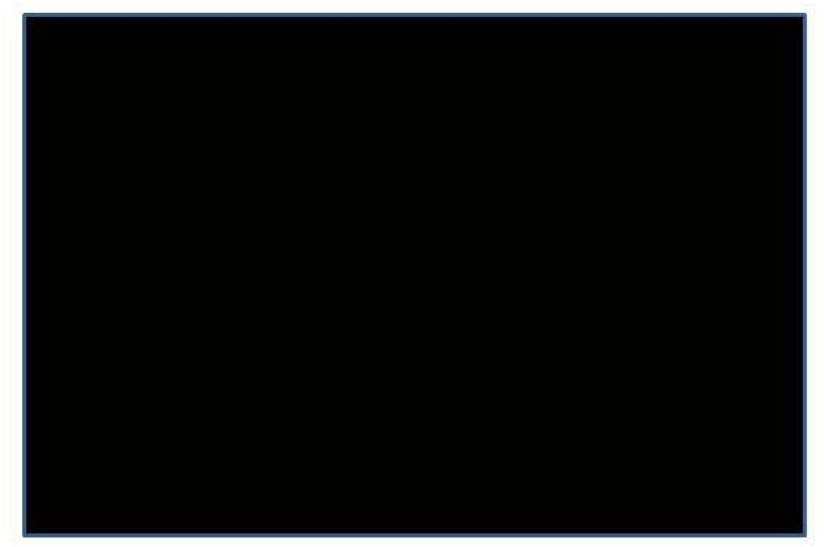

Figure 24. Sensitivity of RCW to UI, for head waves and operationalLowAft damping. 


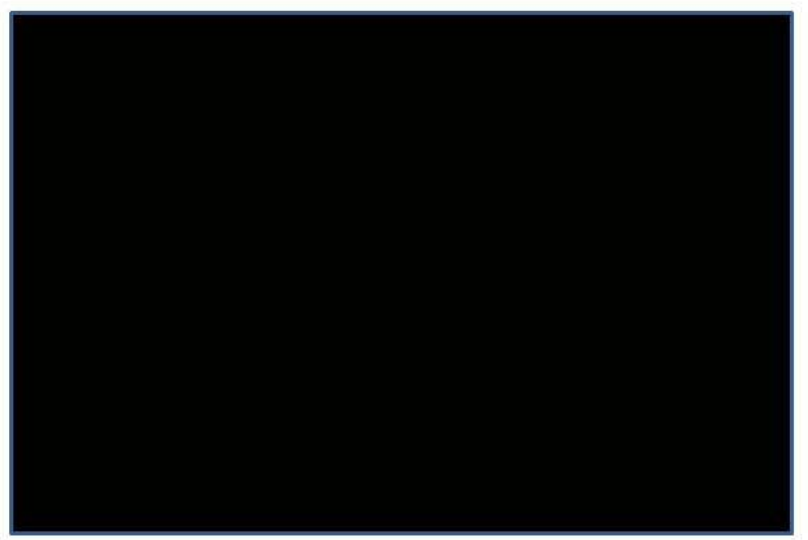

Figure 25. Sensitivity of RCW to fitQ, for head waves and operationalLowAft damping.

The spectral fit quality, fitQ, is a normalized parameter which characterizes how well the measured spectra conform to a JONSWAP form. After fitting a JONSWAP to the measured, RMS error and bias error are calculated. The sum of these two errors is normalized by the zeroth moment of the measured spectrum. A small number indicates a good quality of fit. It seems that, at least in some bins, a measured spectrum that deviates significantly from the JONSWAP form is a predictor of poor performance.

Based upon the sensitivity of performance to directionality (as characterized by UI) and spectral shape (as characterized by fitQ), these two additional dimensions are added to the performance matrices. To avoid an over dilution of the number of trials in a given bin, division along each of these dimensions is limited to two bins. The threshold for directionality was set at UI $=0.3$, and the threshold for spectral shape was set at fitQ $=0.75$. With the inclusion of fitQ and UI into the list of parameters to which performance is sensitive, the performance data can be sorted into a six dimensional matrix.

Performance matrices indicating the central tendency of RCW and bin-wise variability (represented by one std) are given for several cases. In each case the full range of observed $H_{m 0} / T_{e}$ combinations will be given, while the other four dimensions are held constant. The three cases that will be included in this report are:

- Head on, operationalHighAft damping, high UI and good fitQ (Figure 26)

- Head on, operationalHighAft damping, low UI and poor fitQ (Figure 27)

- Head on, operationalLowAft damping, high UI and good fitQ (Figure 28) 
Note the comparison between the performance matrix with high UI and good quality of fit, and the one with low UI and poor quality of fit (Figures 26 and 27). Where there is overlap a clear distinction between performance is evident, with performance significantly better in sea states that are more unidirectional and conform more closely to the JONSWAP shape. The performance matrix shown in Figure 28 (high UI and good quality of fit, but with operationalLowAft damping case) is included to give performance results for more energetic sea states that were observed in the winter months. Where there is overlap Figures 26 and 28 largely show agreement. This is to be expected based on the damping investigation of 4.2.3, in which it was shown that the two operational damping cases showed moderately different performance when the fore and aft floats were investigated separately, but relatively little difference when the total performance was considered. When comparing any of these refined matrices to the primary matrix given in Figure 19, it is seen that the variation within a significant number of bins has been reduced by sorting the data over parameters beyond $H_{m 0}$ and $T_{e}$. That being said, there is still a considerable amount of variation that has not been correlated to any measured parameters.

Figure 26. Performance matrix: head on, low spreading, operationalHighAft damping. 


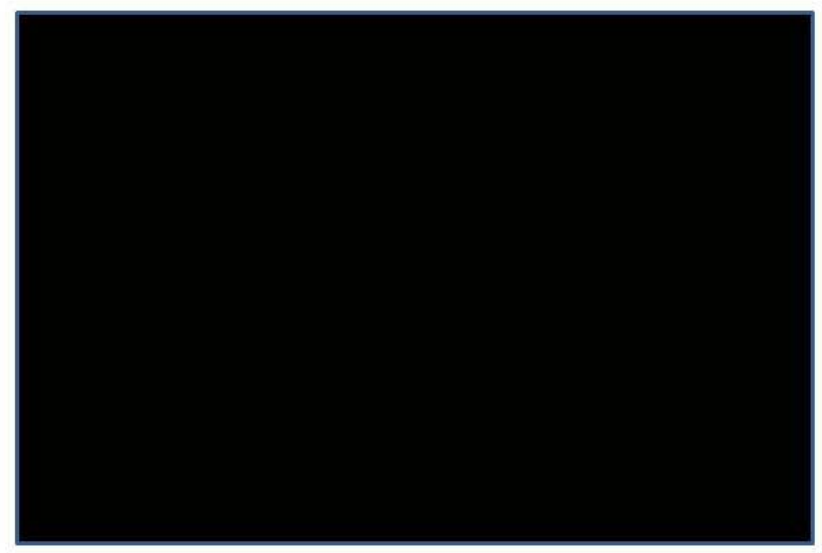

Figure 27. Performance matrix: head on, high spreading, operationalHighAft damping.

Figure 28. Performance matrix: head on, low spreading, operationalLowAft damping.

Columbia Power Technologies, LLC • 4920A SW $3^{\text {rd }}$ St, Corvallis, Oregon 97333 Phone: (541) 368-5033 • Fax (541) 230-1498 • www.columbiapowertechnologies.com 


\subsubsection{Conclusion}

WEC and environmental data was collected for nearly 25,000 trials of 512 s duration. After quality control of sea state data and WEC PTO data, roughly 16,500 trials remained for consideration. These remaining trials were sorted into a six-dimensional matrix (see Table 5). Each of these dimensions is defined by a parameter to which WEC performance has shown a significant experimental sensitivity.

Table 5. Parameters to which WEC performance was observed to be sensitive.

\begin{tabular}{lll}
\hline Parameter & Name in trial table & Binning \\
\hline Significant wave height & Hm0_int & 0.07 m bin width \\
Energy period, observed & Te_obs & $0.35 \mathrm{~s}$ bin width \\
Relative wave heading & relWaveHeading & $45^{\circ}$ bin width \\
PTO damping & - & 4 defined fore/aft ranges \\
Directional spreading & UI & UI $<0.3$ and UI $\geq 0.3$ \\
Spectral shape & fitQ & fitQ $<0.75$ and fitQ $\geq 0.75$ \\
\hline
\end{tabular}

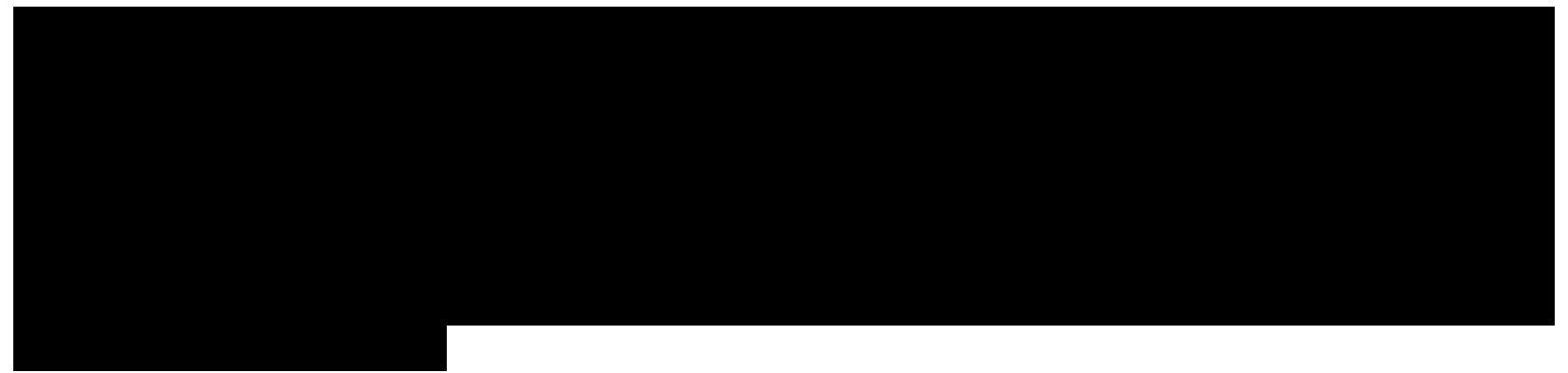

The effect of relative wave heading on WEC performance is more complex. Generally speaking, there is a reduction of performance (as defined by total RCW) as the waves transition from head, to beam, to following. This trend gradually flattens out as the sea states transition from shorter energy period to longer. It is plausible that

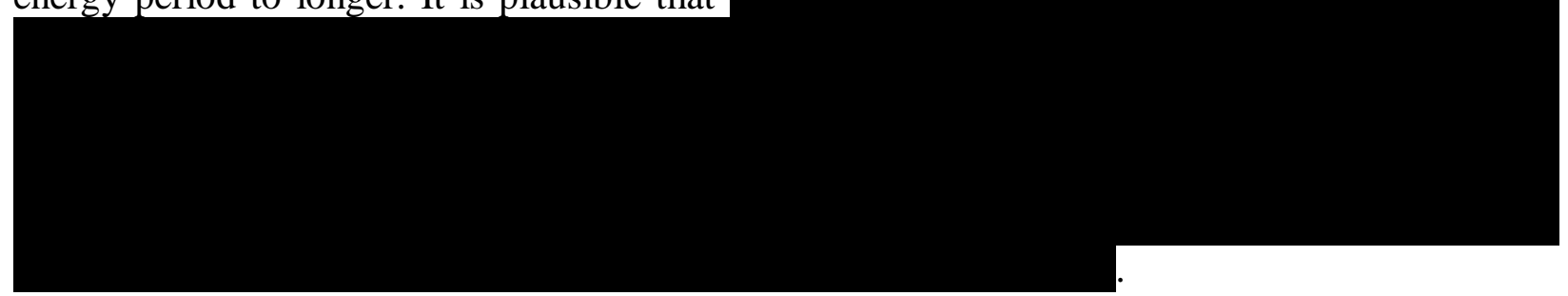

The effect of PTO damping on performance is somewhat difficult to assess, in part because there is not an abundance of trials with significantly differing damping cases. The small number of trials where a 'survival' damping was applied (a damping designed to reduce relative motion) show a significant reduction in performance. The other cases do not show great distinction in performance when the total RCW is used as the metric. However when the two floats are 
investigated independently, it becomes apparent that

The effect of wave directional spreading is characterized using the Unidirectivity Index. As expected, performance in positively correlated with UI; as more of the wave energy is incident from head on, the WEC captures a greater share of the power. This trend diminishes somewhat with increasing energy period, as discussed in a previous paragraph. The correlation of performance with spectral shape is not so easily explained. That being said, performance is negatively correlated with the fitQ statistic, indicating that for sea states that do not conform to the JONSWAP form performance goes down.

After accounting for these six parameters, there is still considerable variation in the performance data that is unexplained. This variation in performance could be related to some factor (WEC or environmental) that is not fully understood, or was unexpected and thus unmeasured. Another possibility is error in measurement; error in measuring torque or speed, or wave height or direction, for example, may be responsible for some or all of the observed variation. Wave direction is a feasible candidate, as the AWAC was unable to resolve wave direction for wave energy propagating with a frequency above $0.45 \mathrm{~Hz}$. Another plausible factor is changes in PTO control strategy and power electronics hardware, or other time-varying changes to the PTO such as changes in friction.

Along with the performance sensitivities that have already been discussed,

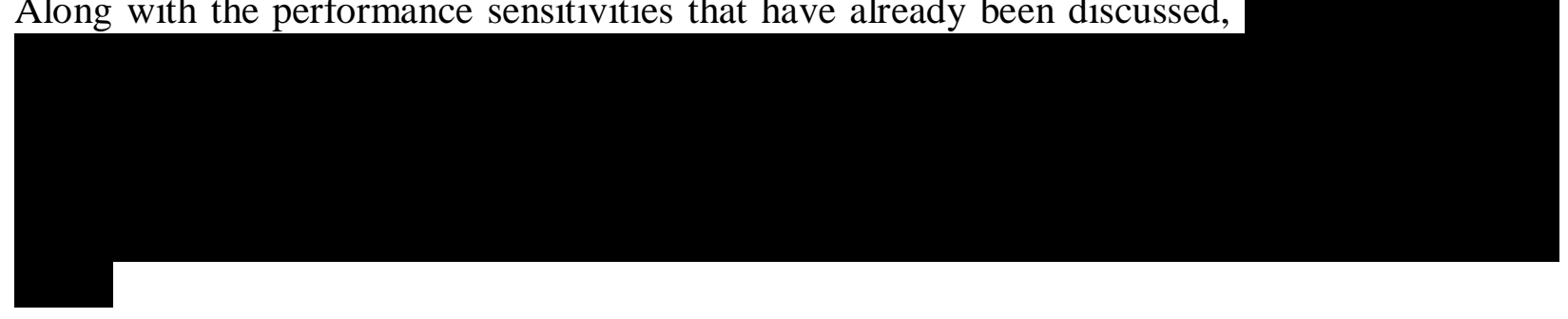

\subsection{Design Data}

\subsubsection{Introduction}

The heavily instrumented SeaRay yields not only performance data, but data that informs design as well. Load cells were installed at the mooring fairleads, in-line with the three mooring lines. A total of eight end stops were mounted on the nacelle, their function to non-destructively stop a float from traveling into the mast, or down into the spar. Load cells were installed in the mounting plates that secured the end stops to the hull. Furthermore, strain gauges were installed on the inner surface of the hull at five locations: two in the spar, and three in the nacelle. All of this data has been quality controlled and archived, and is discussed in the following sections.

\subsubsection{Mooring loads}

As mentioned previously, the SeaRay was deployed twice and the anchor locations were not identical. See Appendix C for documentation of mooring orientation for each of the two deployments (Feb 15, 2011 and March 11, 2011). One effect of the different anchor locations was a slight reorientation of the mooring system, with the second deployment resulting in a clockwise rotation of $10^{\circ}$ w.r.t. the first deployment. Additionally, the pretension of the lines 
during the second deployment was significantly greater (roughly five times greater). The pretension for each line was estimated by the following method. A small number of trials with exceptionally small waves and currents were identified, and mean mooring tension was calculated (see Appendix C for details). Due to the significant change in pretension, only those trials taking place after the second installation are considered in this section. For these trials the south anchor was at a heading of $173^{\circ}$ w.r.t. the centroid of the three anchors. The three anchors were spaced roughly $120^{\circ}$ apart.

At least two additional factors further complicate analysis of the mooring loads. The WEC was able to rotate relative to the mooring system using the Yaw Control System. As such the mooring lines were always oriented to true north in the same manner, but their orientations w.r.t. the WEC changed from trial to trial. Thus the 'south line', for example, was not consistently the 'port line'. Another complication arises from the not infrequent down time experienced in the mooring load signals (see Appendix D for detailed down time listing).

There are many parameters to which the mooring loads may be sensitive. Rather than exploring these all, at this time the investigation is limited to the factors assumed to dominate the response. Mean mooring loads are assumed to be dominated by the current speed and wave driven oscillatory mooring loads. With the three point mooring arrangement, clearly the current and wave direction is also important. Figure 29 depicts the mean mooring load for currents associated with the ebb tide and the flood tide. The median heading from which the ebb tide current arrived at the WEC location was $139^{\circ}$, and for the flood tide current the median heading was $5^{\circ}$. Defining ebb and flood tide currents as those falling within $\pm 22.5^{\circ}$ of these median values, and sorting trials into bins based on current speed of a width of $0.1 \mathrm{~m} / \mathrm{s}$, the mean response of each of three mooring lines was calculated. The mean mooring loads trends shown in Figure 29 follow the patterns expected for the observed current headings; the mooring lines facing the oncoming current trend up with current speed and the one in the lee trends down.

Columbia Power Technologies, LLC $•$ 4920A SW $3^{\text {rd }}$ St, Corvallis, Oregon 97333

Phone: (541) 368-5033 • Fax (541) 230-1498 • www.columbiapowertechnologies.com 

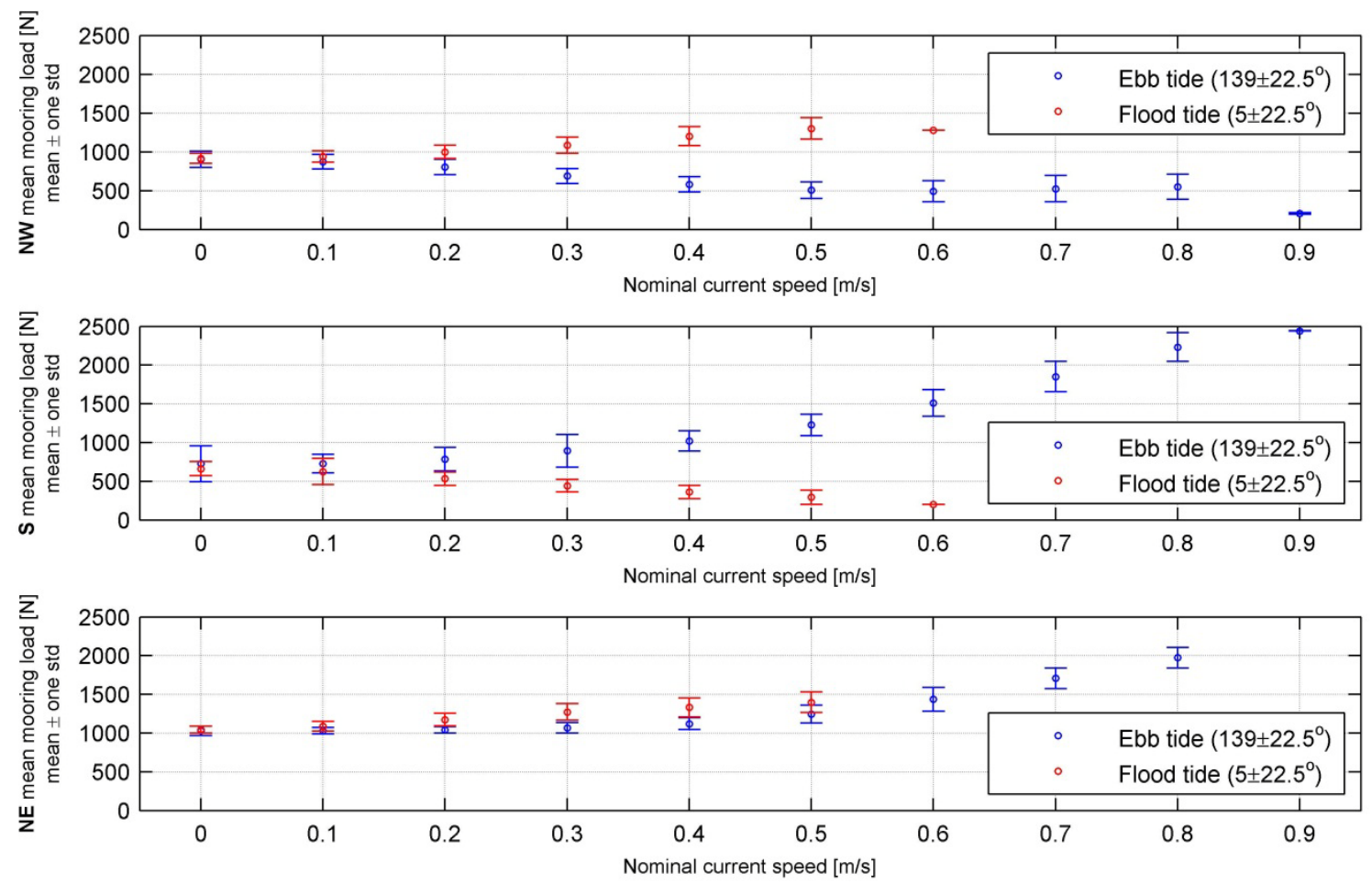

Figure 29. Mean mooring loads versus current speed.

Figure 30 depicts the oscillatory mooring load response as a function of wave heights. To compare seas where the waves were coming up one mooring line, and those where the waves were incident from a heading falling in between two lines (which can then share the load), two nominal directions were selected. South seas were defined as $173 \pm 22.5^{\circ}$ and southwest seas as $233 \pm 22.5^{\circ}$. These central values correspond to the waves coming up the south mooring line, and those arriving between the south and northwest lines. With increasing wave height and south seas, the south mooring line RMS response steadily climbs. With southwest seas, the south mooring line RMS response climbs only a bit more slowly; it is not clear why this distinction is not clearer. The same can be said for all three mooring lines where a mean wave heading shift of $60^{\circ}$ appears to have little effect on the oscillatory load response. In any case we see that the current induced mean loading, in general, is significantly greater than the wave induced oscillatory loading. Note that the south line data is not shown for higher seas because the signal had failed before going into the storms of the second winter (see Appendix D). 

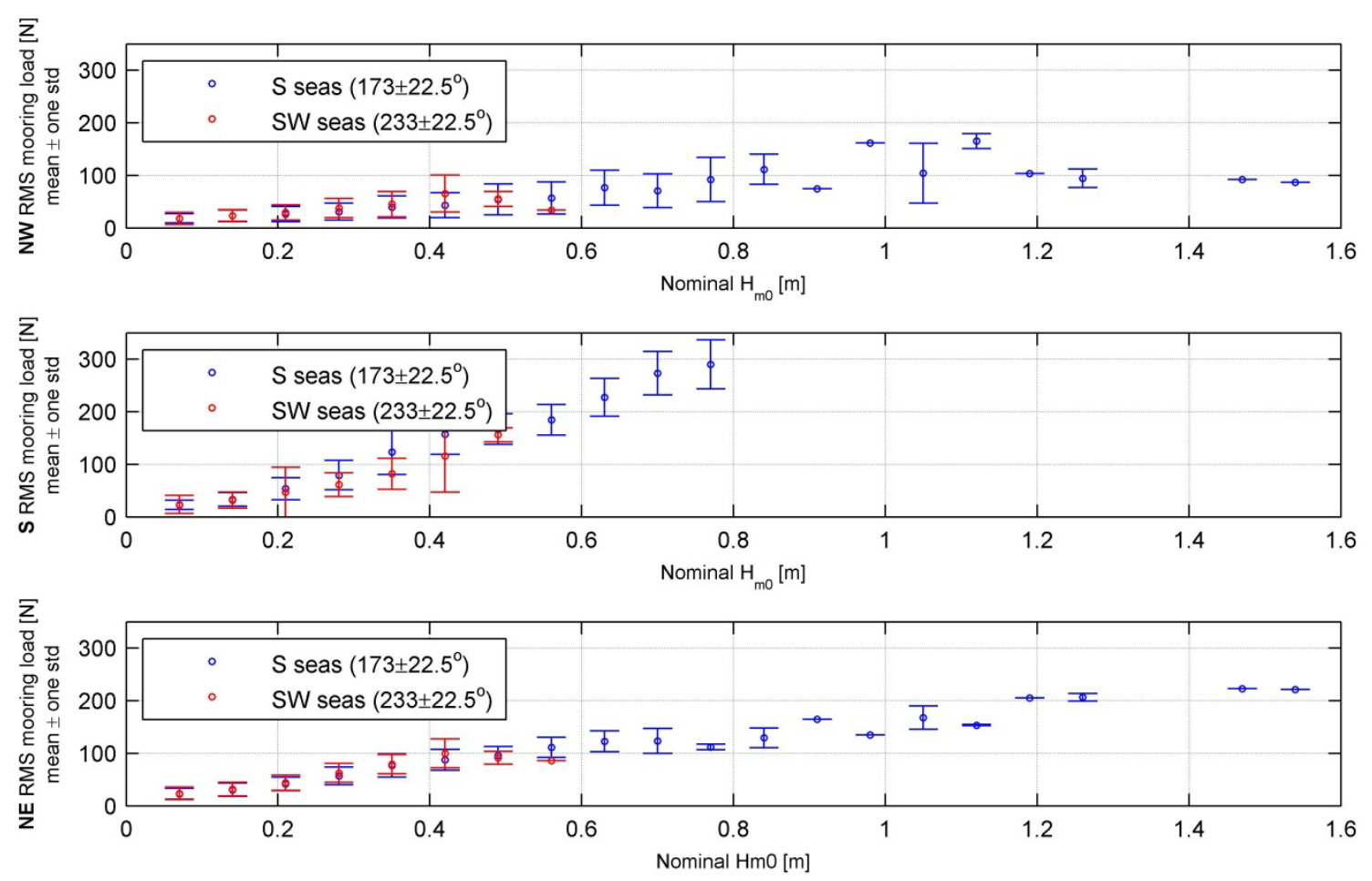

Figure 30. RMS mooring loads versus significant wave height.

\subsection{3. $\quad$ End stop loads}

End stops, manufactured by Galvi of expanded polyurethane foam, were installed on custom made mounting plates fitted with strain gauges. The end stop assemblies were calibrated in the laboratory, resulting in a $3^{\text {rd }}$ order polynomial fit for strain to compressive load conversion. End stops were mounted on the nacelle in port and starboard pairs, their function to non-destructively stop a float if it were to travel up to close to the mast, or down to close to the spar (see Figure 1).

Interpretation of the end stop load signal was somewhat challenging in that the signal was fairly noisy. There was a persistent noise floor present, the level of which was not consistent over the length of the experiment. Additionally, spurious spikes were observed. The float encoder signal can be used to help sort out this confusion, but unfortunately there was some drift in the encoder position. As such the following methodology was employed to quality control the end stop load signal. To filter out the low level noise a lower threshold is established during each trial, for each end stop. This threshold is calculated as the median of the signal plus one $\operatorname{std}_{\mathrm{iqr}}$ of the signal. To filter out the noise showing up as spikes, end stop strikes must correlate with peak position. As such, only those apparent strikes that occur when float position is less than $3^{\circ}$ away from peak position pass quality control. A logical vector is established for each pair of end stops; cotemporal samples from both signals must pass noise threshold and the position test for a one value to be assigned at that time step. Finally, a median end stop signal threshold of $5 \mathrm{kN}$ was established; for any trial in which the median signal did not exceed this threshold, it is assumed that the load signal was erroneous. This last quality control excludes some trials in which the load cells reported a very high bias in the noise floor. 
Figure 31 depicts the maximum end stop load exerted on the float for each trial in which end stop strikes were detected. The load is reported as the sum of the observed loads from the port and starboard pair. Note that the greatest number of events and the greatest loads impinged upon the aft float, in the upwards position. This is almost certainly explained in large part by the range of motion allowed in each of the four quadrants. The fore float was allowed $43^{\circ}$ and $45^{\circ}$, up and down respectively, from still water resting position. The aft float was allowed $33^{\circ}$ and $47^{\circ}$, up and down respectively, from still water resting position. With a greater range of motion the PTO damping has more time to slow down the float, and the float is also allowed to move further from the excitation of the wave. Interestingly, waves coming from the back did not necessarily increase the likelihood or the force of aft float impact. Although nearly all of the impacts with the aft bottom end stops did come from following waves. Finally, early in the experiment the PTOs were allowed to freewheel for the better part of day, during a storm event. As expected, the end stop strikes during this freewheeling period tend to be more extreme. Freewheeling is considered a failure state that must be considered in design and as such this data is invaluable. The most extreme strike observed, in full scale equivalent terms assuming a scale factor of 7 , was roughly $14 \mathrm{MN}$ and occurred in a sea state where $H_{m 0}=4.5 \mathrm{~m}$.
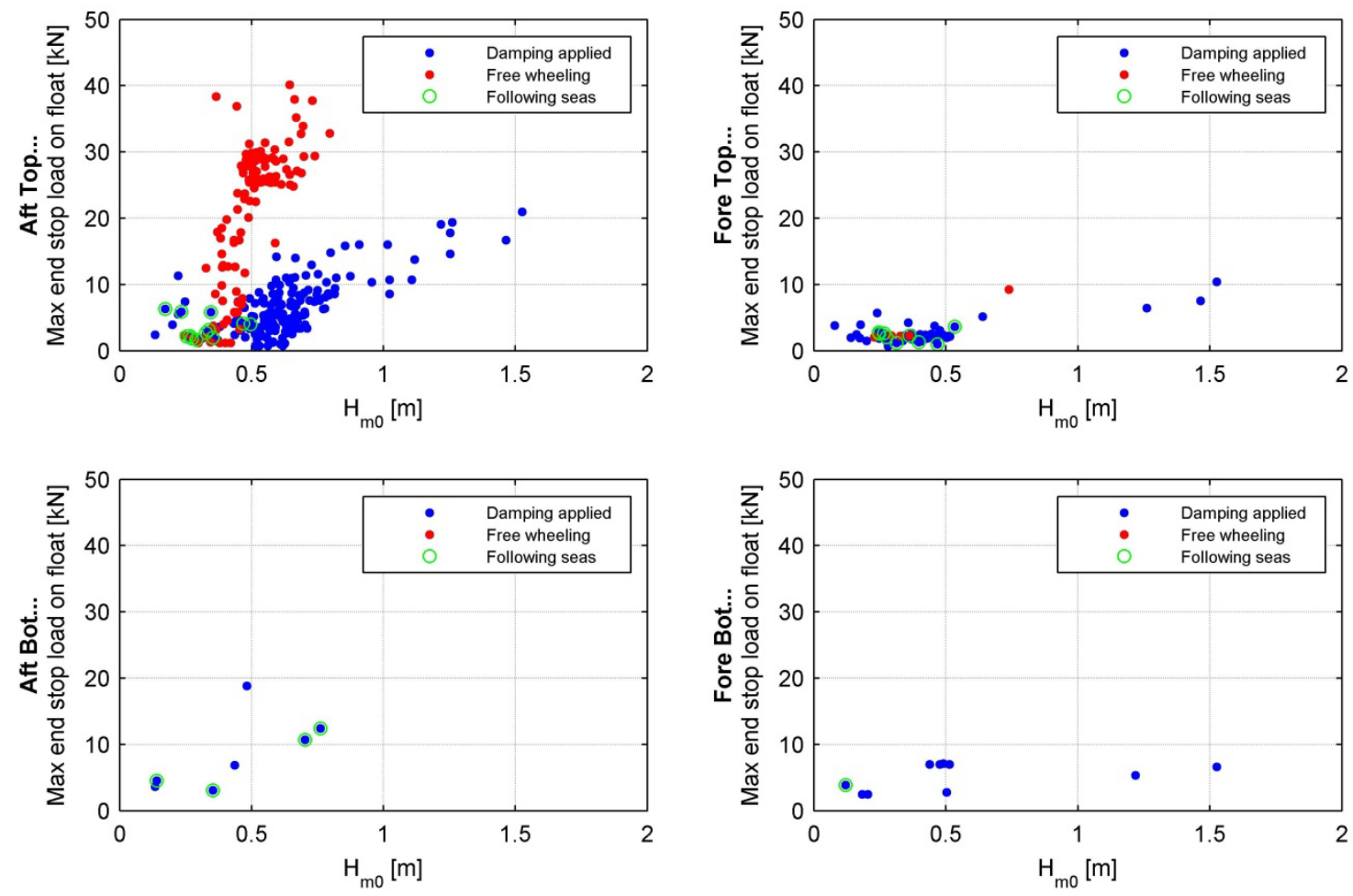

Figure 31. Maximum end stop loads exerted on floats, as a function of $\mathrm{H}_{\mathrm{m} 0}$. 


\subsubsection{Structural strain}

Vishay strain gauges were mounted on the interior surface of the hull in five locations. Single axis strain gauges were installed near to vertical mid-point of the spar, one to the fore and one to the aft. These strain gauges were installed parallel to the vertical axis of the spar. A strain gauge rosette was installed on the fore surface of the nacelle, midway between the port and starboard end walls. It was oriented to read axial and tangential strain. Strain gauge rosettes were also installed on the port and starboard end walls, just below the PTO axis. Per manufacturer guidelines, the FRP was etched away allowing for the strain gauges to be bonded and sealed with a flexible, waterproof material (Figure 32). Custom amplifiers were built and the signals were recorded by the SCADA system.

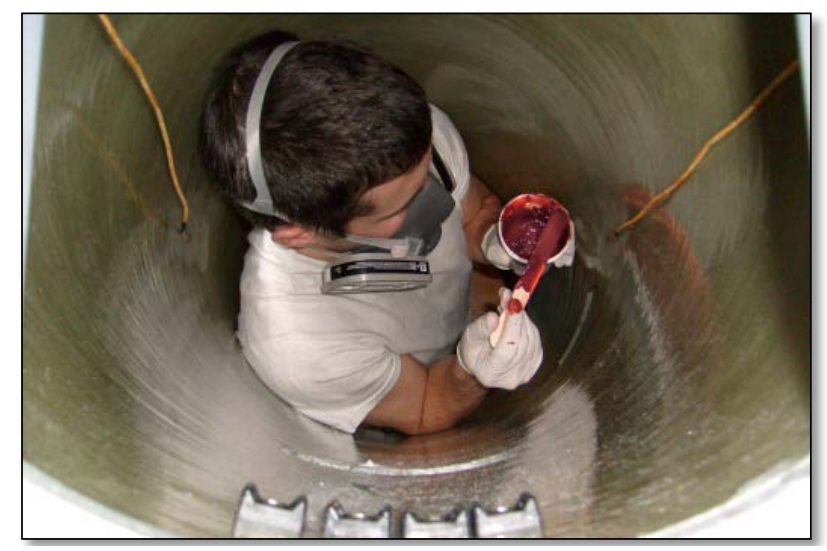

Figure 32. Sealing and waterproofing of structural strain gauges.

The mean strain signal drifted over time. The fore spar mean strain signal is taken as representative and is shown in Figure 33. The cause of this drift is unclear, but due to the drift absolute strain values were ignore and the RMS strain of the demeaned signal is used to characterize the intensity of the strain during a given sea state. The strain oscillations observed in the spar were much greater than those observed in the nacelle. Often the nacelle strain was at the noise level. As such, only the spar strain will be discussed moving forward.

RMS strain for the fore spar is shown as a function of $H_{m 0}$ in Figure 34. Data is only included for trials in which the relative wave heading was either head on or following $\left( \pm 10^{\circ}\right)$. Note that the strain response is much greater for head seas than for following seas. It shoud be noted that the trend is essentially identical for the aft spar signal. One plausible explanation is that WEC excitation is significantly greater in head seas. Indeed the WEC is designed for head seas, and the performance data shows a similar relationship with relative wave heading.

The mean RMS fore spar strain response matrix in head seas is given in Figure 35. The pattern of response is quite clear, with RMS strain increasing significantly with increasing $H_{m 0}$, and decreasing to a lesser extent with increasing energy period. As with the performance data, the dispersion about the mean response is relatively large. Although several factors were investigated, such as spectral width and directional spreading, no significant correlation was observed. 


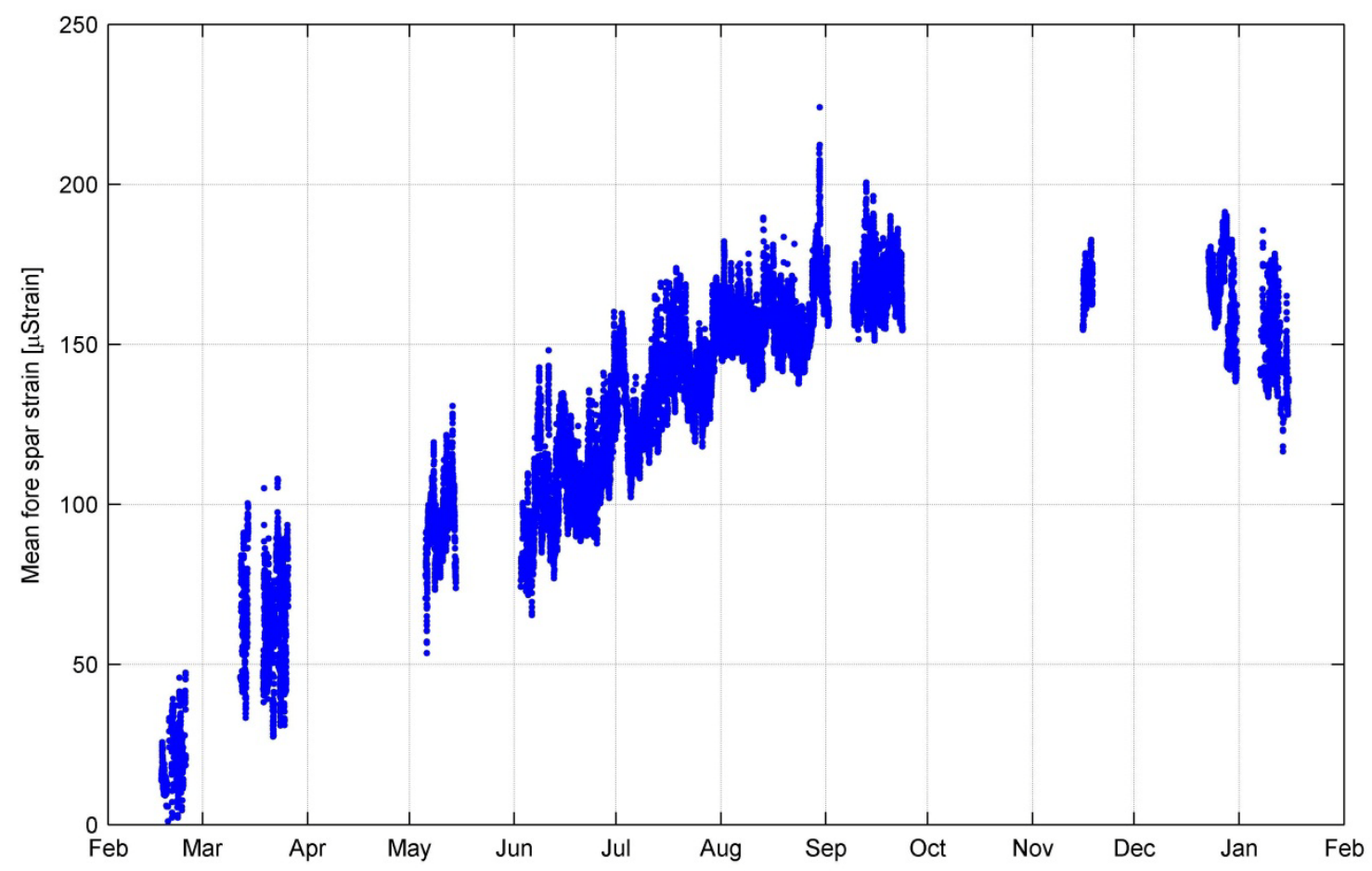

Figure 33. Drift in mean strain over time, for the fore spar gauge.

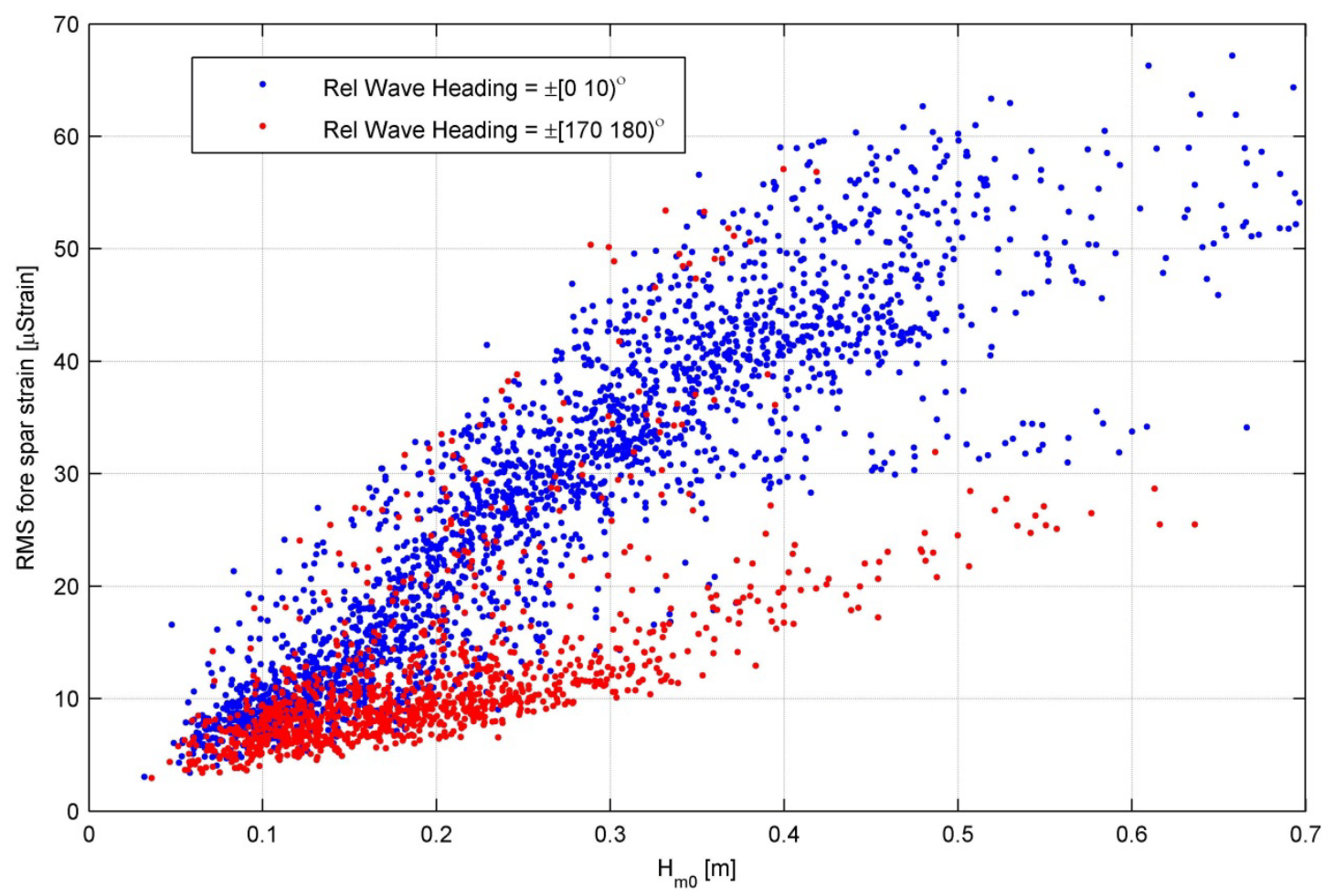

Figure 34. Directional sensitivity of fore spar strain. 


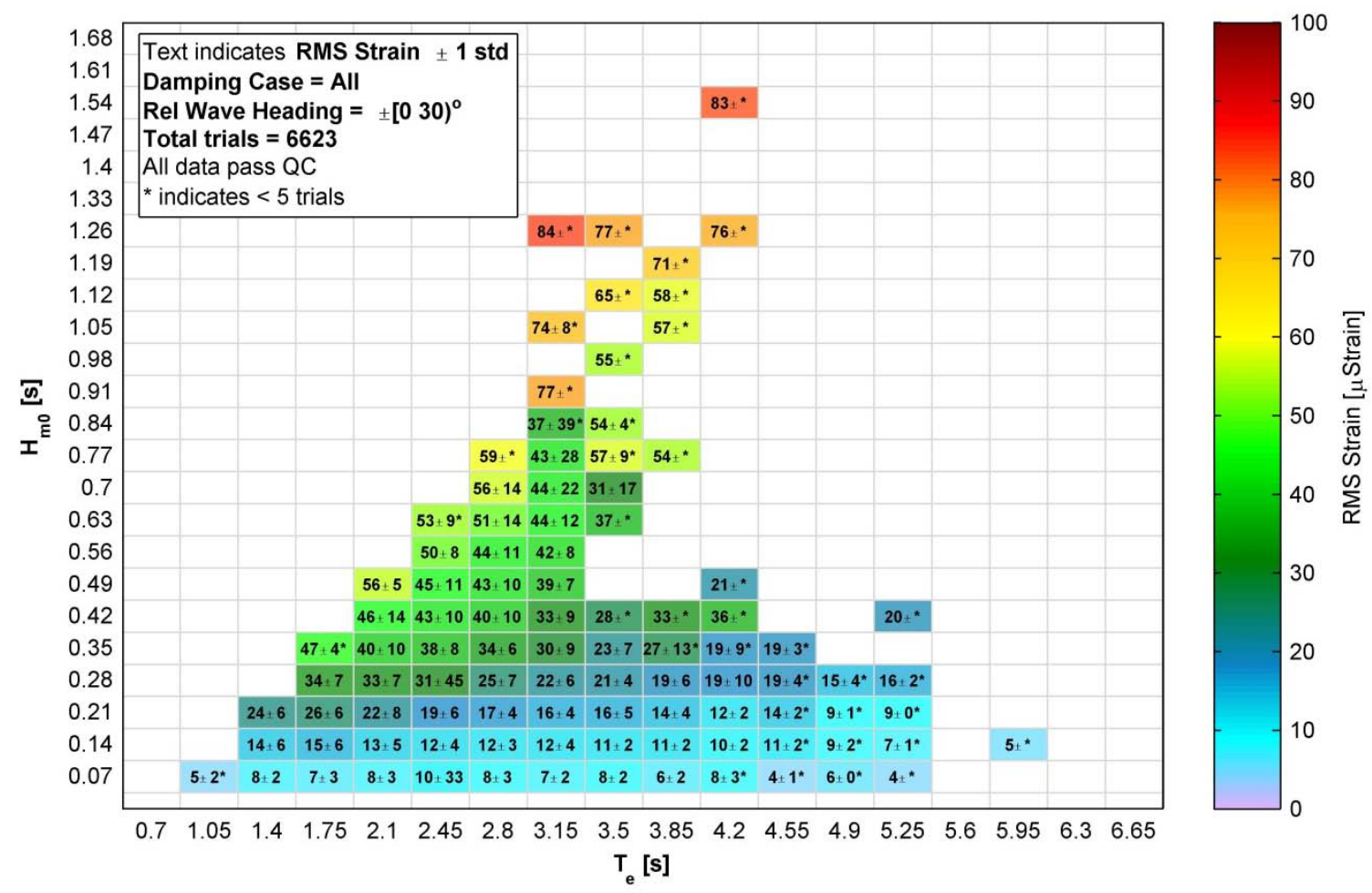

Figure 35. Mean RMS fore spar strain response matrix, head seas.

\subsubsection{Conclusion}

The SeaRay prototype was instrumented to yield a number of design signals during its 13 month deployment, including mooring loads, structural strain and end stop loads. Mean mooring loads were shown to correlate strongly with current speed, and RMS mooring loads with significant wave height. These relationships were expected, and it can be assumed that they will also pertain to future designs. Furthermore, it was shown that the mean mooring loads (from pretension and current loading primarily) in general were much greater than the RMS mooring loads (from wave induced loading). It is not clear that this trend will remain for future designs/deployments. For one thing, mooring designs with much lower pretension are currently being investigated by Columbia Power and our partners. Additionally, the scaled current speeds in the Puget Sound are significantly greater than would be expected in likely deployment scenarios.

Noisy end stop load signals were quality controlled, yielding a dataset of strikes that are trusted. The data shows that the majority of the end stop strikes were on the upper end stops, with only a small number of strikes on the lower end stops. Surprisingly, the vast majority of the strikes involved the aft float. This is likely explained by the relatively small range of motion designed into the upper domain of the aft float. It is observed that the end stop loads correlate well with wave height, and are also strongly influenced by PTO damping. For a period of time where the PTOs were allowed to freewheel, the end stop strike response is much greater. The point highlights one of the weaknesses of end stops: an object whose function is to slow down and stop 
the float occupies finite space, thus reducing the range of motion. Of course it is this motion which generates electricity and as such restrictions should be avoided.

The strain sensor signals showed considerable drift over time that has not been explained. As such absolute strains are not analyzed, but rather the RMS of the demeaned signal. Strain in the spar was shown to be significantly greater than strain in the nacelle, as was expected. The strain response was correlated with significant wave height, energy period and wave direction. With an eye on reducing hull cost, future deployments should invest in more reliable strain sensor networks. More strain sensors would also be helpful, with a focus on sensors where stress and strain are expected to be critical.

\section{Model Validation}

\subsection{Introduction}

The primary use of the SeaRay data is to validate numerical models. There are several reasons to use simulation results rather than the experimental results. Firstly, a commercial WEC will have a mass distribution that differs significantly from that of the SeaRay. The mass distribution of the SeaRay was not ideal, as the scale and heavy instrumentation necessitated certain trade-offs in the design space. Furthermore, additional design changes can be investigated with validated modeling tools, after the SeaRay experiment is over. Similarly, validated modeling tools can be used to test out various control strategies and PTO configurations. Lastly, the range of conditions tested in the SeaRay experiment does not encompass all expected conditions in the open ocean. For example, very few sea states were observed with a full scale equivalent energy period beyond $10 \mathrm{~s}$, whereas longer period sea states would be fairly common off the coast of Oregon.

\subsection{Methodology}

A numerical model was developed using ANSYS AQWA version 14.0. The first step in developing the numerical model was the calculation of frequency and directionally dependent hydrodynamic parameters using AQWA's frequency domain panel method solver (AQWA LINE). The WEC was modeled with as-built dimensions. A water depth of $21 \mathrm{~m}$ was used, representative of the mean observed depth during the SeaRay experiment. The surface mesh of the WEC (see Figure 36) employed a total of 10,493 diffracting panels. The modeled mass (Table 6) differs somewhat from the prototype (Table 2). AQWA requires mass distributions to be given w.r.t. the bodies respective centers of gravity, and as such the values used to describe the moments of inertia are different. Furthermore, the necessary simplification of the geometry (compare Figure 36 to Figure 1) resulted in a change in displaced volume. To account for the change in displace volume one would need to accept a change in the draft line, or a balancing change in the mass. The spar/nacelle/damper displace volume was kept similar to the as-built prototype, but the fore and float displaced volumes remain 3 and 5\% below as-built. The loss of displace volume in the float models is explained by the removal of the end stop 'strike pads' and the float arms. These features exist very close to the nacelle and their inclusion leads to fatal computational errors; thus they were remove from the model. It was assumed that making up the lost volume by increasing the width (axial length) of the floats would unfairly improve performance and changing the cross-sectional area would affect performance in an unpredictable

Columbia Power Technologies, LLC • 4920A SW $3^{\text {rd }}$ St, Corvallis, Oregon 97333 Phone: (541) 368-5033 • Fax (541) 230-1498 • www.columbiapowertechnologies.com 
manner. The LINE model was run using a combination of 39 discrete directions and 50 discrete frequencies.

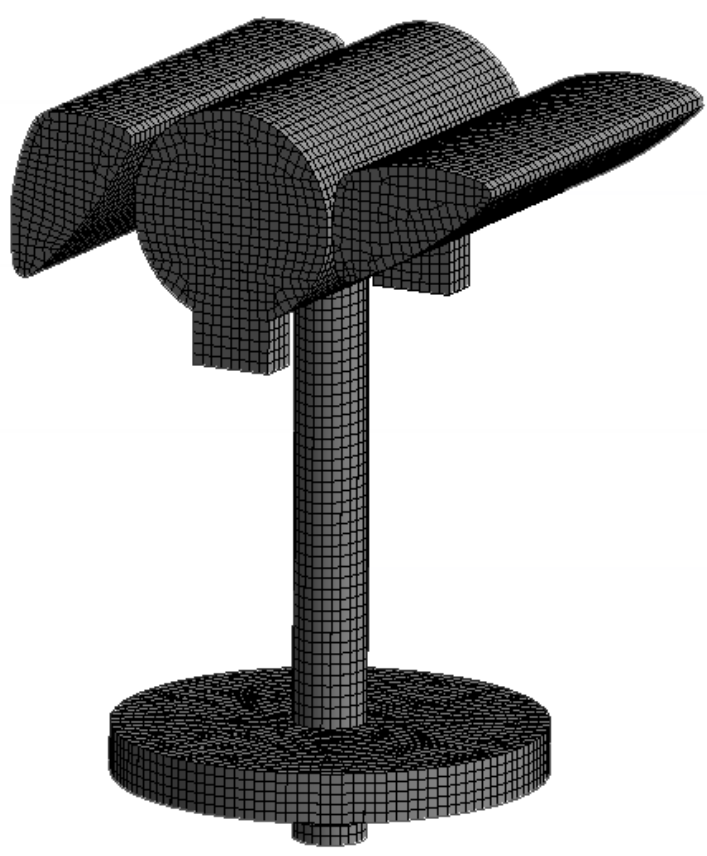

Figure 36. Surface mesh for AQWA model, with a total of 10,500 diffracting panels.

Table 6. SeaRay mass distribution as used by AQWA.

\begin{tabular}{|c|c|c|c|c|}
\hline & \multicolumn{2}{|c|}{ Mass } & \multirow{2}{*}{$\begin{array}{c}\text { Center of gravity } \\
{[\text { [m] }}\end{array}$} & \multirow{2}{*}{$\begin{array}{c}\text { Moment of inertia } \\
\text { wrt center of gravity } \\
{\left[\mathrm{kg} \mathrm{m}^{2}\right]}\end{array}$} \\
\hline & $\begin{array}{l}\text { AQWA } \\
\text { [kg] }\end{array}$ & $\begin{array}{c}\text { error } \\
\text { wrt as- } \\
\text { built [\%] }\end{array}$ & & \\
\hline Total system & 6460 & -1 & $\mathbf{x}, \mathbf{y}, \mathbf{z}$ & $\mathbf{I}_{\mathrm{xx}}, \mathbf{I}_{\mathrm{yy}}, \mathbf{I}_{\mathrm{zz}}$ \\
\hline Spar & & & & \\
\hline Fore float & & & & \\
\hline Aft float & & & & \\
\hline
\end{tabular}


The LINE model outputs are then used to set up time domain simulations using AQWA NAUT. This time domain solver handles diffraction and radiation forces using the standard linear assumptions of small amplitude motions and waves. However, the Froude-Krylov and hydrostatic force calculations take the instantaneous wetted surface into account. In addition to the hydrodynamic forces, PTO torque is applied with a linear damping model where torque is proportional to relative pitch velocity. The PTO model is limited to $\square \mathrm{Nm}$ and $\square \mathrm{Nm}$ for the fore and aft floats respectively, just as the SeaRay. The rotor inertia experienced by the floats is magnified via the gear box; the effect of the rotor and gearbox inertia on float motion is accounted for via an applied torque which is linearly dependent upon relative pitch acceleration. The rotor inertia and PTO torques are applied via a custom .dll module developed by Columbia Power.

Additionally included in the simulation are the mooring system and viscous drag. A polynomial force-displacement relationship is applied at three positions around the damper tank to model the physical mooring system employed in the Puget Sound. AQWA allows the user to input a $6 \times 6$ matrix of viscous drag coefficients for each rigid body. The viscous drag force in the $i^{\text {th }}$ DOF, $F_{d, i}$, due to a velocity in the $j^{\text {th }} \mathrm{DOF}, v_{j}$, is calculated at each time step as

$$
F_{d, i}=-C_{D, i j} v_{j}\left|v_{j}\right|
$$

where $C_{D, i j}$ is the $i j^{\text {th }}$ entry in the $6 \times 6$ drag coefficient matrix. $C_{D}$ values are the product of several factors: fluid density, cross-sectional surface area perpendicular to velocity vector, and a dimensionless drag coefficient, $C_{d}$. The damping coefficients used were first determined through a heuristic investigation involving data from a previously undertaken tank test of a $33^{\text {rd }}$ scale Generation 3.1 WEC [6]. These values were simply modified by substituting the SeaRay crosssectional areas for the $33^{\text {rd }}$ scale cross-sectional areas.

One factor that cannot be simulated in AQWA NAUT is directional spreading. However as stated previously in this report the directional spreading observed in the Puget Sound was substantial, with spreading indices on the order of 1 . Furthermore it is understood that directional spreading decreases the performance of the WEC. Fortunately, the $33^{\text {rd }}$ scale tank testing previously mentioned investigated the effects of directional spreading. As a part of the tank testing a number of head on, real seas were simulated with spreading indices of 10 and 4, and with no spreading at all. The range of sea states tested at that time does not fully encompass the incident sea states from the Puget Sound, but they were similar in significant wave height and energy period. As such a reasonable approach to accounting for heavily spread seas in the Puget Sound simulations is to apply a 'directional efficiency' factor to the results simulated in unidirectional seas (i.e. seas with no directional spreading). The directional efficiency number used is $71 \%$, which is the average ratio of experimentally derived RCWs in seas with spreading index of 4 and unidirectional seas [6]. As all real seas exhibit directional spreading to some degree, the development of a suitable, commercially available modeling tool that accounts for directionally spread seas in the time domain is essential for the accurate estimations of power production. 
Having developed a suitable model for the SeaRay in the Puget Sound, the next step was to establish a sub-set of the experimental data against which to validate. Several requirements were established to down-select the data. Firstly, all trials had to pass the quality control flags for metocean characterization (3.1.8) and linearity of PTO damping (3.2.9). Then the six factors to which performance was found to be sensitive were employed. Only those trials that were head on (absolute value of relative wave heading less than $22.5^{\circ}$ ), with relatively little directional spreading (UI $\geq 0.3$ ), were reasonably well represented by the JONSWAP spectral shape (fitQ $\leq$ 0.75), and were within the operationalHighAft PTO damping case were considered. The remaining 779 remaining trials were sorted into bins by $\mathrm{Hm} 0$ and Te. A total of 18 of these bins were populated by at least 5 down-selected trials, and the mean conditions for each of these were taken as AQWA validation cases. These cases are all indicated in Figure 26, as the highlighted cases from that are not marked with an asterisk.

For each of the 18 validation cases, a mean incident wave spectrum was calculated. While these spectra could be represented by fitted JONSWAP spectra, to most accurately represent the incident conditions the observed spectra were used. These mean observed spectra needed to be represented by a mere 25 points, as this is the format AQWA accepts for user defined spectra. The AQWA spectra all ranged from 0.05 to $1.9 \mathrm{~Hz}$. Within these bounds the remaining 23 points were selected algorithmically, with inflection points being favored. The resulting AQWA spectra were scaled such that the zeroth moment was equivalent to the mean observed spectra they were representing. An example observed/AQWA pair is depicted in Figure 37, illustrating the generally excellent representation achieved. It should be noted that the use of the term observed here indicates that the Doppler shifted spectra were used, rather than the intrinsic spectra. Current is not included in the simulation, and thus the Doppler shifting is the only manner in which the current is accounted for.

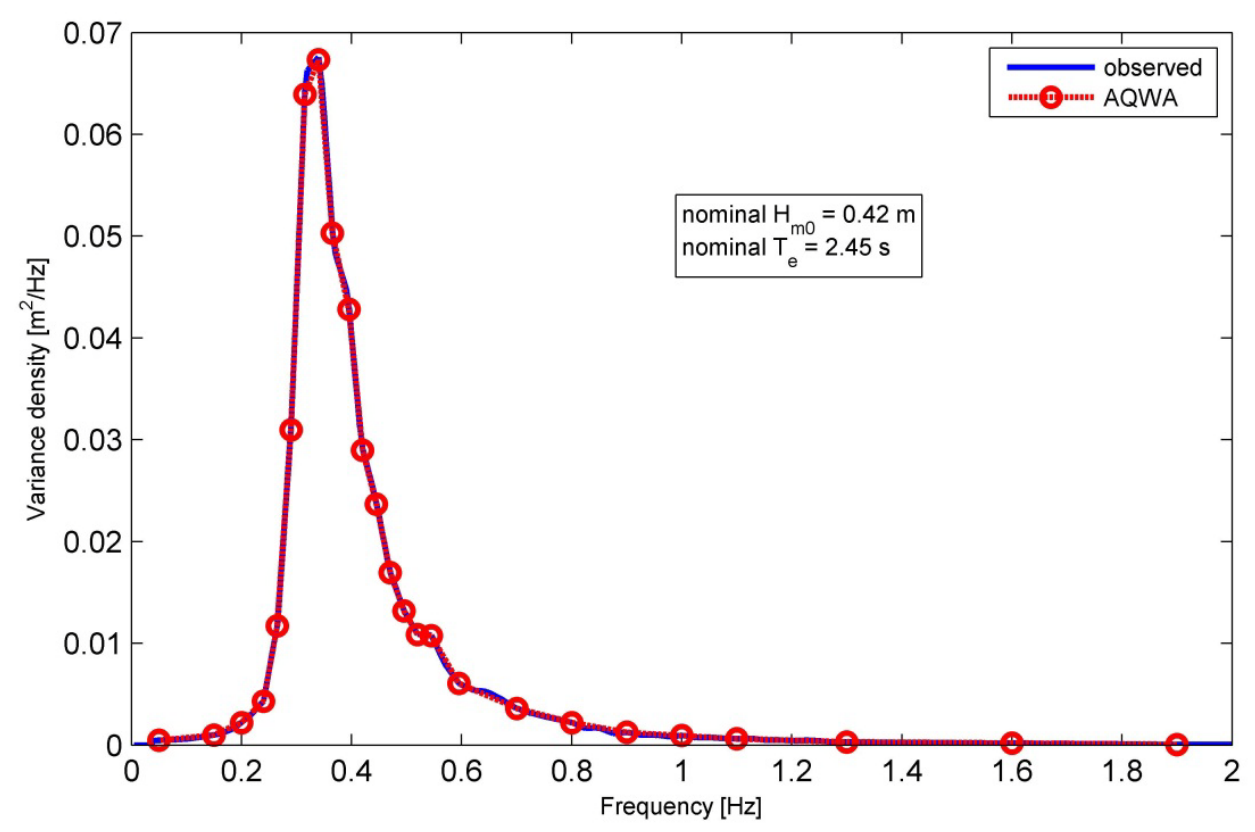

Figure 37. Example mean observed /AQWA spectra pair. 
The 18 validation cases with mean characteristic parameters are given in Table 7 . Note that each case is represented by the mean incident conditions and WEC response of between 6 and 122 unique trials. The only data from this table that was used as input to the individual AQWA NAUT models are the PTO damping values. The depth was modeled at $21 \mathrm{~m}$, the direction as head on and the mean spectra were input as described in the previous paragraph. Each validation case was run for a simulation time of $10 \mathrm{~min}$, with a time step of $0.006 \mathrm{~s}$.

Table 7. AQWA validation data set.

\begin{tabular}{|c|c|c|c|c|c|c|c|c|c|c|c|c|c|}
\hline \multicolumn{2}{|c|}{ Nominal } & \multicolumn{11}{|c|}{ Observed } & \multirow{2}{*}{$\begin{array}{l}\text { AQWA } \\
\text { Total } \\
\text { RCW }\end{array}$} \\
\hline $\begin{array}{c}\mathrm{HmO} 0 \\
{[\mathrm{~m}]}\end{array}$ & $\begin{array}{l}\mathrm{Te} \\
{[\mathrm{s}]}\end{array}$ & $\begin{array}{c}\text { \# of } \\
\text { trials }\end{array}$ & $\begin{array}{c}\mathrm{Hm0} \\
{[\mathrm{m}]}\end{array}$ & Te $[\mathrm{s}]$ & $\begin{array}{c}\text { Water } \\
\text { depth } \\
{[\mathrm{m}]}\end{array}$ & UI & $\begin{array}{c}\text { Rel } \\
\text { wave } \\
\text { dir }\left[^{\circ}\right] \\
\end{array}$ & fitQ & $\begin{array}{c}\text { Fore } \\
\text { damping } \\
{[\mathrm{N} \mathrm{m} \mathrm{s}]}\end{array}$ & $\begin{array}{c}\text { Aft } \\
\text { damping } \\
{[\mathrm{N} \mathrm{m} \mathrm{s}]}\end{array}$ & $\begin{array}{l}\text { Total } \\
\text { RCW } \\
\end{array}$ & $\begin{array}{l}\text { Total } \\
\text { RCW } \\
\text { std } \\
\end{array}$ & \\
\hline 0.21 & 1.75 & 12 & 0.237 & 1.84 & 20.7 & 0.34 & 2 & 0.34 & & & & & \\
\hline 0.21 & 2.10 & 28 & 0.227 & 2.12 & 20.7 & 0.34 & -4 & 0.45 & & & & & \\
\hline 0.21 & 2.45 & 7 & 0.203 & 2.45 & 20.7 & 0.36 & 4 & 0.46 & & & & & \\
\hline 0.28 & 1.75 & 20 & 0.280 & 1.89 & 20.8 & 0.37 & 0 & 0.37 & & & & & \\
\hline 0.28 & 2.10 & 122 & 0.288 & 2.08 & 20.9 & 0.38 & 0 & 0.40 & & & & & \\
\hline 0.28 & 2.45 & 39 & 0.285 & 2.42 & 21.4 & 0.42 & 0 & 0.52 & & & & & \\
\hline 0.35 & 2.10 & 108 & 0.347 & 2.16 & 20.9 & 0.44 & 2 & 0.37 & & & & & \\
\hline 0.35 & 2.45 & 66 & 0.351 & 2.40 & 21.3 & 0.45 & 2 & 0.42 & & & & & \\
\hline 0.35 & 2.80 & 8 & 0.353 & 2.66 & 21.6 & 0.49 & -6 & 0.49 & & & & & \\
\hline 0.42 & 2.10 & 28 & 0.405 & 2.22 & 20.9 & 0.51 & -1 & 0.37 & & & & & \\
\hline 0.42 & 2.45 & 109 & 0.421 & 2.41 & 20.8 & 0.49 & 3 & 0.43 & & & & & \\
\hline 0.42 & 2.80 & 8 & 0.429 & 2.66 & 21.2 & 0.46 & -1 & 0.45 & & & & & \\
\hline 0.49 & 2.45 & 100 & 0.482 & 2.49 & 20.9 & 0.55 & 1 & 0.39 & & & & & \\
\hline 0.49 & 2.80 & 27 & 0.494 & 2.70 & 20.9 & 0.53 & 0 & 0.48 & & & & & \\
\hline 0.56 & 2.45 & 11 & 0.547 & 2.54 & 20.7 & 0.61 & 5 & 0.36 & & & & & \\
\hline 0.56 & 2.80 & 36 & 0.556 & 2.76 & 21.3 & 0.60 & -4 & 0.44 & & & & & \\
\hline 0.63 & 2.80 & 6 & 0.623 & 2.87 & 20.6 & 0.64 & 7 & 0.50 & & & & & \\
\hline 0.63 & 3.15 & 6 & 0.616 & 3.14 & 21.1 & 0.60 & 10 & 0.56 & & & & & \\
\hline
\end{tabular}

\subsection{Results and discussion}

The total RCW calculated from the simulation results is given in Table 7, and can be compared to observed total RCW and the standard deviation of the total RCWs of the individual trials comprising the validation case. The observed mean total RCWs and associated standard deviations are also given in Figure 26. The percent error of the total RCW, for the simulation results w.r.t. observed, is depicted in Figure 38. The mean error over all 18 cases for total RCW is $-2 \%$. For the fore and aft PTOs considered separately, the mean errors are $7 \%$ and $-2 \%$ respectively. For total, fore and aft RCW the mean absolute errors are 17\%, 26\% and 22\% respectively. These results are quite encouraging; while the error in total WEC performance for any one case simulated can be as extreme as $\pm 30 \%$, on the average the result can be expected to be less than $20 \%$ off and on the whole the results are unbiased. 


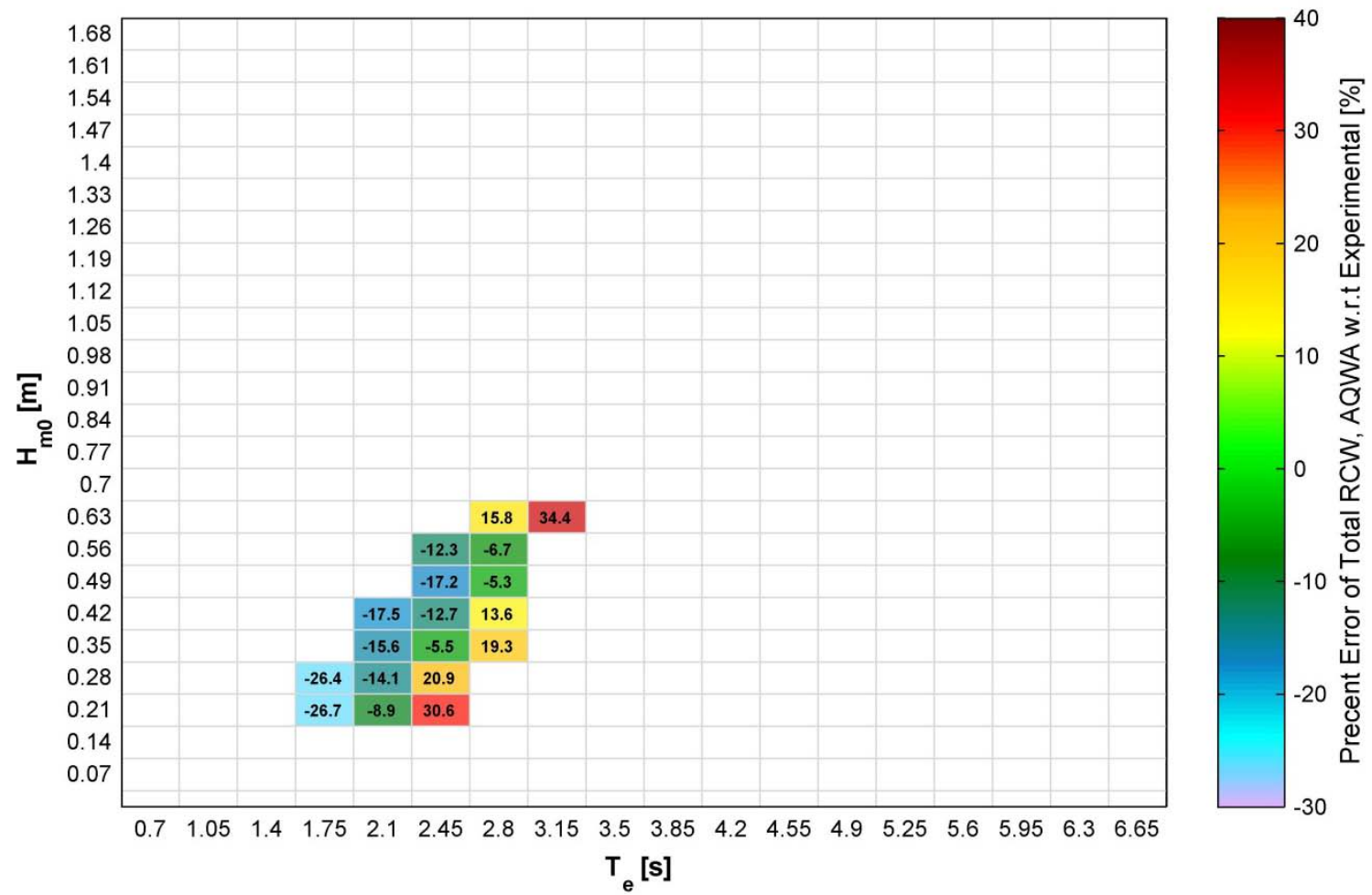

Figure 38. Percent error of total RCW, for simulation w.r.t. experimental.

Total WEC power is plotted versus incident wave power in Figure 39. Total WEC power is depicted in the upper plot, followed by fore power in the center plot and aft power in the lower plot. Here we see that the AQWA simulation results follow the observed performance quite well, particularly when considering total WEC performance. For the more energetic seas we see that AQWA tended to over predict fore float power and under predict aft float power.

Figure 40 depicts total, fore and aft RCW as given by experiment and simulation, as a function of energy period. Separate subplots are given for each nominal significant wave height. While it has been shown that the simulations generally follow the experimental results, for several of these subplots the trends in experimental and simulated data are opposite one another. The experimental results typically show performance dropping with increasing energy period, while the simulation results show performance increasing. Note that these simulations were carried out over a fairly narrow range of energy periods and the trends observed should not be assumed to continue beyond the range depicted. Looking back at Figure 38 this error trend is apparent, with AQWA under predicting along the wave steepness limited diagonal, and then slowly trending towards over predicting as the periods increase. It is unclear to what this error should be attributed. 


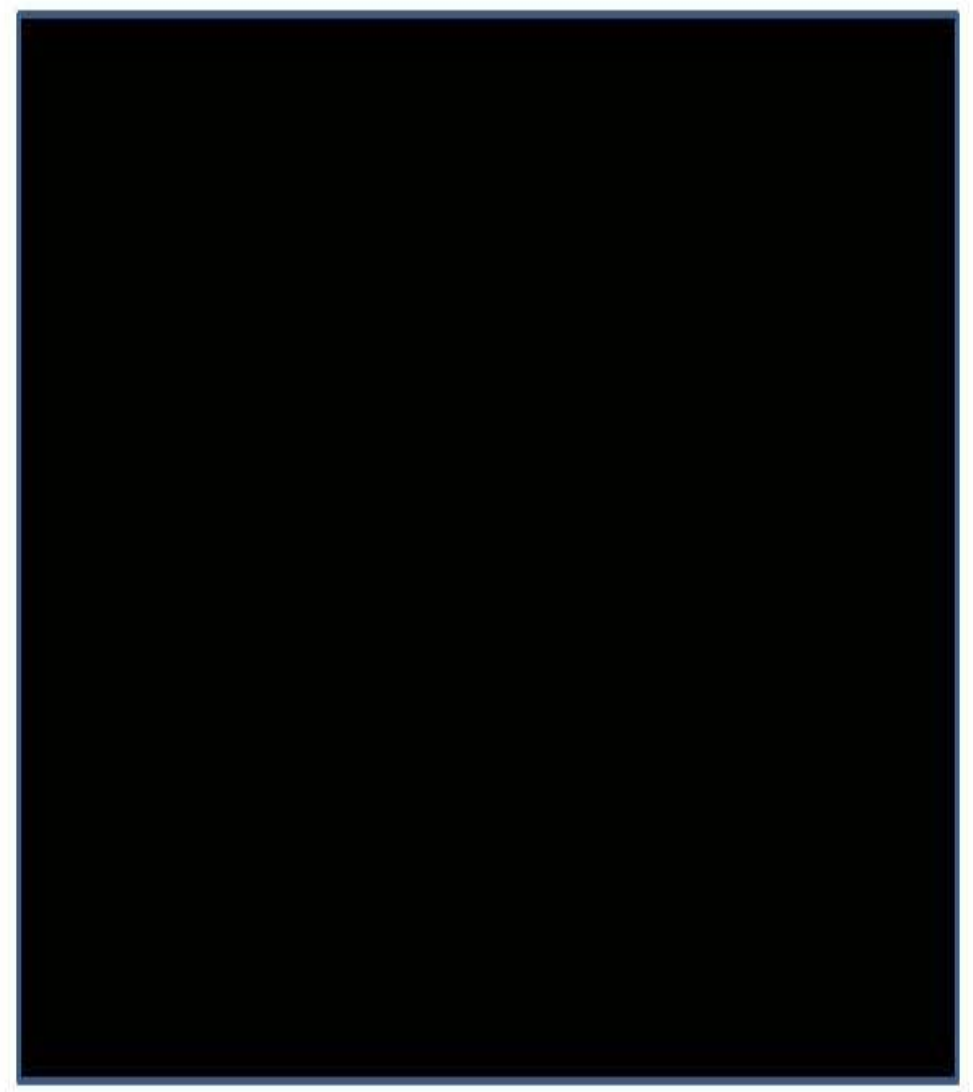

Figure 39. WEC power versus wave power, comparison of experiment and simulation.

Columbia Power Technologies, LLC $\bullet 4920$ A SW $3^{\text {rd }}$ St, Corvallis, Oregon 97333 Phone: (541) 368-5033 • Fax (541) 230-1498 • www.columbiapowertechnologies.com 


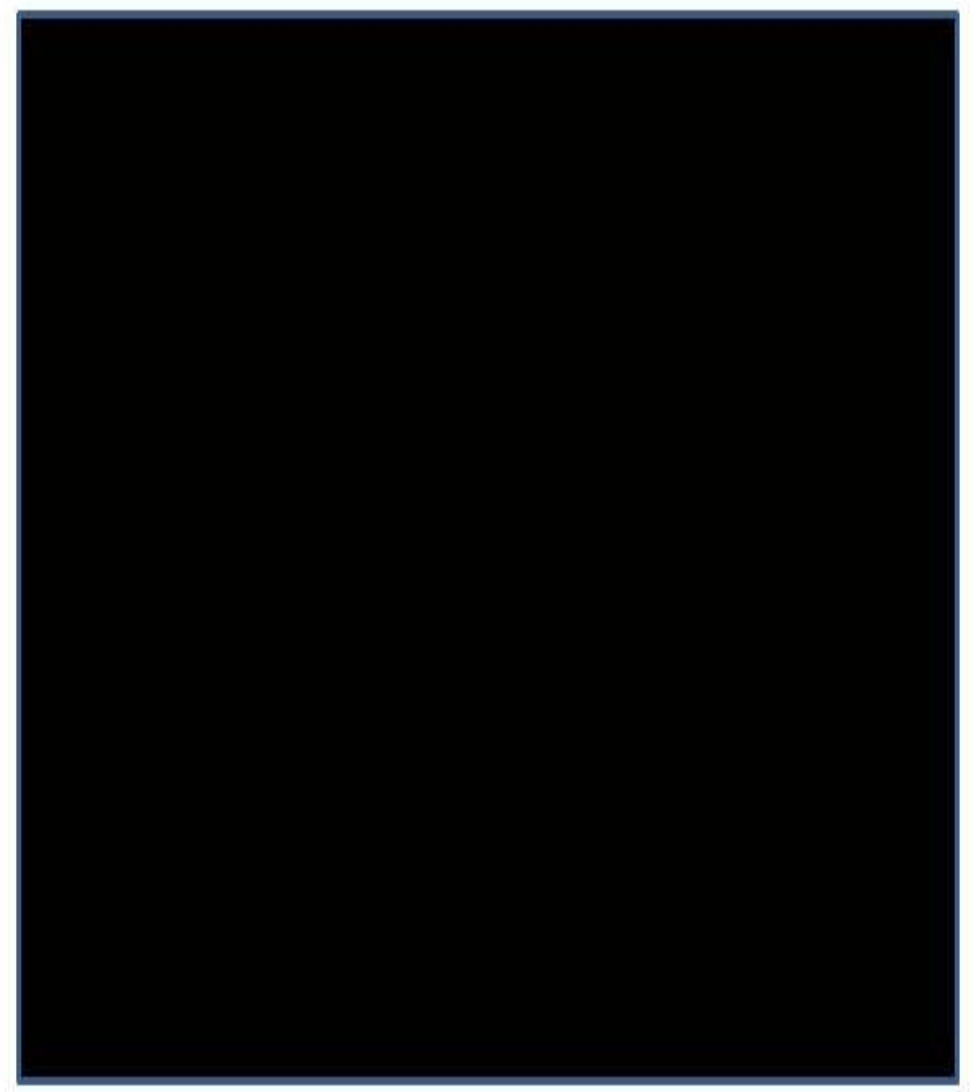

Figure 40. RCW versus Te, comparison of experiment and simulation.

Columbia Power Technologies, LLC $\bullet 4920$ A SW $3^{\text {rd }}$ St, Corvallis, Oregon 97333 Phone: (541) 368-5033 • Fax (541) 230-1498 • www.columbiapowertechnologies.com 
There are several sources of error to be considered, both in the simulation and in the measured data. Not the least among these is the crude approximation of the performance effects of directional spreading discussed earlier, taken from previous tank testing results. Another potential source of error is the difference between as-built float mass and AQWA model float mass ( 4\%). The simplified geometry employed in the model is another, as is the implementation of viscous damping. Additionally, the PTOs exhibit friction and cogging and other nonlinearities, but are modeled as simple torque limited, linear devices. Furthermore, one must consider the physical characteristics left out of the model, including but not limited to ocean current, relative wave heading and changing water depth. Another potential source of error comes from the hard limit AQWA has on the number of panels in the model; the panel size employed limited AQWA's analysis to frequencies less than or equal to $1.5 \mathrm{~Hz}$, while in general the observed spectra had some small amount of energy up to $2 \mathrm{~Hz}$. On top of this there is the model itself, which relies on linear wave theory and therefore assumes infinitesimal wave amplitude and WEC motion. Beyond the potential modeling errors, one must also consider the physical data. As discussed in great detail in 3.1.3 there is some uncertainty in WEC heading. Linked to this is uncertainty in wave heading, as the AWAC was limited by its placement in the water column to directionally resolving wave energy at $0.45 \mathrm{~Hz}$ and below (see 3.1.7), leaving a large portion of the spectrum unexamined. One more potential source of error is the presence of significant ship generated waves. Much care was put into excluding such trials from the database, but it is certainly possible that some trials not excluded were affected by non-stationary ship waves. Aside from these and other possible errors in measurement, it is altogether possible that some physical characteristic of sea or WEC to which performance is sensitive simply was not measured. Error cannot be entirely excluded from an experiment or altogether accounted for, but considerable effort was made to reduce and/or mitigate error in these trials. The mean error of $-2 \%$ in total RCW discussed previously is the satisfactory result of a project well done.

Separately, individual trials were used by InterMoor to validate their OrcaFlex model of Columbia Power's Ray series WECs. Several trials were selected, including one extreme case. Mean mooring loads and maximum mooring loads were compared. Reasonable agreement was found between the simulated and experimental mooring loads in most cases. Tabulated comparison can be found in Appendix M.

\subsection{Conclusion}

A subset of the extensive SeaRay data set has been used to validate numerical models. The validated models will be used to inform the performance optimization and design of prototype WECs. The numerical model utilized to assess performance was developed using ANSYS AQWA version 14.0. The simulations were carried out in the time domain, and accounted for some nonlinearity, such as PTO torque limiting and viscous drag. AQWA utilizes a 3-D panel method to calculate diffraction and radiation forces, and uses the instantaneous wetted surface to calculate hydrostatic and Froude-Krylov excitation forces. Columbia Power has made extensive use of AQWA, and has successfully validated this modeling tool in the past.

To select validation cases the quality controlled data was first sorted into a six dimensional matrix. Four of these dimensions were held constant, leaving a two dimensional $H_{m 0} / T_{e}$ matrix

Columbia Power Technologies, LLC $\bullet 4920 \mathrm{~A}$ SW $3^{\text {rd }}$ St, Corvallis, Oregon 97333

Phone: (541) 368-5033 • Fax (541) 230-1498 • www.columbiapowertechnologies.com 
that only included trial data where the PTO damping fell within a single operational case, and the seas were described as head on with relatively low directional spreading and whose spectra conformed reasonably well to the JONSWAP shape. The resulting bins that were populated by at least five trials were retained, and the mean conditions for each of these were taken as AQWA validation cases. For each of the 18 validation cases, a mean incident wave spectrum was calculated and used as the forcing input for the time domain simulations. The mean error over all 18 cases for total RCW is $-2 \%$. For the fore and aft PTOs considered separately, the mean errors are $7 \%$ and $-2 \%$ respectively.

\section{Experience Gained}

This section illustrates the practical experiences gained during the deployment of the SeaRay WEC. Detailed recommendations on full scale design modifications to these and other experiences can be found in the Project Influence on Full Scale Design section of the final DOE EE0002647 report.

\subsection{General Requirements (0050)}

\subsubsection{Assembly, Operations, \& Maintenance (0054)}

\section{Assembly:}

Generally the assembly of the SeaRay prototype went very well. Good communication and special built assembly jigs ensured that all hardware matched up well for assembly. Once the FRP nacelle was completed with the PTO alignment frame installed it was shipped it to Columbia Power for further assembly. This modular assembly process worked well and gave Columbia Power full access nacelle while Ershigs completed the rest of the WEC. With the nacelle at Columbia Power, the PTOs, electrical enclosures, end stops, bilge pumps, and instrument mast were installed with people familiar with the design in the convenience of their own facility. The then completed nacelle assembly was returned to Ershigs in Bellingham Washington for final WEC assembly. Columbia Power and Ershigs staff working together to finalize the assembly. The final ballast testing was performed at Bellingham Bay. Once complete, the entire assembly shown in Figure 1, was trucked to Lake Union Dry Dock where operations commenced.

\section{Operations:}

There were numerous operations during the 13 month deployment in the Puget Sound. The operations for deployment, recovery and maintenance were all complex and required skilled team members and planning. The team members included the crew of the SeaHorse crane barge, All Star charters, personnel from Sound \& Sea Technologies and Columbia Power. The operations went smoothly with many valuable experiences gained regarding the difficulties of servicing buoys at sea. Though difficult, Columbia Power and contractors performed many ongoing operations task on the WEC while deployed. It was quickly learned that having easily accessible and replaceable buoy component and modules was highly desirable. Performing repairs on electronic assemblies at sea in a rain storm has its challenges. Overall all the 
operations were safe and executed with precision within the planned window of good weather. On the other hand, finding a window of good weather often presented delays.

\section{Maintenance:}

During deployment a few hardware failures did occur. The accessible items were quickly and easily repaired or replaced with little trouble. Other items however, were housed in consecutive watertight enclosures and difficult to access requiring large crews, specialized service vessels, and a full day of effort. Such critical systems need to be made accessible and modular so that replacements can be quickly changed out at sea and time consuming troubleshooting work can be followed up on shore. Having quick and convenient access to these high risk components will reduce the time, required hardware, and the ultimate cost of failure events.

\subsection{2. $\quad$ Cost Reduction (0053)}

Costs for intermediate scale testing increased due to extending the deployment period from 4 months to thirteen. Cost considerations include charter boats supporting this area of Puget Sound that range between $\$ 150-\$ 200$ per hour, tug boats and barges costing between $\$ 2500$ and \$5000 per day, travel to and from Seattle, food and lodging, repair parts and equipment to support repairs and regular battery charges when waves were too small. Additionally, diver support services were hired to assist with hull cleaning after 6 months of deployment, mooring inspections and anchor recovery.

Intermediate scale test cost reduction can be accomplished in a few ways:

- Design for sufficient energy to support 100\% of the WEC's instrumentation 7 days x 24 hours for the entire deployment duration. This will reduce costs to charge batteries.

- Five months or more of data is important to assure statistical significance on the data. Plan for a full 6-month period of energetic waves and assure the system will collect data with $>90 \%$ availability..

- Maximize the use of remote cameras and remote data collection to minimize costly "hands-on" visits to the WEC.

- Remotely monitor WEC no-less than hourly to identify and address problems in a timely manner to allow for early intervention and possible cost reductions.

- WEC design and testing is a specialty and often times requires one-off designs; however, commercial off the shelf solutions will improve reliability and reduce design/build costs.

- Pre-testing of all "watertight rated" hardware and equipment is a prudent recommendation. Several expensive repairs were required because of watertight equipment not meeting specification.

\subsection{Hull (0100)}

During the ballasting operations a leak was detected in the nacelle. Upon closer inspection it was noticed that the FRP plate that made up the end of the nacelle was defective and had a leak path through the layers of FRP. The leak was fixed and tighter quality control was implemented to ensure the manufacturing of FRP plates met or exceeded the designed engineering standard.

The aluminum access hatch was attached to the deck plate of the nacelle using a fiberglass adhesive. Once deployed, temperature fluctuations between the dissimilar aluminum and 
fiberglass caused stresses which cracked the brittle adhesive. This led to leakage when waves overtopped the nacelle. The mounting flange of the hatch was later drilled, bolted in place, and sealed with a compliant marine sealant providing a watertight seal throughout the deployment.

During a routine inspection there was evidence that the WEC suffered a collision with a presumed recreational vessel. Surveillance evidence showed this event occurred on New Year's Eve. The FRP survived the collision fairly well but the collision left a hole roughly the size of a golf ball in the aft float. A service crew led by Columbia Power promptly repaired the hole with a boat plug and marine sealant.

From the data we see that the aft float did not contribute as much to the overall energy capture of the WEC. There are a number of theories that may explain this result. Improving the aft float performance is a major focus of subsequent shape optimization work covered in the final DOE EE0002647 report.

We are very pleased with the performance of the fiberglass Hull structure. It was subjected to extreme conditions in open water for 13 months and showed no deterioration. It was both tough and flexible and was easily modified. It remained waterproof and did not corrode, crack, or leak after initial repairs were made. The fully submerged FRP battery compartments were completely dry after 13 months in water at a depth of approximately 12 feet.

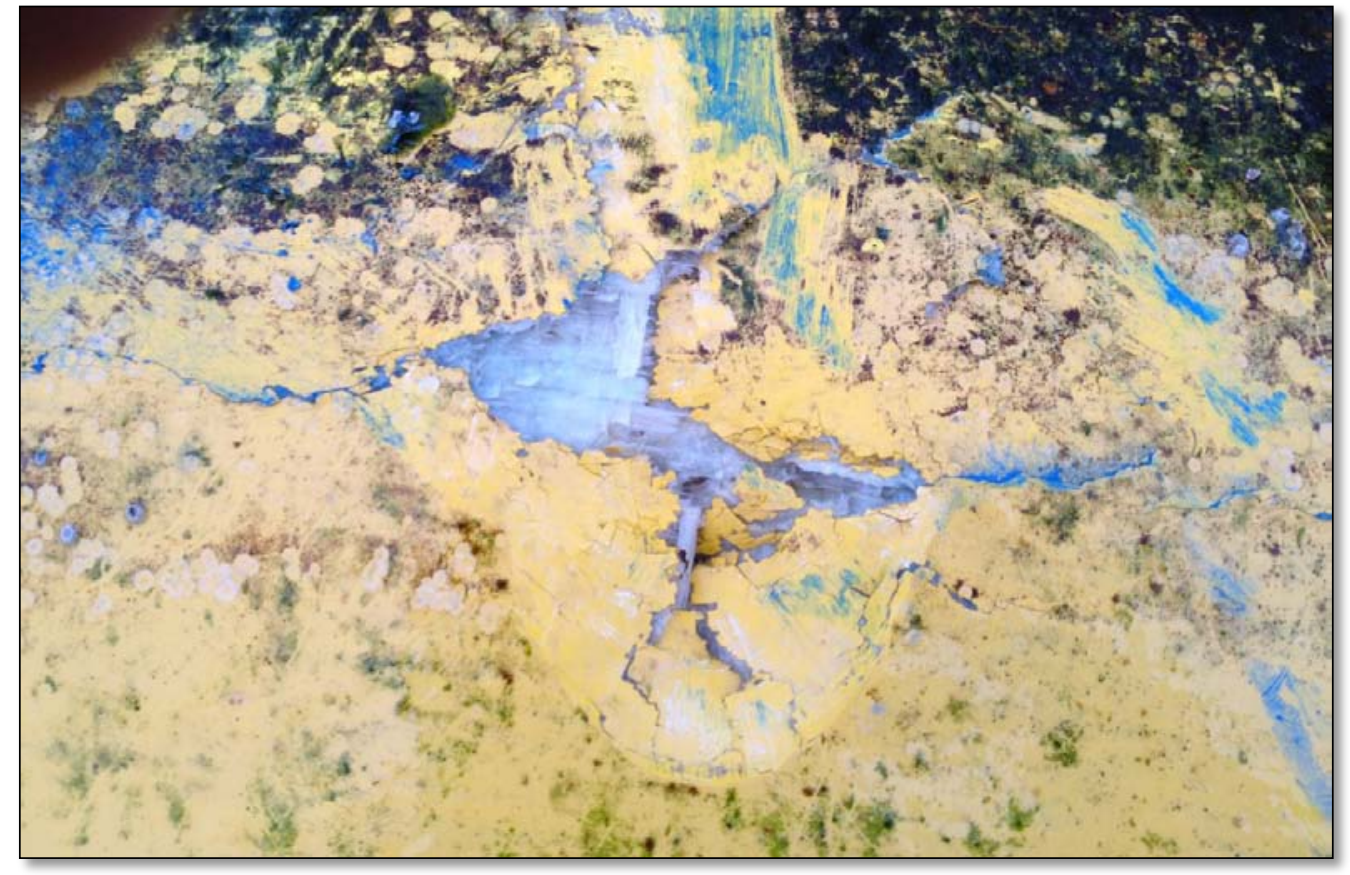

Figure 41 - Collision damage to the aft float FRP structure.

\subsection{Power Take-Off (PTO) (0200)}

The PTO design operated as planned and reliably converted the WEC motion into electric power. This PTO could be developed into a small commercial WEC product with only minor changes. The torque transducer provided accurate torque measurement for performance assessment, but 
was designed for accuracy in the normal range of torque and did not cover the ranges of extreme events. With linear damping, the torque transducer often saturated at high speeds and extreme events. The slip clutch protected the torque transducer against damage but restricted the WEC from utilizing full torque potential of the PTO. Follow on design iteration could include higher torque sensor specifications, at the cost of lower resolution, or use PMG torque/current characterizations to elimination of the torque sensor completely. One of the encoders, a corrosion resistant IP66 device failed after being wetted with sea water. The manufacture replaced it under warranty; however the replacement procedure in the Puget Sound was quite extensive.

\subsection{Electric Plant (0300)}

The initial deployment of the Electric Plant worked quite well but went through several design iterations as power electronic hardware failures occurred. The major current switching components of the Electric Plant are IGBTs. These high power switches are prone to failure in many power electronic applications and the intermittent high voltage variable frequency operation of the wave energy converter adds additional stresses. There was much experience gained in developing DC-DC converters which had the ability to operate over a very large range of voltages on both input and output. Using separately sourced control power was vital to maintain the power electronic controls.

Accessing the power electronic enclosures inside the nacelle while at sea presented a great deal of effort involving a large crew, an anchor handling tug, and a full day's work. This obstacle led us to developing an upgraded Electric Plant module that was externally mounted on the topside of the Nacelle. This 'Fix Box' bypassed the original Electric Plant and provided full load control with better accessibility and improved battery charging capability. 


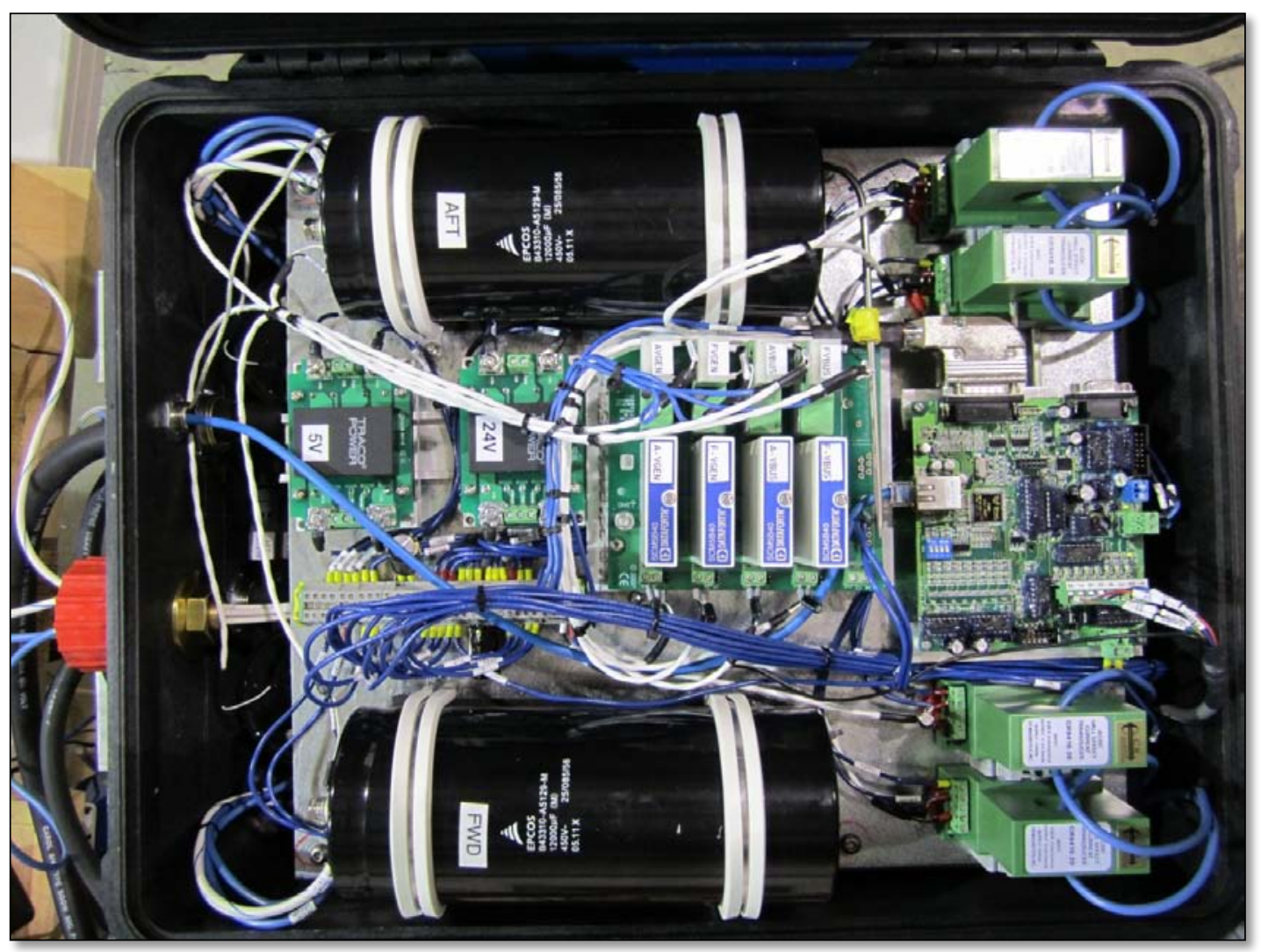

Figure 42 - Power Electronic components inside the waterproof Fix Box.

During deployment the Electric Plant would charge the onboard battery bank to provide power to the Station Power system. When the wave resource was sufficient this worked extremely well and surplus power was often needed to be dumped by the burn-off resistor. However, during low summer wave conditions the incoming energy did not meet the Station Power load levels. This disparity was initially addressed through support vessel based charging operations. A small craft support vessel with an onboard generator and battery charger would dock alongside the WEC and perform a battery charge that would last for 4-8 hours.

With sunny summer conditions approaching, three 220W solar panels and an onboard solar charge controller were added to help maintain station battery levels. This worked extremely well during peak summer months and eliminated charging requirements altogether. As fall approached the solar power contribution fell dramatically. Fortunately however, the waves regained strength and the Electric Plant again provided power to the onboard batteries.

\subsection{Control \& SCADA (0400)}

Early in the testing period the most prevalent issue encountered was related to remote communications. The USB connected 3G wireless modem worked wonderfully during single day testing prior to deployment however after several days of deployment, issues arose; the modem would lose a link with the network and often failed to reconnect. Additionally, software updates and USB port lockouts prevented connection. This became a real burden since this wireless connection was our only way of remotely connecting to the WEC. Eventually, a $3 G$ network 
router was installed to replace the $3 G$ modem which operated flawlessly for the rest of the 13 month deployment. This connection allowed operators real-time access to SeaRay and transferred operational data to land based servers.

A number of events occurred that prevented the data collection PC from capturing data continuously. The data collection software did not live up to expectations and would occasionally cease data collection requiring human intervention in order to restart the process. On several occasions this same software would fail to recognize its own hardware resources which required a difficult and time consuming reinstallation onto the remote PC.

On a few occasions the onboard PC would power down and could not be restarted remotely. This was soon corrected by setting the PC to automatically power up anytime voltage was restored. Recommended solutions to these and other experiences can be found in the final DOE EE0002647 report.

\subsection{Auxiliary Systems (0500)}

\subsubsection{Ballast System (0510)}

The unanticipated collision between the WEC and a presumed recreational vessel resulted in a leak in the ballast chamber of the aft float and allowed air to enter and water to exit the float. This change in ballast meant that the aft float was lighter than desired. Future ballast chamber designs should account for such a contingency.

\subsubsection{Station Power (0540)}

With the station power being supplied by onboard battery systems it is crucial that all power supplies feeding off of the battery bank have a wide input voltage range to accommodate higher than normal voltages during charging events and low voltages associated with depleted batteries. Additionally, the Station Power should have remotely controllable breakers on each subsystem to allow remote shut down and restart.

In a rare event, the input power supply to one I/O module failed short which would normally blow the time delay fuse and isolate the defective unit. This unit was equipped with a low voltage protection relay that opened when the supply voltage dropped and closed when it recovered. This led to uncontrolled relay cycling which affected adjacent modules. If this type of input supply must be used, it should be protected by a fast response fuse.

\subsubsection{Solar (0545)}

The solar electric collection system proved to be highly valuable addition during our intermediate scale deployment, providing good base load capability during low wave summer conditions. At one point a large sea lion hauled out onto the float causing structural damage to the forward solar panel. This damaged panel continued to produce usable levels of energy. By the end of the deployment there was strong evidence of corrosion on the solar power cable plugs.

\subsubsection{Navigation (0550)}

The navigational beacon required by the U.S. Coast Guard required a minimum visible range of 2 NM. In adverse weather this light intensity was not considered adequate by several seasoned 
mariners. A significantly higher intensity 12 NM light which replaced the original beacon demonstrated far better performance in adverse weather and was deemed well worth the investment to protect the WEC from collision. Additionally, the original 8" passive radar reflector did not provide definitive identification when viewed with a support vessel's radar. This was upgraded with an 8" and a 12" radar reflector mounted atop a 3' tower extension to increase the height over the water. This was marked with reflective tape and topped with a highly visible flag. The new radar signature when viewed by the support vessel was now large and unmistakable.

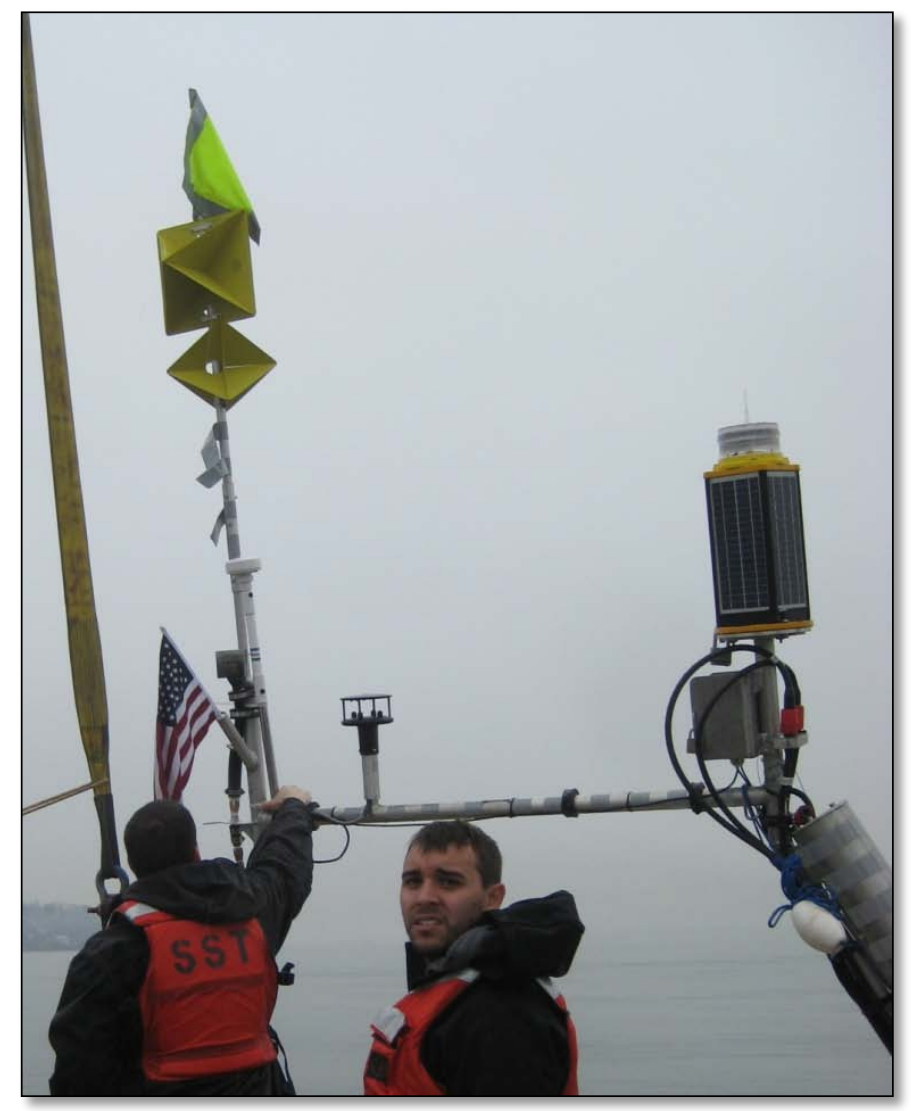

Figure 43 - Upgraded navigational aids to increase visibility against a grey Seattle sky.

\subsubsection{Bilge System (0570)}

Our dual redundant bilge system performed well, once the hull system was demonstrated water tight the bilge pumps almost never ran. Only a few seconds of run time were every recorded by SCADA over the entire deployment duration. During low power conditions in the summer months the onboard battery banks were occasionally depleted. Though this never became an issue, it did leave the bilge pumps vulnerable to low supply voltage until the batteries could be recharged. 


\subsubsection{Surveillance (0580)}

The shore based webcam was used on a daily basis to check on the WEC. The convenience of checking the webcam proved invaluable when there were questions as to the condition of the WEC. There were a few occasions when a camera with higher zoom capabilities to see certain parts of the WEC were needed, but this one worked well and within budget. Even at night the webcam could capture the navigational beacon confirming the WEC's continued presence. At times Seattle's foul weather prevented the webcam from seeing the WEC at all.

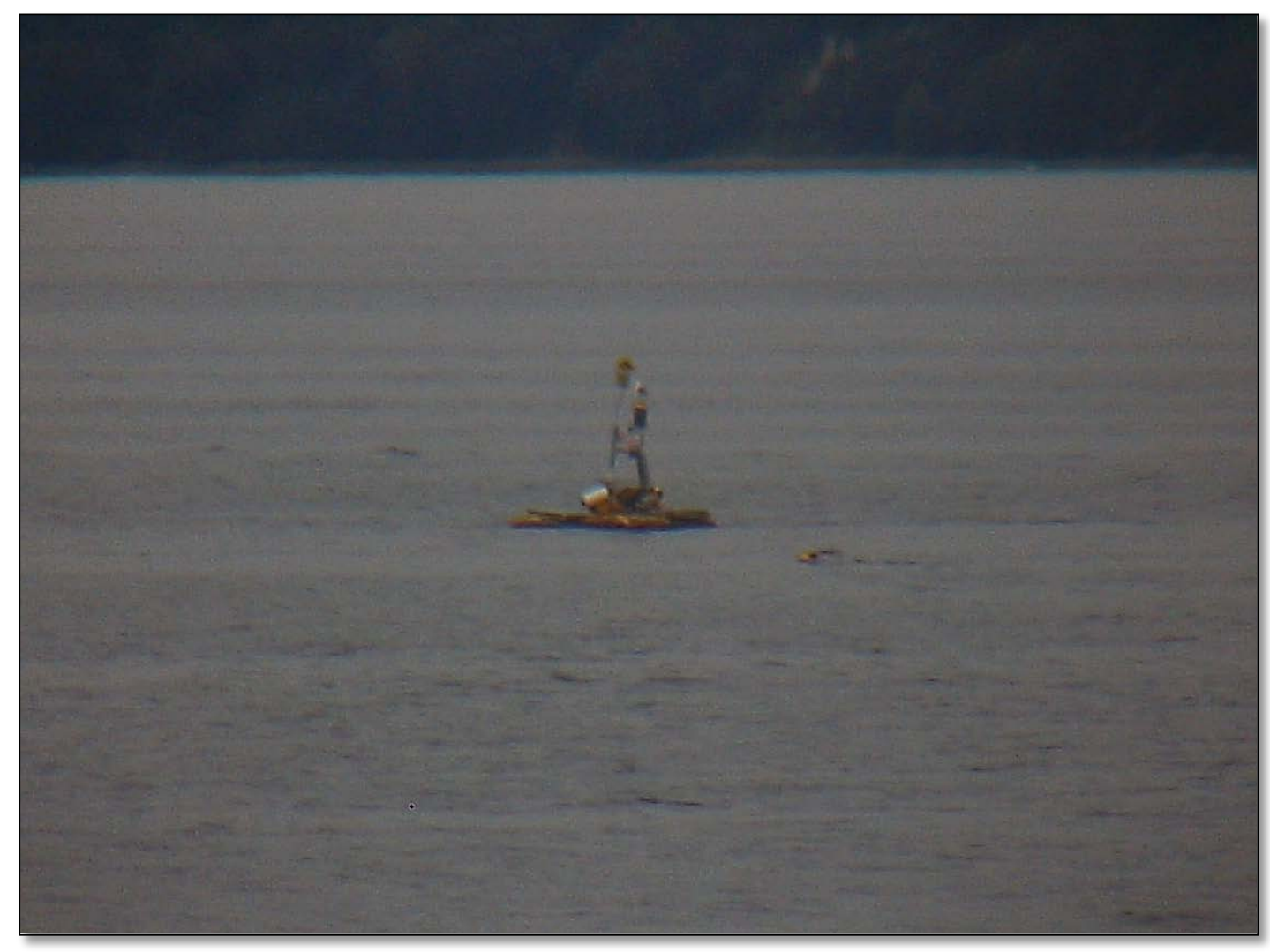

Figure 44 - Typical webcam image from lighthouse 500 yards away.

\subsection{Outfit \& Furnishings (0600)}

\subsubsection{Hull Fittings (0620)}

The attachment points designed into the WEC were heavily used. Various methods were used to tie off to the WEC. The overhead instrument mast became a valuable hand hold while working on the WEC and securing tools and equipment too. Aluminum frames made to mount the solar panels conveniently attached to the existing lifting points on the floats. The nacelle lifting eyes also served as an attachment point for the Fix Box. Boat bumpers were left attached to the sides of the nacelle to protect service vessels when tied off.

\subsubsection{Corrosion \& Biofouling (0640)}

The zinc anodes proved extremely useful. Upon recovery the anodes had lost roughly half of their mass. This steel load arms did not show significant corrosion and all electrically connected 
components fared well. Several live electrical connectors exposed to marine conditions did show signs of corrosion and would have ultimately led to failure in time.

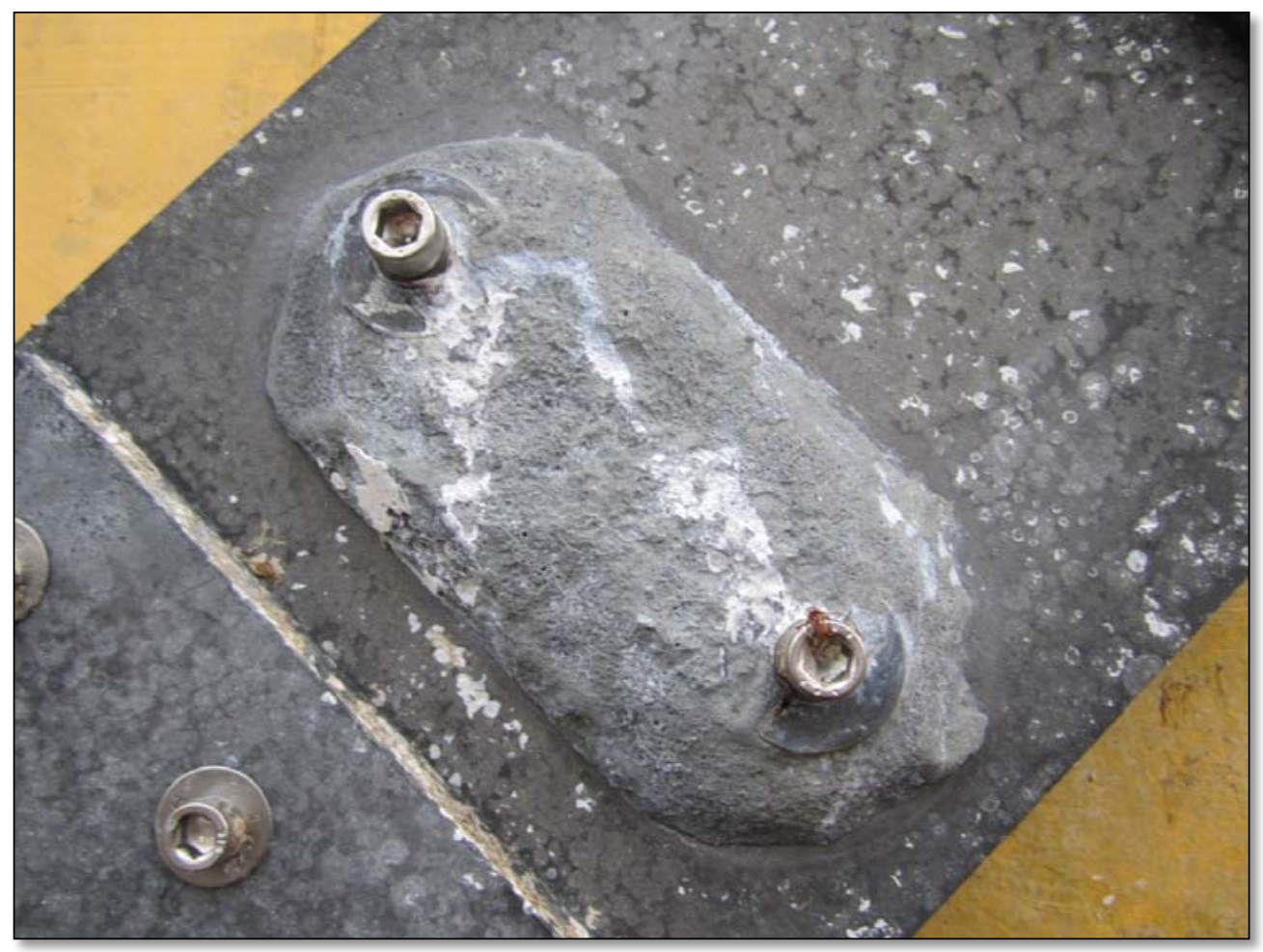

Figure 45 - Degraded zinc anode after deployment.

Three small IP67 rated enclosures housing the mooring load cell amplifier were made from thin walled aluminum of unknown alloy. These boxes where coated with zinc paint, but did not survive well. In the last weeks of testing two of these enclosures failed completely. The original design was based on a three month deployment which was achieved.

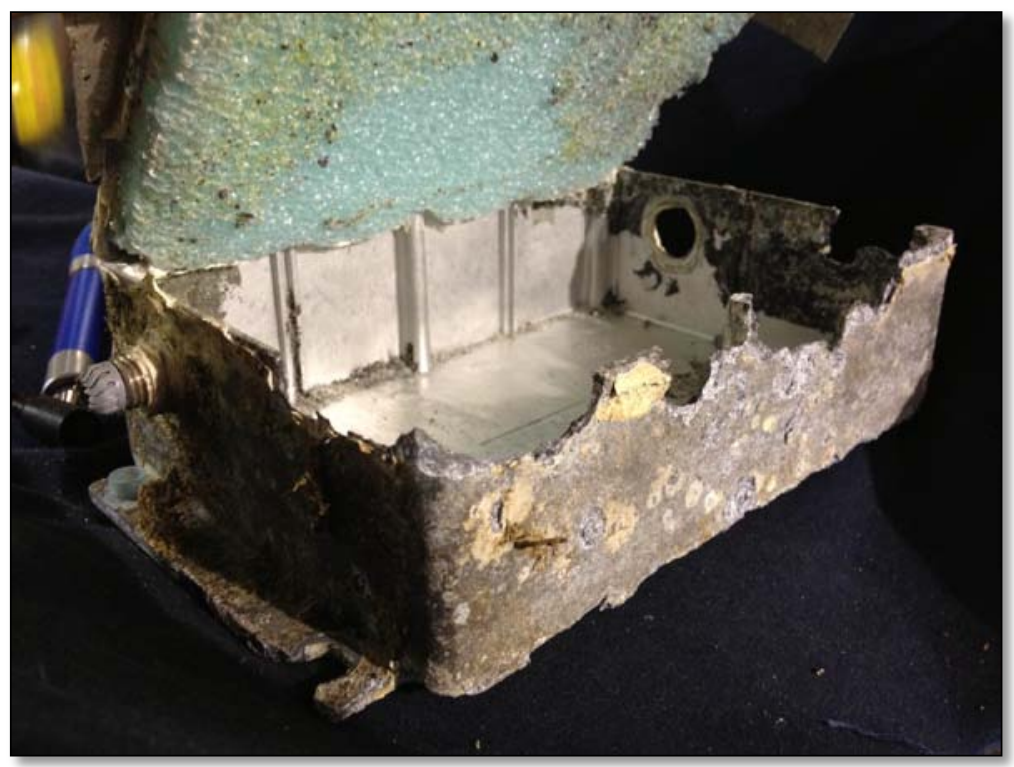

Columbia Power Technologies, LLC $\bullet 4920$ A SW $3^{\text {rd }}$ St, Corvallis, Oregon 97333 Phone: (541) 368-5033 • Fax (541) 230-1498 • www.columbiapowertechnologies.com 
Figure 46 - Corrosion caused failure of mooring load cell amplifier enclosure.

The external paint on the FRP components was a standard gel coat with no anti-fouling additives. The WEC was cleaned by divers once over the year long deployment. A biofouling coating may have prevented some growth on the surface and reduced the need for maintenance. The main surface of the acoustic modem was coated in petroleum jelly which reduced bio-fouling.

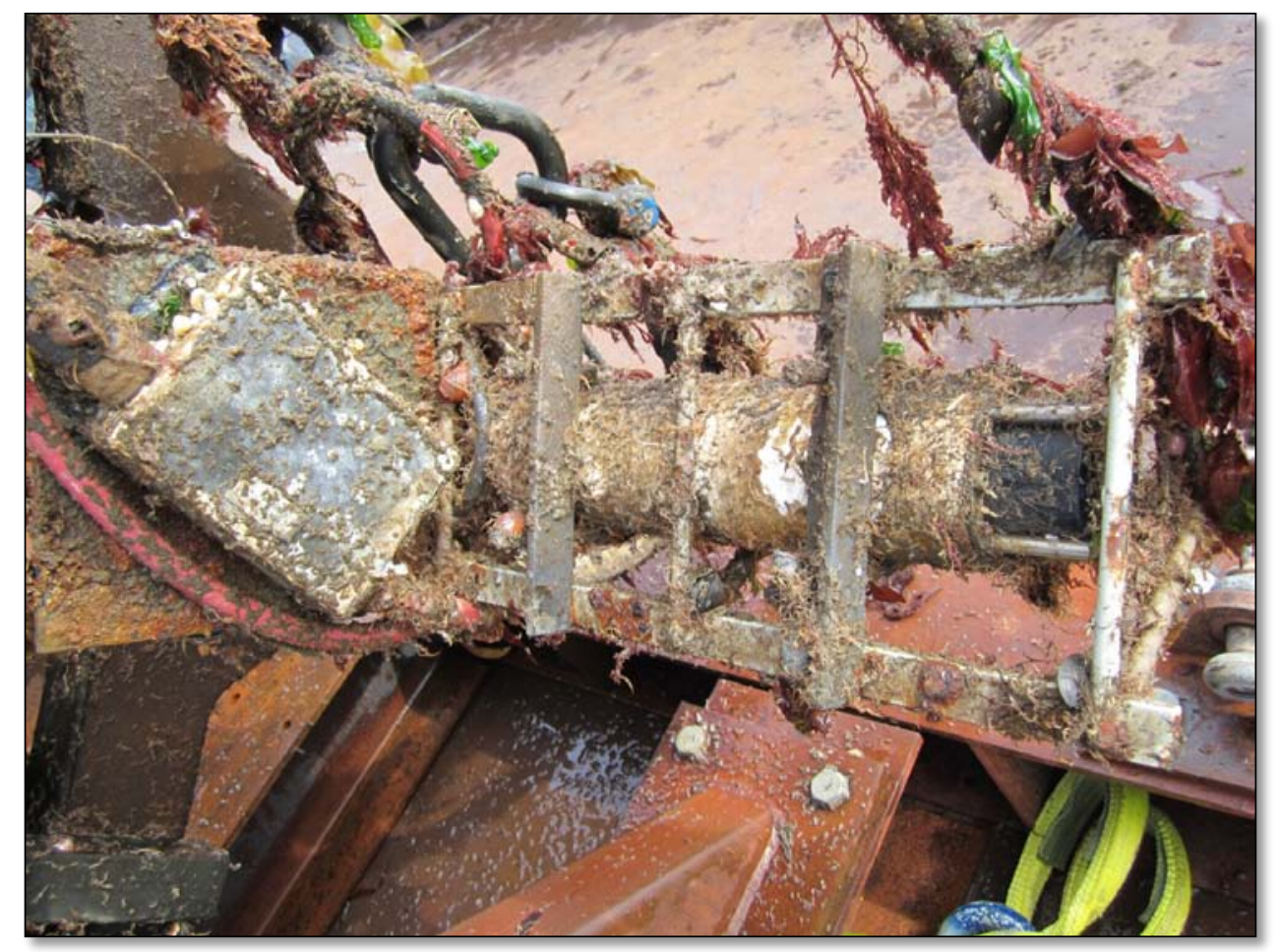

Figure 47 - Typical biofouling on acoustic modem and mooring hardware. 


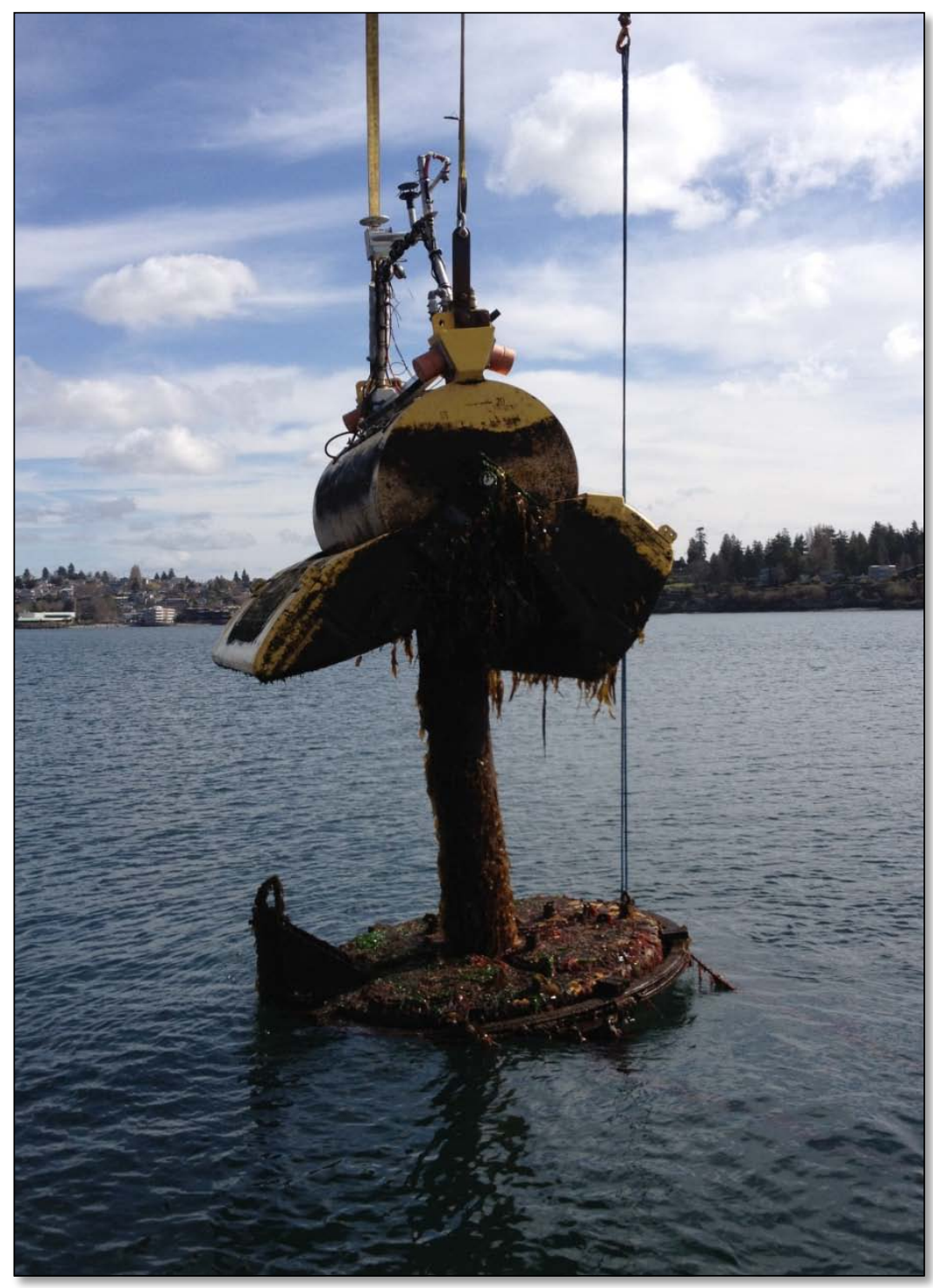

Figure 48 - SeaRay during recovery, not a sea monster.

\subsection{Mooring (0700)}

SeaRay's three point mooring system worked well at keeping the WEC on station and limiting mooring loads. The yaw control system encountered a number of failures. Initially binding between the outer ring and the guide roller systems required careful adjustments and yielded higher than anticipated friction. Ultimately the original cable driven approach did not have enough traction or torque to drive the yaw control system. Shortly after the initial deployment SeaRay was recovered and the cable was replaced with a chain and sprocket solution with a lower gear ratio. This new solution overcame the binding issues and worked well to the point that the chain broke. The chain was replaced by divers and operated well thereafter. 


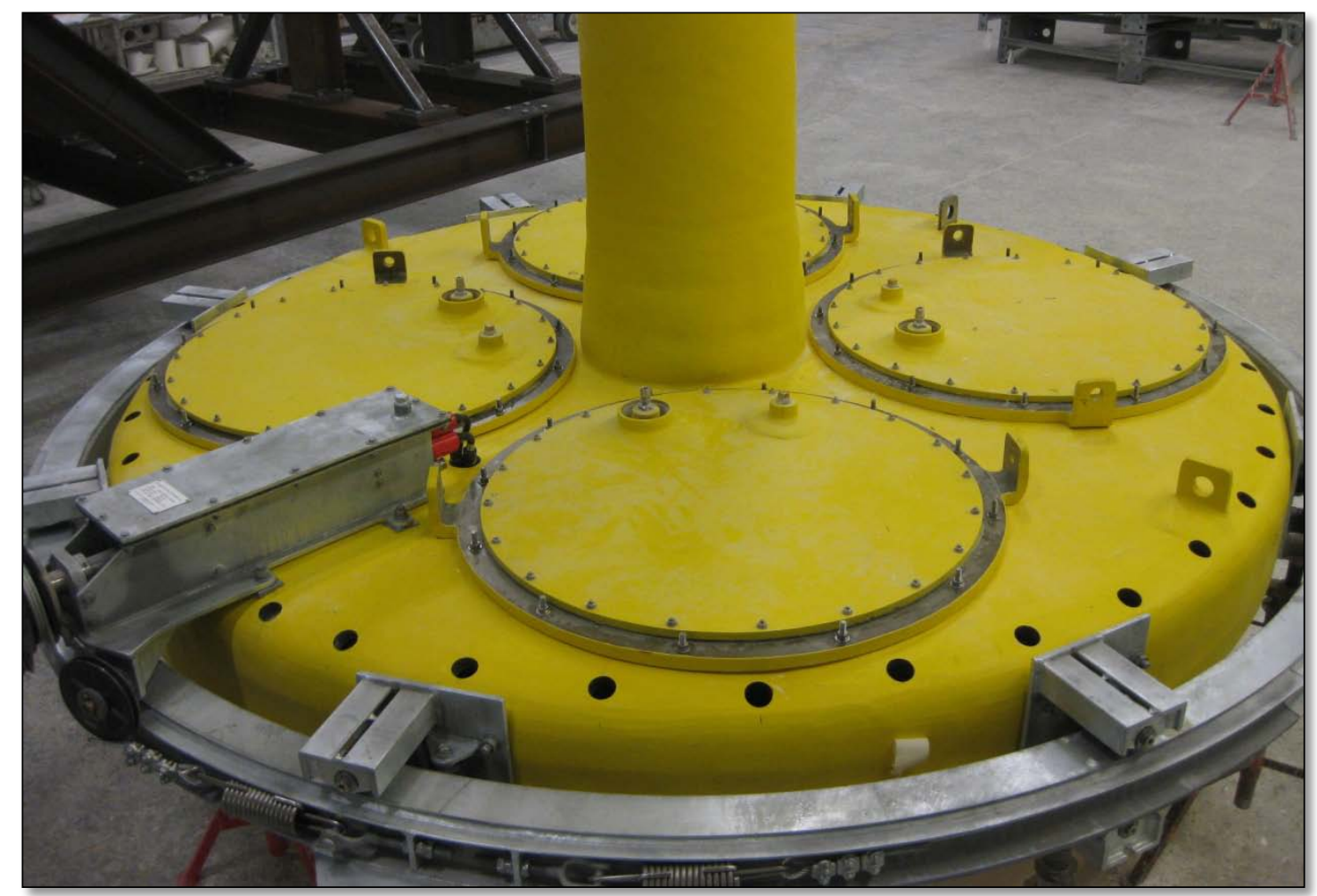

Figure 49 - SeaRay damper tank showing the yaw control system and yaw drive motor.

On recovery day the $6,000 \mathrm{lb}$ anchors were to be recovered by the vertical mooring lines which were $3 / 4$ " galvanized steel cables rated at 25,000 lb. During recovery two out of three of these cables parted before the anchor was lifted from the sea bed. Further inspection showed that the cables had prematurely rusted almost completely through. Follow on research will be conducted prior to the utilization of steel cables in any future mooring designs. Divers were dispatched to attach recovery lines and the two remaining anchors were removed the following day.

\subsection{Logistics (0900)}

Even an intermediate scale test requires that considerable details are addressed in the preparation, permitting, build, transportation, deployment, recovery and decommissioning of the WEC, and while those plans associated with a 7-ton device differ in magnitude from an 1100-ton WEC, the necessities are the same.

\subsubsection{Permitting (0930)}

Permitting was initiated early in order to get a head start on the effort. Even though the project was not grid connected, a categorical exclusion and Nationwide permit for scientific instrumentation (NWP-5) were obtained, the process took over twelve months. An early start on permitting is essential. 


\subsubsection{Site Planning (0950)}

Site selection (appendix H), wave resource assessments and scale selection (appendix I and J) were performed prior to contract start and at the beginning of the project. The site at Puget Sound provided the best-combined solution for an intermediate test site on the west coast.

Contractors for transporting, deploying, operations, maintenance, metocean instrumentation design and installation, mooring location selection and acoustic studies were established. Mooring placement was driven by depth, current, wave, wind considerations, the expectation that a yaw control system could provide preferred WEC-wave heading and ultimately a depth of approximate $60-70$ feet was selected.

\subsection{Operations (1100)}

Normal operations included automated remote download and recording of WEC data, remote connection and review of WEC instrumentation status, Web cam observations, checking battery voltage against battery performance curves to determine if charge was needed. The frequency of these activities varied from hourly, 4-hours, or 8-hours, seven days per week, depending on WEC status and weather conditions.

\subsubsection{WEC and Mooring Installation (1110)}

The installation procedures are covered in detail in appendix L.

\subsubsection{Emergency Operations (1130)}

A number of emergent events occurred during the first deployment of the WEC.

A Kline wire rope grip was used to lift the anchor assembly pictured in Figure 7 in the procedure. During this lift the grip detached from the wire rope and the anchor and subsurface float fell to the deck. No personnel were injured and only minor damage was done to equipment. This event did present a potential hazard to personnel safety and equipment integrity. The cause was twisting of the wire rope as it was loaded and the picking up of multiple loads not directly in line. Corrective actions was to avoid use of a wire rope grip by designing the anchor assembly with multiple in line pick up points as required.

During movement of the crane barge from the NE anchor position to the South anchor position the crane barge hit and damaged the WEC. Damages were minor and easily repaired on site. This occurred because personnel were not assigned to each specific task and some areas were understaffed. Personnel left their assigned stations to assist other personnel. The hazard of a slow moving barge was underestimated. Corrective action is to treat buoy like a man over board situation- one man equipped with a radio, should be dedicated to watching the buoy. All personnel involved in the operation should have radios. Personnel should stay on assigned stations until tasks are completed. Procedure changes include assigning specific personnel to be assigned to monitor buoy location and communicate with crane barge bridge.

The small boat (Zodiac) could not maintain headway in wind and had to be recovered by the other skiff. The wind had picked up during the operation and the battery powering the electric motor on the Zodiac was partially discharged causing an inability of the Zodiac maintain 
headway. Corrective actions included; supplying extra batteries on board main vessel to insure fresh battery for electric motor; insure that all personnel operating skiffs have radios and/or cell phones aboard; and limit operations of Zodiac to manageable wind and current conditions.

\subsubsection{Maintenance, Repair \& Overhaul (MRO) (1140)}

As identified in the report, repairs made to the power electronics, wireless communications and yaw control repairs systems. Normal battery charges, solar panel installation and a WEC heading calibration was also performed. Two diver inspections were made and the hull was cleaned over the thirteen month period. These are reasonable examples of on station activities to consider when planning future deployments.

\section{Conclusion}

Columbia Power Technologies deployed a scaled prototype WEC in the Puget Sound. The SeaRay was kept on station using a 3-point mooring system, and could be actively controlled to yaw with respect to the mooring system, such that it could face any direction. The SeaRay prototype is a dedicated research platform instrumented with a suite of data collection equipment and sensors. Additionally, a subsurface buoy mounted AWAC allowed for characterization of waves and currents.

The SeaRay was designed as a 1:7 scale prototype of the Generation 3.1 Ray series WEC. However, due to practical limitations associated with scale and the data requirements, the mass distribution of the SeaRay differs substantially from the commercial scale design. As such, the observed performance is indicative, but does not accurately describe the response of the commercial scale device. The primary use of the data is validation of numerical models, and as such the mass differences are not seen as problematic.

With the scale factor of 7, typical full scale equivalent (FSE) $H_{m 0}$ values observed in the Puget Sound range from roughly 0.5 to $3.5 \mathrm{~m}$, covering the range of operational seas expected in open oceans. The maximum observed value of $1.55 \mathrm{~m}$ scales to $10.9 \mathrm{~m}$, which is on the order of design wave height expected in open oceans. FSE $T_{e}$ was typically between 5 and $9 \mathrm{~s}$, which is marginally higher frequency than what would be considered representative of expected open ocean conditions. Thus the waves on the whole tend to be steeper than expected open ocean conditions. The seas were generally much more directionally spread than would be expected in open oceans (with a spreading index typically between 1 and 2), and with stronger currents than would be typical for a candidate utility WEC location (FSE currents up to $2 \mathrm{~m} / \mathrm{s}$ ). All things considered, the deployment location was a good choice, offering many seas in the operational range and several in the design range. WEC and environmental data was collected for more nearly 25,000 trials of $512 \mathrm{~s}$ duration. After quality control of sea state data and WEC PTO data, roughly 16,500 trials remained for consideration.

WEC performance was characterized primarily using the Relative Capture Width (RCW). Performance was shown to correlate with six parameters characterizing either the WEC or metocean conditions: energy period, significant wave height, wave heading, PTO damping, Unidirectivity Index, and fitQ (a parameter describing the spectral shape). The strongest correlation is with the frequency content of the incident waves. In general the observed 
frequencies had been Doppler shifted by the pervasive tidal currents and as such the observed, rather than intrinsic, spectrum was used to calculate the energy period. Performance declined noticeably with increasing energy period. Though not as drastic, performance also declined with increasing wave heights. As expected, performance was best for head seas. This trend, however, flattened significantly with increasing energy period. The effect of PTO damping is harder to quantify, primarily because the testing of significantly different damping cases was fairly limited. That being said, performance dropped noticeably when very heavy damping was applied. Although the incident seas were in general extremely short-crested (i.e. heavily spread directionally), the positive correlation of performance with increasing Unidirectivity (i.e. longcrestedness) is clear. Finally, performance was shown to be negatively correlated with fitQ, implying that performance is generally improved when the incident wave spectrum conforms to JONSWAP shape.

The heavily instrumented SeaRay yields not only performance data, but data that informs design as well. Design data includes mooring loads, end stop loads and structural strain. Mean mooring loads were shown to correlate with current speed, and oscillatory loads with significant wave height. Furthermore, it was found that mean loads were in general significantly greater than the oscillatory loads. Only the oscillatory strain was analyzed, as signal drift made analysis of absolute strain problematic. Oscillatory strain was seen to correlate with wave height, and was significantly greater for head seas as compared to following seas. End stop loads were found to correlate with significant wave height, and were most numerous and forceful for the aft float at the 'top' position where the range of motion was most restricted. The strikes were particularly forceful when the PTOs were undamped (i.e. freewheeling). WEC design has since evolved to alleviate the need for end stops.

The SeaRay was a well conceived prototype that was well built through collaboration with several key partners. As a $1: 7^{\text {th }}$ scale model of the version 3.1 WEC all efforts were made to match the physical parameters as closely as possible to their FSE values. The use of fiberglass reinforced plastic (FRP) proved very valuable as it was rugged, corrosion resistant, and was easily modified. FRP will continue to be utilized in all of Columbia Powers future deployments. The commercial off the shelf PTO worked extremely well during the testing. The low cost gearbox and low speed PMG reliably converted the WEC motion into electric power. As power electronic hardware failures occurred, the Electric Plant went through several design iterations. The final Electric Plant configuration proved very effective and reliable while benefiting from being readily accessible for repairs and maintenance.

The control and SCADA system aboard the SeaRay worked well but also taught us a lot about communication, accessibility, and reliability. The importance of having multiple communication paths cannot be overstated. The sensor network suite successfully gathered an enormous amount of operational data during the deployment. A few sensors experienced failure during the deployment, most due to corrosion and waterproofing failures. At times the data collection software failed to achieve high reliability, but with incremental improvements and careful operation it was able to capture tens of thousands of trials during its 13 months of operation. The Auxiliary Systems worked extremely well at supporting the WEC with station power, navigational aid, bilge pumping, and surveillance among other responsibilities.

Columbia Power Technologies, LLC • 4920A SW $3^{\text {rd }}$ St, Corvallis, Oregon 97333

Phone: (541) 368-5033 • Fax (541) 230-1498 • www.columbiapowertechnologies.com 
SeaRay's three point mooring system worked well at keeping the WEC on station and limiting mooring loads. A serious concern still exists regarding the failure of two galvanized steel cables on recovery day which warrants further investigation. When functional, the yaw control system worked to turn the WEC into any given heading. The YCS did however encounter a number of failures that required careful attention and repair.

A subset of the extensive SeaRay data set has been used to validate numerical models in AQWA. The simulations were carried out in the time domain, and accounted for some nonlinearity, such as PTO torque limiting and viscous drag. The mean error over all 18 cases for total RCW is $-2 \%$. For the fore and aft PTOs considered separately, the mean errors are $7 \%$ and $2 \%$ respectively. 


\section{References}

[1] B. Holmes, “Tank Testing of Wave Energy Conversion Systems,” EMEC Standards. European Marine Energy Centre. Orkney, UK, 2009.

[2] T. Pedersen, A. Lohrmann, and H. E. Krogstad, "Wave measurements from a subsurface platform,” Proceedings WAVES 2005, 2005.

[3] M. J. Tucker and E. Pitt, Waves in ocean engineering, vol. 5. Elsevier Science Ltd, 2001.

[4] L. H. Holthuijsen, Waves in Oceanic And Coastal Waters. Cambridge University Press, 2007.

[5] D. Mollison, "Wave climate and the wave power resource," Hydrodynamic of Ocean WaveEnergy Utilization, pp. 133-156, 1986.

[6] P. Lenee-Bluhm, “33rd Scale Tank Testing: Single WEC Assessment," Columbia Power Technologies, Jan. 2012.

\section{Appendix A. Prototype WEC dimensions}

\section{SeaRay prototype dimensions (m)}



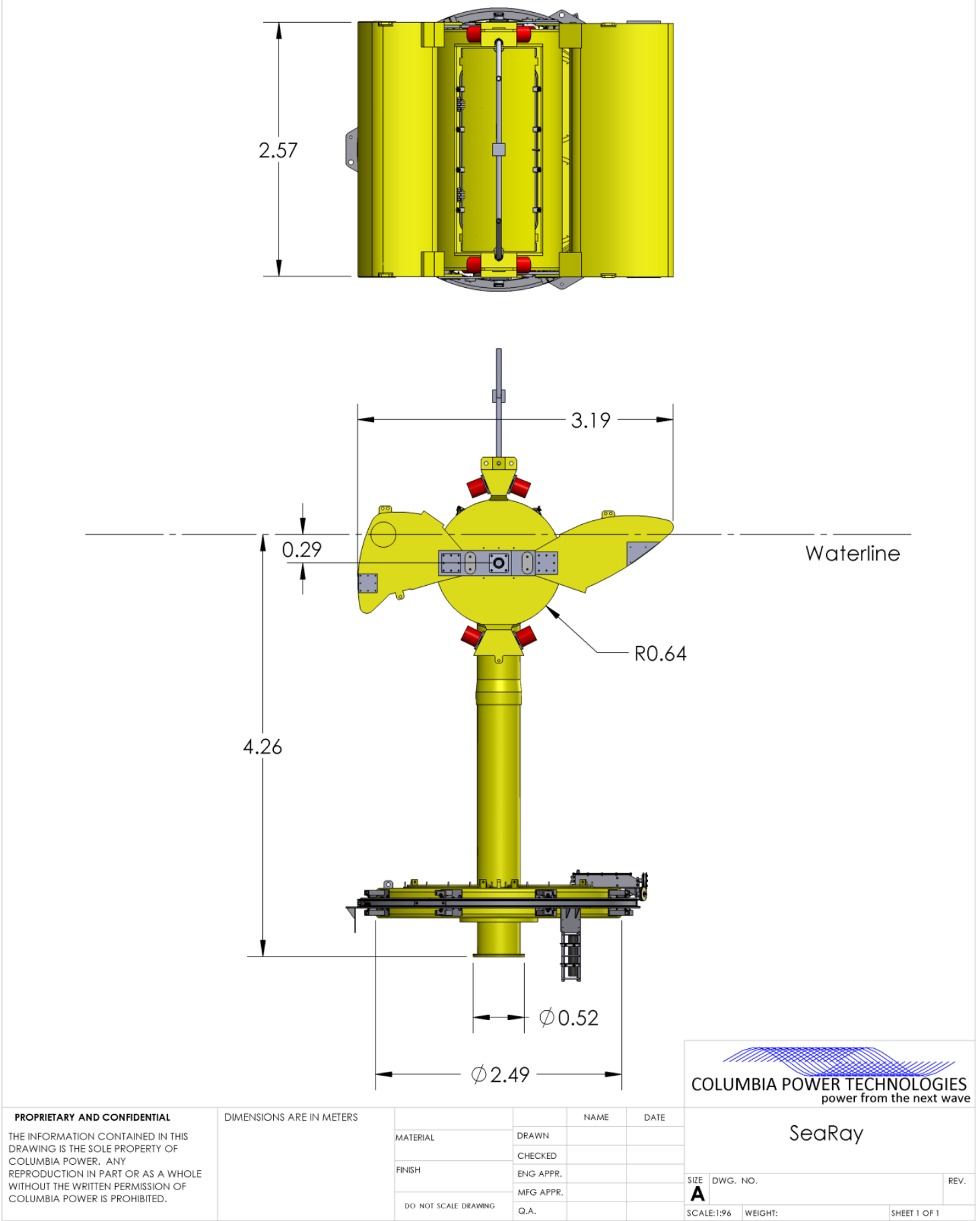

Columbia Power Technologies, LLC • 4920A SW $3^{\text {rd }}$ St, Corvallis, Oregon 97333 Phone: (541) 368-5033 • Fax (541) 230-1498 • www.columbiapowertechnologies.com 


\section{Appendix B. Prototype data collection equipment and sensors}

The SeaRay prototype is a dedicated research platform instrumented with a suite of data collection equipment and sensors. Each sensor was provided with factory calibration or calibrated by engineers at Columbia Power. To ensure proper calibration a documented validation process was conducted by Columbia Power which tested each sensor over its entire range of operation. The sensor network is organized into the major WEC subsystems with a complete sensor list below. In total there are 98 unique data sets collected by these sensors and onboard set points.

\begin{tabular}{|c|c|}
\hline Signal Group & Signal Name \\
\hline 100 - Hull Structure & $\begin{array}{l}\text { End Stop Load Ch01 } \\
\text { End Stop Load Ch02 } \\
\text { End Stop Load Ch03 } \\
\text { End Stop Load Ch04 } \\
\text { End Stop Load Ch05 } \\
\text { End Stop Load Ch06 } \\
\text { End Stop Load Ch07 } \\
\text { End Stop Load Ch08 } \\
\text { Struct_Strain_Gauge_Ch09 } \\
\text { Struct_Strain_Gauge_Ch10 } \\
\text { Struct_Strain_Gauge_Ch11 } \\
\text { Struct_Strain_Gauge_Ch12 } \\
\text { Struct_Strain_Gauge_Ch13 } \\
\text { Struct_Strain_Gauge_Ch14 } \\
\text { Struct_Strain_Gauge_Ch15 } \\
\text { Struct_Strain_Gauge_Ch16 }\end{array}$ \\
\hline $\begin{array}{l}\mathbf{2 0 0} \text { - PTO } \\
\text { Power Take-Off }\end{array}$ & $\begin{array}{l}\text { Fwd_Measured_Torque } \\
\text { Aft_Measured_Torque } \\
\text { Fwd_AC_Voltage_A } \\
\text { Fwd_AC_Voltage_B } \\
\text { Fwd_AC_Voltage_C } \\
\text { Aft_AC_Voltage_A } \\
\text { Aft_AC_Voltage_B } \\
\text { Aft_AC_Voltage_C } \\
\text { Fwd_Proximity_Sensor } \\
\text { Aft_Proximity_Sensor } \\
\text { Fwd_Encoder } \\
\text { Aft_Encoder } \\
\text { Fwd_Slip_Switch } \\
\text { Aft_Slip_Switch } \\
\text { Oil Sensor }\end{array}$ \\
\hline
\end{tabular}

\begin{tabular}{|l|l|}
\hline Signal Group & \multicolumn{1}{|c|}{ Signal Name } \\
\hline $\mathbf{3 0 0}$ - Power Electronics & Fwd_Passive_Bus_Voltage \\
& Aft_Passive_Bus_Voltage \\
& Active_Bus_Voltage \\
& Fwd_Passive_Bus_Current \\
& Aft_Passive_Bus_Current \\
& Active_Bus_Current \\
& Fwd_A_PWM \\
& Fwd_C_PWM \\
& Aft_A_PWM \\
& Aft_C_PWM \\
\hline $\mathbf{4 0 0}$ - SCADA & IMU - Accelerometer \\
& GPS \\
& Heading \\
& Fwd Generator Temperature \\
& Aft Generator Temperature \\
& Fwd Gearbox Temperature \\
& Aft Gearbox Temperature \\
& SCADA Temperature \\
& Power Electronics Temperature \\
& 24V Box Temperature \\
& Capacitor Temperature \\
\hline $\mathbf{7 0 0}$ - Mooring & Battery_Bus_Charge_Current \\
& Battery_Bus_Discharge_Current \\
& Battery_Bus_Voltage \\
& Bilge_1_Status \\
& Bilge_2_Status \\
& Wind_Speed \\
& Wind_Direction \\
& AWAC-Wave information \\
\hline & Stbd_Mooring_Tension \\
& Port_Mooring_Tension \\
& Aft_Mooring Tension \\
& Yaw Drive Command \\
Yaw Position Encoder \\
\hline
\end{tabular}

Columbia Power Technologies, LLC • 4920A SW $3^{\text {rd }}$ St, Corvallis, Oregon 97333 Phone: (541) 368-5033 • Fax (541) 230-1498 • www.columbiapowertechnologies.com 
The data collection and human machine interface was accomplished with a dSPACE in vehicle rapid prototyping hardware known as the MicroAutoBox. This hardware takes in outputs from all field sensors which are used in making control decisions and prepared for data collection. An onboard embedded PC is loaded with Matlab/Simulink and ControlDesk software. The MicroAutoBox is programmed with a special real-time interface through Matlab/Simulink. This allows control and processing codes to be developed and tested offline and quickly programmed into hardware for use in the SeaRay WEC. ControlDesk provides a convenient graphical user interface (GUI) to visualize and store data collected by the MicroAutoBox. Together the embedded PC, the MicroAutoBox, and various signal conditioning modules make up the SCADA system. This system is secured in a watertight enclosure to ensure safe operation during the SeaRay deployment.

\section{Appendix C. Anchor locations}

SeaRay deployed position

Anchor locations and mean WEC location in 'null' conditions

Deployment $1 \quad$ 2/16/11 to 3/1/11

Deployment 2 3/11/11 to 3/20/12

Note that the WEC position and anchor locations were measured using different GPS devices. This may explain the mean WEC position not being centered on the anchors.

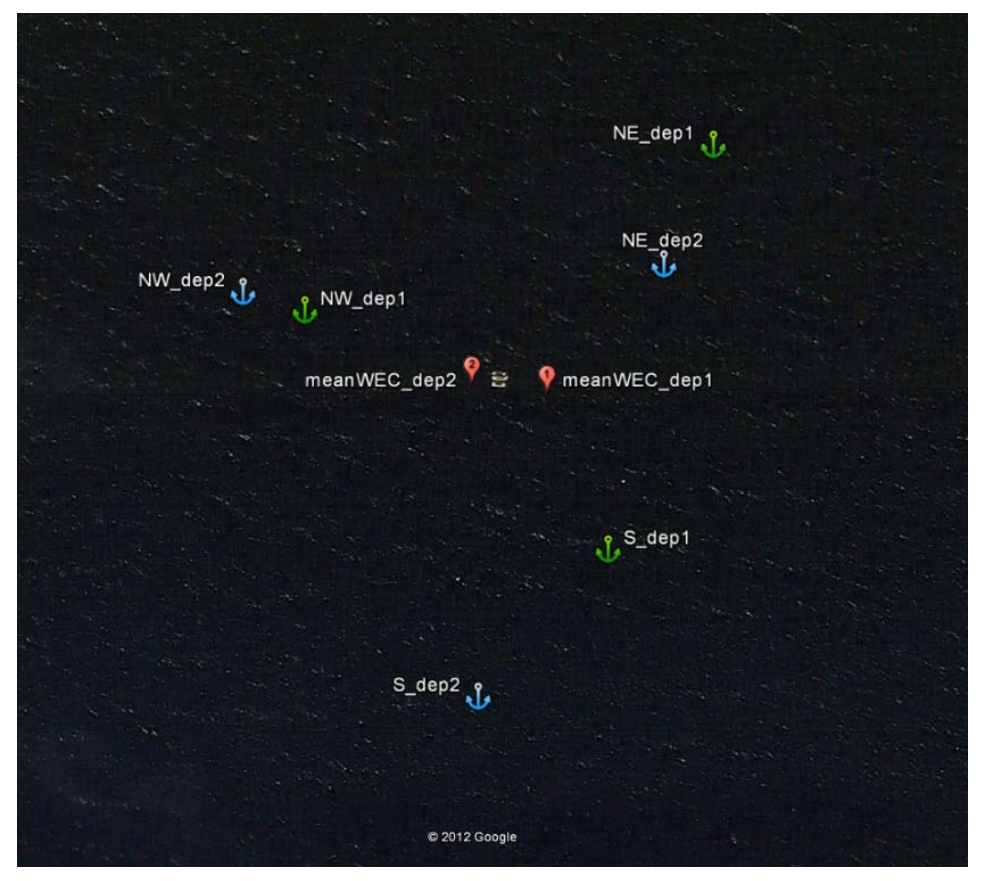

Anchor locations for $1^{\text {st }}$ deployment (2/16/11)

Measurements taken by Ryan Gowler of Sound and Sea on 2/18/11 and 2/22/11

Columbia Power Technologies, LLC • 4920A SW $3^{\text {rd }}$ St, Corvallis, Oregon 97333

Phone: (541) 368-5033 • Fax (541) 230-1498 • www.columbiapowertechnologies.com 


\begin{tabular}{ccc}
\hline anchor & lat (deg N) & long (deg W) \\
\hline port s & 47.6580000 & 122.4402833 \\
stbd nw & 47.6584333 & 122.4411000 \\
aft ne & 47.6587333 & 122.4400000 \\
\hline
\end{tabular}

Anchor locations for $2^{\text {nd }}$ deployment (3/11/11)

Measurements taken by Bill Daly of Sound and Sea on 3/27/11

\begin{tabular}{ccc}
\hline anchor & lat (deg N) & long (deg W) \\
\hline port s & 47.657733333 & 122.440633333 \\
stbd nw & 47.658466667 & 122.441266667 \\
aft ne & 47.658516667 & 122.440133333 \\
\hline
\end{tabular}

Null condition mooring loads

Note: These mean mooring loads in nearly null conditions are the best representation of pretension. An X indicates a failed signal.

\begin{tabular}{|c|c|c|c|c|c|c|c|c|}
\hline & $\mathrm{Hmo}[\mathrm{m}]$ & $\begin{array}{l}\text { direction } \\
\text { [deg CW } \\
\text { from } \mathrm{N} \text { ] }\end{array}$ & $\begin{array}{c}\text { curr } \\
\text { speed } \\
{[\mathrm{m} / \mathrm{s}]}\end{array}$ & $\begin{array}{l}\text { direction } \\
\text { [deg CW } \\
\text { from N] }\end{array}$ & $\begin{array}{c}\text { water } \\
\text { depth } \\
{[\mathrm{m}]}\end{array}$ & $\begin{array}{c}\text { mea } \\
\text { nw } \\
\text { (stbd) }\end{array}$ & oring te & ne (aft) \\
\hline \multicolumn{9}{|l|}{ 1st deployment } \\
\hline seaRayTrial_y2011_m02_d21_12_10_00 & 0.059 & 37 & 0.048 & 10 & 22 & $\mathrm{X}$ & 30 & 199 \\
\hline seaRayTrial_y2011_m02_d21_12_20_00 & 0.056 & 67 & 0.043 & 10 & 22.2 & $\mathrm{X}$ & 32 & 199 \\
\hline MEAN & & & & & & & 31 & 199 \\
\hline \multicolumn{9}{|l|}{ 2nd deployment } \\
\hline seaRayTrial_y2011_m07_d17_09_30_00 & 0.038 & 299 & 0.020 & 2 & 20.7 & 883 & 635 & 1003 \\
\hline seaRayTrial_y2011_m09_d19_10_50_00 & 0.038 & 28 & 0.014 & 132 & 19.1 & 941 & $x$ & 1026 \\
\hline seaRayTrial_y2011_m11_d16_12_30_00 & 0.039 & 138 & 0.022 & 122 & 20.5 & 1109 & $x$ & 1137 \\
\hline seaRayTrial_y2011_m11_d16_12_50_00 & 0.035 & 307 & 0.008 & 338 & 20.7 & 1111 & $x$ & 1139 \\
\hline seaRayTrial_y2011_m11_d16_13_00_00 & 0.034 & 93 & 0.021 & 86 & 20.9 & 1109 & $x$ & 1138 \\
\hline MEAN & & & & & & 1031 & 635 & 1089 \\
\hline
\end{tabular}

\section{Appendix D. Data availability}

\section{Manual corrections to the Yaw Control System encoder heading signal}

\begin{tabular}{|c|c|c|c|}
\hline $\begin{array}{l}\text { From [UTC } \\
\text { time] }\end{array}$ & $\begin{array}{l}\text { To [UTC } \\
\text { time] }\end{array}$ & $\begin{array}{l}\text { Heading } \\
\text { [deg N] }\end{array}$ & Notes \\
\hline $2 / 15 / 11$ & $3 / 3 / 11$ & 204 & Known anchor locations, 1st deployment. \\
\hline $8: 00$ & $8: 00$ & & \\
\hline $3 / 11 / 11$ & $6 / 28 / 11$ & $-5.4 *$ & Bias of -5.4 deg applied to all YCS encoder readings. \\
\hline $8: 00$ & 21:00 & & $\begin{array}{l}\text { Measured anchor locations indicated mooring orientation } \\
\text { that differed from assumed orientation. Bias only applied to } \\
\text { signal directly reported by encoder, not to values entered } \\
\text { manually. From beginning of } 2 \text { nd deployment until encoder }\end{array}$ \\
\hline
\end{tabular}

Columbia Power Technologies, LLC • 4920A SW $3^{\text {rd }}$ St, Corvallis, Oregon 97333

Phone: (541) 368-5033 • Fax (541) 230-1498 • www.columbiapowertechnologies.com 
failure.

\begin{tabular}{|c|c|c|c|}
\hline 5/4/11 8:00 & $\begin{array}{l}5 / 9 / 11 \\
20: 00\end{array}$ & 175 & $\begin{array}{l}\text { Erroneous YCS encoder position... recreated from } \\
\text { handwritten log book. }\end{array}$ \\
\hline $\begin{array}{l}6 / 6 / 11 \\
18: 00\end{array}$ & $\begin{array}{l}6 / 7 / 11 \\
16: 00\end{array}$ & 175 & $\begin{array}{l}\text { YCS encoder position not recorded... recreated from } \\
\text { handwritten log book. }\end{array}$ \\
\hline $\begin{array}{l}6 / 7 / 11 \\
16: 00\end{array}$ & $\begin{array}{l}6 / 10 / 11 \\
16: 00\end{array}$ & 165 & $\begin{array}{l}\text { YCS encoder position not recorded... recreated from } \\
\text { handwritten log book. }\end{array}$ \\
\hline $\begin{array}{l}6 / 10 / 11 \\
16: 00\end{array}$ & $\begin{array}{l}6 / 11 / 11 \\
17: 20\end{array}$ & 355 & $\begin{array}{l}\text { YCS encoder position not recorded... recreated from } \\
\text { handwritten log book. }\end{array}$ \\
\hline $\begin{array}{l}6 / 11 / 11 \\
17: 20\end{array}$ & $\begin{array}{l}6 / 20 / 11 \\
16: 20\end{array}$ & 176 & $\begin{array}{l}\text { YCS encoder position not recorded... recreated from } \\
\text { handwritten log book. }\end{array}$ \\
\hline $\begin{array}{l}6 / 28 / 11 \\
21: 00\end{array}$ & $\begin{array}{l}7 / 6 / 11 \\
3: 40\end{array}$ & 175 & Persistence of YCS encoder position reading pre-failure. \\
\hline $7 / 6 / 113: 40$ & $\begin{array}{l}7 / 14 / 11 \\
1: 20\end{array}$ & 337 & $\begin{array}{l}\text { Heading measured by Sound and Sea Technology (SST) using } \\
\text { hand held GPS unit. }\end{array}$ \\
\hline $\begin{array}{l}7 / 14 / 11 \\
1: 20\end{array}$ & $\begin{array}{l}8 / 16 / 11 \\
20: 30\end{array}$ & 154 & Heading measured by Island Marine using pelorus. \\
\hline $\begin{array}{l}8 / 16 / 11 \\
20: 30\end{array}$ & $\begin{array}{l}8 / 16 / 11 \\
23: 00\end{array}$ & various & Heading calibration exercise carried out by SST and CPT. \\
\hline $\begin{array}{l}8 / 16 / 11 \\
23: 00\end{array}$ & $\begin{array}{l}9 / 2 / 11 \\
0: 00\end{array}$ & 179 & Heading measured by Island Marine using pelorus. \\
\hline
\end{tabular}

Columbia Power Technologies, LLC $\bullet 4920$ A SW $3^{\text {rd }}$ St, Corvallis, Oregon 97333

Phone: (541) 368-5033 • Fax (541) 230-1498 • www.columbiapowertechnologies.com 


\section{Mooring load signal availability}

Failure was determined by visual inspection of the mean and RMS values of reported mooring loads for each trial.

\begin{tabular}{|c|c|c|c|c|}
\hline UTC date & $\begin{array}{l}\text { UTC } \\
\text { time }\end{array}$ & NW (aka starboard) & S (aka port) & NE (aka aft) \\
\hline $2 / 16 / 2011$ & 19:00 & \multicolumn{3}{|c|}{ deployment 1 , pretension on order of $100 \mathrm{~N}$} \\
\hline $2 / 19 / 2011$ & $12: 00$ & fail high, low, high & & \\
\hline $2 / 23 / 2011$ & $20: 00$ & & & fail mixed, then low \\
\hline $3 / 12 / 2011$ & 0:00 & \multicolumn{3}{|c|}{ deployment 2, pretension on order of $800 \mathrm{~N}$} \\
\hline $3 / 12 / 2011$ & 0:00 & good & & \\
\hline $3 / 16 / 2011$ & 4:40 & fail low & fail low & \\
\hline $3 / 17 / 2011$ & 22:30 & good & good & \\
\hline $5 / 5 / 2011$ & $18: 00$ & & & good \\
\hline $5 / 10 / 2011$ & $3: 10$ & fail low & fail low & fail low \\
\hline $5 / 10 / 2011$ & 20:00 & good & good & good \\
\hline $8 / 25 / 2011$ & 3:00 & & fail high, then mixed & \\
\hline $11 / 15 / 2011$ & 21:00 & & & fail high \\
\hline $11 / 16 / 2011$ & $0: 40$ & & & good \\
\hline $12 / 23 / 2011$ & 0:00 & & & fail high \\
\hline $12 / 23 / 2011$ & 7:00 & & & good \\
\hline $12 / 31 / 2011$ & $21: 20$ & fail low & & fail low \\
\hline $1 / 6 / 2012$ & $18: 00$ & good & & good \\
\hline $1 / 15 / 2012$ & $15: 20$ & fail low & & fail low \\
\hline $1 / 15 / 2012$ & 18:00 & \multicolumn{3}{|c|}{ dSpace failure, no more mooring data } \\
\hline
\end{tabular}


PTO Characterization 1 of 4

Summary

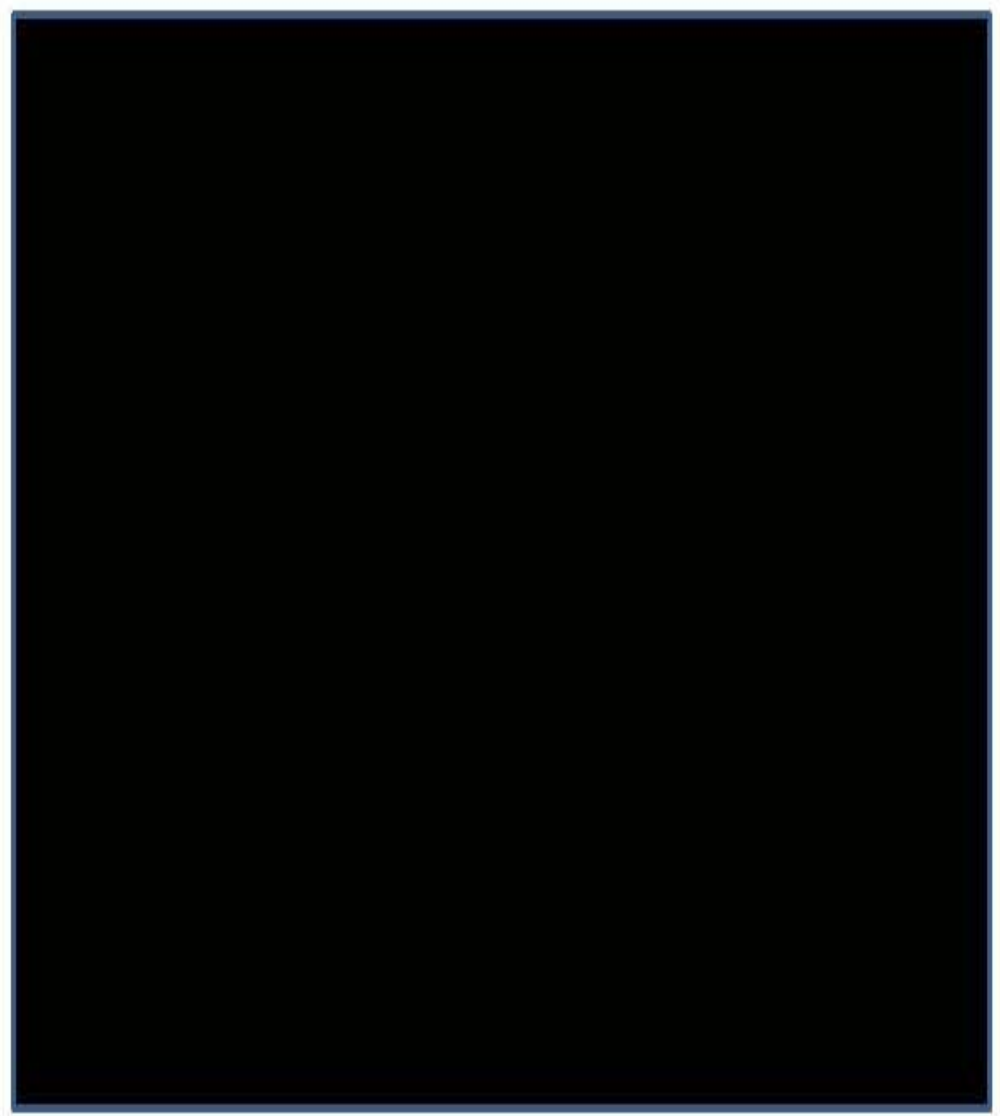

Columbia Power Technologies, LLC $\bullet 4920$ A SW $3^{\text {rd }}$ St, Corvallis, Oregon 97333 Phone: (541) 368-5033 • Fax (541) 230-1498 • www.columbiapowertechnologies.com 
PTO Characterization 2 of 4

Time and energy tabulation for various PTO states

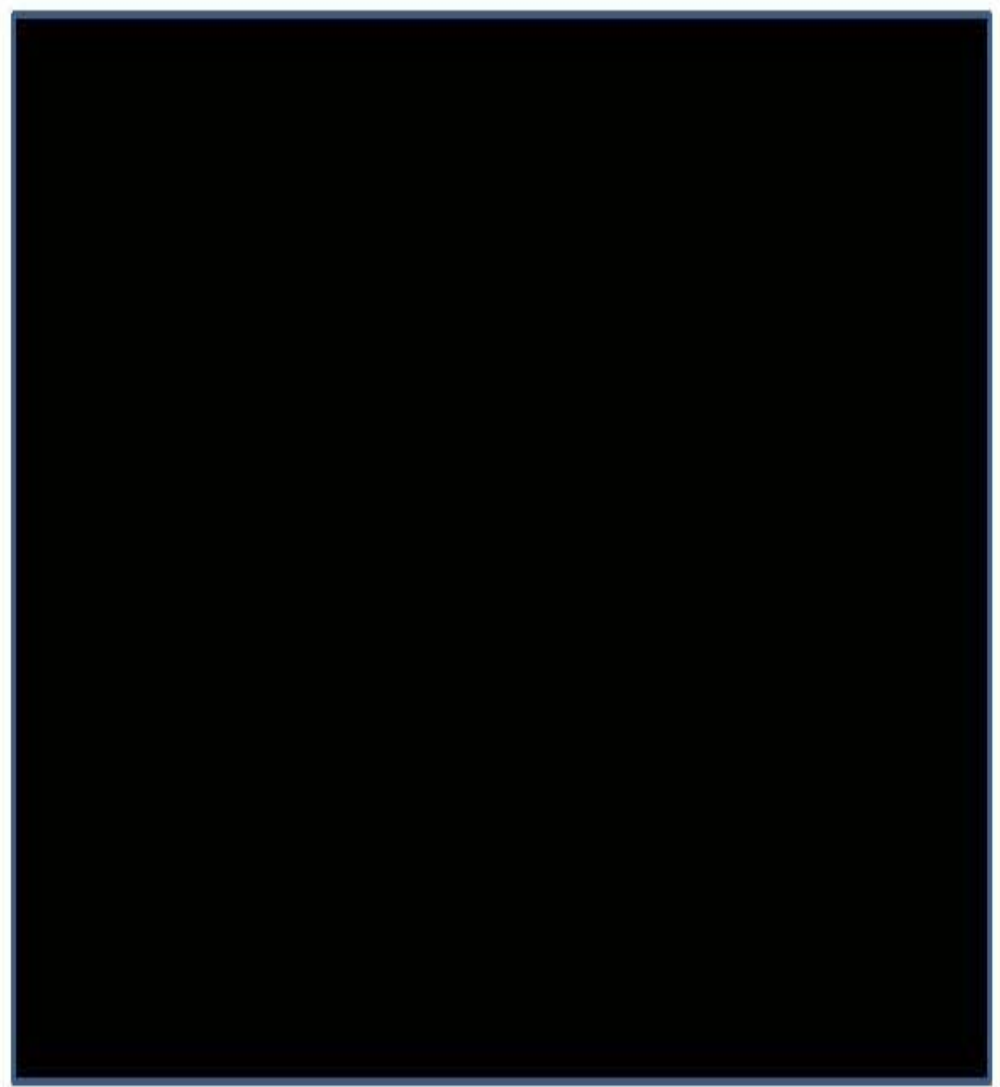

Columbia Power Technologies, LLC $\bullet 4920$ A SW $3^{\text {rd }}$ St, Corvallis, Oregon 97333 Phone: (541) 368-5033 • Fax (541) 230-1498 • www.columbiapowertechnologies.com 


\section{PTO Characterization 3 of 4}

Timeline for fore and aft PTO states
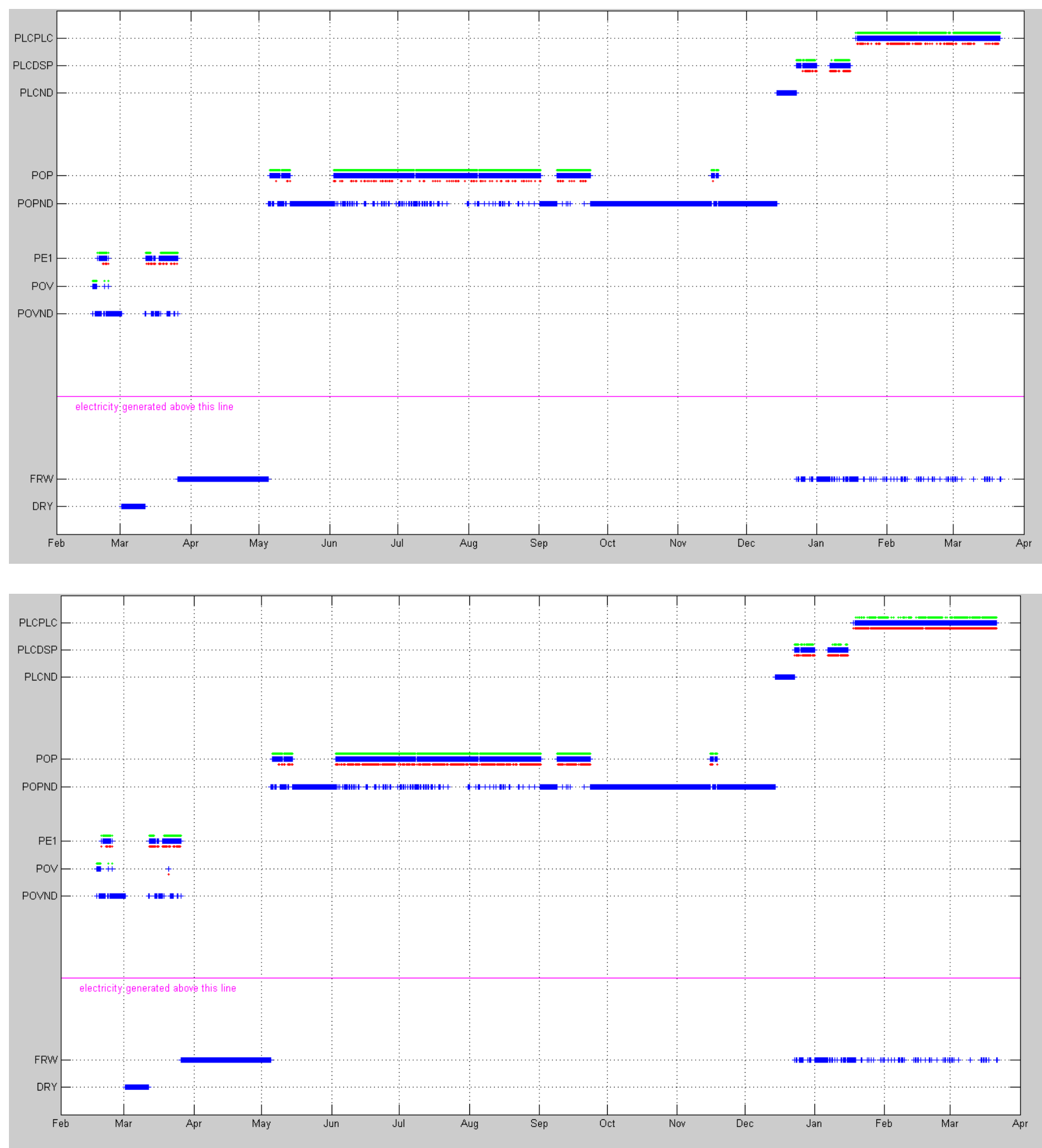

Columbia Power Technologies, LLC • 4920A SW $3^{\text {rd }}$ St, Corvallis, Oregon 97333

Phone: (541) 368-5033 • Fax (541) 230-1498 • www.columbiapowertechnologies.com 
PTO Characterization 4 of 4

Seasonal mechanical power production during data collection phases

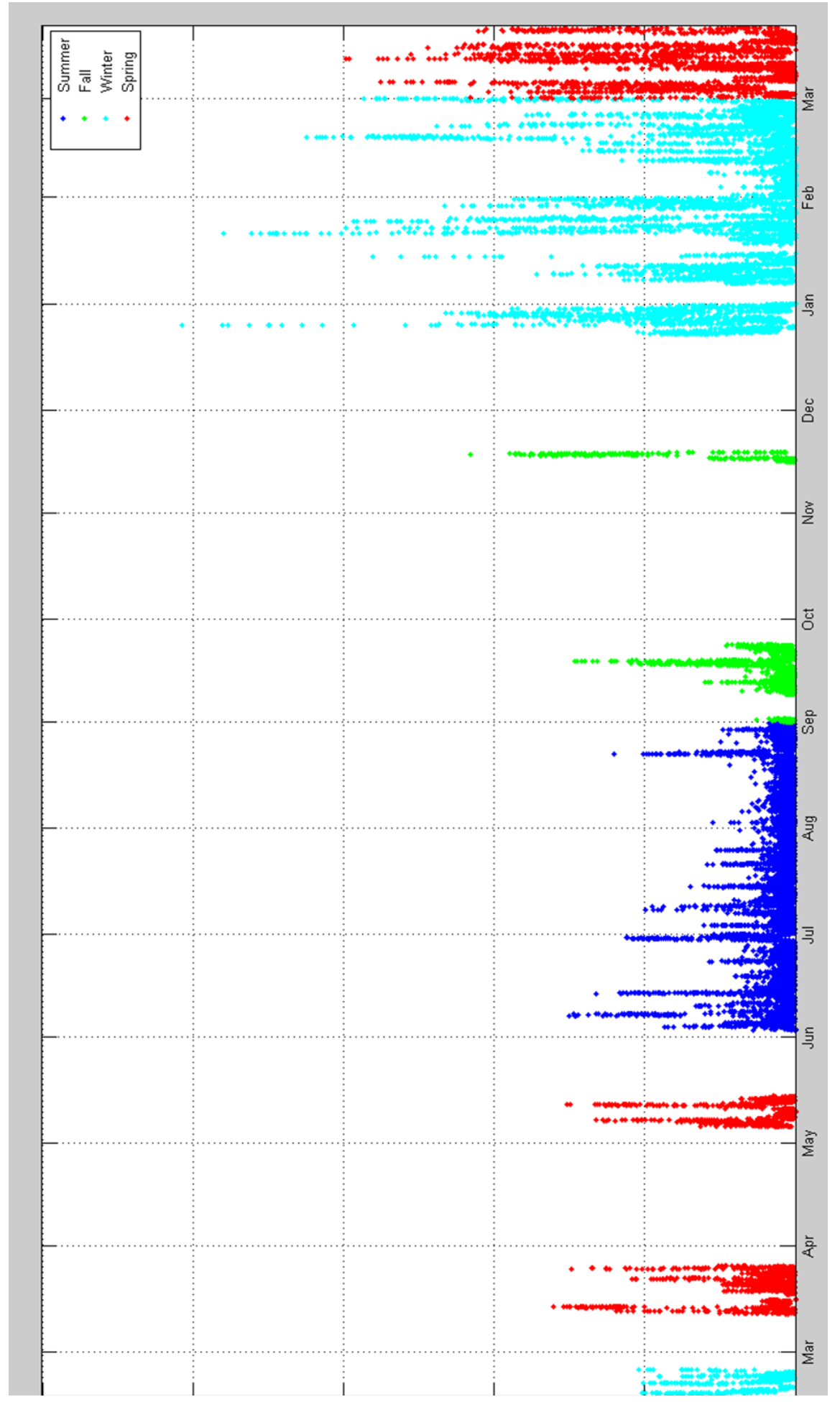

Columbia Power Technologies, LLC • 4920A SW $3^{\text {rd }}$ St, Corvallis, Oregon 97333 Phone: (541) 368-5033 • Fax (541) 230-1498 • www.columbiapowertechnologies.com 


\section{Appendix E. Corrected raw data format}

\begin{tabular}{|c|c|c|c|}
\hline structure name & variable & units & description \\
\hline & startTime & days & fractional days since start of 2011 \\
\hline & startTimeNom & days & start time, rounded to $10 \min$ (eg. $12: 10: 00,12: 20: 00$, etc) \\
\hline & elapsedTime & $\mathrm{s}$ & elapsed time during record \\
\hline & totalTime & $\mathrm{s}$ & total time in record \\
\hline & dampingFore & Nms & linear damping estimate (leasat squares linear regression) \\
\hline & dampingAft & Nms & linear damping estimate (leasat squares linear regression) \\
\hline dampingFitR2 & fore & - & $\mathrm{R}^{\wedge} 2$ measure of goodness of fit for dampingFore \\
\hline \multirow[t]{34}{*}{ dampingFitR2 } & aft & - & $\mathrm{R}^{\wedge} 2$ measure of goodness of fit for dampingAft \\
\hline & rawS001_Fwd_Msrd_Torq & $\mathrm{Nm}$ & torque measured by Interface torque transducer \\
\hline & rawS002_Aft_Msrd_Torq & $\mathrm{Nm}$ & torque measured by Interface torque transducer \\
\hline & S001_Fwd_Msrd_Torq & $\mathrm{Nm}$ & corrected torque, see report \\
\hline & S002_Aft_Msrd_Torq & $\mathrm{Nm}$ & corrected torque, see report \\
\hline & S003_Fwd_AC_Voltage_A & Volts & Forward generator - phase A voltage \\
\hline & S004_Fwd_AC_Voltage_B & Volts & Forward generator - phase B voltage \\
\hline & S005_Fwd_AC_Voltage_C & Volts & Forward generator - phase C voltage \\
\hline & S006_Aft_AC_Voltage_A & Volts & Forward generator - phase A voltage \\
\hline & S007_Aft_AC_Voltage_B & Volts & Forward generator - phase B voltage \\
\hline & S008_Aft_AC_Voltage_C & Volts & Forward generator - phase C voltage \\
\hline & S009_Fwd_Pass_V & Volts & Passive bus voltage \\
\hline & S010_Aft_Pass_V & Volts & Passive bus voltage \\
\hline & S011_Active_V & Volts & Active bus voltage \\
\hline & S012_Fwd_Pass_I & Amps & Passive bus current \\
\hline & S013_Aft_Pass_I & Amps & Passive bus current \\
\hline & S014_Active_I & Amps & Active bus current \\
\hline & S015_Charge_I & Amps & battery charge current \\
\hline & S016_Discharge_I & Amps & battery discharge current \\
\hline & S017_Charge_P & Watts & battery charge power \\
\hline & S018_Discharge_P & Watts & battery discharge power \\
\hline & S019_Batt_V & Volts & $24 \mathrm{~V}$ battery voltage \\
\hline & S020_Stbd_Mooring & $\mathrm{N}$ & mooring tension \\
\hline & S021_Port_Mooring & $\mathrm{N}$ & mooring tension \\
\hline & S022_Aft_Mooring & $\mathrm{N}$ & mooring tension \\
\hline & S023_Ch01_ES_Fwd_Port_Top & $\mathrm{kN}$ & endstop loads - saturate at $70 \mathrm{kN}$ \\
\hline & S024_Ch02_ES_Fwd_Port_Bot & $\mathrm{kN}$ & endstop loads - saturate at $70 \mathrm{kN}$ \\
\hline & S025_Ch03_ES_Fwd_Stbd_Top & $\mathrm{kN}$ & endstop loads - saturate at $70 \mathrm{kN}$ \\
\hline & S026_Ch04_ES_Fwd_Stbd_Bot & $\mathrm{kN}$ & endstop loads - saturate at $70 \mathrm{kN}$ \\
\hline & S027_Ch05_ES_Aft_Port_Top & $\mathrm{kN}$ & endstop loads - saturate at $70 \mathrm{kN}$ \\
\hline & S028_Ch06_ES_Aft_Port_Bot & $\mathrm{kN}$ & endstop loads - saturate at $70 \mathrm{kN}$ \\
\hline & S029_Ch07_ES_Aft_Stbd_Top & $\mathrm{kN}$ & endstop loads - saturate at $70 \mathrm{kN}$ \\
\hline & S030_Ch08_ES_Aft_Stbd_Bot & $\mathrm{kN}$ & endstop loads - saturate at $70 \mathrm{kN}$ \\
\hline & S031_Ch09_Body_Spar_Fwd & micro_strain & Body stain \\
\hline
\end{tabular}

Columbia Power Technologies, LLC $•$ 4920A SW $3^{\text {rd }}$ St, Corvallis, Oregon 97333

Phone: (541) 368-5033 • Fax (541) 230-1498 • www.columbiapowertechnologies.com 


\begin{tabular}{|c|c|c|c|}
\hline structure name & variable & units & description \\
\hline & S032_Ch10_Body_Spar_Aft & micro_strain & Body stain \\
\hline & S033_Ch11_Body_Nac_Port_Z & micro_strain & Body stain \\
\hline & S034_Ch12_Body_Nac_Port_X & micro_strain & Body stain \\
\hline & S035_Ch13_Body_Nac_Stbd_Z & micro_strain & Body stain \\
\hline & S036_Ch14_Body_Nac_Stbd_X & micro_strain & Body stain \\
\hline & S037_Ch15_Body_Nac_Fwd_Z & micro_strain & Body stain \\
\hline & S038_Ch16_Body_Nac_Fwd_Y & micro_strain & Body stain \\
\hline & S039_Bilge_1_Status & $1=\mathrm{On}, 0=\mathrm{Off}$ & Primary bilge pump status \\
\hline & S040_Bilge_2_Status & $1=\mathrm{On}, 0=\mathrm{Off}$ & Secondary bilge pump status \\
\hline & S041_Wind_Speed & $\mathrm{m} / \mathrm{s}$ & Wind Speed \\
\hline & S042_Wind_Direction & $\begin{array}{l}\operatorname{deg} \text { CW from } \\
\text { FWD }\end{array}$ & Wind Direction \\
\hline & S043_Temp_1_Fwd_Gen & $\operatorname{deg} \mathrm{F}$ & Forward Generator Temperature \\
\hline & S044_Temp_2_Fwd_Gear & $\operatorname{deg} \mathrm{F}$ & Forward Gearbox Temperature \\
\hline & S045_Temp_3_Aft_Gen & $\operatorname{deg} F$ & Aft Generator Temperature \\
\hline & S046_Temp_4_PE_Box & $\operatorname{deg} F$ & Power Electronics Box Temperature \\
\hline & S047_Temp_5_Aft_Gear & $\operatorname{deg} F$ & Aft Gearbox Temperature \\
\hline & S048_Temp_6_24V_Box & $\operatorname{deg} F$ & 24V Box Temperature \\
\hline & S049_Temp_7_SCADA & $\operatorname{deg} F$ & SCADA Temperature \\
\hline & S050_Temp_8_MABX & $\operatorname{deg} F$ & MABXII Temperature \\
\hline & S051_Fwd_Prox & $1=\mathrm{On}, 0=\mathrm{Off}$ & Fwd_Proximity_Sensor \\
\hline & S052_Aft_Prox & $1=\mathrm{On}, 0=\mathrm{Off}$ & Aft_Proximity_Sensor \\
\hline & S053_Fwd_Slip & $1=\mathrm{On}, 0=\mathrm{Off}$ & Fwd_Slip_Switch \\
\hline & S054_Aft_Slip & $1=\mathrm{On}, 0=\mathrm{Off}$ & Aft_Slip_Switch \\
\hline & S055_Oil Sensor & $1=\mathrm{On}, 0=\mathrm{Off}$ & Oil detection in the oil pan \\
\hline & S056_Fwd_Gen_Position & Radians & Fwd_Encoder_Position \\
\hline & S057_Fwd_Gen_Speed & $\mathrm{Rad} / \mathrm{s}$ & Fwd_Encoder_Speed \\
\hline & S058_Fwd_Gen_Acceleration & $\mathrm{Rad} / \mathrm{s}^{\wedge} 2$ & Fwd_Encoder_Acceleration \\
\hline & S059_Aft_Gen_Position & Radians & Aft_Encoder_Position \\
\hline & S060_Aft_Gen_Speed & $\mathrm{Rad} / \mathrm{s}$ & Aft_Encoder_Speed \\
\hline & S061_Aft_Gen_Acceleration & $\mathrm{Rad} / \mathrm{s}^{\wedge} 2$ & Aft_Encoder_Acceleration \\
\hline & S062_Yaw_Encoder_Position & degrees & Yaw_Encoder_Position \\
\hline & S063_Yaw_Fault & $1=\mathrm{OK}, 0=$ Fault & Fault signal coming form yaw \\
\hline & S064_Yaw_Wake & $1=\mathrm{On}, 0=\mathrm{Off}$ & yaw system wake command \\
\hline & S065_Yaw_CW & $1=\mathrm{On}, 0=\mathrm{Off}$ & clockwise command \\
\hline & S066_Yaw_CCW & $1=\mathrm{On}, 0=\mathrm{Off}$ & counterclockwise command \\
\hline & S067_Active_Mode & $1=$ Act, $0=$ Pass & Active mode status $1=$ Active, $0=$ Passive \\
\hline & S068_Pass_Burn & $1=\mathrm{Off}, 0=\mathrm{On}$ & Passive_Load_Switch \\
\hline & S069_Act_Burn & $1=\mathrm{On}, 0=\mathrm{Off}$ & Active_Load_Switch \\
\hline & S070_Batt_Charge & $1=\mathrm{On}, 0=\mathrm{Off}$ & Battery_Charger_Enable \\
\hline & S071_Bus_Charge & $1=\mathrm{On}, 0=\mathrm{Off}$ & Bus_Charger_Enable \\
\hline & S072_5V_Sleep & $1=\mathrm{On}, 0=\mathrm{Off}$ & +5 Volt Sleep \\
\hline & S073_24V_Sleep & $1=\mathrm{On}, 0=\mathrm{Off}$ & +24 Volt Sleep \\
\hline
\end{tabular}

Columbia Power Technologies, LLC $\bullet$ 4920A SW $3^{\text {rd }}$ St, Corvallis, Oregon 97333 Phone: (541) 368-5033 • Fax (541) 230-1498 • www.columbiapowertechnologies.com 


\begin{tabular}{llll}
\hline structure name & variable & units & description \\
\hline S074_PWM_Enable & $1=\mathrm{On}, 0=\mathrm{Off}$ & Enable PWMs \\
S075_Spar_Surge & $\mathrm{m} / \mathrm{s}^{\wedge} 2$ & Surge acceleration from IMU \\
S076_Spar_Sway & $\mathrm{m} / \mathrm{s}^{\wedge} 2$ & Sway acceleration from IMU \\
S077_Spar_Heave & $\mathrm{m} / \mathrm{s}^{\wedge} 2$ & Heave acceleration from IMU \\
S078_Spar_Roll & $\mathrm{rad}$ & Roll angle from IMU \\
S079_Spar_Pitch & $\mathrm{rad}$ & Pitch angle from IMU \\
S080_Spar_Yaw & rad & Yaw angle from IMU \\
S081_WEC_Latitude & deg N & Latitude from GPS \\
S082_WEC_Longitude & deg W & Longitude from GPS \\
S083_WEC_Heading & deg CW from & Heading from GPS \\
S091_PC_Time & $\mathrm{N}$ & Days since Jan 1, 2011 - UTC \\
S095_Yaw_Cmd & days & Desired Yaw heading wrt wave heading \\
S096_Fwd_D & - & The 0-100 buck/boost ratio \\
\hline S097_Aft_D & - & The 0-100 buck/boost ratio \\
\hline
\end{tabular}

Columbia Power Technologies, LLC • 4920A SW $3^{\text {rd }}$ St, Corvallis, Oregon 97333 Phone: (541) 368-5033 • Fax (541) 230-1498 • www.columbiapowertechnologies.com 


\section{Appendix F. Trial structure format}

AWAC parameters

\begin{tabular}{|c|c|c|c|}
\hline structure & variable & units & description \\
\hline & startTime & days & fractional days since start of 2011 \\
\hline & startTimeNom & days & start time, rounded to 10 min mark (eg. $12: 10: 00,12: 20: 00$, etc) \\
\hline & magDelcination & $\operatorname{deg}$ & magnetic declination at site \\
\hline & waterDepth & $\mathrm{m}$ & mean water depth during trial \\
\hline & currSpeed & $\mathrm{m} / \mathrm{s}$ & current speed, from near surface measurement cell \\
\hline & currDir & $\operatorname{deg}$ & direction current is coming from, cw from true north \\
\hline $\mathrm{HmO}$ & int & $\mathrm{m}$ & significant wave height, from intrinsic spectrum \\
\hline \multirow[t]{2}{*}{$\mathrm{Hmo}$} & obs & $\mathrm{m}$ & significant wave height, from observed spectrum \\
\hline & Hs & $\mathrm{m}$ & mean of highest $1 / 3$ waves, from time series analysis \\
\hline Te & int & $\mathrm{s}$ & energy period, calculated from intrinsic spectrum \\
\hline $\mathrm{Te}$ & obs & $\mathrm{s}$ & energy period, calculated from observed spectrum \\
\hline $\mathrm{Tp}$ & int & $\mathrm{s}$ & peak period, intrinsic \\
\hline $\mathrm{Tp}$ & obs & $\mathrm{s}$ & peak period, observed \\
\hline $\mathrm{Tz}$ & int & $\mathrm{s}$ & mean upcross period, intrinsic \\
\hline $\mathrm{Tz}$ & obs & $\mathrm{s}$ & mean upcross period, observed \\
\hline eps0 & int & - & spectral width (in frequency space), intrinsic \\
\hline \multirow[t]{3}{*}{ eps0 } & obs & - & spectral width (in frequency space), observed \\
\hline & Ss & - & significant steepness \\
\hline & wavePower & $\mathrm{W} / \mathrm{m}$ & omnidirectional wave power, intrinsic \\
\hline waveDir & Jweight & $\operatorname{deg}$ & wave heading (coming from), weighted by wave power, int \\
\hline waveDir & peak & $\operatorname{deg}$ & wave heading (coming from), mean direction at $f=1 / T p$, int \\
\hline spreadAngle & Jweight & $\operatorname{deg}$ & mean spreading angle, weighted by wave power, int \\
\hline spreadAngle & peak & $\operatorname{deg}$ & spreading angle, at $\mathrm{f}=1 / \mathrm{Tp}$, int \\
\hline spreadIndex & Jweight & - & mean spreading index, weighted by wave power, int \\
\hline \multirow[t]{2}{*}{ spreadIndex } & peak & - & spreading index, at $f=1 / T p$, int \\
\hline & UI & - & unidirectivity index, based on mean direction as function of $f$ \\
\hline relCurrSpeed & peak & $\mathrm{m} / \mathrm{s}$ & relative current speed w.r.t. waveDir.peak \\
\hline relCurrSpeed & Jweight & $\mathrm{m} / \mathrm{s}$ & relative current speed w.r.t. waveDir.Jweight \\
\hline jsFit & $\mathrm{HmO}$ & $\mathrm{m}$ & significant wave height of JONSWAP spectra fit to intrinsic spectra \\
\hline jsFit & $\mathrm{Tp}$ & $\mathrm{s}$ & peak period of JONSWAP spectra fit to intrinsic spectra \\
\hline jsFit & Te & $\mathrm{s}$ & energy period of JONSWAP spectra fit to intrinsic spectra \\
\hline jsFit & gamma & - & peakedness enhancement factor of JONSWAP spectra fit to intrinsic spectra \\
\hline jsFit & fitTotalError & & wave energy total error (bias error plus rms error) of fit spectra \\
\hline jsFit & fitBiasError & & wave energy bias error of fit spectra \\
\hline jsFit & fitJError & & normalized wave energy bias error of fit spectra \\
\hline jsFit & fitQ & & normalized wave energy total error (bias error plus rms error) of fit spectra \\
\hline
\end{tabular}

Columbia Power Technologies, LLC • 4920A SW $3^{\text {rd }}$ St, Corvallis, Oregon 97333

Phone: (541) 368-5033 • Fax (541) 230-1498 • www.columbiapowertechnologies.com 
AWAC time series

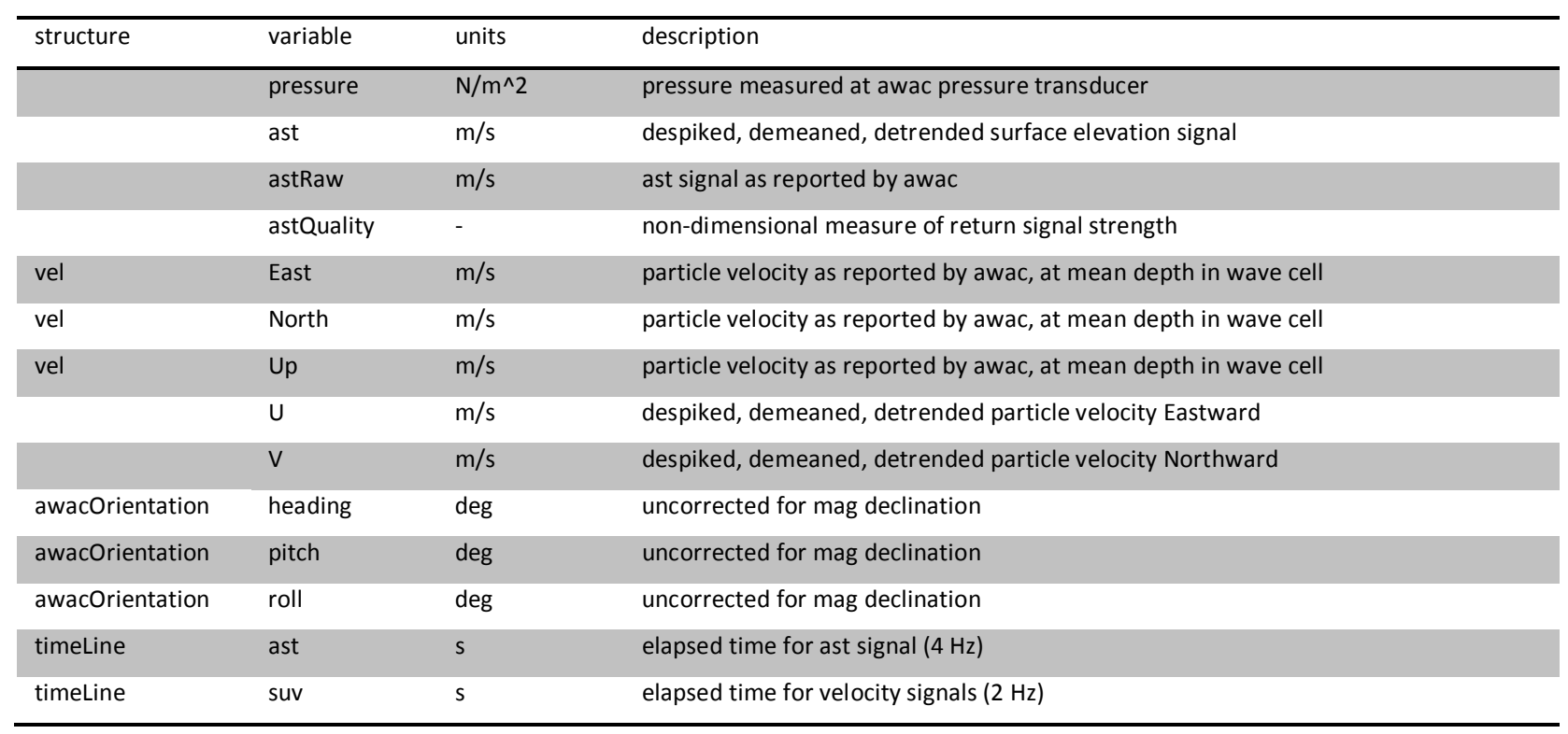

\section{AWAC prolog}

Note: All prolog variables are from 1024 s records, and thus are common to a pair of $512 \mathrm{~s}$ records. These variables are calculated by AWAC internal processer and are not used in analysis.

\begin{tabular}{|c|c|c|c|}
\hline structure & variable & units & description \\
\hline prolog & battVolt & V & awac battery voltage \\
\hline prolog & currSpeed & $\mathrm{m} / \mathrm{s}$ & current speed, near surface \\
\hline prolog & currDir & deg & current direction, near surface \\
\hline prolog & errorCode & - & error code \\
\hline prolog & $\mathrm{HmO}$ & $\mathrm{m}$ & significant wave height \\
\hline prolog & T02 & $\mathrm{s}$ & mean zero crossing period \\
\hline prolog & $\mathrm{Tp}$ & $\mathrm{s}$ & peak period \\
\hline prolog & dirPeak & deg & mean wave direction at peak \\
\hline prolog & dirMean & deg & mean wave direction \\
\hline prolog & sprTp & deg & directional spreading at peak \\
\hline prolog & $f$ & $\mathrm{~Hz}$ & frequency \\
\hline prolog & Sf & $m^{\wedge} 2 / H z$ & variance density spectrum \\
\hline
\end{tabular}

Columbia Power Technologies, LLC • 4920A SW $3^{\text {rd }}$ St, Corvallis, Oregon 97333

Phone: (541) 368-5033 • Fax (541) 230-1498 • www.columbiapowertechnologies.com 
AWAC spectral

\begin{tabular}{|c|c|c|c|}
\hline structure & variable & units & description \\
\hline unSpec.raw & fObs & $\mathrm{Hz}$ & untruncated, observed spectrum... no fitting of tail \\
\hline unSpec.raw & SfObs & $\mathrm{m}^{\wedge} 2 / \mathrm{Hz}$ & untruncated, observed spectrum... no fitting of tail \\
\hline unSpec & fObs & $\mathrm{Hz}$ & observed spectrum, truncated.... then high freq tail fit \\
\hline unSpec & SfObs & $\mathrm{m}^{\wedge} 2 / \mathrm{Hz}$ & observed spectrum, truncated.... then high freq tail fit \\
\hline unSpec & flnt & $\mathrm{Hz}$ & intrinsic spectrum, truncated.... then high freq tail fit \\
\hline unSpec & SfInt & $m^{\wedge} 2 / H z$ & intrinsic spectrum, truncated.... then high freq tail fit \\
\hline unSpec & $\mathrm{cg}$ & $\mathrm{m} / \mathrm{s}$ & group velocity of unSpec.fint \\
\hline unSpec.common & f & $\mathrm{Hz}$ & spectra interpolated to a common scale \\
\hline unSpec.common & SfObs & $\mathrm{m}^{\wedge} 2 / \mathrm{Hz}$ & spectra interpolated to a common scale \\
\hline unSpec.common & SfInt & $m^{\wedge} 2 / H z$ & spectra interpolated to a common scale \\
\hline unSpec.common & $\mathrm{cg}$ & $\mathrm{m} / \mathrm{s}$ & group velocity of unSpec.common.f \\
\hline unSpec.dir & $\mathrm{C} 3 \times 3$ & varying & cross spectra \\
\hline unSpec.dir & A1 & - & coefficients of Fourier decomposition of directional distribution \\
\hline unSpec.dir & B1 & - & coefficients of Fourier decomposition of directional distribution \\
\hline unSpec.dir & A2 & - & coefficients of Fourier decomposition of directional distribution \\
\hline unSpec.dir & B2 & - & coefficients of Fourier decomposition of directional distribution \\
\hline unSpec.dir & theta & deg & mean direction as function of frequency \\
\hline unSpec.dir & sigma & deg & spreading angle as function of frequency \\
\hline unSpec.dir & s & - & spreading index as function of frequency \\
\hline cutOff & index.high & - & $\begin{array}{l}\text { high frequncy limits... a high frequency tail is fit above this for calculation of } \\
\text { parameters }\end{array}$ \\
\hline cutOff & flnt.high & $\mathrm{Hz}$ & $\begin{array}{l}\text { high frequncy limits... a high frequency tail is fit above this for calculation of } \\
\text { parameters }\end{array}$ \\
\hline cutOff & fObs.high & $\mathrm{Hz}$ & $\begin{array}{l}\text { high frequncy limits... a high frequency tail is fit above this for calculation of } \\
\text { parameters }\end{array}$ \\
\hline cutOff & index.low & - & low frequency cutOffs \\
\hline cutOff & flnt.low & $\mathrm{Hz}$ & low frequency cutOffs \\
\hline cutOff & fObs.low & $\mathrm{Hz}$ & low frequency cutOffs \\
\hline cutOff.dir & index.high & - & $\begin{array}{l}\text { high frequency cutOffs for directional spectrum... low freq cutOffs same as } 1 D \\
\text { spectrum }\end{array}$ \\
\hline cutOff.dir & flnt.high & $\mathrm{Hz}$ & $\begin{array}{l}\text { high frequency cutOffs for directional spectrum... low freq cutOffs same as } 1 D \\
\text { spectrum }\end{array}$ \\
\hline cutOff.dir & fObs.high & $\mathrm{Hz}$ & $\begin{array}{l}\text { high frequency cutOffs for directional spectrum... low freq cutOffs same as } 1 D \\
\text { spectrum }\end{array}$ \\
\hline jsFit & f & $\mathrm{Hz}$ & frequency scale for fit JONSWAP spectrum \\
\hline jsFit & Sf & $\mathrm{m}^{\wedge} 2 / \mathrm{Hz}$ & fit JONSWAP spectrum (fit to intrinsic spectrum) \\
\hline
\end{tabular}

Columbia Power Technologies, LLC • 4920A SW $3^{\text {rd }}$ St, Corvallis, Oregon 97333 Phone: (541) 368-5033 • Fax (541) 230-1498 • www.columbiapowertechnologies.com 
AWAC QC

\begin{tabular}{|c|c|c|c|}
\hline structure & variable & units & description \\
\hline & astVarRatioMax & - & maximum ast variance ratio (see report) \\
\hline & astlndexOutBad & - & indices of data points replaced during despiking \\
\hline badCount & ast & - & indicates how many data points replaced during despiking \\
\hline badCount & $\mathrm{U}$ & - & indicates how many data points replaced during despiking \\
\hline badCount & V & - & indicates how many data points replaced during despiking \\
\hline flag & whiteNoise & - & see report for flag definitions \\
\hline flag & shipWave & - & see report for flag definitions \\
\hline flag & lowFreqNoise & - & see report for flag definitions \\
\hline flag & unusualDepth & - & see report for flag definitions \\
\hline flag & waveCrestClipped & - & see report for flag definitions \\
\hline flag & highAstDataLoss & - & see report for flag definitions \\
\hline flag & excessiveTilt & - & see report for flag definitions \\
\hline flag & unusualWaveConditions & - & see report for flag definitions \\
\hline flag & lowHighCutOff & - & see report for flag definitions \\
\hline
\end{tabular}

Columbia Power Technologies, LLC $\bullet 4920$ A SW $3^{\text {rd }}$ St, Corvallis, Oregon 97333 Phone: (541) 368-5033 • Fax (541) 230-1498 • www.columbiapowertechnologies.com 


\section{WEC parameters}

\begin{tabular}{|c|c|c|c|}
\hline structure & variable & units & description \\
\hline ogFileWec & fileName & - & file name of 'correctedRawData' file this trial is taken from \\
\hline ogFileWec & indices & - & bounding indices of trial data extracted from 'correctedRawData' \\
\hline wecTime & startTime & days & fractional days since start of 2011 \\
\hline wecTime & samplingFrequency & $\mathrm{Hz}$ & sampling frequency \\
\hline wecTime & $\mathrm{dt}$ & $\mathrm{s}$ & inverse of sampling frequency \\
\hline \multirow[t]{7}{*}{ wecTime } & totalTrialTime & $\mathrm{s}$ & total elapsed trial time \\
\hline & mechPowerFore_mean & W & mean mechanical power of fore generator \\
\hline & mechPowerAft_mean & W & ... of aft generator \\
\hline & wecHeading_mean & deg & direction wec is facing, cw from true north, mean over trial \\
\hline & flagMeanWecHeadingSource & - & 'YCS'=yawControlSystemEncoder, 'GPS'=gpsHeading \\
\hline & dampingFore & Nms & $\begin{array}{l}\text { linear damping estimate, obtained using least squares linear } \\
\text { regression }\end{array}$ \\
\hline & dampingAft & Nms & $\begin{array}{l}\text { linear damping estimate, obtained using least squares linear } \\
\text { regression }\end{array}$ \\
\hline dampingFitR2 & fore & - & $\mathrm{R}^{\wedge} 2$ measure of goodness of fit for dampingFore \\
\hline dampingFitR2 & aft & - & $R^{\wedge} 2$ measure of goodness of fit for dampingAft \\
\hline torqLim & fore & $\mathrm{Nm}$ & mechanical or generator controlled maximum torque, fore \\
\hline torqLim & aft & $\mathrm{Nm}$ & mechanical or generator controlled maximum torque, aft \\
\hline qc.wec & torqFit.fore & percent/100 & $\begin{array}{l}\text { percent of torq signal linearly fit (beyond instrument saturation } \\
\text { limit) }\end{array}$ \\
\hline qc.wec & torqFit.aft & percent/100 & $\begin{array}{l}\text { percent of torq signal linearly fit (beyond instrument saturation } \\
\text { limit) }\end{array}$ \\
\hline qc.wec & torqSat.fore & percent/100 & percent of torq signal saturated \\
\hline qc.wec & torqSat.aft & percent/100 & percent of torq signal saturated \\
\hline qc.wec & speedDespiked.fore & percent/100 & percent of speed signal despiked \\
\hline qc.wec & speedDespiked.aft & percent/100 & percent of speed signal despiked \\
\hline genPos & mean.fore & rad & $\begin{array}{l}\text { mean position of fore generator (note 'up' is negative for both } \\
\text { floats) }\end{array}$ \\
\hline genPos & mean.aft & rad & mean position of aft generator \\
\hline genPos & max.fore & rad & max position of fore generator \\
\hline genPos & max.aft & rad & max position of aft generator \\
\hline genPos & min.fore & rad & min position of fore generator \\
\hline genPos & min.aft & rad & min positio of aft generator \\
\hline mooringLoads & max.stbd & $\mathrm{N}$ & max mooring load signal \\
\hline mooringLoads & max.port & $\mathrm{N}$ & max mooring load signal \\
\hline mooringLoads & max.aft & $\mathrm{N}$ & max mooring load signal \\
\hline mooringLoads & mean.stbd & $\mathrm{N}$ & mean mooring load signal \\
\hline mooringLoads & mean.port & $\mathrm{N}$ & mean mooring load signal \\
\hline mooringLoads & mean.aft & $\mathrm{N}$ & mean mooring load signal \\
\hline mooringLoads & rms.stbd & $\mathrm{N}$ & rms of demeaned mooring load signal \\
\hline mooringLoads & rms.port & $\mathrm{N}$ & rms of demeaned mooring load signal \\
\hline mooringLoads & rms.aft & $\mathrm{N}$ & rms of demeaned mooring load signal \\
\hline endStopLoads & max.forePortTop & $\mathrm{kN}$ & max end stop load for trial \\
\hline endStopLoads & max.forePortBot & $\mathrm{kN}$ & max end stop load for trial \\
\hline
\end{tabular}

Columbia Power Technologies, LLC • 4920A SW $3^{\text {rd }}$ St, Corvallis, Oregon 97333

Phone: (541) 368-5033 • Fax (541) 230-1498 • www.columbiapowertechnologies.com 


\begin{tabular}{|c|c|c|c|}
\hline structure & variable & units & description \\
\hline endStopLoads & max.foreStbdTop & $\mathrm{kN}$ & max end stop load for trial \\
\hline endStopLoads & max.foreStbdBot & $\mathrm{kN}$ & max end stop load for trial \\
\hline endStopLoads & max.aftPortTop & $\mathrm{kN}$ & max end stop load for trial \\
\hline endStopLoads & max.aftPortBot & $\mathrm{kN}$ & max end stop load for trial \\
\hline endStopLoads & max.aftStbdTop & $\mathrm{kN}$ & max end stop load for trial \\
\hline endStopLoads & max.aftStbdBot & $\mathrm{kN}$ & max end stop load for trial \\
\hline endStopLoads & med.forePortTop & $\mathrm{kN}$ & median end stop load for trial \\
\hline endStopLoads & med.forePortBot & $\mathrm{kN}$ & median end stop load for trial \\
\hline endStopLoads & med.foreStbdTop & $\mathrm{kN}$ & median end stop load for trial \\
\hline endStopLoads & med.foreStbdBot & $\mathrm{kN}$ & median end stop load for trial \\
\hline endStopLoads & med.aftPortTop & $\mathrm{kN}$ & median end stop load for trial \\
\hline endStopLoads & med.aftPortBot & $\mathrm{kN}$ & median end stop load for trial \\
\hline endStopLoads & med.aftStbdTop & $\mathrm{kN}$ & median end stop load for trial \\
\hline endStopLoads & med.aftStbdBot & $\mathrm{kN}$ & median end stop load for trial \\
\hline endStopLoads & num.foreTop & [] & number of strike events \\
\hline endStopLoads & num.foreBot & [] & number of strike events \\
\hline endStopLoads & num.aftTop & [] & number of strike events \\
\hline \multirow[t]{5}{*}{ endStopLoads } & num.aftBot & [] & number of strike events \\
\hline & windSpeed_mean & $\mathrm{m} / \mathrm{s}$ & mean wind speed, as measured by wec anemometer \\
\hline & windDir_mean & deg & $\begin{array}{l}\text { mean direction wind is coming from, as measured by wec } \\
\text { anemometer }\end{array}$ \\
\hline & flagESvar & & 1 indicates ES is only noise, while the other shows hits \\
\hline & flagPosVar & & $\begin{array}{l}1 \text { indicates identically no motion for the float associated with the } \\
\text { end stop }\end{array}$ \\
\hline
\end{tabular}

Columbia Power Technologies, LLC • 4920A SW $3^{\text {rd }}$ St, Corvallis, Oregon 97333 Phone: (541) 368-5033 • Fax (541) 230-1498 • www.columbiapowertechnologies.com 
WEC time series

\begin{tabular}{|c|c|c|c|}
\hline structure & variable & units & description \\
\hline wecTime & pcTime & days & fractional days since start of 2011 \\
\hline \multirow[t]{7}{*}{ wecTime } & elapsedTime & s & elapsed time during trial \\
\hline & genTorqFore & $\mathrm{Nm}$ & generator torque, fore float \\
\hline & genTorqAft & $\mathrm{Nm}$ & generator torque, aft float \\
\hline & genSpeedFore & $\mathrm{rad} / \mathrm{s}$ & generator speed, fore float \\
\hline & genSpeedAft & $\mathrm{rad} / \mathrm{s}$ & generator speed, aft float \\
\hline & mechPowerFore & w & mechanical power, fore float \\
\hline & mechPowerAft & W & mechanical power, aft float \\
\hline rawGenTorqSpeed & torqFore & $\mathrm{Nm}$ & raw values, no replacement above torque transducer saturation limit \\
\hline rawGenTorqSpeed & torqAft & $\mathrm{Nm}$ & raw values, no replacement above torque transducer saturation limit \\
\hline rawGenTorqSpeed & speedFore & $\mathrm{rad} / \mathrm{s}$ & raw values, no despiking \\
\hline \multirow[t]{3}{*}{ rawGenTorqSpeed } & speedAft & $\mathrm{rad} / \mathrm{s}$ & raw values, no despiking \\
\hline & genPositionFore & $\mathrm{rad}$ & generator position, fore float \\
\hline & genPostionAft & $\mathrm{rad}$ & generator position, aft float \\
\hline sparlmu6dof.acc & surge & $\mathrm{m} / \mathrm{s}^{\wedge} 2$ & imu signal \\
\hline sparlmu6dof.acc & sway & $\mathrm{m} / \mathrm{s}^{\wedge} 2$ & imu signal \\
\hline sparlmu6dof.acc & heave & $\mathrm{m} / \mathrm{s}^{\wedge} 2$ & imu signal \\
\hline sparlmu6dof.pos & roll & $\operatorname{deg}$ & imu signal \\
\hline sparlmu6dof.pos & pitch & $\operatorname{deg}$ & imu signal \\
\hline sparlmu6dof.pos & yaw & $\operatorname{deg}$ & imu signal \\
\hline wecMotion.acc & surge & $\mathrm{m} / \mathrm{s}^{\wedge} 2$ & filtered imu signal \\
\hline wecMotion.acc & sway & $\mathrm{m} / \mathrm{s}^{\wedge} 2$ & filtered imu signal \\
\hline wecMotion.acc & heave & $\mathrm{m} / \mathrm{s}^{\wedge} 2$ & filtered imu signal \\
\hline wecMotion.vel & surge & $\mathrm{m} / \mathrm{s}$ & filtered, integrated imu signal \\
\hline wecMotion.vel & sway & $\mathrm{m} / \mathrm{s}$ & filtered, integrated imu signal \\
\hline wecMotion.vel & heave & $\mathrm{m} / \mathrm{s}$ & filtered, integrated imu signal \\
\hline wecMotion.pos & surge & $\mathrm{m}$ & filtered, double integrated imu signal \\
\hline wecMotion.pos & sway & $\mathrm{m}$ & filtered, double integrated imu signal \\
\hline wecMotion.pos & heave & $\mathrm{m}$ & filtered, double integrated imu signal \\
\hline wecMotion.pos & pitch & $\operatorname{deg}$ & filtered imu signal \\
\hline wecMotion.pos & roll & $\operatorname{deg}$ & filtered imu signal \\
\hline wecMotion.pos & yaw & deg & filtered imu signal \\
\hline wecMotion.pos & foreFloatRel & $\operatorname{deg}$ & filtered relative float position signal (encoder) \\
\hline wecMotion.pos & aftFloatRel & $\operatorname{deg}$ & filtered relative float position signal (encoder) \\
\hline wecMotion.pos & foreFloatAbs & $\operatorname{deg}$ & filtered absolute float position signal (encoder and imu) \\
\hline wecMotion.pos & aftFloatAbs & $\operatorname{deg}$ & filtered absolute float position signal (encoder and imu) \\
\hline wecMotion.pos & pitchRaw & deg & raw imu signal \\
\hline wecMotion.pos & rollRaw & $\operatorname{deg}$ & raw imu signal \\
\hline wecMotion.pos & yawRaw & $\operatorname{deg}$ & raw imu signal \\
\hline wecMotion.pos & foreFloatRelRaw & $\operatorname{deg}$ & raw relative float position signal (encoder) \\
\hline wecMotion.pos & aftFloatRelRaw & $\operatorname{deg}$ & raw relative float position signal (encoder) \\
\hline
\end{tabular}

Columbia Power Technologies, LLC • 4920A SW $3^{\text {rd }}$ St, Corvallis, Oregon 97333

Phone: (541) 368-5033 • Fax (541) 230-1498 • www.columbiapowertechnologies.com 


\begin{tabular}{|c|c|c|c|}
\hline structure & variable & units & description \\
\hline wecMotion.pos & foreFloatAbsRaw & deg & raw absolute float position signal (encoder and imu) \\
\hline wecMotion.pos & aftFloatAbsRaw & deg & raw absolute float position signal (encoder and imu) \\
\hline wecMotion.stepChange & foreFlag & - & time series flag identifying 'step changes' in encoder signal \\
\hline \multirow[t]{9}{*}{ wecMotion.stepChange } & aftFlag & - & time series flag identifying 'step changes' in encoder signal \\
\hline & proxFore & - & $1=$ proximity sensor tripped, fore float \\
\hline & proxAft & - & $1=$ proximity sensor tripped, aft float \\
\hline & slipFore & - & $1=$ slipClutch activated, fore float \\
\hline & slipAft & - & $1=$ slipClutch activated, aft float \\
\hline & wecHeadingYCS & deg & wec heading as referenced to the yaw control system ring \\
\hline & wecHeadingGPS & deg & wec heading as measured by GPS \\
\hline & weclat & $\operatorname{deg} N$ & wec latitude as measured by GPS \\
\hline & wecLong & $\operatorname{deg} W$ & wec longitude as measure by GPS \\
\hline wecLocation & east & $\mathrm{m}$ & gps based location relative to reference location, smoothed \\
\hline wecLocation & north & $\mathrm{m}$ & gps based location relative to reference location, smoothed \\
\hline wecLocation & eastRaw & $\mathrm{m}$ & gps based location relative to reference location \\
\hline \multirow[t]{3}{*}{ wecLocation } & northRaw & $\mathrm{m}$ & gps based location relative to reference location \\
\hline & windDir & $\operatorname{deg}$ & wind direction, $\mathrm{CW}$ from true north \\
\hline & windSpeed & $\mathrm{m} / \mathrm{s}$ & wind speed \\
\hline mooringLoads & stbd & $\mathrm{N}$ & mooring loads \\
\hline mooringLoads & port & $\mathrm{N}$ & mooring loads \\
\hline mooringLoads & aft & N & mooring loads \\
\hline endStopLoads & forePortTop & kN & end stop loads \\
\hline endStopLoads & forePortBot & $\mathrm{kN}$ & end stop loads \\
\hline endStopLoads & foreStbdTop & $\mathrm{kN}$ & end stop loads \\
\hline endStopLoads & foreStbdBot & $\mathrm{kN}$ & end stop loads \\
\hline endStopLoads & aftPortTop & $\mathrm{kN}$ & end stop loads \\
\hline endStopLoads & aftPortBot & $\mathrm{kN}$ & end stop loads \\
\hline endStopLoads & aftStbdTop & $\mathrm{kN}$ & end stop loads \\
\hline endStopLoads & aftStbdBot & $\mathrm{kN}$ & end stop loads \\
\hline endStopLoads.logicalFilter & foreTop & 0 or 1 & $0=$ no striking, $1=$ strike is occurring \\
\hline endStopLoads.logicalFilter & foreBot & 0 or 1 & $0=$ no striking, $1=$ strike is occurring \\
\hline endStopLoads.logicalFilter & aftTop & 0 or 1 & $0=$ no striking, 1 = strike is occurring \\
\hline endStopLoads.logicalFilter & aftBot & 0 or 1 & $0=$ no striking, $1=$ strike is occurring \\
\hline endStopLoads.num & foreTop & - & number of strike events \\
\hline endStopLoads.num & foreBot & - & number of strike events \\
\hline endStopLoads.num & aftTop & - & number of strike events \\
\hline endStopLoads.num & aftBot & - & number of strike events \\
\hline structStrain & sparFore & micro strain & structural strain, see documentation for location \\
\hline structStrain & sparAft & micro strain & structural strain, see documentation for location \\
\hline structStrain & nacellePortZ & micro strain & structural strain, see documentation for location \\
\hline structStrain & nacellePortX & micro strain & structural strain, see documentation for location \\
\hline structStrain & nacelleStbdz & micro strain & structural strain, see documentation for location \\
\hline structStrain & nacelleStbdX & micro strain & structural strain, see documentation for location \\
\hline
\end{tabular}

Columbia Power Technologies, LLC • 4920A SW $3^{\text {rd }}$ St, Corvallis, Oregon 97333 Phone: (541) 368-5033 • Fax (541) 230-1498 • www.columbiapowertechnologies.com 


\begin{tabular}{llll}
\hline structure & variable & units & description \\
\hline structStrain & nacelleForeZ & micro strain & structural strain, see documentation for location \\
\hline structStrain & nacelleForeY & micro strain & structural strain, see documentation for location \\
\hline powerElectronics & S003_Fwd_AC_A & V & Forward generator - phase A voltage \\
\hline powerElectronics & S004_Fwd_AC_B & V & Forward generator - phase B voltage \\
\hline powerElectronics & S005_Fwd_AC_C & V & Forward generator - phase C voltage \\
\hline powerElectronics & S006_Aft_AC_A & V & Forward generator - phase A voltage \\
\hline powerElectronics & S007_Aft_AC_B & V & Forward generator - phase B voltage \\
\hline powerElectronics & S008_Aft_AC_C & V & Forward generator - phase C voltage \\
\hline powerElectronics & S009_Fwd_Pass_V & V & Passive bus voltage \\
\hline powerElectronics & S010_Aft_Pass_V & V & Passive bus voltage \\
\hline powerElectronics & S011_Act_V & V & Active bus voltage \\
\hline powerElectronics & S012_Fwd_Pass_I & A & Passive bus current \\
\hline powerElectronics & S013_Aft_Pass_I & A & Passive bus current \\
\hline powerElectronics & S014_Act_I & A & Active bus current \\
\hline powerElectronics & S015_Charge_I & A & battery charge current \\
\hline powerElectronics & S016_Discharge_I & A & battery discharge current \\
\hline powerElectronics & S019_Batt_V & V & 24V battery voltage \\
\hline
\end{tabular}

Columbia Power Technologies, LLC • 4920A SW $3^{\text {rd }}$ St, Corvallis, Oregon 97333 Phone: (541) 368-5033 • Fax (541) 230-1498 • www.columbiapowertechnologies.com 


\section{WEC spectral}

\begin{tabular}{|c|c|c|c|}
\hline structure & variable & units & description \\
\hline specResponse & $f$ & $\mathrm{~Hz}$ & frequency scale \\
\hline specResponse.Sf & pos.heave & $m^{\wedge} 2 / H z$ & wec response variance density spectrum \\
\hline specResponse.Sf & pos.surge & $m^{\wedge} 2 / H z$ & wec response variance density spectrum \\
\hline specResponse.Sf & pos.sway & $\mathrm{m}^{\wedge} 2 / \mathrm{Hz}$ & wec response variance density spectrum \\
\hline specResponse.Sf & pos.pitch & $\operatorname{deg}^{\wedge} 2 / H z$ & wec response variance density spectrum \\
\hline specResponse.Sf & pos.roll & $\operatorname{deg}^{\wedge} 2 / H z$ & wec response variance density spectrum \\
\hline specResponse.Sf & pos.yaw & $\operatorname{deg}^{\wedge} 2 / H z$ & wec response variance density spectrum \\
\hline specResponse.Sf & pos.foreFloatRel & $\operatorname{deg}^{\wedge} 2 / H z$ & wec response variance density spectrum \\
\hline specResponse.Sf & pos.aftFloatRel & $\operatorname{deg}^{\wedge} 2 / H z$ & wec response variance density spectrum \\
\hline specResponse.Sf & pos.foreFloatAbs & $\operatorname{deg}^{\wedge} 2 / H z$ & wec response variance density spectrum \\
\hline specResponse.Sf & pos.aftFloatAbs & $\operatorname{deg}^{\wedge} 2 / H z$ & wec response variance density spectrum \\
\hline specResponse.Sf & wave & $m^{\wedge} 2 / H z$ & wave elevation variance density spectrum \\
\hline specResponse.af & pos.heave & $\mathrm{m}$ & wec response amplitude spectrum \\
\hline specResponse.af & pos.surge & $\mathrm{m}$ & wec response amplitude spectrum \\
\hline specResponse.af & pos.sway & $\mathrm{m}$ & wec response amplitude spectrum \\
\hline specResponse.af & pos.pitch & deg & wec response amplitude spectrum \\
\hline specResponse.af & pos.roll & deg & wec response amplitude spectrum \\
\hline specResponse.af & pos.yaw & deg & wec response amplitude spectrum \\
\hline specResponse.af & pos.foreFloatRel & deg & wec response amplitude spectrum \\
\hline specResponse.af & pos.aftFloatRel & deg & wec response amplitude spectrum \\
\hline specResponse.af & pos.foreFloatAbs & $\operatorname{deg}$ & wec response amplitude spectrum \\
\hline specResponse.af & pos.aftFloatAbs & $\operatorname{deg}$ & wec response amplitude spectrum \\
\hline specResponse.af & wave & $\mathrm{m}$ & wave elevation amplitude spectrum \\
\hline specResponse.rao & pos.heave & $\mathrm{m} / \mathrm{m}$ & wec response amplitude operator \\
\hline specResponse.rao & pos.surge & $\mathrm{m} / \mathrm{m}$ & wec response amplitude operator \\
\hline specResponse.rao & pos.sway & $\mathrm{m} / \mathrm{m}$ & wec response amplitude operator \\
\hline specResponse.rao & pos.pitch & $\mathrm{deg} / \mathrm{m}$ & wec response amplitude operator \\
\hline specResponse.rao & pos.roll & $\mathrm{deg} / \mathrm{m}$ & wec response amplitude operator \\
\hline specResponse.rao & pos.yaw & $\mathrm{deg} / \mathrm{m}$ & wec response amplitude operator \\
\hline specResponse.rao & pos.foreFloatRel & $\mathrm{deg} / \mathrm{m}$ & wec response amplitude operator \\
\hline specResponse.rao & pos.aftFloatRel & $\mathrm{deg} / \mathrm{m}$ & wec response amplitude operator \\
\hline specResponse.rao & pos.foreFloatAbs & $\mathrm{deg} / \mathrm{m}$ & wec response amplitude operator \\
\hline specResponse.rao & pos.aftFloatAbs & $\mathrm{deg} / \mathrm{m}$ & wec response amplitude operator \\
\hline wecMotion.filter & cutOffLow & $\mathrm{Hz}$ & pass band frequencies \\
\hline wecMotion.filter & cutOffHigh & $\mathrm{Hz}$ & pass band frequencies \\
\hline
\end{tabular}

Columbia Power Technologies, LLC • 4920A SW $3^{\text {rd }}$ St, Corvallis, Oregon 97333 Phone: (541) 368-5033 • Fax (541) 230-1498 • www.columbiapowertechnologies.com 


\section{Joint parameters}

\begin{tabular}{llll}
\hline structure & variable & units & description \\
\hline rcw & total & - & relative capture width, total \\
rcw & fore & - & relative capture width, fore \\
rcw & aft & - & relative capture width, aft \\
& relWaveHeading & deg & wave heading w.r.t. wec, deg cw from true north, coming from \\
& relCurrentHeading & deg & current heading w.r.t. wec, deg cw from true north, coming from
\end{tabular}

Columbia Power Technologies, LLC $•$ 4920A SW $3^{\text {rd }}$ St, Corvallis, Oregon 97333 Phone: (541) 368-5033 • Fax (541) 230-1498 • www.columbiapowertechnologies.com 


\section{Appendix G. Trial table format}

\begin{tabular}{|c|c|c|}
\hline trial structure variable & units & description \\
\hline trialName & - & file name containing trial information \\
\hline startTimeNom & days in 2011 & $\begin{array}{l}\text { start time, rounded to } 10 \text { min mark (eg. 12:10:00, 12:20:00, etc), in datenum } \\
\text { format }\end{array}$ \\
\hline totalTrialTime & s & elapsed time in seconds \\
\hline mechPowerTotal & W & mean mechanical power \\
\hline mechPowerFore & W & mean mechanical power \\
\hline mechPowerAft & W & mean mechanical power \\
\hline dampingFore & Nms & damping value determined by linear regression \\
\hline dampingAft & Nms & damping value determined by linear regression \\
\hline R2dampingFore & - & $R^{\wedge} 2$ goodness of fit \\
\hline R2dampingAft & - & $R^{\wedge} 2$ goodness of fit \\
\hline slipCountFore & - & slip count (based on $25 \mathrm{~Hz}$ sampling rate) \\
\hline slipCountAft & - & slip count (based on $25 \mathrm{~Hz}$ sampling rate) \\
\hline wecHeading & deg & mean wec heading \\
\hline windSpeed & $\mathrm{m} / \mathrm{s}$ & mean wind speed \\
\hline windDir & deg & mean wind direction, $\mathrm{CW}$ from true north \\
\hline genPosMeanFore & rad & mean position of float \\
\hline genPosMeanAft & rad & mean position of float \\
\hline genPosMaxFore & rad & extreme position of float \\
\hline genPosMaxAft & rad & extreme position of float \\
\hline genPosMinFore & rad & extreme position of float \\
\hline genPosMinAft & rad & extreme position of float \\
\hline mooringLoadsMaxStbd & $\mathrm{N}$ & max mooring load \\
\hline mooringLoadsMaxPort & $\mathrm{N}$ & max mooring load \\
\hline mooringLoadsMaxAft & $\mathrm{N}$ & max mooring load \\
\hline mooringLoadsMeanStbd & $\mathrm{N}$ & mean mooring load \\
\hline mooringLoadsMeanPort & $\mathrm{N}$ & mean mooring load \\
\hline mooringLoadsMeanAft & $\mathrm{N}$ & mean mooring load \\
\hline mooringLoadsRmsStbd & $\mathrm{N}$ & rms of demeaned mooring load signal \\
\hline mooringLoadsRmsPort & $\mathrm{N}$ & rms of demeaned mooring load signal \\
\hline mooringLoadsRmsAft & $\mathrm{N}$ & rms of demeaned mooring load signal \\
\hline endStopLoadsMax_forePortTop & $\mathrm{kN}$ & max end stop load \\
\hline endStopLoadsMax_forePortBot & $\mathrm{kN}$ & max end stop load \\
\hline endStopLoadsMax_foreStbdTop & $\mathrm{kN}$ & max end stop load \\
\hline endStopLoadsMax_foreStbdBot & $\mathrm{kN}$ & max end stop load \\
\hline endStopLoadsMax_aftPortTop & $\mathrm{kN}$ & max end stop load \\
\hline endStopLoadsMax_aftPortBot & $\mathrm{kN}$ & max end stop load \\
\hline endStopLoadsMax_aftStbdTop & $\mathrm{kN}$ & max end stop load \\
\hline endStopLoadsMax_aftStbdBot & $\mathrm{kN}$ & max end stop load \\
\hline endStopLoadsMed_forePortTop & $\mathrm{kN}$ & median end stop load \\
\hline endStopLoadsMed_forePortBot & $\mathrm{kN}$ & median end stop load \\
\hline endStopLoadsMed_foreStbdTop & $\mathrm{kN}$ & median end stop load \\
\hline
\end{tabular}

Columbia Power Technologies, LLC • 4920A SW $3^{\text {rd }}$ St, Corvallis, Oregon 97333 Phone: (541) 368-5033 • Fax (541) 230-1498 • www.columbiapowertechnologies.com 


\begin{tabular}{|c|c|c|}
\hline trial structure variable & units & description \\
\hline endStopLoadsMed_foreStbdBot & $\mathrm{kN}$ & median end stop load \\
\hline endStopLoadsMed_aftPortTop & $\mathrm{kN}$ & median end stop load \\
\hline endStopLoadsMed_aftPortBot & $\mathrm{kN}$ & median end stop load \\
\hline endStopLoadsMed_aftStbdTop & $\mathrm{kN}$ & median end stop load \\
\hline endStopLoadsMed_aftStbdBot & $\mathrm{kN}$ & median end stop load \\
\hline endStopLoadsNum_forePortTop & - & number of strikes \\
\hline endStopLoadsNum_forePortBot & - & number of strikes \\
\hline endStopLoadsNum_foreStbdTop & - & number of strikes \\
\hline endStopLoadsNum_foreStbdBot & - & number of strikes \\
\hline endStopLoadsNum_aftPortTop & - & number of strikes \\
\hline endStopLoadsNum_aftPortBot & - & number of strikes \\
\hline endStopLoadsNum_aftStbdTop & - & number of strikes \\
\hline endStopLoadsNum_aftStbdBot & - & number of strikes \\
\hline torqFitFore & percent/100 & proportion of torq signal that was replaced with linear fit \\
\hline torqFitAft & percent/100 & proportion of torq signal that was replaced with linear fit \\
\hline torqSatFore & percent/100 & proportion of torq signal that was replaced with saturated value \\
\hline torqSatAft & percent/100 & proportion of torq signal that was replaced with saturated value \\
\hline speedDespikedFore & percent $/ 100$ & percent of speed signal that was replaced during the despiking process \\
\hline speedDespikedAft & percent/100 & percent of speed signal that was replaced during the despiking process \\
\hline wecHeadingSource & ycs or gps & yaw control system encoder or dual gps unit \\
\hline wavePower & $\mathrm{W} / \mathrm{m}$ & incident wave power \\
\hline Hm0_int & $\mathrm{m}$ & significant wave power, intrinsic \\
\hline $\mathrm{Hs}$ & $\mathrm{m}$ & significant wave power, time series analysis \\
\hline H10 & $\mathrm{m}$ & mean of largest $1 / 10$ of waves, time series analysis \\
\hline Hmax & $\mathrm{m}$ & max wave height \\
\hline Te_int & s & energy period, intrinsic \\
\hline Te_obs & s & energy period, observed \\
\hline Tp_int & s & peak period, intrinsic \\
\hline Tp_obs & s & peak period, observed \\
\hline eps0_int & - & spectral width, intrinsic \\
\hline epsO_obs & - & spectral width, observed \\
\hline waveDir_Jweight & deg & mean wave heading, wave power weighted \\
\hline waveDir_peak & deg & mean wave heading at peak frequency \\
\hline UI & - & unidirectivity index, based on mean direction as function of $f$ \\
\hline spreadAngle_Jweight & $\operatorname{deg}$ & mean spreading angle, weighted by wave power, intrinsic \\
\hline spreadlndex_Jweight & - & spreading angle, at $f=1 / T p$, intrinsic \\
\hline Ss & - & significant steepness, intrinsic \\
\hline currSpeed & $\mathrm{m} / \mathrm{s}$ & mean current speed from near surface cell \\
\hline currDir & deg & mean current direction from near surface cell \\
\hline relCurrSpeed_peak & $\mathrm{m} / \mathrm{s}$ & relative current speed w.r.t. waveDir.peak \\
\hline relCurrSpeed_Jweight & $\mathrm{m} / \mathrm{s}$ & relative current speed w.r.t. waveDir.Jweight \\
\hline waterDepth & $\mathrm{m}$ & mean water depth during trial \\
\hline astVarRatioMax & - & maximum ast variance ratio (see $x . x x$ ) \\
\hline
\end{tabular}

Columbia Power Technologies, LLC • 4920A SW $3^{\text {rd }}$ St, Corvallis, Oregon 97333

Phone: (541) 368-5033 • Fax (541) 230-1498 • www.columbiapowertechnologies.com 


\begin{tabular}{|c|c|c|}
\hline trial structure variable & units & description \\
\hline astLowQcount & - & number of ast points with relatively low signal to noise ratio \\
\hline astDespikeCount & - & number of ast points replaced during despiking process \\
\hline flagWhiteNoise & - & see report for flag definitions \\
\hline flagShipWave & - & see report for flag definitions \\
\hline flagLowFreqNoise & - & see report for flag definitions \\
\hline flagUnusualDepth & - & see report for flag definitions \\
\hline flagWaveCrestsClipped & - & see report for flag definitions \\
\hline flagHighAstDataLoss & - & see report for flag definitions \\
\hline flagExcessiveTilt & - & see report for flag definitions \\
\hline flagUnusualWaveConditions & - & see report for flag definitions \\
\hline flagLowHighCutOff & - & see report for flag definitions \\
\hline cutOff_flnt_high & $\mathrm{Hz}$ & highest frequency (intrinsic) for which measured data is reliable \\
\hline awacFileNames & - & file name containing awac data for this trial \\
\hline rcwTotal & - & relative capture width, total \\
\hline rcwFore & - & relative capture width, fore \\
\hline rcwAft & - & relative capture width, aft \\
\hline relWaveHeading & deg & wave heading w.r.t. wec, deg cw from true north, coming from \\
\hline relCurrHeading & deg & current heading w.r.t. wec, deg cw from true north, coming from \\
\hline wecLocationEast & $\mathrm{m}$ & mean wec location (plan view) w.r.t. arbitrary reference location \\
\hline wecLocationNorth & $\mathrm{m}$ & mean wec location (plan view) w.r.t. arbitrary reference location \\
\hline Hmo_fit & $\mathrm{m}$ & significant wave height of JONSWAP spectra fit to intrinsic spectra \\
\hline Tp_fit & s & peak period of JONSWAP spectra fit to intrinsic spectra \\
\hline gamma_fit & - & peakedness enhancement factor of JONSWAP spectra fit to intrinsic spectra \\
\hline fitQ & - & normalized wave energy total error (bias error plus rms error) of fit spectra \\
\hline fitJError & - & normalized wave energy bias error of fit spectra \\
\hline
\end{tabular}

Columbia Power Technologies, LLC • 4920A SW $3^{\text {rd }}$ St, Corvallis, Oregon 97333 Phone: (541) 368-5033 • Fax (541) 230-1498 • www.columbiapowertechnologies.com 


\section{Appendix H. Site selection}

Please see enclosed file for this appendix.

\section{Appendix I. Wave climate study}

Please see enclosed file for this appendix.

\section{Appendix J. Wave climate study and scale selection}

Please see enclosed file for this appendix.

\section{Appendix K. Acoustic study}

Please see enclosed file for this appendix.

\section{Appendix L. Deployment plan}

Please see enclosed file for this appendix.

\section{Appendix M. Mooring load model validation (redacted)}

Redacted

\section{Appendix N. Site bathymetry survey}

Please see enclosed file for this appendix. 


\section{CPT Scaled Test Site Selection}

Task Order number 1131

\section{Objective:}

To gather data/information on possible test locations for a $1 / 5$ scale test of test of a novel direct-drive rotary wave energy converter (DDR WEC).

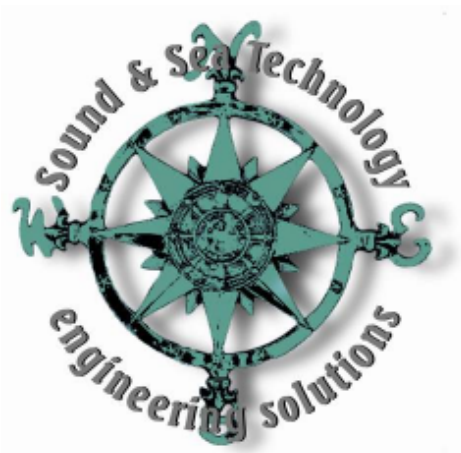

\section{Site specifications:}

Wave conditions at test site should have Hs and Tp to $1 / 5^{\text {th }}$ scale power of Stonewall Banks site. The average low range of Hs for this location should be $0.32 \mathrm{~m}$, and the average high range Hs would be $0.76 \mathrm{~m}$, thus average Hs of $0.5 \mathrm{~m}$ was preferred. The $1 / 5$ scale of Tp at the Stonewall Banks site is 1.78 seconds. Hs should not exceed $2.0 \mathrm{~m}$. For these reasons sites in protected bays with adequate fetch distances to produce wind waves were examined as possible test locations. The depth required for mooring the DDR WEC is $30 \mathrm{~m}$ or greater.

\section{Conditions required for wind wave:}

Data provided by Jim Thomson, Ph.D. a University of Washington, Applied Physics Lab study in Puget Sound, WA showed that wind speeds $>8 \mathrm{~m} / \mathrm{s}$ (15 knots), with a fetch of $20 \mathrm{~km}$ and a duration of several hours produced wind waves with $\mathrm{Hs}=0.5 \mathrm{~m}$ and $\mathrm{Tp}$ that varied between 2 and 4 seconds. Jim Thompson stated that Tp was not well correlated with wind speed as Tp was likely affected by currents and other features within the Puget Sound basin, and as a result ranged from 2 to 4 seconds.

Data provided from SST engineer, Bob Taylor, from the Coastal Protection Manual provided data tables predicting the period of wind waves produced by various wind speeds (see figure below). These data are for $10 \mathrm{~km}$ of fetch and 100 foot water depths. They are expected to produce lower Hs and $\mathrm{Tp}$ than are typically found in areas of greater fetch and depth (conditions in Puget Sound).

Wind data was gathered from National Data Buoy Center (NDBC) Met station locations in the Puget Sound, WA, San Francisco Bay, CA and near Tomales Bay, CA as these were 3 bays along the US west coast that had $20 \mathrm{~km}$ of fetch in the direction of prevailing winds. When longterm data sets were available (10 years), analysis could be made on the average number of wind events per year that meet or exceed threshold conditions. Three threshold conditions were selected; $8-12 \mathrm{~m} / \mathrm{s}, 12-16 \mathrm{~m} / \mathrm{s}$ and $>16 \mathrm{~m} / \mathrm{s}$.

From this initial analysis of wind events at possible test locations it was determined that West Point, Puget Sound, WA would be the central focus of this effort as it had a high number of wind PROPRIETARY DOCUMENT, COLUMBIA POWER TECHNOLOGIES NOT FOR DISTRIBUTION, KR 11/20/2009 
events meeting each of the three threshold conditions listed (see Tab 3). This location also had a long-term data set on wind conditions, and satisfied other logistical considerations for the deployment of a WEC device (adequate depth, dry dock and boat yard facilities close by). A detail analysis of the average number of wind events per month from October through January was also produced for the West Point site (see Tab 2). These months were chosen as this is the expected time of the year the WEC device will be tested.

It was only possible to make estimates of Hs during wind events as there was no available wave buoy information at any of these locations. Rough estimates of Tp can be made from the table in the figure below, but it should be noted that these tables do not account for the effect of strong currents and variable bathymetry found in the Puget Sound.
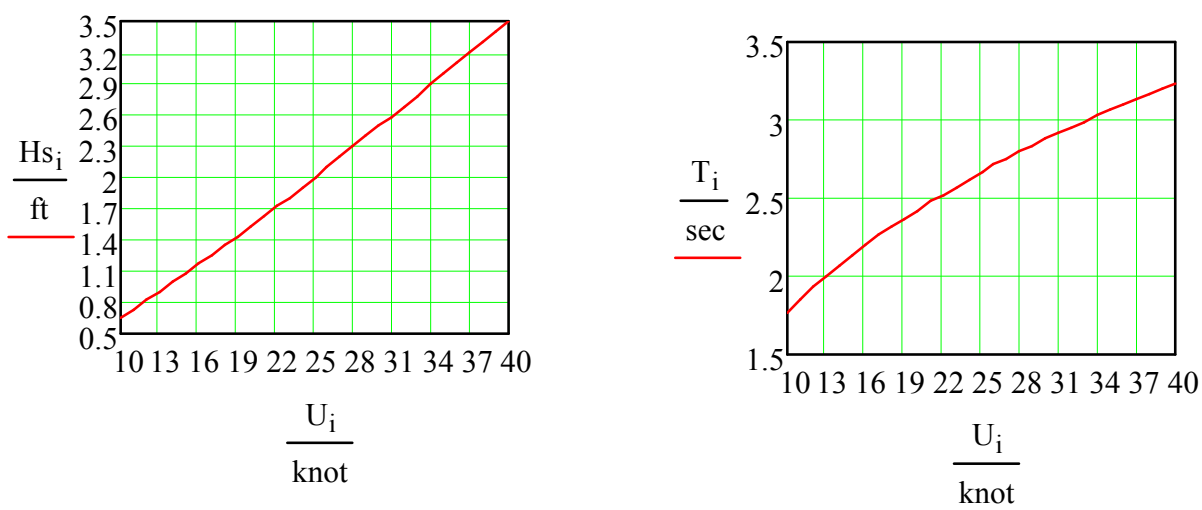

\section{Recommendations:}

The West Point location near Seattle, WA meets many of the logistical specifications for this study. There are several marine facilities within 5 miles of this location that provide dry docks, cranes and other facilities required for the deployment of a small scale WEC device. Although the NDBC Met station location at West Point (site WPOW1) provides an excellent long-term data set on wind speeds, there is no wave buoy data available for this location. It is not likely that any site within a protected bay will have an established wave buoy as wave buoy programs are concerned with collecting data on open ocean waves. Therefore to adequately measure the Hs and Tp for wind waves near the West Point location we recommend placing a wave buoy at this location during the time of the year of the planned test (October through January) to obtain measured data of the wave spectrum at this location. 


\section{Field Experiments}

\section{Puget Sound Feb 2008}

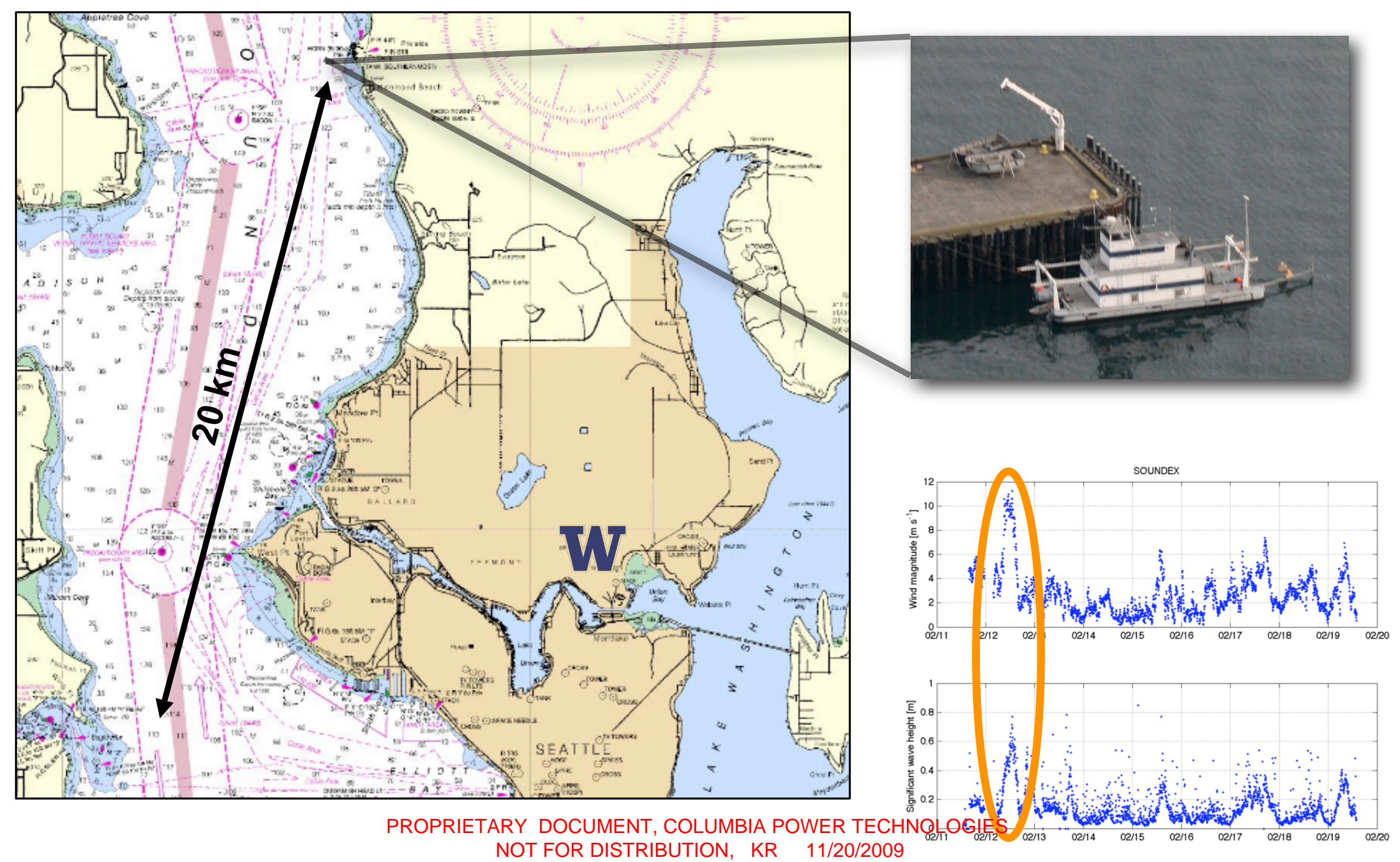




\begin{tabular}{|c|c|c|c|c|c|c|c|c|c|c|c|c|c|c|c|}
\hline \multirow[b]{2}{*}{ Site Name / Description } & \multicolumn{2}{|c|}{ Scale Estimates } & \multicolumn{4}{|c|}{ Location } & \multicolumn{9}{|c|}{ Wave Spectrum \& Bottom Conditions } \\
\hline & $\begin{array}{c}\text { Scale } \\
\text { Factor } \\
\text { Low High }\end{array}$ & $\begin{array}{c}\text { Scaled } \\
\text { Power } \\
\text { (Peak kW) } \\
\text { Low } \\
\text { High }\end{array}$ & $\begin{array}{l}\text { Country } \\
\text { \& State }\end{array}$ & City & Latitude / Long. & $\begin{array}{c}\text { Avalladole } \\
\text { Facilities } \\
\text { (DD, SY, } \\
\text { CR, DK, } \\
\text { PT) }\end{array}$ & $\begin{array}{c}\text { Lowest } \\
\text { Ave. } \\
\text { monthly } \\
\text { Hs (m) }\end{array}$ & $\begin{array}{c}\text { Highest } \\
\text { Ave. } \\
\text { monthly } \\
\text { Hs }(m)\end{array}$ & $\begin{array}{l}\text { Tz } \\
\text { (s) }\end{array}$ & $\begin{array}{l}\mathrm{Tp} \\
\text { (s) }\end{array}$ & $\begin{array}{c}\text { Max } \\
\text { Wave } \\
(\mathrm{m})\end{array}$ & $\begin{array}{c}\text { Depth } \\
(\mathrm{m})\end{array}$ & $\begin{array}{c}\text { Sand, } \\
\text { mud, } \\
\text { rock }\end{array}$ & $\begin{array}{l}\text { Dist. } \\
\text { from } \\
\text { shore } \\
(\mathrm{NM})\end{array}$ & $\begin{array}{c}\text { Years } \\
\text { of data } \\
\text { studied }\end{array}$ \\
\hline $\begin{array}{l}\text { Stonewall Banks, Baseline } \\
\text { West Point, Puget Sound } \\
\text { Alki Point, Puget Sound } \\
\text { Maury Point, Vashon Island } \\
\text { Dabob Bay, Hood Canal } \\
\text { Tomales Bay, Hog Island } \\
\text { San Pablo, S.F. Bay } \\
\text { North Lummi Island }\end{array}$ & \begin{tabular}{|l|l}
$*$ & \\
$*$ & $*$ \\
$*$ & $*$ \\
$*$ & $*$ \\
$*$ & $*$ \\
$*$ & $*$ \\
$*$ & $*$ \\
&
\end{tabular} & \begin{tabular}{c|c}
\multicolumn{2}{c}{400} \\
$*$ & $*$ \\
$*$ & $*$ \\
$*$ & $*$ \\
$*$ & $*$ \\
$*$ & $*$ \\
$*$ & $*$ \\
$*$ & $*$ \\
& \\
\end{tabular} & $\begin{array}{l}\text { US, OR } \\
\text { US, WA } \\
\text { US, WA } \\
\text { US, WA } \\
\text { US, WA } \\
\text { US, CA } \\
\text { US, CA } \\
\text { US, WA }\end{array}$ & $\begin{array}{c}\text { Newport } \\
\text { Seattle } \\
\text { Seattle } \\
\text { Seattle } \\
\text { Seattle } \\
\text { Tomales } \\
\text { San Francisco } \\
\text { Bellingham }\end{array}$ & $\begin{array}{l}44.641 \mathrm{~N} 124.5 \mathrm{~W} \\
47.66 \mathrm{~N} 122.44 \mathrm{~W} \\
47.57 \mathrm{~N} 122.42 \mathrm{~W} \\
47.39 \mathrm{~N} 122.37 \mathrm{~W} \\
47.69 \mathrm{~N} 122.94 \mathrm{~W} \\
38.20 \mathrm{~N} 122.94 \mathrm{~W} \\
37.93 \mathrm{~N} 122.40 \mathrm{~W} \\
48.76 \mathrm{~N} 122.73 \mathrm{~W}\end{array}$ & $\begin{array}{c}\text { all } \\
\text { all } \\
\text { all } \\
\mathrm{dk} \\
\mathrm{dk} \\
\text { all } \\
\mathrm{dk}, \mathrm{cr}\end{array}$ & $\begin{array}{l}1.6 \\
0.0 \\
0.0 \\
0.0 \\
0.0 \\
0.0 \\
0.0 \\
0.0 \\
{ }^{*} \text { Req } \\
* \\
*\end{array}$ & $\begin{array}{c}3.8 \\
* \\
* \\
* \\
* \\
* \\
* \\
* \\
\text { y's wave } \\
\text { See Tab }\end{array}$ & $\begin{array}{l}7.4 \\
* \\
* \\
* \\
* \\
* \\
* \\
* \\
\text { data } \\
2\end{array}$ & $\begin{array}{l}8.9 \\
* \\
* \\
* \\
* \\
* \\
* \\
*\end{array}$ & $\begin{array}{l}18 \\
2 \\
2 \\
2 \\
2 \\
1 \\
2 \\
2\end{array}$ & $\begin{array}{c}123 \\
37 \\
37 \\
46 \\
46 \\
16 \\
\mathrm{n} / \mathrm{a} \\
37\end{array}$ & $\begin{array}{l}\text { rock } \\
\text { Sand } \\
\text { Sand } \\
\text { Sand } \\
\text { Sand } \\
\text { Sand } \\
\text { unk. } \\
\text { rock }\end{array}$ & $\begin{array}{l}20 \\
0.1 \\
0.1 \\
0.1 \\
1.0 \\
0.6 \\
0.2 \\
0.6 \\
\quad \text { wi }\end{array}$ & $\begin{array}{c}18 \\
10 \\
10 \\
10 \\
10 \\
10 \\
2 \\
10 \\
\text { ind data }\end{array}$ \\
\hline & & & & & & $\begin{array}{l}\text { DD = Dry do } \\
\text { SY = shipya } \\
\text { CR=Crane } \\
\text { DK = Docks } \\
\text { PT = Port o } \\
\text { etc }, \text { define a }\end{array}$ & $\begin{array}{l}\mathrm{k} \\
\mathrm{d} \\
\text { Terminal } \\
\text { needed }\end{array}$ & to descril & 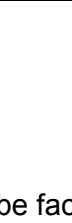 & 更 & & & & & \\
\hline
\end{tabular}

http://www.ndbc.noaa.gov/station page.php?station=46050

http://www.marine.ie/home/aboutus/organisationstaff/researchfacilities/Ocean+Energy+Test+Site.htm 
West Point $\mathrm{N}$ wind defined as 315 - 45 degrees $\mathrm{T}$ (center point 0 degrees $\mathrm{T}$ )

West Point $S$ wind defined as $140-230$ degrees $T$ (center point 185 degrees $\mathrm{T}$ )

Wind conditions producing Hs: $8-12 \mathrm{~m} / \mathrm{s}=1.0 \mathrm{~m}, 12-16 \mathrm{~m} / \mathrm{s}=1.5 \mathrm{~m}, 16+\mathrm{m} / \mathrm{s}=2.0 \mathrm{~m}$

(Based on Jim Thompson / UW Study)

Freq. of $\mathrm{Hs}=1 \mathrm{~m}$ produced by South winds

October November December January

$\begin{array}{rrrrr}2008 & 3 & 11 & 14 & 15 \\ 2007 & 15 & 7 & 11 & 16 \\ 2006 & 7 & 16 & 10 & 18 \\ 2005 & 7 & 10 & 2 & 5 \\ 2004 & 5 & 4 & 3 & 7 \\ 2003 & 9 & 3 & 12 & 5 \\ 2002 & 1 & 10 & 11 & 15 \\ 2001 & 13 & 11 & 9 & 7 \\ 2000 & 7 & 7 & 2 & 10 \\ 1999 & 7 & 12 & 11 & 7 \\ \text { Average } & \mathbf{7 . 4} & \mathbf{9 . 1} & \mathbf{8 . 5} & \mathbf{1 0 . 5}\end{array}$

Freq. of $\mathrm{Hs}=1.5 \mathrm{~m}$ produced by South winds

October November December January

$\begin{array}{rrrrr}2008 & 2 & 2 & 0 & 6 \\ 2007 & 2 & 3 & 8 & 6 \\ 2006 & 0 & 5 & 2 & \\ 2005 & 2 & 2 & 1 & \\ 2004 & 1 & 2 & 3 & \\ 2003 & 5 & 1 & 2 & 2 \\ 2002 & 0 & 0 & 3 & 4 \\ 2001 & 2 & 2 & 6 & \\ 2000 & 0 & 0 & 1 & \\ 1999 & 3 & 0 & 10 & \\ \text { erage } & 1.7 & \mathbf{1 . 7} & \mathbf{3 . 6} & \mathbf{4 . 2}\end{array}$

Freq. of $\mathrm{Hs}=2 \mathrm{~m}$ produced by South winds

October November December January

$\begin{array}{ll}2008 & 0 \\ 2007 & 0 \\ 2006 & 0 \\ 2005 & 0 \\ 2004 & 0 \\ 2003 & 0 \\ 2002 & 0 \\ 2001 & 1 \\ 2000 & 0 \\ 1999 & 0\end{array}$

Average
0.1
15

18

5

7

5
15

15

10

7
0.5
Freq. of $\mathrm{Hs}=1 \mathrm{~m}$ produced by North winds

October November December January

$\begin{array}{rrrrr}2008 & 4 & 3 & 5 & 1 \\ 2007 & 2 & 2 & 3 & 2 \\ 2006 & 3 & 2 & 0 & 0 \\ 2005 & 0 & 0 & 0 & 5 \\ 2004 & 1 & 2 & 4 & 3 \\ 2003 & 2 & 3 & 1 & 0 \\ 2002 & 3 & 0 & 3 & 2 \\ 2001 & 2 & 1 & 2 & 0 \\ 2000 & 1 & 0 & 2 & 1 \\ 1999 & 0 & 0 & 0 & 1 \\ \text { erage } & 1.8 & 1.3 & 2 & 1.5\end{array}$

Freq. of $\mathrm{Hs}=1.5 \mathrm{~m}$ produced by North winds

October November December January

$\begin{array}{lllll}2008 & 0 & 0 & 0 & 0 \\ 2007 & 0 & 0 & 0 & 0 \\ 2006 & 0 & 1 & 0 & 0 \\ 2005 & 0 & 0 & 0 & 0 \\ 2004 & 0 & 0 & 0 & 1 \\ 2003 & 0 & 0 & 0 & 0 \\ 2002 & 0 & 0 & 0 & 0 \\ 2001 & 0 & 0 & 0 & 0 \\ 2000 & 0 & 0 & 0 & 0 \\ 1999 & 0 & 0 & 0 & 0 \\ \text { rage } & \mathbf{0} & \mathbf{0 . 1} & \mathbf{0} & \mathbf{0 . 1}\end{array}$

\section{Freq. of $\mathrm{Hs}=\mathbf{2 m}$ produced by North winds}

October November December January

$\begin{array}{cllll}2008 & 0 & 0 & 0 & 0 \\ 2007 & 0 & 0 & 0 & 0 \\ 2006 & 0 & 0 & 0 & 0 \\ 2005 & 0 & 0 & 0 & 0 \\ 2004 & 0 & 0 & 0 & 0 \\ 2003 & 0 & 0 & 0 & 0 \\ 2002 & 0 & 0 & 0 & 0 \\ 2001 & 0 & 0 & 0 & 0 \\ 2000 & 0 & 0 & 0 & 0 \\ 1999 & 0 & 0 & 0 & 0 \\ \text { erage } & 0 & 0 & 0 & 0\end{array}$




\begin{tabular}{|c|c|c|c|}
\hline $\begin{array}{l}\text { Wind } \\
\text { events } \\
\text { per year }\end{array}$ & $\begin{array}{l}\text { Wind } \\
\text { events } \\
\text { per year }\end{array}$ & $\begin{array}{l}\text { Wind } \\
\text { events } \\
\text { per year }\end{array}$ & $\begin{array}{l}\text { Wind } \\
\text { events } \\
\text { per year }\end{array}$ \\
\hline$>=8 \mathrm{~m} / \mathrm{s}$ & $>=12 \mathrm{~m} / \mathrm{s}$ & $>=16 \mathrm{~m} / \mathrm{s}$ & $>=18 \mathrm{~m} / \mathrm{s}$ \\
\hline 104 & 23 & 1.8 & 0.6 \\
\hline 104 & 23 & 1.8 & 0.6 \\
\hline 104 & 23 & 1.8 & 0.6 \\
\hline 104 & 23 & 1.8 & 0.6 \\
\hline 144 & 69 & 4.2 & 0.4 \\
\hline $2^{*}$ & 0 & 0 & 0 \\
\hline $2^{*}$ & 0 & 0 & 0 \\
\hline 16 & 3 & 0 & 0 \\
\hline
\end{tabular}

\section{CPT Scaled Test sites}

\section{State Depth at site}

West Point, Puget Sound

Alki Point, Puget Sound

WA

Maury Point, Vashon Island WA

Dabob Bay, Hood Canal WA

Tomales Bay, Hog Island CA

San Pablo North, S.F. Bay CA

San Pablo South, S.F. Bay CA

N. of Lummi Island*

WA

*est. from Smith Island data
16 to 30 fathoms, Chart 18441

44+ fathoms, Chart 18441

25+ fathoms, Chart 18448

$24+$ fathoms, south end, Chart 18441

54 feet deepest N. Hog Island, 18643

Chart 18642 not avail for free view

depth unknown

30 fathoms N. Lummi, Chart 18421

\section{Location}

West Point, Puget Sound Alki Point, Puget Sound

Maury Point, Vashon Island Dabob Bay, Hood Canal

Tomales Bay, Hog Island San Pablo North, S.F. Bay San Pablo South, S.F. Bay N. of Lummi Island*

*est. from Smith Island data

\section{Wind data notes}

N. July - Sept, SW Nov - Mar "

NW Apr - June

NW Apr - June

NW-N winds May - July

\section{notes}

Exposed to frieghter traffic, wind chop $\mathrm{N}$ and $\mathrm{S}$

Freighter traffic, Ferry traffic, wind chop $\mathrm{N}$ and $\mathrm{S}$

NW and SW exposure good, near shipping lanes, restricted channel

North wind, if deployed at $S$ end of bay at point

8 foot deep at bar entrance, moving buoys over a problem

only 3 years of wind data, NDBC event summary seem too low

N. Lummi site very close to ship lanes, N. Alden Bank more area 


\title{
Wave Conditions on Puget Sound During Winter
}

- A report for Columbia Power Technologies -

\author{
J. Thomson (jthomson@apl.washington.edu) \\ Applied Physics Laboratory, University of Washginton \\ 1013 NE 40th St, Seattle, WA 98105
}

May 27, 2010

\section{Abstract}

Surface-gravity waves, generated by local winds, are observed in the main basin of Puget Sound, WA, from November 2009 to April 2010. A climatology a wave conditions is assembled. Wave conditions are dominated by synoptic weather patterns, which in winter storms with southerly winds on the order of $20 \mathrm{~m} / \mathrm{s}$ produce waves of $1 \mathrm{~m}$ significant wave height and 3 s period (nominal values). These wind waves are young, fetch-limited, and highlyforced. Waves steepness and inferred whitecap breaking rates are consistent with previous observations. In addition to the naturally generated waves, ship wakes from commercial traffic are common and are larger than all but the biggest natural waves.

\section{Introduction}

Puget Sound is a fjord-type estuary in the Pacific Northwest region of the United States. It is connected to the Pacific via the Strait of Juan de Fuca, however swell waves from the Pacific do not propagate to Puget Sound (a result of the complex geometry). Previous observations have shown that waves in fjords exhibit fetch-limited growth and are aligned with the wind (Thomson et al., 2009; Pettersson, 2004; Atakturk and Katsaros, 1999). These waves are always young, compared with the open ocean, and cannot evolve or propagate much beyond the local wind forcing.

In the following sections, a four-month long dataset of waves on Puget Sound is described, analyzed for climatology, and compared with numerical simulations. Consistent with previous observations, winter storms produce waves that are approximately $1 \mathrm{~m}$ height and 3 s period. Wind climatologys show that summer months are comparatively calm, although individual events may be equally strong. 


\section{Observations}

Water surface elevations and wind speeds were recorded from 11 December 2009 to 4 April 2010 at the southern end of the Paramount Petroleum pier off of Point Wells in the main basin of Puget Sound (N 47.7799, W 122.3991). In addition, a week of pilot data was collected from 19-24 November 2009 at the same location. The site was selected to maintain deepwater conditions (depth is $16 \mathrm{~m}$ ref. MLLW) for short-period waves ( $<20 \mathrm{~m}$ wavelength), and for an open fetch towards the prevailing southerly winds. At summary of the wind and wave observations is shown in Figure 1.

\subsection{Sampling: waves}

Water surface elevations were measured with a down-looking sonic range finder (Miltronics AirRanger SPL) cantilevered out $2 \mathrm{~m}$ from the south end of pier. Piling spacing under the pier is approximately $3 \mathrm{~m}$ and the average piling diameter is $0.4 \mathrm{~m}$, resulting in a blockage ratio of $13 \%$ that is unlikely to significantly alter the incoming wave field. This is visually confirmed by a lack of standing-wave or diffraction patterns in the vicinity of the pier.

Water surface elevations were sampled at $1.4 \mathrm{~Hz}$ for a 20 min burst at the beginning of each hour. This sampling was limited by the serial data acquisition (Acumen SDR) and windgenerated power supply (Southwest Windpower Air-X). The resulting Nyquist frequency $f_{N}=0.7 \mathrm{~Hz}$ is sufficient to resolve the short-period waves, and the $20 \mathrm{~min}$ bursts have strict stationarity for ensemble averaging. Based on previous observations on Puget Sound (Thomson et al., 2009; Gemmrich, 2010), the unresolved highest frequencies are expected to be small, because of the persistence of an $f^{-4}$ equilibrium (Banner, 1990). The $f^{-4}$ dependence at high frequencies is sufficiently steep that estimates of peak period $T_{p}$ or energy period $T_{e}$ are not expected to be biased by the unresolved portion of the spectra above $f_{N}=0.7 \mathrm{~Hz}$.

Wave directions are not measured.

\subsection{Sampling: winds}

Wind speed and direction were measured with a tri-cup and vane anemometer (Onset SWCA-M003) colocated with the wave gage. The anemometer height was $7.5 \mathrm{~m}$ ref MLLW. Wind speeds were sampled at $1 \mathrm{~Hz}$, with averages and maximum gusts recorded every 5 minutes to an integrated logger (Onset U10). Winds are interpolated to hourly values for comparison with wave results. It is expected, and well-demonstrated in previous work, that wave directions would be similar to the wind directions in the absence of swell. 
APL-UW wave monitoring at Pt Wells
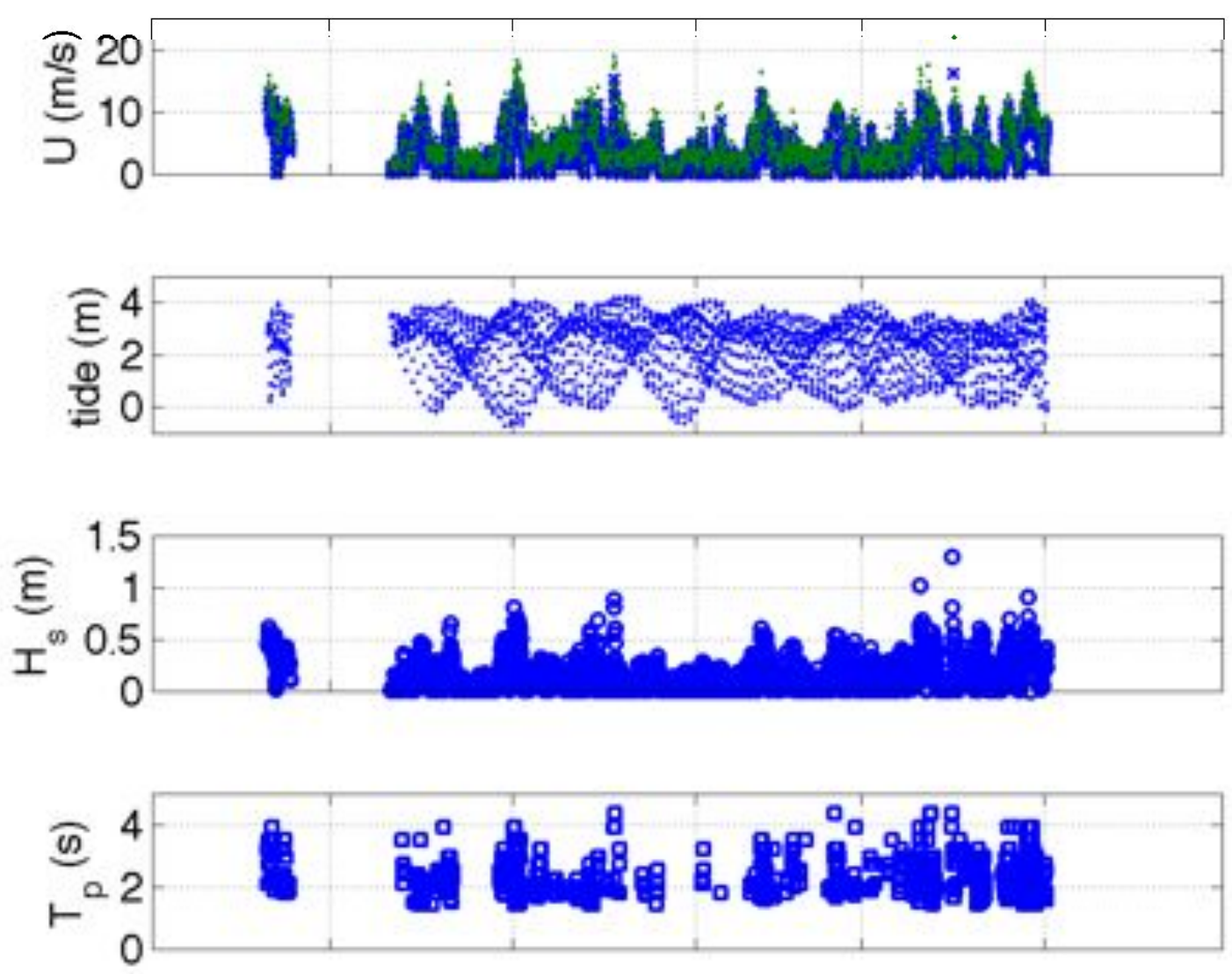

Spectral energy density $\left(\log \left[\mathrm{m}^{2} / \mathrm{Hz}\right]\right)$

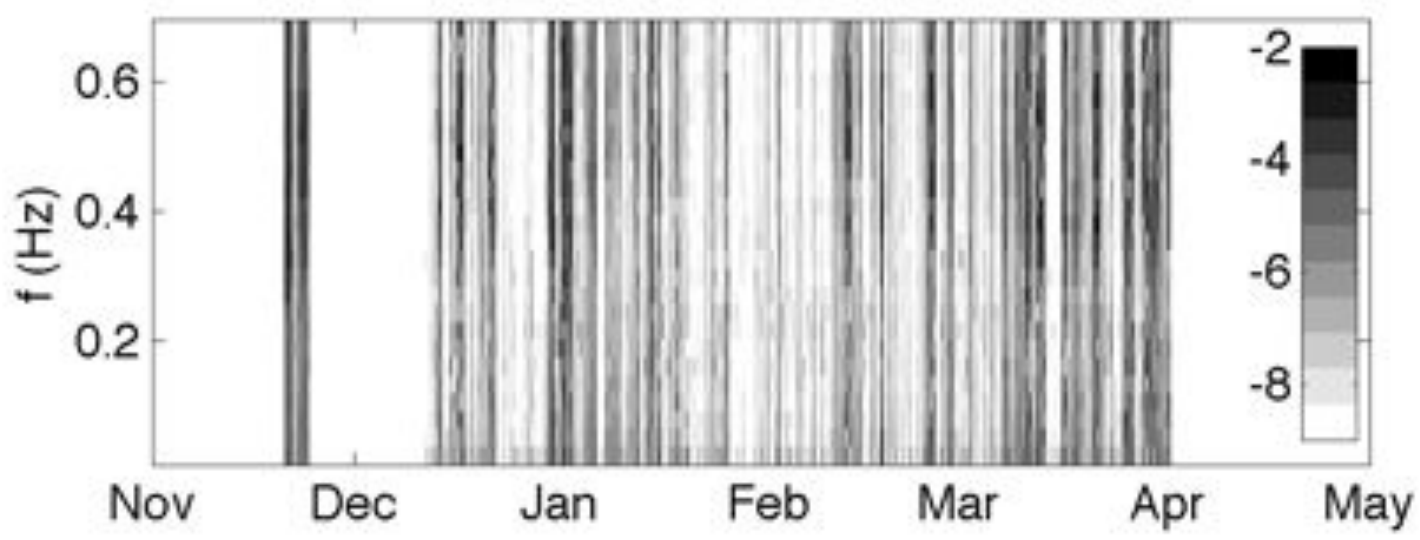

Figure 1: Summary of hourly wind speeds (blue crosses) and gusts (green dots), wave heights (blue circles), wave periods (blue squares), and wave energy spectral densities (grayscale). 


\section{Analysis}

\subsection{Spectra}

Wave energy spectra are generated for each 20 min burst by first dividing into 12 windows of $50 \%$ overlap. The windows are detrended to remove the tide and tapered to reduce signal leakage. A normalized Fast Fourier Transform converts each window to frequency space, and the windows are then ensemble averaged to improved statistical confidence. In addition, each five neighboring frequency bands are merged. The resulting spectra have 60 degrees of freedom, compared with 2 degrees of freedom for raw spectra. The final frequency resolution is $0.027 \mathrm{~Hz}$. Average spectral energy densities $S(f)$ are shown in Figure 2.

Peak period $T_{p}$ and energy period $T_{e}$ are estimated by determining the location of the peak and the centroid, respectively, in the spectral energy densities. For very small waves, the spectra are relatively flat and there are not peaks significant at 95\% confidence (using 60 degrees of freedom). Thus, periods are not reported during low wave conditions. The relatively flat spectra are likely the result of low frequency motions (seiches, tides), episodic motions (ship-wakes), and aliasing of higher frequency fluctuations. The effective cutoff used is $0.2 \mathrm{~m}$ significant wave height, which corresponds to cases when the standard deviation of the water surface elevation is less than $0.05 \mathrm{~m}$. The apparent peak around $f=0.1 \mathrm{~Hz}$ in Figure 2 during low wave conditions appears to be related to ship wakes, but a rigorous study on this effect has not been completed.

\subsection{Significant wave heights}

The significant wave height, corresponding to the largest $1 / 3$ of the waves in Rayleigh distribution, is given by

$$
H_{s}=4 \int_{f_{2}}^{f_{1}} S(f) d f
$$

where $f_{1}=0.1 \mathrm{~Hz}$ and $f_{2}=0.7 \mathrm{~Hz}$ delineate the wave frequencies and the spectral estimate is approximately equivalent to four times the standard deviation of the elevation time series (assuming wave motions dominate the signal).

The average significant wave height observed is $0.13 \mathrm{~m}$, but can reach $1.3 \mathrm{~m}$ during winter storms. A histogram of wave heights is shown in Figure 3, where significant wave heights above $0.5 \mathrm{~m}$ are observed only $5 \%$ of the time. In addition, ship wakes are common in the area and may include instantaneous wave heights of a few meters (Curtiss et al., 2009). The significant wave heights are somewhat correlated with peak period, as shown in the joint occurrence histogram in Figure 4, presumably because of wave evolution during the longer storms that produce larger waves. 


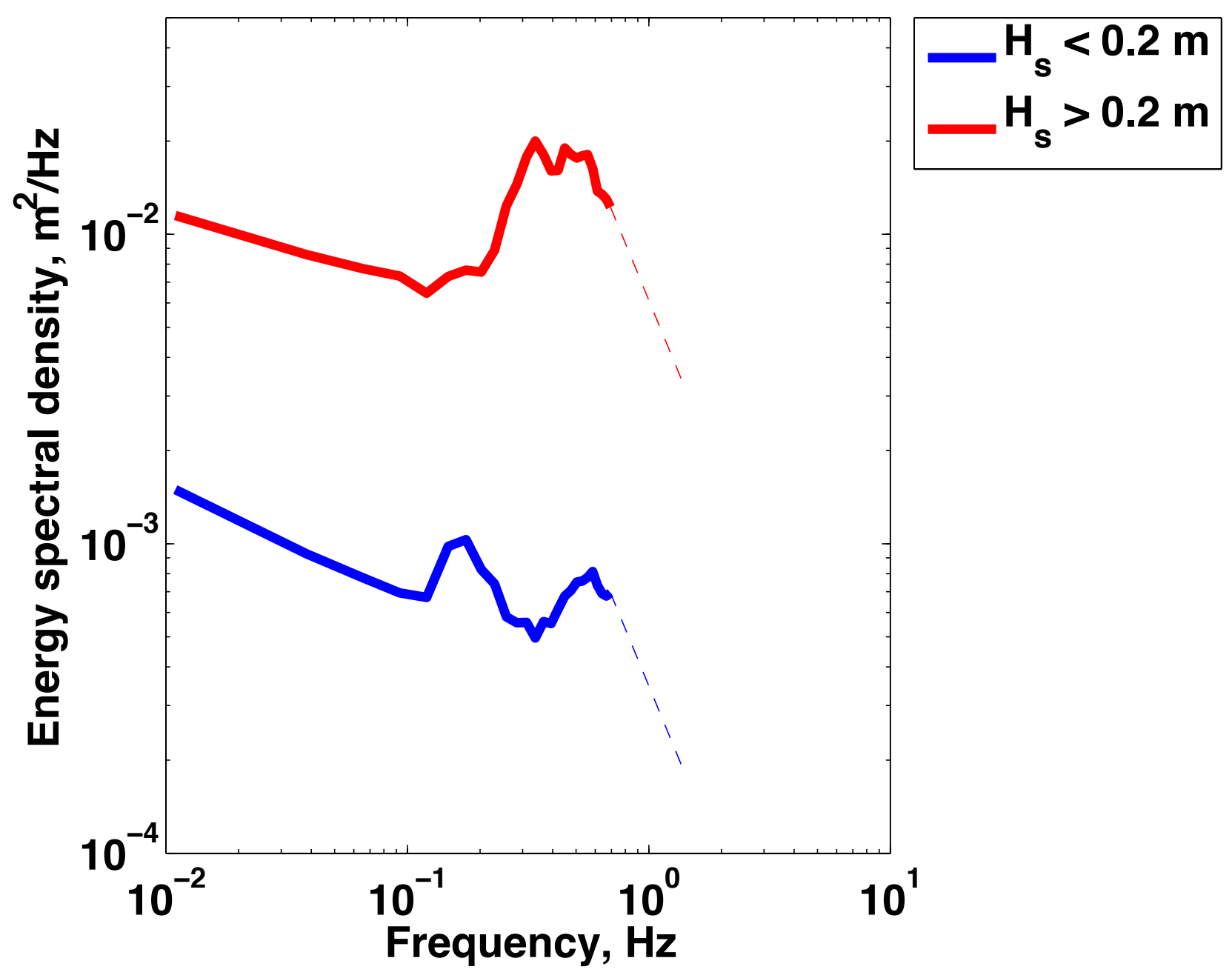

Figure 2: Mean spectral energy density versus frequency for small waves (blue) and large waves (red). The expected high frequency tail $f^{-4}$, determined during previous observations at the site, is shown by the dashed line. 

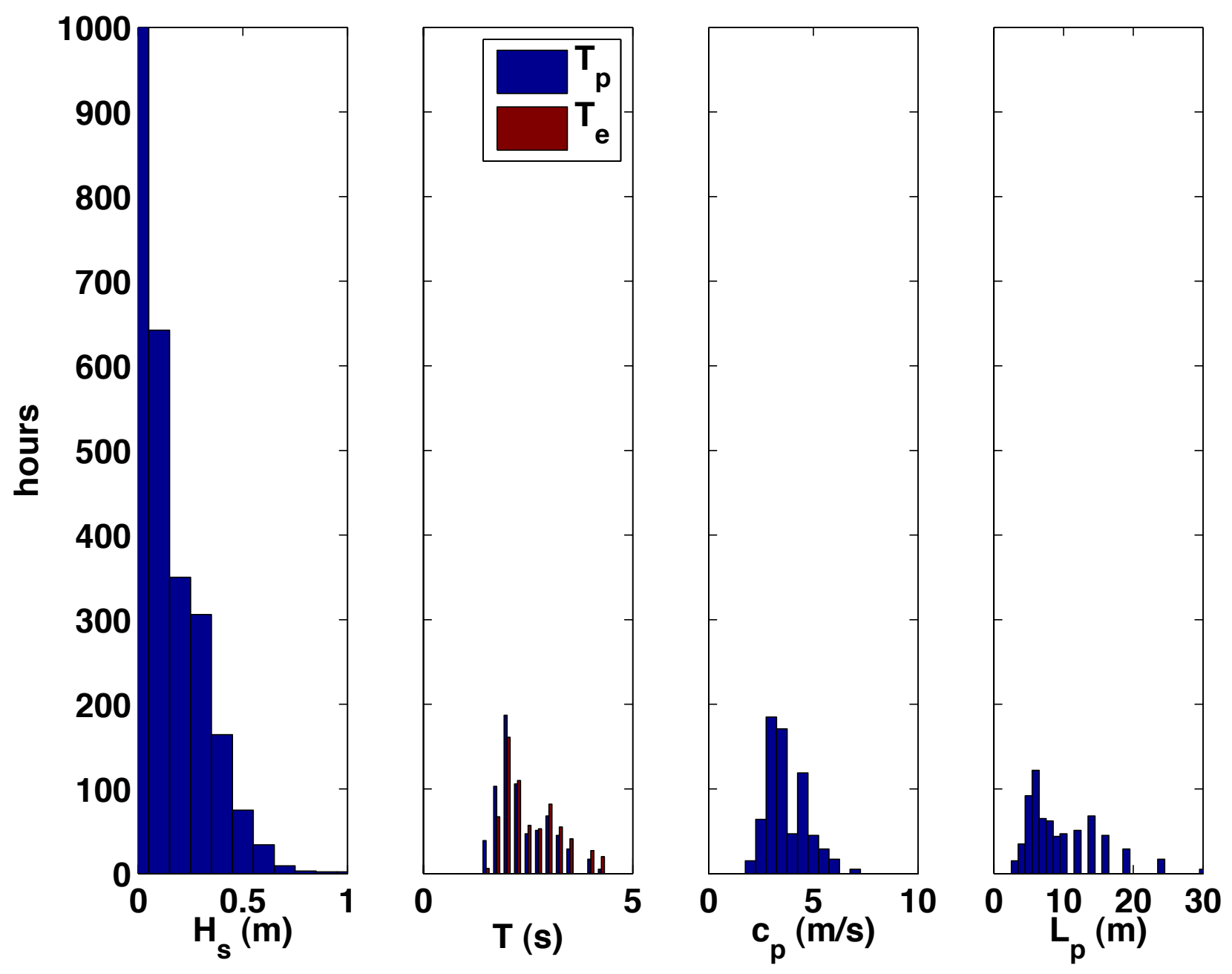

Figure 3: Histograms, by hour, of significant wave height, peak period, peak phase speed, and peak wavelength. 


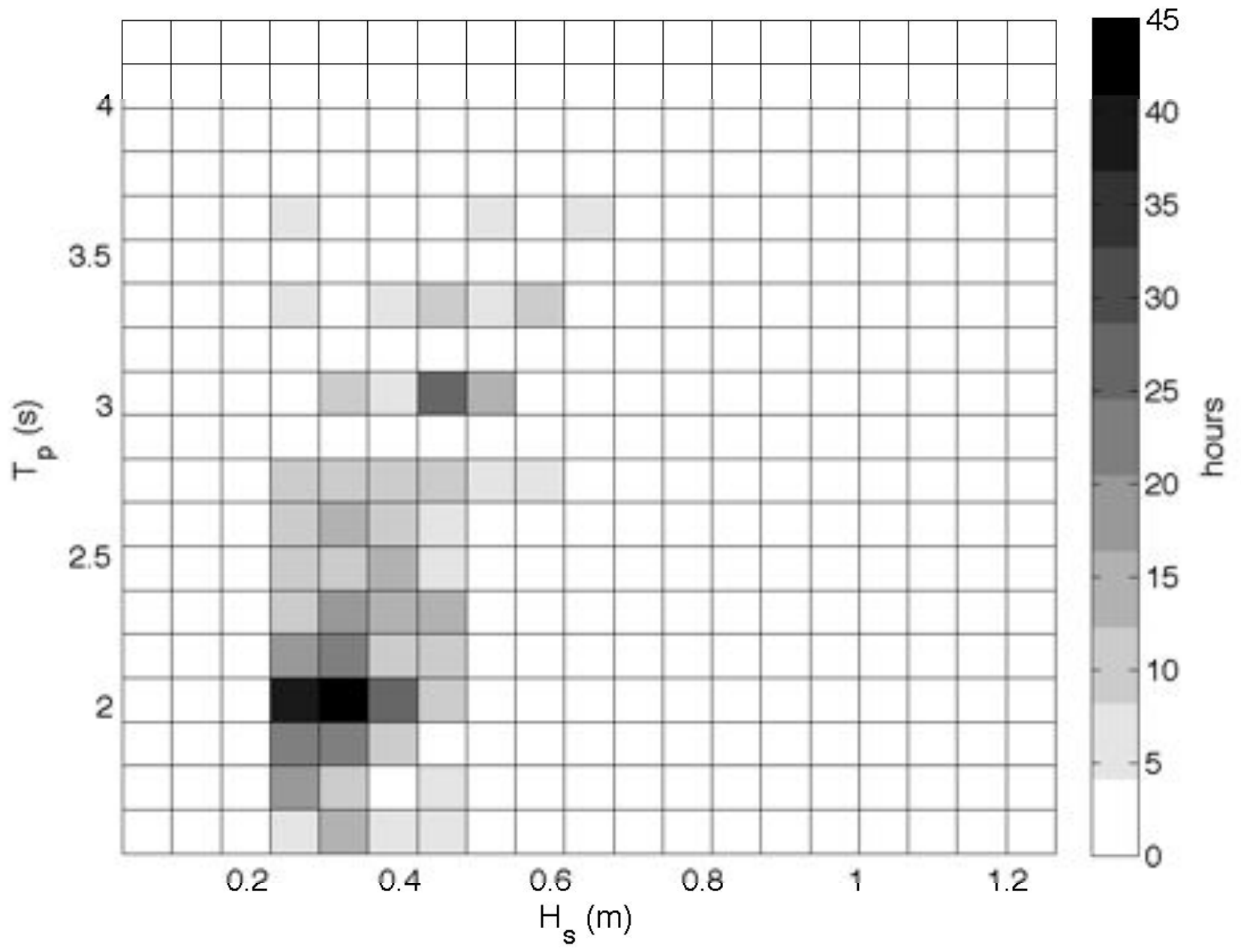

Figure 4: Joint histogram showing the hours of occurrence for wave heights at a given peak period. 


\subsection{Power density}

The energy flux $F$ of linear propagating waves is equivalent to the power per unit crest-length. For monochromatic waves, this is given by

$$
F=\frac{1}{8} \rho g H_{s}^{2} c_{g}
$$

where $g$ is gravity, $\rho$ is water density, and $c_{g}=\frac{g}{4 \pi f}$ is the group velocity at a given frequency according to deep-water wave dispersion ( $M e i, 1989)$.

For natural broad-band waves, a more accurate description of the energy flux is given by

$$
F=\frac{1}{8} \rho g \int_{f_{2}}^{f_{1}} S(f) c_{g} d f
$$

where $c_{g}$ varies with frequency inside the integral.

A shown in Figure 5, typical power densities on Puget Sound are less than $400 \mathrm{~W} / \mathrm{m}$, and the monochromatic estimate of energy flux is typically biased high by $45 \%$.

\subsection{Wave evolution}

At the onset of a wind event, waves are known to form first as small capillary waves and then grow in size and extent. These waves are initially quite steep, as quantified by $A k_{p}$, where $A=H_{s} / 2$ and $k_{p}$ is the wavenumber at the peak of the spectrum. At increased wave ages, estimated by the ratio of peak phase speed to wind speed $\frac{c_{p}}{U}$, wave steepness becomes limited. As shown in Thomson et al. (2009), this is likely a result of whitecaping, which limits the steepness of older waves to be less then $A k_{p} \approx 0.12$.

A simple energy budget for the evolution of total wave energy (thus neglecting nonlinear interactions between various components) is (Terray et al., 1996; Gemmrich et al., 1994)

$$
\rho g \int_{f_{2}}^{f_{1}} \frac{\partial S}{\partial t} d f=c_{e} \tau-\epsilon,
$$

where $c_{e} \tau / \rho$ is the energy input by the wind stress $\tau$ on a surface moving at an effective speed $c_{\text {eff }}$ and $\epsilon$ is dissipation due to whitecaping. Using the observed dependence of $\epsilon$ on wave steepness from Thomson et al. (2009), this energy budget is consistent with the observations.

\subsection{Climatology}

Local wind forcing conditions are compared with a climatology based on 24 years of wind observations at nearby West Point (NOAA station WPOW1, N 47.662 W 122.436) during winter months (Nov, Dec, Jan, Feb, Mar). Prior to comparison with climatology, the Point Wells wind data are corrected to the standard height of $10 \mathrm{~m}$, assuming neutral conditions (Large and Pond, 1981; Hoffman). 


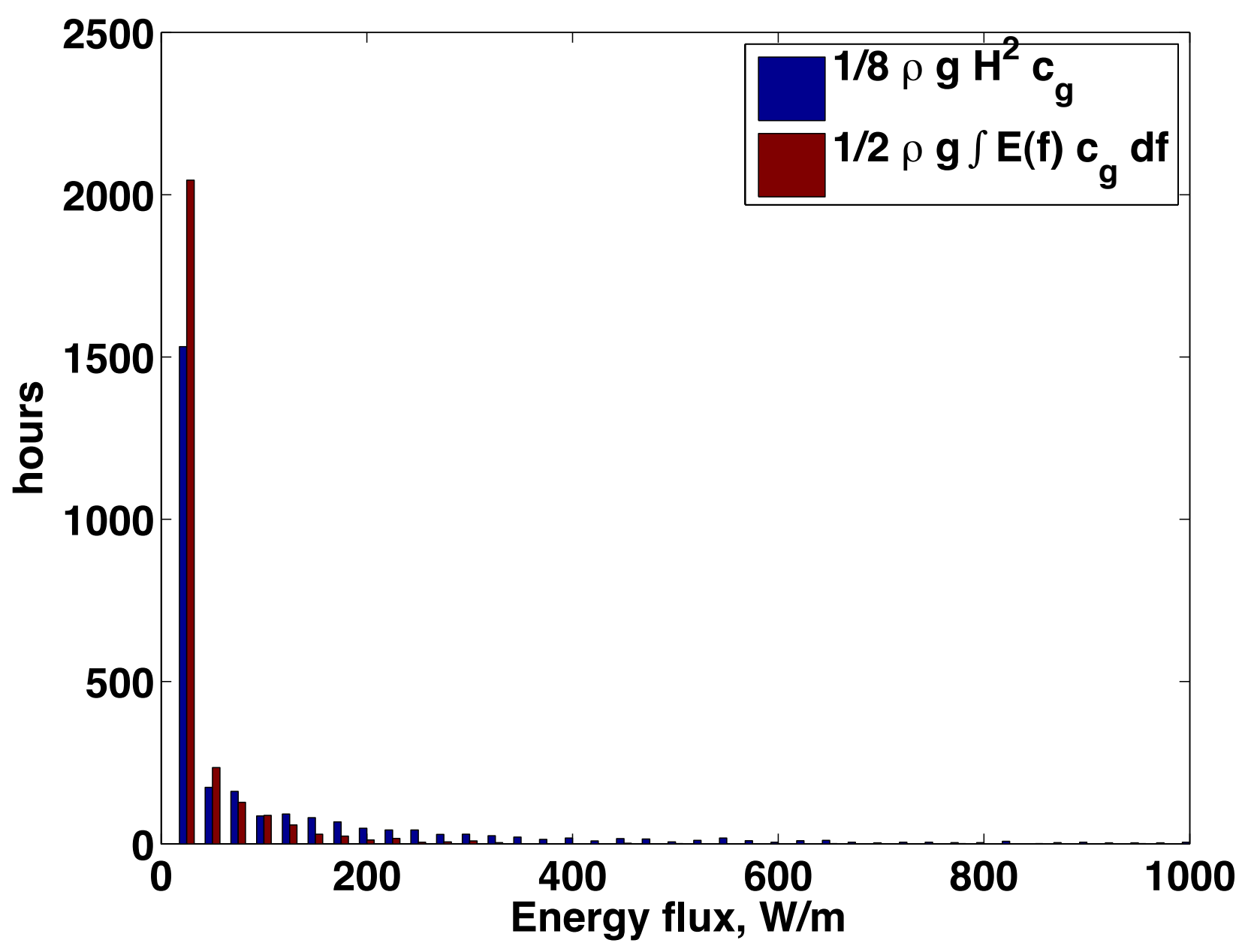

Figure 5: Histograms, by hour, of wave energy flux estimated from monochromatic values (blue) and from spectral energy densities (red). 


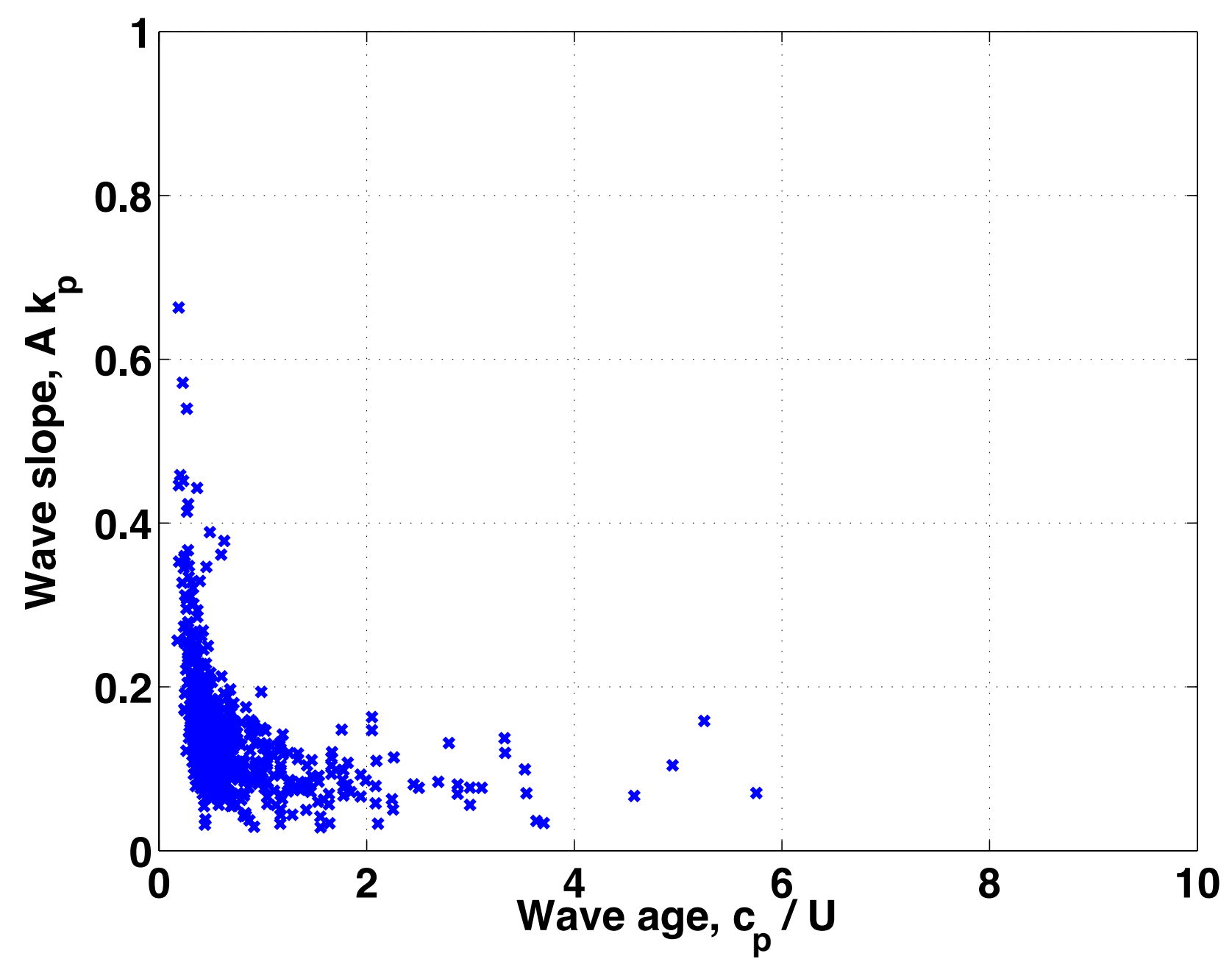

Figure 6: Hourly estimates of average wave slope versus wave age. Young waves are typically steep during strongly forced periods of growth, and then limited by whitecaping at later stages of development. 
As shown in Figure 7 the 2009-2010 data from Pt Wells are consistent with climatology, especially for higher winds, suggesting that the waves observed this winter are typical of Puget Sound. The details of wind direction and storm duration are absent from this comparison, but recent work (Pettersson, 2004) has described significant wave directionality in fjords.

\subsection{Wind-wave regression}

A multi-variate linear regression is used to form an empirical relation between the wind and wave observations. This relation can be used to extrapolate historical wave conditions from previous winters when only wind observations were recorded. These extrapolated wave values are much lower quality than the actual observed values, but are useful in confirming climatology. The resulting empirical prediction for significant wave height $(\mathrm{m})$ is

$$
H_{s}=0.04+0.0033 U_{10}^{2}+0.024 F+0.0016 D
$$

where $U_{10}$ is hourly mean wind speed at $10 \mathrm{~m}$ height $(\mathrm{m} / \mathrm{s}), F$ is fetch $(\mathrm{km})$ for a given wind direction, and $D$ is the duration of a wind event (hrs). The average residual (i.e., a measure of the error in the linear regression) in the $H-S$ regression is $0.07 \mathrm{~m}$. The resulting empirical prediction for energy period $(\mathrm{s})$ is

$$
T_{e}=1.9+2.1 H_{s}
$$

with an average residual of $0.5 \mathrm{~s}$.

\section{Model-data comparison}

As shown in Figure 8, numerical wave simulations provided by the US Geological Survey are consistent with the observations at Point Wells. In addition, the model output shows similar wave conditions between Point Wells (location of observations) and West Point (location of wind climatology). The model employed is SWAN (Simulating Waves Accurately Nearshore), which provides high spatial resolution wave height and period, as shown in Figure 9. 


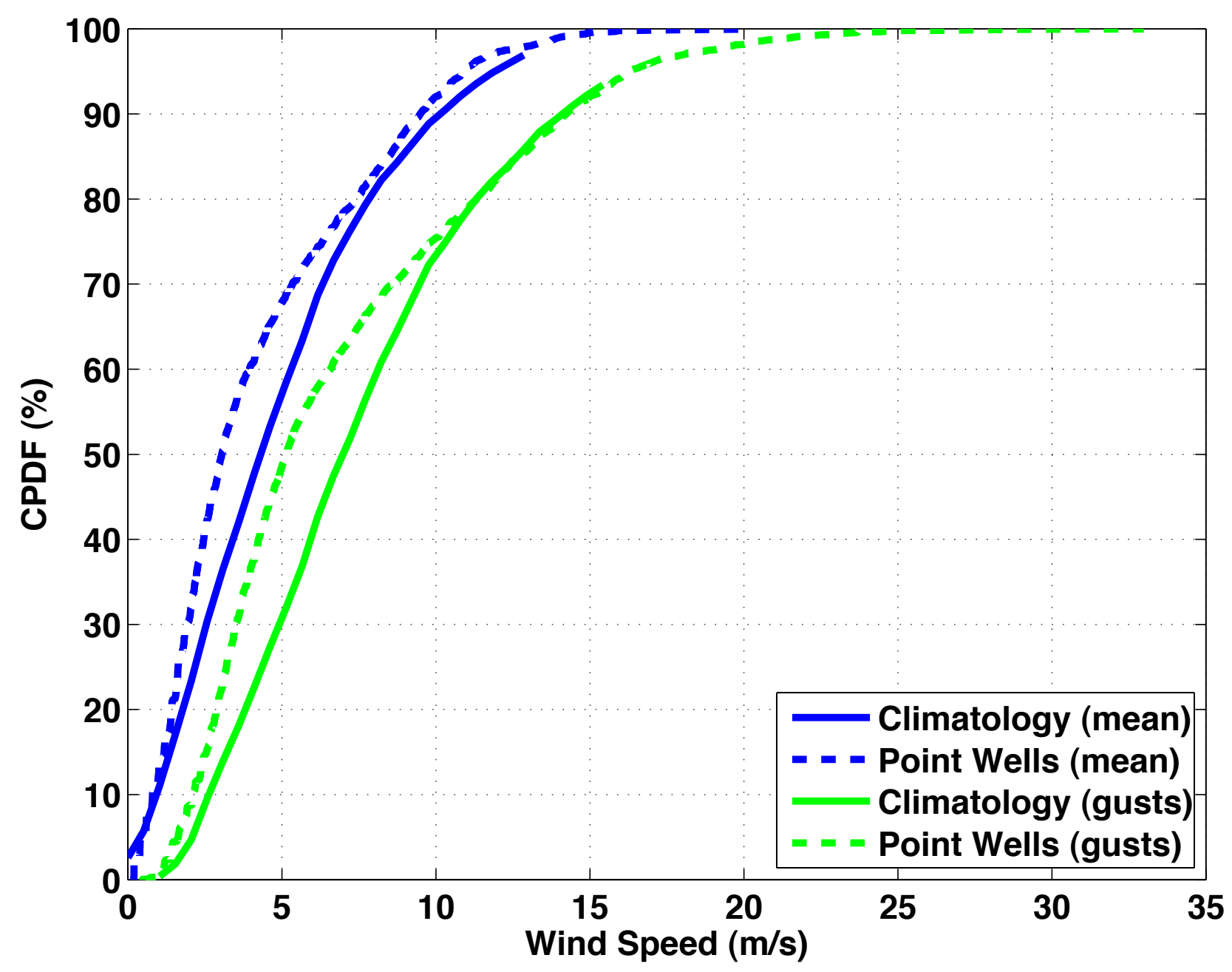

Figure 7: Cumulative probability distribution functions for hourly mean (blue) and gust (green) wind speeds during winter. Solid lines are a 24-year climatology from NOAA station WPOW1 at West Point, dashed lines are 2009-2010 data recorded a Point Wells. The distance between sites is $12 \mathrm{~km}$, and both are open to the predominantly southern winds.

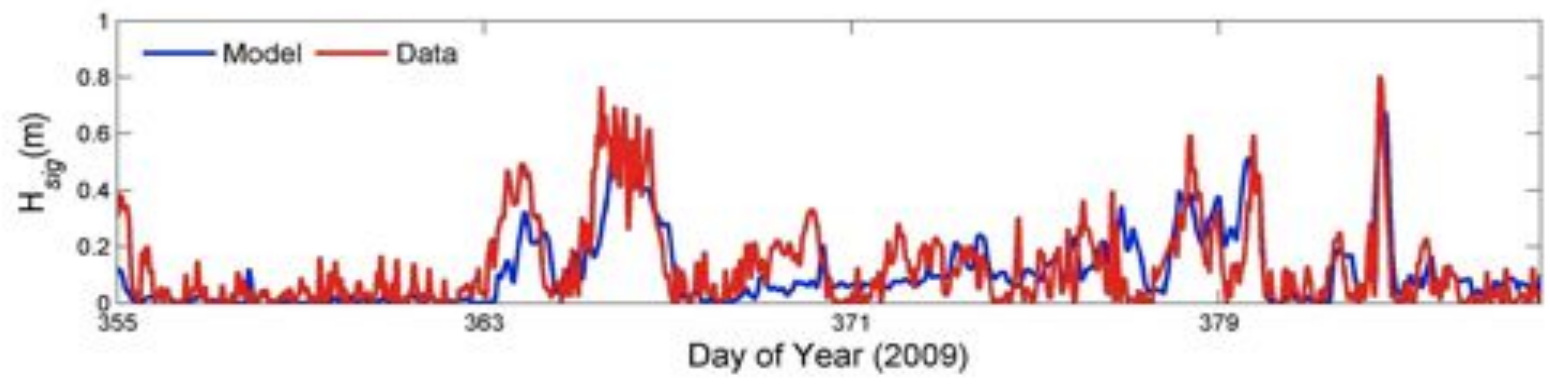

Figure 8: Comparison of modeled and observed wave heights. 

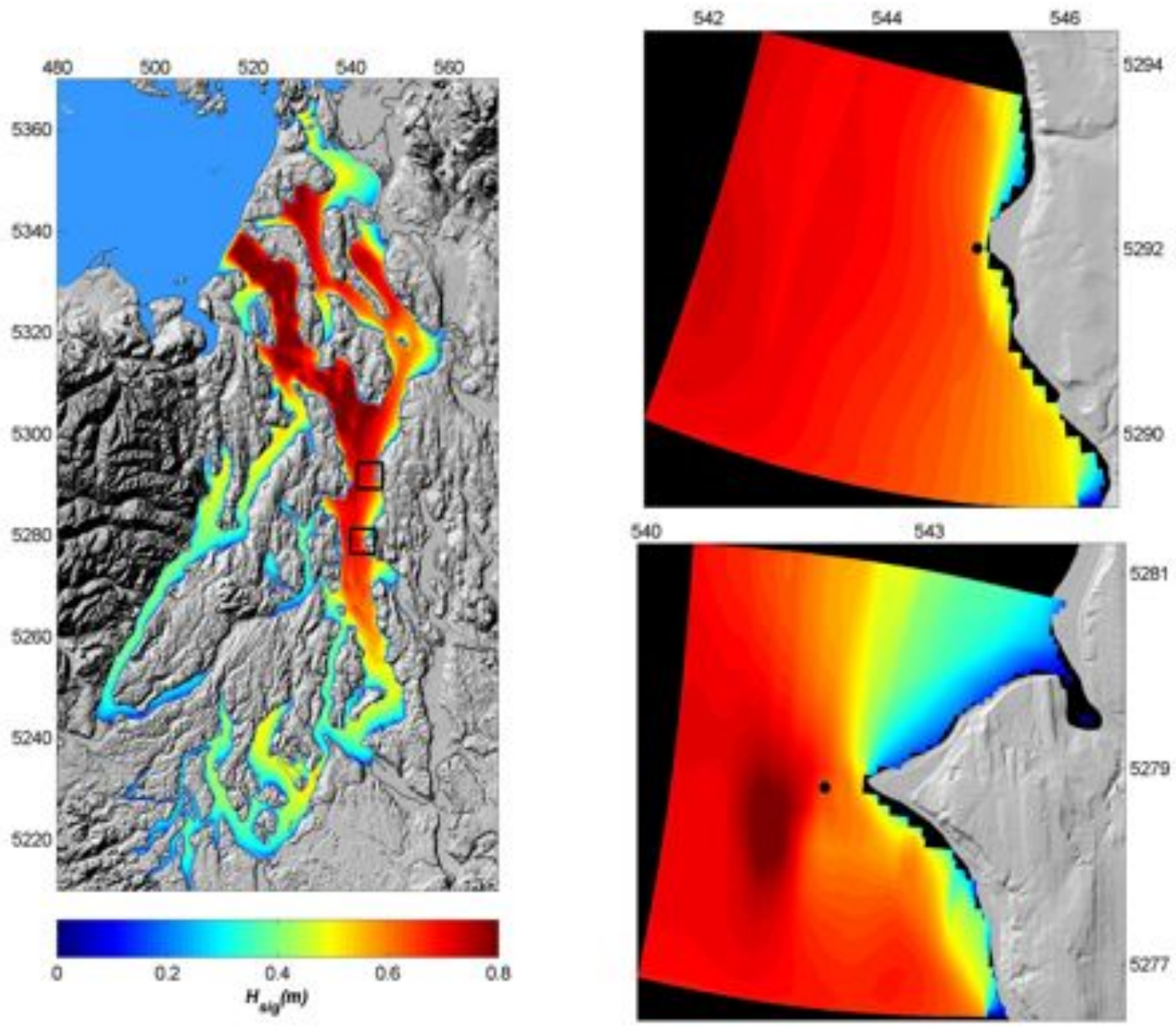

Figure 9: Spatial distribution of wave heights in Puget Sound (left) and detailed simulations for Point Wells (upper right) and West Point (lower right). Results are for an average winter storm. 


\section{References}

Atakturk, S. S., and K. B. Katsaros, Wind stress and surface waves observed on lake washington, J. Phys. Oceanogr., 29, 633-650, 1999.

Banner, M. L., Equilibrium spectra of wind waves, J. Phys. Oceanogr., 20, 966-984, 1990.

Curtiss, G., P. Osborne, and A. Horner-Devine, Seasonal patterns of coarse sediment transport on a mixed sand and gravel beach due to vessel wakes, wind waves, and tidal currents, Mar. Geo., 259, 73-85, 2009.

Gemmrich, J., Strong turbulence in the wave crest region, J. Phys. Oceanogr., 40, 583-595, 2010.

Gemmrich, J., T. Mudge, and V. Polonichko, On the energy input from wind to surface waves, J. Phys. Oceanogr., 24, 2413-2417, 1994.

Hoffman, R., Neutral stability height correction for ocean winds, http://sivo.gsfc.nasa.gov/oceanwinds/src/height-correct.pdf.

Large, W., and S. Pond, Open ocean momentum flux measurements in moderate to strong winds, J. Phys. Oceanogr., 11, 324-336, 1981.

Mei, C., The Applied Dynamics of Ocean Surface Waves, Advanced Series on Ocean Engineering, vol. 1, World Scientific, 1989.

Pettersson, H., Wave growth in a narrow bay, Ph.D. thesis, University of Helsinki, 2004.

Terray, E., M. Donelan, Y. Agrawal, W. Drennan, K. Kahma, A. Williams, P. Hwang, and S. Kitaigorodskii, Estimates of kinetic energy dissipation under breaking waves, J. Phys. Oceanogr., 26, 792-807, 1996.

Thomson, J., A. Jessup, and J. Gemmrich, Energy dissipation and the spectral distribution of whitecaps, Geophys. Res. Let., 36, 2009.

\section{Acknowledgments}

Joe Talbert contributed to field/deployment engineering. USGS provided SWAN model results and figures. Paramount Petroleum granted permission to work on the pier, in cooperation with the Brightwater project. Funded by Columbia Power Technologies, via DOE. 
Garrad Hassan America, Inc.

9665 Chesapeake Dr, Suite 435

Tel: +1 8588363370

San Diego, CA 92123, USA

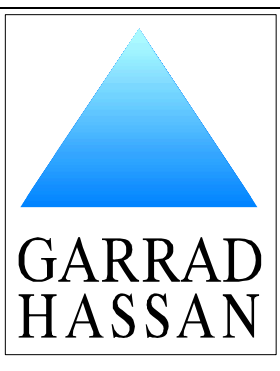

Document Details

\begin{tabular}{|l|l|}
\hline Title & Review of site data for CPT Puget Sound project \\
\hline Document & 41080AR01 \\
\hline Author & E Mackay \\
\hline Date & 21 May 2010 \\
\hline Rev & 3 \\
\hline Checked & J Cruz \\
\hline Approved & R I Rawlinson-Smith \\
\hline
\end{tabular}

\begin{tabular}{|c|c|c|}
\hline Iistory & & \\
\hline Rev & Date: & Summary \\
\hline 1 & 09 April 2010 & Initial draft \\
\hline 2 & 13 May 2010 & $\begin{array}{l}\text { Revised following CPT feedback \& updated to include hindcast } \\
\text { data }\end{array}$ \\
\hline 3 & 21 May 2010 & Final version (inc. client feedback) \\
\hline & & \\
\hline & & \\
\hline & & \\
\hline & & \\
\hline & & \\
\hline & & \\
\hline & & \\
\hline & & \\
\hline & & \\
\hline & & \\
\hline & & \\
\hline
\end{tabular}




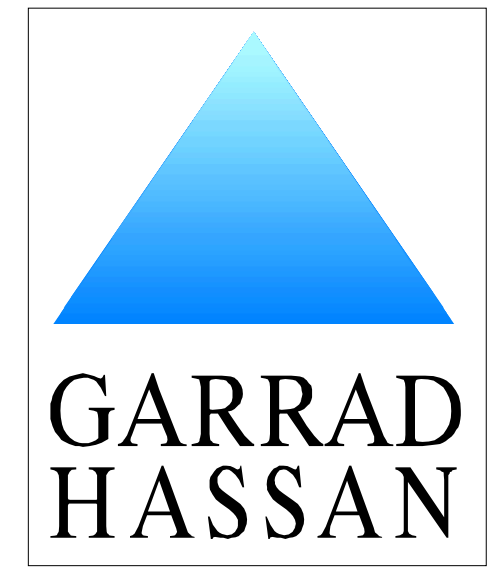

\section{REVIEW OF SITE DATA FOR CPT PUGET SOUND PROJECT}

Client

Contact

Document No

Issue

Status

Classification

Date
Columbia Power Technologies LLC

Ken Rhinefrank

41080AR01

$\mathrm{B}$

Revised draft

Client's Discretion

21 May 2010

Author:

E Mackay

Checked by:

J Cruz

Approved by:

R I Rawlinson-Smith 


\section{Important notice and disclaimer}

This Report shall be for the sole use of the Client for whom the Report is prepared. The document is subject to the terms of the Agreement between the Client and Garrad Hassan America, Inc. and should not be relied on by third parties for any use whatsoever without the express written authority of Garrad Hassan America, Inc. The Report may only be reproduced and circulated in accordance with the Document Classification and associated conditions stipulated in the Agreement, and may not be disclosed in any offering memorandum without the express written consent of Garrad Hassan America, Inc.

Garrad Hassan America, Inc. does not provide legal, regulatory, tax and/or accounting advice. The recipient must make its own arrangements for advice in these areas.

This document has been produced from information at the date of this document and, where applicable, information relating to dates and periods referred to in this document. The Report is subject to change without notice and for any reason including, but not limited to, changes in information, conclusion and directions from the Client.

\section{Key To Document Classification}

$\begin{array}{lll}\text { Strictly Confidential } & : & \text { Recipients only } \\ \text { Private and Confidential } & : & \begin{array}{l}\text { For disclosure to individuals directly } \\ \text { concerned within the recipient's } \\ \text { organization }\end{array} \\ \text { Commercial in Confidence } & : & \begin{array}{l}\text { Not to be disclosed outside the recipient's } \\ \text { organization }\end{array} \\ \text { GH only } & : \quad \begin{array}{l}\text { Not to be disclosed to non GH staff and } \\ \text { affiliates }\end{array} \\ \text { Client's Discretion } & : \quad \begin{array}{l}\text { Distribution at the discretion of the client } \\ \text { subject to contractual agreement }\end{array} \\ \text { Published } & : \quad \text { Available to the general public }\end{array}$

(C) 2010 Garrad Hassan America, Inc. 


\section{Revision History}

\begin{tabular}{|c|c|l|}
\hline Issue & Issue Date & \multicolumn{1}{c|}{ Summary } \\
\hline $\mathrm{A}$ & $13 / 05 / 10$ & Draft release - electronic only \\
\hline $\mathrm{B}$ & $21 / 05 / 10$ & Final release - including client feedback \\
\hline
\end{tabular}

\begin{tabular}{|c|c|}
\hline Distribution: & Copy No: \\
\hline Client & 1 \\
\hline GH Portland & 2 \\
\hline GH Bristol & 3 \\
\hline GH Portugal & 4 \\
\hline
\end{tabular}

Copy No: 


\section{Table of Contents}

1 INTRODUCTION

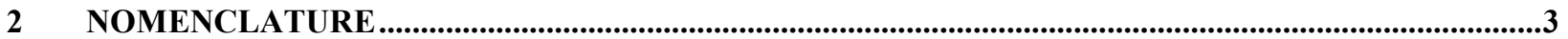

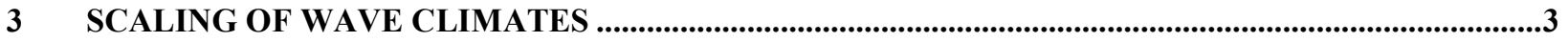

4 ANALYSIS OF POINT WELLS WAVE MEASUREMENTS ....................................................................

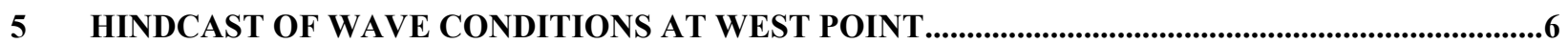

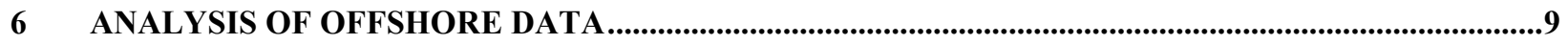

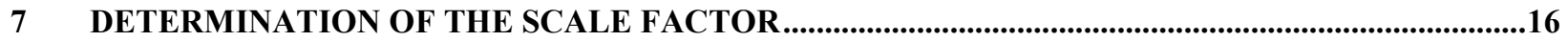

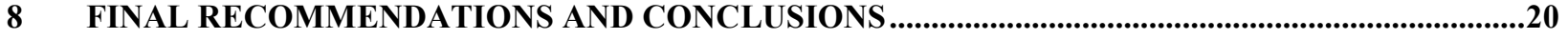

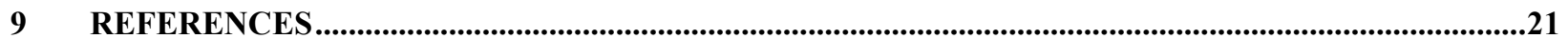




\section{INTRODUCTION}

Columbia Power Technologies LLC (CPT) has contracted Garrad Hassan America (GH) to examine the wave climate for the West Point site in Puget Sound where a scale model wave energy converter (WEC) will be deployed. CPT has provided GH with wave measurements made at Point Wells, a site approximately $14 \mathrm{~km}$ north of West Point. This data has been compared with wave measurements from buoys located in the North Pacific off the coast of Oregon to obtain an approximate scale factor for the site.

\section{NOMENCLATURE}

$\begin{array}{ll}H & \text { Wave height } \\ \lambda & \text { Wave length } \\ f & \text { Wave frequency } \\ g & \text { Acceleration due to gravity } \\ S(f) & \text { Variance density spectrum } \\ m_{n}=\int_{0}^{\infty} S(f) d f & n^{\text {th }} \text { spectral moment } \\ H_{s}=4 \sqrt{m_{0}} & \text { Significant wave height } \\ T_{e}=m_{-1} / m_{0} & \text { Energy period } \\ T_{m}=m_{0} / m_{1} & \text { Mean period } \\ T_{e}=\sqrt{m_{0} / m_{2}} & \text { Zero-crossing period } \\ S=2 \pi H_{s} / g T_{z}^{2} & \text { Significant steepness } \\ U_{10} & \text { Wind speed at 10m above sea level } \\ X & \text { Fetch }\end{array}$

\section{SCALING OF WAVE CLIMATES}

Scale testing of WECs is conducted according to Froude scaling laws. This ensures that scale tests are geometrically, kinematically and dynamically similar to full scale conditions. Under Froude scaling laws time scales with the square root of length. For example a full-scale sea state with $H_{s}=3 \mathrm{~m}$ and $T_{e}=10 \mathrm{~s}$ would be equivalent to a $5^{\text {th }}$ scale sea state with $H_{s}=3 / 5=0.6 \mathrm{~m}$ and $T_{e}=10 / \sqrt{5}=4.47 \mathrm{~s}$.

In deep water the ratio between wave length and period is given by $\lambda=g T^{2} / 2 \pi$. So scaling wave period with the square root of wave length ensures that this ratio remains valid at scale, satisfying the requirement for geometric similarity, i.e. wave steepness is invariant with scale.

There are no fixed rules about how to calculate a scale factor for the wave climate at a test site. In general it is unlikely that the wave climate at a test site will be an exact scale representation of full scale conditions, due to differences in the storm characteristics over the fetches that each site is exposed to. Determining a scale factor for a site is therefore somewhat subjective and will depend on the sea states which are of interest. If the crucial criterion is the extreme wave conditions to which the scale WEC is exposed, then the scale factor for the site may be determined by the ratio of the return values at the two sites. CPT has advised that the test buoy will be designed to survive all possible wave climates at West 
Point and will not be at risk of damage; therefore extreme waves are not used to limit considerations of scale in this report.

Since extreme conditions are not considered critical at this location, it may be advantageous to choose a scale factor so that the scale wave climate is marginally more energetic than the anticipated full scale site, so that there is a greater chance of higher-energy sea states occurring during testing. This will result in a greater proportion of time when tests of real interest can be conducted. In terms of device performance (as opposed to survivability) the most important tests to conduct are those which correspond to the conditions which represent the highest fraction of the available wave energy. For example if the full-scale WEC is to be deployed in an area where $90 \%$ of the available wave energy occurs in sea states with $H_{s}$ in the range $2 \mathrm{~m}-6 \mathrm{~m}$, then it would be advisable to choose the scale factor so that there is a high likelihood of these conditions occurring during the scale model deployment.

GH recommends that the criteria which should be used to determine the scale factor for the site are the frequencies of occurrence of scaled $H_{s}$ at various levels. This will inform how much data is likely to be collected for each sea state. Although the wave period also has a significant effect on the device response, it is not possible to scale the period independently of the wave height (since steepness is invariant with scaling), therefore only $H_{s}$ is used to determine the scale factor.

\section{ANALYSIS OF POINT WELLS WAVE MEASUREMENTS}

Wave measurements have been conducted by APL at Point Wells, approximately $13 \mathrm{~km}$ north of the proposed deployment site at West Point. The measurements cover the period 20 Nov. 2009 - 1 April 2010. The measurements were made using an acoustic wave sensor located at the end of a pontoon with the following specifications:

Resolution: $0.1 \mathrm{~cm}$,

Accuracy: $\pm 0.05 \mathrm{~cm}$

Sampling frequency: $1.7 \mathrm{~Hz}$,

Sample length: $20 \mathrm{~min} /$ hour.

Sea states with $H_{s}<20 \mathrm{~cm}$ are below the noise level of the sensor and have been excluded from the analysis. Figure 4.1 shows the mean spectral shape measured at Point Wells. It appears that noise is a problem for frequencies below $0.1 \mathrm{~Hz}$ and that the sampling frequency is too low to accurately measure the high frequency tail of the spectrum (for a sampling frequency of $1.7 \mathrm{~Hz}$ the Nyquist frequency is 0.85 $\mathrm{Hz}$ ). Both these factors can cause estimates of wave periods to be biased high, especially $T_{z}$ which is more sensitive to the energy in the high frequency end of the spectrum. A lower limit of $0.1 \mathrm{~Hz}$ has been used in the calculation of the spectral moments, from which the wave parameters are derived, but no correction has been made for the high-frequency cut-off. The effect of neglecting energy at high frequencies can be gauged by considering standard spectral shapes. For a Bretschneider spectrum with peak frequency of $0.4 \mathrm{~Hz}$, curtailing the spectrum at $0.7 \mathrm{~Hz}$ will result in a bias of $7 \%$ in $T_{e}$ and $20 \%$ in $T_{z}$. The bias in wave steepness is even larger, since it depends on the square of $T_{z}$.

The high frequency waves which were not measured by the acoustic wave sensor are not likely to affect the response of the model. However, it is important to obtain accurate measurements of period parameters to validate machine performance. In deep water the level of non-linearity is mainly controlled by the steepness of the waves, so using biased estimates of steepness may impair the comparisons of physical and numerical models. To obtain accurate wave data when testing the scaled model at West Point, GH would recommend the following wave measurement device specifications: 
- Sample Frequency: $4 \mathrm{~Hz}$ or above

- Range: $\pm 3 \mathrm{~m}$ or above

- Accuracy: $<1 \mathrm{~cm}$

- Resolution: $<1 \mathrm{~cm}$

- Data collection: Continuous with records analyzed in 60 minute blocks

GH attempted to mitigate for the effect of the high-frequency cut-off by fitting a high-frequency tail to the spectra. However, the individual spectra were extremely noisy and did not display standard shapes, so it was not possible to fit a reasonable looking tail. Since $H_{s}$ will be the only parameter used to determine the scale factor for the site, the bias in the period parameters and wave steepness is not critical.

Figure 4.2 shows scatter plots of $H_{s}$ against $T_{e}$ and $H_{s}$ against significant steepness (a measure of the average steepness of the waves). For offshore wave measurements a limiting significant steepness of around 0.09 is commonly observed (see Section 4). The maximum significant steepness observed in the Point Wells data is 0.11 which is high, especially considering that this is likely to be an underestimate due to the high-frequency cut-off. The tidal range at the site is almost $5 \mathrm{~m}$ so there is a possibility of strong currents, which may be responsible for increasing the wave steepness. CPT has also noted that these high steepness events may be a result of large and steep waves generated by passing ships which have not been filtered out of the analysis.

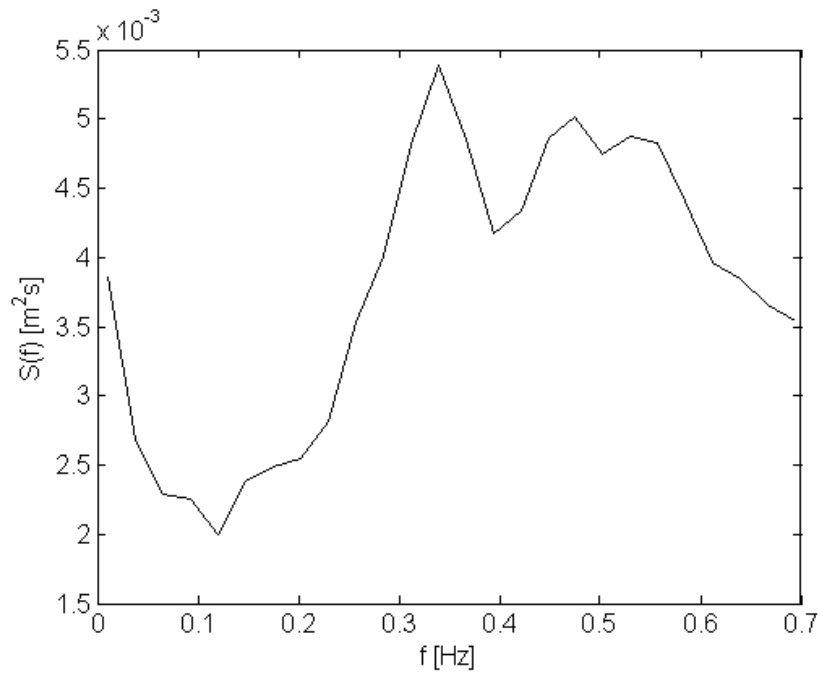

Figure 4.1. Mean spectral shape measured at Point Wells. 

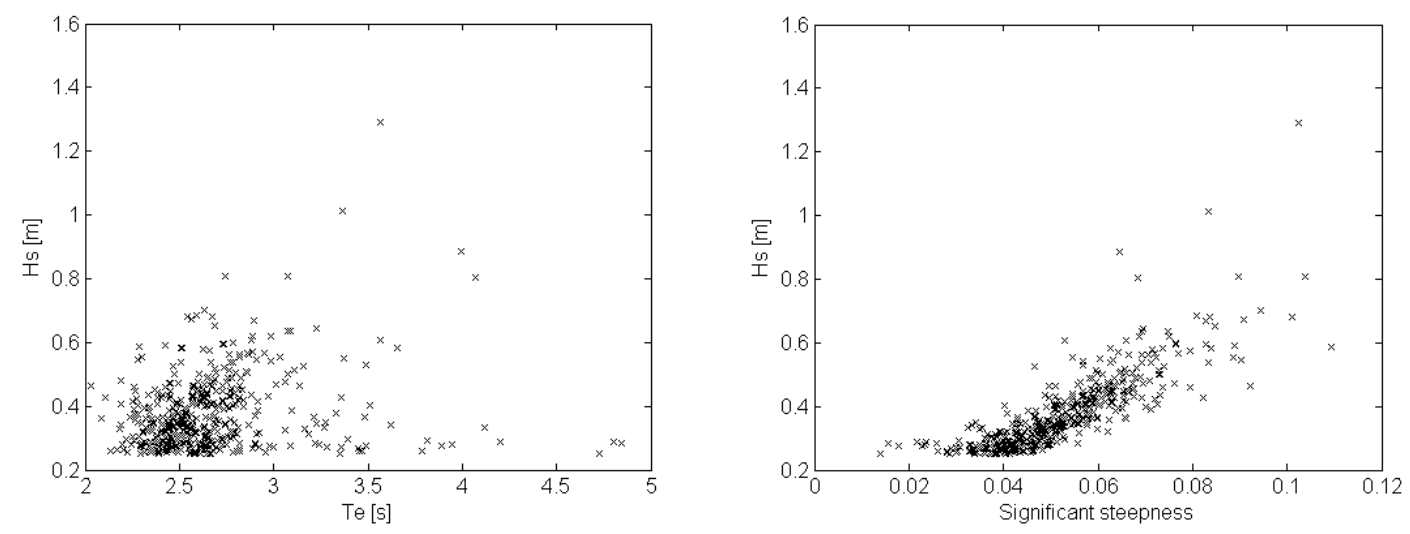

Figure 4.2. Scatter plots of $H_{s}$ against $T_{e}$ and $H_{s}$ against significant steepness

for the Point Wells wave measurements.

\section{HINDCAST OF WAVE CONDITIONS AT WEST POINT}

The wind and wave conditions measured at Point Wells have also been used to determine a relationship between wind speed, fetch and wave height in Puget Sound, from which the long term conditions can be estimated. The procedure has two steps:

1. Estimate relationship between wind speed, fetch and wave height at Point Wells.

2. Apply this relationship to wind data recorded at West Point to estimate long-term wave conditions.

The wind speed measurements at Point Wells and West Point were made using anemometers at different heights. To ensure the relationship between wind speed, fetch and wave height is valid for both locations, both sets of wind data have been adjusted to the same reference level. The anemometer at Point Wells is located $7.5 \mathrm{~m}$ above mean lower low water (MLLW). APL have calculated $U_{10}$, the wind speed at $10 \mathrm{~m}$ above sea level, accounting for the tide, although it is not known what formula has been used. The anemometer at West Point is located $9.8 \mathrm{~m}$ above site elevation, and the site is $3.0 \mathrm{~m}$ above mean water level. GH has estimated $U_{10}$ for West Point under the assumption of neutral atmospheric stability, using the formula [1]:

$$
\frac{U\left(z_{0}\right)}{U\left(z_{r}\right)}=\left(\frac{z_{0}}{z_{r}}\right)^{\alpha}
$$

where $z_{0}$ is the height at which the measurements are made, $z_{r}$ is the reference height and $\alpha=0.11$, a figure typically used for offshore conditions.

The fetches at Point Wells and West Point for various directions have been estimated using Google Earth and are displayed in Figure 5.1.

A formula which is often used to estimate $H_{s}$ under fetch limited conditions is [2]:

$$
H_{s}=0.016 X^{0.5} U_{10}
$$



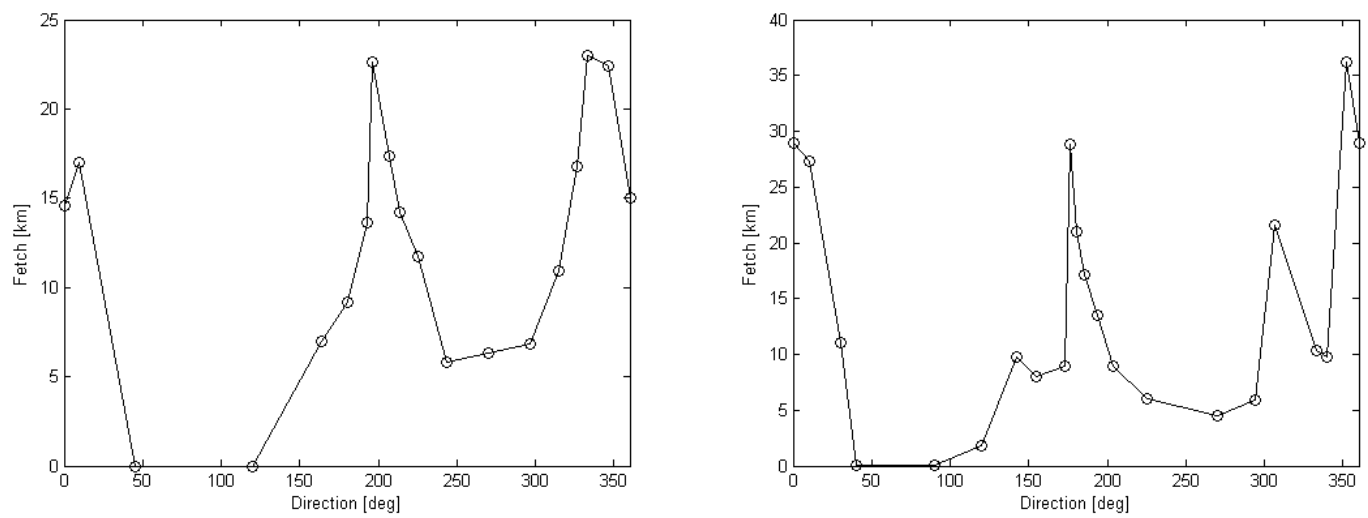

Figure 5.1 Estimated fetch against direction for Point Wells (left) and West Point (right).

The formula was derived from data obtained during the JONSWAP experiment in the North Sea. To test the applicability of the formula under the much shorter fetches in Puget Sound, a comparison of the wind and wave data recorded at Point Wells has been made. Orthogonal regression has been used to determine a linear relationship between $H_{s}$ and $X^{0.5} U_{10}$ in Puget Sound. Orthogonal regression finds the line which minimises the orthogonal distances between the data points and regression line. It differs slightly from ordinary least-squares regression which minimises the vertical distances between the data points and regression line, which in effect assigns all the errors to the ordinate. In contrast, orthogonal regression accounts for errors in both data sets and gives a better approximation of the underlying relationship (for more information see e.g. [3]).

Figure 5.2 shows an orthogonal regression of $H_{s}$ against $X^{0.5} U_{10}$, with the estimated parameters shown above the plot. The correspondence is reasonable, with a correlation coefficient of 0.77 . The standard deviation of the residuals about the regression line is shown in the right hand plot of Figure 5.2. The standard deviation increases approximately linearly with $X^{0.5} U_{10}$ due, in part, to the increase in sampling variability in both $H_{s}$ and $U_{10}$. The distribution of the residuals, normalised by the standard deviation, is shown in Figure 5.3. The distribution is well fitted by a Student-t distribution with 5 degrees of freedom. This gives the following model for $H_{s}$ :

$$
H_{s}=0.03+0.0099 X^{0.5} U_{10}+(0.075+0.016) X^{0.5} U_{10} \varepsilon
$$

where $\varepsilon$ is a random Student-t variable. The inclusion of the Student-t variable in the model accounts for the observed variability of the data about the regression line, evident in Figure 5.2. 

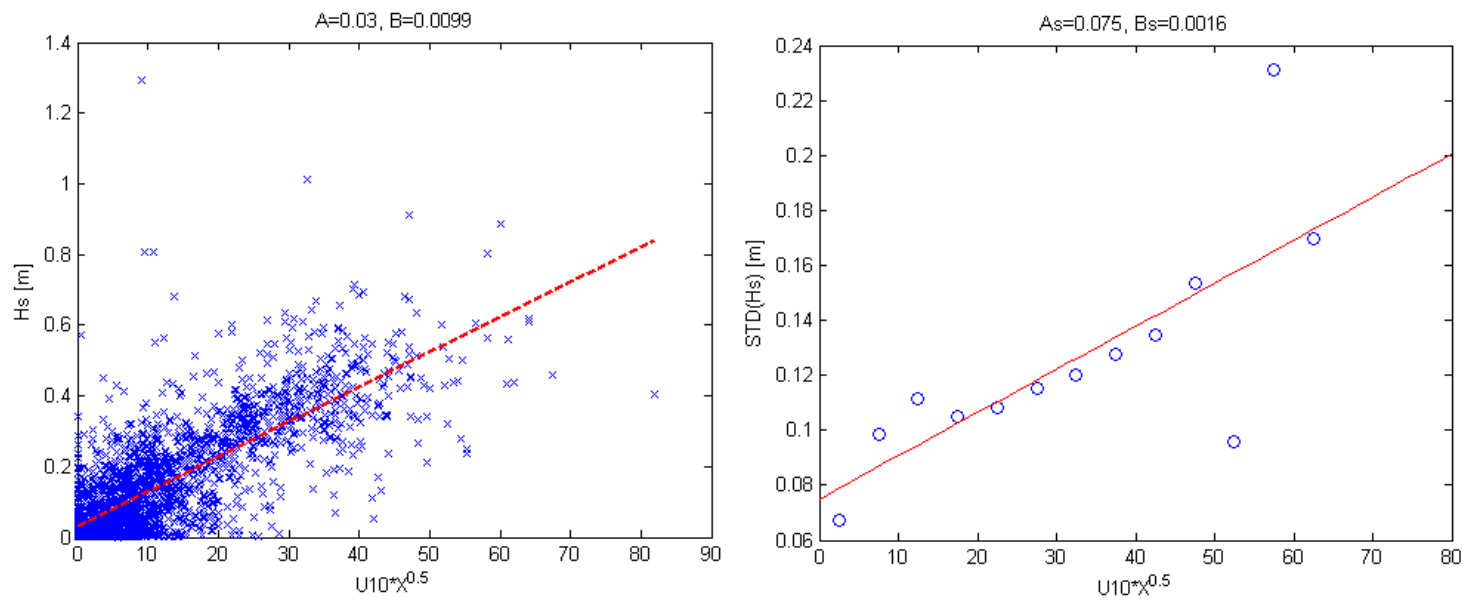

Figure 5.2. Left: Orthogonal regression of $H_{s}$ against $X^{0.5} U_{10}$. Right: Standard deviation of residuals.

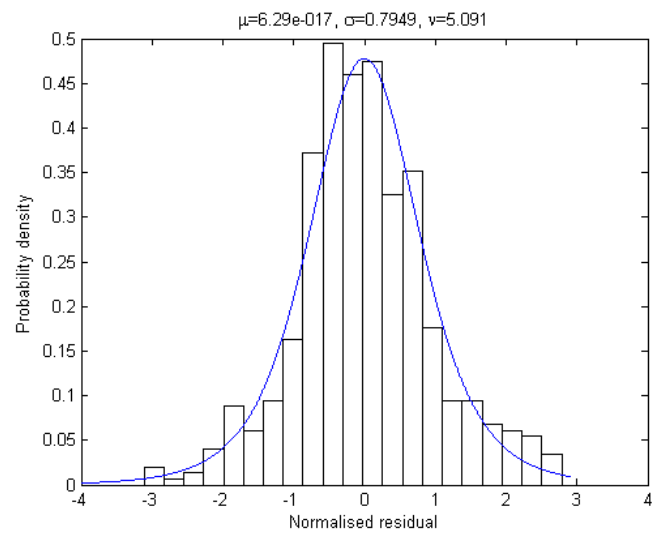

Figure 5.3. Histogram of normalised residuals and fitted Student's-t distribution.

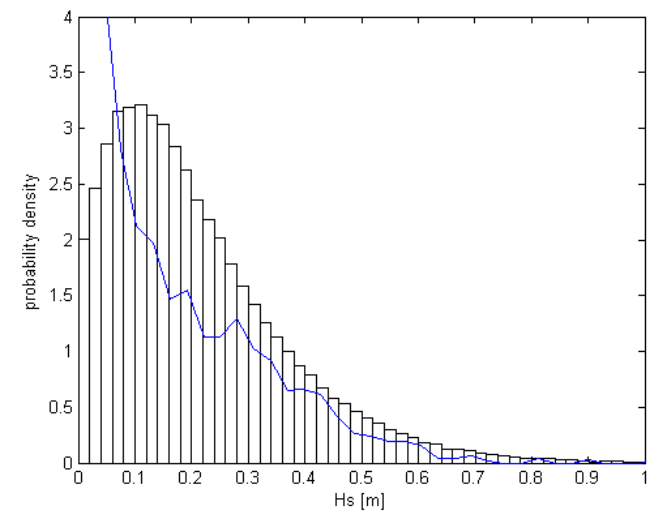

Figure 5.4. Distribution of $H_{s}$. Histogram: derived using Eq. (2). Line: Measured at Point Wells. 


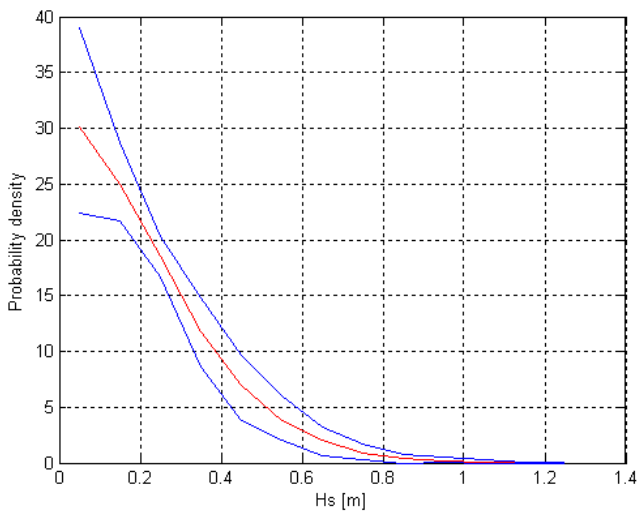

Figure 5.5. Distribution of Hs over period October-April.

Red line: mean value, blue lines: maximum and minimum values for individual years.

The relationship presented in Eq. (3) has been applied to the wind data recorded at West Point to obtain an estimate of the long-term wave conditions. Wind measurements at West Point cover the period 29 Jan. 1984 to 31 Dec 2009 at hourly intervals. $H_{s}$ has been estimated using Eq. (3), with values of $\varepsilon$ generated as random Student-t variables (records which result in $H_{s}<0$ are discarded). The inclusion of a random variable in the hindcast makes each realisation different, but has negligible effect on the long term statistics. Figure 5.4 shows a histogram of occurrence of $H_{s}$ over the entire year, together with the distribution measured at Point Wells. It is clear that there is some discrepancy in the two distributions. This is possibly due to a difference in the wind regime at the two sites, but may also be a result of differing methods used to calculate $U_{10}$ at the two locations.

Since the hindcast displays a different distribution to the measurements, GH would advise that the measurements are used to determine the scale factor. Since the measurements display a lower occurrence of higher sea states than the hindcast, using them to determine the scale factor will result in a lower estimate of the scale factor, but a higher frequency of occurrence of higher energy sea states.

Although the hindcast shows discrepancies with the measurements, it can still be used to estimate the level of interannual variability in the wave conditions. Figure 5.5 shows the distribution of $H_{s}$, over the period October-April together with the maximum and minimum values for individual years. There is relatively little interannual variability in the occurrence of the lower sea states with $H_{s}<0.4 \mathrm{~m}$. However the occurrence of sea states with $H_{s}>0.6 \mathrm{~m}$ can change by as much as $50 \%$ from year to year.

\section{ANALYSIS OF OFFSHORE DATA}

The scale factor for the West Point test site is determined relative to the wave conditions off the coast of Oregon. There are several long datasets for this area from buoys operated by the National Data Buoy Centre (NDBC). These measurements have been downloaded from the National Oceanographic Data Centre (NODC) FTP site ${ }^{1}$. Details of the buoys selected for the analysis are listed in Table 6.1 and their locations are shown in Figure 6.1. The buoys selected are all located on the continental shelf and have record lengths upwards of 5 years.

Figures 6.2-6.7 show the joint distribution of $H_{s}$ and $T_{e}$ and the joint distribution of $H_{s}$ and $s$ for the six buoys considered in the study. Generally, the distributions display similar shapes, since each buoy has a

${ }^{1} \mathrm{ftp}: / / \mathrm{ftp}$. nodc.noaa.gov/pub/f291/

Garrad Hassan America, Inc

9 of 21

GARRAD


similar exposure to the North Pacific. The distribution for buoy 46027 shows a reduction in the occurrence of large and steep sea states compared to the other buoys. This is most likely a consequence of being the located in a marginally more sheltered location, further south than the other buoys. The steepest waves were recorded by buoy 46010 in April 1981, but there are no concurrent measurements from nearby buoys covering this period, which can be used to validate these measurements. Visual inspection of the time series and individual spectra do not show any obvious errors.

As well as the occurrence of various sea states it is important to quantify which sea states represent the highest proportion of the available wave energy. Figures 6.8-6.11 show a comparison between the distribution of occurrence of sea states and the proportion of the total energy which they account for, using data from Buoy 46029. Figure 6.8 shows the distribution binned by both $H_{s}$ and $T_{e}$, Figure 6.9 shows the distributions binned by $H_{s}$ only, Figure 6.10 shows the distributions binned by $T_{e}$ only, and Figure 6.11 shows the distributions binned by significant steepness only. These distributions are also presented numerically in Tables 6.2 and 6.3. It can be seen that although $90 \%$ of the sea states have $H_{s}<4 \mathrm{~m}$, this accounts for only $60 \%$ of the available energy. Approximately $75 \%$ of the total energy occurs in sea states with $H_{s}$ between $2 \mathrm{~m}$ and $6 \mathrm{~m}$, and $90 \%$ of the energy occurs in seas with $T_{e}$ between $6 \mathrm{~s}$ and $14 \mathrm{~s}$. The scale factor for the model to be deployed at West Point should be chosen so that there is a sufficient probability of occurrence of scaled equivalents of these sea states.

\begin{tabular}{|c|c|c|c|c|c|c|c|c|}
\hline $\begin{array}{c}\text { Buoy } \\
\text { number }\end{array}$ & $\begin{array}{c}\text { Buoy } \\
\text { type }\end{array}$ & $\begin{array}{c}\text { Latitude } \\
{\left[{ }^{\circ} \mathbf{N}\right]}\end{array}$ & $\begin{array}{c}\text { Longitude } \\
{\left[{ }^{\circ} \mathbf{W}\right]}\end{array}$ & $\begin{array}{c}\text { Water } \\
\mathbf{d e p t h} \\
{[\mathbf{m}]}\end{array}$ & $\begin{array}{c}\text { Start } \\
\text { date }\end{array}$ & $\begin{array}{c}\text { End } \\
\text { date }\end{array}$ & $\begin{array}{c}\text { Max. } \boldsymbol{H}_{\boldsymbol{s}} \\
{[\mathbf{m}]}\end{array}$ & $\begin{array}{c}\text { Max. } \\
\text { steepness }\end{array}$ \\
\hline 46010 & $\begin{array}{c}10 \mathrm{~m} \\
\text { discus }\end{array}$ & 46.2 & 124.2 & 64 & $11 / 1979$ & $04 / 1991$ & 10.2 & 0.091 \\
\hline 46015 & $\begin{array}{c}3 \mathrm{~m} \\
\text { discus }\end{array}$ & 42.75 & 124.82 & 422 & $07 / 2002$ & $11 / 2009$ & 11.9 & 0.080 \\
\hline 46027 & $\begin{array}{c}3 \mathrm{~m} \\
\text { discus }\end{array}$ & 41.85 & 124.38 & 48 & $09 / 1983$ & $11 / 2009$ & 9.96 & 0.074 \\
\hline 46029 & $\begin{array}{c}3 \mathrm{~m} \\
\text { discus }\end{array}$ & 46.14 & 124.51 & 135 & $03 / 1984$ & $11 / 2009$ & 13.8 & 0.082 \\
\hline 46040 & $\begin{array}{c}3 \mathrm{~m} \\
\text { discus }\end{array}$ & 44.8 & 124.3 & 112 & $05 / 1987$ & $06 / 1992$ & 11.7 & 0.083 \\
\hline 46050 & $\begin{array}{c}3 \mathrm{~m} \\
\text { discus }\end{array}$ & 44.64 & 124.5 & 123 & $11 / 1991$ & $11 / 2009$ & 14.1 & 0.082 \\
\hline
\end{tabular}

Table 6.1. Details of wave buoys shown in Figure 6.1. 
Review of site data for CPT Puget Sound Project

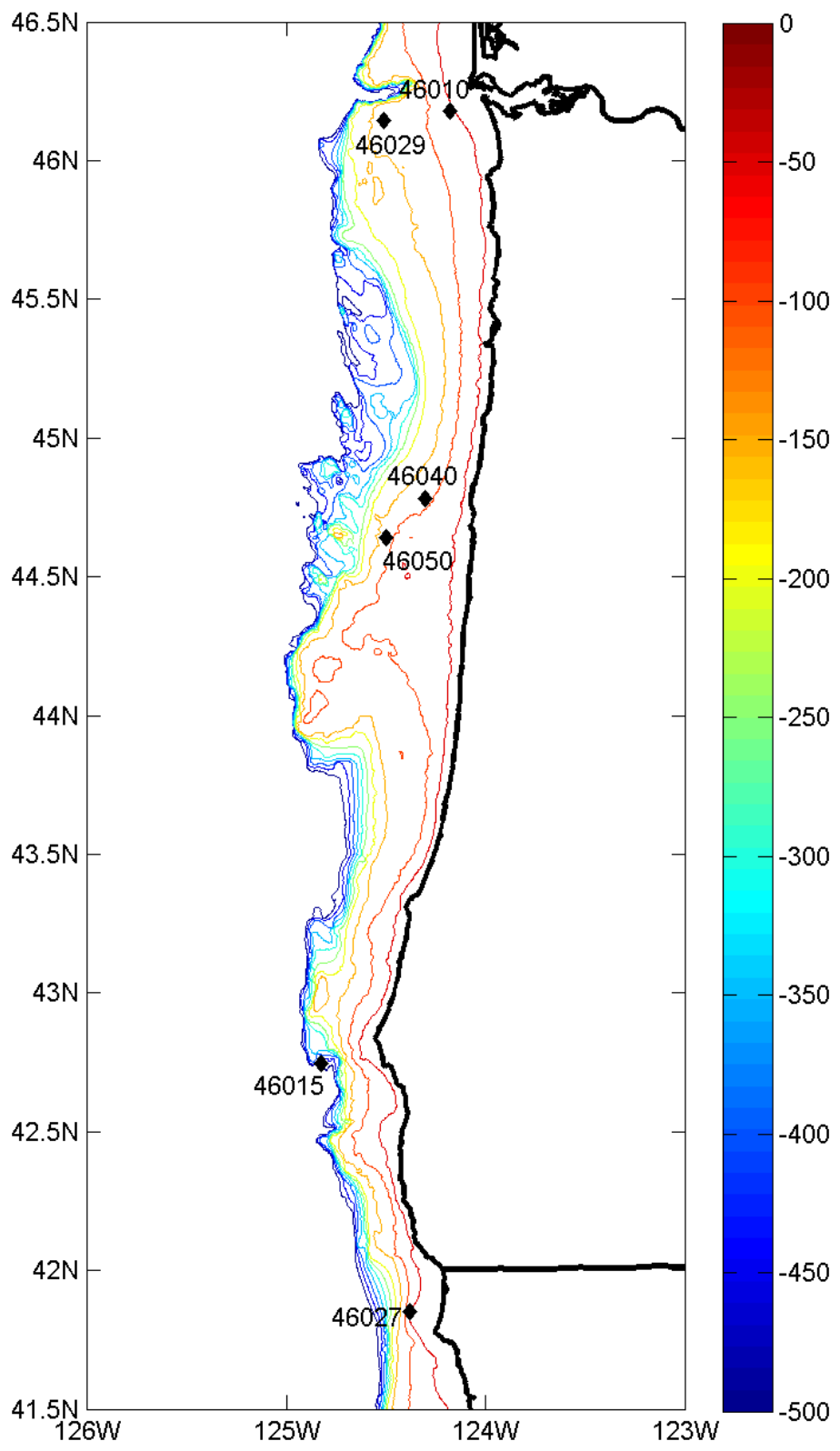

Figure 6.1. Locations of NDBC wave buoys considered in this report. Coloured contours show bathymetry at $50 \mathrm{~m}$ intervals. 

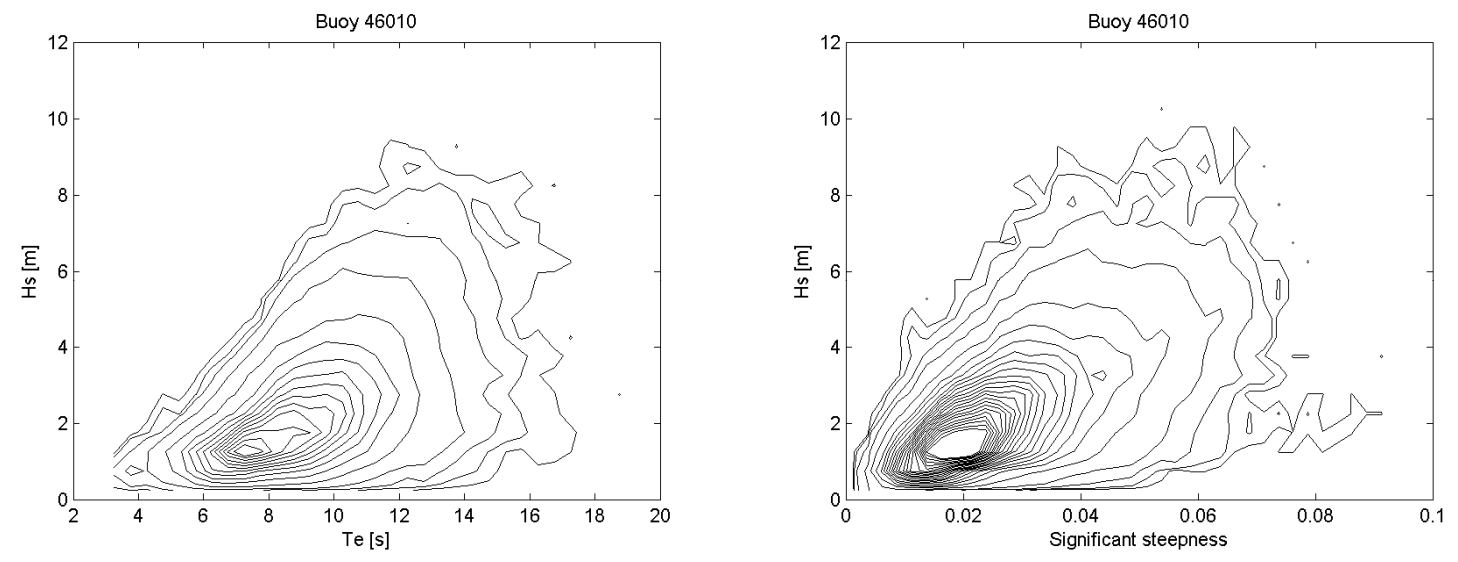

Figure 6.2. Buoy 46010: Joint distribution of $H_{s}$ and $T_{e}$ (left) and joint distribution of $H_{s}$ and significant steepness (right).
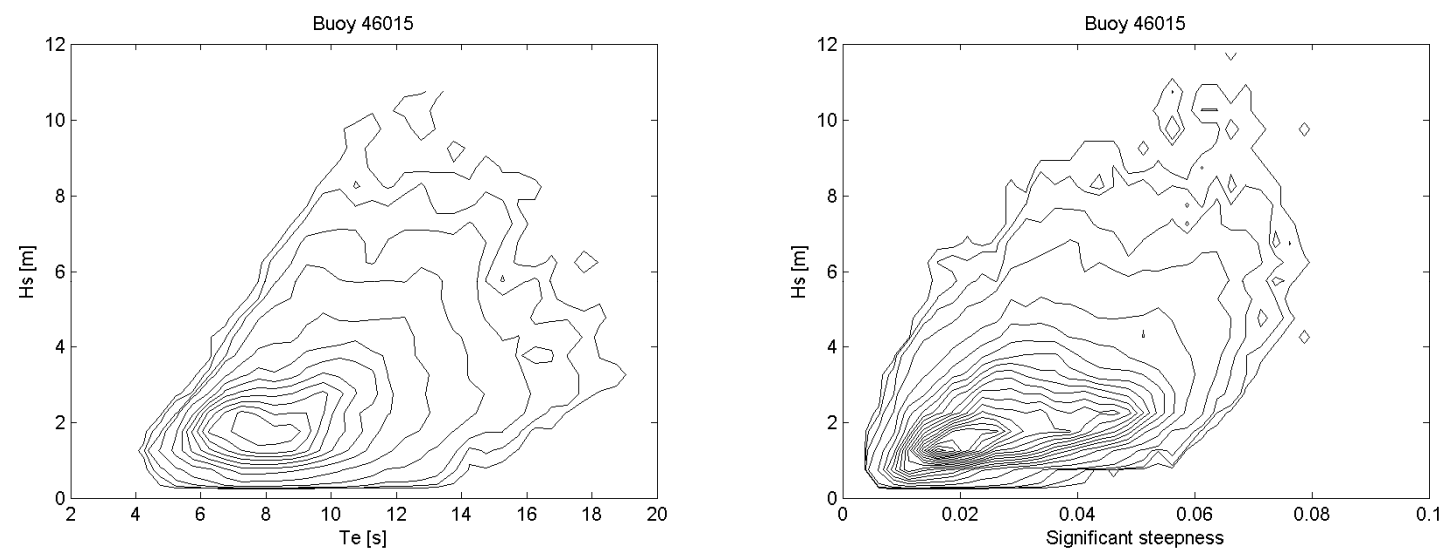

Figure 6.3. Buoy 46015: Joint distribution of $H_{s}$ and $T_{e}$ (left) and joint distribution of $H_{s}$ and significant steepness (right).
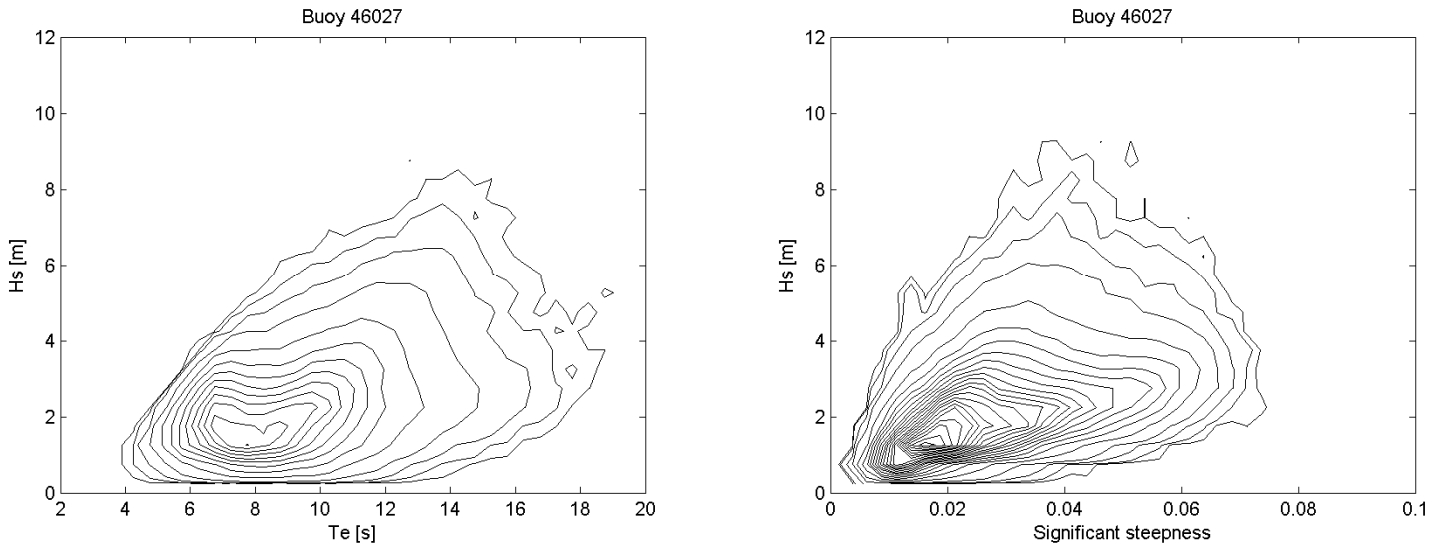

Figure 6.4. Buoy 46027: Joint distribution of $H_{s}$ and $T_{e}$ (left) and joint distribution of $H_{s}$ and significant steepness (right). 

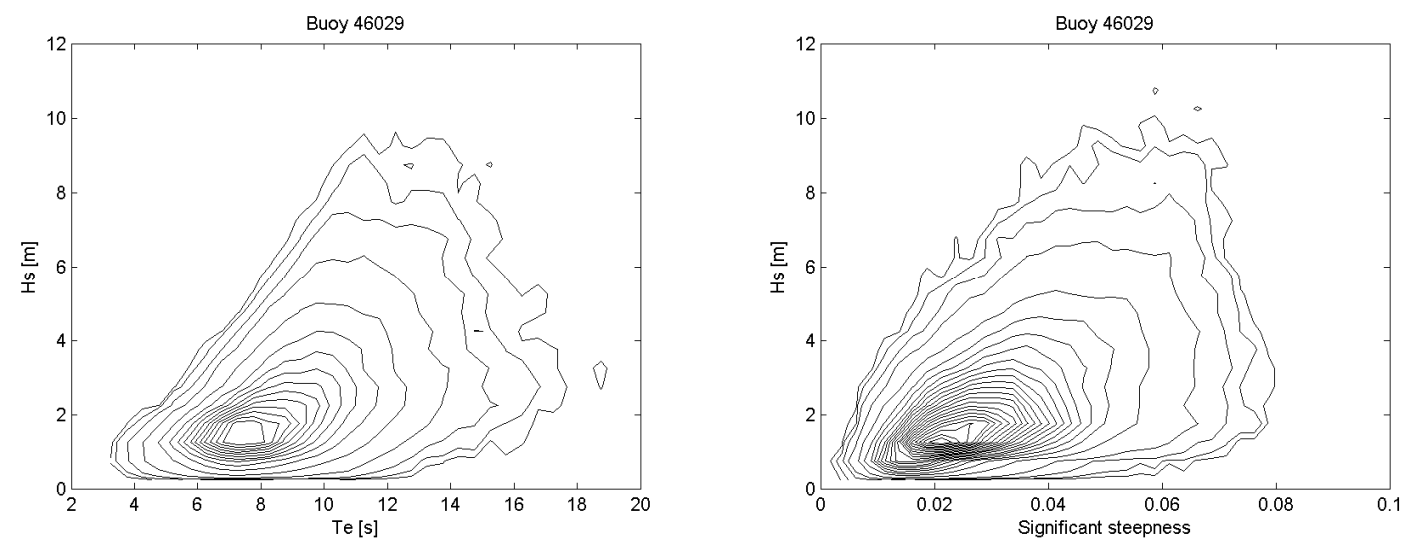

Figure 6.5. Buoy 46029: Joint distribution of $H_{s}$ and $T_{e}$ (left) and joint distribution of $H_{s}$ and significant steepness (right).
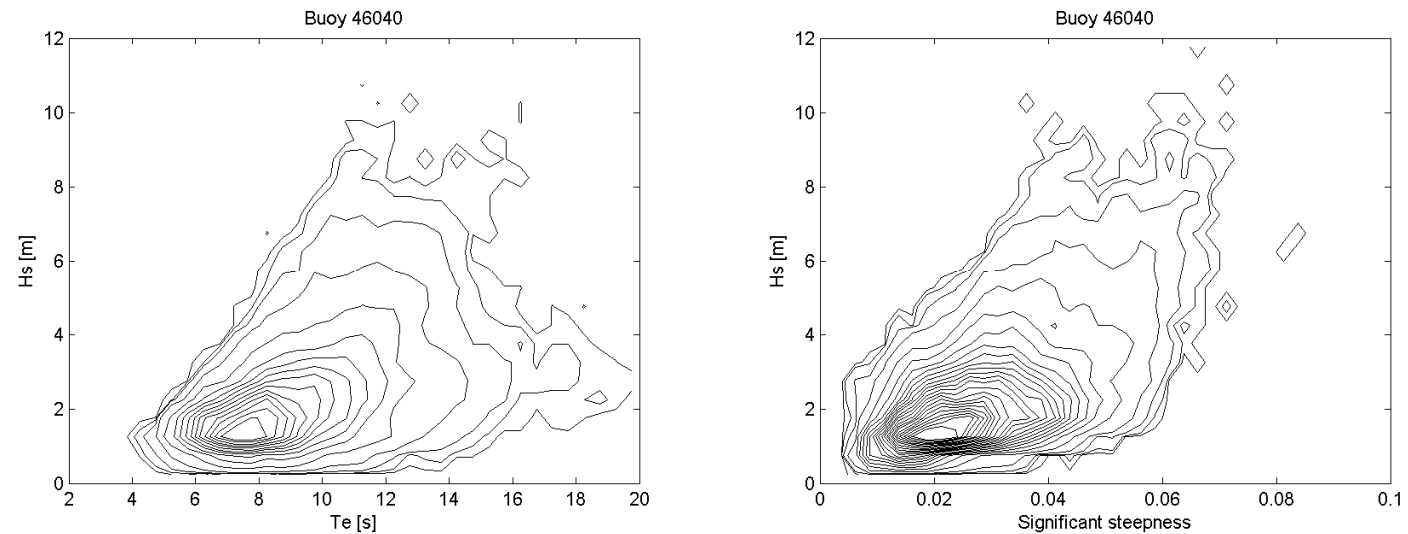

Figure 6.6. Buoy 46027: Joint distribution of $H_{s}$ and $T_{e}$ (left) and joint distribution of $H_{s}$ and significant steepness (right).
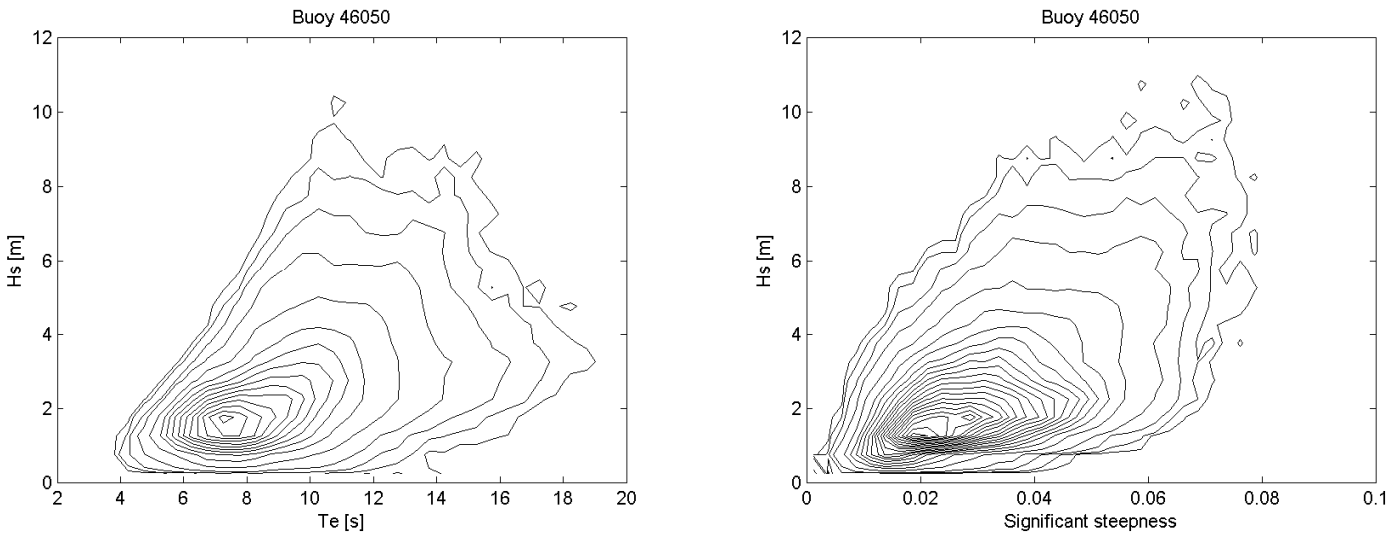

Figure 6.7. Buoy 46050: Joint distribution of $H_{s}$ and $T_{e}$ (left) and joint distribution of $H_{s}$ and significant steepness (right). 

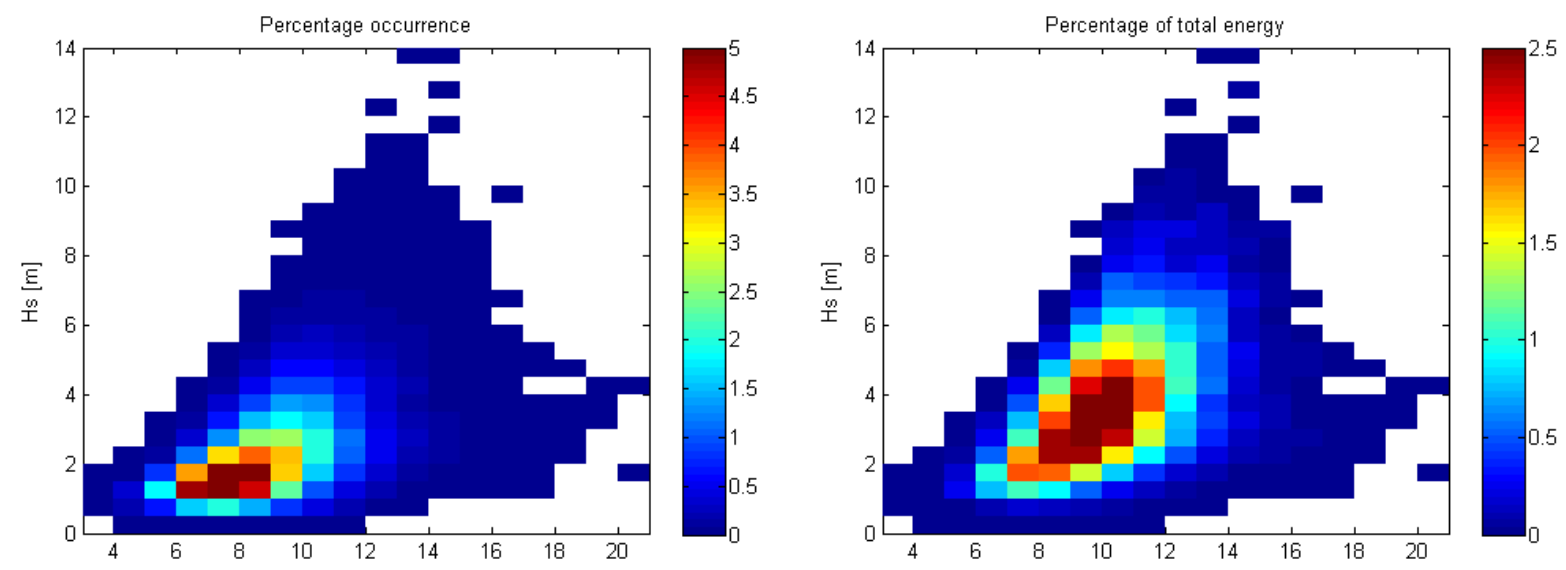

Figure 6.8. Left: Percentage occurrence of sea states, binned by $H_{s}$ and $T_{e}$. Right: Percentage of total available energy, binned by $H_{s}$ and $T_{e}$. Both plots for data from Buoy 46029.
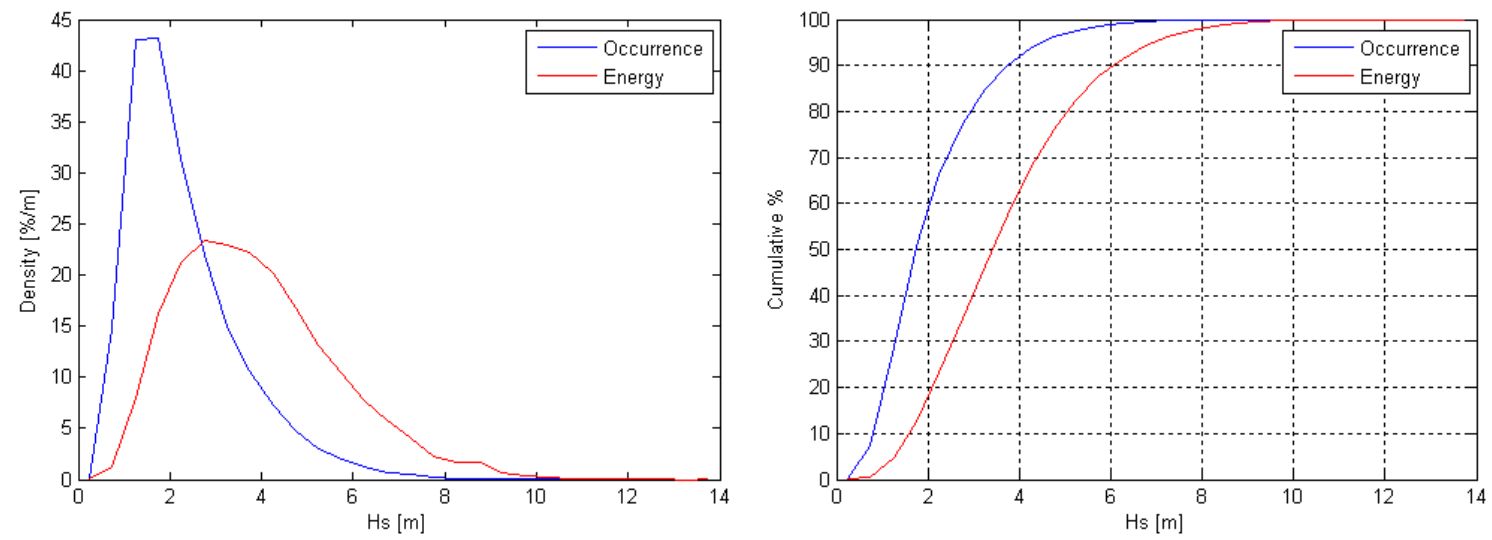

Figure 6.9. Percentage occurrence and percentage of total energy, binned by $H_{s}$ (left: density; right: cumulative). Both plots for data from Buoy 46029.
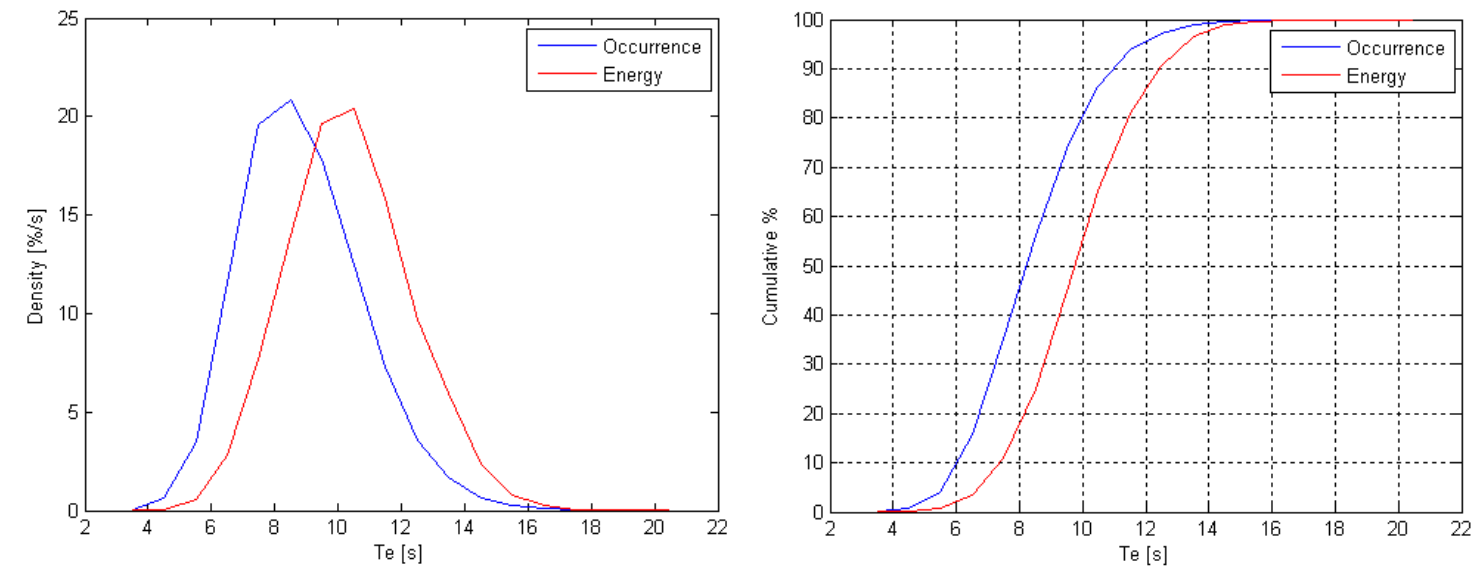

Figure 6.10. Percentage occurrence and percentage of total energy, binned by $T_{e}$ (left: density; right: cumulative). Both plots for data from Buoy 46029 . 

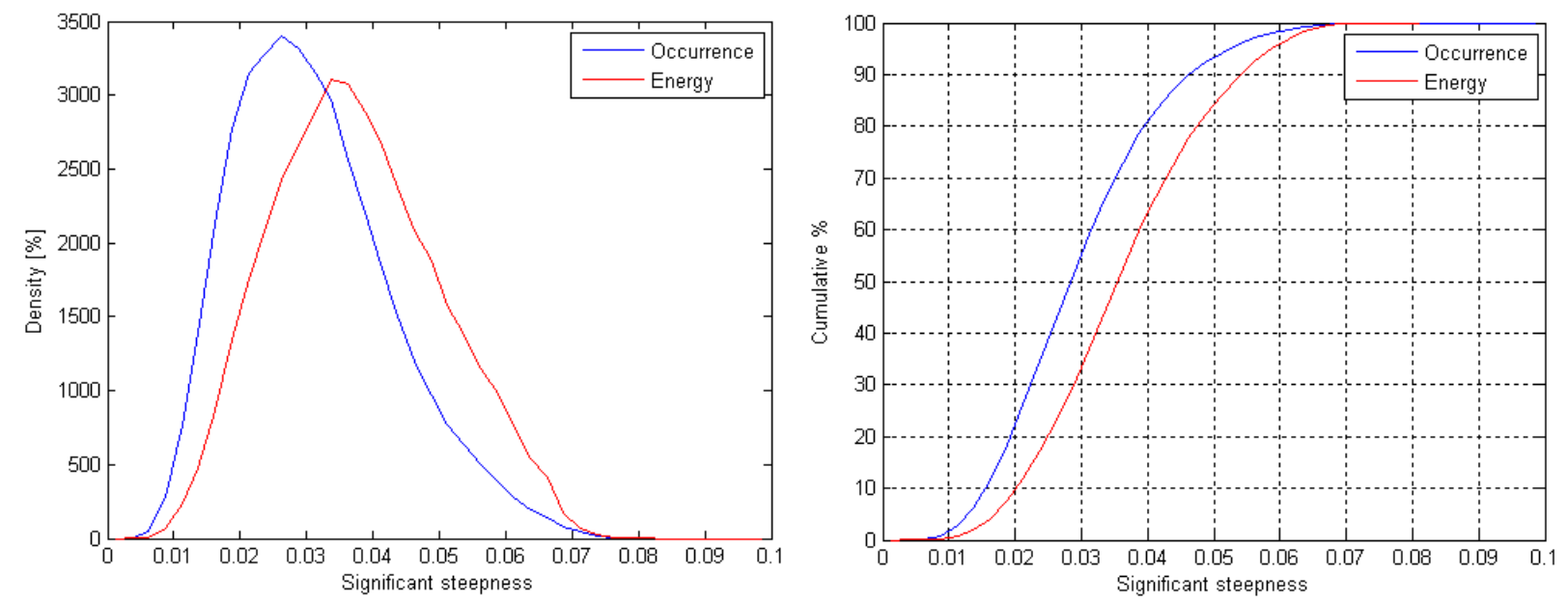

Figure 6.11. Percentage occurrence and percentage of total energy, binned by significant steepness (left: density; right: cumulative). Both plots for data from Buoy 46029.

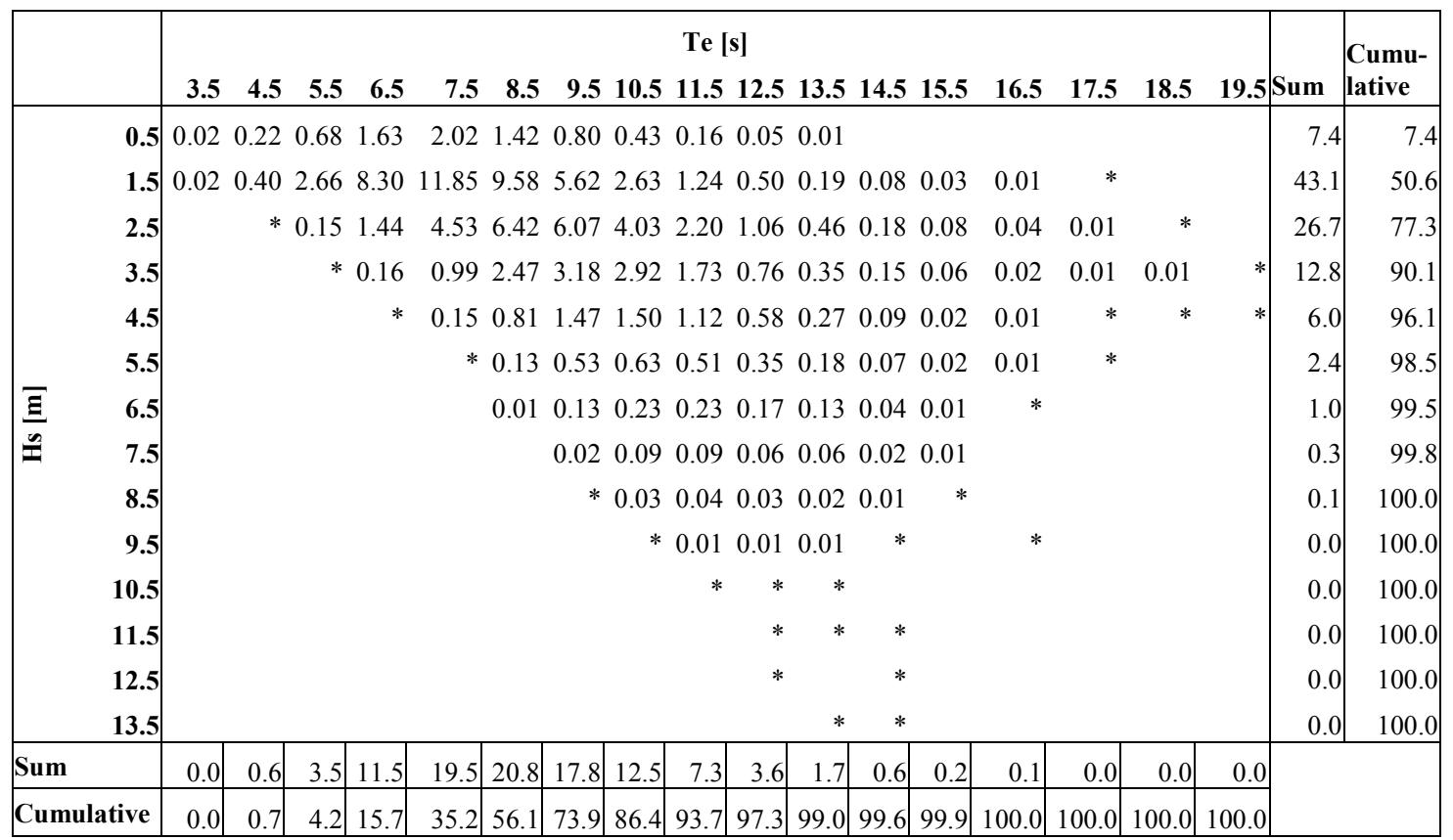

Table 6.2. Percentage occurrence of sea states binned by $H_{s}$ and $T_{e}$ for buoy 46029 . Cells with percentage occurrence $>0 \%$ but less than $0.01 \%$ are denoted with a star. 


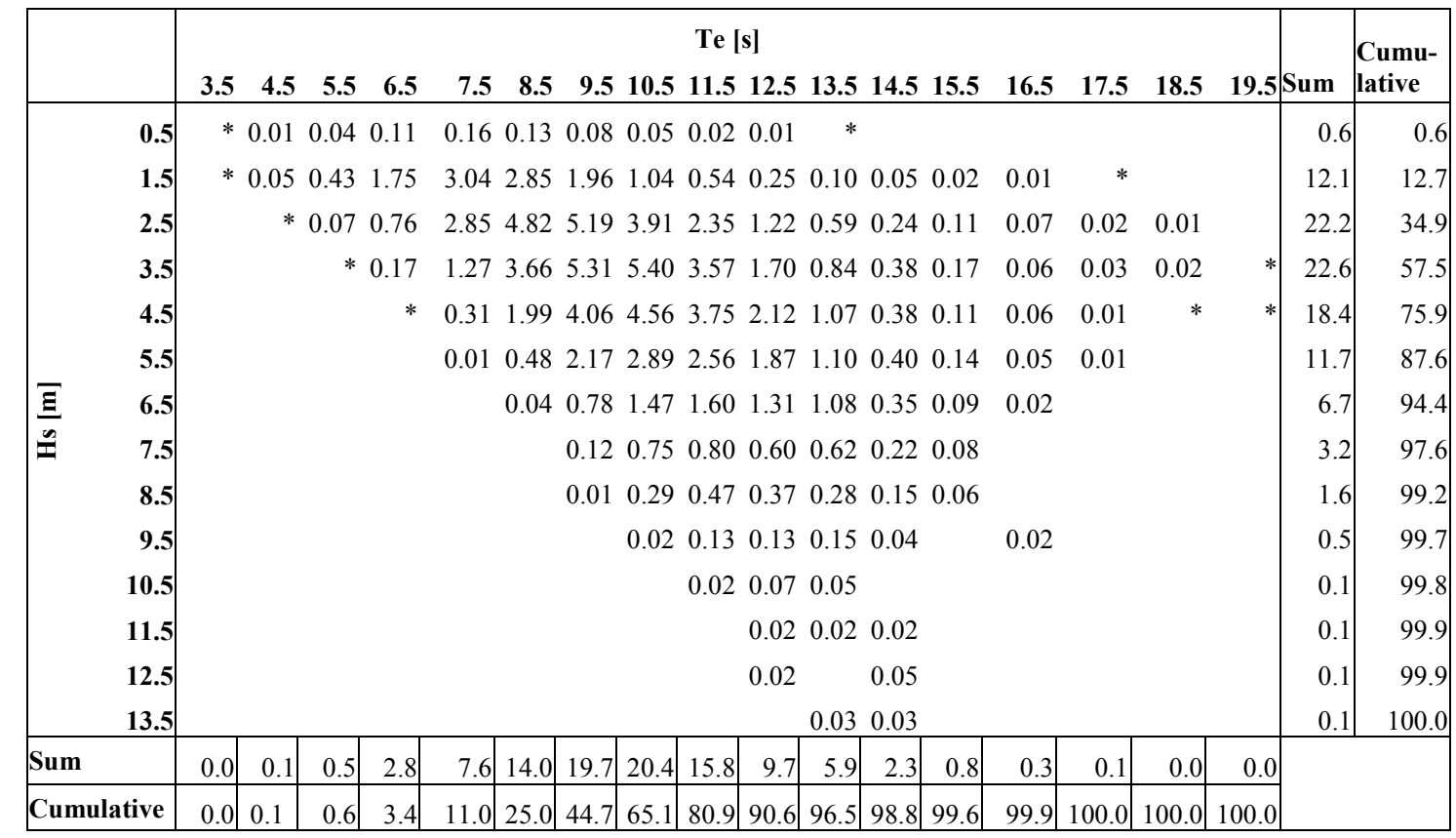

Table 6.3. Percentage of total available wave energy by Hs and Te for buoy 46029. Cells with percentage occurrence $>0 \%$ but less than $0.01 \%$ are denoted with a star.

\section{DETERMINATION OF THE SCALE FACTOR}

Buoy 46029 has been chosen for comparison with the Point Wells wave data, due to the long record available. Since the wave climates at each buoy considered in the previous section were similar, the choice of a particular wave buoy is not deemed critical.

The intended deployment period for the scale model at West Point is October 2010 - April 2011. The period covered by the measurements at Point Wells covers a similar period (Nov-April). However, since the measurements are for only one year, the hindcast will be used to check whether the measurements are representative of the long term conditions.

Figures 7.1-7.6 show scatter plots of $H_{s}$ against $T_{e}$ and $H_{s}$ against $s$ for the Point Wells measurements, using scale factors between 5 and 10, overlaid on the distributions derived from NDBC buoy 46029. It is evident that scaled conditions only cover a limited range of the offshore conditions under scaling factors of 5 or 6 . Using a scaling factor between 7 and 8 gives a reasonable coverage of the higher energy sea states with $H_{s}$ between $2 \mathrm{~m}$ and $6 \mathrm{~m}$. However it should be noted that the distribution of $H_{s}$ and $T_{e}$ is skewed towards steeper conditions than the offshore data. Since steepness is invariant with scaling it is not possible to adjust for this. Moreover, as explained in Section 4, the estimate of steepness in the Point Wells measurements may be underestimates.

The percentage occurrence of scaled $H_{s}$ in bins of width $1 \mathrm{~m}$ is shown in Table 7.1 for various scaling factors. As noted in the previous section, the conditions which account for the largest proportion of the total energy in Oregon waters have $H_{s}$ in the range $2 \mathrm{~m}-6 \mathrm{~m}$. It is clear that using a scale factor of 5 or 6 gives a very low probability of the higher sea states occurring. Using a scale factor of 8 gives approximately $22 \%$ of the time when $H_{s}$ exceeds $2 \mathrm{~m}, 9 \%$ exceeding $3 \mathrm{~m}$, and $3 \%$ exceeding $4 \mathrm{~m}$. The choice of scaling factor will depend on how much time it is anticipated is needed to conduct experiments 
in each sea state. The percentage occurrence can be converted to hours per month, e.g. an occurrence of $3 \%$ corresponds to $0.03 * 24 * 31=22.3$ hours per month. These figures can then be used to determine whether using a certain scaling factor is likely to give enough time in the required conditions for data to be gathered.
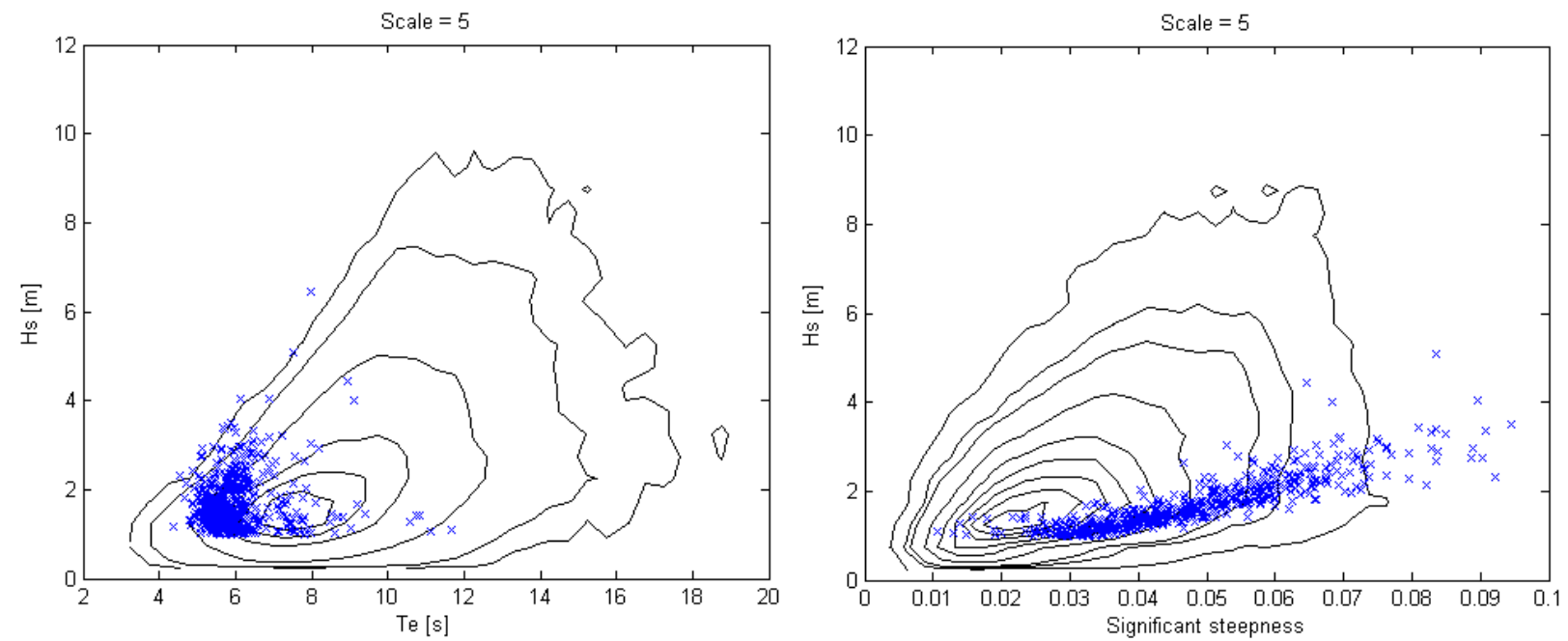

Figure 7.1. Comparison of joint distributions of $H_{s}$ and $T_{e}$ (left) and $H_{s}$ against $s$ (right) for offshore buoy data (contours) and Point Wells (crosses) scaled by a factor of 5.
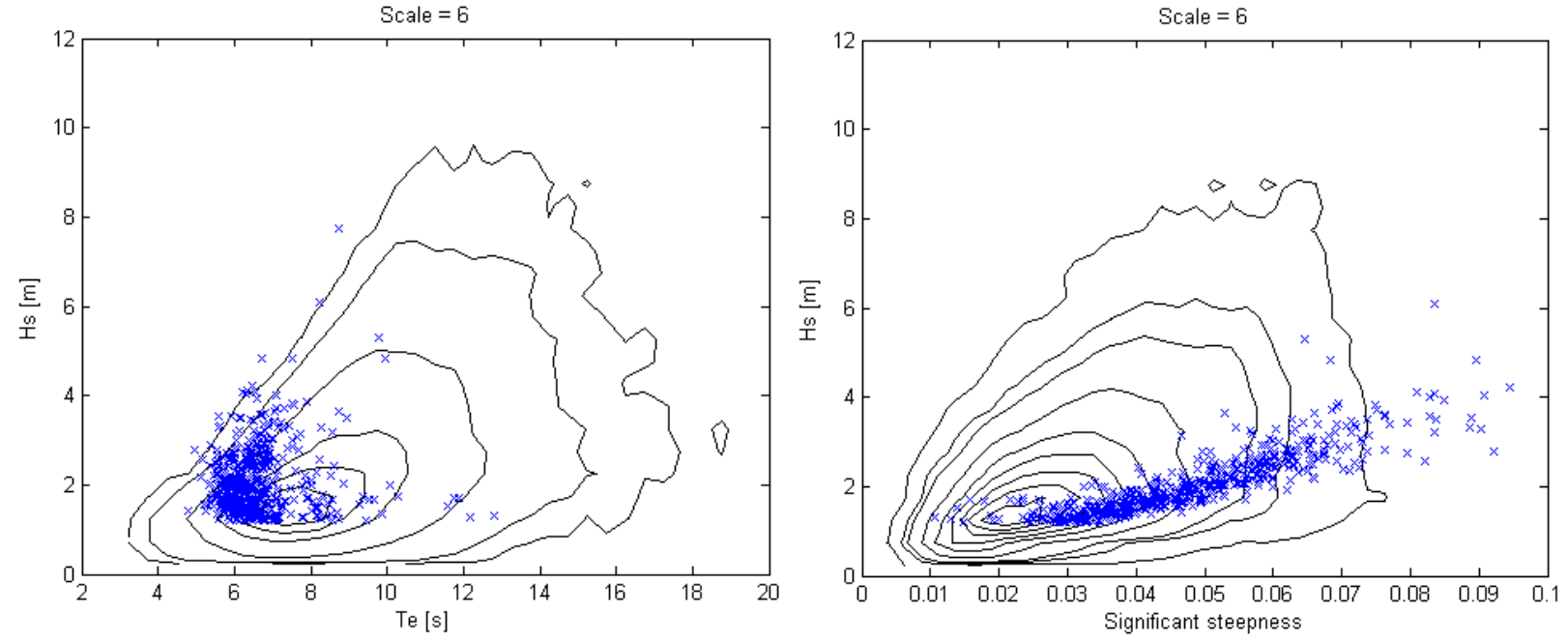

Figure 7.2. As previous figure but for a scale factor of 6 . 

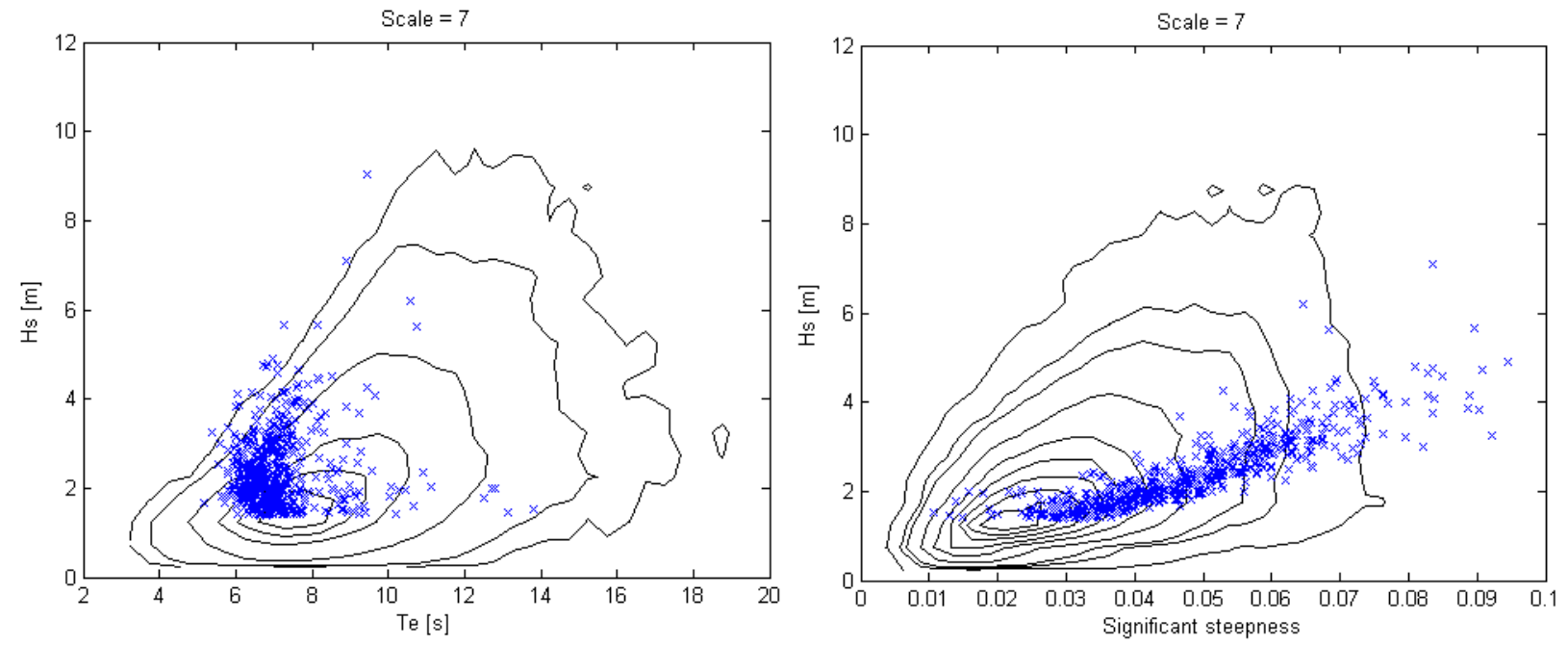

Figure 7.3. As previous figure but for a scale factor of 7 .
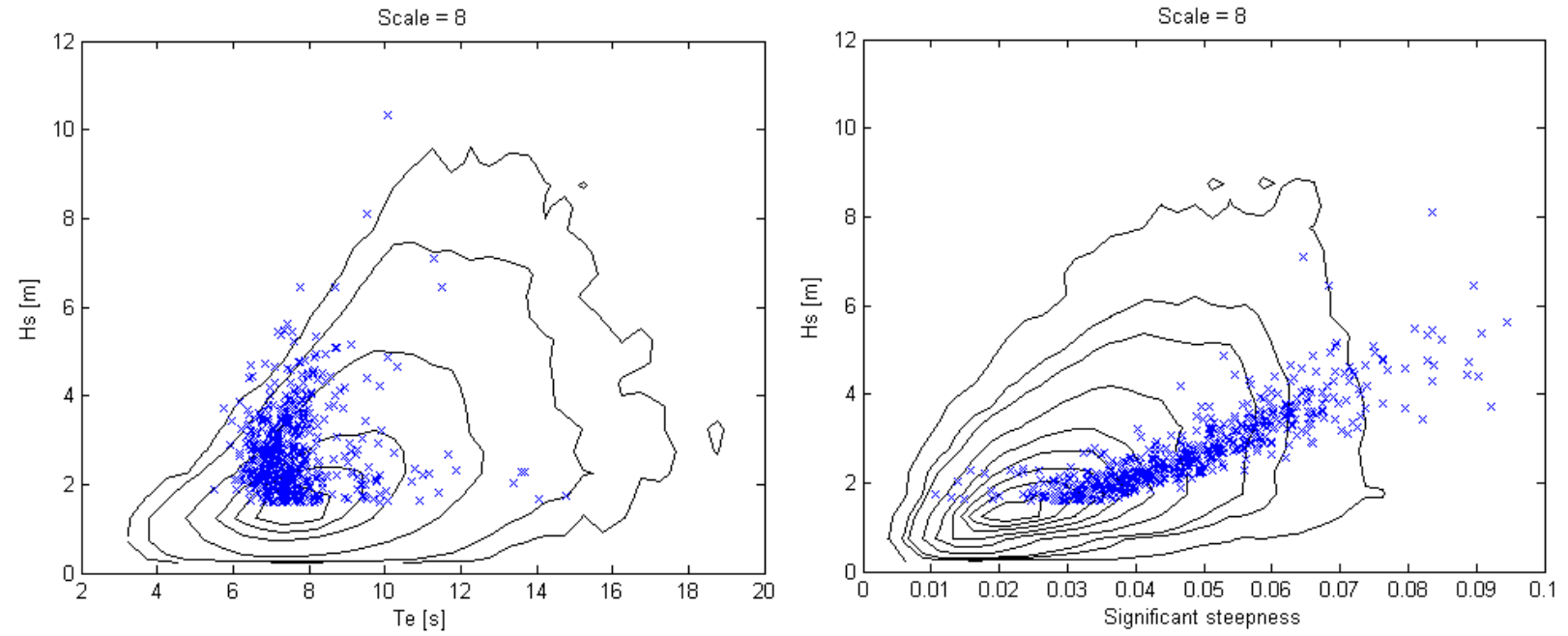

Figure 7.4. As previous figure but for a scale factor of 8 . 

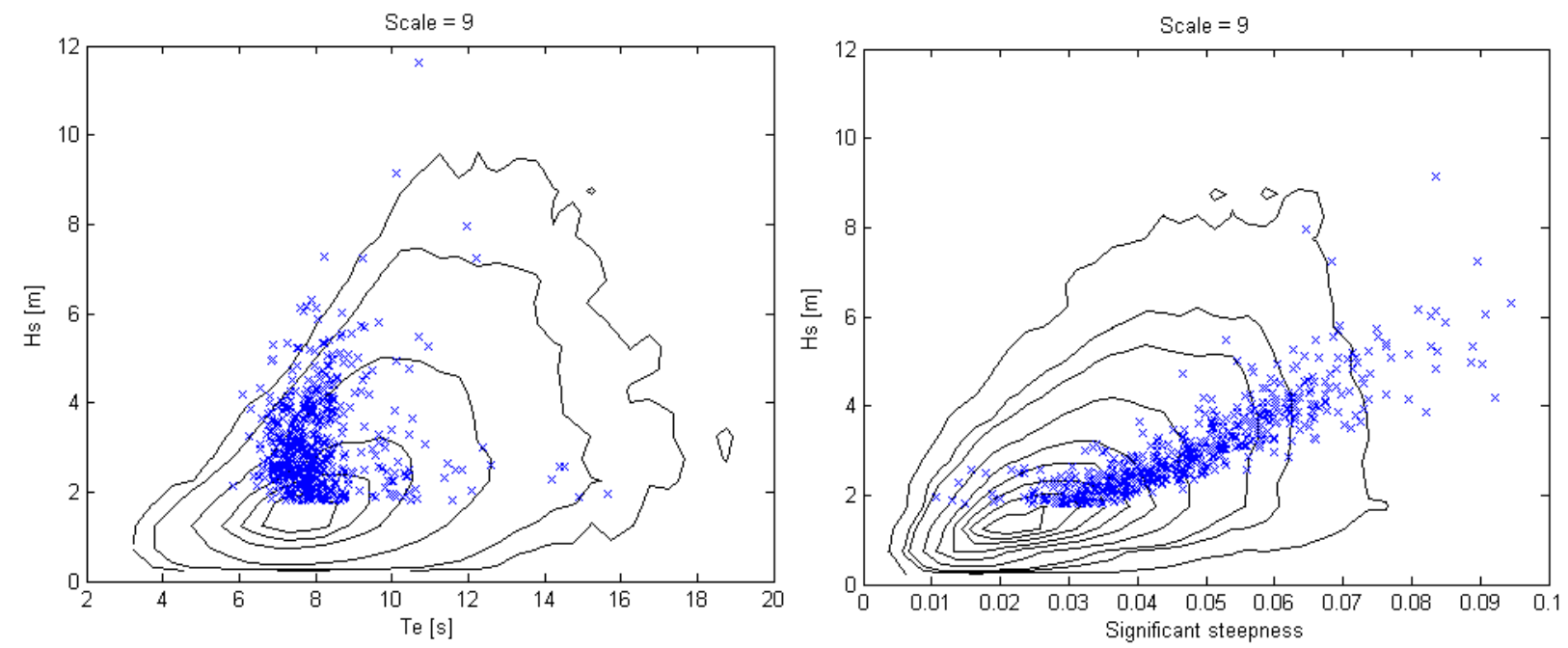

Figure 7.5. As previous figure but for a scale factor of 9 .
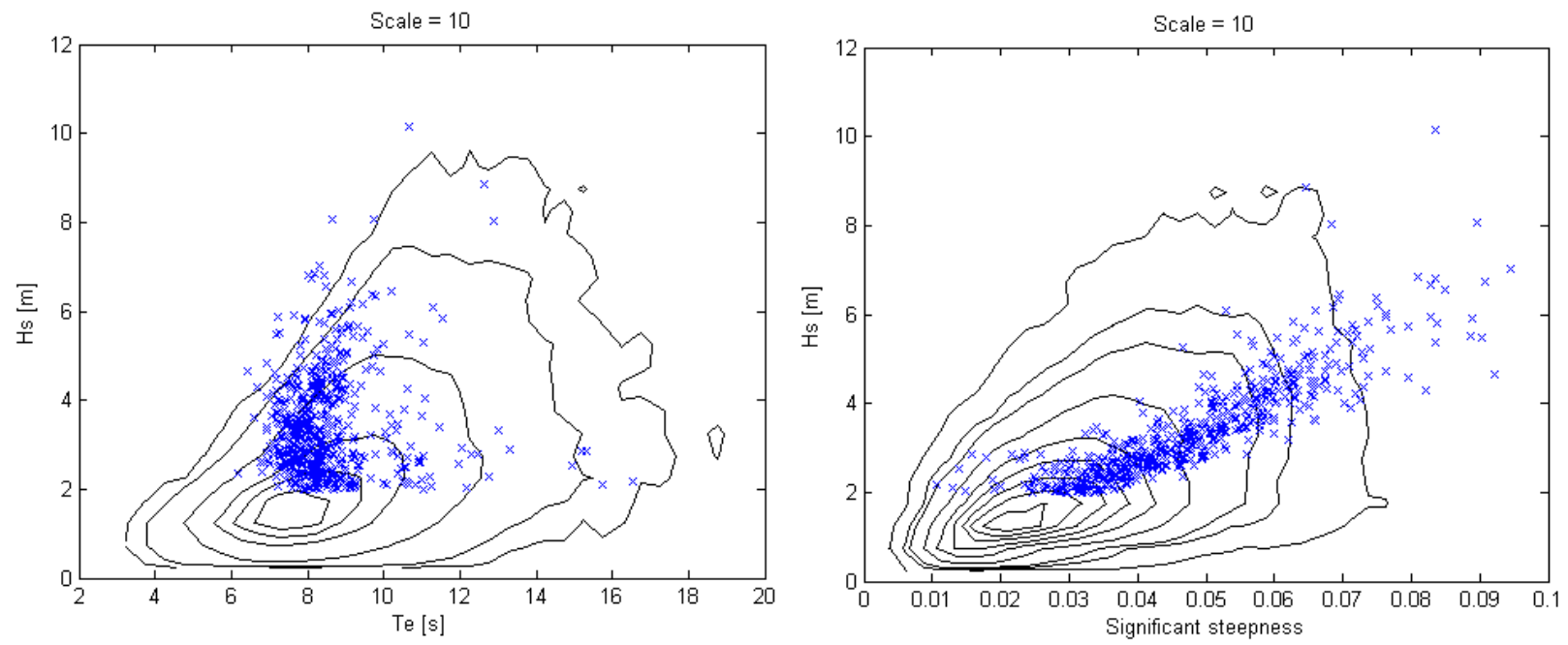

Figure 7.6. As previous figure but for a scale factor of 10 . 


\begin{tabular}{|c|c|c|c|c|c|c|c|}
\hline & \multicolumn{6}{|c|}{ scale factor } \\
\hline & & 5 & 6 & 7 & 8 & 9 & 10 \\
\hline & 0.5 & 72.1 & 67.0 & 63.2 & 59.8 & 57.1 & 54.8 \\
\hline & 1.5 & 20.1 & 20.3 & 19.4 & 17.9 & 17.9 & 17.3 \\
\hline & 2.5 & 6.7 & 9.6 & 11.5 & 13.1 & 12.4 & 12.0 \\
\hline & 3.5 & 0.8 & 2.6 & 4.4 & 6.1 & 7.7 & 8.1 \\
\hline $\boldsymbol{\Xi}$ & 4.5 & 0.2 & 0.4 & 1.2 & 2.4 & 3.3 & 4.6 \\
\hline$\infty$ & 5.5 & 0.0 & 0.1 & 0.1 & 0.4 & 1.2 & 2.1 \\
\hline & 6.5 & 0.0 & 0.0 & 0.1 & 0.1 & 0.3 & 0.7 \\
\hline & 7.5 & 0.0 & 0.0 & 0.0 & 0.1 & 0.1 & 0.1 \\
\hline & 8.5 & 0.0 & 0.0 & 0.0 & 0.0 & 0.0 & 0.1 \\
\hline & 9.5 & 0.0 & 0.0 & 0.0 & 0.0 & 0.0 & 0.0 \\
\hline
\end{tabular}

Table 7.1. Percentage occurrence of scaled Hs for various scaling factors using Point Wells measurements.

\section{FINAL RECOMMENDATIONS AND CONCLUSIONS}

Wave measurements from Point Wells have been compared to buoy data recorded off the coast of Oregon. Under the assumption that the wave conditions at Point Wells are similar to West Point, a scale factor between 7 and 8 would seem appropriate for the CPT test site at West Point. The hindcast described in Section 5 indicates that the wave conditions may be slightly more energetic at West Point than at Point Wells, however this may be a result of the different methods used to estimate $U_{10}$ at the to locations. It is recommended that the Point Wells measurements are used to determine the scale factor, since this results in more conservative estimates.

The choice of scale factor is a compromise. Using a low scale factor will enable a larger model to be tested, which is more representative of the full scale device, but a smaller range of sea states will be covered. Conversely, using a higher scale factor will mean that a greater range of sea states will be covered, but the scaled PTO and moorings may be less representative of the full scale systems. For example small scale PTO components may operate with different efficiencies to large scale components. However this may not be critical to CPT, since PTO systems can be tested on a dry rig. For the purpose of validating the hydrodynamic performance of the model a scale factor of $7-8$ is recommended.

The marginal differences between the percentage of occurrence of the performance related sea states $\left(H_{s}\right.$ between 1.5 and $3.5 \mathrm{~m}$ in Table 7.1$)$ for the $7^{\text {th }}$ scale $(35.3 \%)$ and the $8^{\text {th }}$ scale $(37.1 \%)$ designs lead to the conclusion that a final decision regarding the scale factor, should, excluding non-technical aspects such as cost, address also the cut-in (i.e. minimum $H_{s}$ to excite the model WEC) and cut-off (i.e. maximum performance related $H_{s}$ ) regimes. Again the differences are marginal, thus as a risk mitigation measure (i.e. to reduce the probability of exposing the scaled model to more energetic seas) the priority should be given to the cut-off regime. It is therefore recommended that the scale factor is set at 7.

This recommendation is in-line with the existing protocols (e.g. [4]) that outline the necessary steps when developing a novel WEC. Using [4], the ocean testing of a $7^{\text {th }}$ scale model will be classified as a 'Process Model' (phase 3), immediately after the validation (phase 1) and design (phase 2) stages (for which CPT built and tested a $33^{\text {rd }}$ and a $15^{\text {th }}$ scale model, respectively). It precedes the 'prototype' and 'demonstration' stages (phases 4 and 5, respectively), which can be merged if the next selected scale is 1:1. GH recommends that the way forward (post $7^{\text {th }}$ scale deployment) should include the onshore test of 
full-scale components, in particular critical components such as the PTO. This follows the recommendations outlined in [5]. This will allow, among other aspects, the mitigation of some of the critical risks associated with the 'prototype', the test of the SCADA system in a controlled environment and the calibration of all systems prior to deployment.

It is also recommended that additional instrumentation is deployed alongside the scaled model. Particular emphasis should be given to the wave measurements, to ensure that the measuring device meets the specifications outlined in Section 4.

\section{REFERENCES}

[1] Komen GJ, Cavaleri L, Donelan M, Hasselmann K, Hasselmann S, Janssen PAEM. Dynamics and Modelling of Ocean Waves. Cambridge University Press, 1994.

[2] Hasselmann K, Ross DB, Muller P, Sell W. A parametric wave prediction model. J. Physical Oceanography (1976), 6, 200-228.

[3] Cheng CL and Van Ness JW. Statistical Regression with Measurement Error. Arnold, London, 1999.

[4] Hydraulics and Maritime Research Centre, University College Cork, Ocean Energy: Development and Evaluation Protocol, 2003.

www.sei.ie/Renewables/Ocean_Energy/OceanEnergyIndustryForum/Forum_Archive/Development_and Evaluation_Protocol.pdf

[5] Sarmento, A. and Thomas, G., EU Wave Energy Converters Generic Technical Evaluation Study, Annex Report B1: Device Fundamentals Hydrodynamics, 1993, University College Cork. 


\title{
Northwest National Marine Renewable Energy Center University of Washington \\ Noise Measurements of Columbia Power Technologies 1/7 Scale Prototype (SeaRay)
}

\author{
Jim Thomson, Brian Polagye, \& Chris Bassett
}

\section{August 2011}

\section{Summary}

Field measurements of the underwater acoustic signature of the Columbia Power Technologies (Columbia Power) SeaRay prototype indicate periodic sound generation that is correlated with the peak period of the waves. Under extremely energetic wave conditions, the received sound pressure levels attributed to the SeaRay prototype were periodic between 116 to $126 \mathrm{~dB}$ (re. $1 \mu \mathrm{Pa}$, integrated from $60 \mathrm{~Hz}$ to $20 \mathrm{kHz}$ ) at distances from 10 to $1500 \mathrm{~m}$. Peaks in the pressure spectral densities are identified at approximately $20,100,300$, and $700 \mathrm{~Hz}$, as well as higher harmonics. Test conditions were significant wave heights from 0.4 to $0.7 \mathrm{~m}$ and peak wave periods from 2.9 to 3.2 seconds, which are approximately twice the amplitude and four times the energy of typical operating conditions for the SeaRay in Puget Sound. Shipping traffic activity was typical and received as noise levels up to $132 \mathrm{~dB}($ re. $1 \mu \mathrm{Pa}$, integrated from $20 \mathrm{~Hz}$ to $20 \mathrm{kHz}$ ). In broadband terms, noise from the SeaRay accounts for only a small fraction of the total noise budget at any given range and background noise from ship traffic dominates the overall broadband ( $20 \mathrm{~Hz}$ to $20 \mathrm{kHz}$ ) sound pressure levels, as determined from relative distances and acoustic spectral characteristics. Fully characterizing the SeaRay noise levels was not possible due to persistent background noise produced from ship traffic and other sources. This masking by ship traffic is expected for Puget Sound, and is consistent with UW-NNMREC ambient noise data from Admiralty Inlet, Puget Sound (Bassett et al., in prep). These results should be considered in the context of the existing sound in the region. Acoustic data from a similar environment in Northern Puget Sound with comparable levels of vessel traffic show mean broadband sound pressure levels ( $20 \mathrm{~Hz}$ to $30 \mathrm{kHz}$ ) of $120 \mathrm{~dB}$ (Bassett et al., in prep). Thus, it is difficult to isolate the noise produced by the SeaRay when it is cotemporal with louder sources of similar frequency.

\section{Methods: data collection}

Hydrophone recordings were collected on 30 March 2011 from 09:08 to 13:20 PDT in the vicinity of West Point (Puget Sound, WA). Two types of hydrophone data were collected: cabled drifter and autonomous drifter. Both types of hydrophones were deployed near the SeaRay (Fig. 1) in a series of drifts.

For the cabled drifts, two Cetacean Research Technology C54XRS (-185 dB re $1 \mathrm{~V} / \mu \mathrm{Pa}$ sensitivity, $16 \mathrm{~Hz}$ to $44 \mathrm{kHz}$ ) were deployed at 5 and $15 \mathrm{~m}$ depths from a 
research vessel drifting with the southerly winds. The drifts were intended to minimize flow noise over the hydrophone (as opposed to anchoring or actively holding station). In addition, cable strum was minimized using drag filaments every $20 \mathrm{~cm}$ along the hydrophone cables and an isolator float at the surface. Recordings were collected for 1 minute at $96 \mathrm{kHz}$ continuously, except during repositioning for the drifts.

For the autonomous drifts, a Loggerhead DSG $(-185 \mathrm{~dB}$ re $1 \mathrm{~V} / \mu \mathrm{Pa}$ sensitivity, $20 \mathrm{~Hz}$ to $30 \mathrm{kHz}$ ) was deployed at $1 \mathrm{~m}$ depth on a free drifting buoy (APL-UW 'SWIFT'). Recordings were collected for 1 minute at $80 \mathrm{kHz}$ continuously.

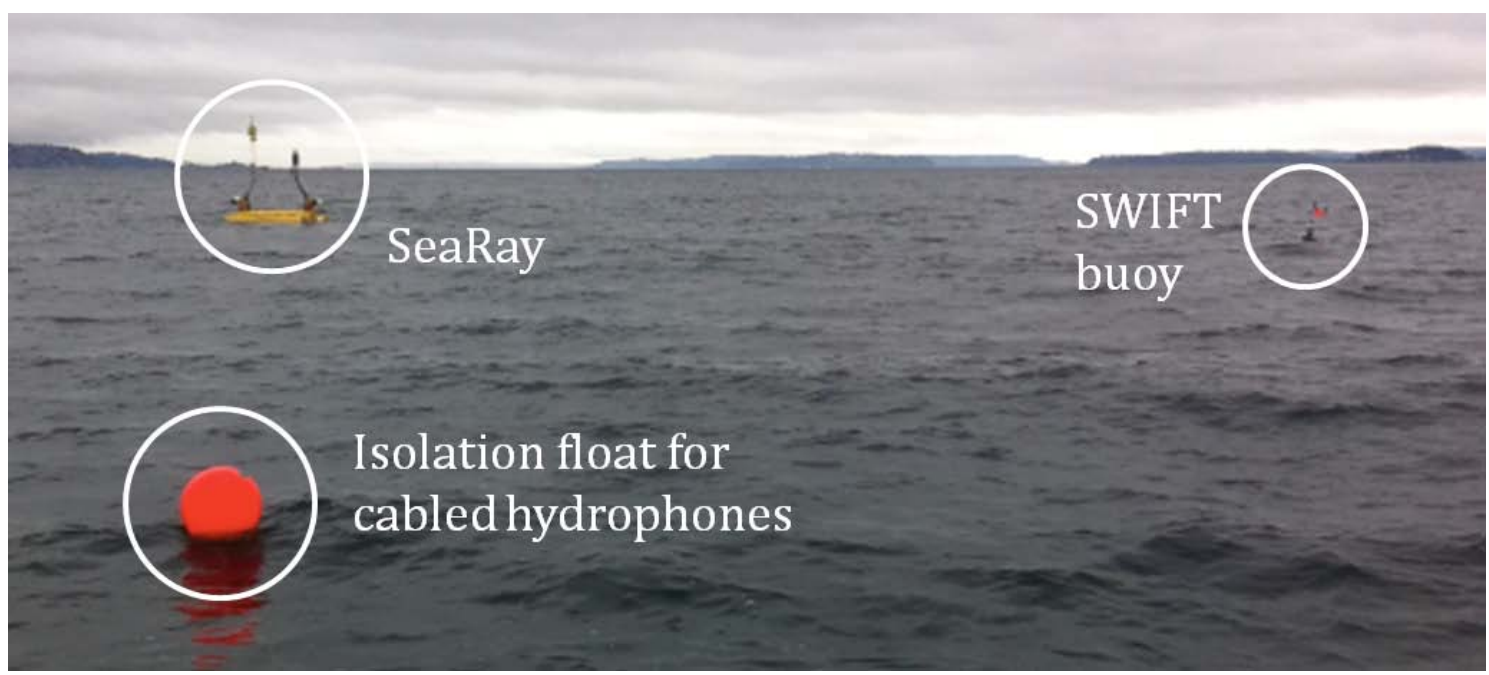

Figure 1. SeaRay (upper left), cabled hydrophone isolator float (lower left), and autonomous drifting hydrophone (upper right).

Ancillary data include GPS logs for the position and range to the SeaRay for each recording, and a ship traffic Automated Identification System (AIS) was used to quantify range to nearby vessels. Wave heights $(0.4$ to $0.7 \mathrm{~m})$, wave periods $(2.9$ to $3.2 \mathrm{~s}$ ), and winds (5-8 m/s, southerly), were measured from the APL-UW SWIFT buoy. Digital Video Recordings (DVR) of the SeaRay in operation during hydrophone recordings indicate full travel on the buoy surge mechanism.

\section{Methods: data processing}

The minute-long hydrophone recordings are divided into windows (8192 points), tapered, overlapped 50\%, Fast Fourier Transformed, and normalized to preserve variance. A hydrophone calibration is applied and 700 windows are ensemble averaged to obtain pressure spectral densities (PSD) with high statistical confidence. The resulting pressure spectral densities describe the frequency content of the recordings. The minimum and maximum resolvable frequencies are dependent on the hydrophone response and data acquisition rate, respectively. The spectra are evaluated for quality control and integrated from $20 \mathrm{~Hz}$ to $20 \mathrm{kHz}$ to determine broad-band sound pressure levels (SPL) given in $\mathrm{dB}$ re. $1 \mu \mathrm{Pa}$. The 
broadband SPL is defined as root mean square (rms) pressure squared divided by the reference pressure squared. In addition, hydrophone recordings were reviewed audibly, and example .wav files are available upon request.

\section{Results: spatial distribution of SPLS}

Sound Pressure Levels for all measurements are shown in Fig. 2, where the drift tracks are south to north because of 5-8 m/s southerly winds during data collection. SPLs are typically around $120 \mathrm{~dB}$, and only exceed this level when a ship is nearby. The max SPL observed is $132 \mathrm{~dB}$ and corresponds to a tugboat passing within $500 \mathrm{~m}$ of the site. For comparison, assuming practical $15 \mathrm{Log}$ spreading losses, the max SPL attributed to the SeaRay is $126 \mathrm{~dB}$ and is equivalent to the same tugboat passing at $1.25 \mathrm{Km}$ range.

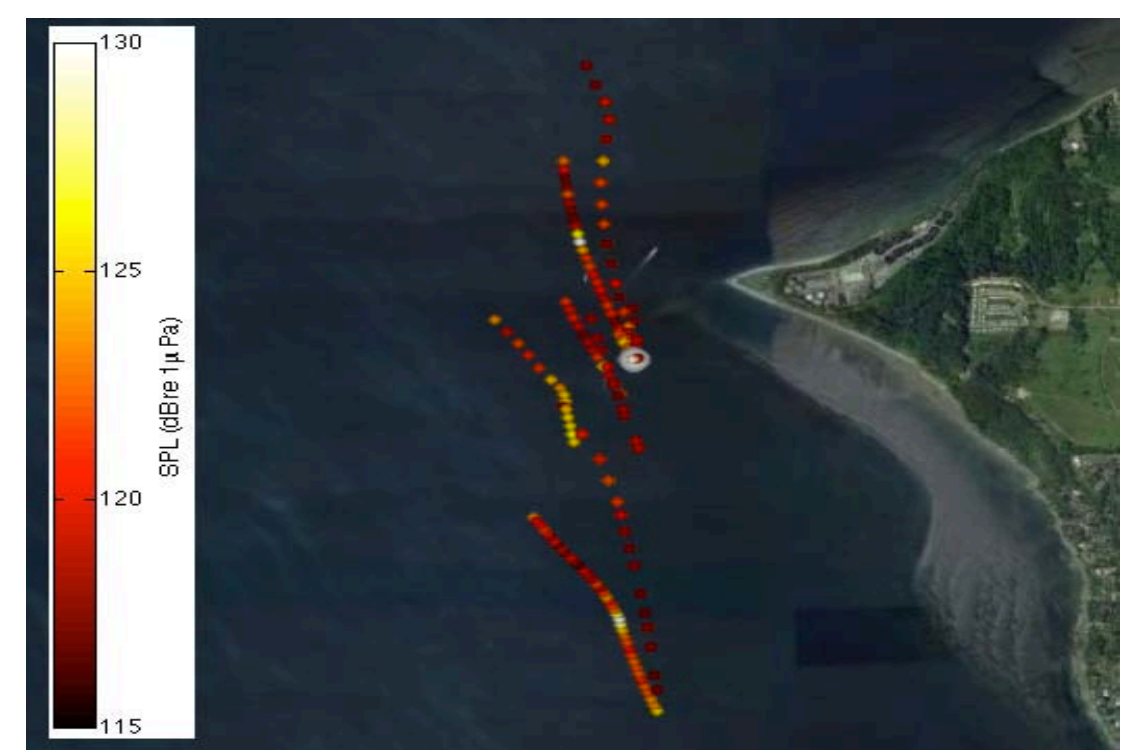

Figure 2. Spatial distribution of recorded broad-band SPLs ( $20 \mathrm{~Hz}-20 \mathrm{kHz}$ in dB re. $1 \mathrm{uPa})$. The white circle at the center indicates the location of the SeaRay near West Point (Puget Sound, WA), and the region shown is $3 \times 3 \mathrm{~km}$.

As shown in Fig. 3, which presents SPLs as function of radial distance to the buoy and recording depth, there is no trend in the spatial data. Even when screening the data for times without ships nearby, there is not a clear spatial pattern relative to the SeaRay. This is in contrast to the expectation that SPL will decrease away from the SeaRay as a result of transmission loss. It is likely that the high level of ambient noise in the region masks the expected transmission loss pattern. 


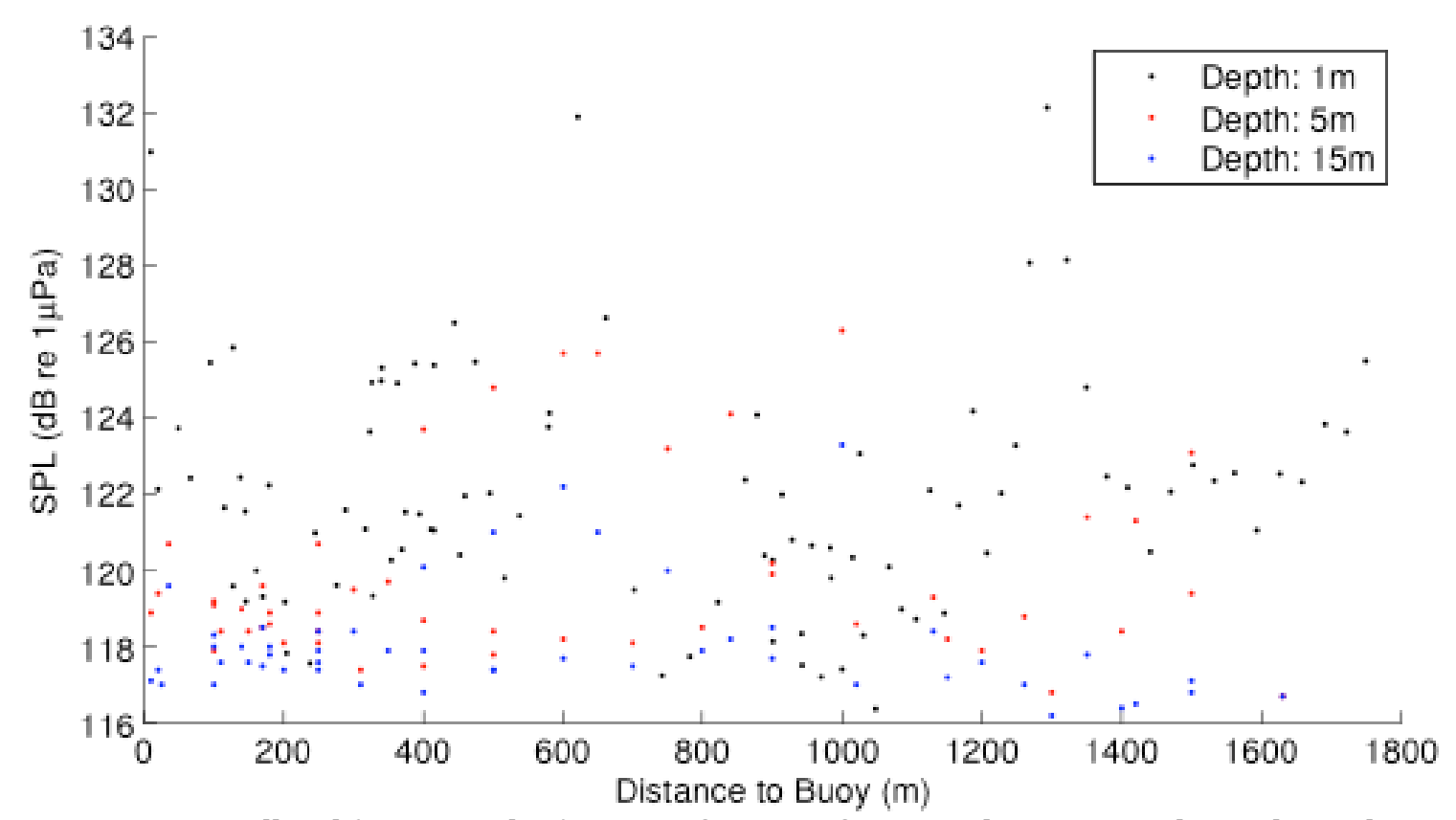

Figure 3. Broadband $(20 \mathrm{~Hz}-20 \mathrm{kHz})$ SPL as a function of range to the SeaRay. Colors indicate the different hydrophones.

\section{Results: spectral characteristics}

Although the contribution to total SPL from the SeaRay is not evident in the spatial patterns, it is possible to detect the buoy (and to hear it audibly in the recordings) at close range and in the absence of ship traffic. Pressure spectral densities, such as the examples in Fig. 4, show persistent peaks at 20, 100, 300, 700, and $1500 \mathrm{~Hz}$. These peaks are most evident within $500 \mathrm{~m}$ of the SeaRay and during lulls in ship traffic. These noise spikes at specific frequencies may be caused by the intermittent start and stop of the drive shaft with each passing wave. The source might also be harmonics of the sound produced by the over-torque limiter or gearbox onboard the SeaRay. When a ship passes nearby (red line in Fig. 4), the peaks are obscured and the pressure spectral densities are elevated at all frequencies (note the logarithmic scale). Another source of noise is wave breaking, which typically contributes at frequencies above a few $\mathrm{kHz}$ (e.g., gray line in Fig. 4.). The frequency of breaking during data collection was $0.5-4$ waves per minute, as measured by video onboard the SWIFT drifter. 


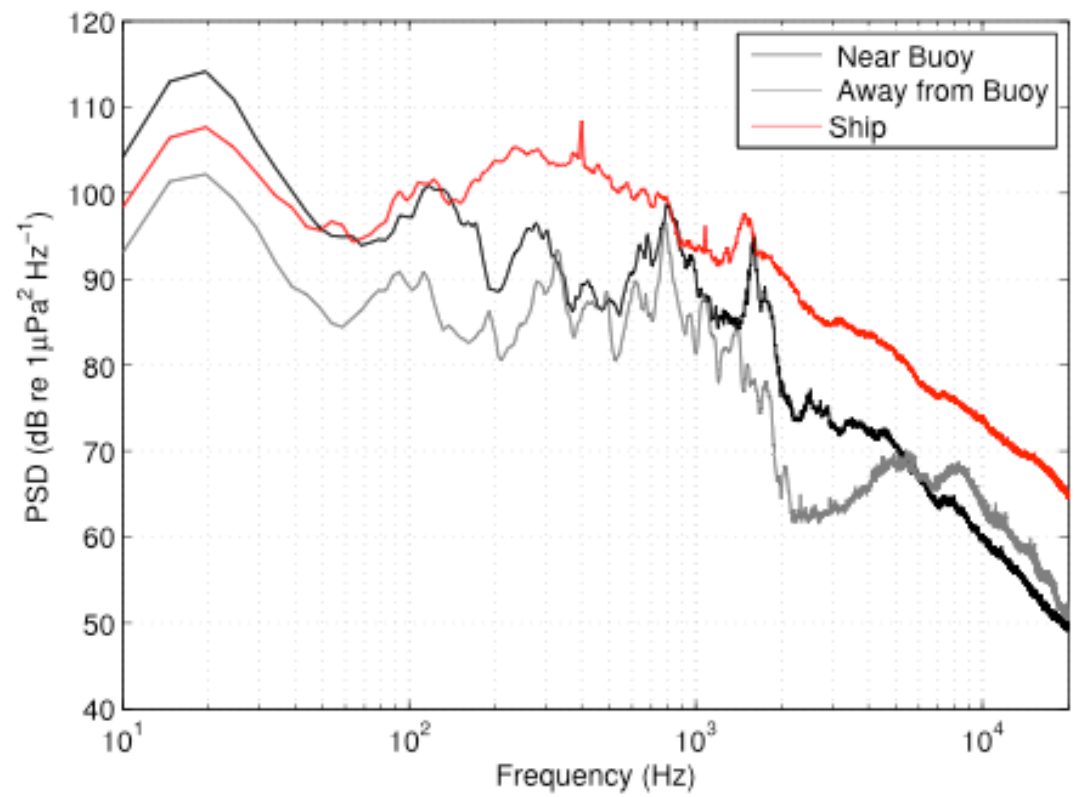

Figure 4. Example pressure spectral densities showing the source and harmonics of the SeaRay (black line and gray lines), as well as typical ship traffic (red line).

The spectral characteristics can be seen more clearly in a short times series, such as the example in Fig. 5. In the absence of ship traffic noise, the SeaRay is observed to produce distinct spectral peaks on a regular cycle with the peak wave period (approximately $3 \mathrm{~s}$ ). Integrating the pressure spectral densities over selected frequencies ranges, the received sound pressure levels from the SeaRay are periodic pulses of approximately $120 \mathrm{~dB}$ (black line, lower panel of Fig. 5).
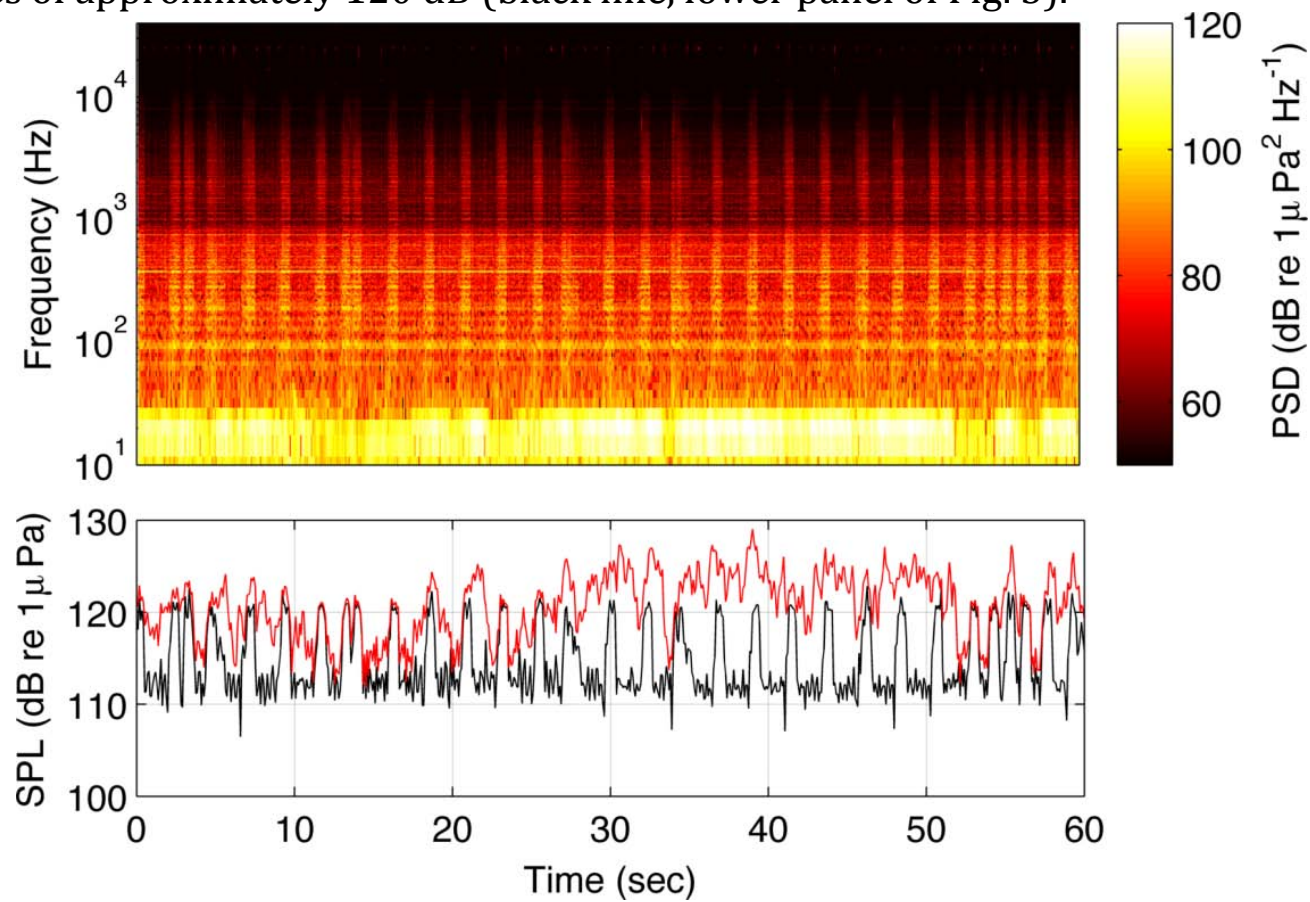

Figure 5. Example time series of pressure spectra densities (color scale, upper panel) and bandintegrated sound pressure levels (lines, lower panel) showing regular sound generation at wave periods. The black line is the SPL integrated from $0.08-2 \mathrm{kHz}$, and the red line is integrated from $0.02-20 \mathrm{kHz}$. Spectra are from the cabled CRT hydrophone at $15 \mathrm{~m}$ depth and $1.4 \mathrm{~km}$ distance from the SeaRay. 


\section{Results: received levels}

The large scatter in the SPL as a function of range (Fig. 3) prevents extrapolation, via the sonar equation, to estimate a source level for the SeaRay at the conventional $1 \mathrm{~m}$ reference. This is because, in broadband terms, noise from the SeaRay accounts for only a small fraction of the total noise budget at any given range. Measurements of received sound pressure levels, particularly integrated over the $80 \mathrm{~Hz}$ to $2 \mathrm{kHz}$ range associated with the SeaRay, can be used to quantify the effect of the SeaRay on the acoustic environment. As shown in Fig. 5 (black line), with each passage of a wave (approximately $3 \mathrm{~s}$ ), the SeaRay produces regular signals approximately $10 \mathrm{~dB}$ over the background levels in frequency bands from $0.08 \mathrm{~Hz}$ to $20 \mathrm{kHz}$. Note, however, that when the frequency range of analysis is increased to $0.02 \mathrm{~Hz}$ to 20 $\mathrm{kHz}$ (red line), the periodic acoustic emission from the SeaRay cannot always be discerned from the background, even very close to the SeaRay. This occurs at all distances from the SeaRay.

These results point to a general challenge in characterizing the acoustic emissions from wave energy converters. Because acoustic emissions are periodic with wave frequencies, sound pressure level is sensitive to the analysis window. For example, sound pressure level for an analysis window restricted to the time of maximum power output from the buoy will be significantly higher (at least a few $\mathrm{dB}$ ) than one in which the analysis window contains several periodic signals. Applying a precautionary principal, the received level discussed here is for the period of maximum power output. For the SeaRay, this received level is typically $120 \mathrm{~dB}$, and varies from 116 to $126 \mathrm{~dB}$.

\section{Conclusions}

In general, noise from the SeaRay accounts for only a small fraction of the total noise at any given range. SeaRay noise is produced on regular intervals, corresponding to wave periods, at multiple harmonic frequencies spanning from $80 \mathrm{~Hz}$ to $2 \mathrm{kHz}$. The integrated sound pressure levels showed background levels of approximately 116 $\mathrm{dB}$ and SeaRay levels intermittently peaking to approximately $126 \mathrm{~dB}$. By contrast, received sound pressure levels from ship traffic are up to $132 \mathrm{~dB}$. The ship noise causes significant masking, such that the signal from the SeaRay is only detectable during times when there are no vessels within approximately $1 \mathrm{~km}$ of the site. Observations do not support trends with depth or distance (i.e. transmission loss), which likely is a result of masking by high levels of ambient shipping noise in the urban waterway of Puget Sound.

The inability to observe a decrease in SPL as the distance increased from the SeaRay prototype is likely caused by the high level of ambient noise in the region, which masks the expected transmission loss pattern. The wide spectral range of frequencies sampled is dominated by noise created by other human and natural sources at frequencies other than the SeaRay. While the SeaRay itself exhibits broadband levels up to $126 \mathrm{~dB}$ levels periodically (it is unlikely that source levels are close to $126 \mathrm{~dB}$ at any particular frequency), the frequency spectrum is dominated by other noise sources. This is consistent with recent UW-NNMREC 
propagation tests with a $120 \mathrm{~dB}$ re $1 \mu \mathrm{Pa}$ at $1 \mathrm{~m}$ source in northern Admiralty Inlet in Puget Sound, an area also dominated by shipping traffic. During those experiments, the tonal source is difficult to detect at ranges greater than $500 \mathrm{~m}$ (Bassett et al., in prep). The acoustic signature of the SeaRay, which is a broadband source, is even more subject to masking by stronger sources in its vicinity. 


\section{CPT $1 / 7^{\text {th }}$ Scale Buoy Deployment Plan}

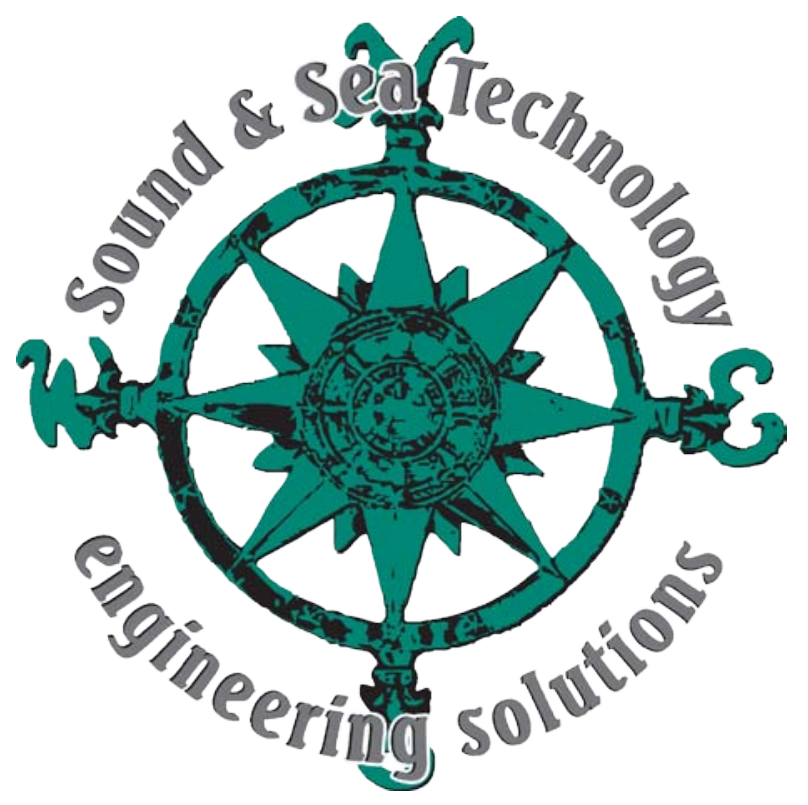

Sound and Sea Technology

2-2-2011

Prepared By:

Sam Gooch 


\section{CPT $1 / 7^{\text {th }}$ Scale Buoy Deployment Plan Revisions}

\begin{tabular}{|l|l|l|}
\hline Version & Date & Reason for Changes \\
\hline 1.0 & & Original DRAFT \\
\hline 2.0 & & $1^{\text {st }}$ Revision \\
\hline 3.0 & & $2^{\text {nd }}$ Revision \\
\hline 4.0 & & $3^{\text {rd }}$ Revision \\
\hline 5.0 & & $4^{\text {th }}$ Revision \\
\hline 6.0 & & $5^{\text {th }}$ Revision \\
\hline 7.0 & 10 January 2011 & $7^{\text {th }}$ Revision \\
\hline 8.0 & 18 January 2011 & $8^{\text {th }}$ Revision \\
\hline 9.0 & 24 January 2011 & $9^{\text {th }}$ Revision \\
\hline 10.0 & 2 February 2011 & $10^{\text {th }}$ Revision \\
\hline 11.0 & & \\
\hline
\end{tabular}




\section{Contents}

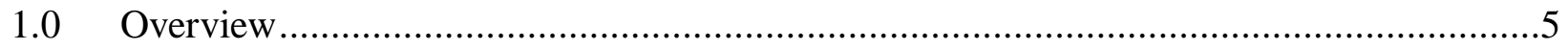

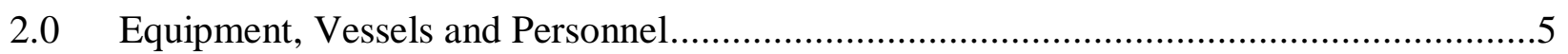

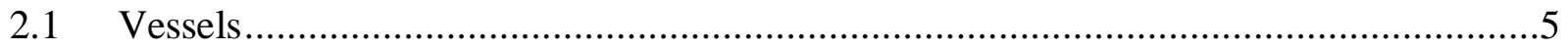

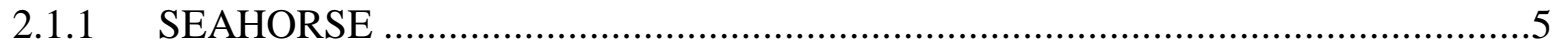

2.1.2 Maintenance Vessel: RV Neper .......................................................................

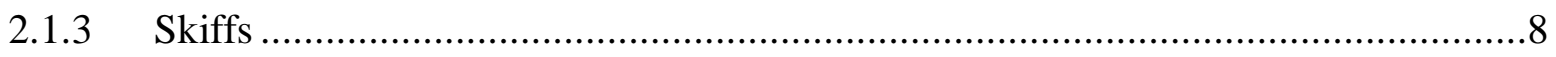

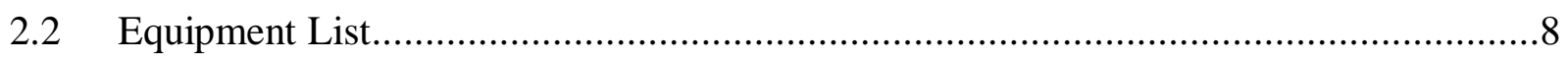

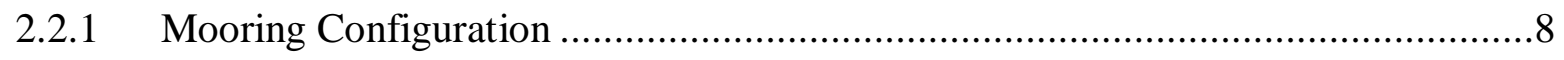

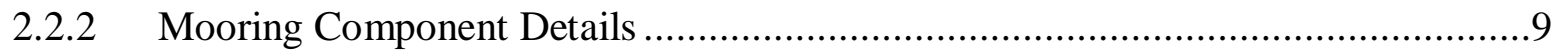

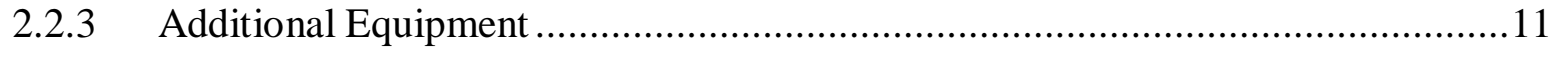

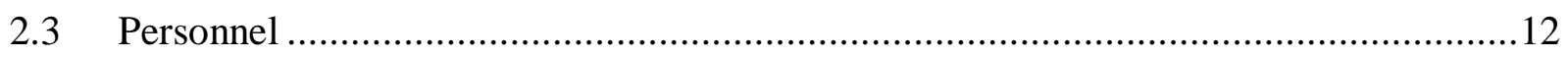

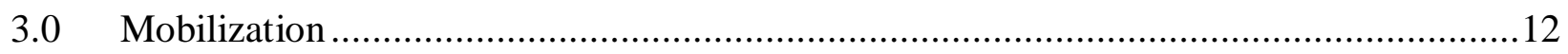

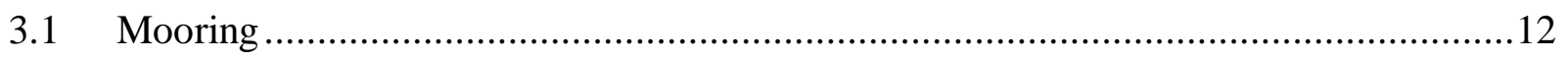

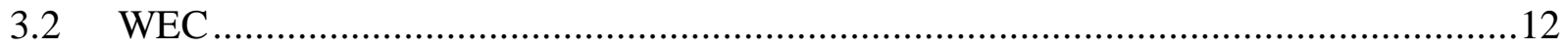

3.3 Loading the SEAHORSE ......................................................................................13

3.4 Mooring Set Up ...............................................................................................14

4.0 GPS Calibration, Float Acoustic Test and Lifting Exercise ............................................14

4.1.1 Transit and Preliminary Testing .........................................................................14

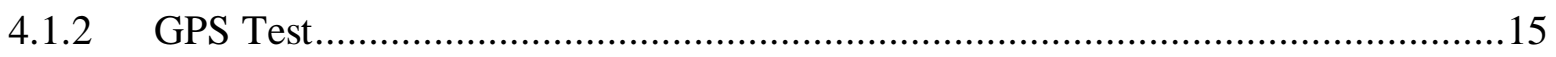

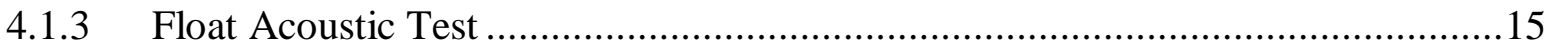

4.1.4 Additional Testing and Recovery ……………................................................16

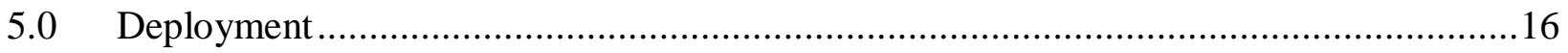

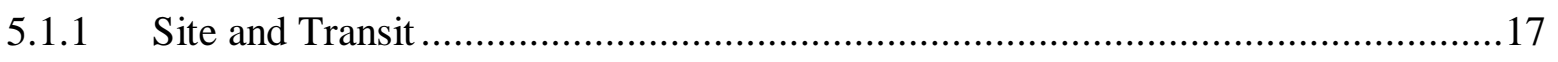

5.1.2 Northwest Mooring Leg Deployment ...................................................................19

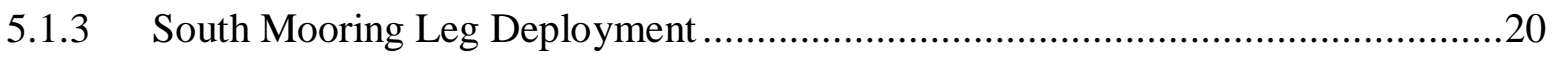

5.1.4 Optional Steps for Overnight Stopping Point.......................................................22

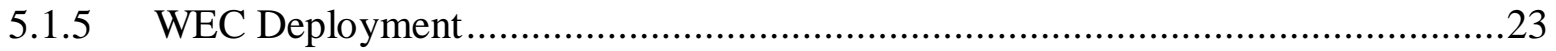

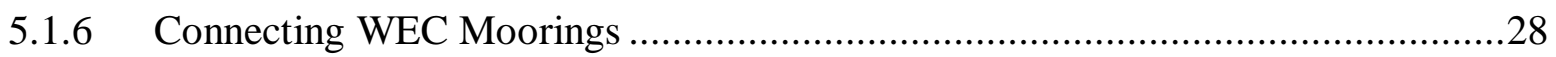

5.1.7 Northeast Anchor Deployment and WEC Pretensioning .........................................30

5.1.8 Target Pretension Table ...................................................................................32

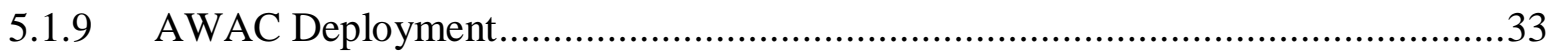

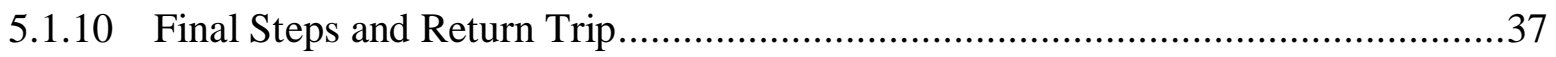

Columbia Power Technologies - Confidential and Proprietary 3

Not for distribution - 2 February 2011 


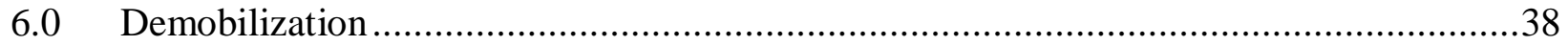

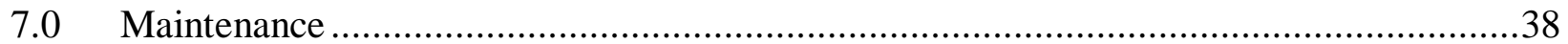

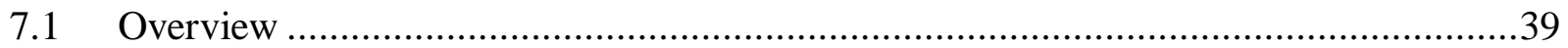

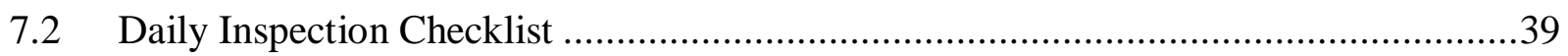

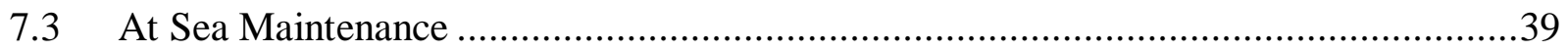

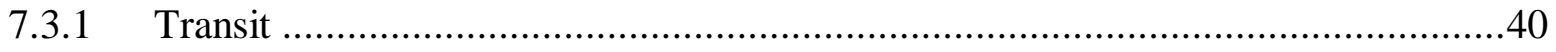

7.3.2 Preparing for Maintenance Operations ……….....................................................40

7.3.3 Maintenance Operations..................................................................................

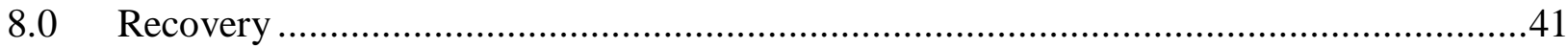

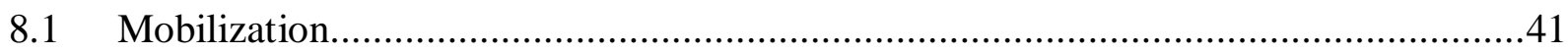

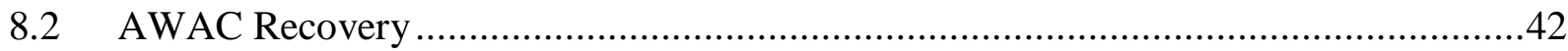

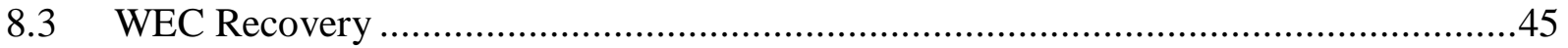

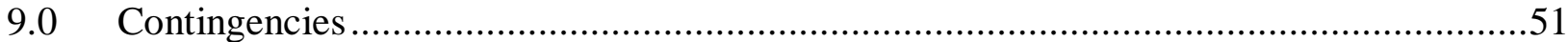

9.1 Retrieving the WEC for Service/Maintenance (If Necessary) ………………..............52

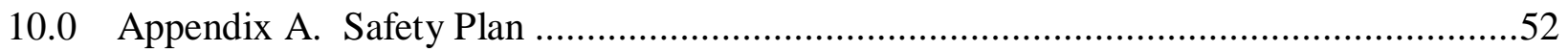

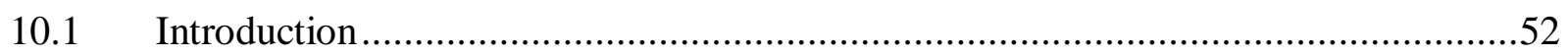

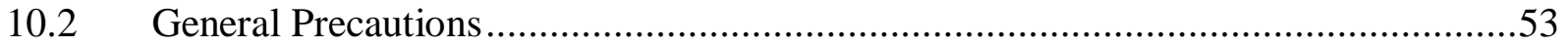

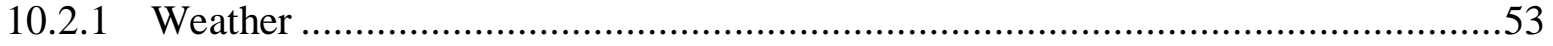

10.2.2 Personal Protective and Safety Equipment ………..............................................53

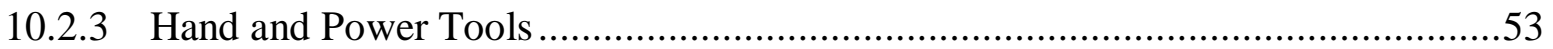

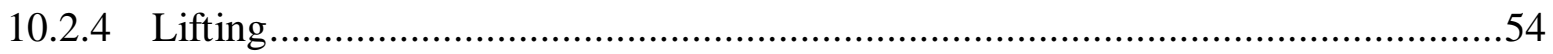

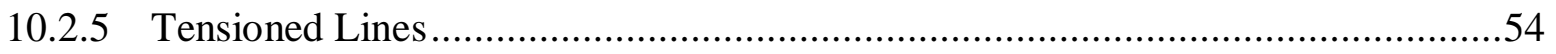

10.2.6 SST Lifting Equipment .................................................................................5

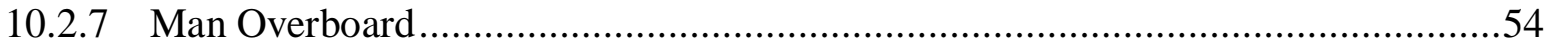

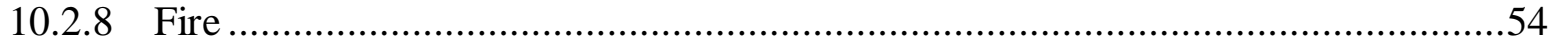

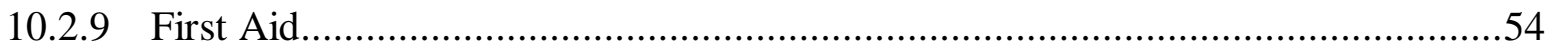

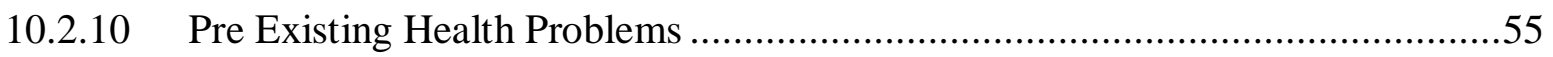

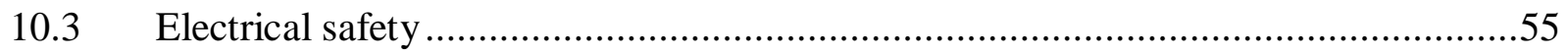

10.3.1 Electrical Safety Procedures...............................................................................5

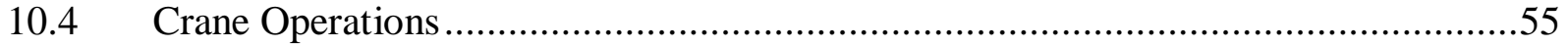

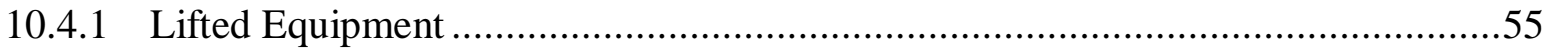

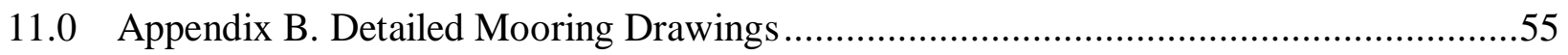

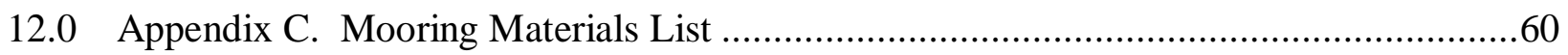

13.0 Appendix D. Component Specifications ....................................................................61

Columbia Power Technologies - Confidential and Proprietary 4

Not for distribution - 2 February 2011 


\subsection{Overview}

This plan covers the deployment of CPT's $1 / 7^{\text {th }}$ scale wave energy converter (WEC), slated for installation beginning 27 January 2011. The deployment will occur off West Point, Puget Sound at approximately $66 \mathrm{ft}$ MLLW. The buoy will be deployed using a crane barge and held in a 3point moor. The buoy deployment is scheduled to last for approximately four months, and is intended to collect data to be used for the design of CPT's full scale WEC.

\subsection{Equipment, Vessels and Personnel}

\subsection{Vessels}

\subsubsection{SEAHORSE}

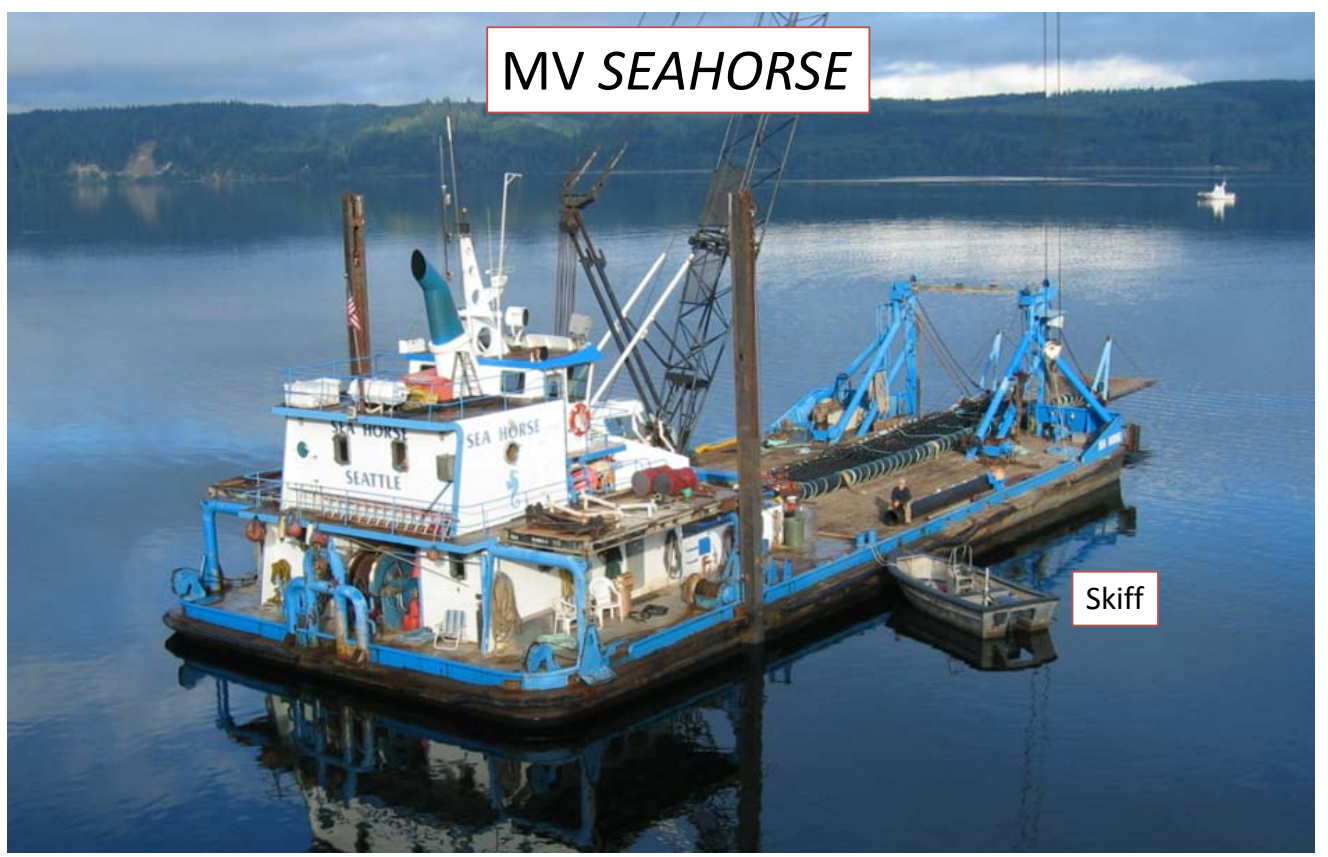

Figure 1. SEAHORSE and one of the provided skiffs. 


\section{SEAHORSE}

- Northern Marine Salvage Co. (Seattle)

- Length Overall

107.6'

- Main Engines:

Twin CATD343 T/A 415 HP

- Breadth Over Guards

42

- Depth Molded

7

- Deck:

3/8" Steel with 4" X 12“" Plank, 40' X 60'

- 4 - 1,000 lb Danforth Anchors

- 1 - 6,000 lb Danforth Anchor

- 4 - Gearmatic Winches $(25,000 \mathrm{lb})$ with 1000 ' of $3 / 4$ " cable capacity

- 1 - stern towing winch $(150,000 \mathrm{lb})$

- $90,000 \mathrm{lb}$. "D" Rings every 10' on Deck

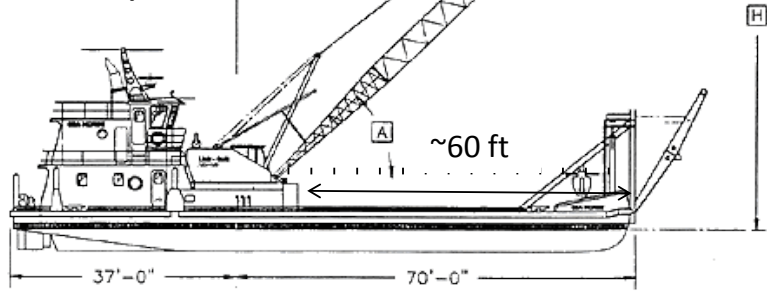

Figure 2.

MV SEAHORSE Crane Capacity
\begin{tabular}{|l|l|l|l|}
\hline \multicolumn{1}{|c|}{ R (ft) } & \multicolumn{1}{c|}{ L (\#) } & \multicolumn{1}{c|}{ H (ft) } & \multicolumn{1}{|c|}{ ( (') } \\
\hline 30 & 52,300 & 137 & 78.1 \\
\hline 35 & 44,800 & 135 & 75.8 \\
\hline 40 & 38,200 & 133 & 73.5 \\
\hline 50 & 29,000 & 130 & 68.9 \\
\hline 60 & 23,100 & 125 & 64.1 \\
\hline 70 & 18,800 & 120 & 59.1 \\
\hline 80 & 15,700 & 113 & 53.8 \\
\hline 90 & 13,200 & 104 & 48.1 \\
\hline 100 & 11,300 & 93 & 41.8 \\
\hline 100 & 9,700 & 80 & 34.7 \\
\hline 120 & 8,400 & 60 & 26 \\
\hline 130 & 7,200 & 10 & 12.7 \\
\hline
\end{tabular}

Note: Seahorse Maximum Crane Extension $(\mathrm{R})$ and Height $(\mathrm{H})$ will be limited to 100 feet (Extension arm is removed). Allowable max load will be higher, although not necessary for this installation.

Figure 3. 


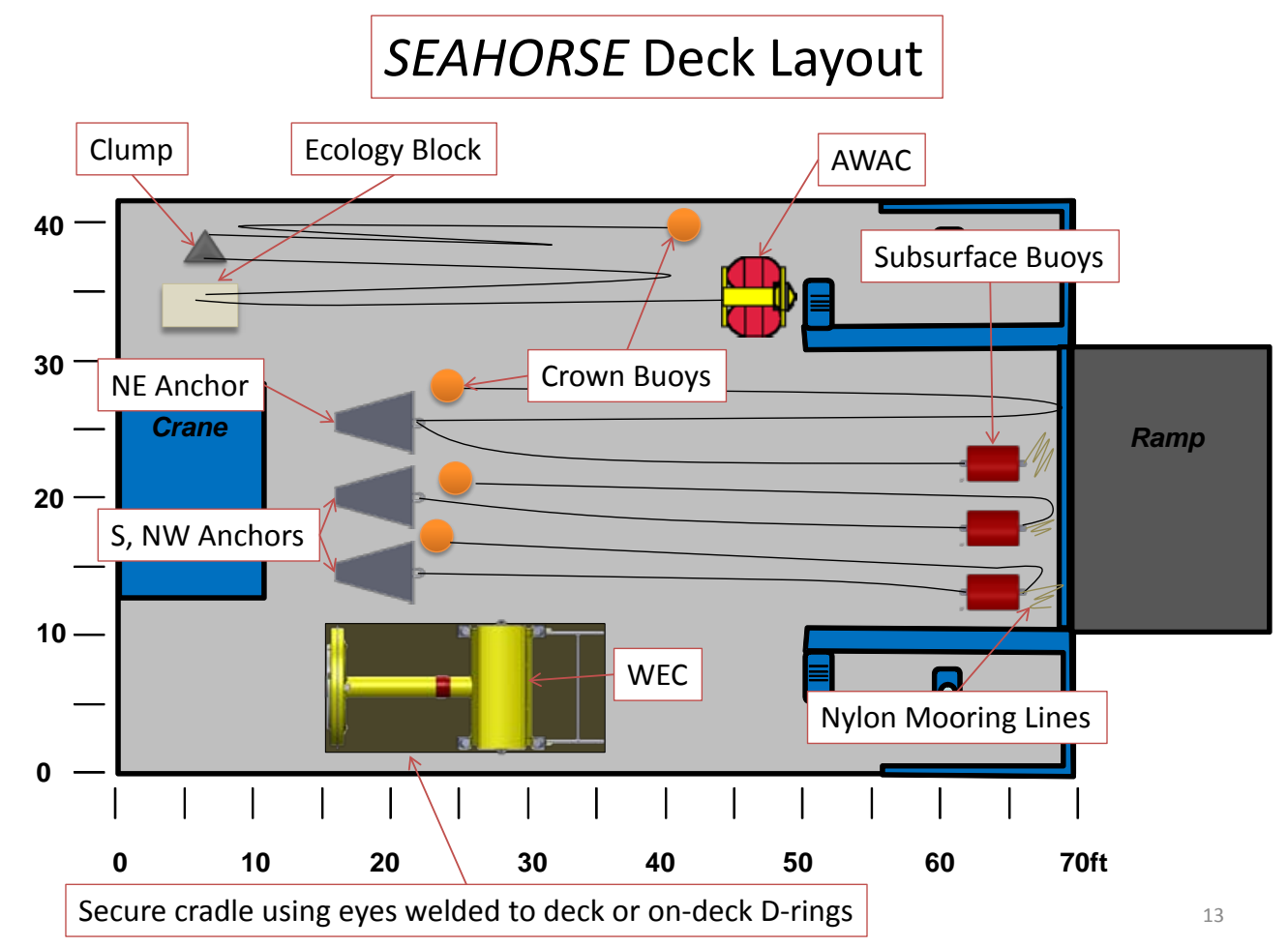

Figure 4.

\subsubsection{Maintenance Vessel: $R V$ Neper}

Sound Support Marine will provide the RV Neper (Figure 5) for maintenance and charging operations. The $R V$ Neper is $22.5 \mathrm{ft}$ in length and is powered by a $5 \mathrm{~L}$ gasoline engine. Gordon Roberts is the captain and will be present for all operations involving the vessel. The Sound Support Research Vessel $R V$ Neper will be made available to perform the tasks defined in the maintenance section of this deployment plan. The Research Vessel $R V$ Neper and vessel operator will be available on-call 24 hours a day 7 days a week during scheduled the periods of operation. Although not anticipated, the Research Vessel RV Neper could experience equipment malfunction that results in it not being fully available. Sound Support will provide at no extra cost to the Charterer a backup vessel Figment Too to support WEC Buoy operations until $R V$ Neper is returned to service. Carl Gowler (SST) is a licensed captain and will be available to drive the $R V$ Neper in the event that Gordon is not able.

The RV Neper will be stored at Brichard-Agee dry storage near the Ballard Locks. It will be trailered to the Shilshole Guest Launch for maintenance and inspection trips. 


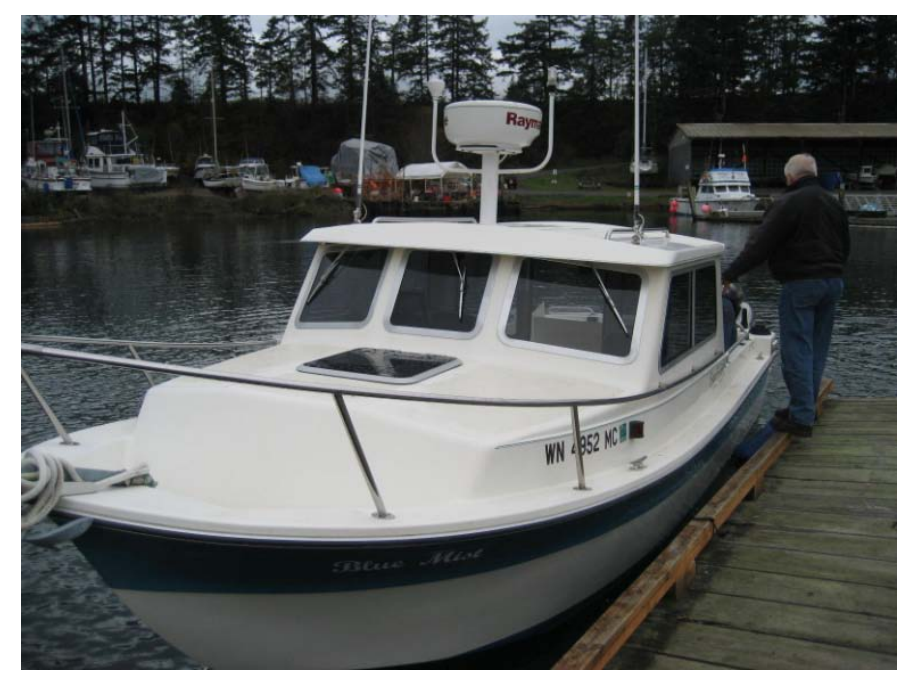

Figure 5. RV Neper Maintenance Vessel.

\subsubsection{Skiffs}

The SEAHORSE will provide 18' and 15' aluminum skiffs to be used during the WEC deployment.

\subsection{Equipment List}

\subsubsection{Mooring Configuration}

Figure 6 shows the WEC mooring configuration. Detailed component views are given in Figure 7, Figure 8, Figure 9, Figure 10 and Figure 11. The AWAC mooring configuration is given in Figure 12. Detailed component specifications and a bill of materials are given in section 13.0.

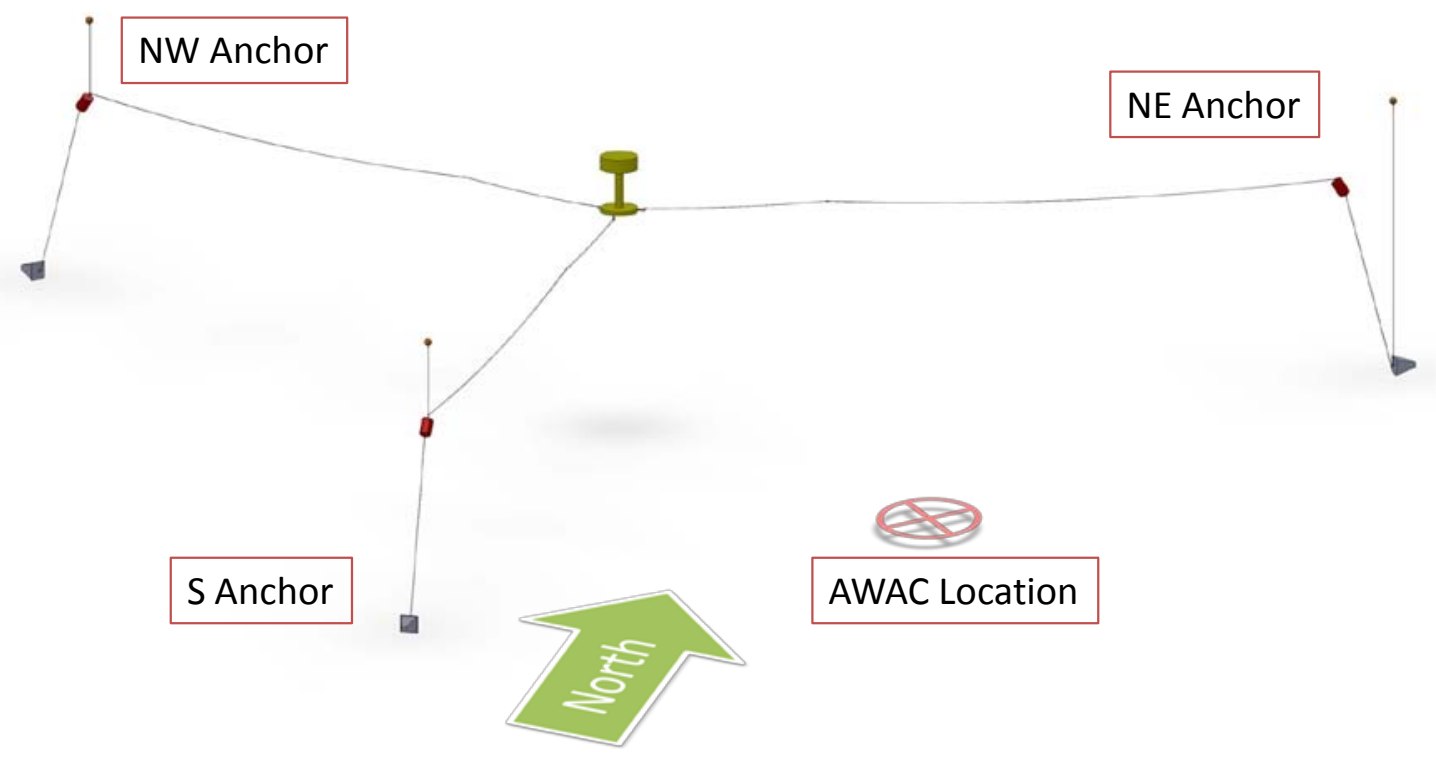

Figure 6. Mooring Configuration. 
NE Mooring Leg Configuration

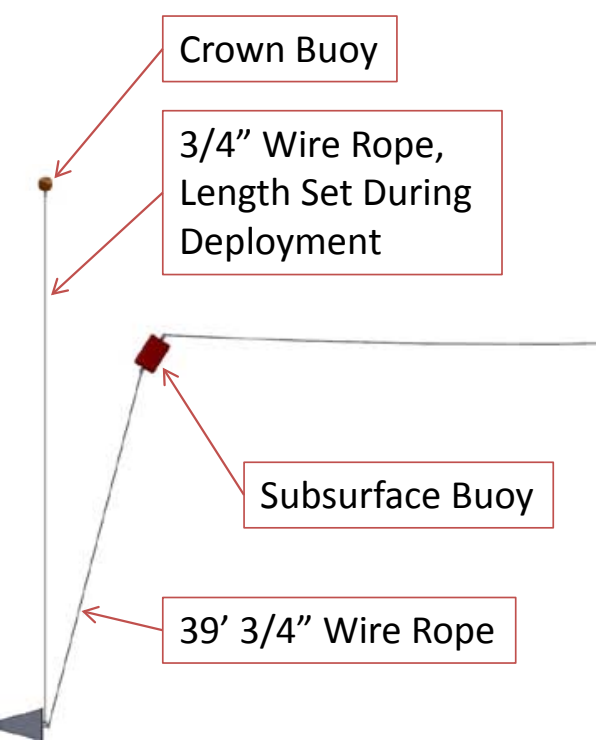




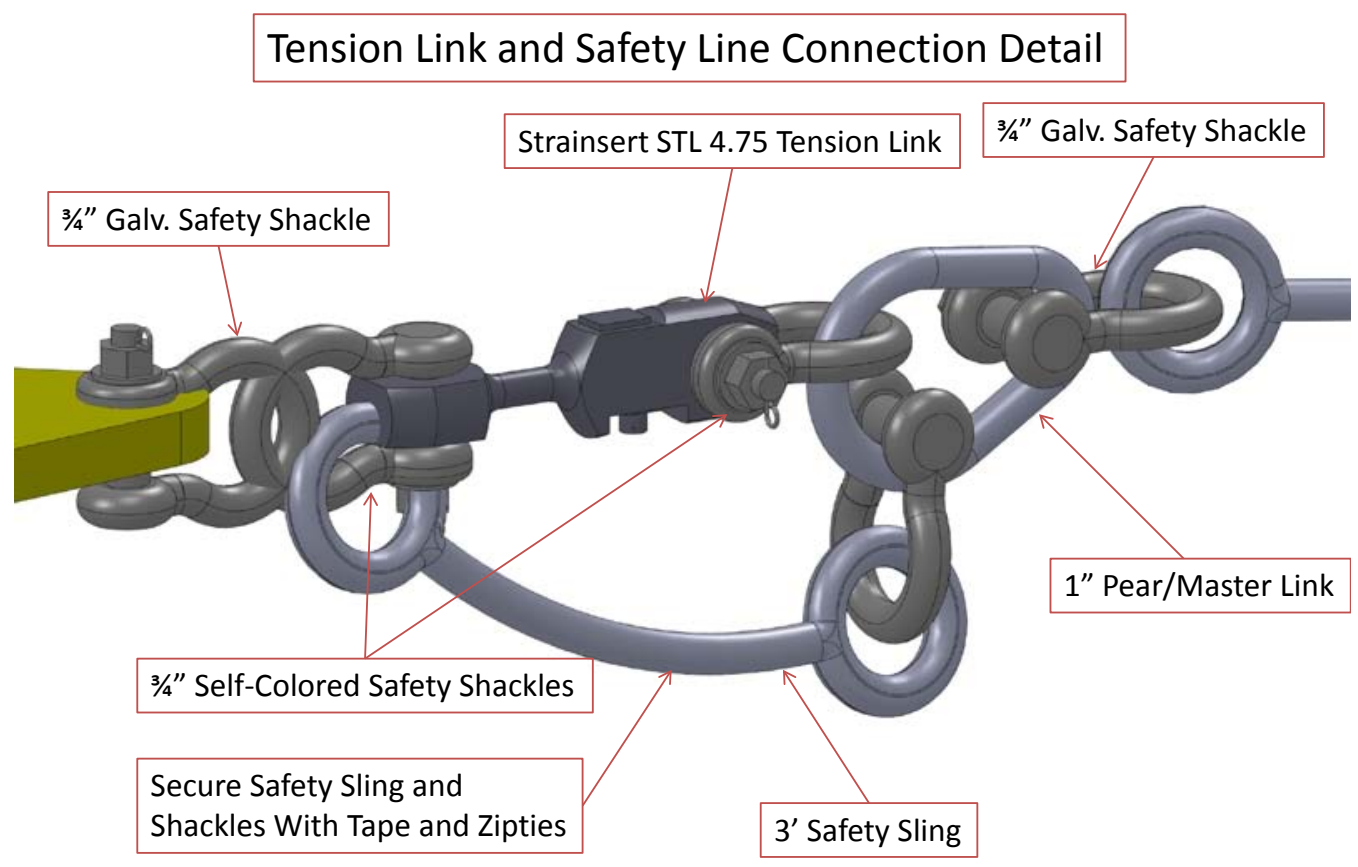

Figure 9. Tension link and Safety Line Connection Detail. Note that the safety sling will be attached to a separate padeye on the WEC (Not shown).

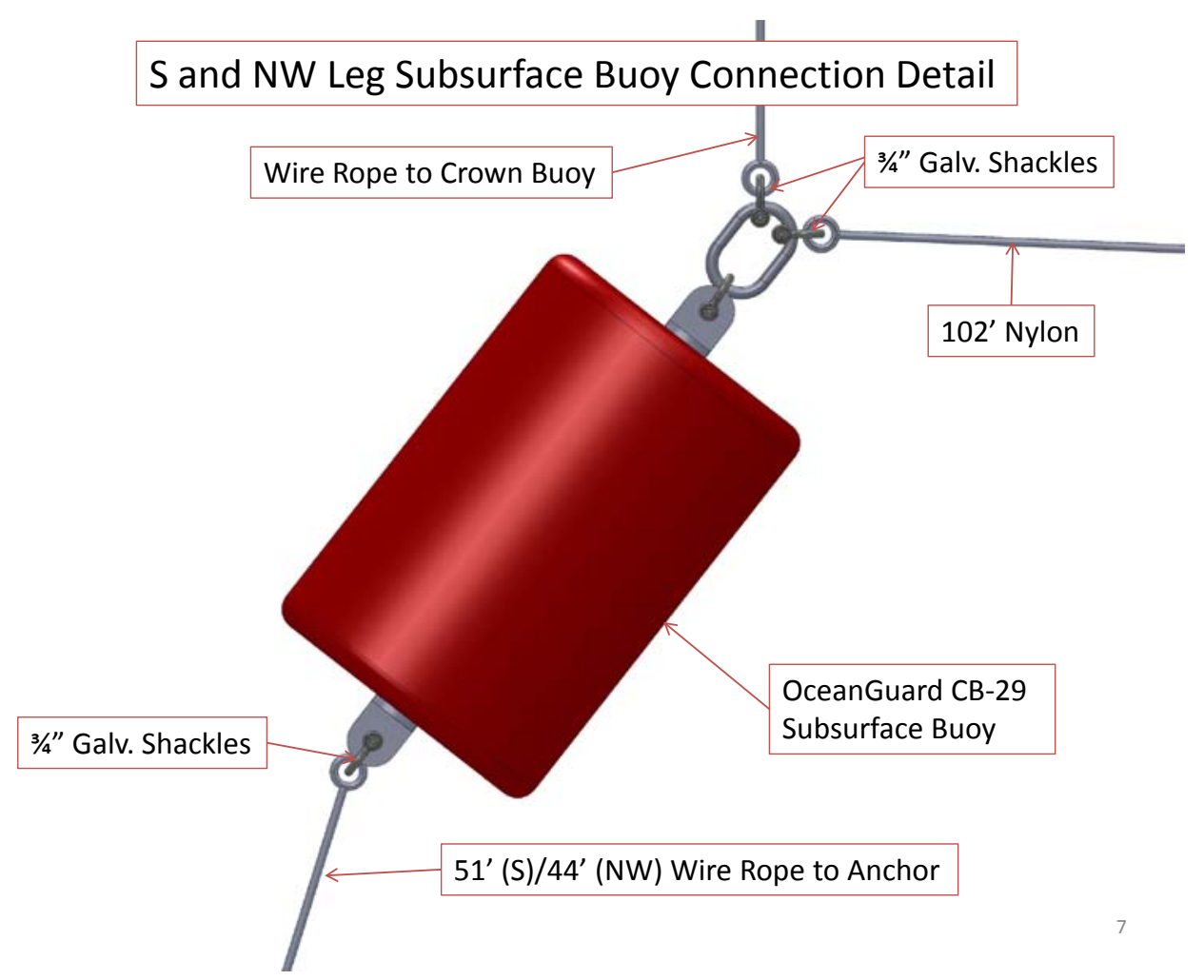

Figure 10. Subsurface buoy detail. Note that this only applies to the $S$ and NW legs, as the recovery line is not attached to the subsurface buoy on the NE leg.

Not for distribution - 2 February 2011 


\section{Mooring to WEC Nylon Line Connection Detail}

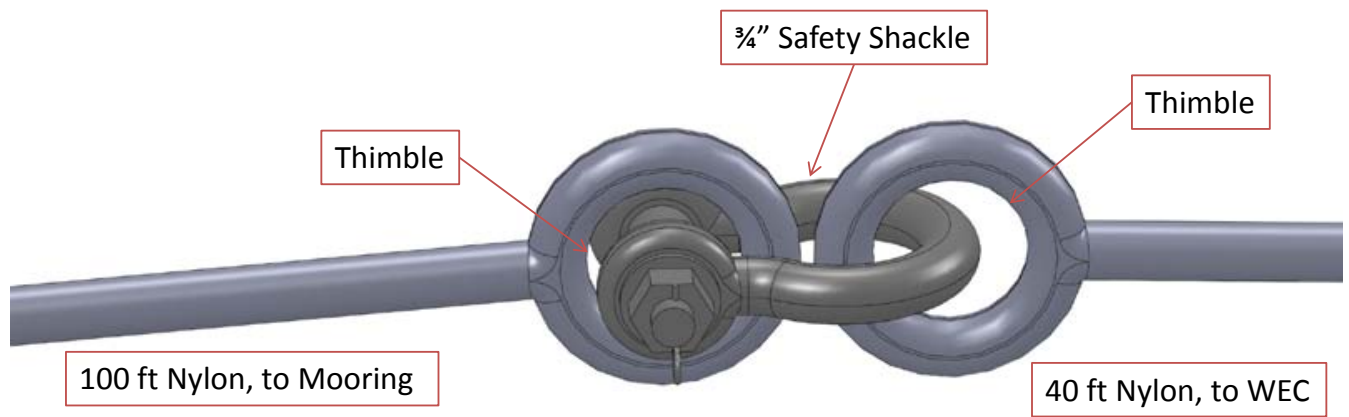

Figure 11. Connection between $40 \mathrm{ft}$ nylon (initially connected to the WEC) and $100 \mathrm{ft}$ nylon lines (initially connected to the subsurface buoy). The connection is made at this point because the connection point on the WEC damper plate will be submerged.

\section{AWAC Mooring Configuration}

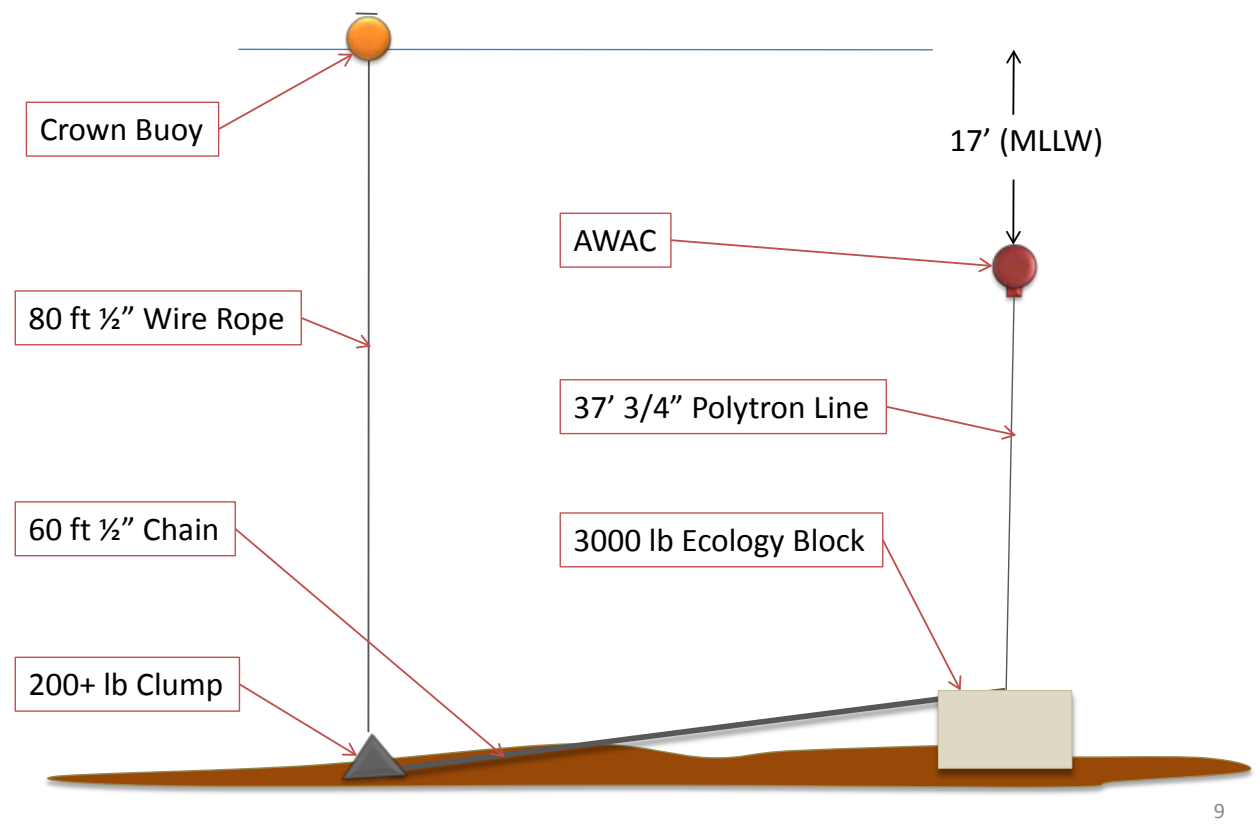

Figure 12. AWAC Mooring configuration. A separate recovery line is used to avoid having lines in the AWAC's field of view, and to avoid the use of acoustic releases or grappling.

\subsubsection{Additional Equipment}

The following equipment will be required. The party responsible for providing equipment is also given.

- Northstar 951 GPS/Antenna (SST)

- $\quad$ PFD’s, Steel Toed Boots, Hardhats (SST/CPT)

- Lunch/Snacks (TBD)

- $\quad$ Shackle Mousing Wire (SST) 
- Tools: Pliers, Adjustable Wrenches, Wire Cutters, etc. (SST)

\subsection{Personnel}

The following people will be required for the deployment:

\begin{tabular}{lll}
\hline Company & Employee & Role(s) \\
\hline Columbia Power & Ken Rhinefrank & Test Director \\
& Joe Prudell & Rigging/Electrical \\
& Al Schacher & SCADA Systems \\
& Erik Hammagren & Rigging/Electrical \\
& Ted Schacher & Standby \\
& Mark Brown & Independent Observer \\
\hline Sound and Sea & Carl Gowler & Safety Officer, Deck Supervisor \\
& Sam Gooch & Operations Supervisor \\
& Matt Ramey & Deck Operations \\
& Ryan Gowler & Deck Operations \\
\hline Northern Marine Salvage & Brian Carlson & SEAHORSE Operator \\
& Charlie & Crane Operator \\
\hline Sound Support Marine & Gordon Roberts & Maintenance Vessel Captain/Operator \\
\hline Navy & Warren Bartel & Guest \\
& Brian Cable & Guest \\
& Alexandra Devisser & Guest
\end{tabular}

\subsection{Mobilization}

\subsection{Mooring}

Anchors will be brought down from the Bangor Sub Base on a flatbed truck and brought to the west wall during the deployment mobilization. Subsurface buoys (stored at the SST warehouse in Lynnwood) will be brought down using a SST furnished trailer, along with the mooring hardware.

\subsection{WEC}

The Wave Energy Converter (WEC) will be trucked from Ershig's to the Seahorse, and transferred at the west wall at Fisherman's Terminal, shown in Figure 13. 


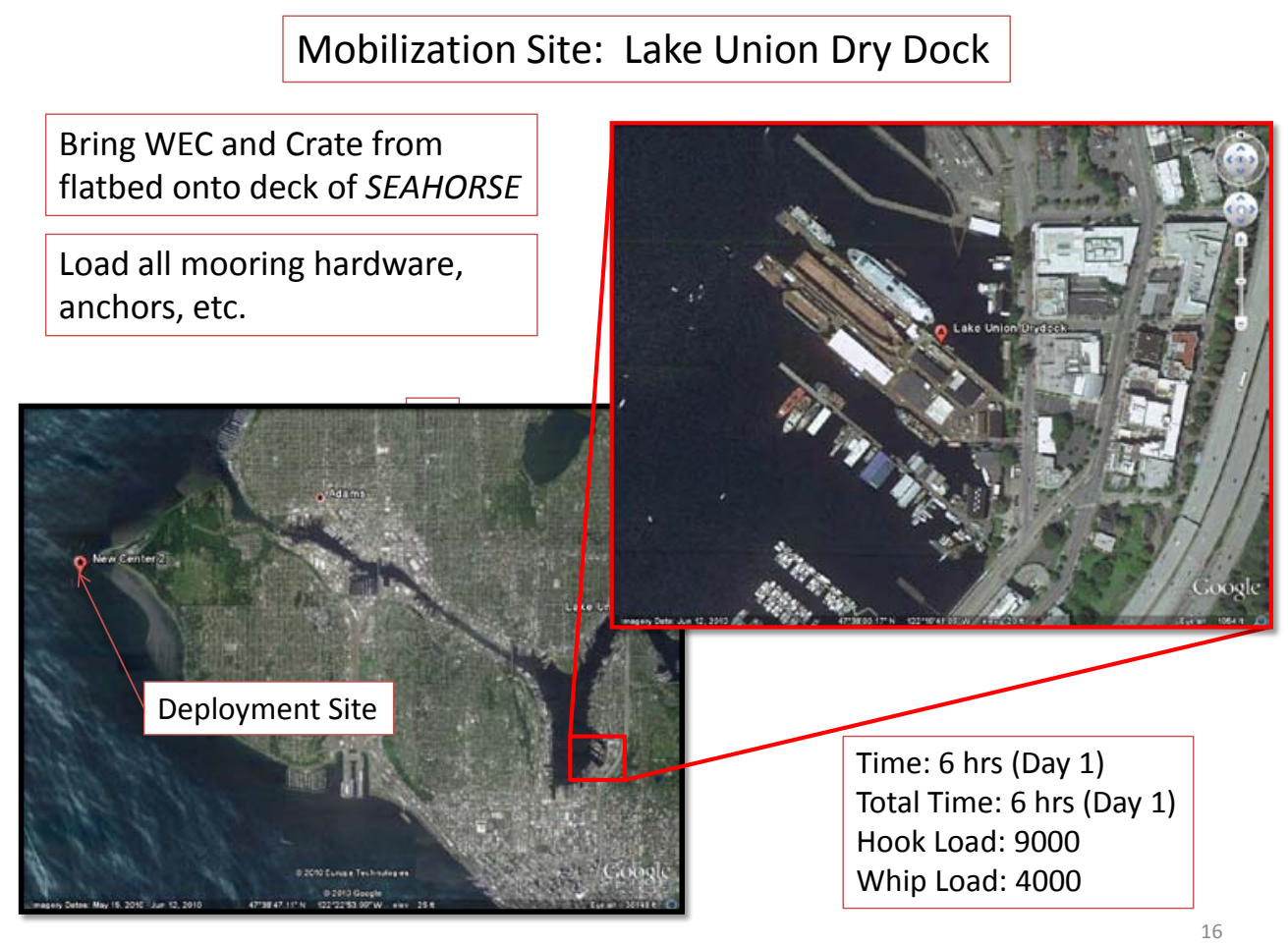

Figure 13. Seahorse loading location at the West Wall, Fisherman's Terminal. Deployment site shown in red.

\subsection{Loading the SEAHORSE}

Mobilization will occur at the Lake Union Dry Dock. During the mobilization day, all components requiring use of the SEAHORSE's crane will be brought onboard, including the WEC/Cradle, AWAC, all anchors and subsurface buoys. Mooring hardware will be brought down from the SST warehouse; the $6000 \mathrm{lb}$ anchors, ecology block and AWAC clump will be brought from Bangor Navy base on a flatbed truck. A diagram of the WEC cradle and rigging is shown in Figure 14. The WEC cradle will be secured to the deck using the D-rings available on the SEAHORSE. 


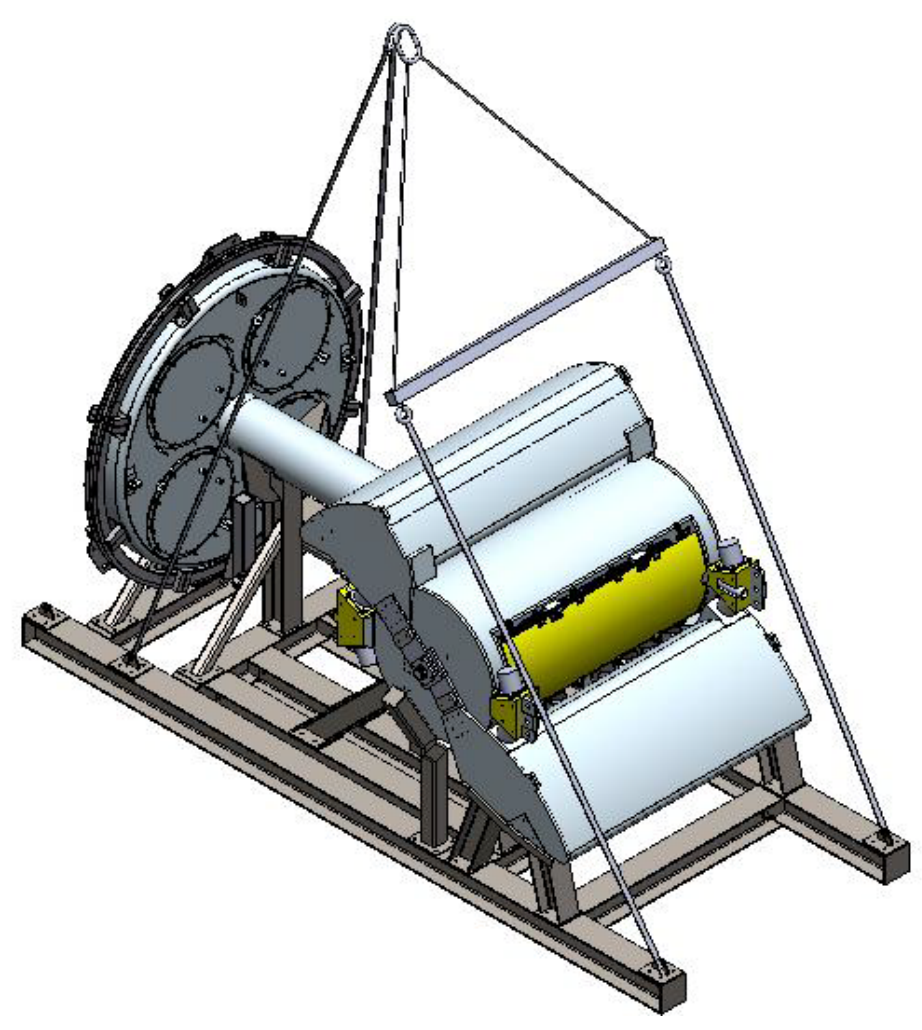

Figure 14. WEC, Crate and Rigging Hardware.

\subsection{Mooring Set Up}

After the mooring hardware and anchors are brought onto the SEAHORSE, the components must be assembled into the complete mooring leg assemblies. Drawings of these assemblies are given in section 11.0. These should be assembled so that all major components, especially the subsurface buoys, remain safely secured to the deck until they are ready to be deployed. Each mooring leg should be secured so that it may be accessed without moving the other legs.

\subsection{GPS Calibration, Float Acoustic Test and Lifting Exercise}

Prior to deployment, the WEC will be run through a GPS calibration, float acoustic test and lifting exercise. The GPS test consists of rotating the WEC several times to calibrate the onboard unit. The float acoustic test involves taking acoustic measurements of the WEC's floats while they are moved by the SEAHORSE's crane. The tests will occur at Lake Washington. The procedure will occur as follows. Prior to departure:

- Confirm all hardware \& personnel on board

- Power on SCADA and confirm all IO and signals function properly. Use Columbia Power SCADA/electrical checklist.

\subsubsection{Transit and Preliminary Testing}

- Load WEC onto SEAHORSE in cradle

- SEAHORSE transit to Lake Washington: 45 min

- Reconfirm SCADA Systems

- SEAHORSE deploys WEC, releases from crane (shown in deployment procedure)

- WEC floats unlocked (shown in deployment procedure)

- Check WEC waterline, taking fresh/saltwater density into consideration 


\subsubsection{GPS Test}

- $\quad$ SEAHORSE stands off $\sim 500 \mathrm{ft}$ (to avoid interference with calibration)

- Tie small skiff (with electric motor) to float, rotate 3x. Details TBD by Carl Gowler

- Bring SEAHORSE back to WEC

\subsubsection{Float Acoustic Test}

- Attach whip to CPT-provided spreader bar, slings and pear link assembly

- Reattach crane to SST-provided spreader sling assembly, shown in Figure 15

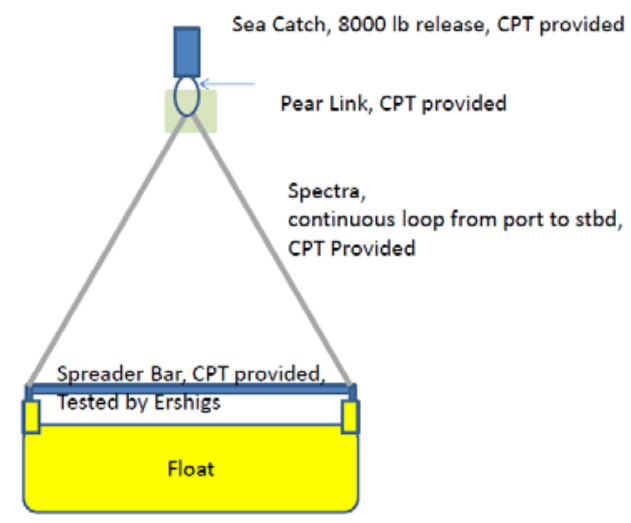

Figure 15. CPT Provided Spreader Sling Assembly.

- Move crane over WEC and attach SST spreader to WEC nacelle (shown in recovery procedure)

- Attach CPT Spreader to forward float. Float “drop” will be performed using a rigging setup shown in Figure 16. Release of the float will be initiated by pulling on the Spectra line from a skiff.

- Using the aforementioned technique, follow CPT provided test procedures:

o This is performed with the buoy raised out of the water at the nacelle lifting points by the crane so that the floats do not touch the water when the floats are down against the lower end stops. Floats are raised and lowered using the whip, a spreader bar, 30 foot spectra lifting bridle, pear link (with floatation/padding) and Seacatch.

o Forward float range of motion testing. Raise lower speed NTE ?? ft/sec

o Forward float PTO power testing. Active and Passive

o Forward float end stop testing

o Perform float motion IO checks IAW CPT document.

o Repeat b, c, d, e for aft float. 


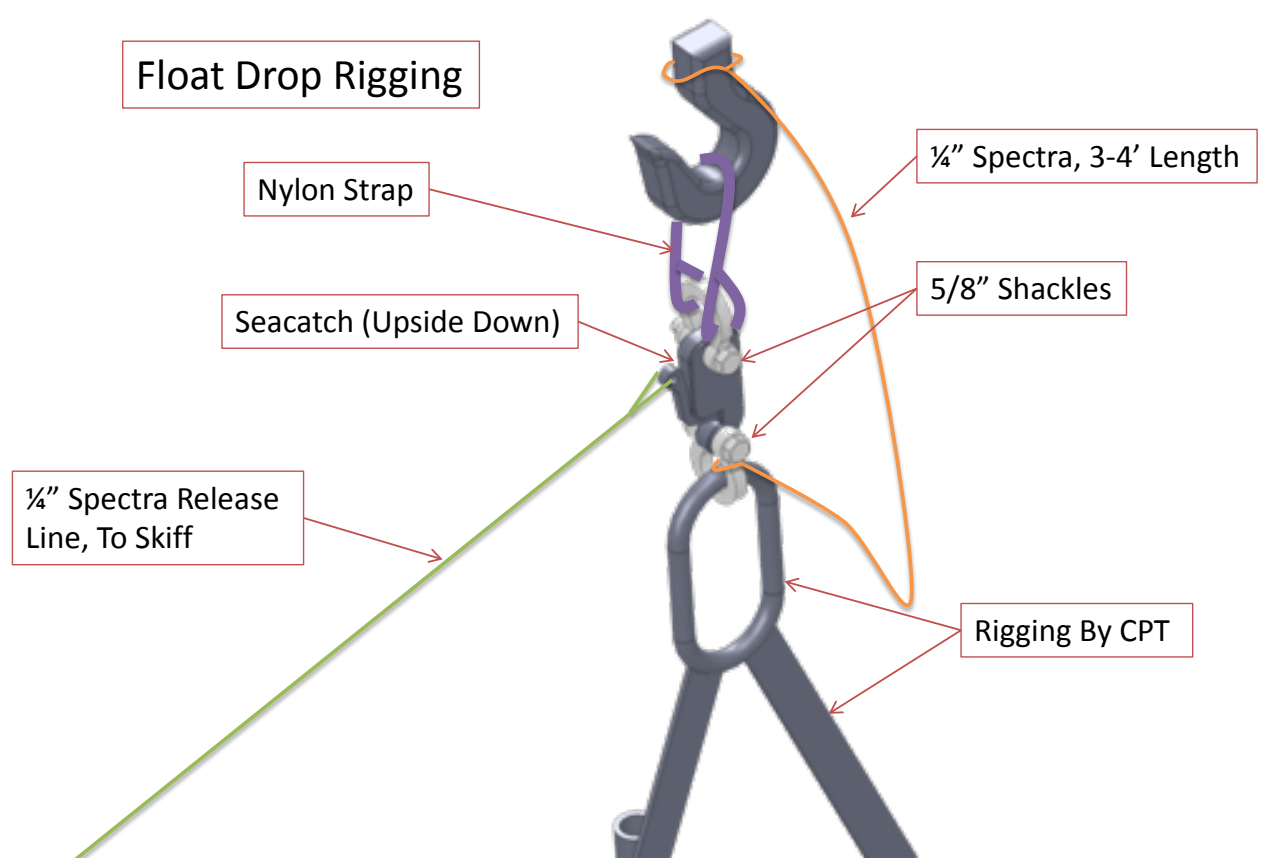

Figure 16. Float "Drop" Rigging Diagram. Pulling on the release line will cause the Seacatch to release the upper shackle. The 3-4' Spectra will catch the rigging hardware, ensuring the float is not damaged. (The Seacatch is upside down to provide the proper angle to release the squib).

\subsubsection{Additional Testing and Recovery}

Perform Yaw Control Test; use crane, SEAHORSE bow/stern and crane to create 3-point moor (if time allows)

IO and other testing per CPT recommendations

- Follow WEC recovery procedure, including forward float lockout

- SEAHORSE transit to deployment site or Lake Union Drydock, TBD

\subsection{Deployment}

After mobilization and pre-deployment testing are complete, the SEAHORSE will transit to the deployment site. This may occur on the same day as the pre-deployment testing, weather and time permitting. The proceeding slides detail the deployment procedure, which occurs as follows:

- Transit to deployment site

- Set NE anchor (live boat)

- Put SEAHORSE into two point moor

- Set $\mathrm{S}$ anchor (in two point moor)

- Rig S, NW anchors to WEC

- Deploy WEC

- Bring WEC to NE anchor

- Set pretension by moving NW anchor into position

- Deploy AWAC 
- Transit back to Lake Union Drydock

Each diagram shows the estimated time to complete that step, along with a running estimate of the total time, and crane and whip loads.

\subsubsection{Site and Transit}

\section{SEAHORSE Transit From Lake Union Dry Dock to Deployment Site}

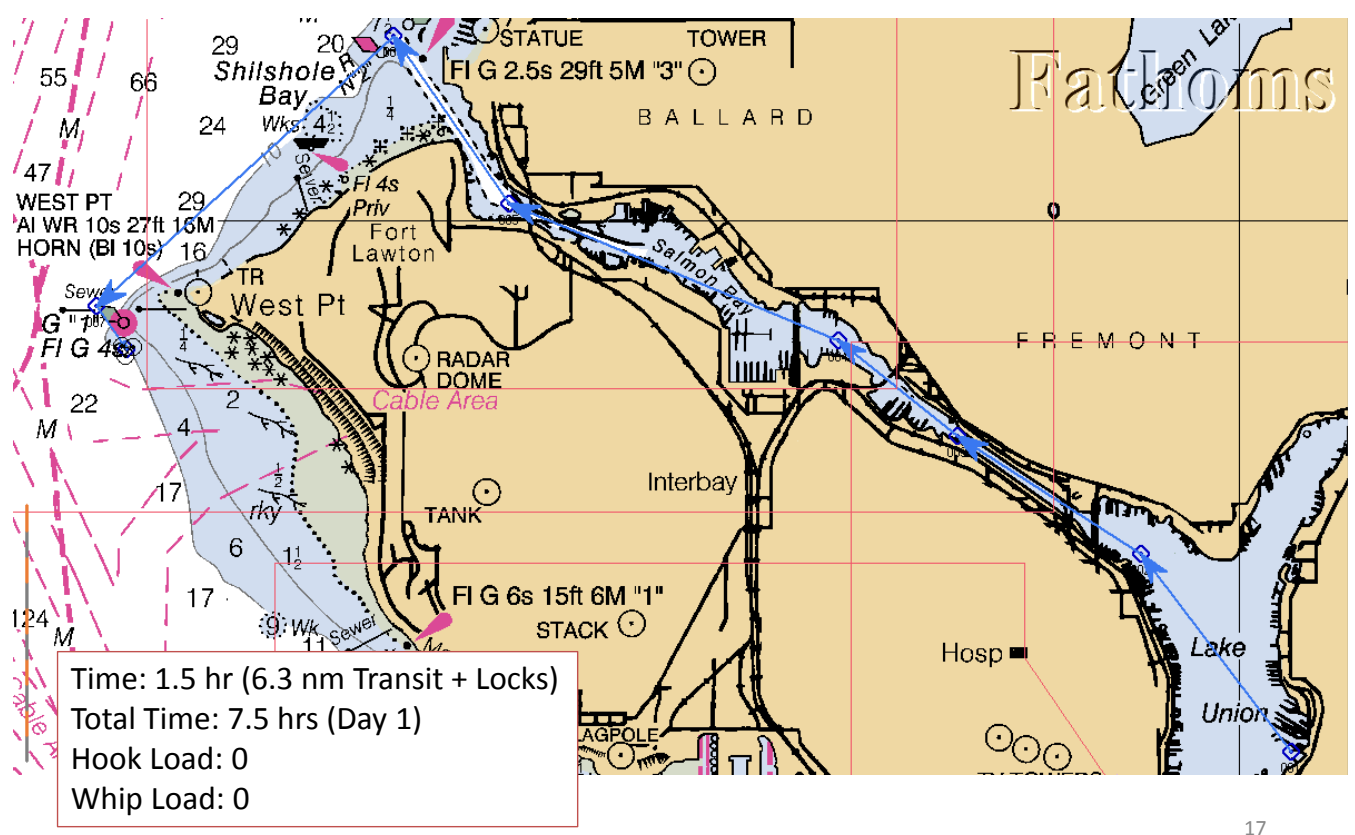

Figure 17. Transit time. Locks will take $\sim 30$ minutes to cross, in addition to travel time. 


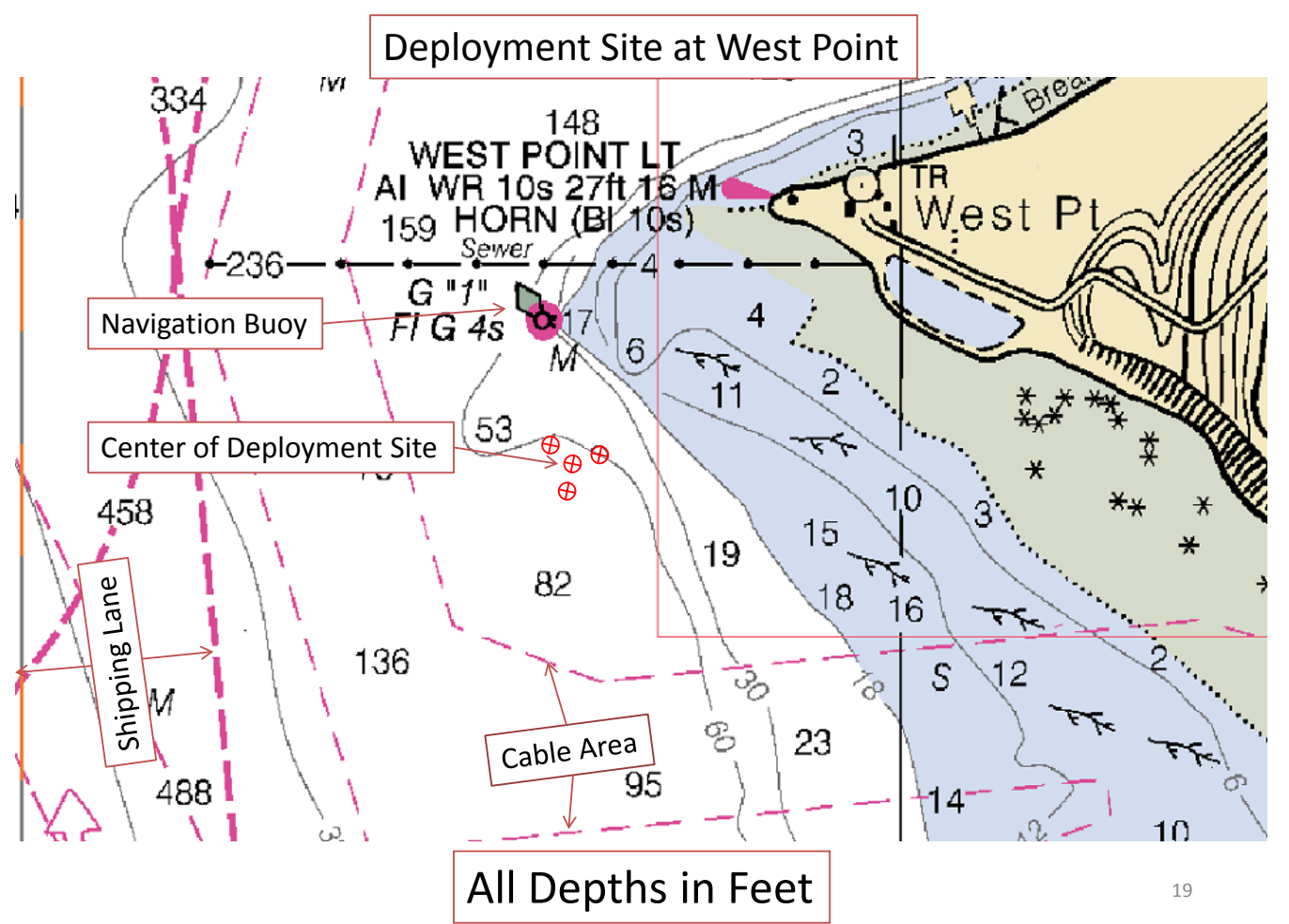

Figure 18. Close up of deployment site.

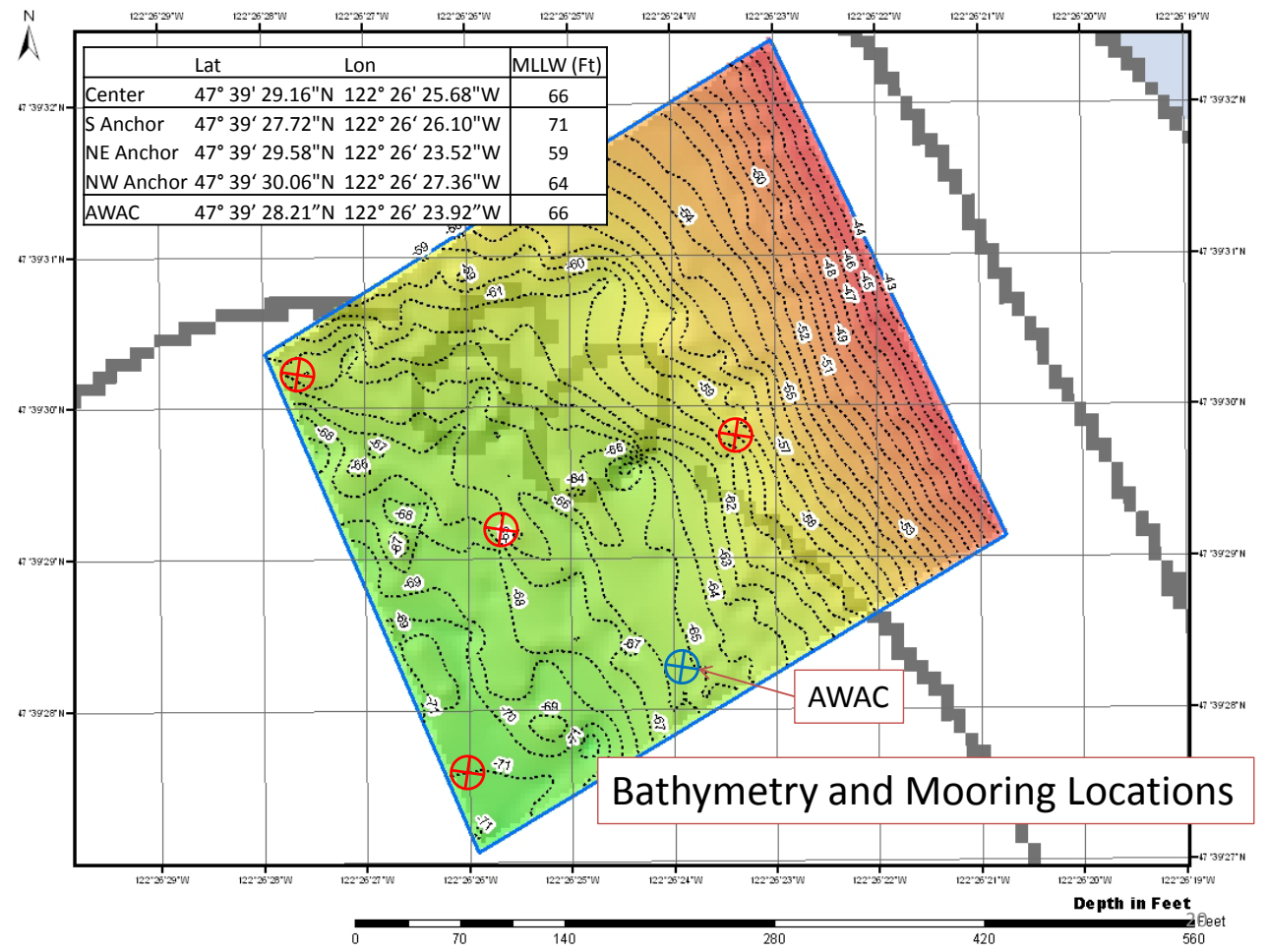

Figure 19. Bathymetry at deployment site. Accurate to within $+/-1.5 \mathrm{ft}$. Data collected by SST. 

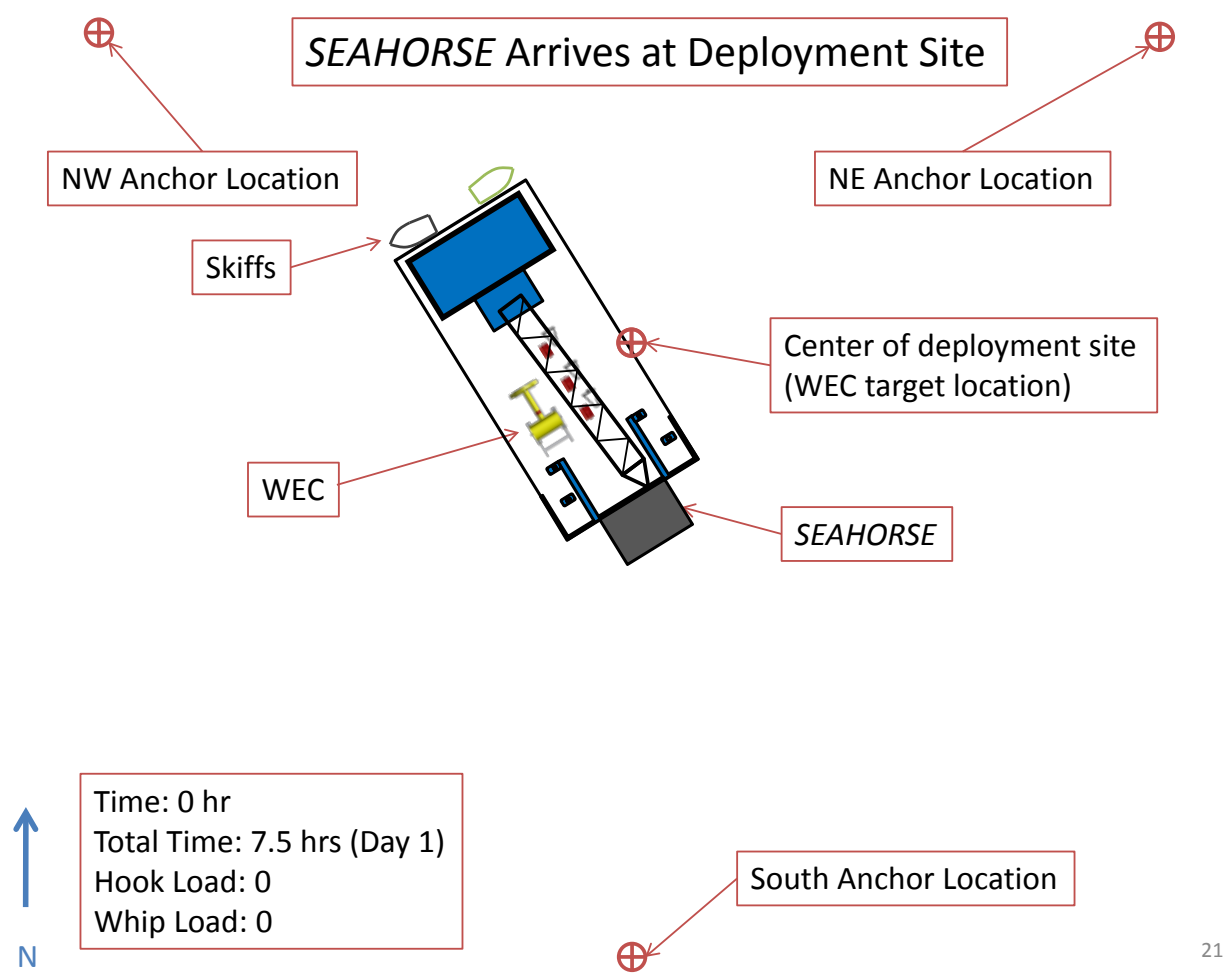

Figure 20. SEAHORSE arriving at deployment site. SEAHORSE will provide the 18' and 15' aluminum skiffs.

\subsubsection{Northwest Mooring Leg Deployment}

\section{Deploying S and NW Mooring Legs: SEAHORSE Crane Rigging}

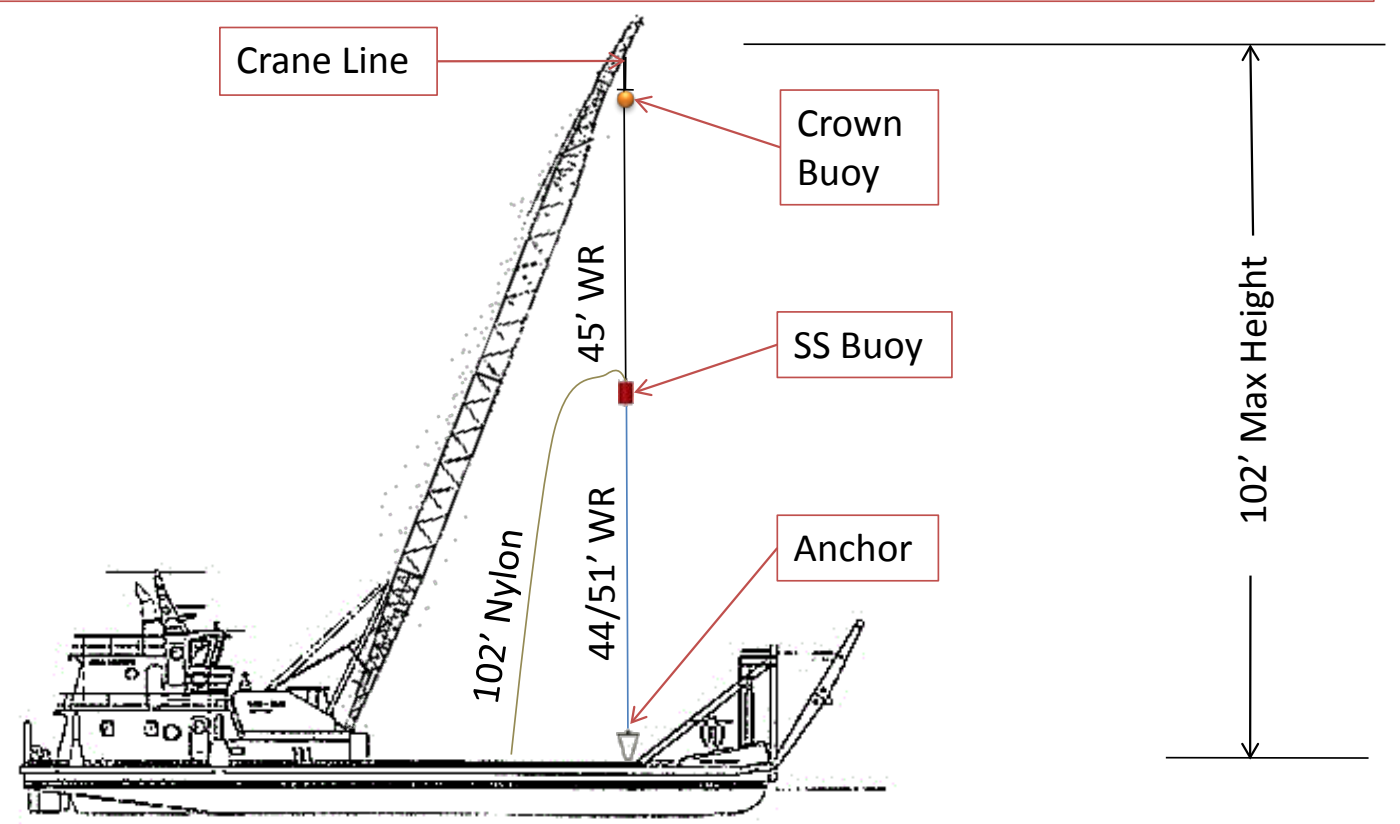

Figure 21. Rigging configuration for S, NW mooring legs. Crane is tall enough to lift entire leg in one pass. 


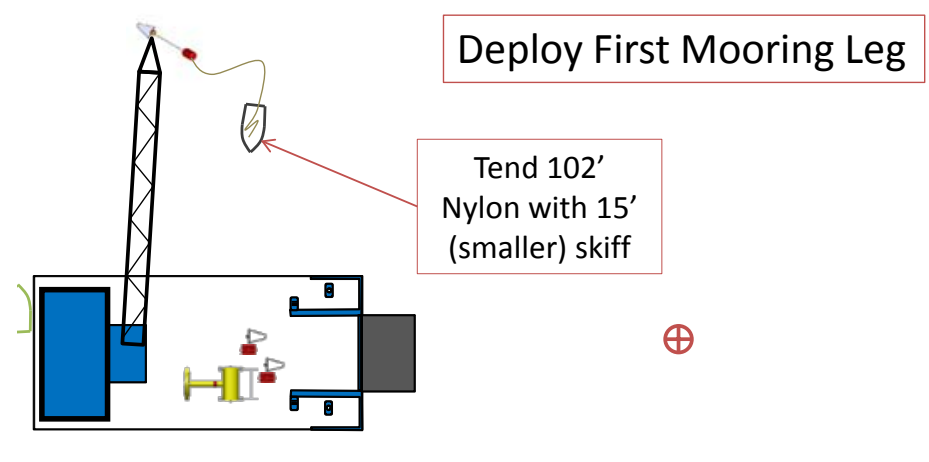

BE SURE TO RECORD ACTUAL ANCHOR GPS COORDINATES!

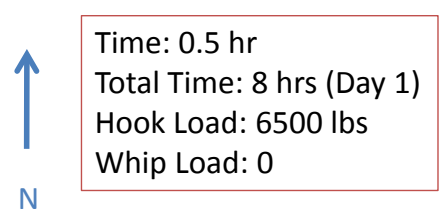

Figure 22. Deploying the first mooring leg. Orient the boat so that the mooring is in the lee. Tend the 102' nylon section with a skiff to avoid fouling the line.

\subsubsection{South Mooring Leg Deployment}

The placement of the Southern mooring leg is relative to the actual placement of the NW leg, nominally given in Figure 19. Using the OpenCPN navigational program and the input from the Magellan 315 GPS (mounted at the apex of the SEAHORSE crane), the exact locations of the second (S) and third (NE) mooring legs can be determined using the offsets shown in Figure 23 relative to the northwest mooring leg.. This will ensure even line tensions in the mooring legs.

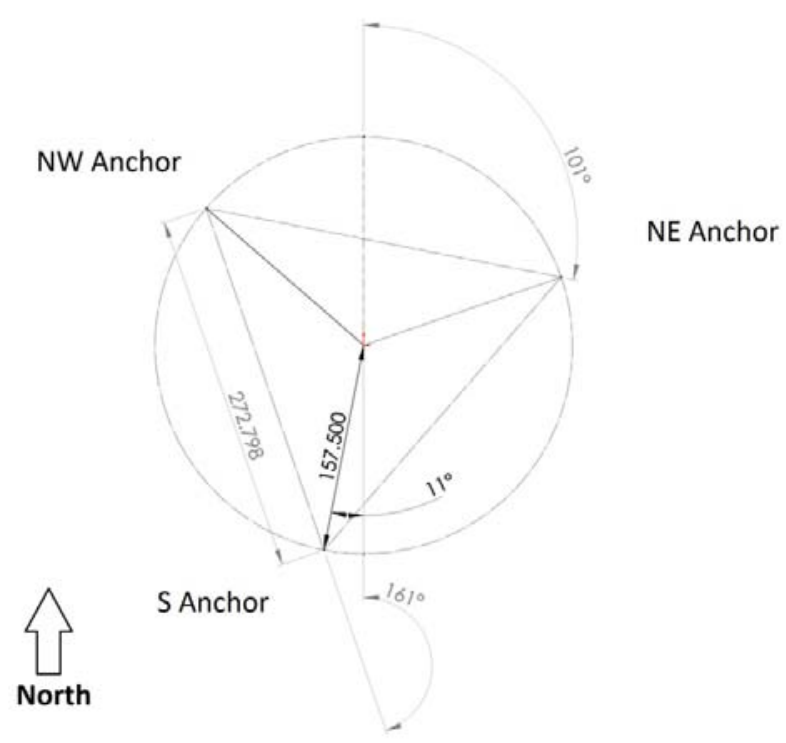

Figure 23. Angles and distances of S and NE anchors relative to NW anchor location. All lengths are in feet. 


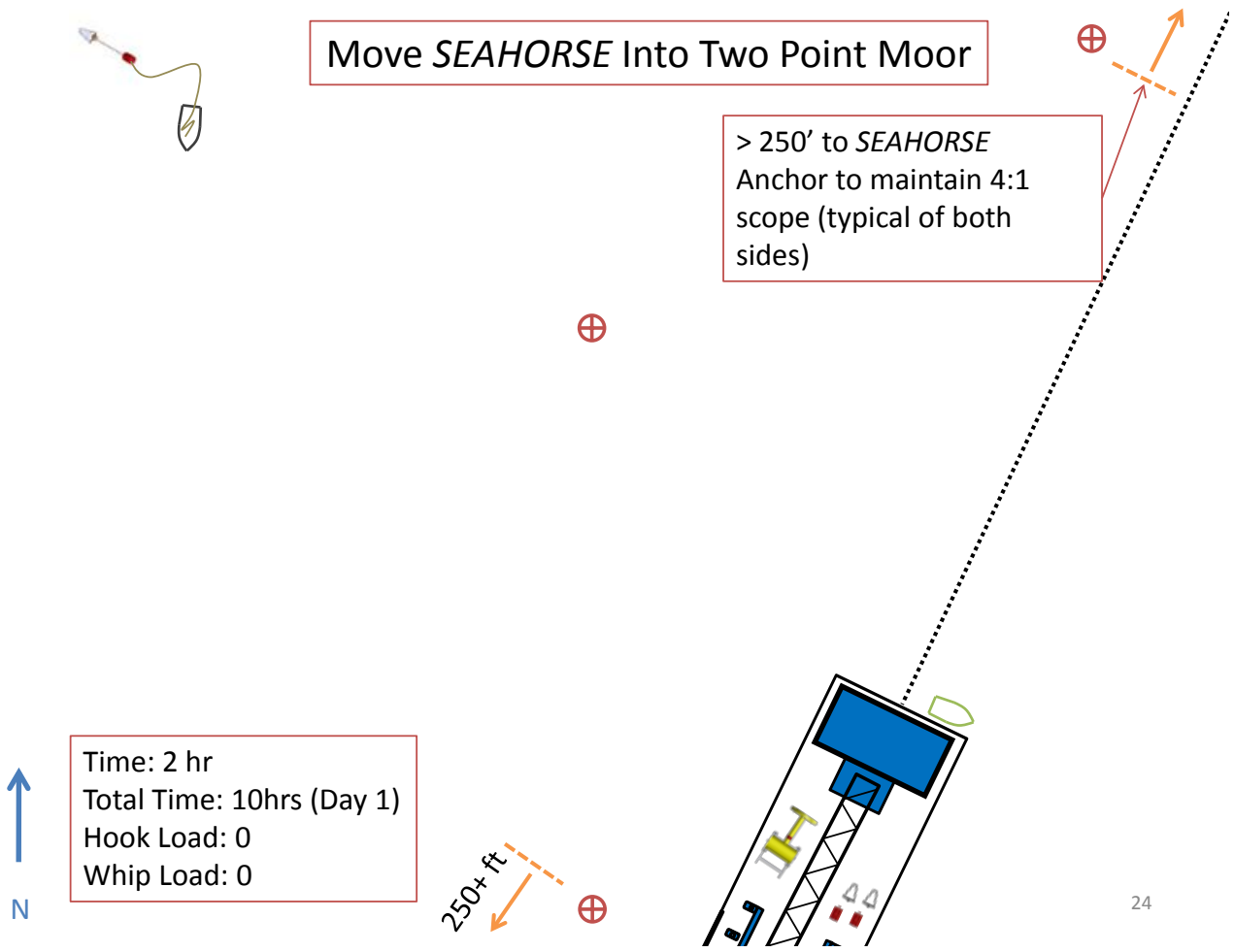

Figure 24. Putting the SEAHORSE into a 2-point moor. The SEAHORSE can set its own anchors. However, if there is a time constraint, a tug can be employed. The anchors must be placed greater than 250 feet away from the target anchor locations to maintain scope.

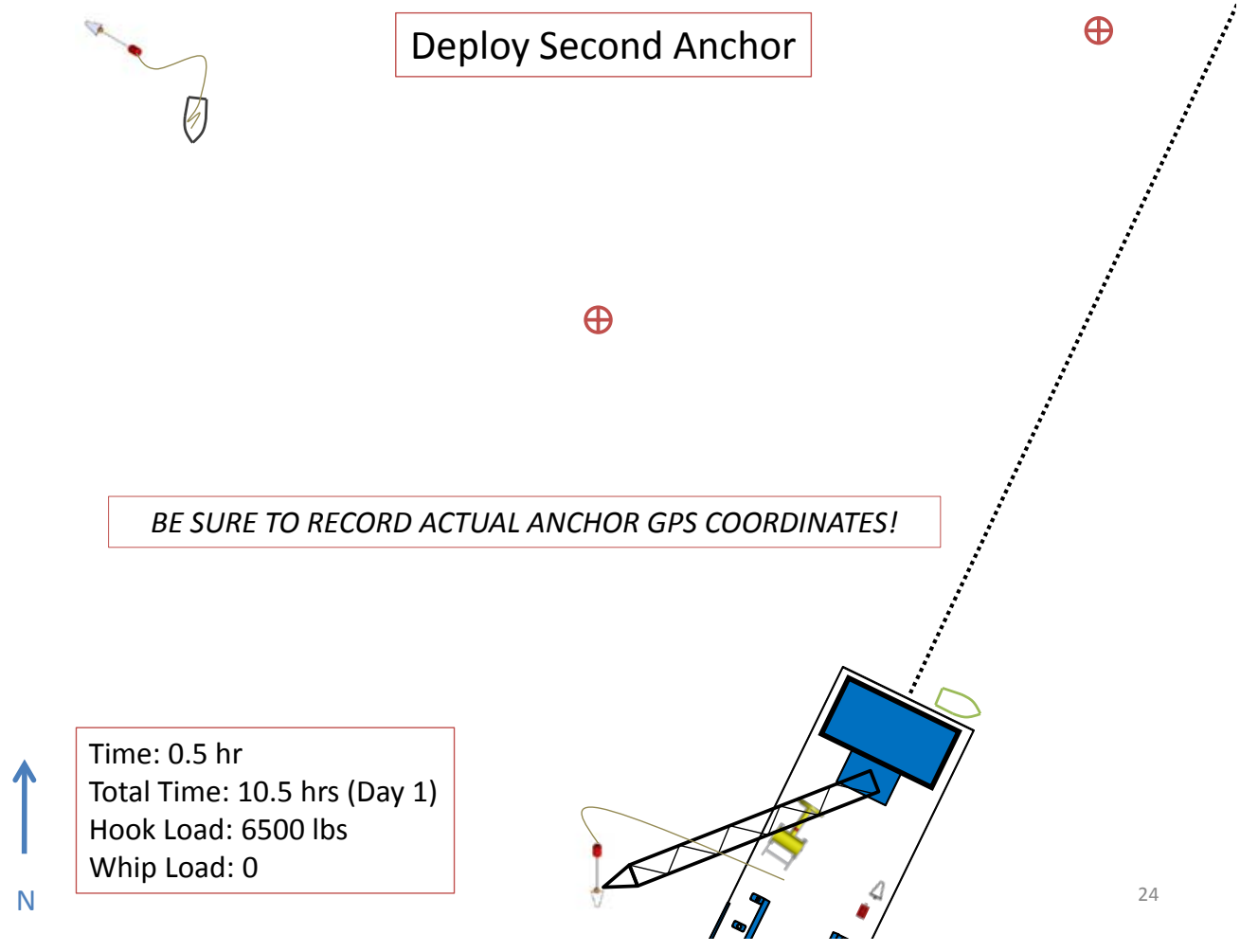

Figure 25. Setting the second anchor. Since the SEAHORSE is in a 2-point moor, this anchor can be placed very accurately. It should be placed relative to the exact position of the first anchor. 


\subsubsection{Optional Steps for Overnight Stopping Point}

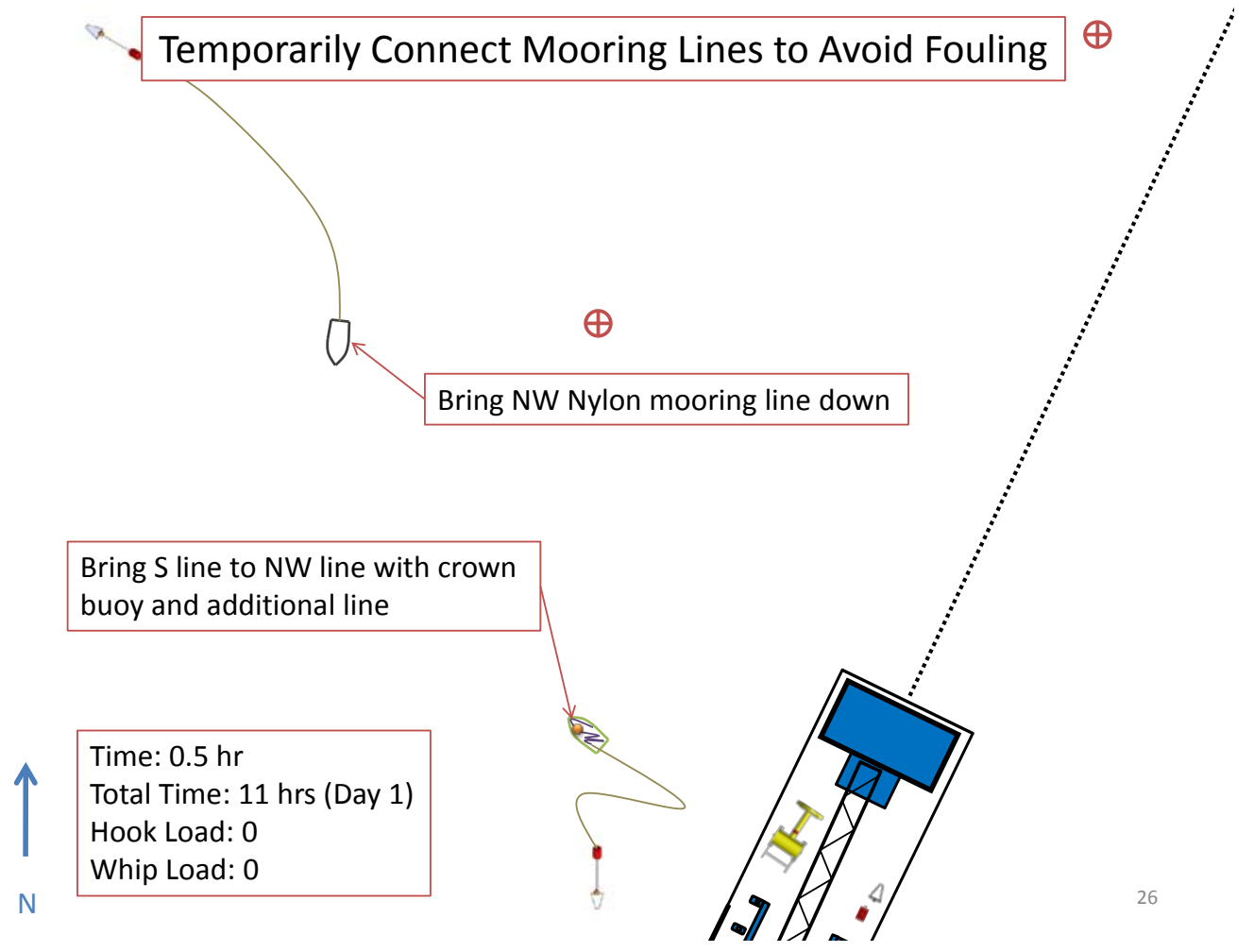

Figure 26. Connecting the mooring lines together with a crown buoy will ensure they do not become fouled.

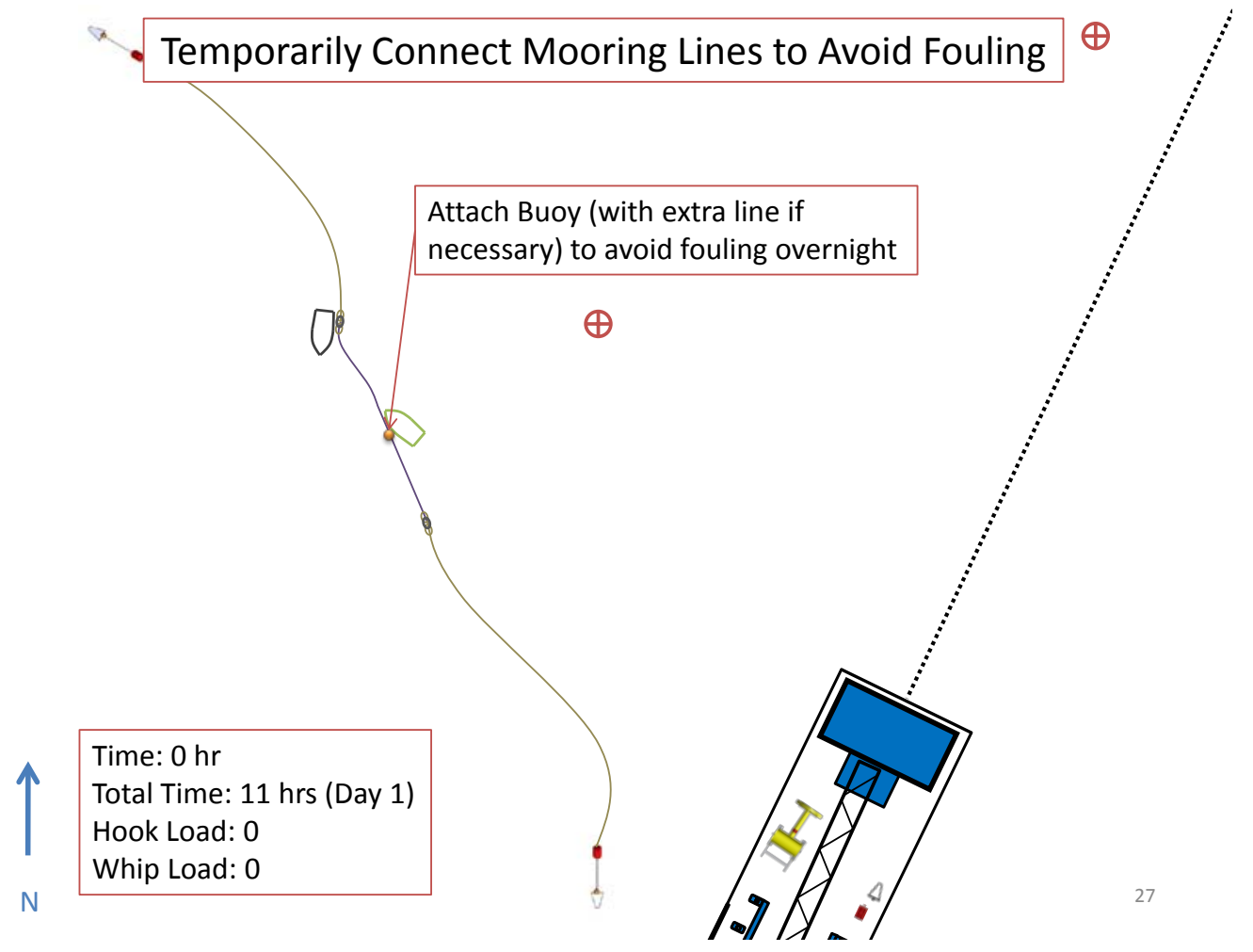

Figure 27. Note: The two mooring lines should only be connected if it is necessary to leave the moorings in place overnight; otherwise, skip to Figure 29. The crown buoy should be kept as taut as possible; however, extra line can be used if required to bring the two mooring lines together. 


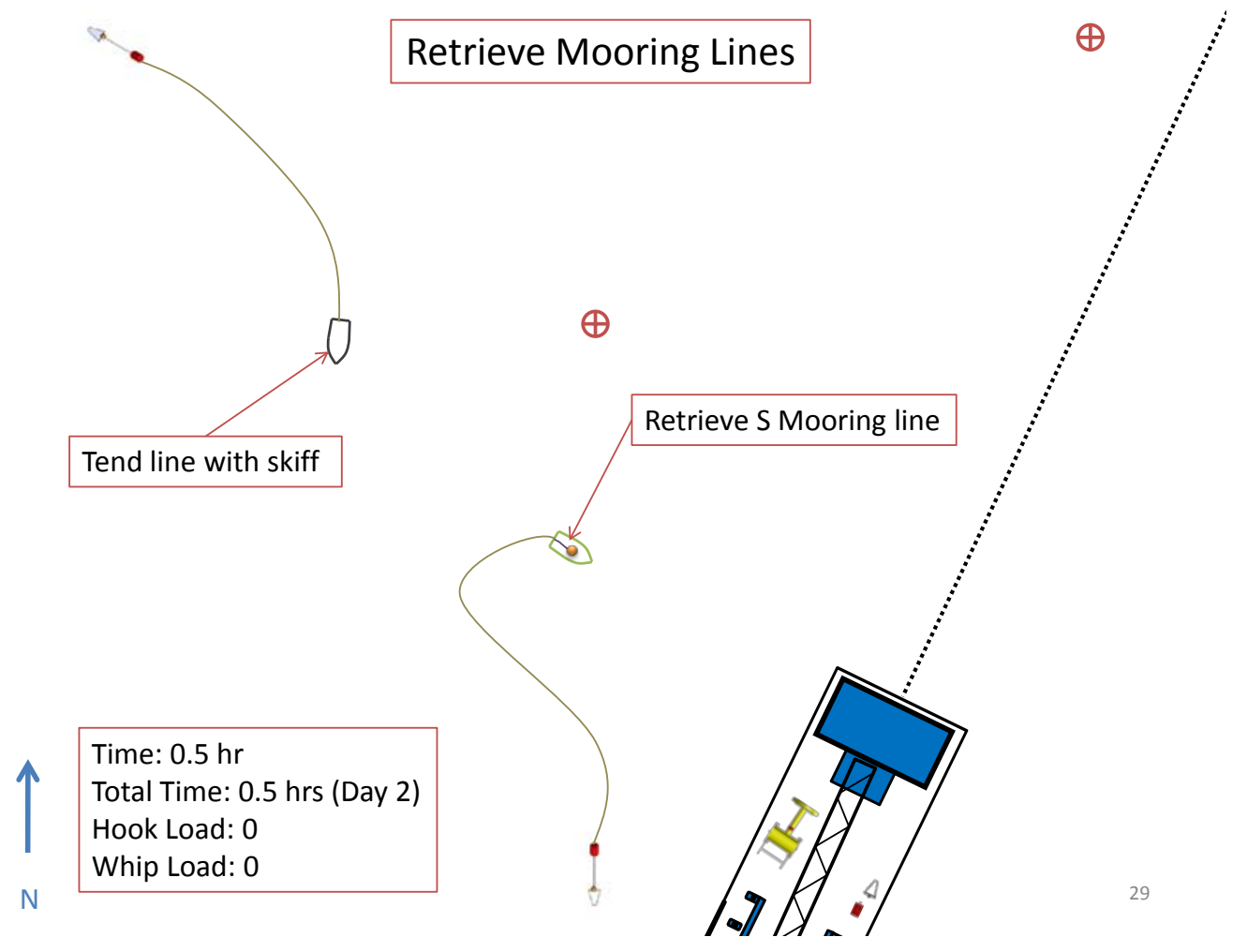

Figure 28. Again, only necessary if the moorings must be left in place overnight.

\subsubsection{WEC Deployment}

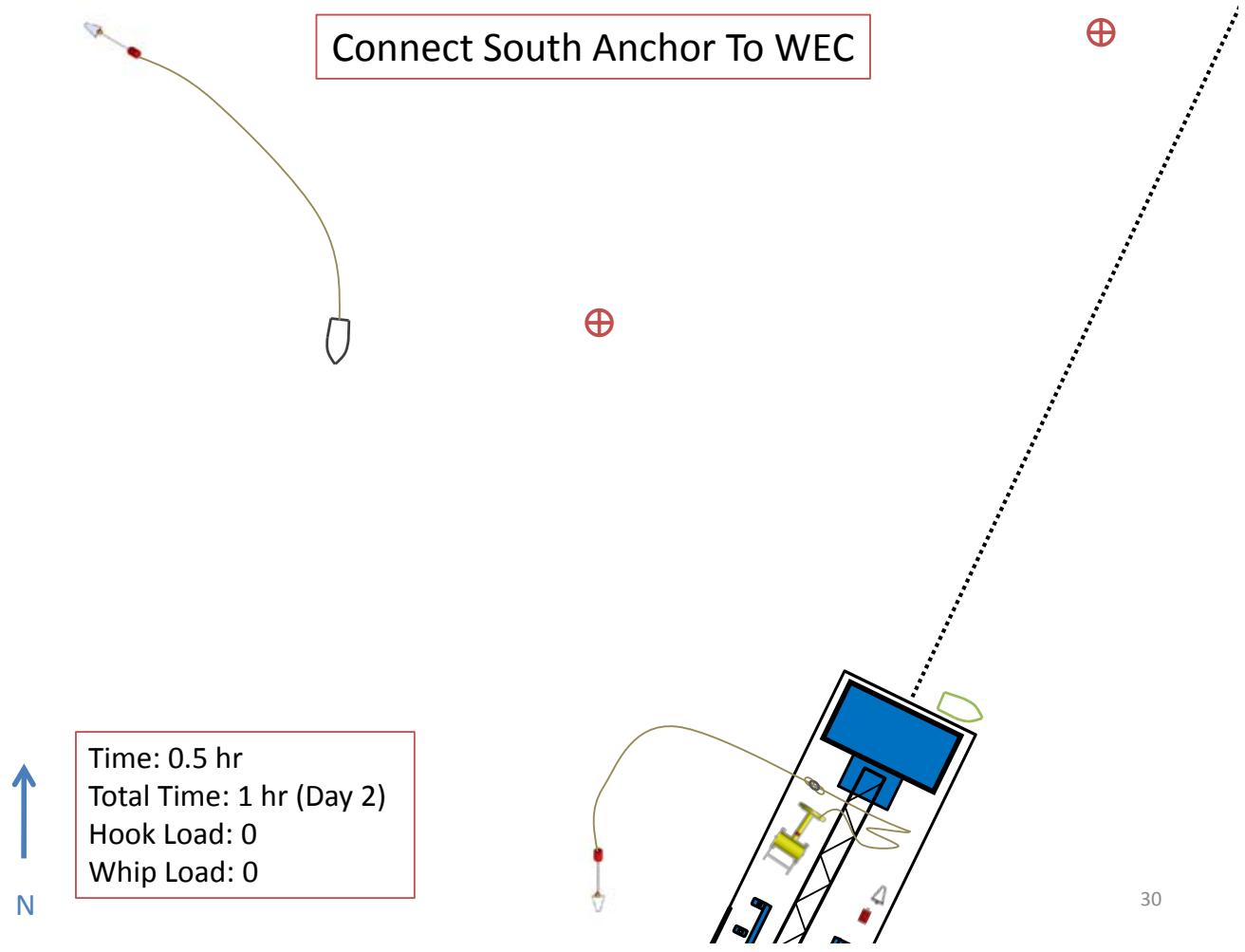

Figure 29. Connecting the $S$ anchor allows for less skiff work and fewer lines in the water.

Columbia Power Technologies - Confidential and Proprietary

Not for distribution - 2 February 2011 


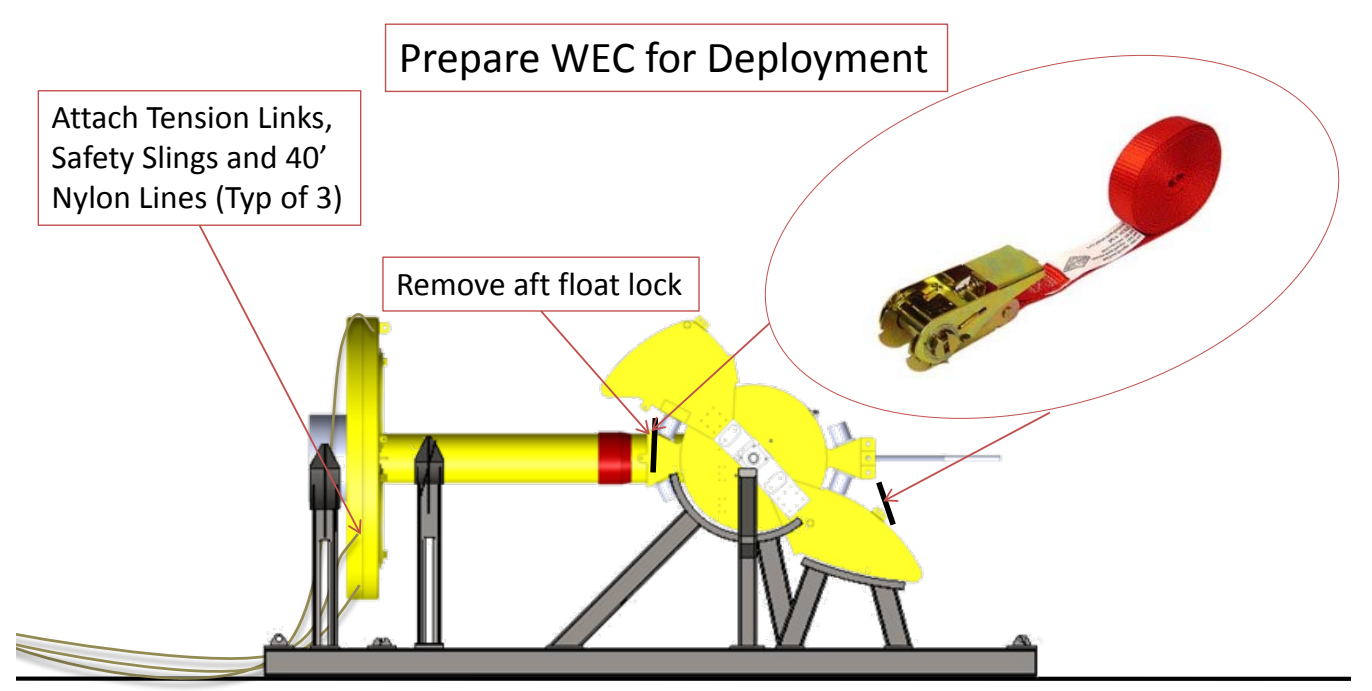

Time: $0.5 \mathrm{hr}$

Total Time: 1.5 hrs (Day 2)

Hook Load: 0

Whip Load: 0
CPT To Check Electronics/

Comms at or before this point

Figure 30. Preparing the WEC for deployment. Float locks will be endless nylon web ratchet straps. All systems should be verified by CPT prior to this point.

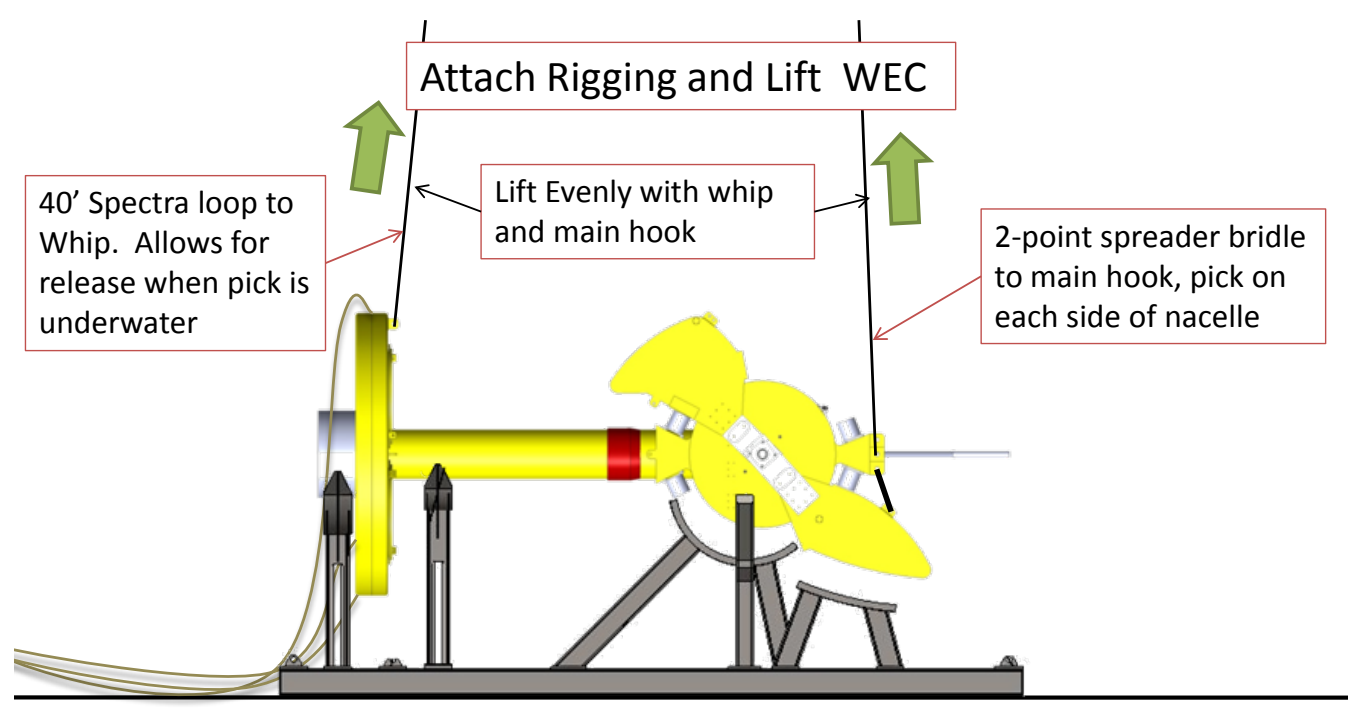

Time: $0.5 \mathrm{hr}$

Total Time: 2 hrs (Day 2)

Hook Load: 9000

Whip Load: 4000

Figure 31. Whip line loop will allow for detachment while submerged. Bridle will attach to both sides of nacelle.

Not for distribution - 2 February 2011 


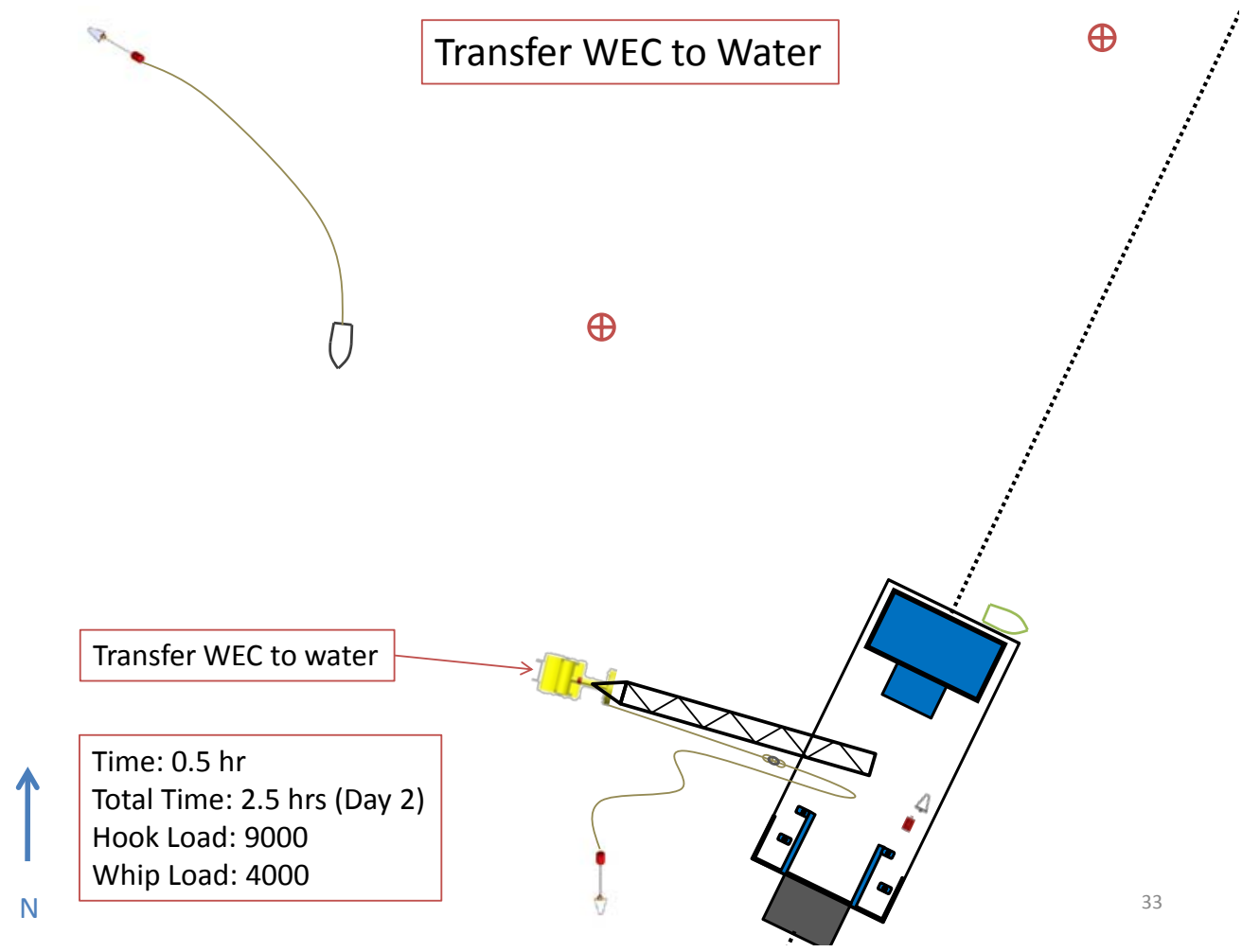

Figure 32. Transfer the WEC in the horizontal position to the water. Tend from SEAHORSE using NE and NW mooring line segments as taglines.

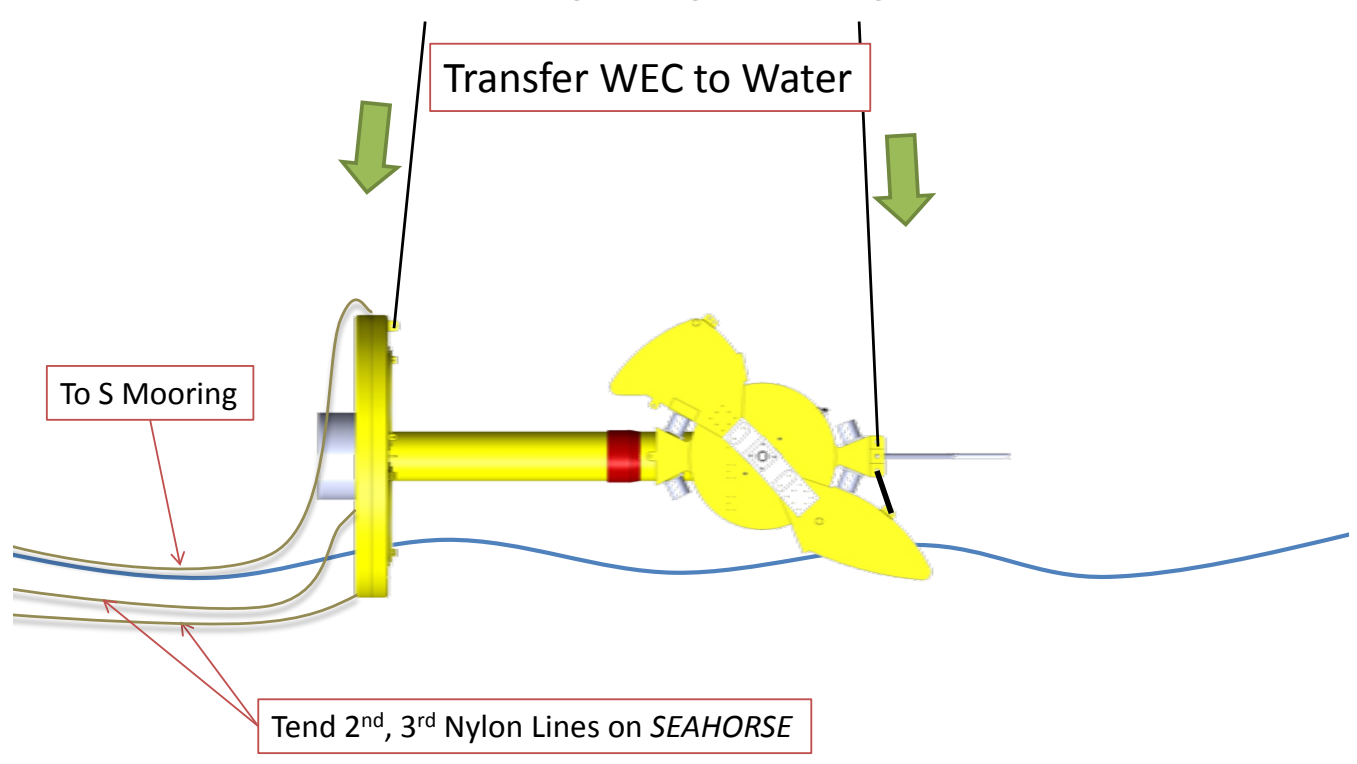

\begin{tabular}{l}
\hline Time: 0 hr \\
Total Time:2.5 hrs (Day 2) \\
Hook Load: 9000 \\
Whip Load: 4000
\end{tabular}

Figure 33.

Columbia Power Technologies - Confidential and Proprietary

Not for distribution - 2 February 2011 


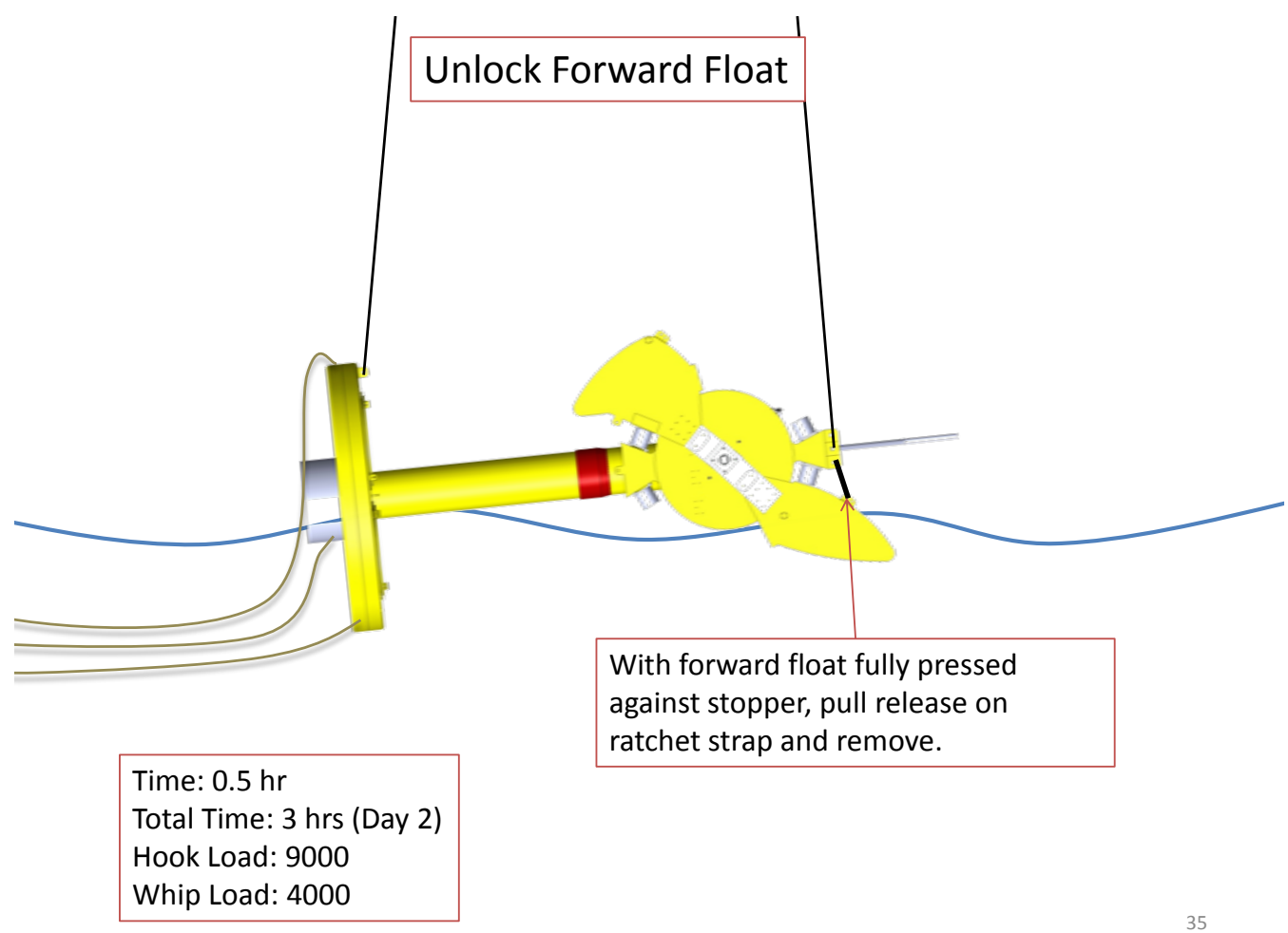

Figure 34. Unlocking the forward float. Use a skiff for this operation.

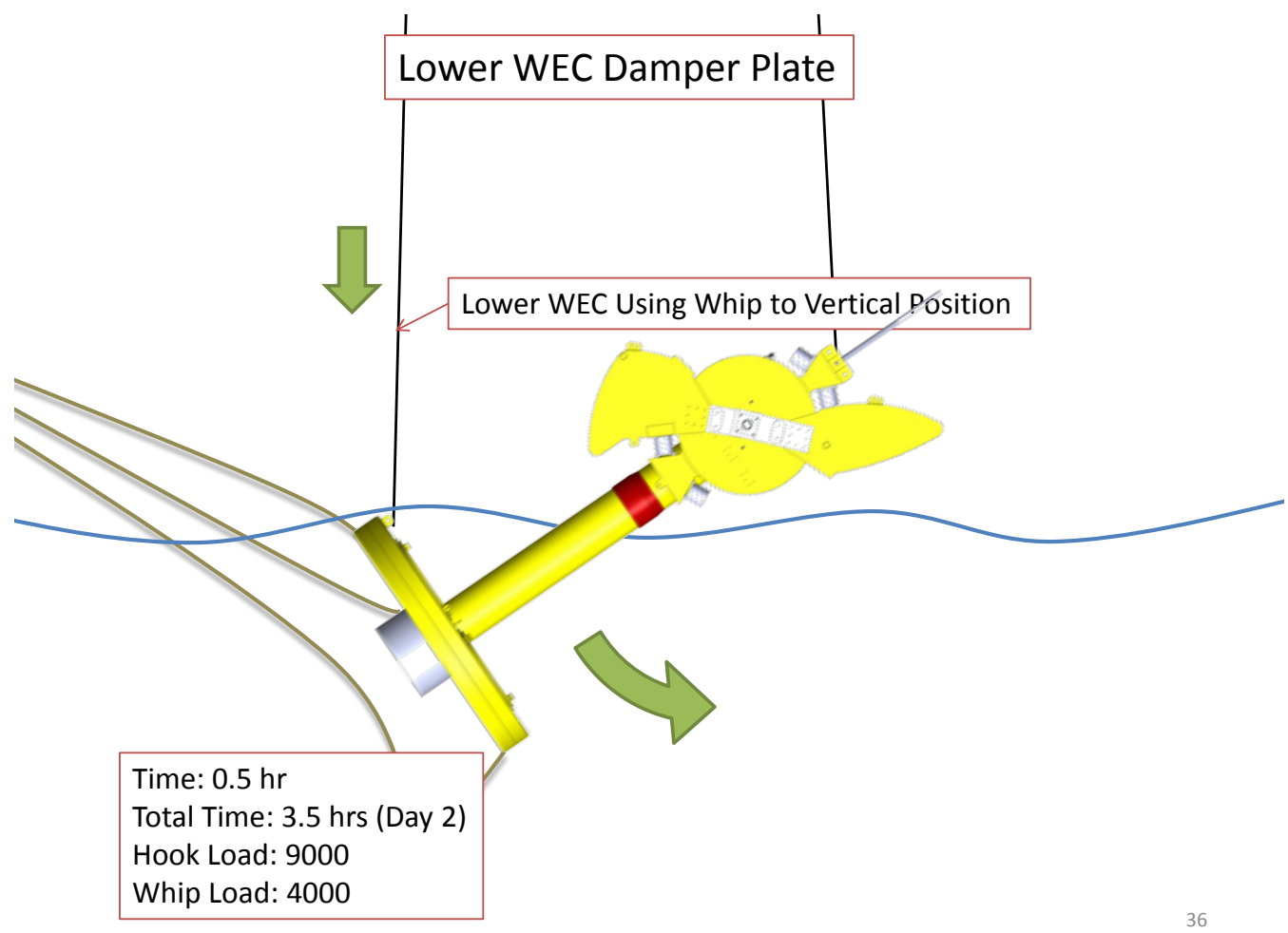

Figure 35. Begin lowering whip line.

Columbia Power Technologies - Confidential and Proprietary

Not for distribution - 2 February 2011 


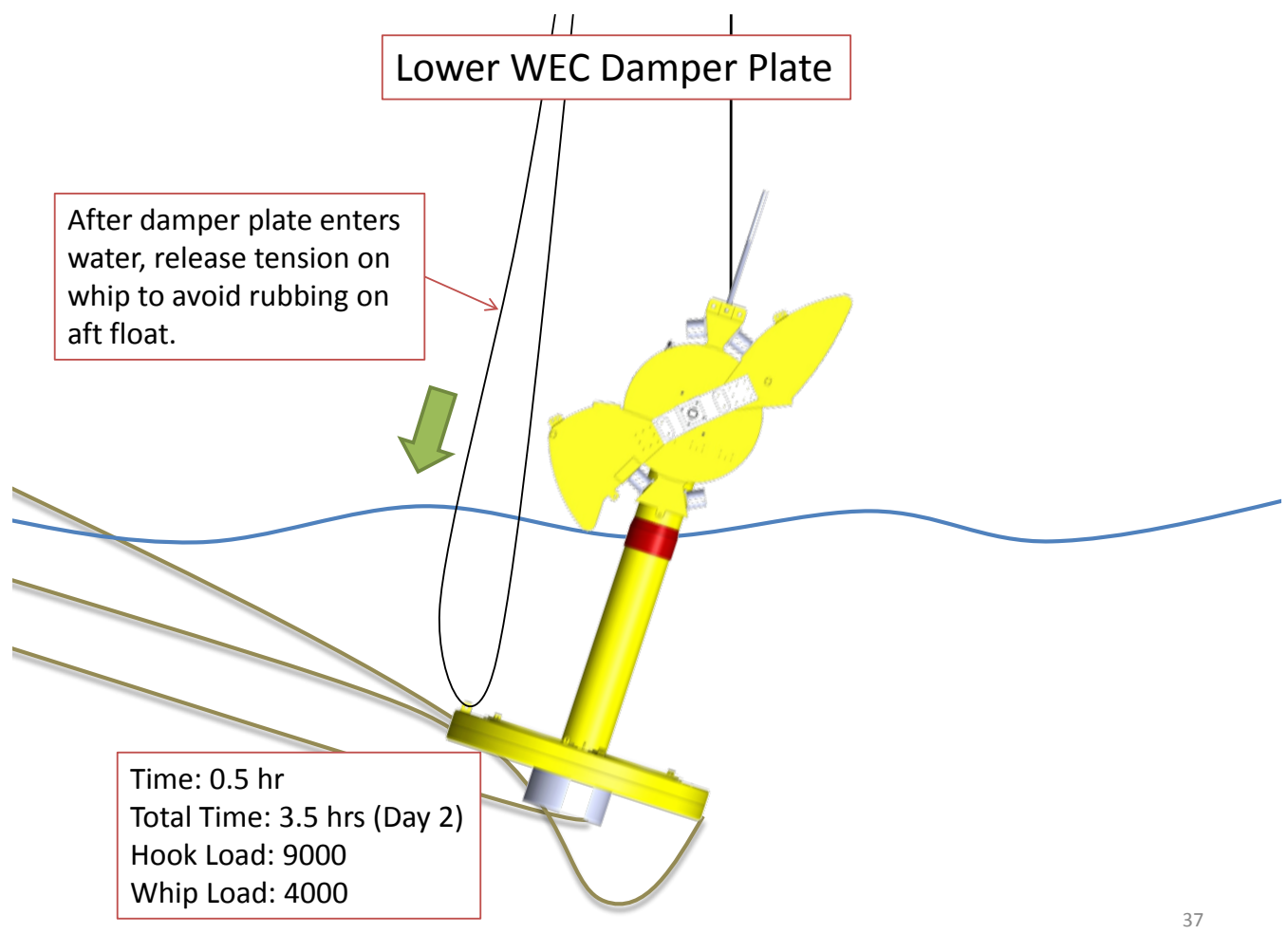

Figure 36. Relieve tension on whip line before WEC becomes vertical to avoid chafing the aft float.

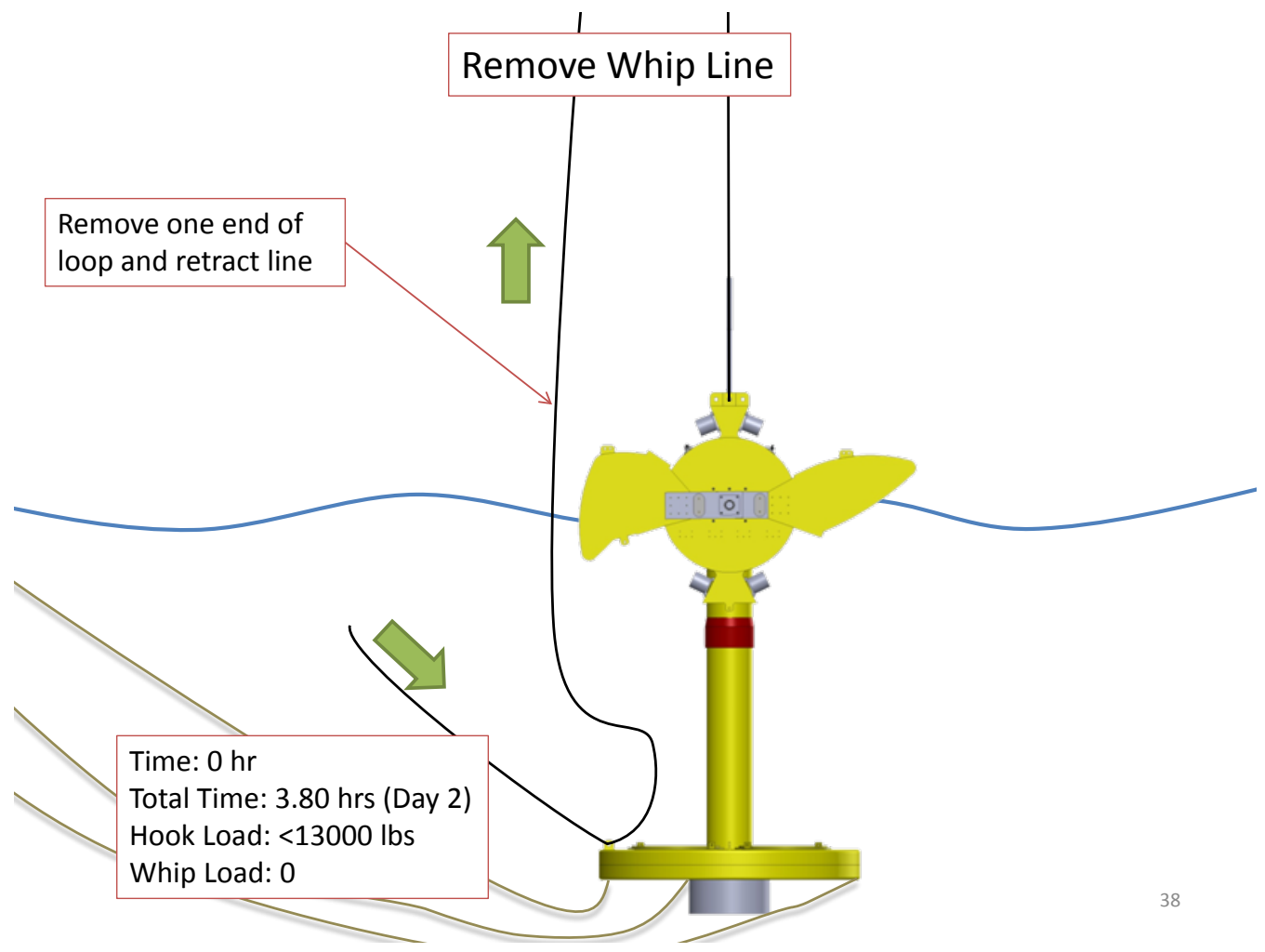

Figure 37. Remove one end of the whip loop to remove. Spectra line should allow for easy removal. 


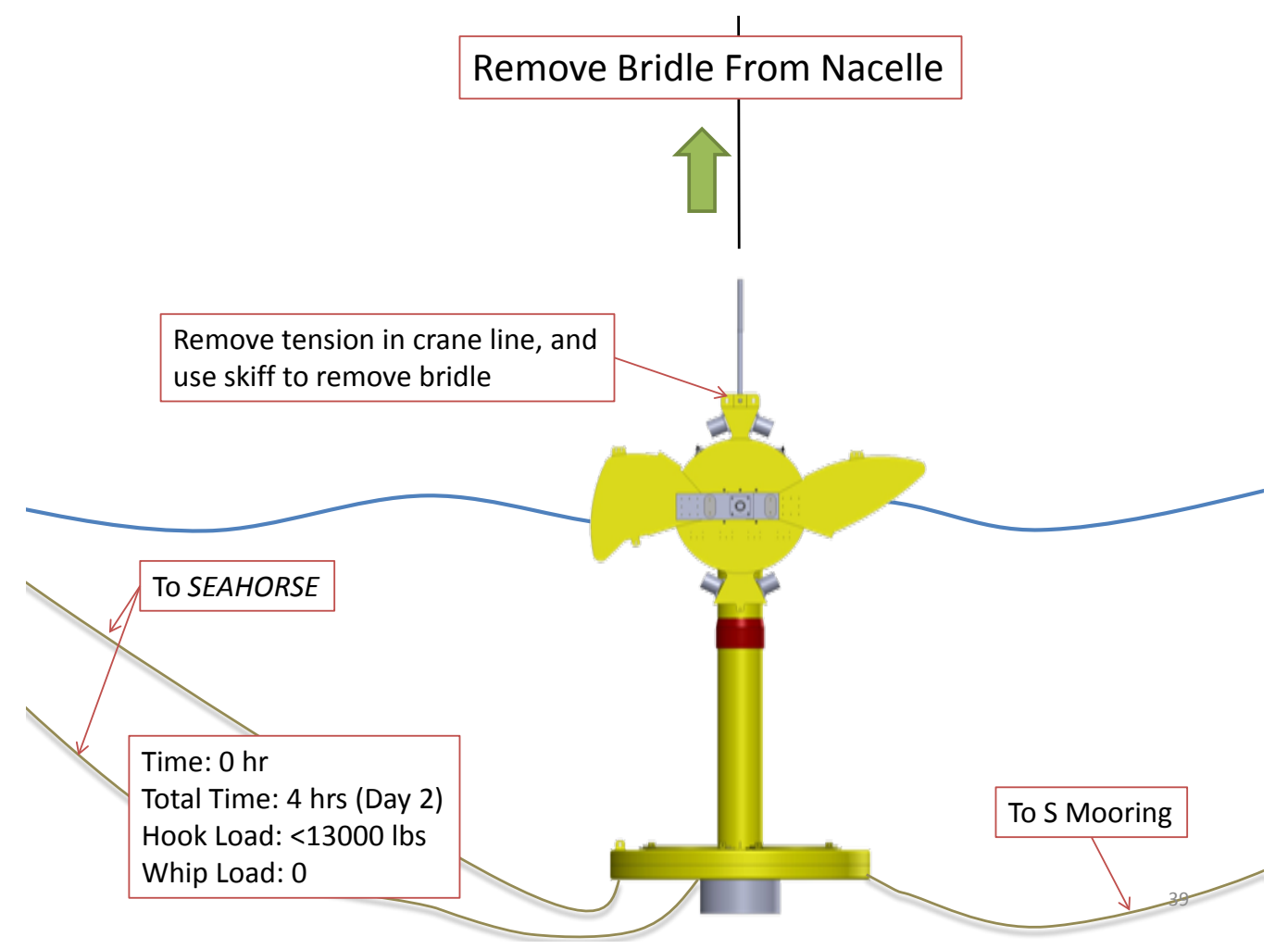

Figure 38. Use caution in the skiff to avoid tagging mooring lines or flaps, which are unlocked at this point.

\subsubsection{Connecting WEC Moorings}

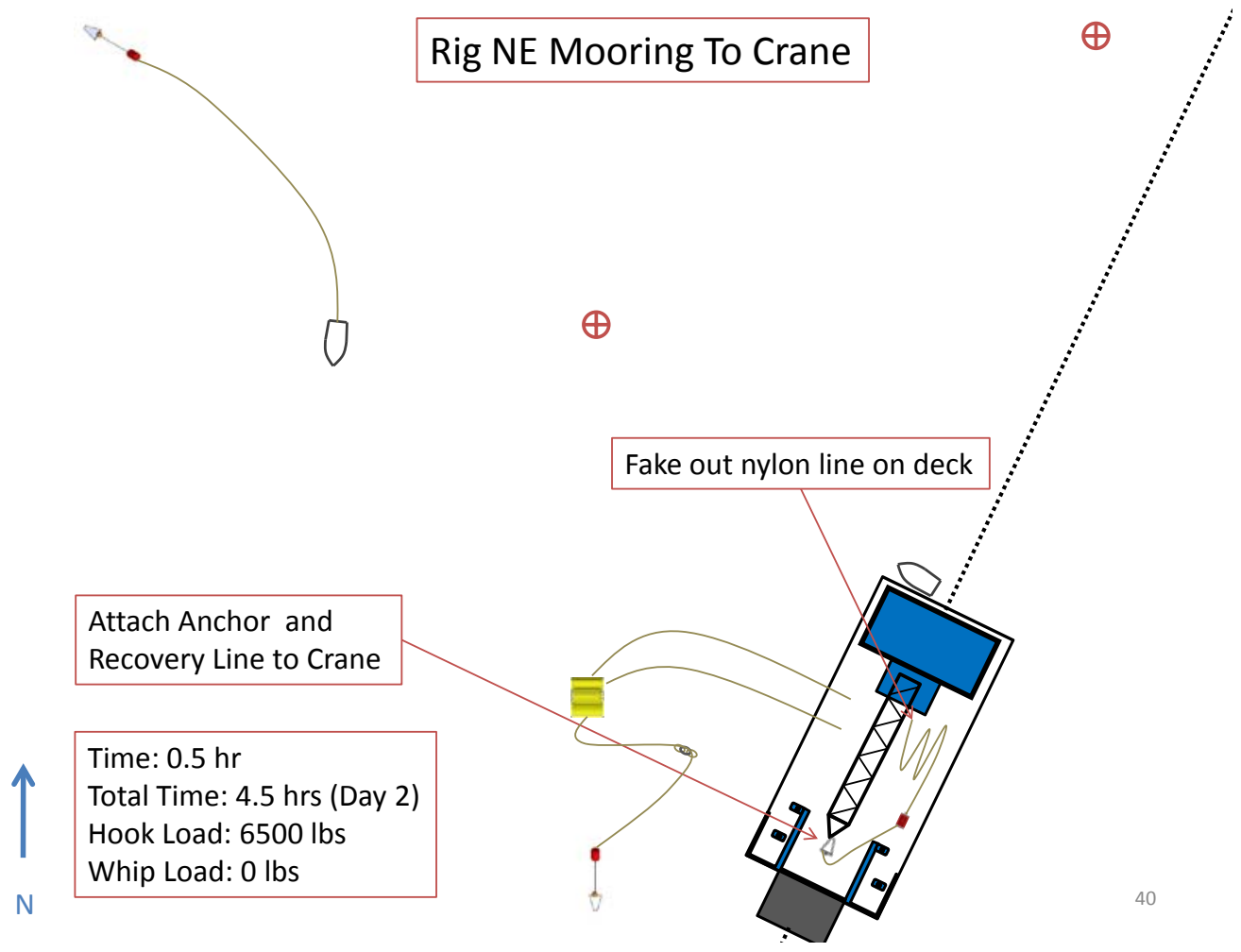

Figure 39. Rigging the NE mooring to the Crane. This step should be accomplished simultaneously with the next.

Columbia Power Technologies - Confidential and Proprietary

Not for distribution - 2 February 2011 


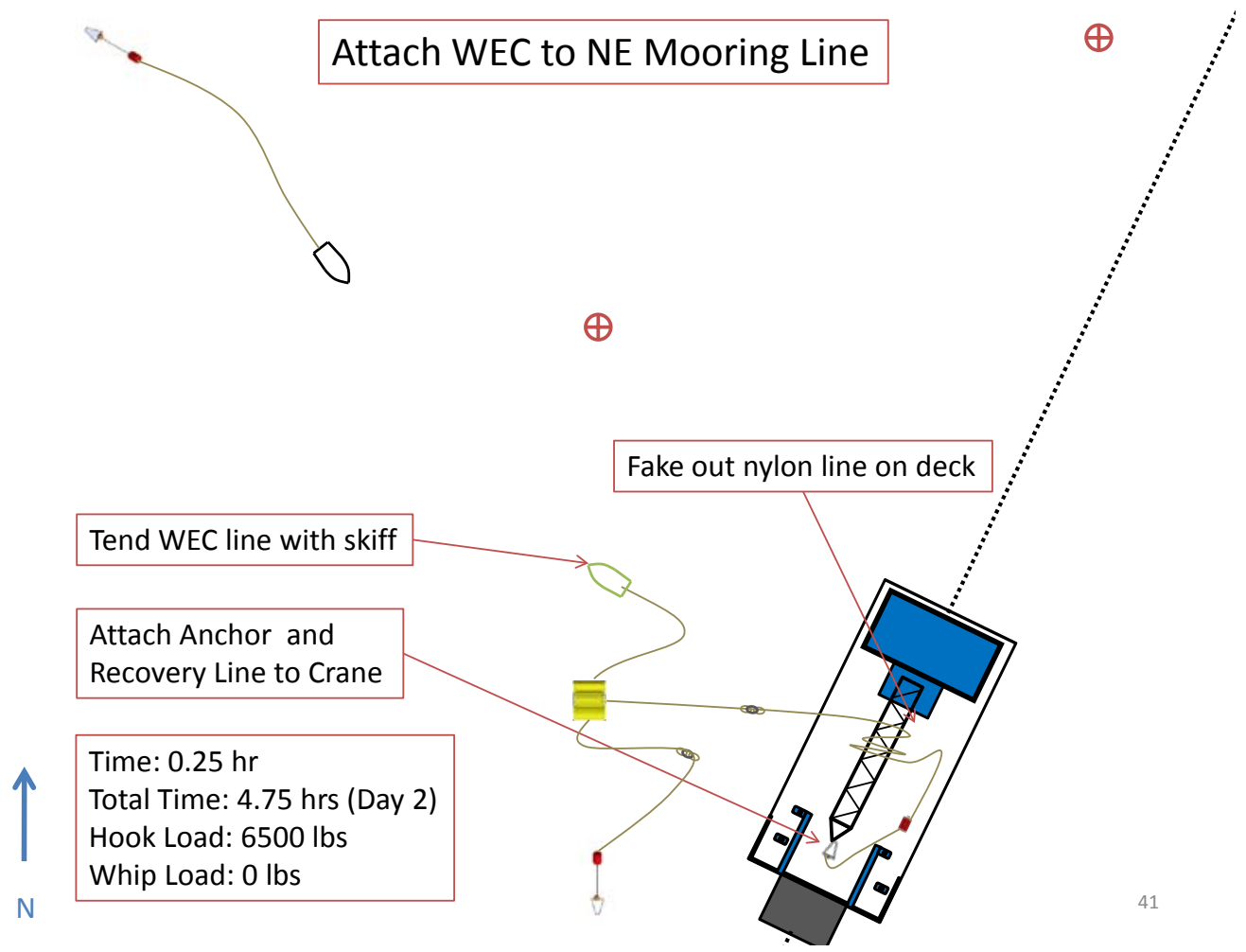

Figure 40. As soon as the NE mooring connection is accomplished on the SEAHORSE, the skiff can begin bringing the WEC towards the NW mooring.

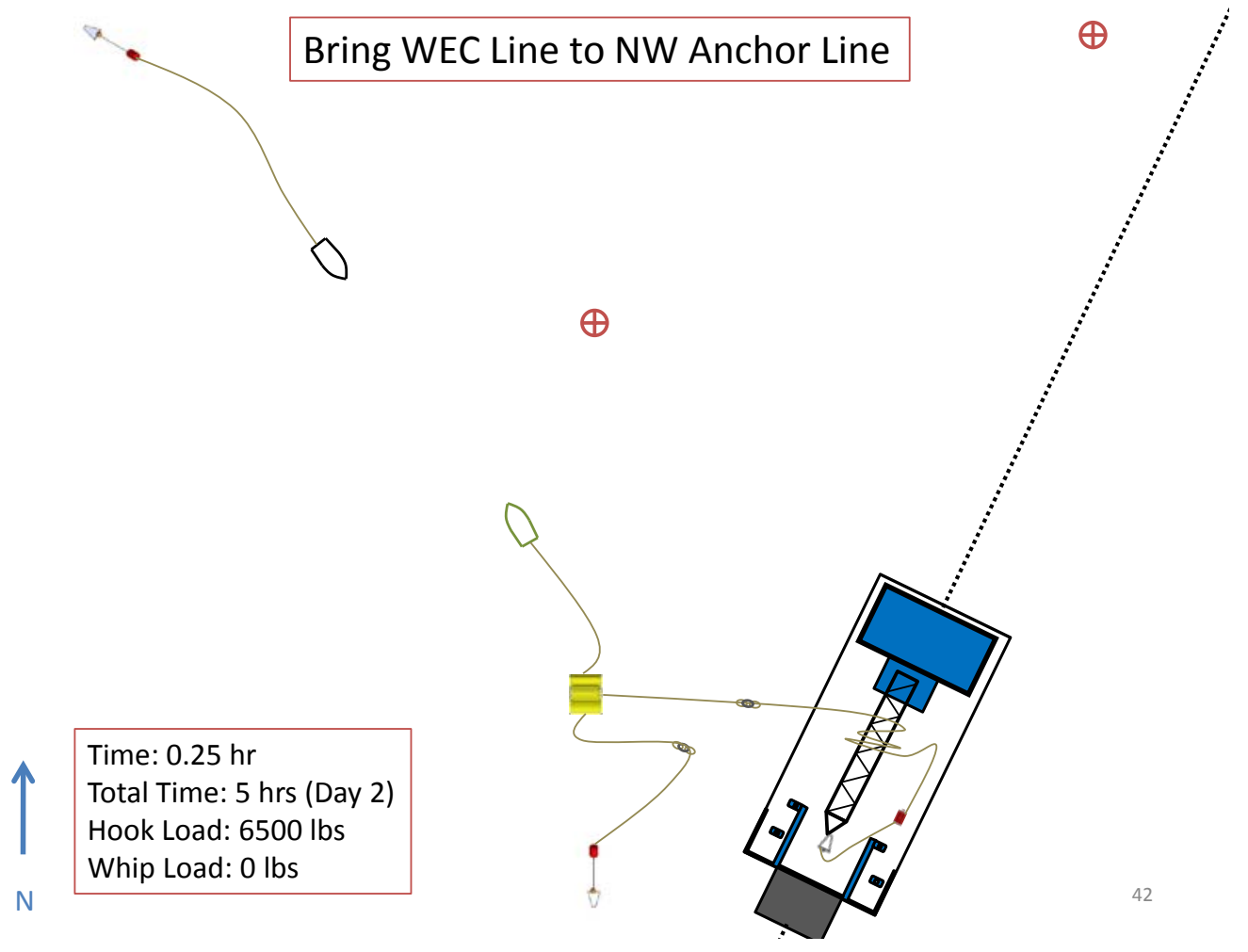

Figure 41.

Columbia Power Technologies - Confidential and Proprietary

Not for distribution - 2 February 2011 


\subsubsection{Northeast Anchor Deployment and WEC Pretensioning}

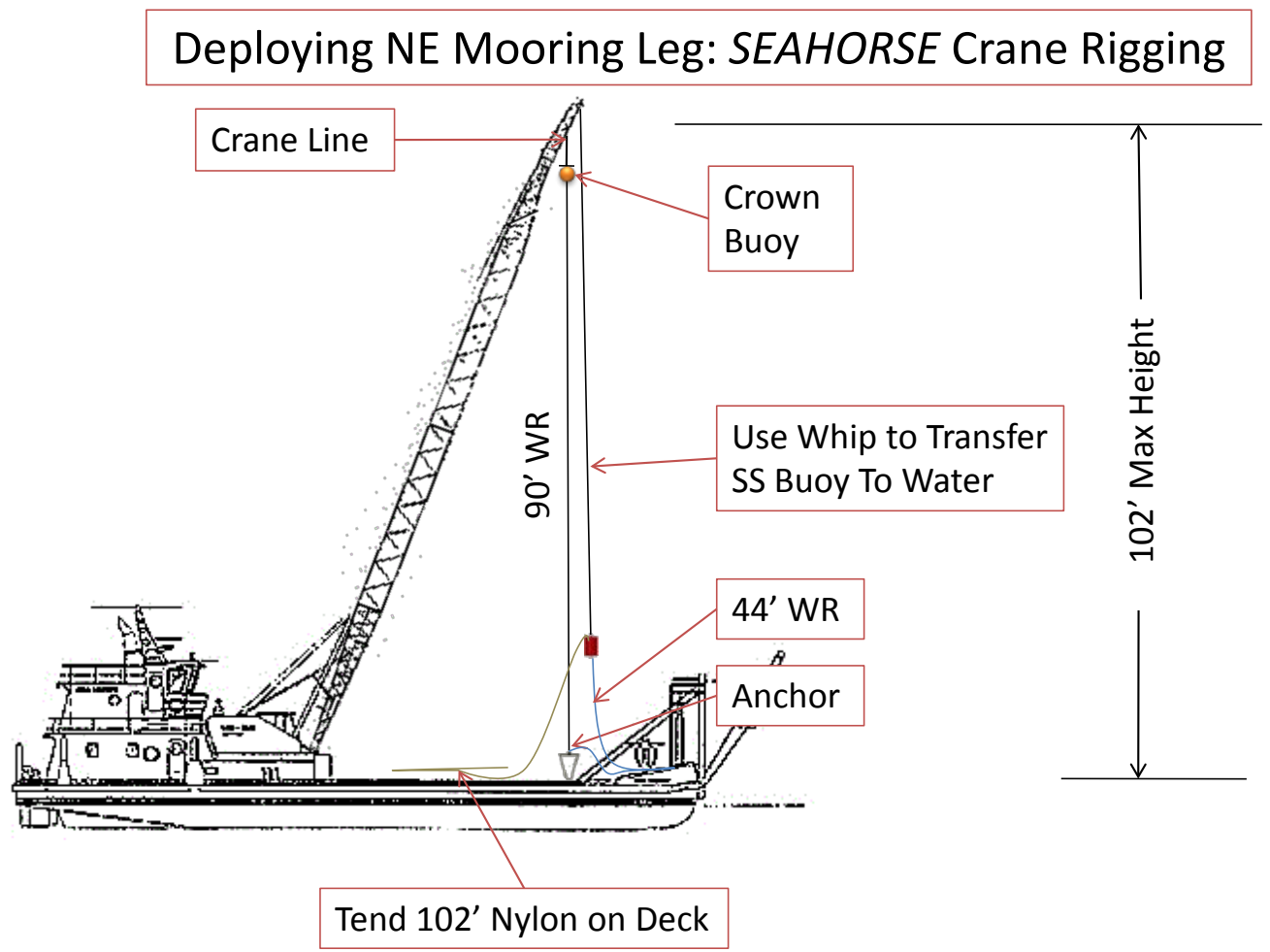

Figure 42. Rigging arrangement for NE anchor. The SS buoy can be disconnected from the whip as soon as it is over the water.

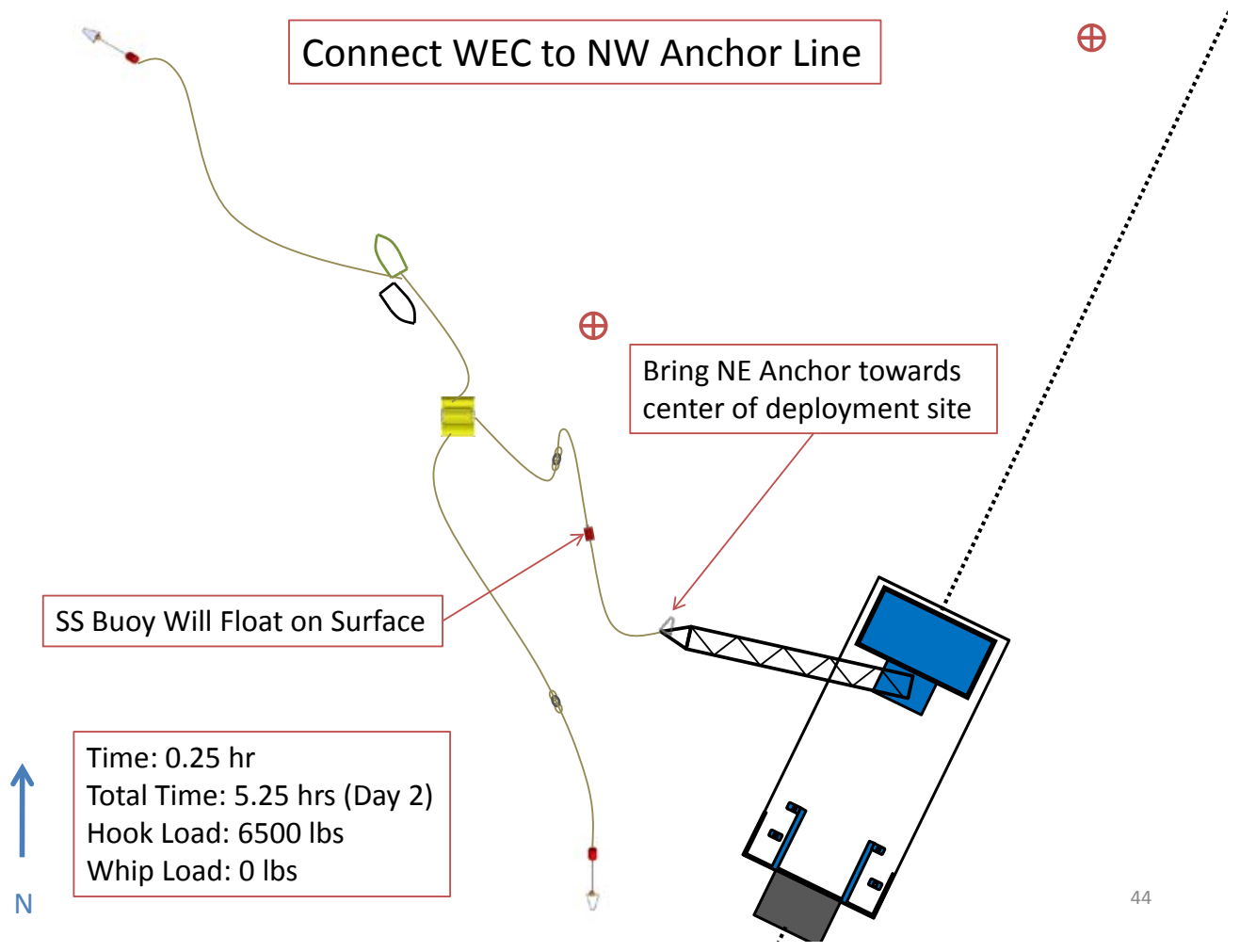

Figure 43.

Columbia Power Technologies - Confidential and Proprietary

Not for distribution - 2 February 2011 


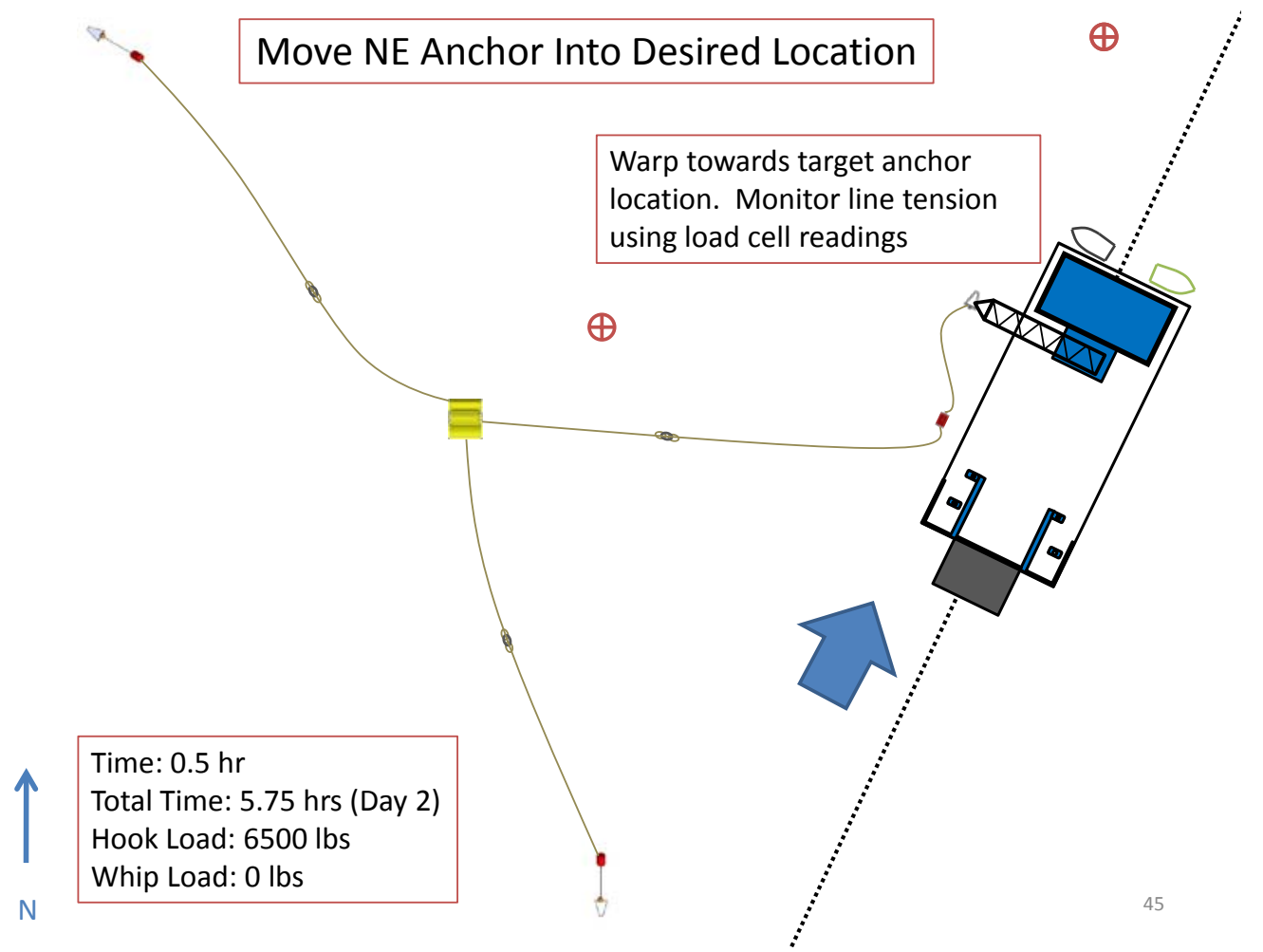

Figure 44. Note: The SEAHORSE crane will be at less of an angle than this to reduce side load.

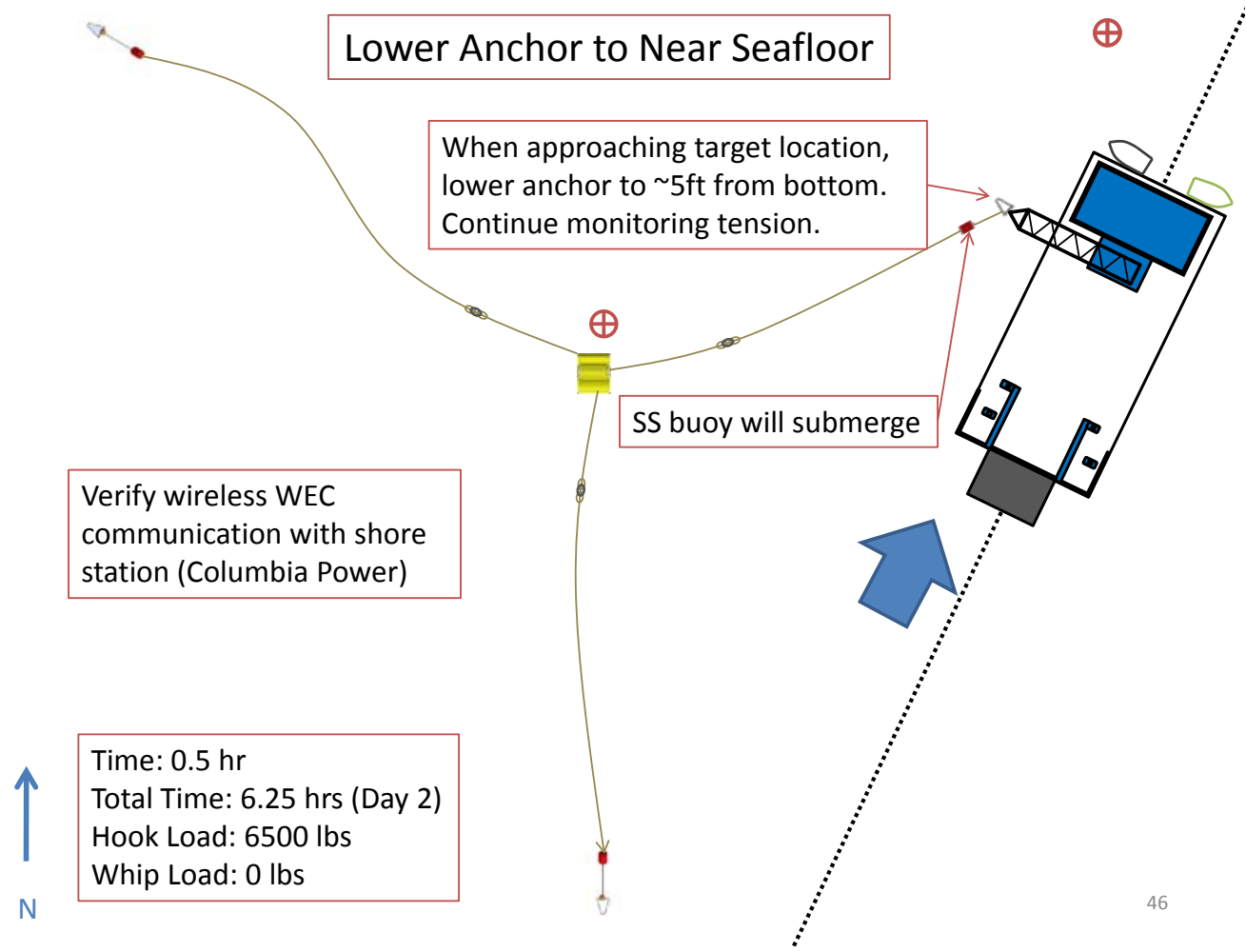

Figure 45. At this point, tension readings should begin being taken from the WEC. Keeping the anchor near the bottom will make the tension readings as realistic as possible. 


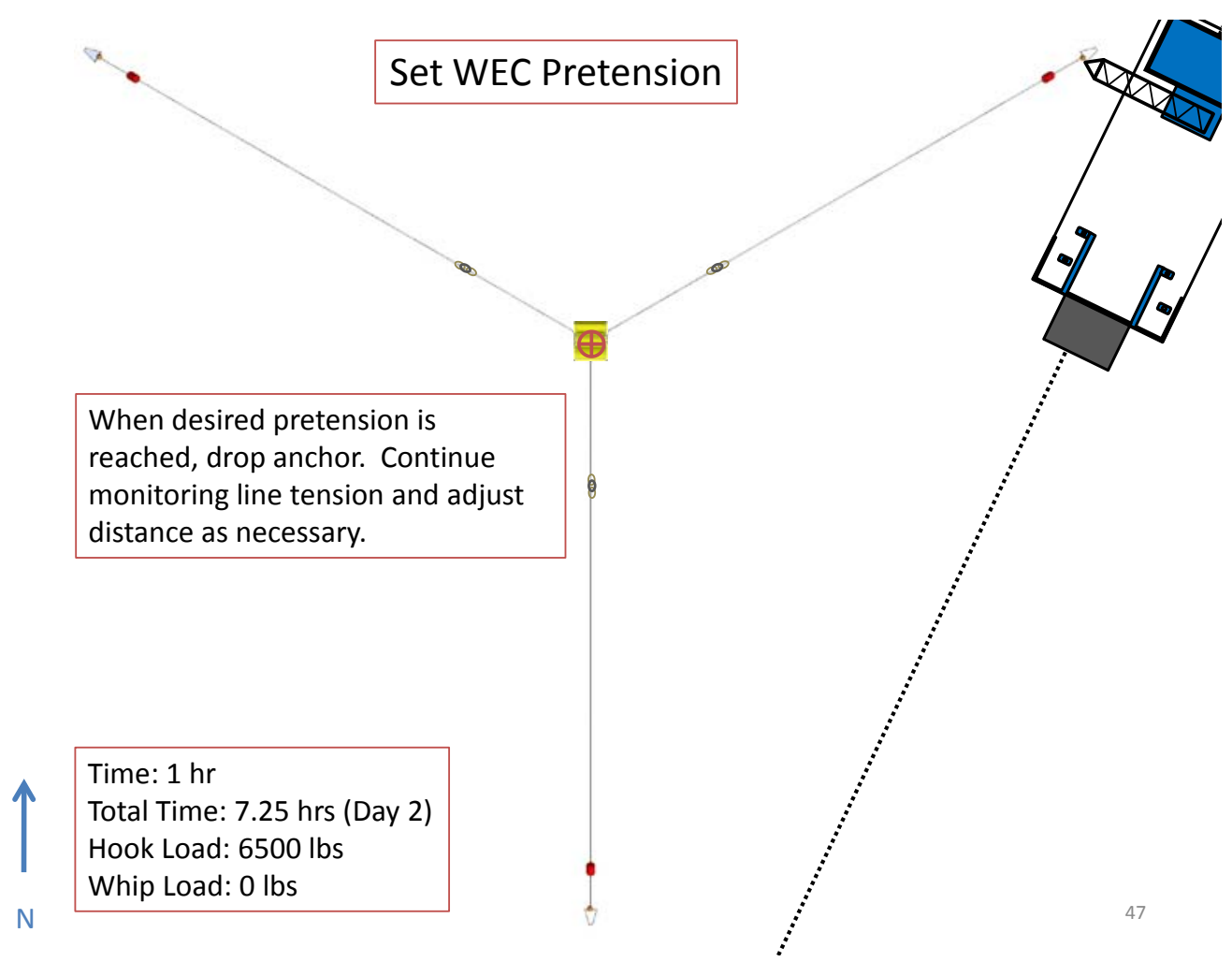

Figure 46. Set the anchor when the desired pretension is reached. (Nominally $150 \mathrm{lbs}$ ), refer to Glosten documentation for details.

\subsubsection{Target Pretension Table}

The distance in the horizontal plane from the center of the WEC to the anchor is $48.0 \mathrm{~m}$. If the anchor can be placed within 1.5-meter radius of the target position, then the pre-tension variation is acceptable. Pre-tension should be measured at slack tide in calm conditions (waves less than 1 foot). The following is a table of target pre-tension as a function of tide level. This allows for the measurement of pre-tension at any slack tide. Tension data will be available in real time from the WEC's tension links via a wireless 4G connection.

Table 1. Target Pretensions at Specified Tidal Conditions

\begin{tabular}{|c|c|}
\hline Tide Level, Relative To MMLW & Target Pre Tension [Kn] For Each Line \\
\hline-4 & 0.649 \\
\hline-3 & 0.649 \\
\hline-2 & 0.650 \\
\hline-1 & 0.651 \\
\hline 0 & 0.653 \\
\hline 1 & 0.655 \\
\hline 2 & 0.658 \\
\hline 3 & 0.660 \\
\hline 4 & 0.664 \\
\hline 5 & 0.667 \\
\hline 6 & 0.672 \\
\hline 7 & 0.676 \\
\hline 8 & 0.681 \\
\hline 9 & 0.686 \\
\hline 10 & 0.692 \\
\hline 11 & 0.698 \\
\hline 12 & 0.705 \\
\hline 13 & 0.711 \\
\hline 14 & 0.719 \\
\hline
\end{tabular}

Columbia Power Technologies - Confidential and Proprietary

Not for distribution - 2 February 2011 


\subsubsection{AWAC Deployment}

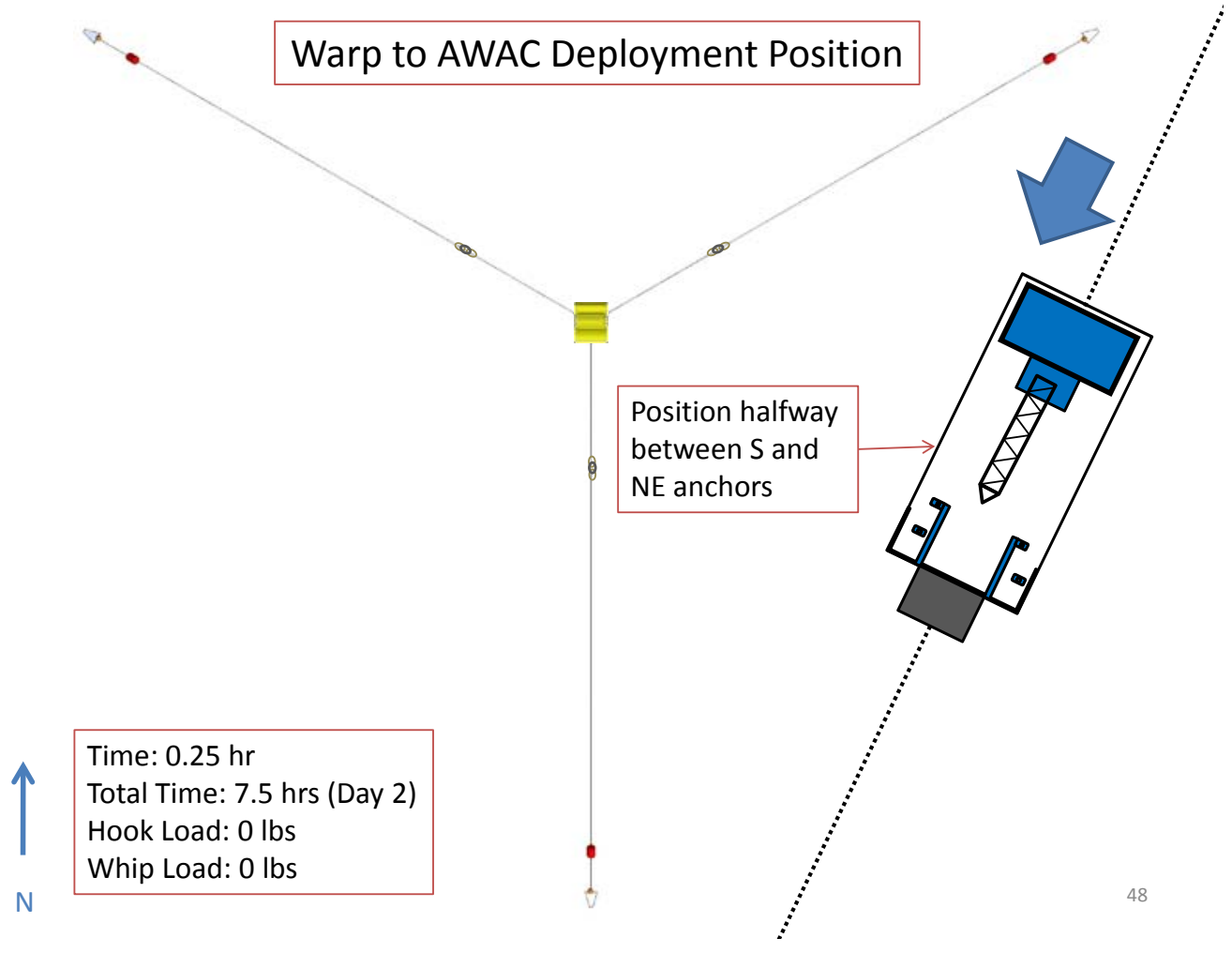

Figure 47.

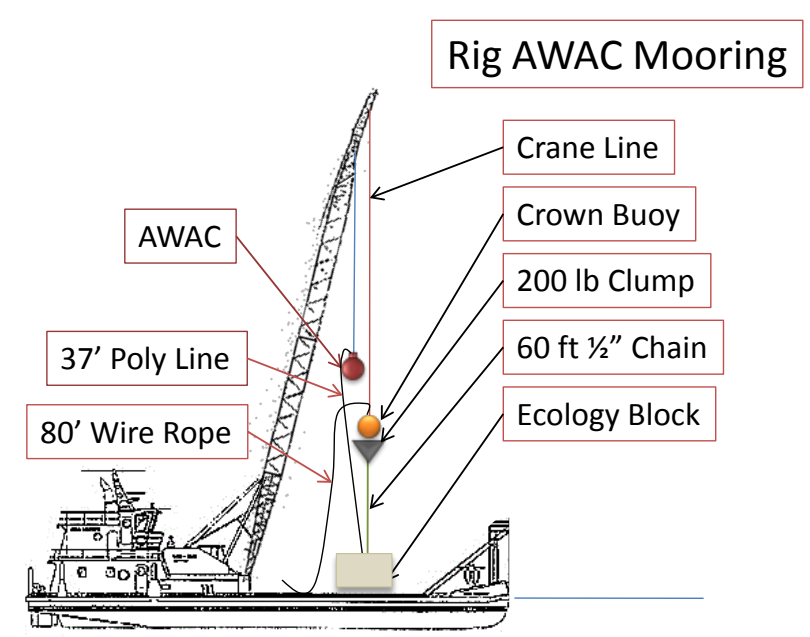

Time: $0.5 \mathrm{hr}$

Total Time: 8 hrs (Day 2)

Hook Load: 0 lbs

Whip Load: 0 lbs

Figure 48.

Columbia Power Technologies - Confidential and Proprietary

Not for distribution - 2 February 2011 


\section{Begin Lowering Ecology Block}

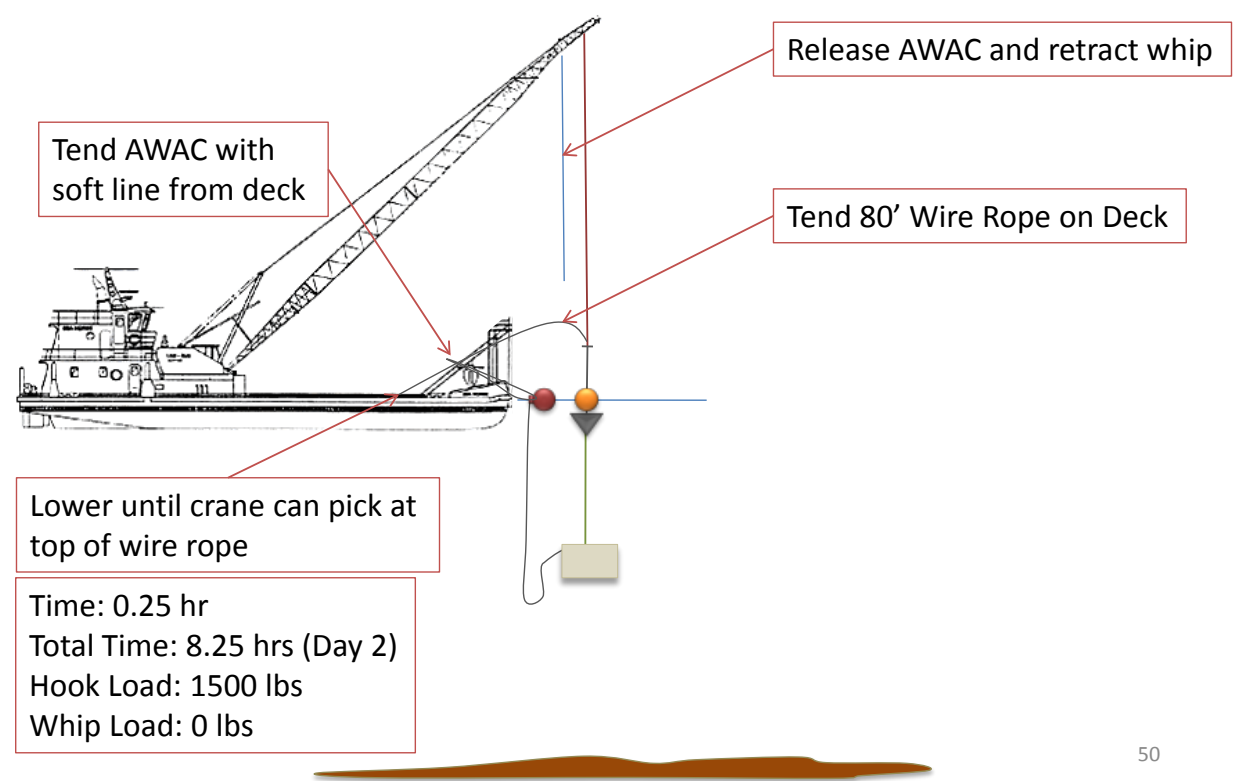

Figure 49. Release AWAC from whip and tend with soft line loop from deck.

\section{Move Pick to Top of Wire Rope}

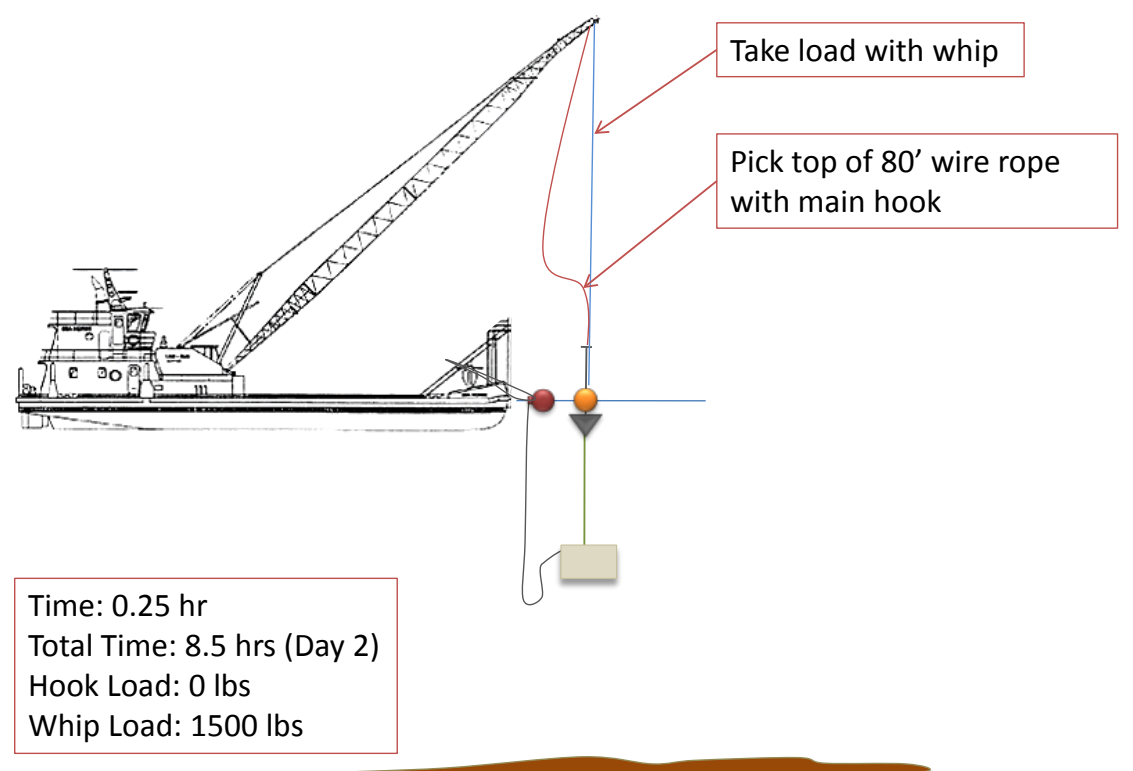

Figure 50. Since this mooring arrangement is in total longer than the SEAHORSE crane is tall, it will be necessary to "repick" it while lowering. This can be accomplished by taking the load with the whip. Since the load is small, the anchors can be lowered using the whip from that point onwards. 


\section{Ecology Block on Bottom}

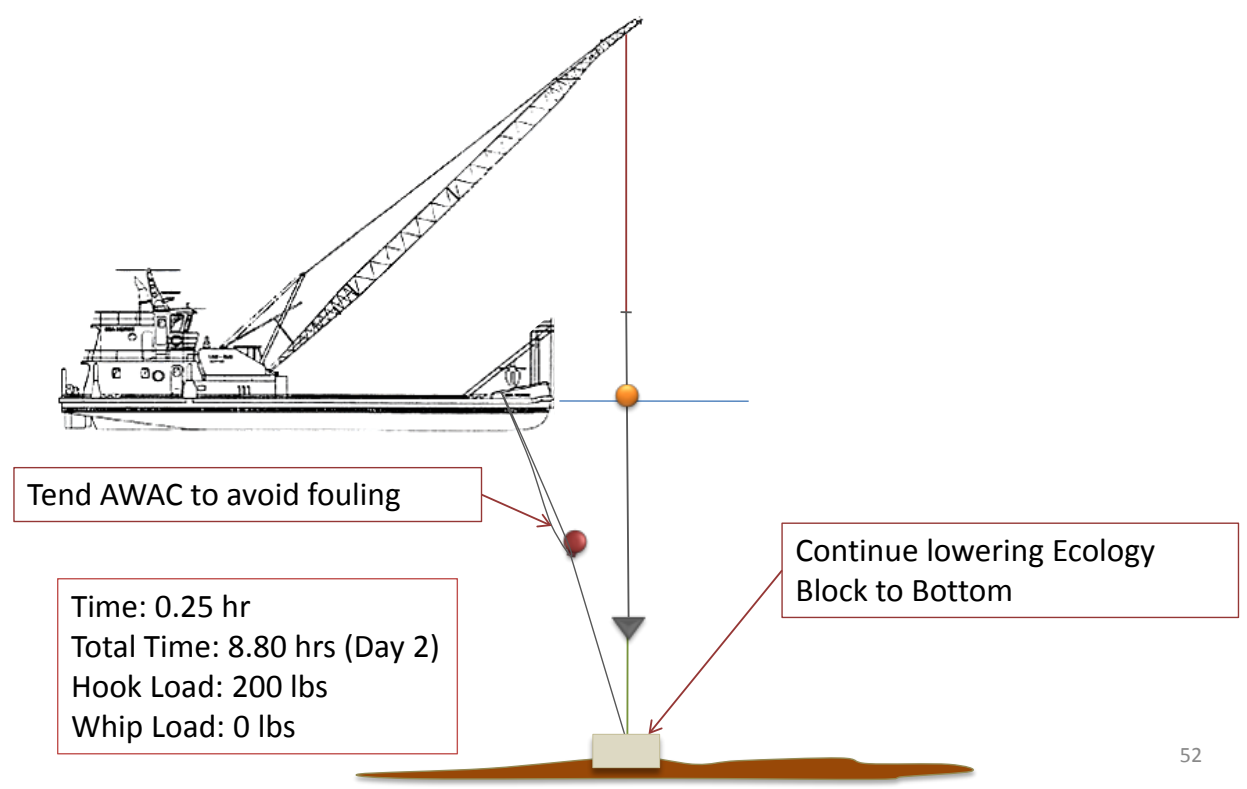

Figure 51.

\section{Move Clump Into Location}

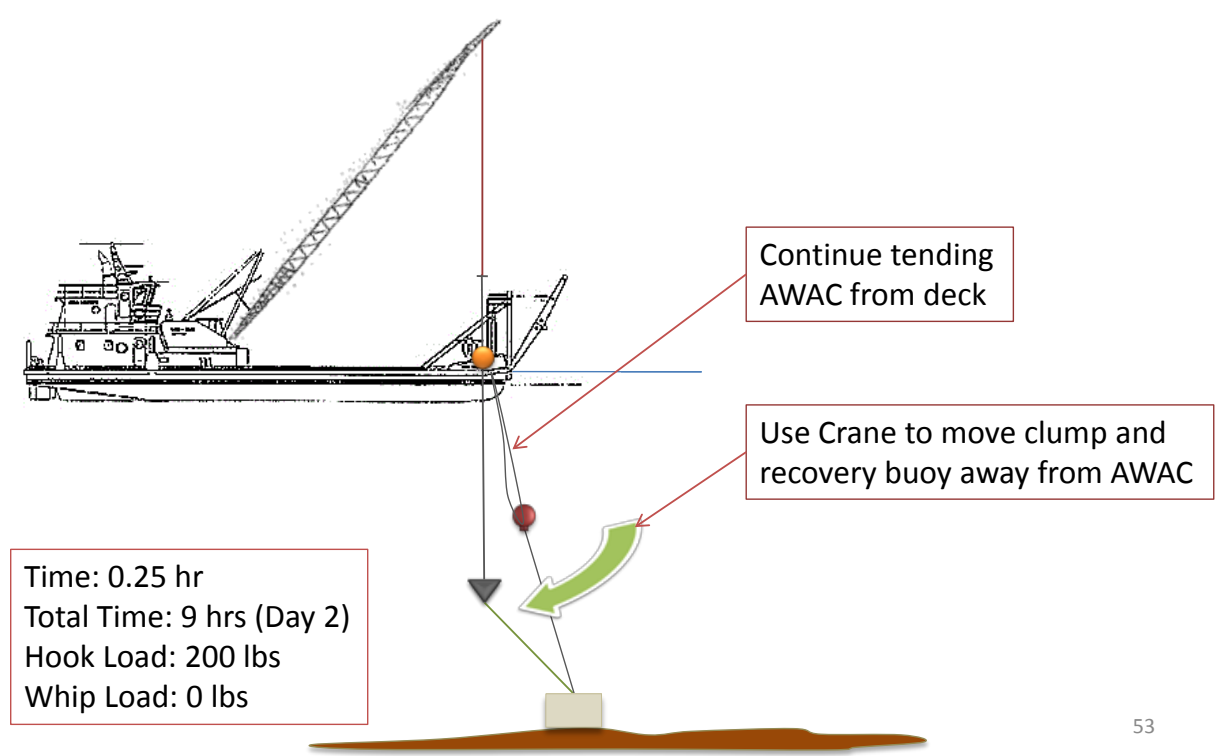

Figure 52. The clump can be moved into position by rotating the crane.

Columbia Power Technologies - Confidential and Proprietary

Not for distribution - 2 February 2011 


\section{Clump on Seafloor}

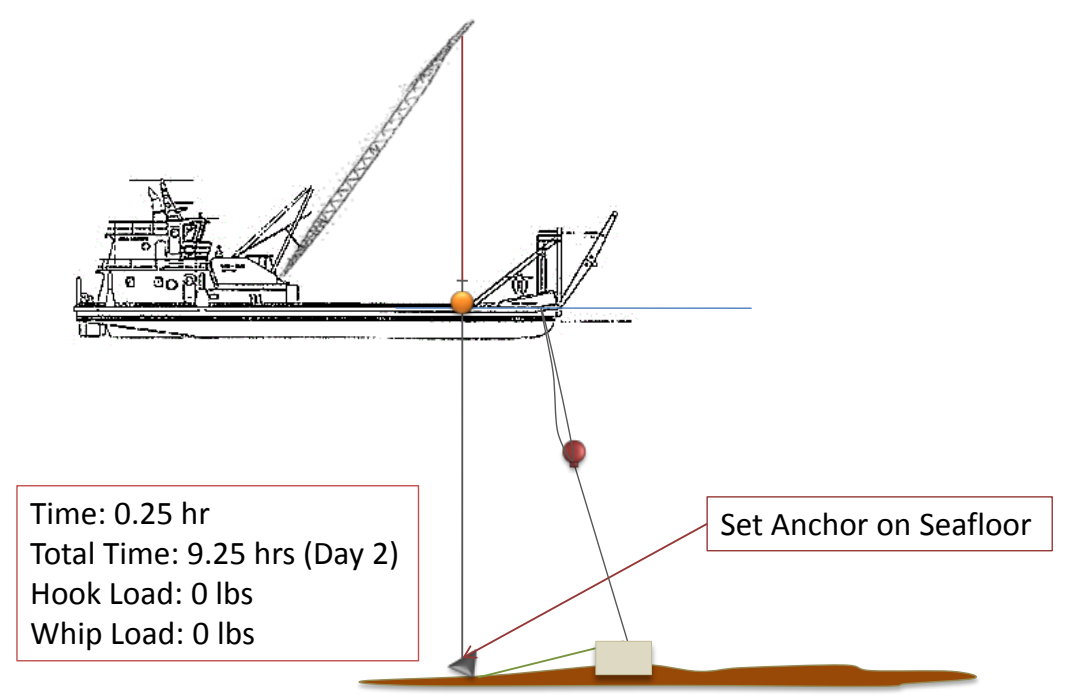

Figure 53. The clump should be lowered when the crane line starts tending off of vertical.

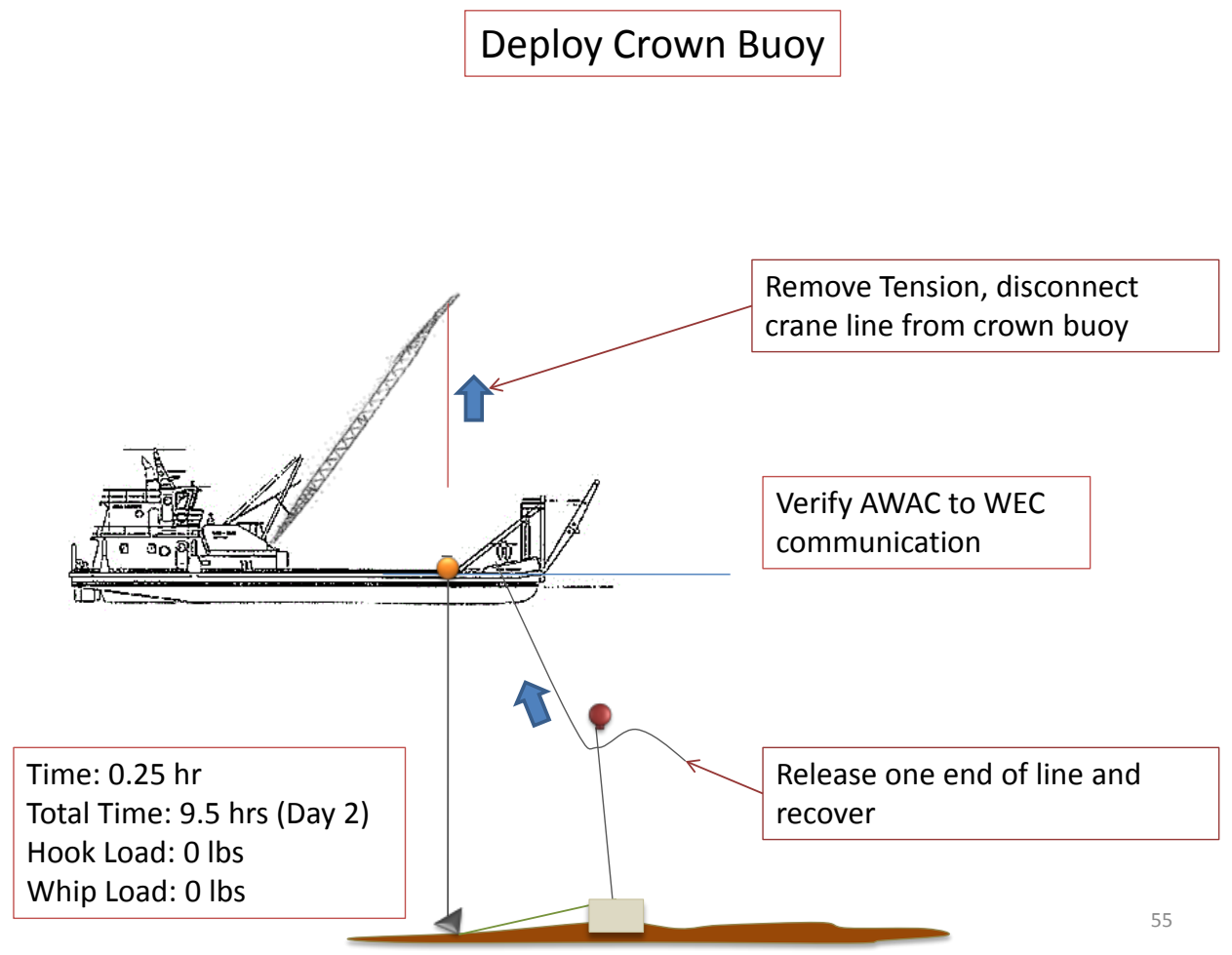

Figure 54. Crown buoy will be secured to recovery line using wire rope clamps and donut plate.

Columbia Power Technologies - Confidential and Proprietary

Not for distribution - 2 February 2011 


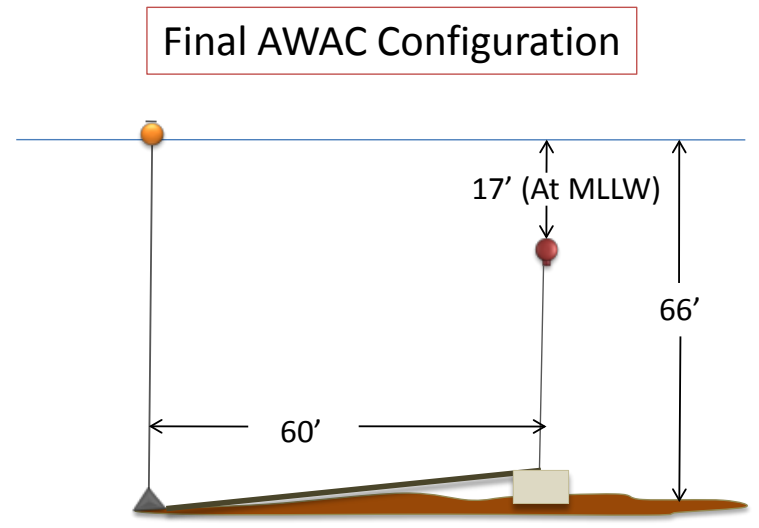

Figure 55. Final AWAC/subsurface buoy configuration. Max crown buoy watch circle: 48'.

\subsubsection{Final Steps and Return Trip}

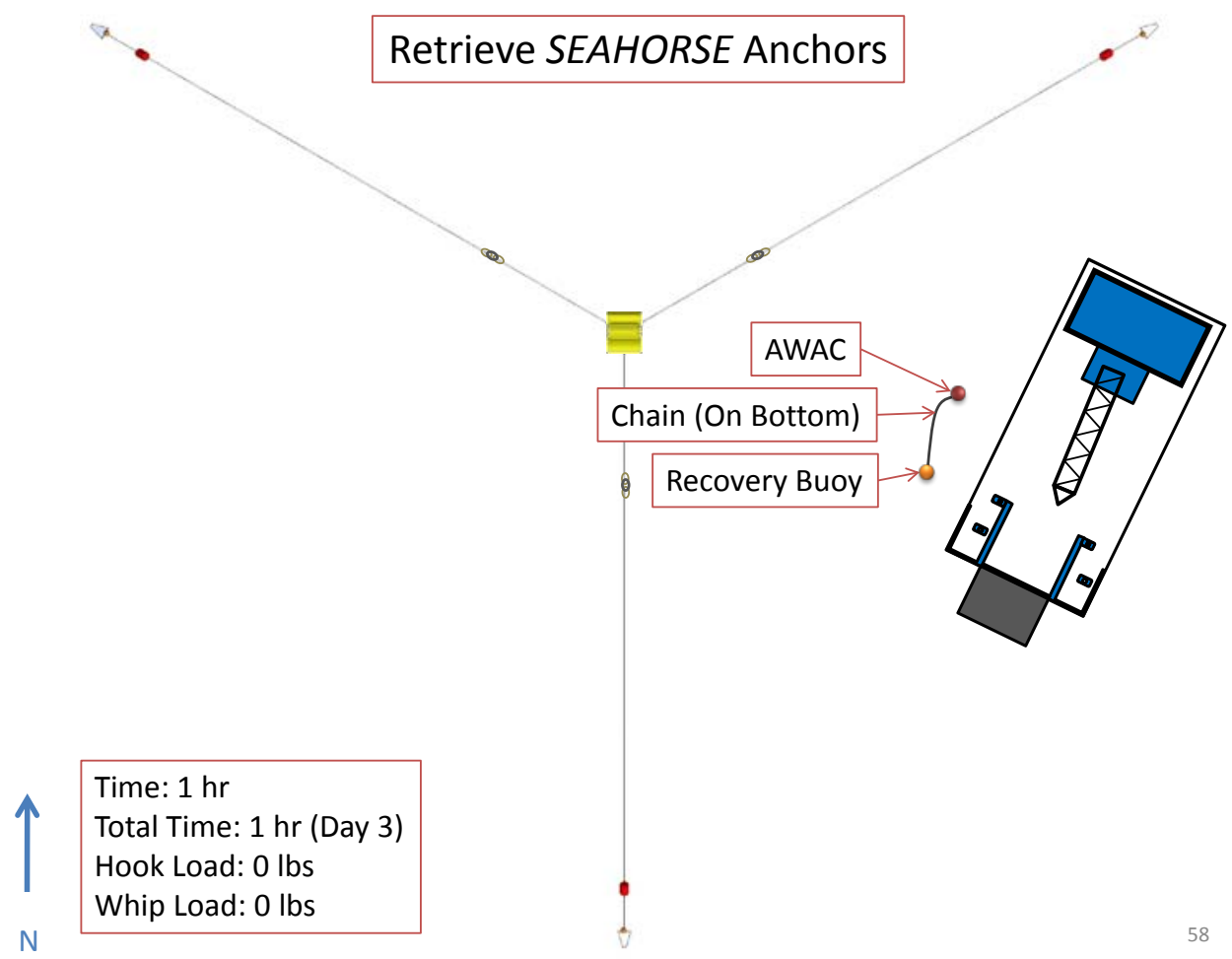

Figure 56. Remove SEAHORSE bow and stern anchors.

Columbia Power Technologies - Confidential and Proprietary

Not for distribution - 2 February 2011 


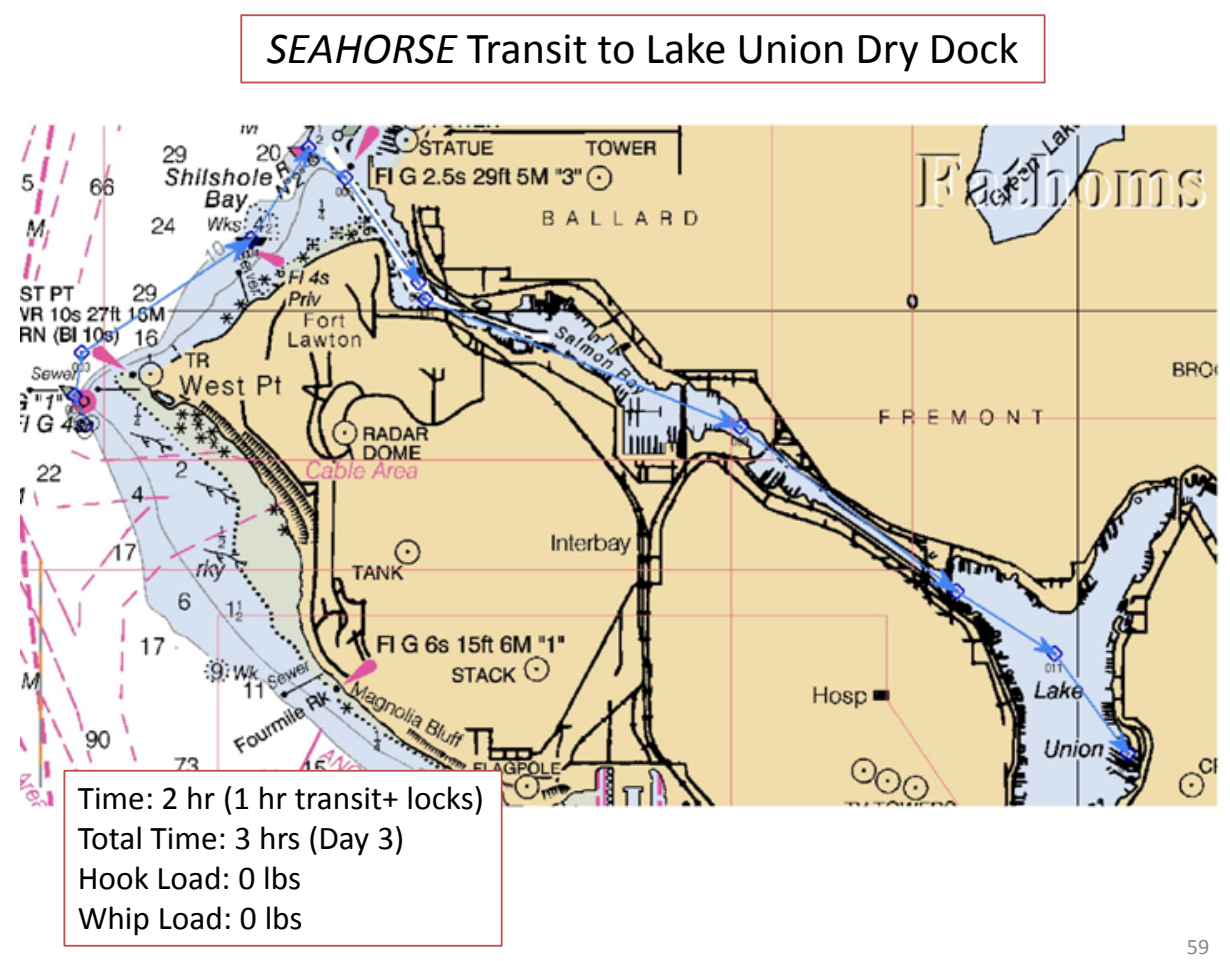

Figure 57. Return to Lake Union Dry Dock. (Seahorse mooring location).

\subsection{Demobilization}

Demobilization will occur at Lake Union Dry Dock. Arrangements will be made ahead of time to have a flatbed ready to remove the cradle and any other gear from the SEAHORSE and move them to a storage location, TBD.

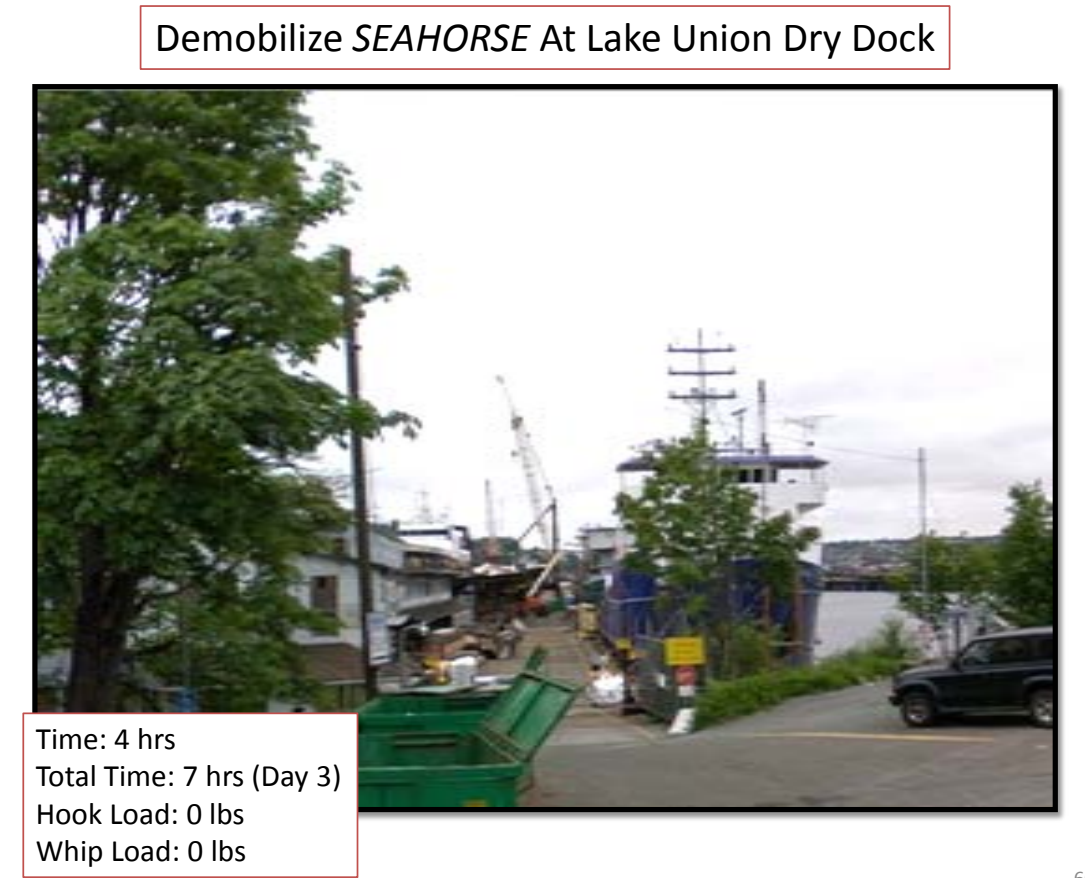

Figure 58. Lake Union Dry Dock can accommodate a truck for cradle transport.

\subsection{Maintenance}

Columbia Power Technologies - Confidential and Proprietary

Not for distribution - 2 February 2011 


\subsection{Overview}

For the first week of deployment, the WEC will be inspected using the $R V$ Neper daily. From then on, the WEC will be inspected using the $R V$ Neper every two weeks, during which charging of the WEC's batteries will take place. Maintenance trips will occur more or less frequently as is deemed necessary. Daily shore-based inspections will be performed by Sam Gooch of SST if deemed necessary. Boat operations will be provided by Gordon Roberts of Sound Support Marine, with support from Sam Gooch and/or Carl Gowler of SST.

\subsection{Daily Inspection Checklist}

The following points will be visually inspected from shore on a daily basis.

- WEC level: Inspect the level the WEC is floating at in the water. A change of more than $2 \mathrm{~cm}$ is considered abnormal and should be reported immediately.

- Damage: Check for damage from floating debris, boats, waves, etc.

- Mooring: Is the WEC in the correct position? Can all of the crown buoys still be seen, and are they in the correct positions? Changing positions could indicate anchor movement and should be reported immediately.

- Navigation Lights: Are the lights functioning? Are they being obstructed by guano or debris?

- Biofouling: Is significant biofouling present? Is it impeding the functioning of the WEC?

\subsection{At Sea Maintenance}

The maintenance boat will be moored at Brichard-Agee Dry Storage, near the Ballard Locks. For maintenance trips, the vessel will be trailered to the Shilshole Marina Boat Launch, shown in Figure 59. From the marina, it is $<15$ minutes by boat to the deployment site. TBD employees from CPT and Sam Gooch and/or Carl Gowler from SST will be present for at-sea maintenance trips. 


\subsubsection{Transit}

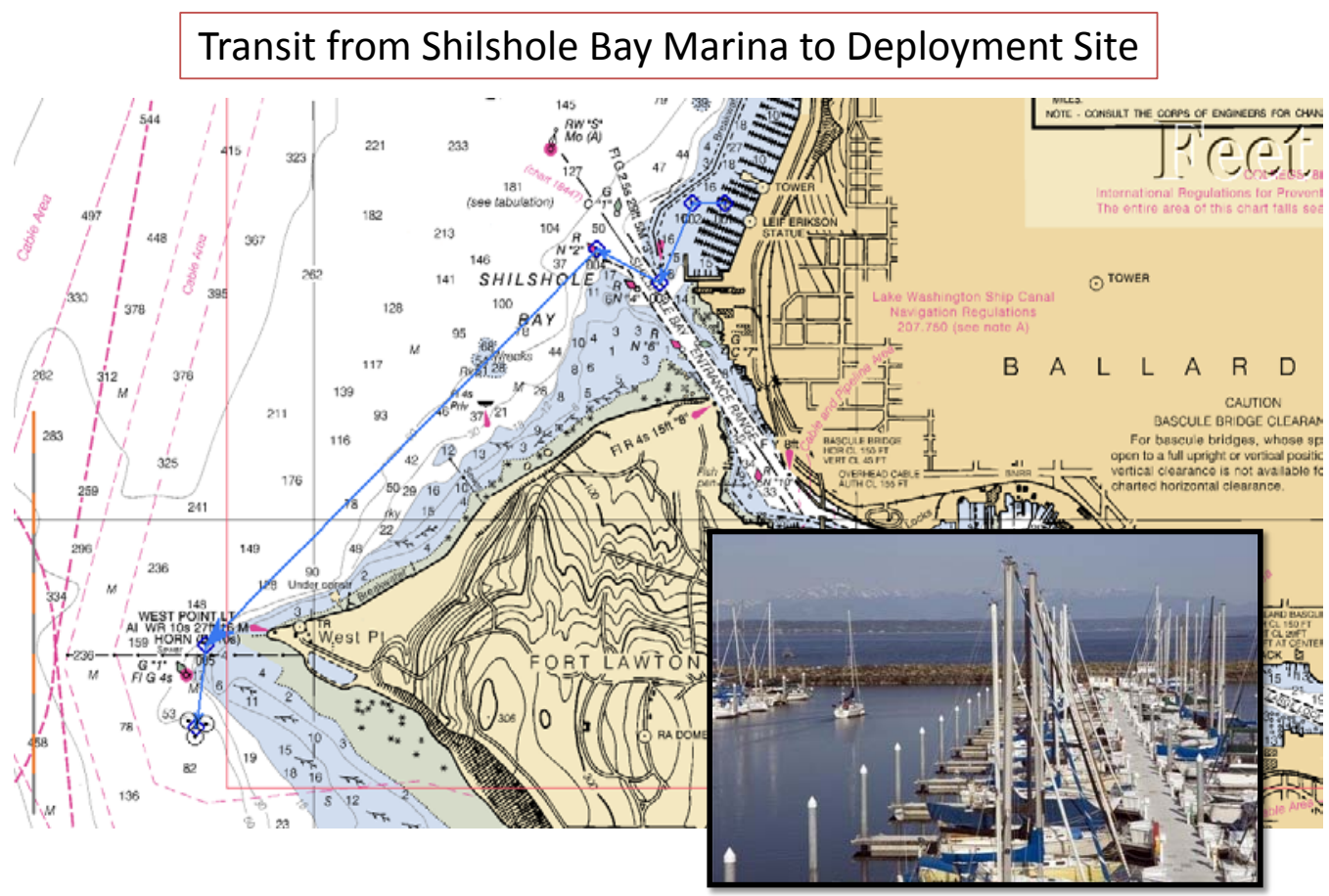

Figure 59. Transit route for maintenance.

\subsubsection{Preparing for Maintenance Operations}

While the exact procedure for tying off the maintenance vessel to the WEC is TBD, it will likely consist of the use of a slip line off the bow through a pad-eye on the buoy. This method allows static adjustment of the vessel to buoy separation, mooring and positioning assistance using the vessel engines, and a rapid method to break moorage in case of emergency. Some guidelines for safety and operations during the charging procedure:

- Don't moor directly to the WEC while charging batteries. The WEC and vessel will have different responses to the wave climate which could cause collision and damage to either.

- Use the fender board arrangement in making up to the WEC during power connection/disconnection. The fender arrangement is shown in Figure 60.

- After hooking up the power cable slip the mooring, heave to using a bow line.

- Moor on the lee side of the WEC with bow into the seas.

- Plan on charging batteries during max flood or ebb currents to maximize standoff between the vessel and the WEC.

- Charge at shorter intervals vs. longer ( 2x4 hr vs. 1x8 hr.).

- Don't rule out charging after dark if conditions are preferred. 


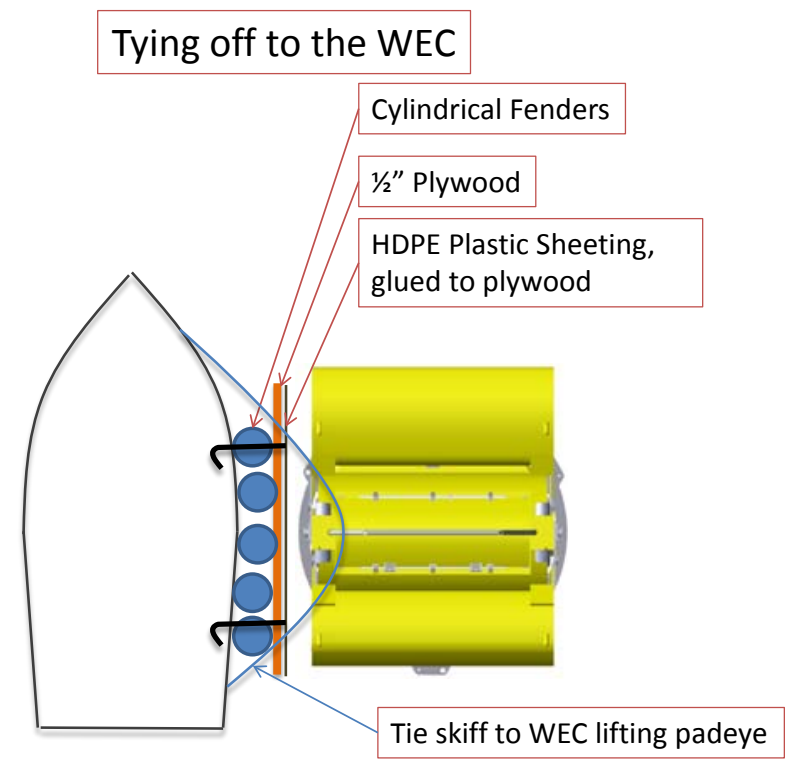

Figure 60. Vessel fendering arrangement. Only to be used for the initial connection of the WEC charging $\operatorname{cord}(\mathrm{s})$.

\subsubsection{Maintenance Operations}

The same points provided in the from-shore checklist will be inspected from the boat. Each maintenance trip will take approximately 8 hours, which is required to charge the WEC's batteries. Additionally, the maintenance and servicing procedures provided by CPT will be carried out.

\subsection{Recovery}

\subsection{Mobilization}

The recovery procedure is similar to the deployment procedure, with events happen in roughly the reverse order. Recovery will be a faster procedure as there is no need for precise anchor setting, pretensioning, or anchor setting on the SEAHORSE. It should be possible to complete all recovery operations in one working day. Mobilization for recovery will occur at the Lake Union Dry dock, where the SEAHORSE is moored. The cradle and will be brought down by truck from TBD storage location and loaded onto the vessel. 


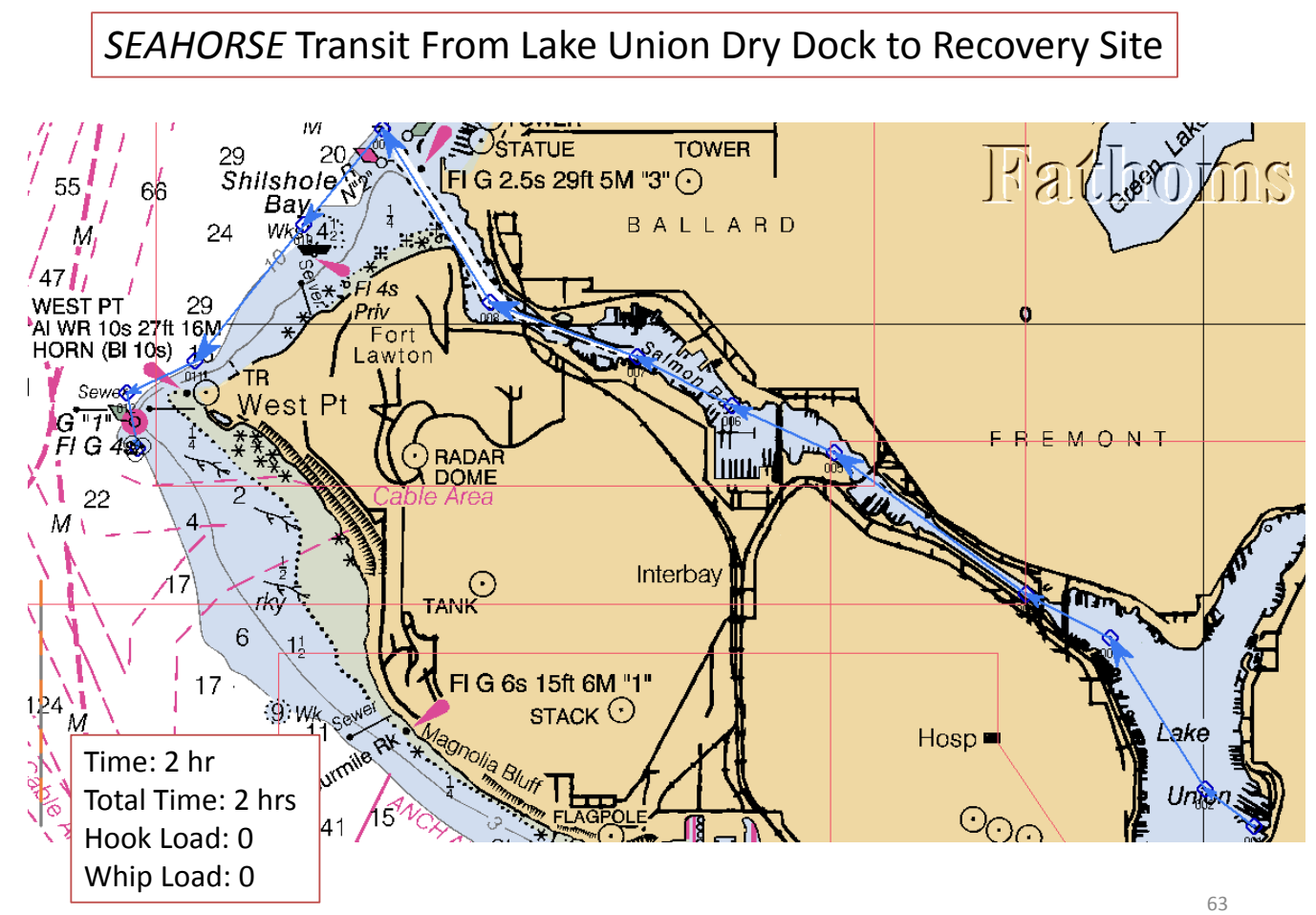

Figure 61.

\subsection{AWAC Recovery}

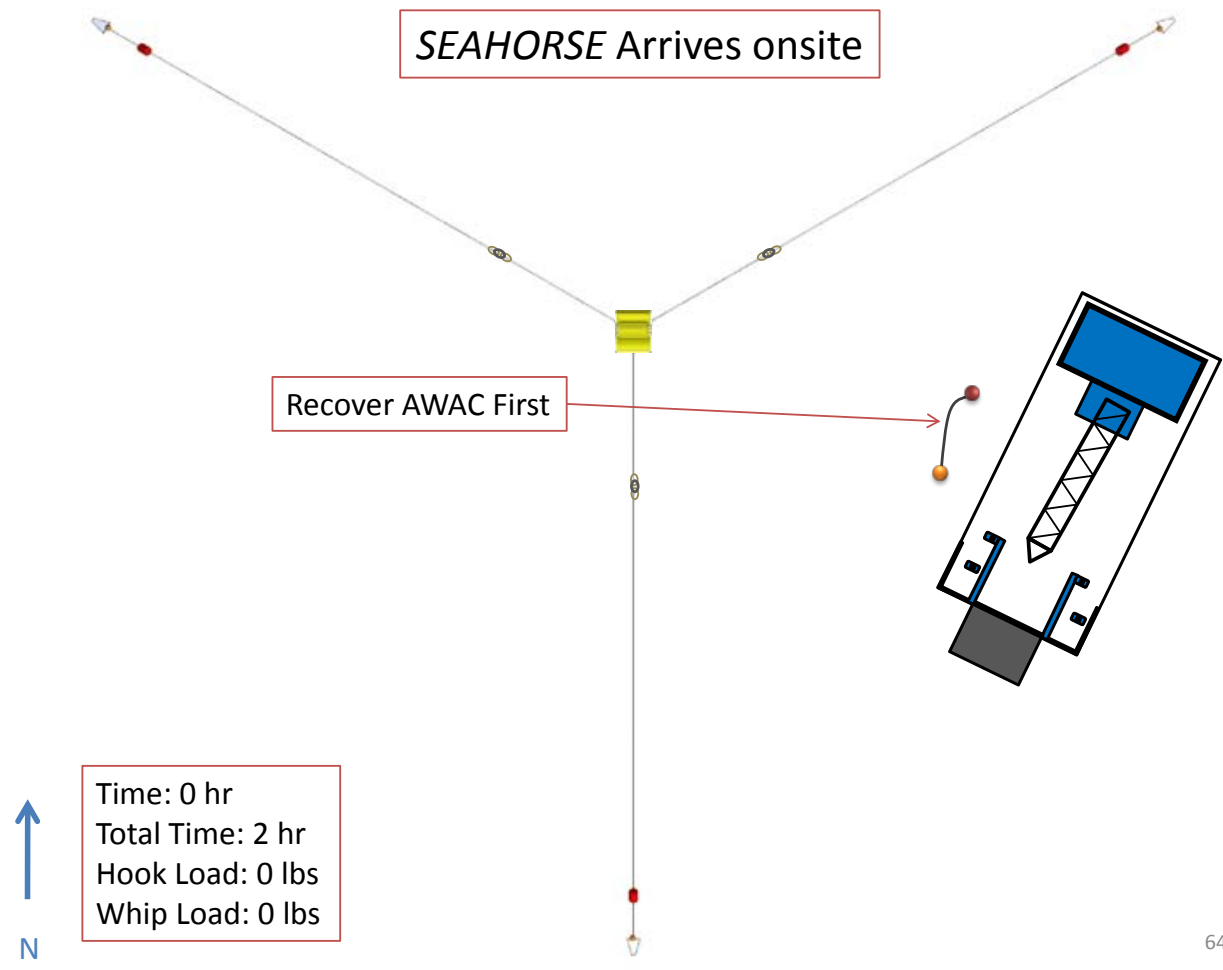

Figure 62. The first recovery step will be removing the AWAC. This will prevent the lines from getting fouled in the mooring when it is being removed. 


\section{Lift AWAC Anchor Through Recovery Buoy}

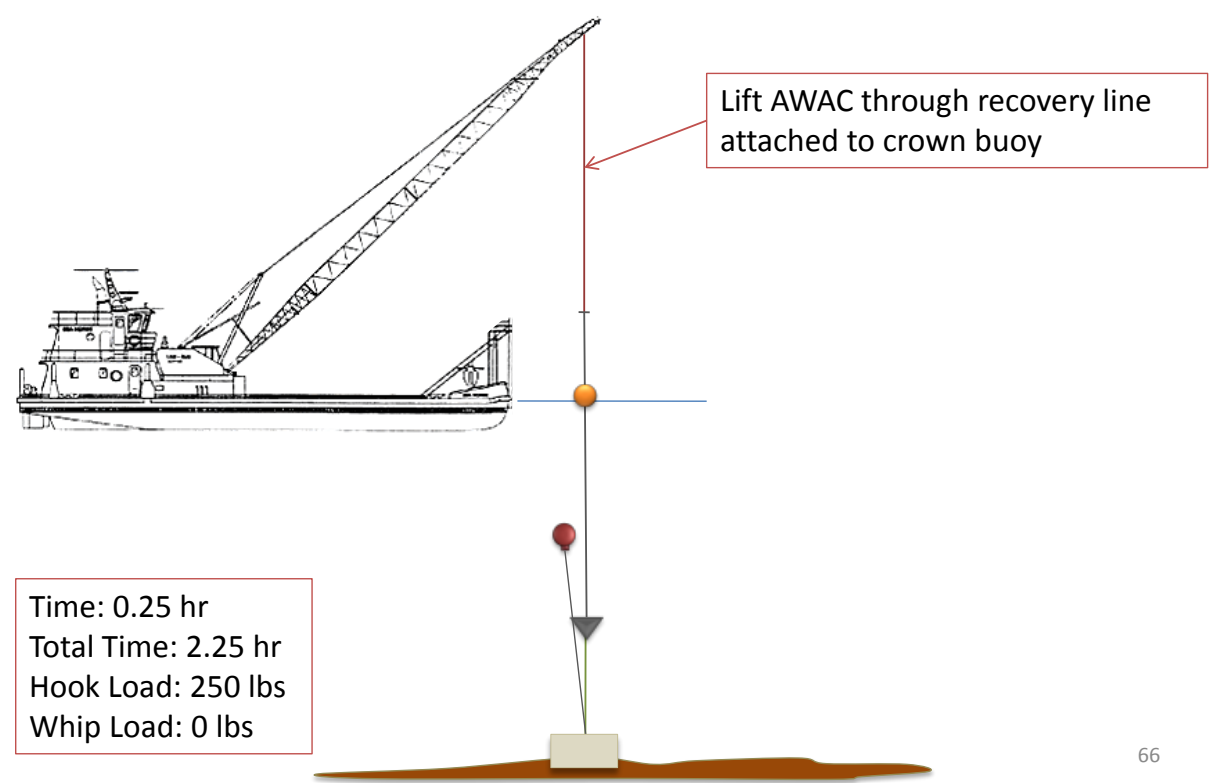

Figure 63. Lifting AWAC mooring through recovery buoy and chain (connecting clump and RR wheel anchor)

\section{Tend AWAC Line}

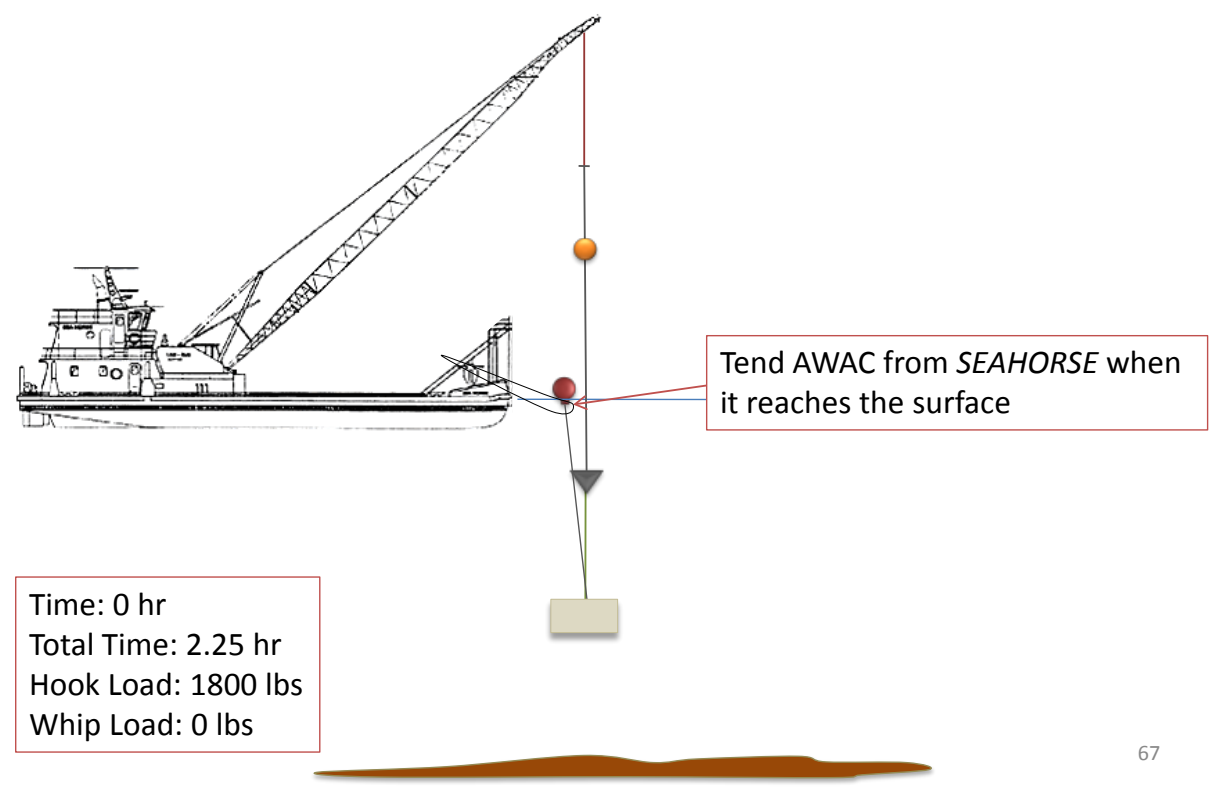

Figure 64. Tend the AWAC from the deck using a tagline. The AWAC will come onboard after the anchors.

Columbia Power Technologies - Confidential and Proprietary

Not for distribution - 2 February 2011 


\section{Transfer Load to Whip, Continue Lifting}

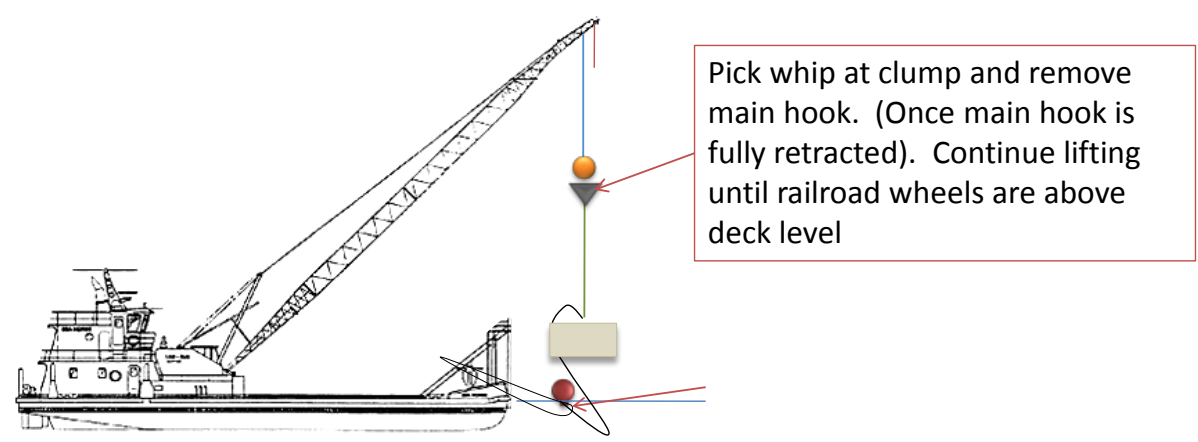

Time: $0.5 \mathrm{hr}$

Total Time: $2.80 \mathrm{hr}$

Hook Load: 0 lbs

Whip Load: 1800 lbs

Figure 65. As in the installation, since the height of the crane is less than the length of the AWAC mooring (including the 40' chain), the load must be transferred to the whip when the crane hook reaches the top of its range of travel. The load can then continue to be lifted with the whip.

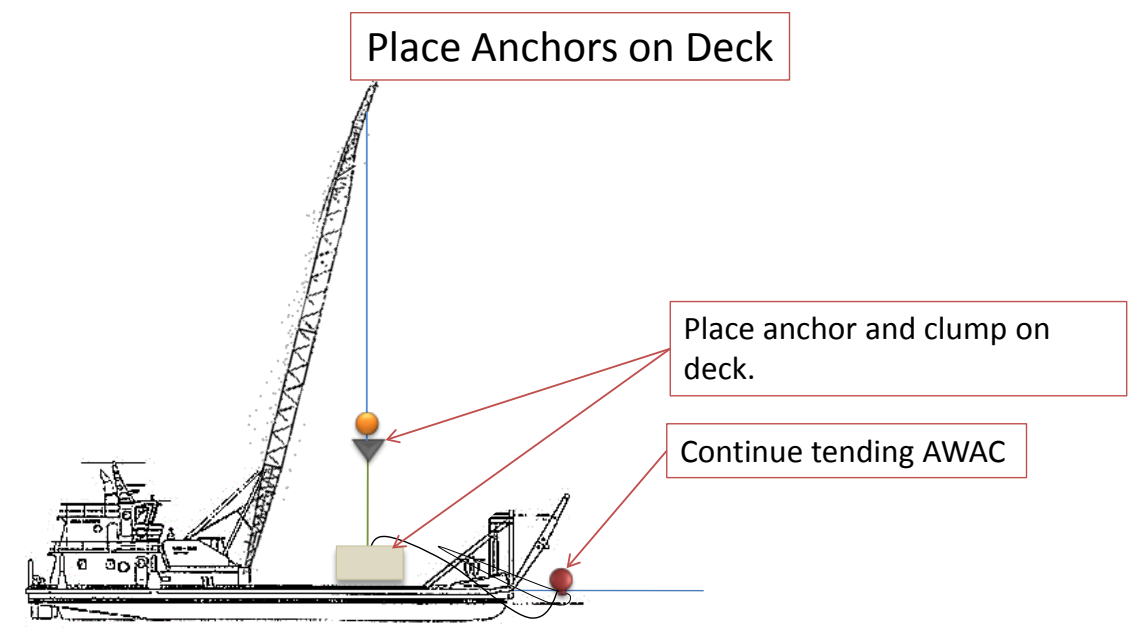

Time: $0.25 \mathrm{hr}$
Total Time: $3 \mathrm{hr}$
Hook Load: $0 \mathrm{lbs}$
Whip Load: $200 \mathrm{lbs}$

Figure 66.

Columbia Power Technologies - Confidential and Proprietary

Not for distribution - 2 February 2011 


\section{Retrieve AWAC}

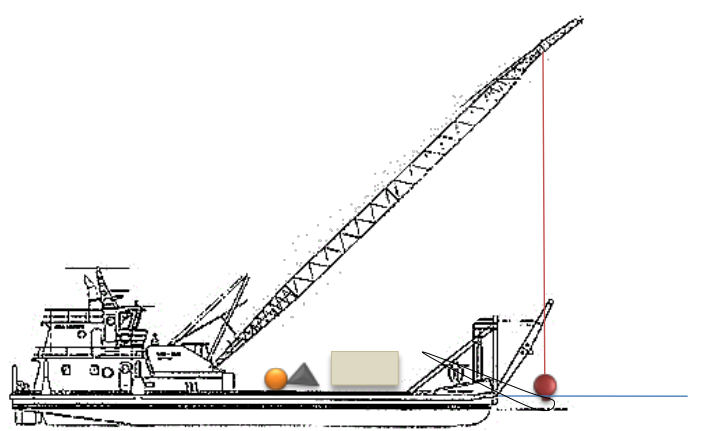

Time: $0.25 \mathrm{hr}$

Total Time: $3.25 \mathrm{hr}$

Hook Load: 200 lbs

Whip Load: 0 lbs

Figure 67. Use the whip or crane to lift the AWAC on deck. The existing loop can be used as a tagline.

\subsection{WEC Recovery}

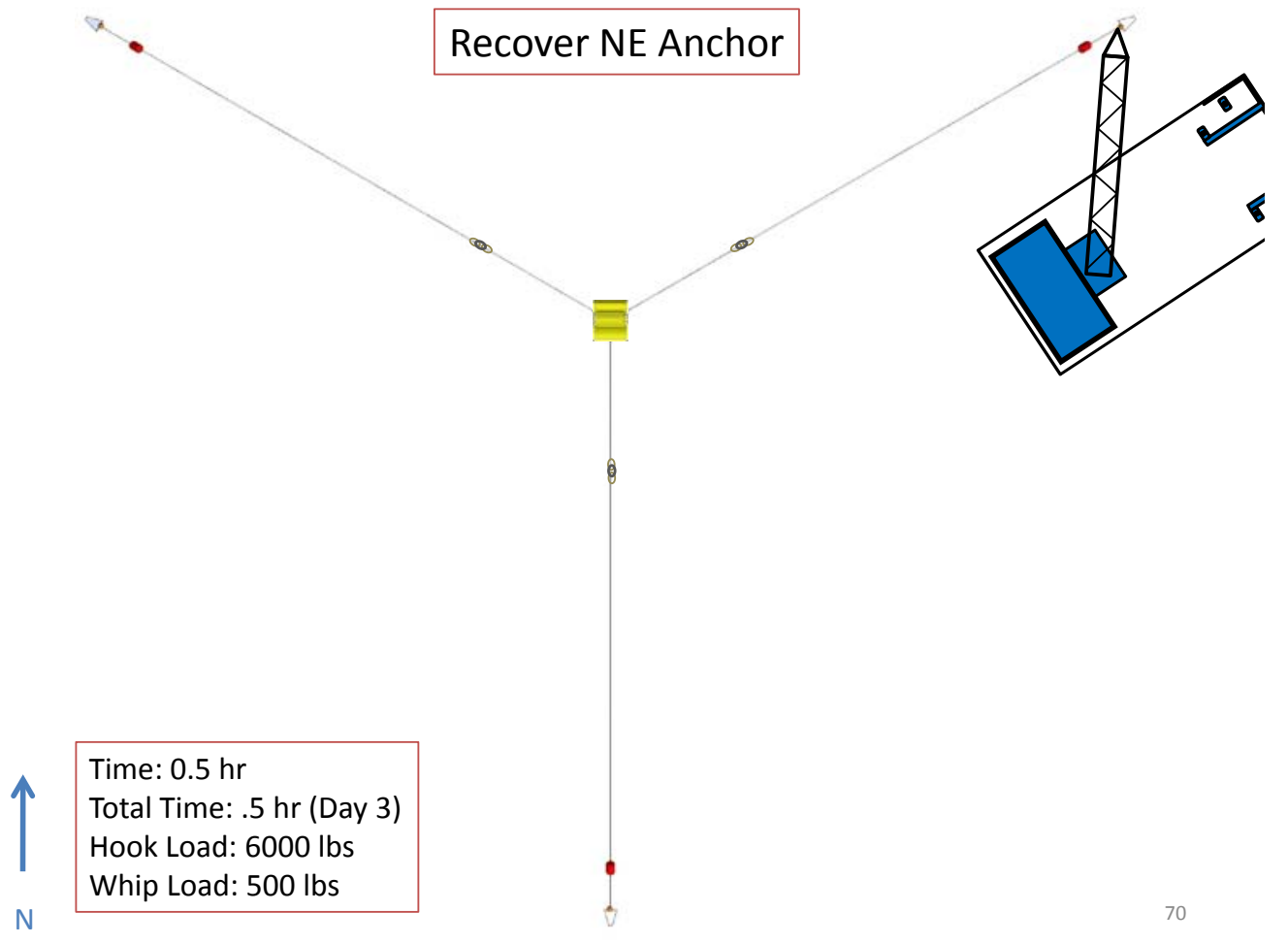

Figure 68. First step in the WEC mooring recovery process.

Columbia Power Technologies - Confidential and Proprietary

Not for distribution - 2 February 2011 


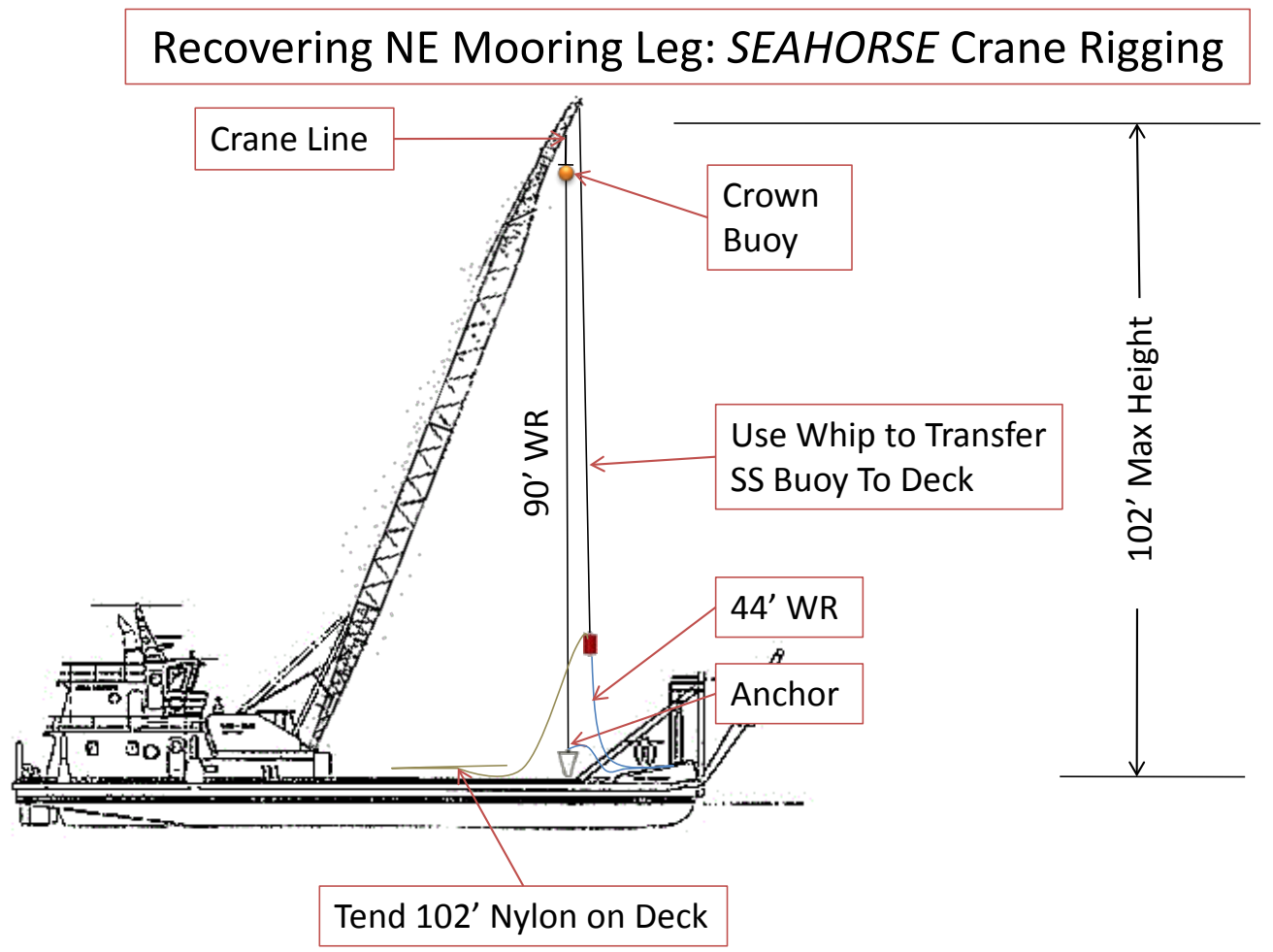

Figure 69. Note that the SS buoy can be brought onboard separately after the anchor is recovered.

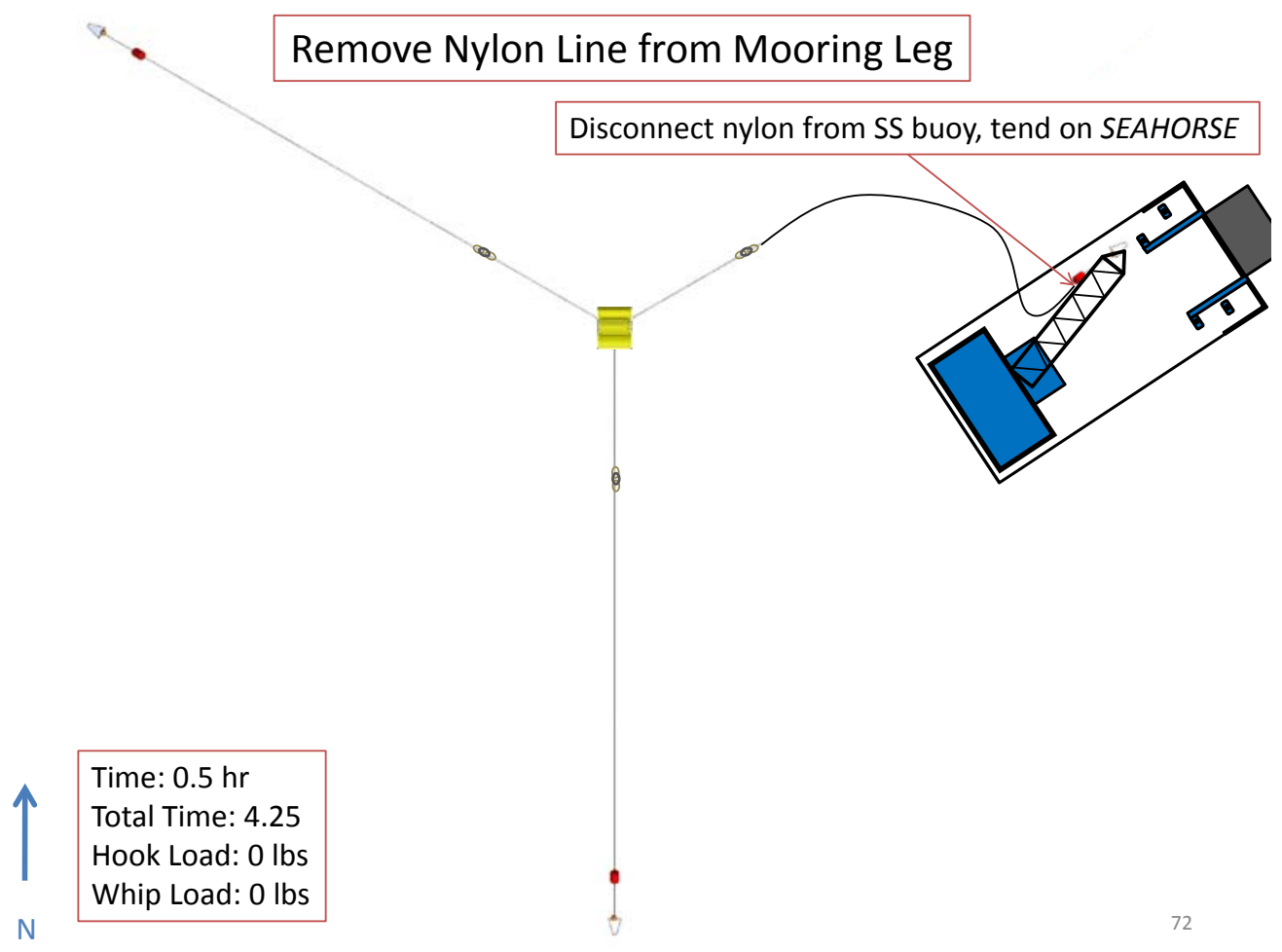

Figure 70. The nylon leading to the WEC will be used as a tagline in the next step. 


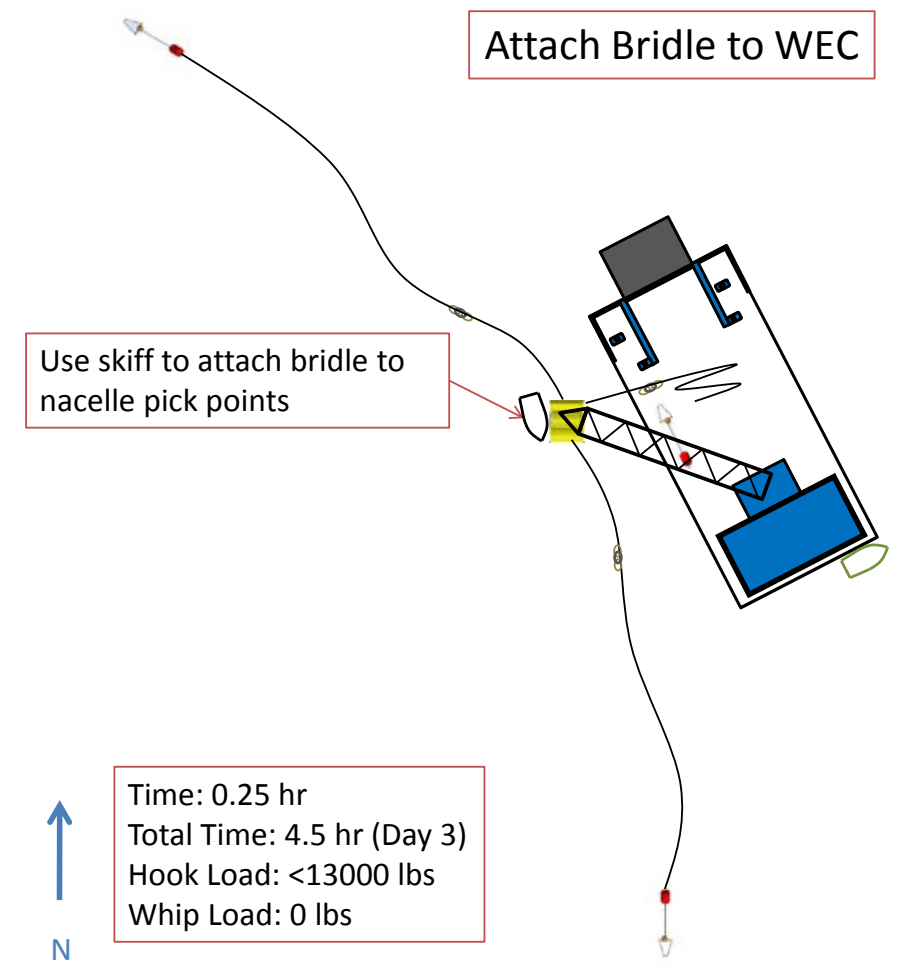

Figure 71.

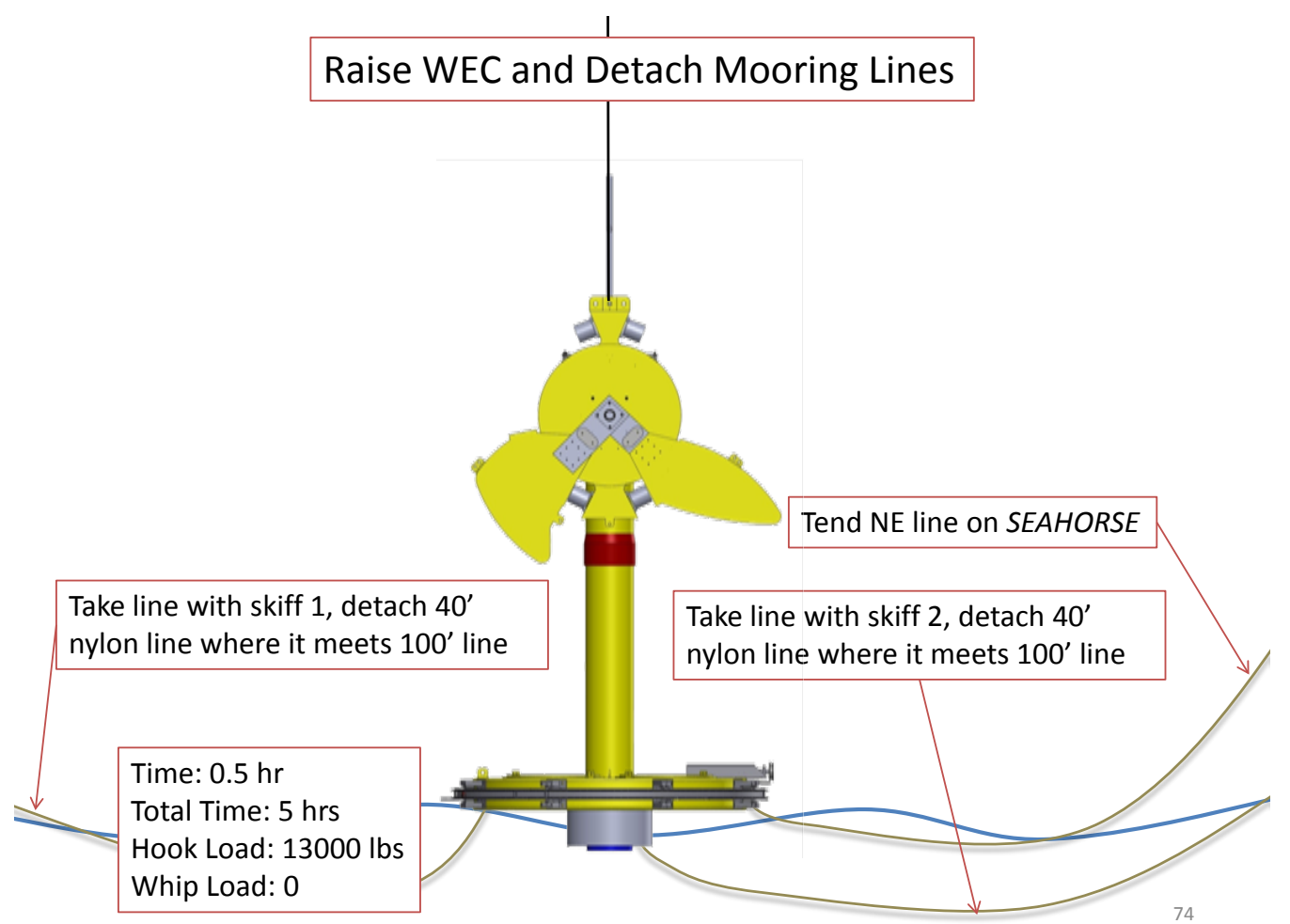

Figure 72. Disconnecting the WEC from the remaining two mooring legs.

Columbia Power Technologies - Confidential and Proprietary

Not for distribution - 2 February 2011 


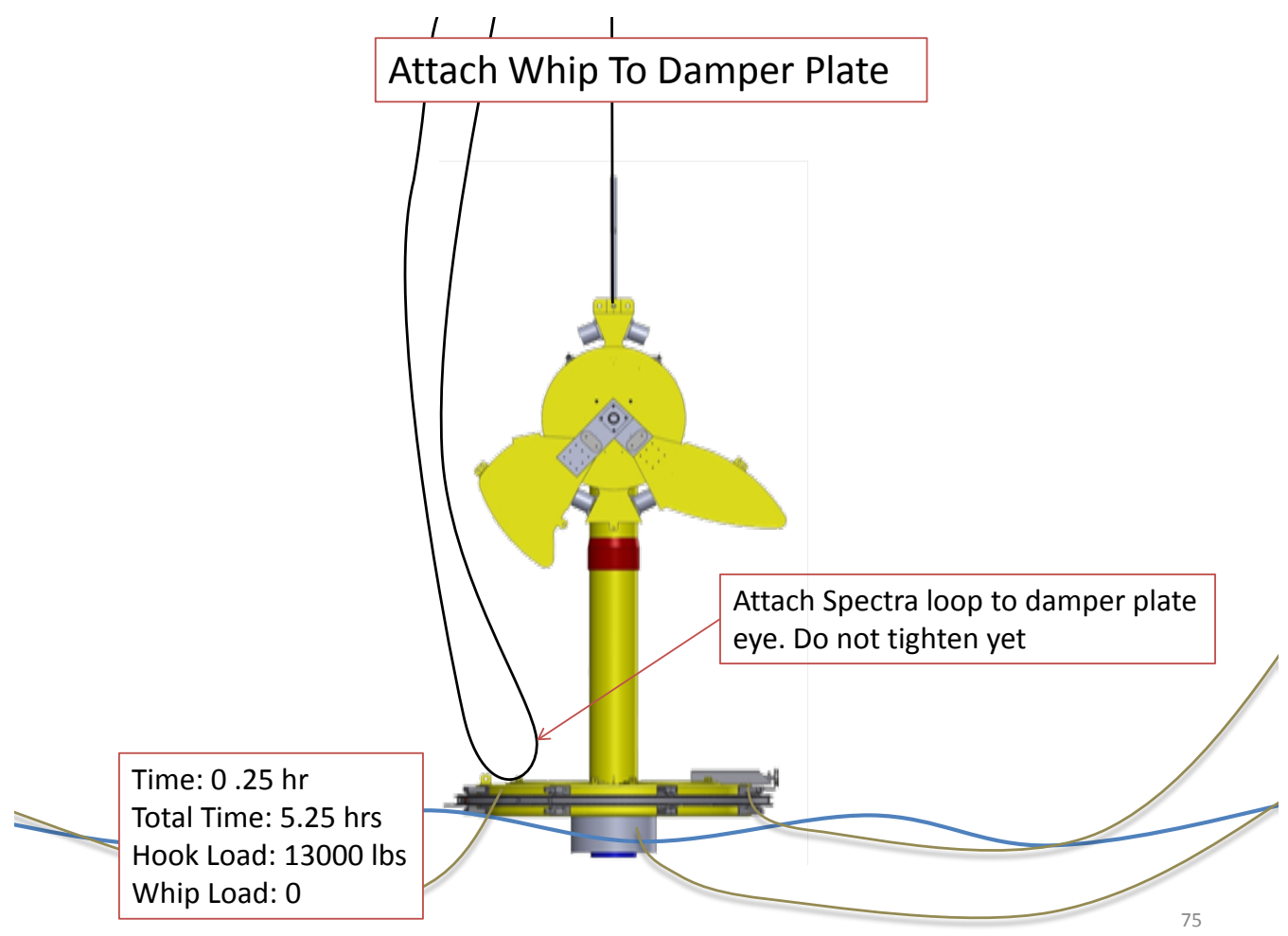

Figure 73.

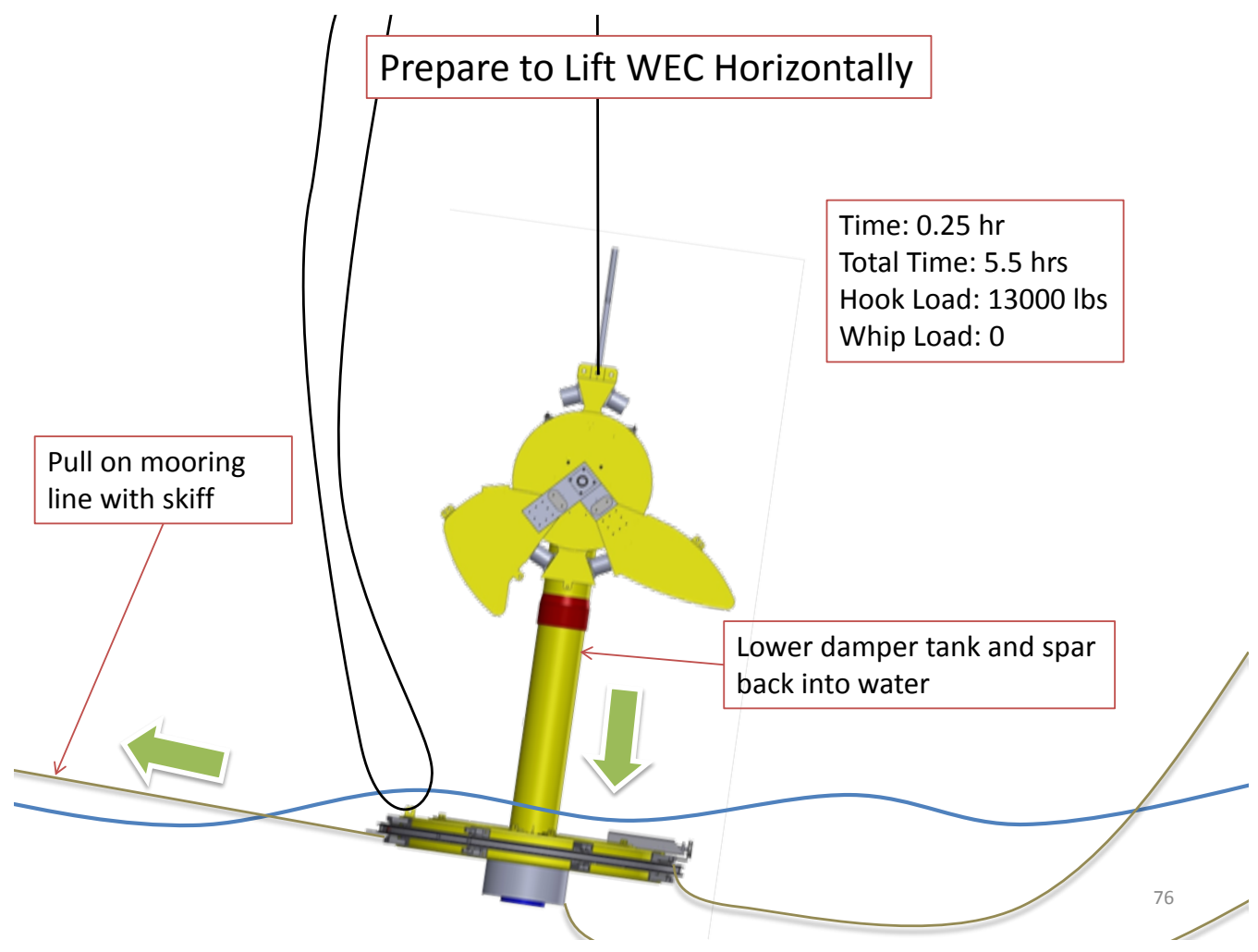

Figure 74. By lowering the WEC partially into the water and pulling on one side with the skiff, the WEC will be brought slightly off vertical. The whip (damper plate) line can then be tensioned without contacting the aft float. 


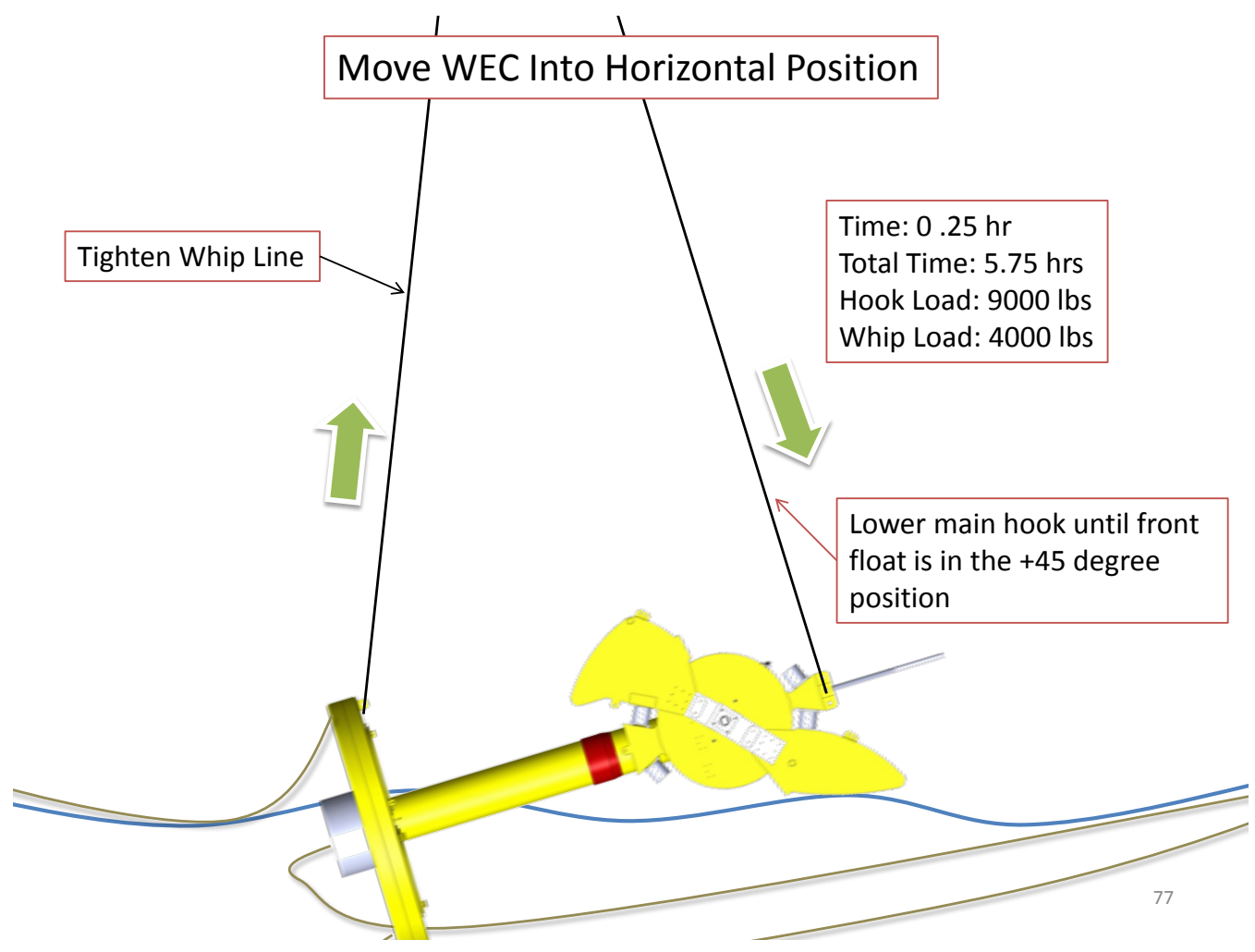

Figure 75.

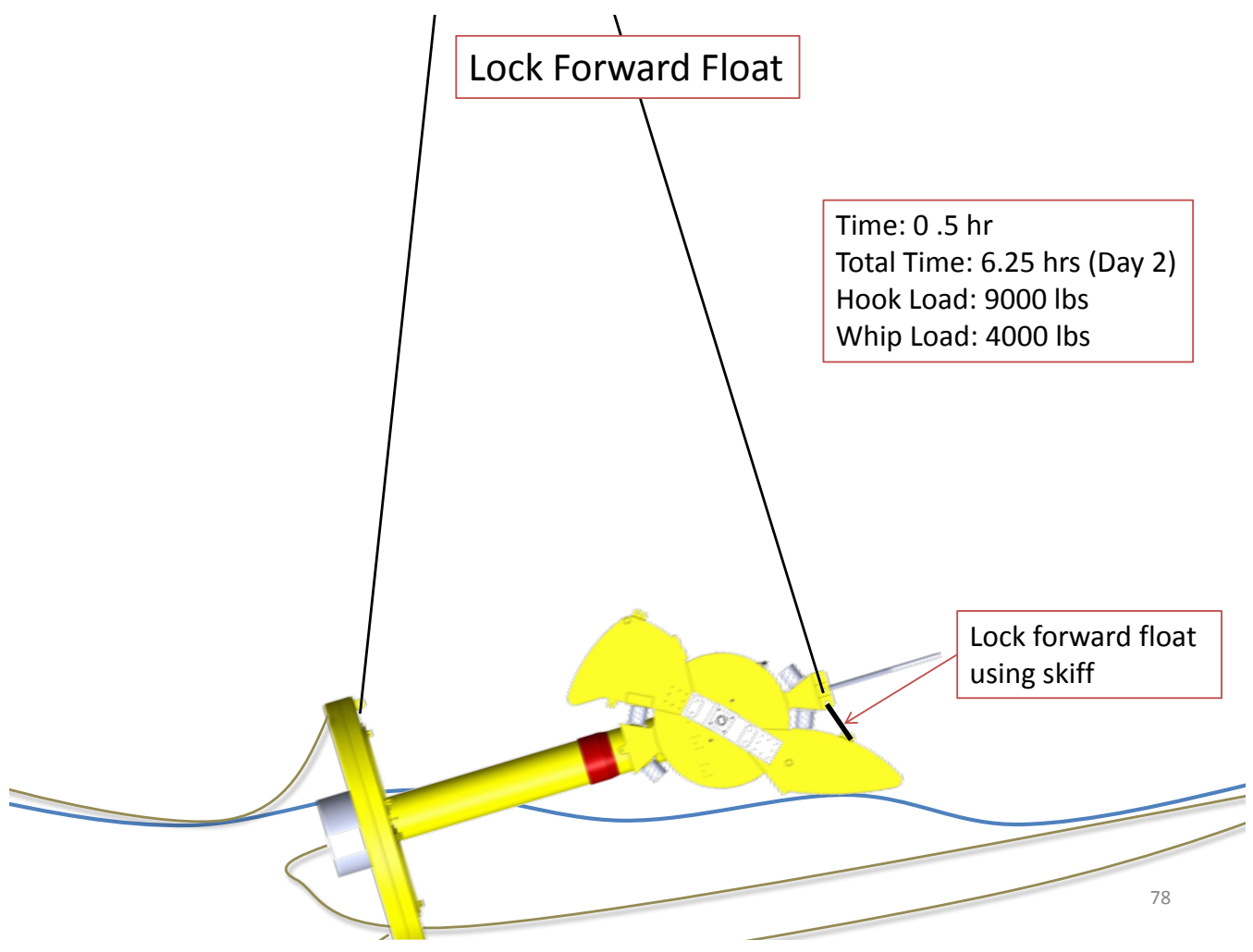

Figure 76.

Columbia Power Technologies - Confidential and Proprietary

Not for distribution - 2 February 2011 


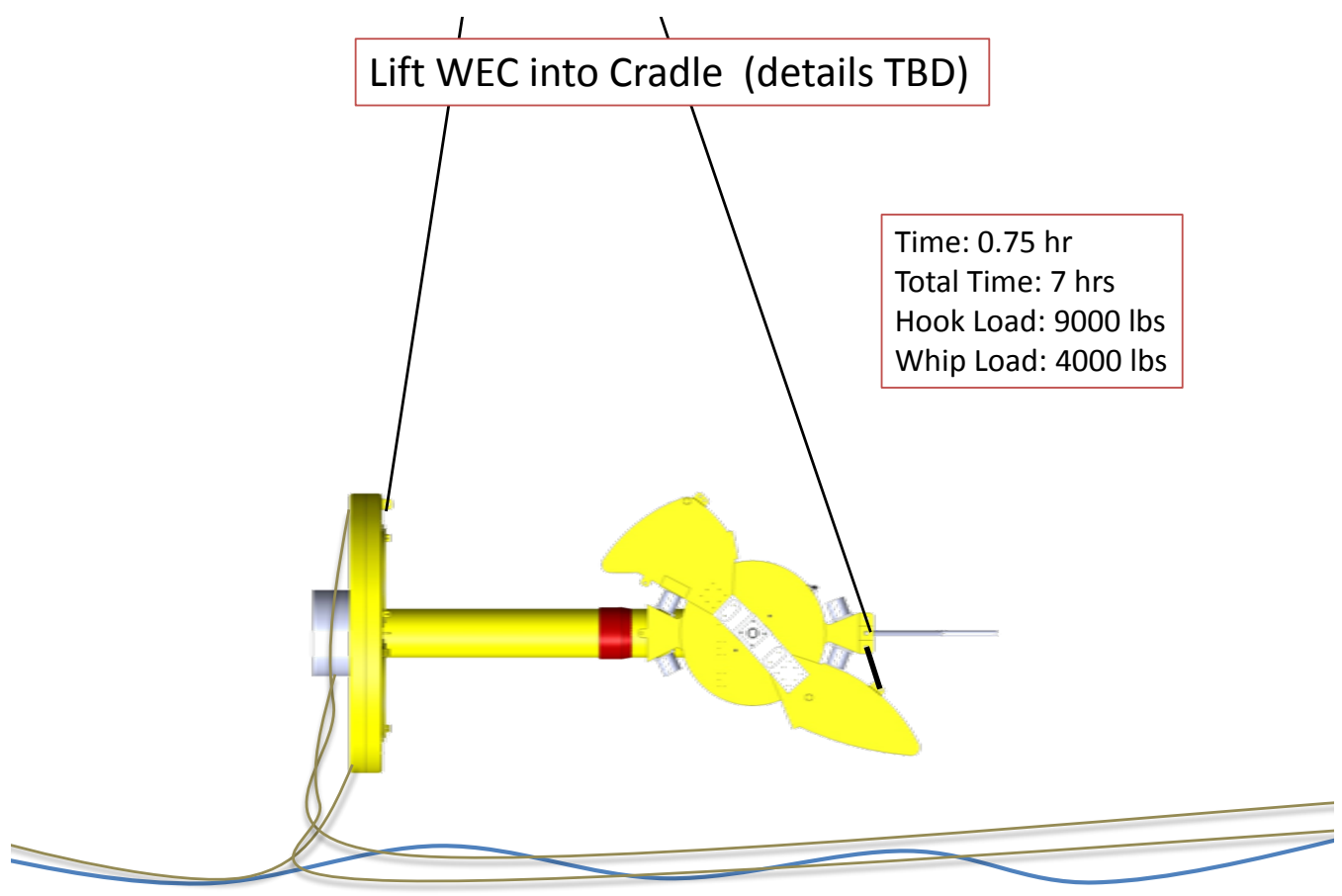

Figure 77.

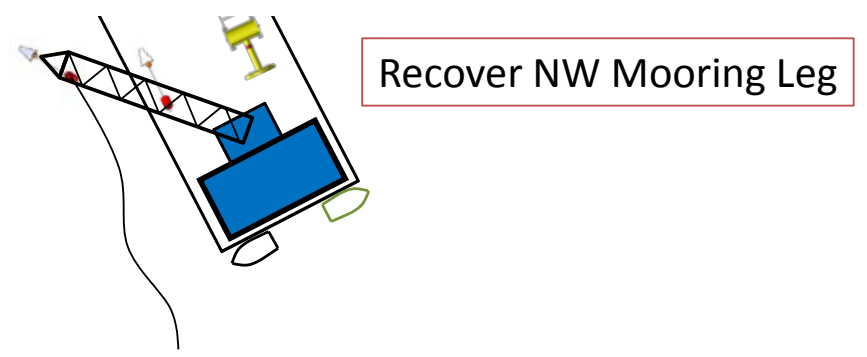

Time: $0.5 \mathrm{hr}$

Total Time: $7.5 \mathrm{hr}$ (Day 3)

Hook Load: $6500 \mathrm{lbs}$

Whip Load: 0 lbs

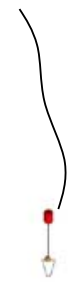

Figure 78.

Columbia Power Technologies - Confidential and Proprietary

Not for distribution - 2 February 2011 

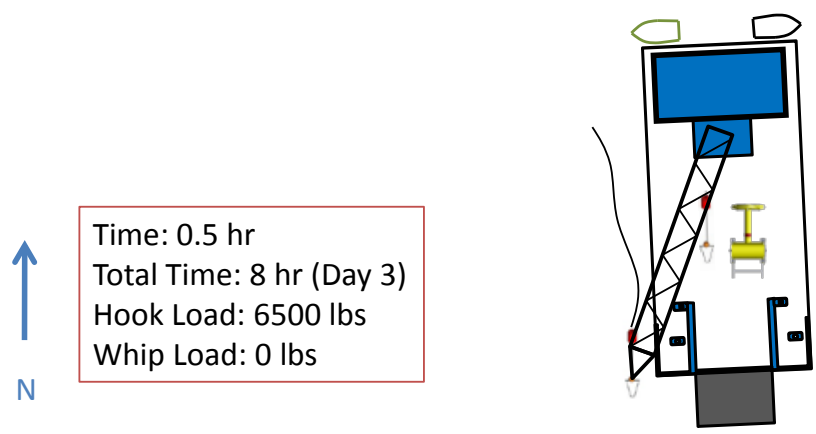

Figure 79.

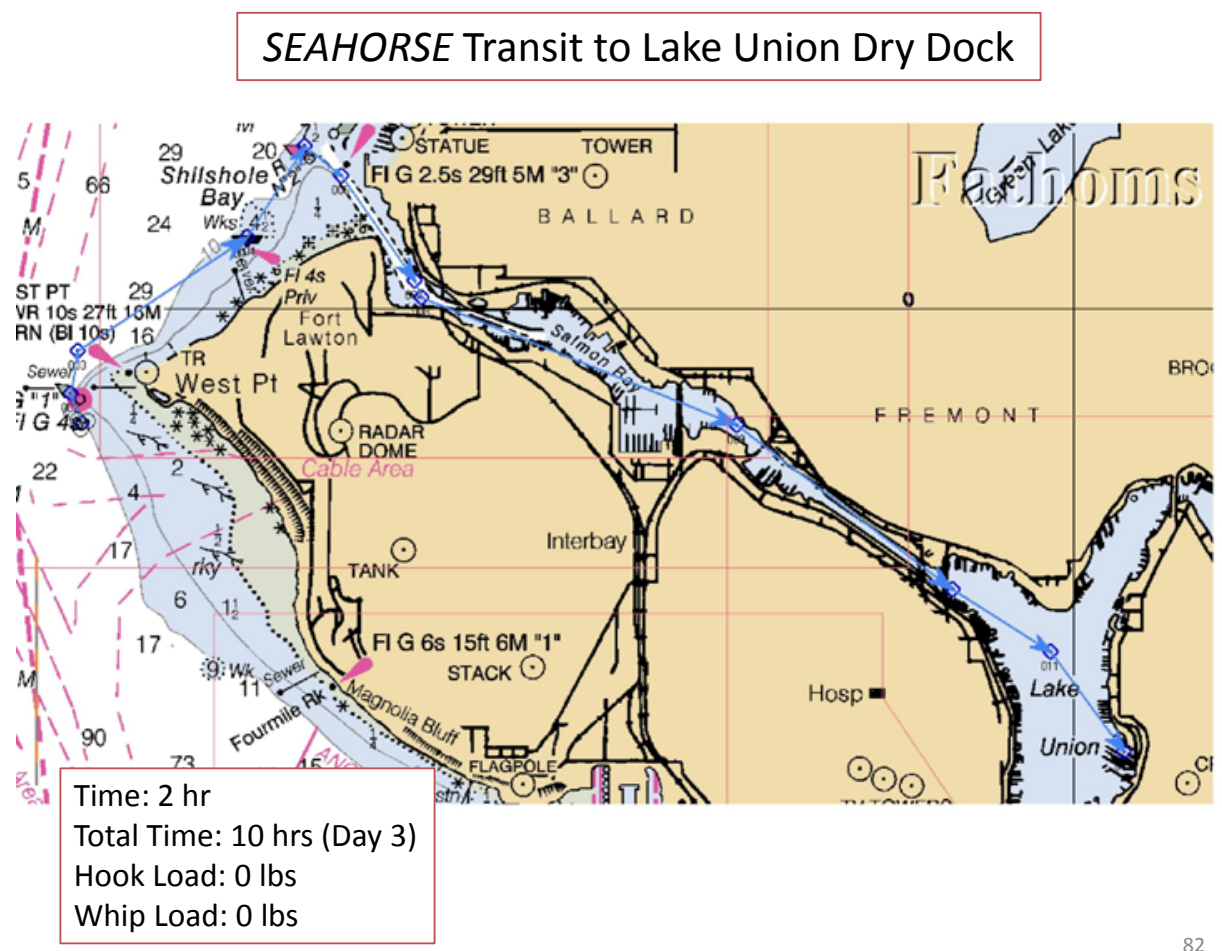

Figure 80. WEC will be unloaded onto flatbed and taken to location TBD. A separate flatbed will be brought down for anchor and SS buoy removal and mooring system disposal/recycling.

\subsection{Contingencies}

Columbia Power Technologies - Confidential and Proprietary

Not for distribution - 2 February 2011 
The following table describes several possible situations where contingency planning could be necessary, and the proposed courses of action:

Description

SEAHORSE Loses Mobility

Rough Seastate

\section{Resolution}

Tugs available with $<1 \mathrm{hr}$ response time. Can use to move SEAHORSE Bow/Stern anchors or WEC Moorings

Process can be "paused” at any point. Contingency (third) day scheduled beforehand with SEAHORSE.

Inclement Weather

Process can be "paused" at any point. Contingency (third) day scheduled beforehand with SEAHORSE.

In order to facilitate stopping in case of inclement weather or equipment failure, several stopping points have been identified, where operations can be suspended indefinitely if necessary:

- After NW (first) anchor is deployed

- After SEAHORSE mooring is set

- After S (second) anchor is deployed

- During WEC deployment:

o WEC can be brought back on deck until third anchor is rigged to crane

- After NE (third) anchor is deployed

- Any point in AWAC deployment

\subsection{Retrieving the WEC for Service/Maintenance (If Necessary)}

In the case of the WEC needing servicing or maintenance during the deployment, the WEC will be retrieved using the SEAHORSE. The exact procedure will vary based on the nature of the issue. A likely retrieval plan would go as follows:

- Retrieve WEC Cradle, bring to Lake Union Drydock and transfer to SEAHORSE

- SEAHORSE transit to deployment site

- Move NE anchor towards WEC to slack off mooring

- Pick WEC by Nacelle

- Detach 120' nylon mooring lines from 40’ lines; attach 120' lines to central crown buoy

- Boom WEC over deck of SEAHORSE

- Attach spectra line to Damper Plate and whip

- Lift WEC horizontally using spectra line/whip, place in cradle (need to determine necessity of locking floats out)

\subsection{Appendix A. Safety Plan}

\subsection{Introduction}

It is the responsibility of Sound \& Sea Technology to maintain a safe working environment for the CPT WEC deployment at West Point, Puget Sound. The USACOE Safety \& Health Requirements Manual (EM385-1-1 15 Sep 2008) was used as a reference when creating this safety plan. 
The Sound \& Sea Technology staff shall verify through written certification that operational personnel have read and understand the Safety Plan \& Safety Training. The written certification shall identify the name of each employee.

\subsection{General Precautions}

Safety precautions set forth in the Safety Plan will be observed during all phases of this test. Safety Officer for this program is Carl Gowler of SST. The Safety Officer will ensure that all operations are conducted in a safe and prudent manner. However, safety is the responsibility of each and every member of this team. Each person will have the obligation to halt any operation they deem unsafe. Each team member shall take their time and speak up if they have any questions. No one is to ever try to save any equipment with the use of their hand or body. Safety always comes first.

Employees are responsible for reporting all injuries or occupationally related illnesses as soon as possible to the Safety Officer.

\subsubsection{Weather}

Installation or maintenance will not commence until sea states are at or below Sea State 3 as determined by the Safety Officer. Additionally, installation will not commence in winds exceeding 10kts. In the event that conditions do not allow the start of operations on a particular day, install team will remain on standby. If weather conditions exceed the prescribed wind speed or seastate, the urgency of the procedure (e.g. battery charging, WEC failure) will be assessed.

\subsubsection{Personal Protective and Safety Equipment}

- PPE Equipment will be provided for all personnel involved in this test.

- When conducting the installation or the operations aboard vessels, all personnel shall wear full PPE (hardhats, gloves, steel-toed shoes, PFD's, etc.).

- Head Protection: Type II head protection (hardhats) will be worn by all personnel present during crane operation, or any other time when loads are moving or suspended overhead.

- Personal Flotation Device (PFD): A US Coast Guard approved Type III safety work vest shall be worn when working over the water in a small boat or on the WEC. At night, a strobe light in working order will be attached to the PFD.

- Protective Footwear: All personnel involved in crane operation or material movement aboard vessels or shore side shall wear protective footwear that meets ASTM F2412 and F2413 standards.

\subsubsection{Hand and Power Tools}

Hand and power tools shall be used, inspected, and maintained in accordance with the manufacturer's instructions and recommendations and shall be used only for the purpose for which designed.

Hand and power tools shall be in good repair and with all required safety devices installed and properly adjusted. Tools, including extension cords, having defects that will impair their strength or render them unsafe shall be removed from service. 


\subsubsection{Lifting}

Assess the load to be lifted. Wherever possible, use a mechanical lifting device, i.e., dolly, gantry crane, forklift. Understand load weight, lifting points, awkward size/shape/sharp edges, hazards to personnel in the area. All are possible considerations. If in doubt, ask for help.

\subsubsection{Tensioned Lines}

Lines that are aboard vessels under tensioned will be monitored by the safety observer and crane director. Personnel will be directed to not stand near any lines or cables under tension. Slack lines being utilized will be monitored by the safety observer. Personnel will be directed to not stand near any working line, reef line or cable, especially in the bight of any line or cable being used.

\subsubsection{SST Lifting Equipment}

All equipment used in conjunction with cranes will follow the requirements of NAVFAC P-307 Section 14. SST will comply with specific activity and regulations pertaining to crane safety and operation for contractors.

Tag lines will be used in conjunction with the crane to assist in the control of the WEC during deployment and recovery.

Personnel not taking part in deployment or recovery will not be permitted aboard the contracted vessel(s).

During Lifting Operations:

- Pay attention to the job at hand

- Do not talk loudly while attending deployment or recovery

- Wear appropriate PPE

- Watch for trip hazards on deck and do not run

- Remain clear of the crane operations when not directly participating in the operation.

\subsubsection{Man Overboard}

Anyone observing a victim falling overboard will yell "MAN OVERBOARD” and notify the Safety Officer or anyone within ear shot.

Anyone seeing the person will continuously point to the man overboard and keep eyes on the victim's location. A USCG-approved Type IV life ring will be thrown to the victim in the water. A small boat will then be dispatched. Medical procedures are followed as appropriate.

\subsubsection{Fire}

If a fire breaks out, attempt to put the fire out or contain it with the Portable type 2 fire extinguisher in the galley, yelling "FIRE" and location.

\subsubsection{First Aid}

A First Aid Kit will be provided onboard the Seahorse. If a person is injured while performing project related duties the following steps will be taken in case of a medical emergency:

Call the Coast Guard (marine band channel 16). 


\subsubsection{Pre Existing Health Problems}

Inform the Safety Officer prior to the test of any health conditions which may be adversely affected by the marine environment (heart or respiratory conditions, previous skeletal or back injuries, etc.),

\subsection{Electrical safety}

\subsubsection{Electrical Safety Procedures}

All electrical service devices, equipment, and cabling shall be in good repair. Any element that appears to be damaged or is suspected of being damaged shall be removed from operation immediately. Care will be taken during battery charging operations to avoid exposing any personnel to hazardous voltages.

All AC power equipment and cabling shall be protected from exposure to water.

Anyone witnessing a potentially dangerous electrical condition shall warn others and report it immediately to the on-site Safety Officer.

\subsection{Crane Operations}

Rigging will be inspected before loads are lifted. Radios will be used for blind lifts. A lift plan for installing and recovering the WEC will be in place with personnel assignments before lifting commences. SST will conduct a safety brief for the lift and associated handling operations.

\subsubsection{Lifted Equipment}

The following is the majority of equipment/material that will be handled with contractor provided cranes:

\begin{tabular}{|ll|}
\hline Item & Weight \\
\hline WEC & $13,500 \mathrm{lbs}$ \\
\hline WEC Crate & $8,000 \mathrm{lbs}$ \\
\hline Lead Cone Anchors & $3 @ 6,000 \mathrm{lbs} /$ each \\
\hline AWAC & $500 \mathrm{lbs}$ \\
\hline AWAC Anchor & $2000 \mathrm{lbs}$ \\
\hline Subsurface Buoys & $3 @ 400 \mathrm{lbs} /$ each \\
\hline AWAC Mooring Clump & $200 \mathrm{lbs}$ \\
\hline
\end{tabular}

\subsection{Appendix B. Detailed Mooring Drawings}




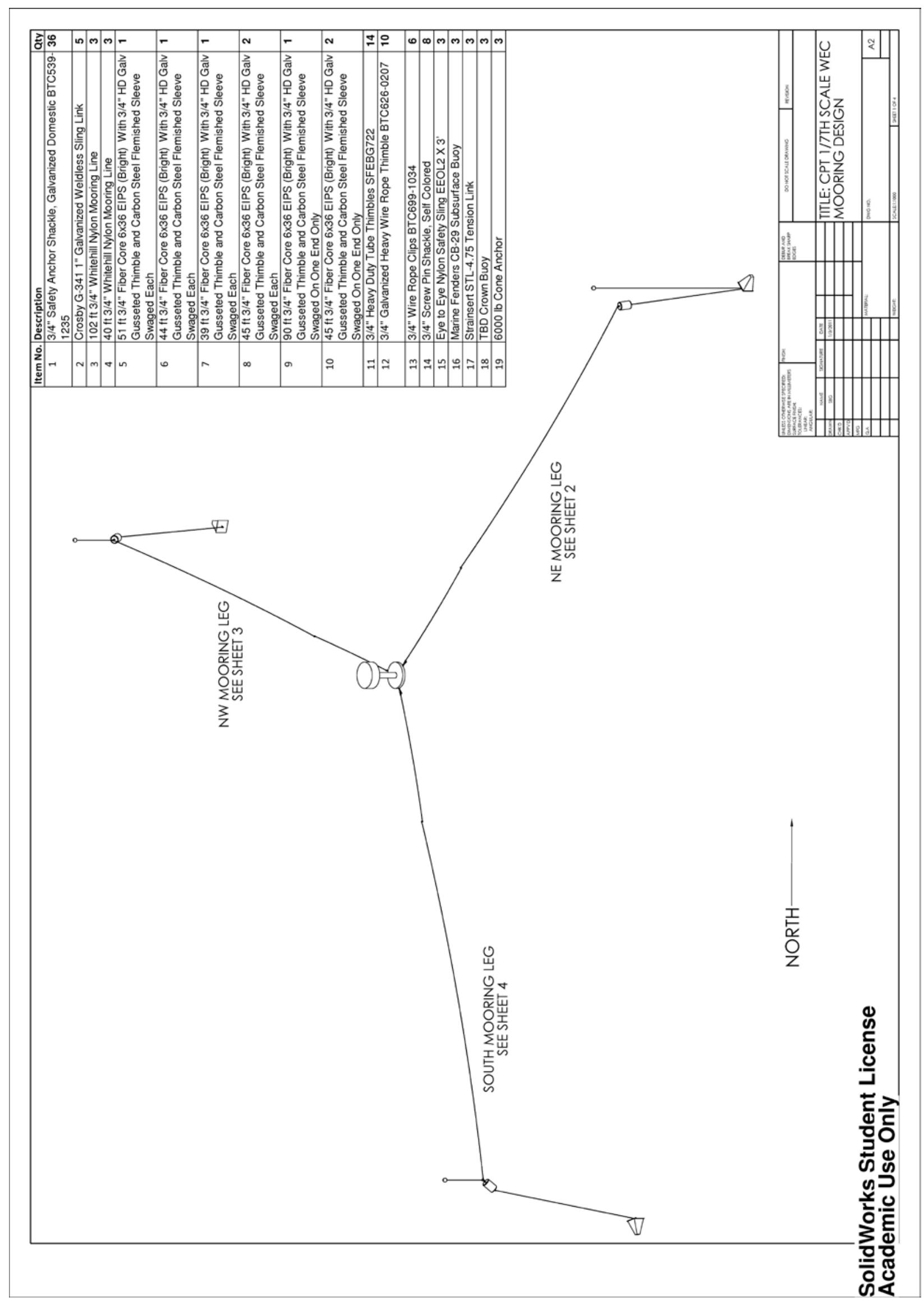

Columbia Power Technologies - Confidential and Proprietary 


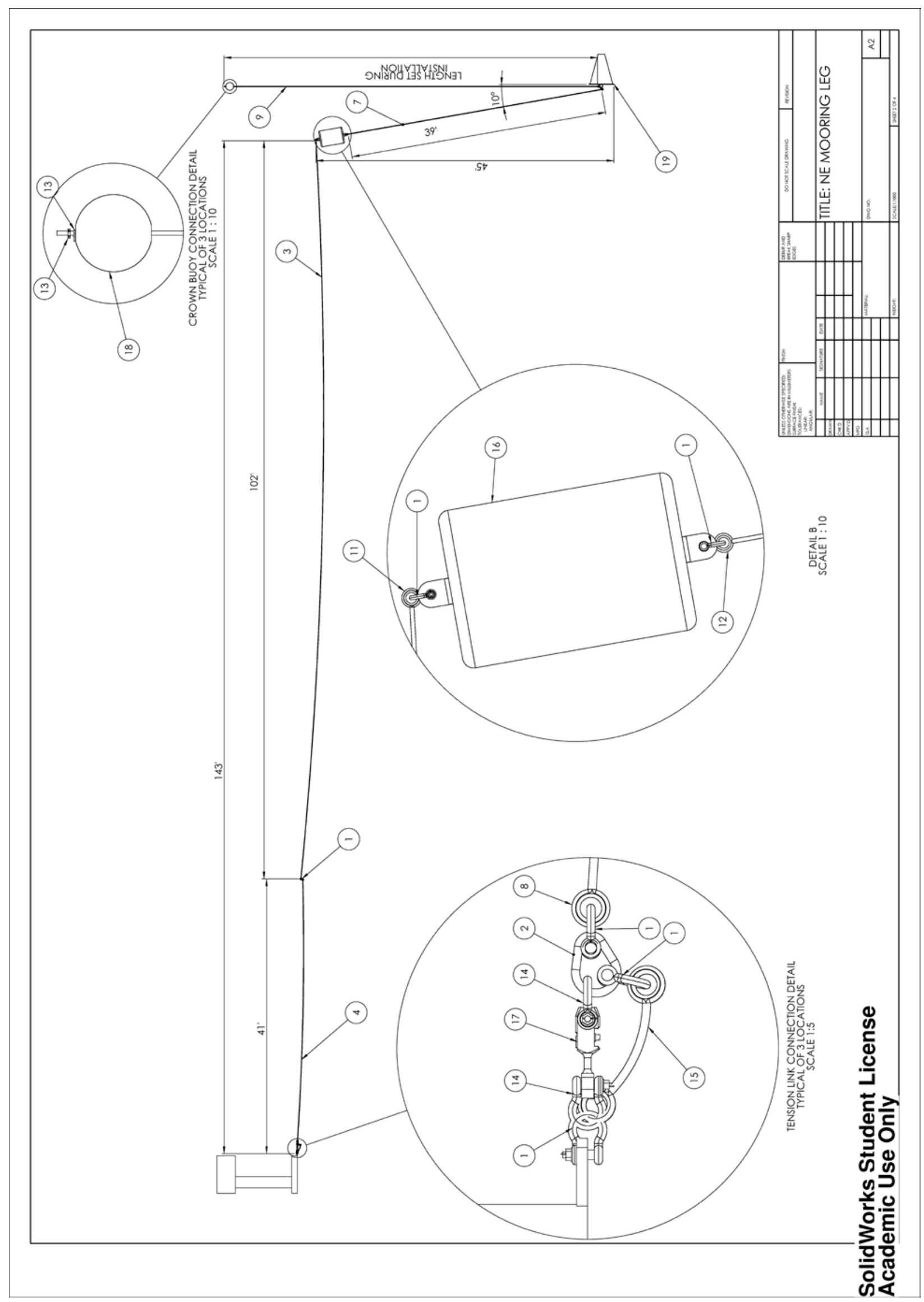

Columbia Power Technologies - Confidential and Proprietary 


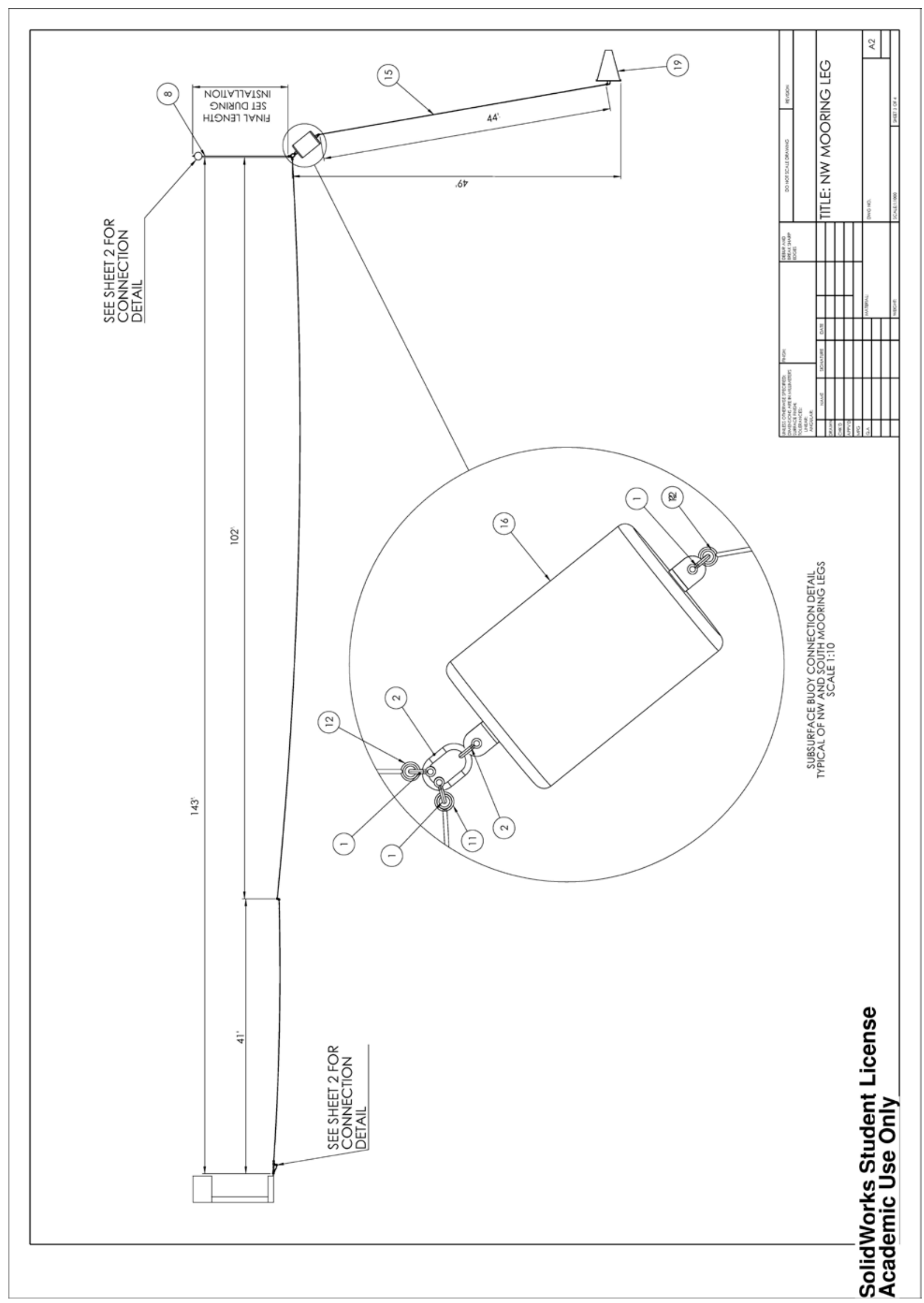

Columbia Power Technologies - Confidential and Proprietary 


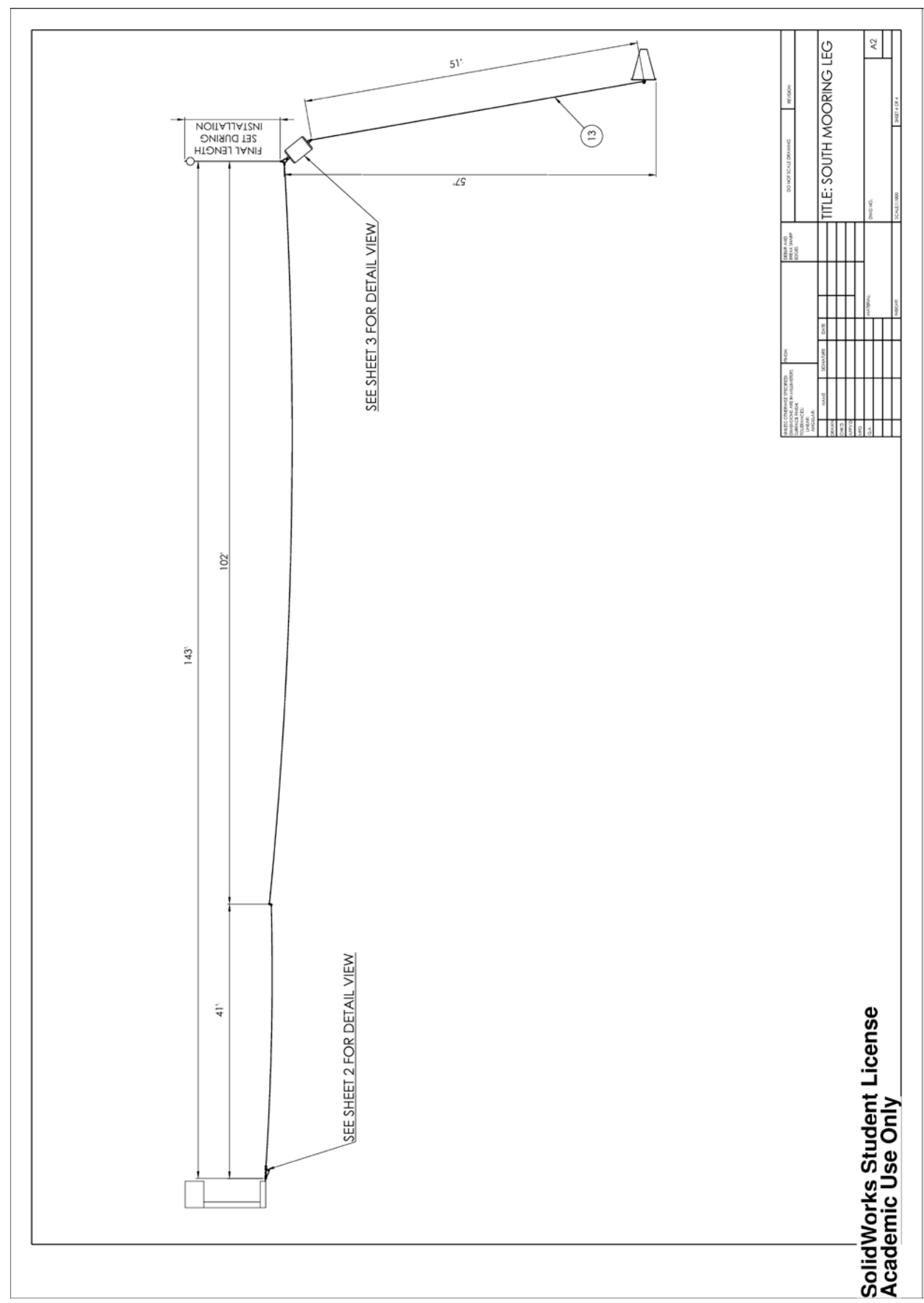

Columbia Power Technologies - Confidential and Proprietary

Not for distribution - 2 February 2011 


\subsection{Appendix C. Mooring Materials List}

\begin{tabular}{|c|c|c|c|}
\hline Item No. & Purpose & Description & Qty \\
\hline 1 & WEC Mooring & $\begin{array}{l}\text { 3/4" Safety Anchor Shackle, Galvanized Domestic BTC539- } \\
1235\end{array}$ & 36 \\
\hline 2 & WEC Mooring & Crosby G-341 1" Galvanized Weldless Sling Link & 5 \\
\hline 3 & WEC Mooring & $102 \mathrm{ft}$ 3/4" Whitehill Nylon Mooring Line & 3 \\
\hline 4 & WEC Mooring & $40 \mathrm{ft}$ 3/4" Whitehill Nylon Mooring Line & 3 \\
\hline 5 & WEC Mooring & $\begin{array}{l}51 \mathrm{ft} \text { 3/4" Fiber Core } 6 \times 36 \text { EIPS (Bright) With 3/4" HD Galv } \\
\text { Gusseted Thimble and Carbon Steel Flemished Sleeve Swaged } \\
\text { Each }\end{array}$ & 1 \\
\hline 6 & WEC Mooring & $\begin{array}{l}44 \mathrm{ft} 3 / 4 " \text { Fiber Core } 6 \times 36 \text { EIPS (Bright) With 3/4" HD Galv } \\
\text { Gusseted Thimble and Carbon Steel Flemished Sleeve Swaged } \\
\text { Each }\end{array}$ & 1 \\
\hline 7 & WEC Mooring & $\begin{array}{l}39 \mathrm{ft} \text { 3/4" Fiber Core } 6 \times 36 \text { EIPS (Bright) With 3/4" HD Galv } \\
\text { Gusseted Thimble and Carbon Steel Flemished Sleeve Swaged } \\
\text { Each }\end{array}$ & 1 \\
\hline 8 & WEC Mooring & $\begin{array}{l}45 \mathrm{ft} \text { 3/4" Fiber Core } 6 \times 36 \text { EIPS (Bright) With 3/4" HD Galv } \\
\text { Gusseted Thimble and Carbon Steel Flemished Sleeve Swaged } \\
\text { Each }\end{array}$ & 2 \\
\hline 9 & WEC Mooring & $\begin{array}{l}90 \mathrm{ft} 3 / 4 " \text { Fiber Core } 6 \times 36 \text { EIPS (Bright) With 3/4" HD Galv } \\
\text { Gusseted Thimble and Carbon Steel Flemished Sleeve Swaged } \\
\text { On One End Only }\end{array}$ & 1 \\
\hline 10 & WEC Mooring & $\begin{array}{l}45 \mathrm{ft} \text { 3/4" Fiber Core } 6 \times 36 \text { EIPS (Bright) With 3/4" HD Galv } \\
\text { Gusseted Thimble and Carbon Steel Flemished Sleeve Swaged } \\
\text { On One End Only }\end{array}$ & 2 \\
\hline 11 & WEC Mooring & 3/4" Heavy Duty Tube Thimbles SFEBG722 & 14 \\
\hline 12 & WEC Mooring & 3/4" Galvanized Heavy Wire Rope Thimble BTC626-0207 & 10 \\
\hline 13 & WEC Mooring & 3/4" Wire Rope Clips BTC699-1034 & 6 \\
\hline 14 & WEC Mooring & 3/4" Screw Pin Shackle, Self Colored & 8 \\
\hline 15 & WEC Mooring & Eye to Eye Nylon Safety Sling EEOL2 X 3' & 3 \\
\hline 16 & WEC Mooring & Marine Fenders CB-29 Subsurface Buoy & 3 \\
\hline 17 & WEC Mooring & Strainsert STL-4.75 Tension Link & 3 \\
\hline 18 & WEC Mooring & TBD Crown Buoy & 3 \\
\hline 19 & WEC Mooring & $6000 \mathrm{lb}$ Cone Anchor & 3 \\
\hline 20 & AWAC Mooring & 37 ft 3/4" Quick Splice Polytron SAM335048 & 1 \\
\hline 21 & AWAC Mooring & $60 \mathrm{ft}$ 1/2" Open Link Mooring Chain & 1 \\
\hline 22 & $\begin{array}{l}\text { AWAC Mooring } \\
\text { (Crown Buoy Line) }\end{array}$ & $\begin{array}{l}90 \mathrm{ft} 1 / 2 \text { " Fiber Core } 6 \times 36 \text { EIPS (Bright) With 1/2" HD Galv } \\
\text { Gusseted Thimble and Carbon Steel Flemished Sleeve Swaged } \\
\text { On One End Only }\end{array}$ & 1 \\
\hline 23 & $\begin{array}{c}\text { WEC Lifting } \\
\text { (Damper Plate Strap) }\end{array}$ & $40 \mathrm{ft} 5 / 8 "$ Spectra (8" eye to 8" eye, inside eye), SAM870040 & 1 \\
\hline 24 & $\begin{array}{l}\text { WEC Lifting } \\
\text { (Bridle) }\end{array}$ & $\begin{array}{l}\text { 7/8" screw Pin Anchor Shackle, Galvanized Domestic BTC539- } \\
1435\end{array}$ & 3 \\
\hline 25 & $\begin{array}{l}\text { WEC Lifting } \\
\text { (Bridle) }\end{array}$ & $\begin{array}{l}\text { 2x } 20 \mathrm{ft} \text { Nylon Strap EE-4-903 sewn to 1-1/4" Alloy Pear Link at } \\
\text { apex }\end{array}$ & 1 \\
\hline
\end{tabular}




\subsection{Appendix D. Component Specifications}
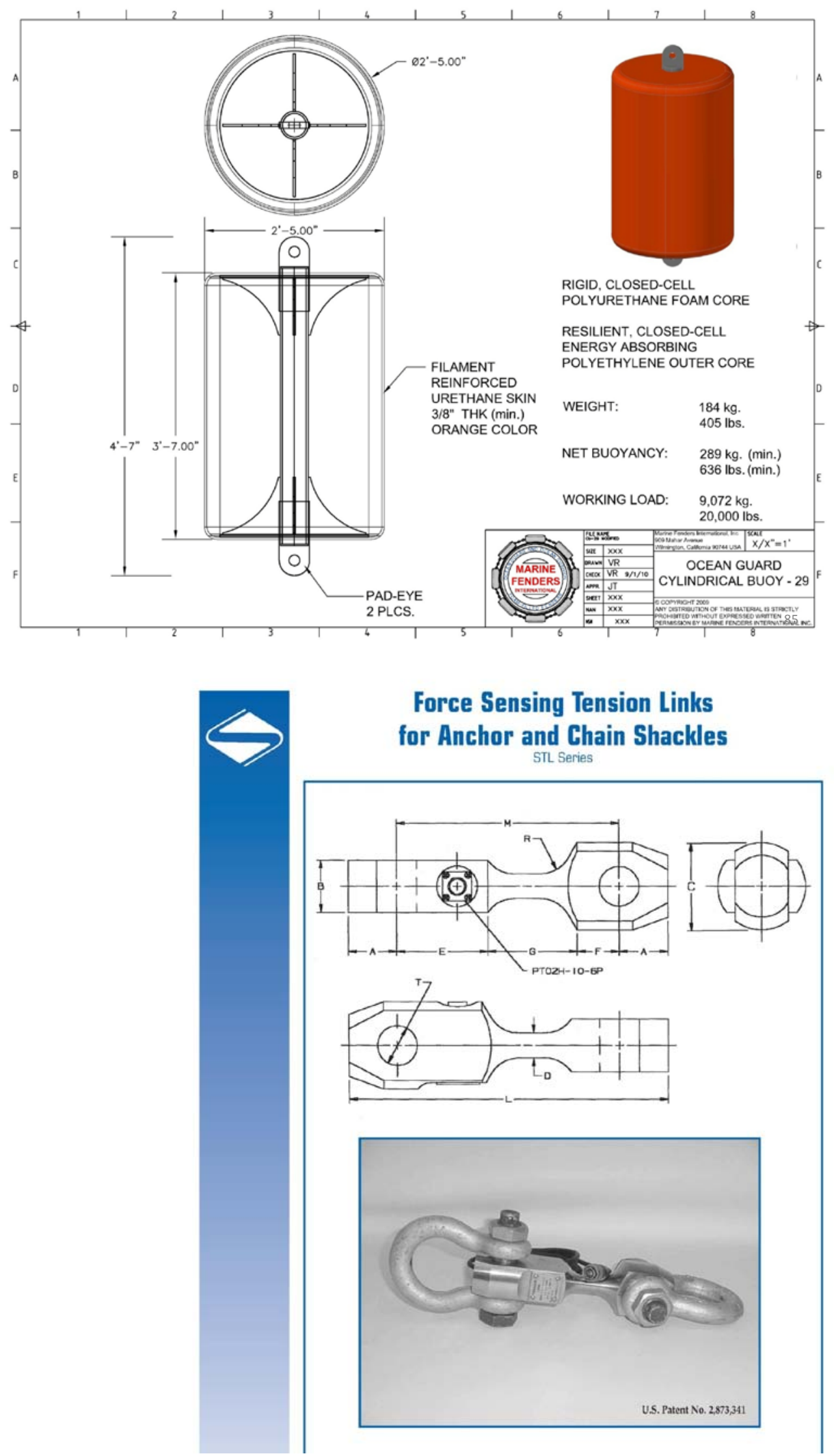

Force Sensing Tension Links for Anchor and Chain Shackles

STL Series

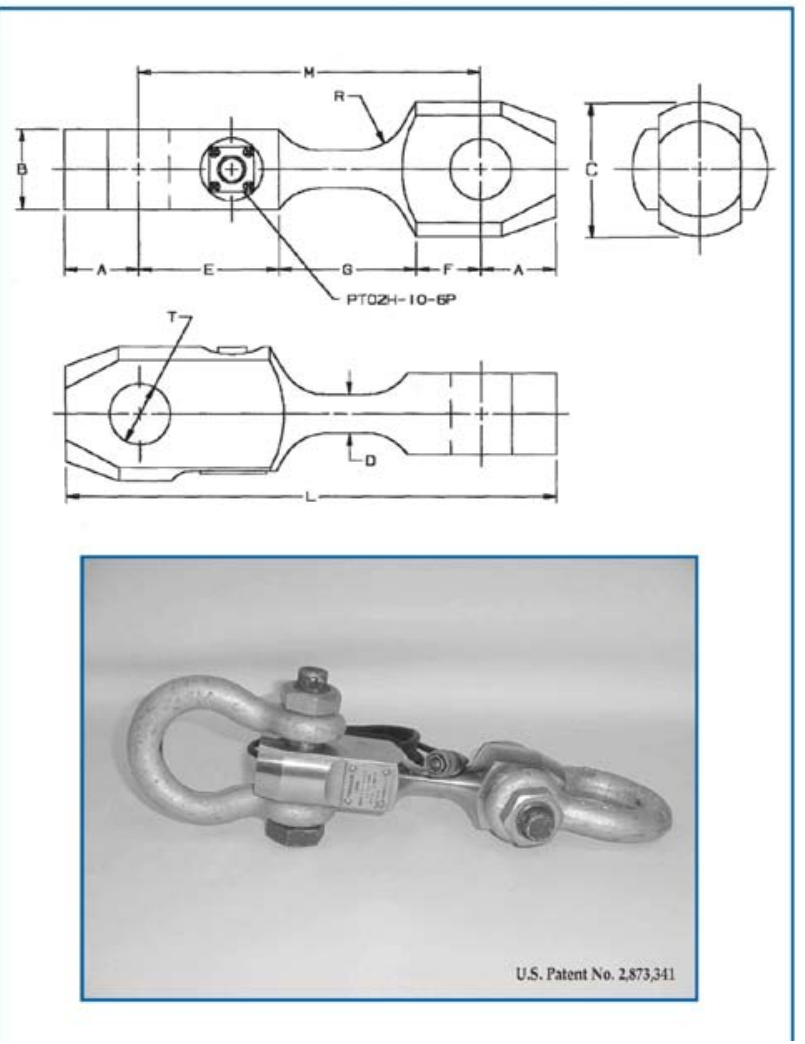


Force Sensing Tension Links for Anchor and Chain Shackles

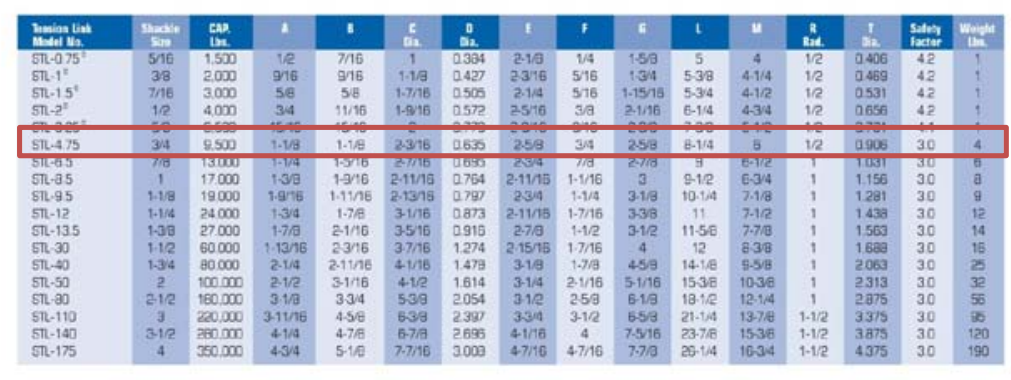

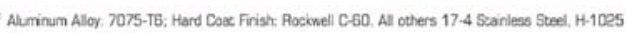
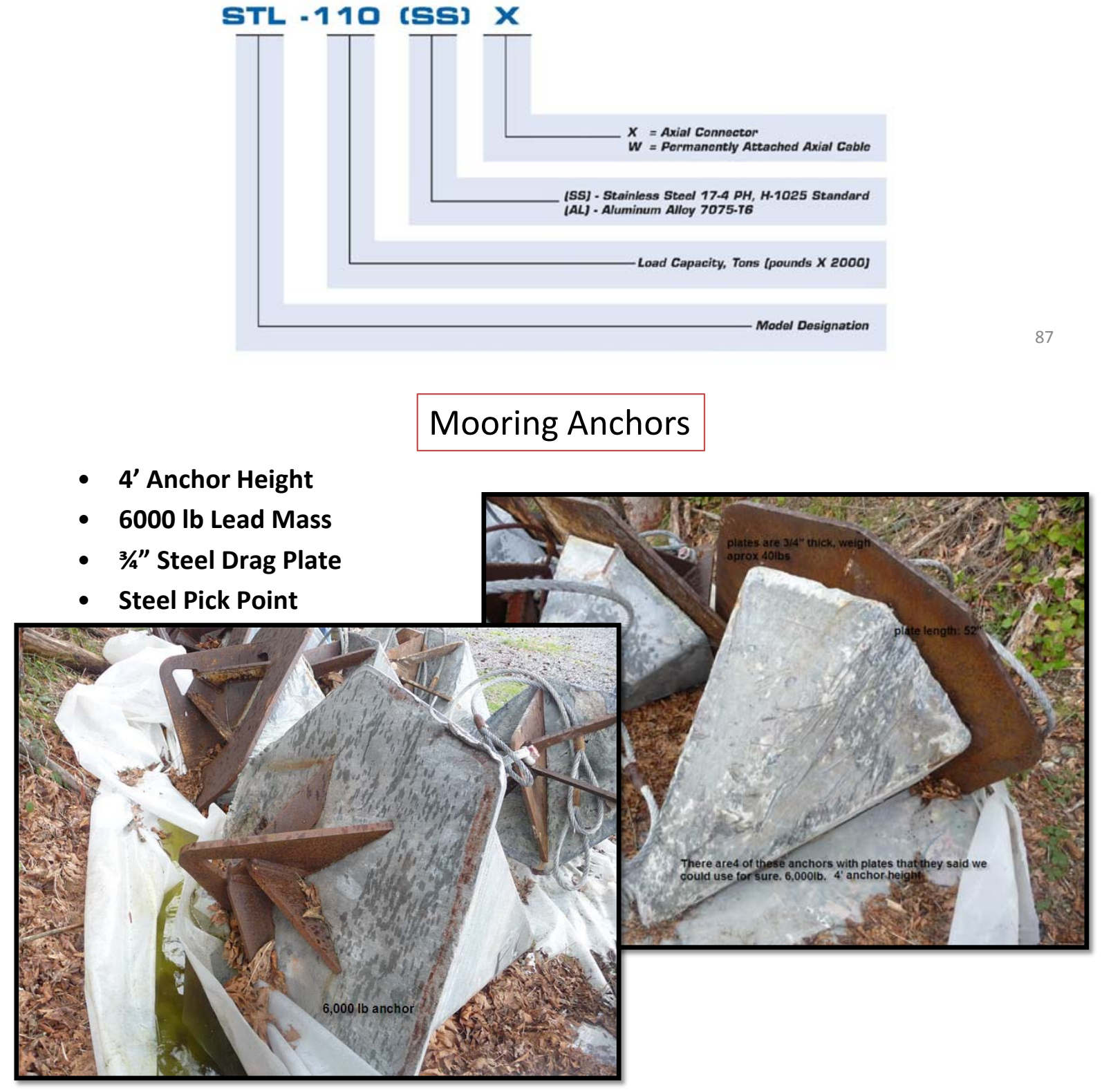

Columbia Power Technologies - Confidential and Proprietary 
Super Strong is a fim but tlexible double braid of hight-tenacity nyion fiber treated with

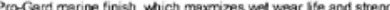

METURE

- Fiber nylon core, nylon cover : Class 1 Double Braid splice

- Standard Color, white with blve - Hgh energy abscrpsonishook

Excellent shock mitigaion $\quad$ Excolont wear resistance
Exclent wet wear

- Remains flevoble and wont $\quad$ Hght strength b weight rafo

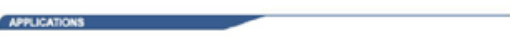

- Dock Lines

- Hakers/Headgear

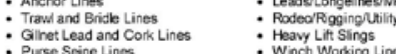

:Purse Scaine Lines $\quad$ - Winch Workirg Lines

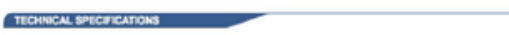

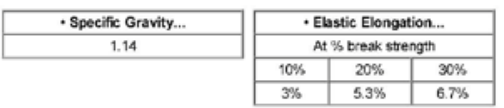

Fipe stabilized from 20002 Ropes

cycled 50 times at aach percent of
average break strength.

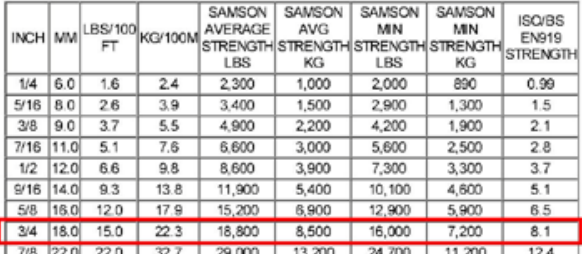

WIRE ROPE

GENERAL PURPOSE

BRIGHT $6 \times 37$ CLASS $(6 \times 29,6 \times 31,6 \times 36,6 \times 37,6 \times 41)$

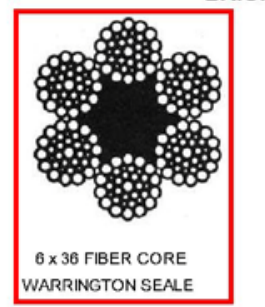

Read important warnings and information on pages 67 and 12 preceding wire rope section.

According to Federal Specifcaticn RR W 410D, preformed, right regular lay. FBER CORE (EIPS) IWRC (EIPS)

Diameter Approx. weight per

Breaking

Approx

weight Breaking pounds in Tons. perfoot in in Tons.

\begin{tabular}{lcccc}
\multicolumn{1}{c}{ 1/4 } & 0.105 & 3.02 & 0.116 & 3.4 \\
$5 / 16$ & 0.164 & 4.69 & 0.18 & 5.27 \\
$3 / 8$ & 0.236 & 6.71 & 0.26 & 7.55 \\
$7 / 16$ & 0.32 & 9.09 & 0.35 & 10.2 \\
$1 / 2$ & 0.42 & 11.8 & 0.46 & 13.3 \\
$9 / 16$ & 0.53 & 14.09 & 0.59 & 16.8 \\
$5 / 8$ & 0.66 & 18.3 & 0.72 & 20.6 \\
\hline $3 / 4$ & 0.95 & 26.2 & 1.04 & 29.4 \\
\hline $7 / 8$ & 1.29 & 35.4 & 1.42 & 39.8 \\
1 & 1.68 & 46 & 1.85 & 51.7 \\
$11 / 8$ & 2.13 & 57.9 & 2.34 & 65 \\
$11 / 4$ & 2.63 & 71 & 2.89 & 79.9 \\
$13 / 8$ & 3.18 & 85.4 & 3.5 & 96 \\
$11 / 2$ & 3.78 & 101 & 4.16 & 114
\end{tabular}




\section{Alloy Master Links}

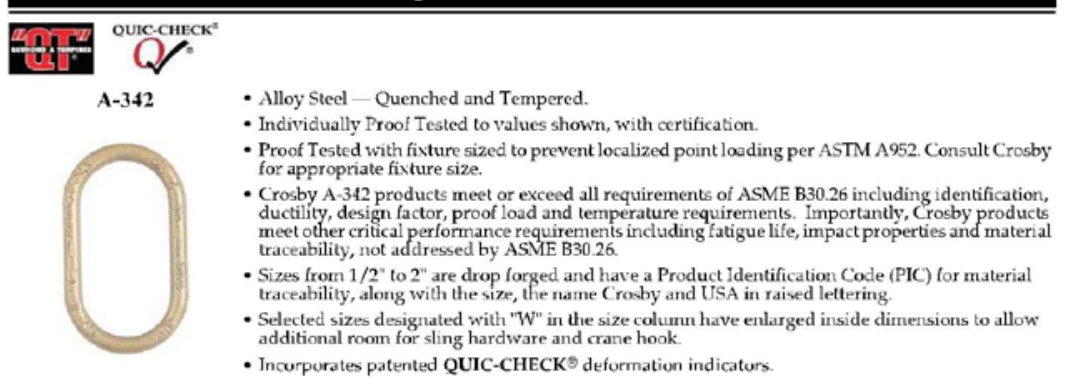

- Incurporates patented QUIC-CHECK\$ defurmation indicaturs

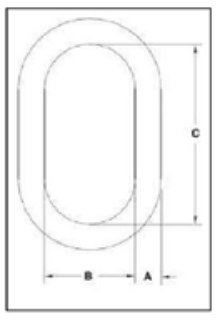

\section{A-342 Alloy Master Links}

\begin{tabular}{|c|c|c|c|c|c|c|c|c|c|}
\hline \multicolumn{2}{|c|}{ Size } & \multirow[b]{2}{*}{$\begin{array}{c}\text { A.342 } \\
\text { Stock No. }\end{array}$} & \multirow{2}{*}{$\begin{array}{l}\text { Weight } \\
\text { Each } \\
\text { (bs.s.) }\end{array}$} & \multirow{2}{*}{$\begin{array}{l}\text { Working } \\
\text { Loasd } \\
\text { Limit } \\
\text { (lbs.j. }\end{array}$} & \multirow{2}{*}{$\begin{array}{l}\text { Proof } \\
\text { Load. } \\
\text { (lbst)" }\end{array}$} & \multicolumn{4}{|c|}{ Dimensions } \\
\hline & $(\mathrm{mm})$ & & & & & $A$ & B & & Deformation \\
\hline & $\frac{\min 13}{13 \mathrm{~W}}$ & 1011255. & $\frac{1 \text { (DS.) }}{1.3}$ & $\frac{1005 .]}{7400}$ & $\frac{1(15)}{17200}$ & $\sqrt{52}$ & $\frac{0}{280}$ & 5.00 & 3.50 \\
\hline 5.8 & 16 & 1011289 & $\frac{7.5}{1.5}$ & 9009 & 18000 & 52 & 300 & 5.00 & 3.50 \\
\hline $3.4 \mathrm{~W}$ & 100 & 1014285 & $\frac{7.5}{20}$ & 12300 & 28400 & 73 & 320 & 600 & 400 \\
\hline $78 \mathrm{sw}$ & $22 \mathrm{~W}$ & 1014319 & 3.0 & 15200 & 35200 & 88 & 3.245 & 6.38 & 4.50 \\
\hline$w$ & $2 E W$ & 1014331 & $\frac{5.1}{6.1}$ & 28000 & 50000 & .1 .10 & 4.30 & 7.50 & 550 \\
\hline $7.14 \mathrm{~W}$ & $32 \mathrm{~W}$ & 1014345 & & 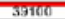 & F(1040) & 738 & $5 x$ & 9.350 & 700 \\
\hline $9 \cdot 1 / 2 \mathrm{~W}$ & $3 \mathrm{FW}$ & 1014365 & 18.5 & 61100 & 141200 & 1.61 & $59 x$ & 10.50 & 7.50 \\
\hline $1-3.4$ & 44 & 1014383 & 25.2 & 84500 & 169300 & 1.75 & 6.00 & 1200 & 7.50 \\
\hline & 51 & 1014404 & 37.0 & 102600 & 205200 & 200 & 7.00 & 14.90 & 5.00 \\
\hline$+2-1 / 4$ & $t+57$ & 1011422 & 54.1 & 143100 & 280200 & 2.2 & 8.00 & 15.00 & \\
\hline$\pi 2-1 / 2$ & $t+63$ & 10114468 & 67.8 & 160000 & 320000 & 2.50 & 8.00 & 16.00 & - \\
\hline$\pi 2-3 / 4$ & +770 & 1014440 & 87.7 & 216900 & 433800 & 2.75 & 8.50 & 15.200 & - \\
\hline
\end{tabular}

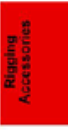

\section{Crosby ${ }^{\circledR}$ Bolt Type Shackles}

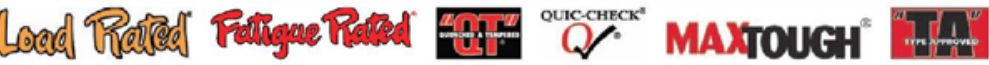

\section{BOLT TYPE - Capacities $1 / 3$ thru 150 metric tons.}

ANCHOR -Working Load Limit permanently shown on every shacke.

SHACR

Forged - Quenched and Iempered, with alloy pins.

Hot Dip galvanized or Self Colored

- Fatigue rated $(1 / 3 t-55$

Shackles 25t and larger are RFID EQUIPPED.

TreD.

and temperature requirements. Importantly, Crosby products meet other

critical performance requirements induding fatigue life, impact

propertes and material traceability, nol addressed by ASML B30.26.

Shackles 55 metric tons and smaller can be fumished proof tested with
certificates to designated standards, such as ABS, DNV, Lloyds, or other

certification when requested at time of order.

G-2130 S-2130

- Shackles 85 metric tons and larger can be provided as follows.

- Non Destructive Tested

- Material Certification (Chemical) Certification must be

ead bolt - nui with oxtler pin. Meets

Speriffortion RR.C.27 DTypeIVA,

ed at time of order.

ms recuind of the ontenctor. Foc - Look for the Red Pin ${ }^{\otimes}$... the mark of genuine Crosby quality

- Type Approval and certification in accordance with ABS2006 Steel Vesse

Rules 1-1-17.7, and ABS Guide for Certification of Cranes.
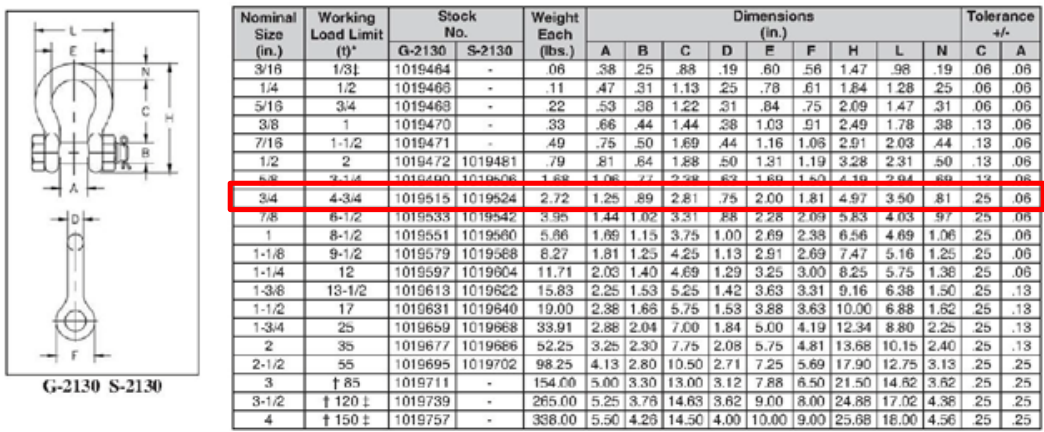


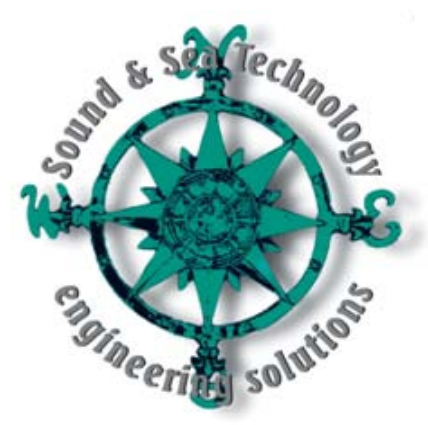

May 18, 2010

Columbia Power Technologies, LLC

3079 Kelley Engineering Center

Corvallis, OR 97331

Attn: Mr. Ken Rhinefrank, VP Research \& Development

Subj: Scale Site Bathymetric Survey Report

Ref: Bathymetry mapping work to determine test site for scaled wave buoy system for Columbia Power, LLC (CPT). Work performed by Sound \& Sea Technology, Inc. (SST).

Work has been completed as described by our original contract for the determination and bathymetry mapping of a selected site for the Scale Site Bathymetric Survey in the area adjacent to West Point - Seattle, WA. The planned approach of the survey was successful and the resulting survey data was processed in accordance with the original description of work outlined in the original contract. The results of this survey work and subsequent data processing resulted in the production of the bathymetry maps delivered with this report.

The following is a description of the process of the work completed. The original area off West Point, Seattle, WA as described in file; “West Point Large Grid.jpg”, was searched with a fathometer mounted on a kayak for several hours to determine a location within these boundaries that best matched the previously determined criteria for a site. This large grid area was evaluated for slope and obstructions until an area was located that best fit the desired criteria for a site survey. Once this best-fit area was determined near the southwest portion of the larger grid area, this area was buoyed off along the perimeters, denoting a square area containing a circular area with a radius of 180 feet. Transects were performed within the buoyed area and depths were recorded in a digital voice recorder at approximately 3 meter intervals. These data were corrected for any offset to MLLW and validated against other bathymetric data of this the area. These validated data points were augmented with bathymetric data resources and interpreted using Natural Neighbors Algorithm in ARCVIEW GIS resulting in 1 meter resolution. The resulting bathymetric data of the site were overlaid against NOAA chart data, and the provided SWAN model data to provide several images delivered with this report. 
If you require additional information, please contact me at 4252481237.

\section{Sincerely,}

SOUND \& SEA TECHNOLOGY, INC.

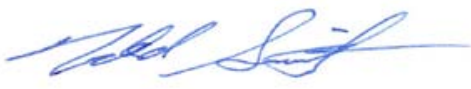

\section{Todd Switzer PhD.,}




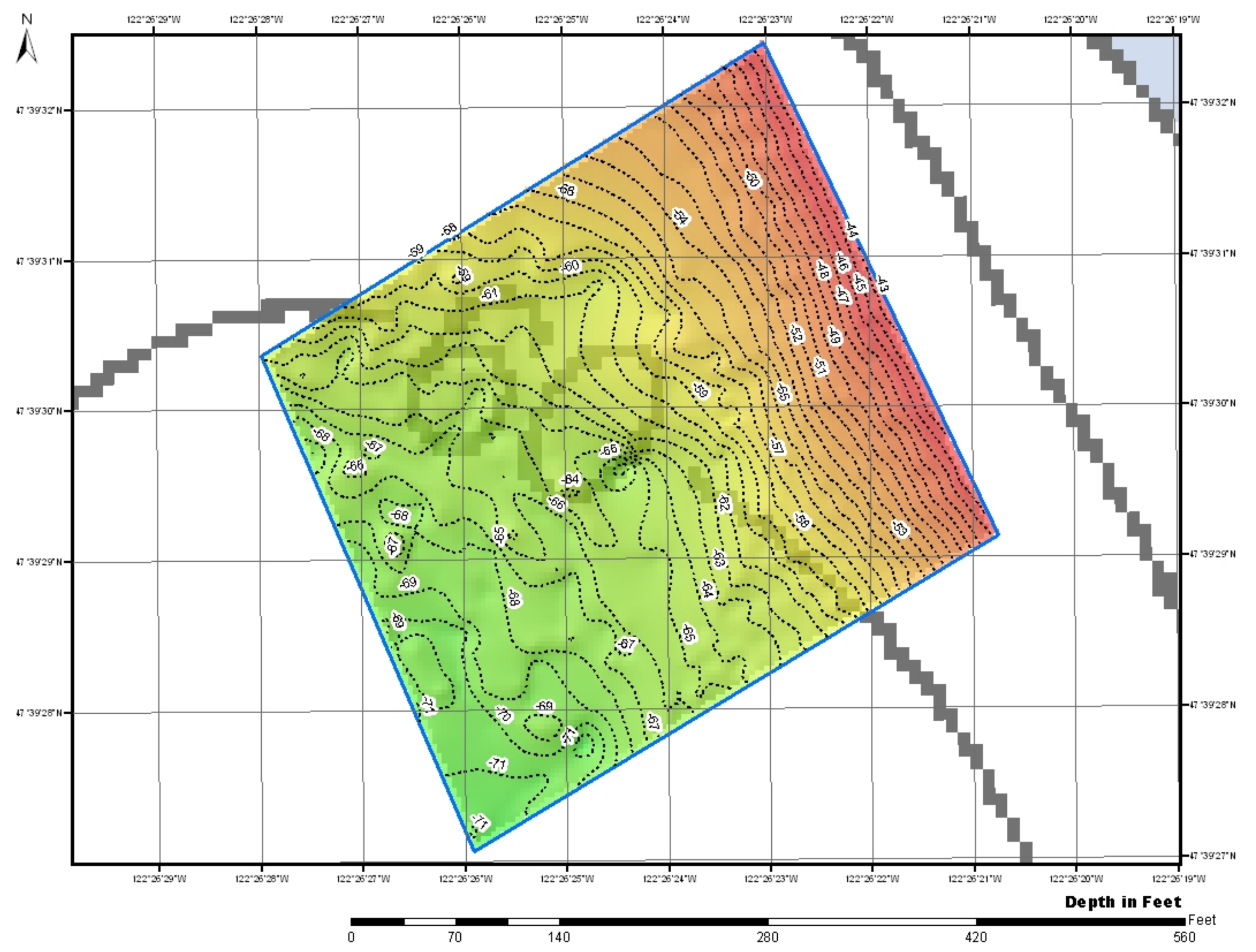




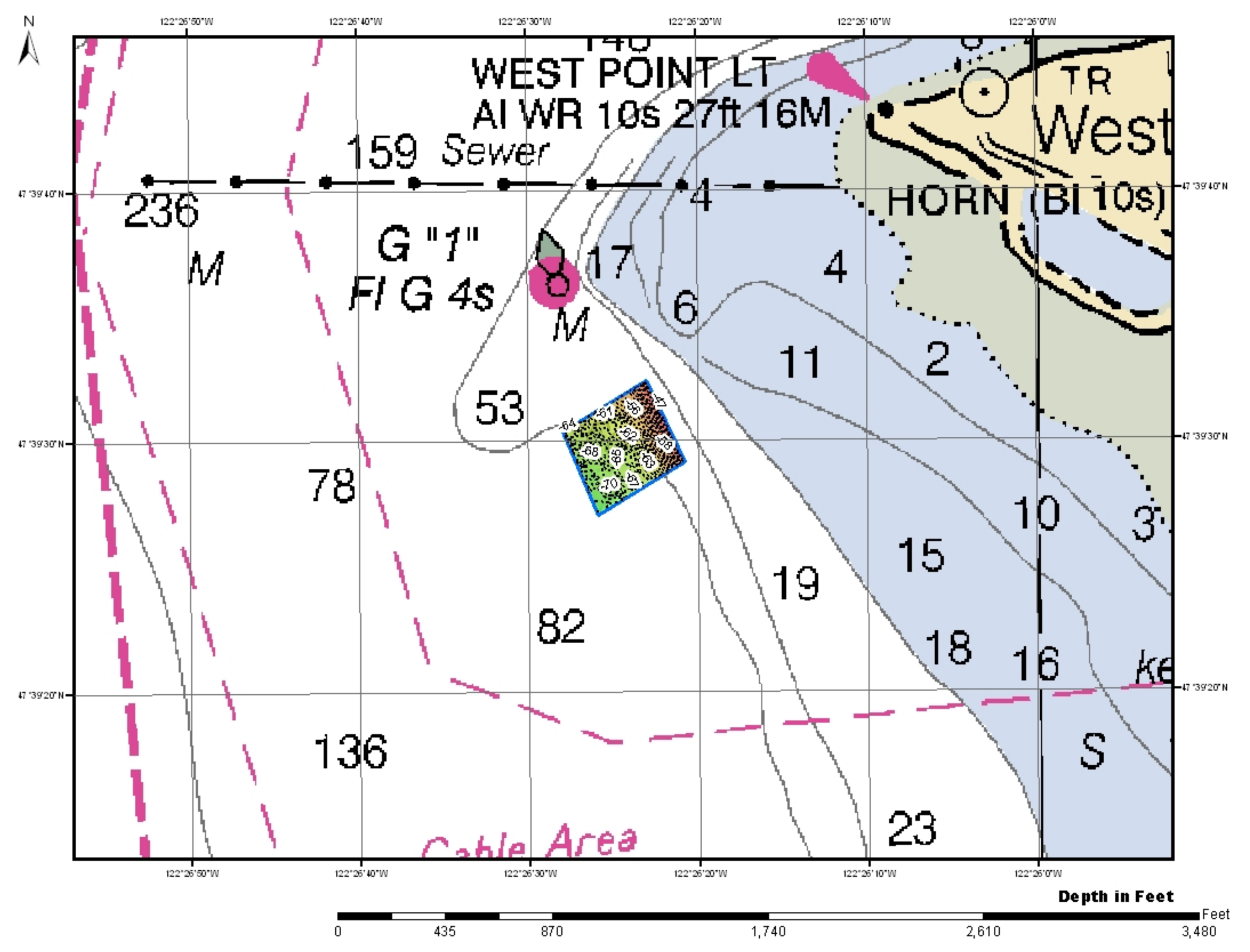




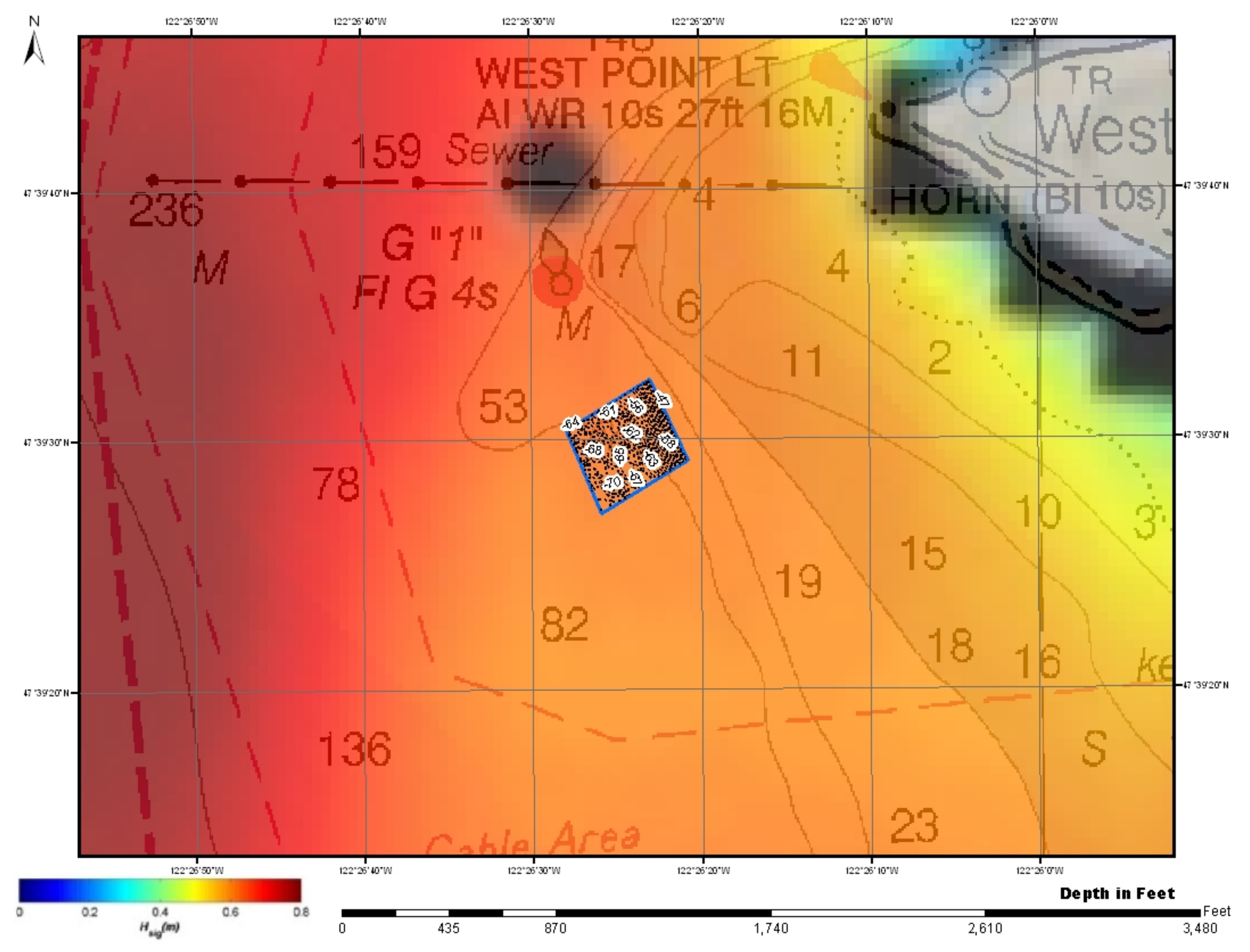

